\title{
Decisions and strategies in drug treatment of epilepsy
}

Citation for published version (APA):

Smeets, R. P. A. M. (1996). Decisions and strategies in drug treatment of epilepsy. [Doctoral Thesis, Maastricht University]. Universiteit Maastricht. https://doi.org/10.26481/dis.19961129rs

Document status and date:

Published: 01/01/1996

DOI:

10.26481/dis.19961129rs

Document Version:

Publisher's PDF, also known as Version of record

\section{Please check the document version of this publication:}

- A submitted manuscript is the version of the article upon submission and before peer-review. There can be important differences between the submitted version and the official published version of record.

People interested in the research are advised to contact the author for the final version of the publication, or visit the DOI to the publisher's website.

- The final author version and the galley proof are versions of the publication after peer review.

- The final published version features the final layout of the paper including the volume, issue and page numbers.

Link to publication

\footnotetext{
General rights rights.

- You may freely distribute the URL identifying the publication in the public portal. please follow below link for the End User Agreement:

www.umlib.nl/taverne-license

Take down policy

If you believe that this document breaches copyright please contact us at:

repository@maastrichtuniversity.nl

providing details and we will investigate your claim.
}

Copyright and moral rights for the publications made accessible in the public portal are retained by the authors and/or other copyright owners and it is a condition of accessing publications that users recognise and abide by the legal requirements associated with these

- Users may download and print one copy of any publication from the public portal for the purpose of private study or research.

- You may not further distribute the material or use it for any profit-making activity or commercial gain

If the publication is distributed under the terms of Article $25 \mathrm{fa}$ of the Dutch Copyright Act, indicated by the "Taverne" license above, 
Decisions and strategies in drug treatment of epilepsy 


\title{
Decisions and strategies in drug treatment of epilepsy
}

\author{
PROEFSCHRIFT
}

ter verkrijging van de graad van doctor aan de Universiteit Maastricht, op gezag van de Rector Magnificus, Prof.mr.M.J.Cohen, volgens het besluit van het College van Dekanen, in het openbaar te verdedigen op

vrijdag 29 november 1996 om 12.00 uur

door

Raymond Pierre Angèle Marie Smeets

geboren te Rothem-Meerssen in 1961 
Promotores:

Co-promotor:

Beoordelingscommissie:
Prof.dr.ir. A. Hasman

Prof.dr. H. Meinardi

Prof.dr. J. Troost

Dr.ir. J.L. Talmon

\author{
Prof.dr. J.A. Knottnerus (voorzitter) \\ Dr. J. van der Lei (Erasmus Universiteit Rotterdam) \\ Prof.dr. P. Pop \\ Dr. W.O. Renier (Katholieke Universiteit Nijmegen) \\ Prof.dr. R.W. Stockbrügger
}

ISBN: $90-9010008-3$

(c) R.P.A.M. Smeets, Maastricht, 1996.

Produktie: Datawyse, Maastricht

Omslag: Consultatie: John van de Poel, Ruud Groothedde.

Foto's: Borders-No Borders, Herbert Rikers, Bunde.

Lay-out: Datawyse, Joop Caris.

Dit proefschrift kwam tot stand in het kader van het instituut ExTra, deeluitmakendvan de landelijke onderzoekschool CaRe (Netherlands School of Primary Care Research), in 1995 erkend door de KNAW.

Het in dit proefschrif gepresenteerde onderzoek werd gesteund door subsidies van het Nationaal Epilepsie Fonds - De Macht van het Kleine en de SWOL.

Met dank aan het Nationaal Epilepsie Fonds - De Macht van het Kleine, Ciba Geigy B.V.. Dumex B.V., Janssen-Cilag B.V., Karwijk Fama B.V., Novo Nordisk Farma B.V., Schering-Plough B.V., Yamanouchi Farma B.V., inzake totstandkoming van dit proefschrift. 


\section{Voor Eline.}





\section{CONTENTS}

\section{CHAPTER 1 INTRODUCTION}

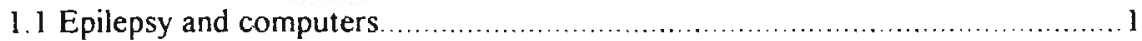

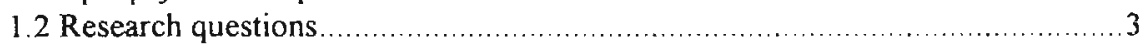

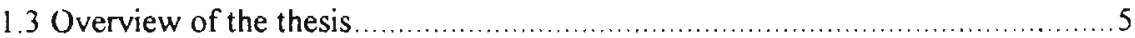

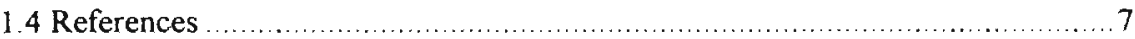

\section{CHAPTER 2 EPILEPSY}

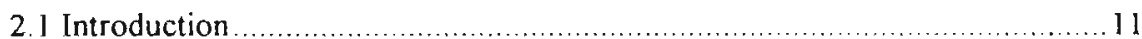

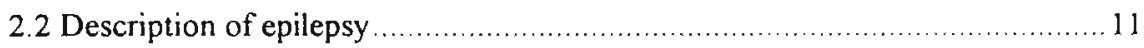

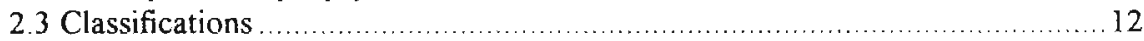

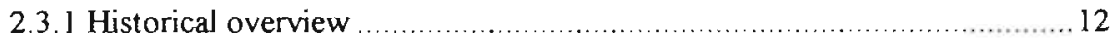

2.3.2 Importance of different classifications ............................................ 13

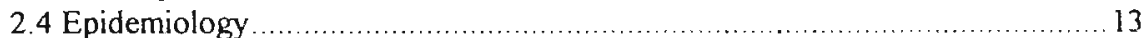

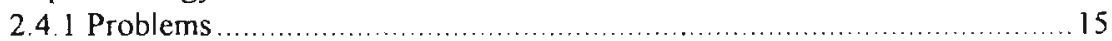

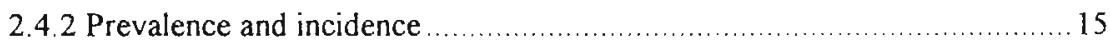

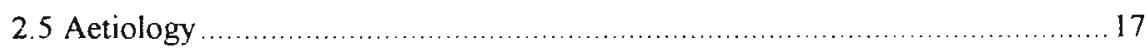

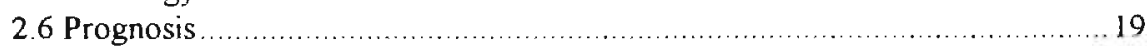

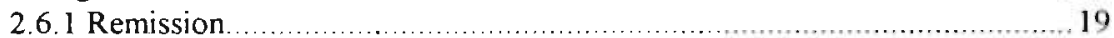

2.6.2 Problems in establishing prognostic factors ................................. 21

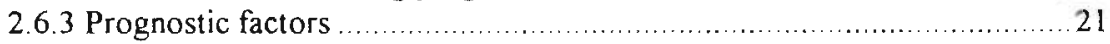

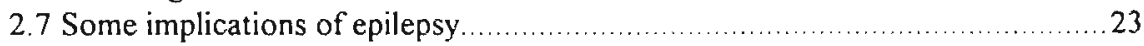

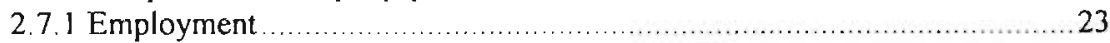

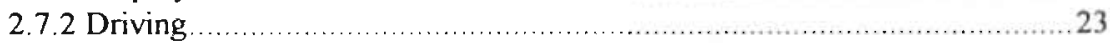

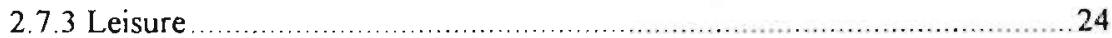

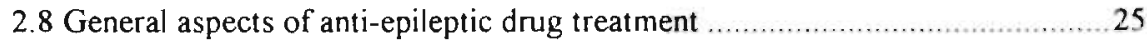

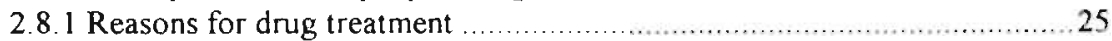

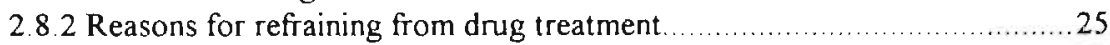

2.8.3 Goals in anti-epileptic drug treatment .....................................26

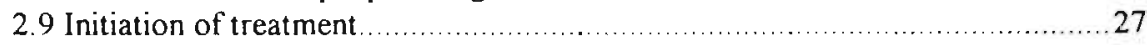

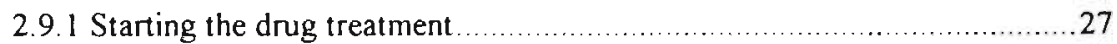

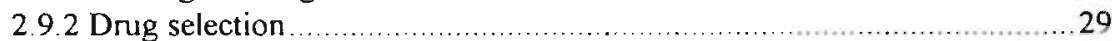

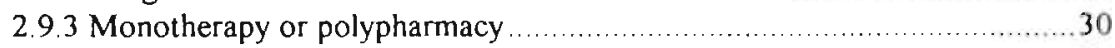

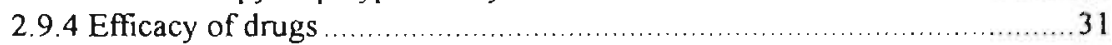

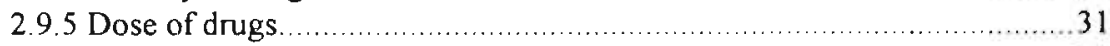

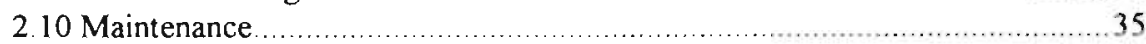

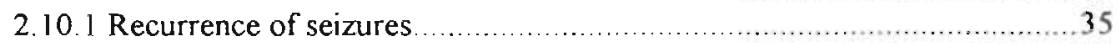

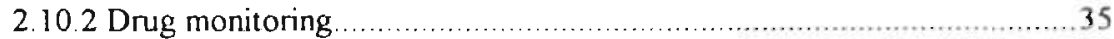

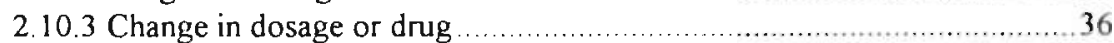

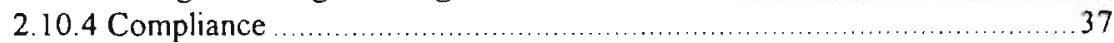

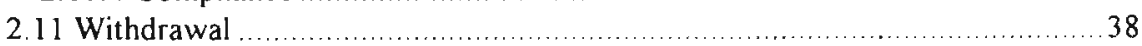

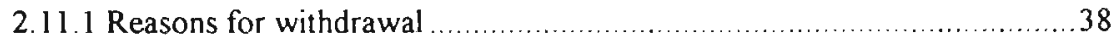

2.11.2 Moment of relapse......................................................... 40

2.12 Summary and conclusion......................................................... 40

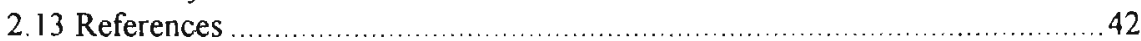




\section{CHAPTER 3 SYSTEM DEVELOPMENT}

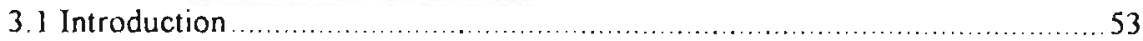

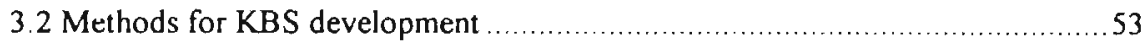

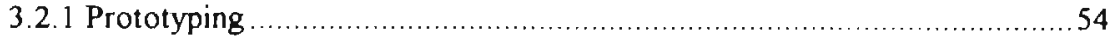

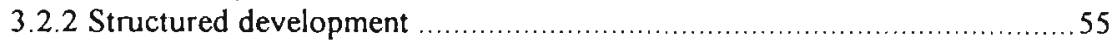

3.2.3 Selection of the development method ........................................ 57

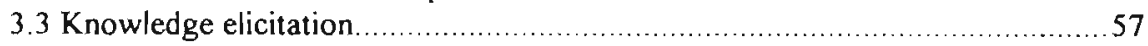

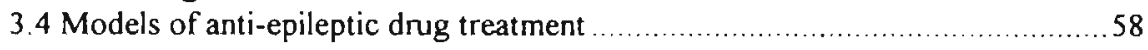

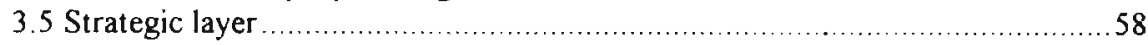

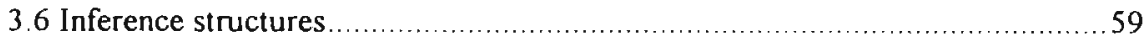

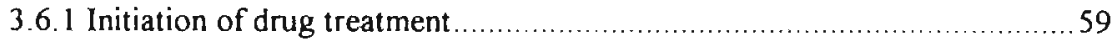

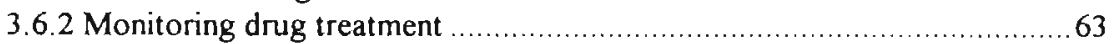

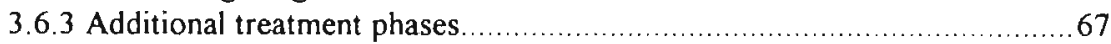

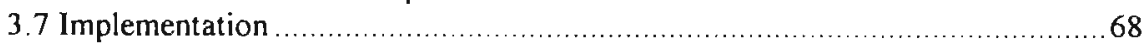

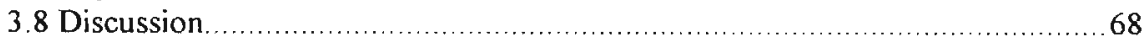

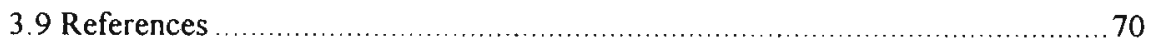

CHAPTER 4 REFERENCE DEVELOPMENT

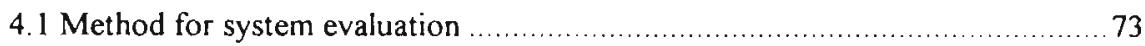

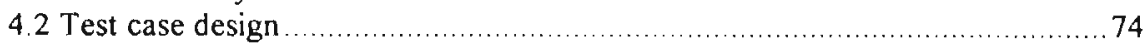

4.2.1 Patient selection .................................................................... 74

4.2.2 Validity of paper cases ............................................................. 74

4,2.3 Description of the test cases ................................................. 75

4.3 Reference development ........................................................... 77

4.3.1 Method for consensus formation .............................................. 77

4.3.2 Reference development/Acceptance testing ...................................78

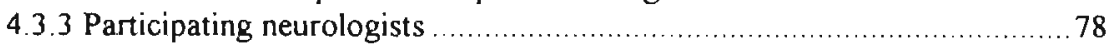

4.3.4 Reference development process ................................................ 79

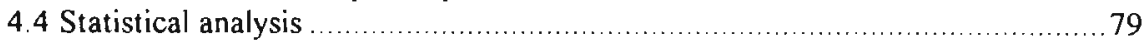

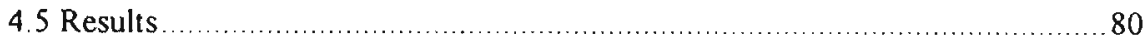

4.5.1 Agreement on the diagnosis, generic drug and daily dose ................... 80

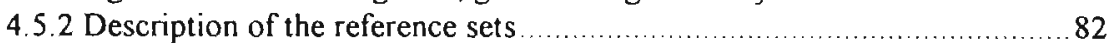

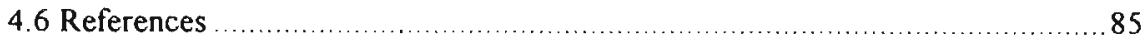

CHAPTER 5 RESULTS ON INITIATION OF TREATMENT

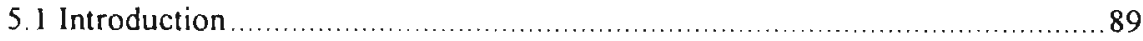

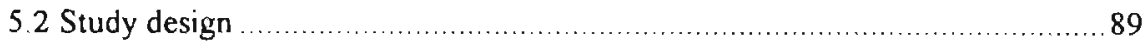

5.3 Comparing the system with Neurologist I and Neurologist II _................... 90

5.3.1 Agreement on the generic drug ................................................. 90

5.3.2 Agreement on tradename ....................................................... 91

5.3 .3 Agreement on daily dose ....................................................... 91

5.4 Comparing the system with an external reference .................................. 92

5.4.1 Agreement about the drug using the diagnostic reference set ................93

5.4 .2 Variability in neurologist's prescriptions ....................................... 94

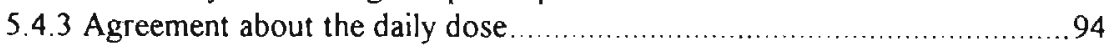

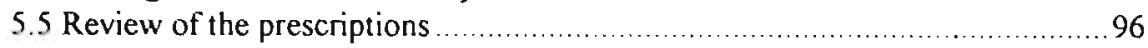


5.5.1 Overview of the results for system and individual neurologists...............96

5.5.2 Comparing overall scores of system and individual neurologists..............98

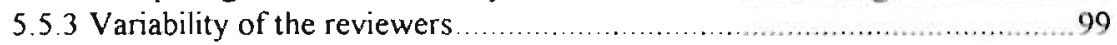

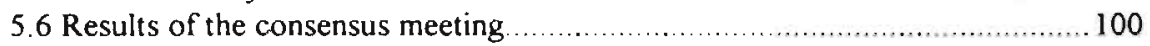

5.6.1 The use of diagnostic classification schemes ............................ 100

5.6 .2 Initiation of drug treatment ..................................................... 100

5.6 .3 Selection of generic drug .................................................... 100

5.6 .4 Generic drug or brand, formulation .......................................... 101

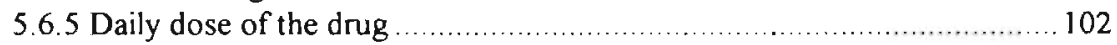

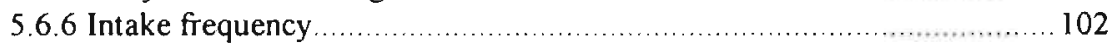

5.6 .7 Gradual increase of the dose ............................................. 103

5.7 Comparing the system and the neurologists with the consensus meeting

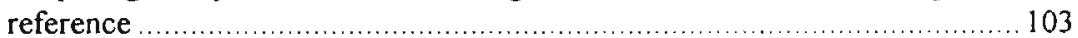

5.7.1 Agreement on the generic drug .............................................. 103

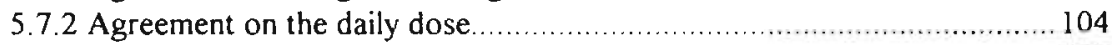

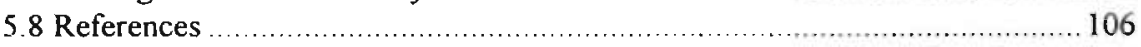

CHAPTER 6 RESULTS ON FOLLOW-UP OF TREATMENT

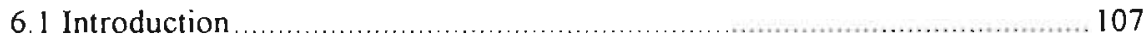

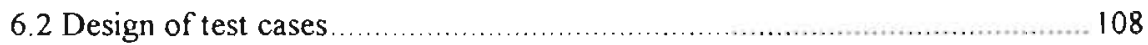

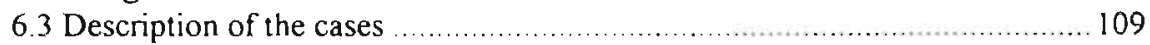

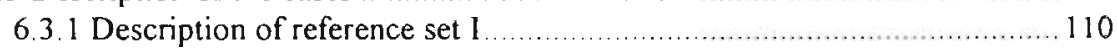

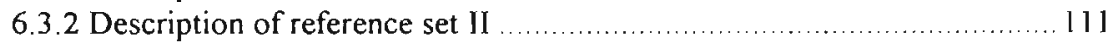

6.3.3 Cases not included in the reference set II .................................. 113

6.3.4 Relevance of characteristics for agreement among neurologists ............. 114

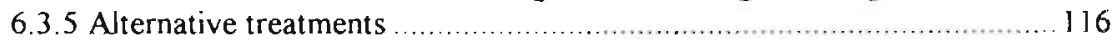

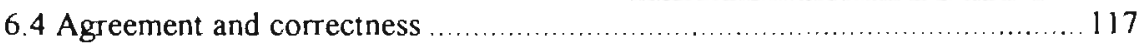

6.4.1 Overview of the decisions in the total set ................................. 117

6.4.2 Differences in treatment approaches ........................................... 118

6.4.3 Agreement between an individual and a group .............................. 119

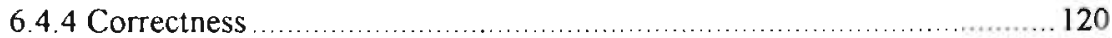

6.4.5 Variability in agreement in set II .......................................... 121

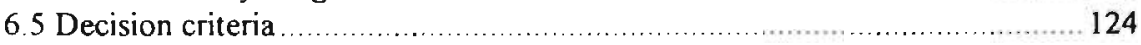

6.5.1 Treatments indicated by a single attribute ................................ 124

6.5.2 Treatments ruled out by a single attribute ................................. 125

6.6 Treatments indicated by combinations of attributes .............................. 126

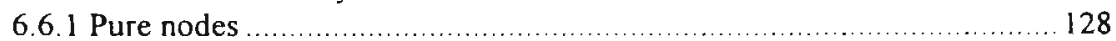

6.6.2 Nearly pure nodes; two decisions ............................................. 129

6.6.3 Mixed nodes; three decisions ................................................ 13 ]

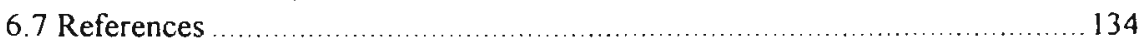


CHAPTER 7 CONCLUSIONS AND DISCUSSION

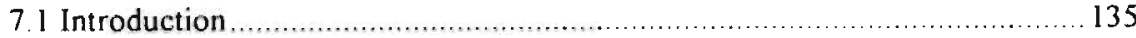

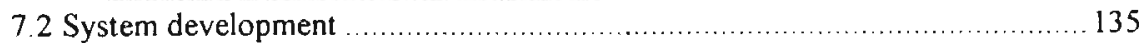

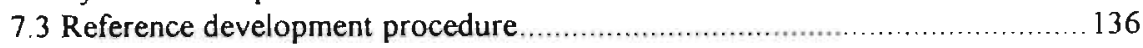

7.4 Initiating anti-epileptic drug treatment ............................................ 137

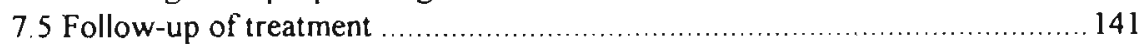

7.6 General conclusion and future prospects ...................................... 143

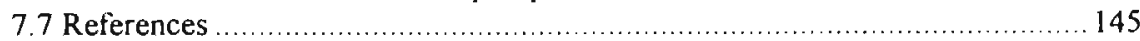

CHAPTER 8 SUMMARY

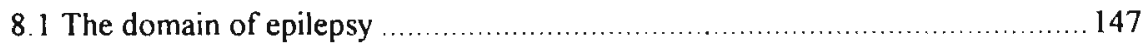

8.2 Building a model of expertise .................................................. 148

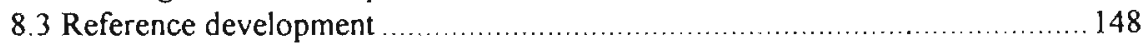

8.4 Evaluation of the initiation of the treatment ...................................... 149

8.5 Evaluation of the follow-up of the treatment ..................................... 150

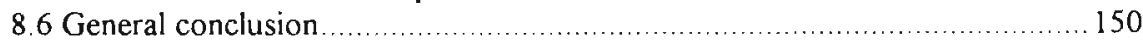

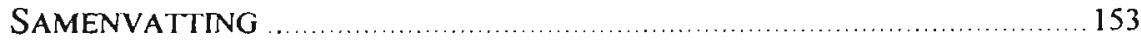

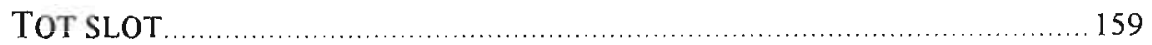

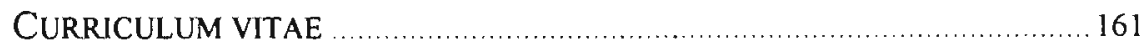




\section{Introduction}

\subsection{Epilepsy and computers}

Epilepsy is a disorder which manifests itself through brief, usually unprovoked stereotypical disturbances of behaviour, emotion, motor function or sensation. The occurrence of these symptoms will have a great impact on the daily life of the patient and his family. Such seizures are normally treated with anti-epileptic drugs. Although doctors may have the same education, it seems that some are more successful than others in the treatment of patients. Part of this difference may be attributed to experience. The use of a 'computer fed by a team of specialists' to support the doctor has been considered an option to improve patient management in the domain of epilepsy (Meinardi, 1985).

Computer programs, either embedded or stand-alone, which are designed to help health professionals in patient management by suggesting or providing patient specific advice or treatment are called decision support systems (DSSs). Evidence, especially the more recent, suggests that this type of computerised decision support can improve physician performance (Sutton 1989, Paterson-Brown and Vipond 1990, Johnston et al. 1994, Sullivan and Mitchell 1995). DSSs analyse and reason about patient data and support the diagnosis, treatment and disease prevention tasks by producing relevant conclusions or by (suggesting) actions for a given problem of a specific patient. This contrasts with clinical information systems and literature reference systems. These latter systems provide information but do not specify how to apply it in the decision making process for the specific problem of an individual patient (Perreault and Wiederhold, 1990).

Taking into account under which circumstances the advice is generated three types of DSSs can be discerned

- Solicited advice systems i.e. systems providing advice upon request of the user

- Unsolicited advice systems i.e systems providing suggestions without a specific request of the user

- Autonomous systems or closed-loop systems i.e. systems undertaking medical actions without having a physician in the loop

Each of these types of systems requires a different involvement from the user. In case of a system providing solicited advice, the physician has to make a request for information and to take a decision on the applicability of the information provided. 
Systems providing unsolicited advice do so on the basis of existing patient data. The user decides whether or not to act on the advice. Autonomous systems both analyse the information and apply the advice for a patient specific problem. The user has to decide whether to apply the system for a specific task.

Studies of the impact of DSSs in various domains have shown that such systems can enhance patient care through (see e.g. Johnston et al. 1994 for a review):

- Improvement of the diagnostic accuracy

- More effective control of the disease by:

a. Having serum levels of therapeutics in accepted ranges more often

b. Earlier achievement of therapeutic doses

c. A reduction of adverse drug reactions

$\mathrm{d}$. Earlier change of ineffective treatments

e. Improvement of uniformity in drug treatment

- Increase in the use of preventive procedures

- Improvement of patient follow-up

In the management of patients with epilepsy three sub-domains can be considered as problem areas for which it may be worthwhile to develop a DSS:

- The diagnostic process

- The decision whether or not to start drug treatment

- Drug treatment

Establishing the correct diagnosis is an essential part in the decision making process. Uncertainty about the fact whether a patient has epilepsy may be a reason for refraining from drug treatment (Chadwick and Reynolds, 1985). Decision support in this domain has been considered. The definitions of disease concepts are not stable or specific enough to enable optimal diagnostic support. For example, recently a new set of definitions has been proposed (Commission on Epidemiology and Prognosis LAE, 1993). Others who developed procedures for diagnostic support encountered problems related to definitions (Principe and Smith 1982, Korpinen et al 1994). Hence a diagnostic decision support system seems less promising.

The decision to start anti-epileptic drug treatment has as a consequence the intake of a drug for at least two years in a regular, daily fashion. The risks of recurrence of seizures has to balance or outweigh the disadvantages of the treatment. In this process of decision making issues such as consequences of the seizures, side-effects, compliance and psycho-social consequences of the drug intake have to be addressed (Dreifuss, 1985). Little guidance was found in the literature on how to incorporate issues such as compliance and psycho-social aspects in the decision making (Chadwick and Reynolds 1985, Jøhannessen et al. 1990). Building decision support for this domain would rely to a large extent on common sense and subtleties that are difficult to formalise. The literature still is not conclusive about the perceived risks of seizures and the moment of commencing treatment (Chadwick 1995, Reynolds 1995).

Once the decision to start treatment has been taken, problems such as the selection of the drug, the determination of an adequate drug dose regime and the adjustment of initiated treatment will have to be addressed. Multiple reasons for adjustment of the treatment can occur (Beardsley et al. 1983, Ward 1989, Meyer 1991, Dichter 1993). 
Insufficient effectiveness of the drug is a common problem. Also side-effects may warrant modification of the treatment. Decision making may be complicated by noncompliance of the patient. More or less formalised but still not uniform procedures for drug treatment are discussed in the literature. Guidance in dosage determination has been provided by models which mainly address the pharmacodynamics of drug treatment (van der Kleijn 1983, Peterson 1983). The domain is relatively stable, as only a few new drugs enter the market. It has been assumed that models which support the physician in treatment may improve patient care (Meinardi 1985, Rimmer and Richens 1988).

It was concluded on the basis of the perceived problems in the three sub-domains that drug treatment was the most suitable domain for decision support. Our study therefore focuses on the elicitation, implementation and assessment of knowledge of treatment procedures for anti-epileptic drugs.

Automated decision support addresses the system of medical practice as a whole including health care professionals and patients (Musen, 1993). However, before one starts to study how a DSS has to be integrated in the system of medical practice it is necessary to establish the efficacy of a DSS. If a DSS has a low efficacy in a domain then one should not put it into routine use. Despite considerable development and evaluation efforts, few DSSs are used in daily practice (Nykänen 1990, O'Moore et al. 1990, Heathfield and Wyatt 1993, de Dombal 1993). Factors hampering the acceptance of DSSs for routine use in medical care may have a technical, economic, organisational, psychological and/or medical origin. It has been suggested that by taking these factors into account the introduction of DSSs in the medical domain may be stimulated (van der Lei, 1993).

A generally accepted evaluation methodology which addresses the above mentioned problem areas is needed. Most existing evaluation methodologies have as a common denominator at least the validation of a system, of which the assessment of the implemented knowledge is considered to be the cornerstone (Spiegelhalter 1983, Green and Keyes 1987, O'Keefe et al. 1987, O'Keefe 1989. O'Leary and O'Keefe 1989, RossiMori et al. 1990, Wyatt and Spiegelhalter 1990, Engelbrecht et al. 1991, Grant et al. 1991, Clarke et al. 1994). We restricted ourselves to this type of assessment.

\subsection{Research questions}

In this thesis the main research question is

"Is it possible to develop decision support that provides adequate advice concerning anti-epileptic drug treatment?"

To answer this question we elicited and analysed the knowledge of an exper neurologist. This knowledge was implemented in a prototype system. The developed system can give advice in two different situations:

- the initiation of the anticonvulsive treatment after the diagnosis has been established and

- the adjustment of the drug treatment after an assessment of its effectiveness. 
The adequacy of the system's advice for initiation of anti-epileptic drug treatment is evaluated by addressing the following questions:

1. Does the system suggest an adequate anti-epileptic drug when initiating drug treatment?

2. Does the system provide an adequate daily dose when initiating drug treatment?

When an objectively correct treatment would be available for each case, such a treatment could serve as reference for answering the above listed questions. In the management of epilepsy there is no such objectively correct treatment (see chapter 2). Therefore we compare the system's advice with the suggestions of a group of neurologists.

For the initiation of drug treatment a Delphi procedure was followed to reduce variability and remove erroneous treatment suggestions of the neurologists. From the set of 60 cases we obtained a diagnostic reference set where the majority of three neurologists agreed on the detailed diagnosis. This diagnostic reference set was reduced in size to a treatment reference set where the majority of the neurologists agreed on the generic drug.

The diagnostic reference set is used for assessment of the proposed generic drug; the treatment reference set for assessment of the proposed daily dose of the drug. For both sets of cases the agreement between the system and individual neurologists was determined. It was decided that the system's advice would be considered adequate when it conforms to the reference as good as or better than the individual neurologists. If the system does not agree with the majority but gave an advice equal to that of one neurologist such an advice can be considered an alternative treatment.

As more than one treatment may be appropriate for a case the acceptability of the prescriptions for cases in the diagnostic reference set was assessed by two independent (non prescribing) neurologists. The question addressed is:

3. What is the overall acceptability of the advice of the system compared with that of the neurologists?

If the prescription was considered of poor quality, the judgement on the appropriateness of the generic drug and the prescribed daily dose was analysed to determine which of these aspects was the reason for the poor quality.

The comparison of the neurologist's prescriptions with the system's advice for the cases in the reference sets and the assessment of the acceptability provided an indication about the performance of the system. However, it did not enable us to make a statement about the correctness of the detailed prescriptions due to the variance in prescribing among the neurologists. To reduce the variability in prescribing among the neurologists we decided to have a consensus meeting in which the prescriptions for each of the cases were discussed. During this discussion global guidelines were agreed upon. These guidelines could be used to determine a reference treatment for each of the cases. The advice of the system as well as the prescriptions of the neurologists are compared with this reference. The question addressed was:

4. What is the correctness of the system's prescriptions compared with the correctness of the neurologists' prescriptions? 
The adjustment of drug treatment is another part of the process for which an advice may be provided by the system. This is evaluated by addressing the question:

5. Does the system take adequate treatment decisions while monitoring drug treatment?

The global decisions to adjust the treatment - i.e. continue, decrease or increase the daily dose or change of drug - were used for comparison.

On the basis of a set of 265 descriptions of follow-up contacts the agreement of the system with each individual neurologist is determined. A comparison between the agreement of an individual neurologist and the remaining group of four neurologists and between the system and this group is made to obtain information about the adequacy of the system.

Based on the 265 follow-up descriptions two reference treatment sets were established to determine the performance of the system. A clinical reference set contained cases for which a majority decision of the five neurologists could be established. The second reference used the majority decision obtained from both the five neurologists and the system. To obtain a more tangible measure, the agreement between the system and the neurologists was expressed as a score, the level of agreement, indicating the number of neurologists which agree or disagree with the system.

To compare the decision making process of the group of neurologists with that of the system a classification tree is constructed based on case characteristics and the treatment decisions. The end-nodes of the tree present sets of cases which share particular case characteristics. The path to an end-node which contains mainly cases with one treatment decision indicates a guideline which may have been applied by the group of neurologists. End-nodes with several treatment decisions indicate categories of cases for which guidelines are difficult to obtain. For each of the major end-nodes the decision making process of the neurologists and the system are compared. The question addressed here is:

6. How do the treatment decisions of the system compare with those of the group of neurologists for the cases that meet the guidelines derived from the neurologists' decisions?

\subsection{Overview of the thesis}

In chapter 2 a review of the literature on epilepsy and its treatment is presented. The chapter is divided into two parts. In the first part a description of epilepsy and an overview of the diagnostic classifications are presented. Studies on prevalence and incidence in the United States and European countries indicate how common epilepsy is. In the second part treatment of epilepsy is discussed including aspects of drug treatment such as initiation, maintenance and withdrawal of drug treatment. This information was used during the design of the system.

Since the literature does not provide enough information about the criteria to be used in the decision making process of drug treatment it was decided also to elicit this knowledge from an expert. The amount of data required for commencing and monitoring the drug treatment makes the use of flow charts unfeasible and calls for 
computer support. Several approaches for knowledge acquisition are reviewed in chapter 3. Models describing the initiation and monitoring part of the treatment process are presented. Problems in analysing expertise using KADS are discussed.

The evaluation of the system focused on the implemented knowledge. As a standard for anti-epileptic dnug treatment was not available, a reference treatment had to be developed for a set of test cases. A Delphi-like approach in combination with a consensus meeting was selected for establishing a reference with which to compare the system. In chapter 4 the construction of the test cases and development of the reference are presented.

Chapter 5 addresses the assessment of the system's advice for initiating anti-epileptic drug treatment. Three analyses are presented in which the advice of the system is compared with the prescriptions of:

- The neurologist who provided the expertise to investigate whether the domain was sufficiently covered during the knowledge elicitation process

- A neurologist from the same department not involved in the development of the system to obtain some information on local differences in treatment

- A group of five neurologists from five different general hospitals in The Netherlands to assess the impact of individual preferences

In each of the experiments the prescribed drug and the daily dose are assessed. A comparison of the performance of individual neurologists and of the system is presented

To be able to determine the correctness of prescriptions a consensus meeting was held to define a treatment reference. The reference determined the required generic drug as well as the daily dose. The advice of the system as well as the prescriptions of the neurologists are compared with this reference.

In chapter 6 the advice of the system is assessed using case descriptions, in which drug treatment has been started, who return for a follow-up. In this chapter the treatment decisions are assessed at a global level: increase, decrease or keep the dose as it is or change the type of drug. The chapter consists of two parts. In the first part the advice of the system is addressed. Three different analyses are presented:

- The degree of agreement between the system and the neurologist(s) is determined to establish how good the system is in relation to the individual neurologist

- A clinical reference based on the majority decision of the group of neurologists was used to assess the correctness of the system and the neurologists

- A comparison using a reference based on the majority decision of both the neurologists and the system provided information on how the system performed when the system is considered as a peer

One may expect that case characteristics determine which treatment decision is taken. The second part of chapter 6 describes the results of an analysis leading to criteria that may have been used by the neurologists in arriving at a treatment proposal. The treatment decisions of the system are compared with them. 
In chapter 7 the final conclusions are presented and discussed. Directions for future research are indicated as well.

\subsection{References}

Beardsley R.S., Freeman J.M., Appel F.A., Anti-convulsant serum levels are useful only if the physician appropriately uses them: an assessment of the impact of providing serum level data to physicians, Epilepsia, 24, 1983, pp. 330-335.

Chadwick D., Reynolds E.H., When do epileptic patients need treatment? Starting and stopping medication, British Medical Journal, 290, 1985, pp. 1885-1888.

Chadwick D., Case for early treatment is not established, British Medical Journal, 1995, 310 , pp.177-178.

Clarke K., O'Moore R., Smeets R., Talmon J., Brender J., McNair P., Nykänen P., Grimson J., Barber B.A., A methodology for evaluation of knowledge-based systems in medicine, Artificial Intelligence in Medicine, 1994, 6, pp. 107-121.

Commission on Epidemiology and Prognosis of the International League Against Epilepsy, Guidelines for epidemiologic studies on epilepsy, Epilepsia, 34, 1993, pp.592-596.

Dichter M.A., The premise, the promise, and the problems with basic research in epilepsy, Epilepsia, 34, 1993, pp.791-799.

Dombal F.T de, Background and current state-of-the-art Technology and Healih Care. European Society for Engineering and Medicine. Vol. I, April 1993, pp. 77-83.

Dreifuss F.E., Risk benefit analysis in the management of the child with epilepsy. In: Modern approach to anti-epileptic drug treatment, improving quality of life, (Dam, Gram, Pedcrsen and Ørum, Eds.), The Danish Epilepsy Society, Kobenhavn, 1985.

Engelbrecht R., Fitter M., Rector A., Requirements for a medical workstation using usercentred design. In: Proceedings MIE '9l (Adlassnig, Graber, Bengtsson and Hansen, Eds.), Lecture Notes in Medical Informatics, vol 45. Springer-Verlag, Berlin, 1991, pp. 140-144.

Grant A., Parker-Jones C., White R., Cramp D, Barreiron A., Mira P., Artal A., Monteror J., Evaluation of knowledge based systems from the user perspective. In: Knowledge-hased systems in Medicine: Methods Applications and Evaluation, (Talmon and Fox, Eds.), Lecture Notes in Mcdical Informatics, vol 47, Springer Verlag, Berlin, 1991, pp. 312-324

Green C.J., Keyes M.M., Verification and validation of expert systems. In: Proceedings Western Conference on expert systems Anaheim, IEEE Computer Socicty Press, New York, 1987, pp. 38-43.

Heathfield H.A., Wyatt J., Philosophies for the design and development of clinical dicision support systems. Methods of Information in Medicine, 32, J993, pp. 1-8

Johannessen S.I., Loyning Y., Munthe-Kaas A.W. General Aspects. In: Comprehensive Epileptology (Dam and Gram, Eds.), Raven Press, New York, 1990, pp 505-524.

Johnston M.E., Langton K.B., Hayncs R.B., Mathicu A., Effects of computer-based clinical decision support systems on clinician performance and patient outcome, a critical appraisal of research, Annals of Internal Medicine, 120, 1994, pp. $135-142$.

Kleyn E. van der, Continuous monitoring of plasma anti-epileptic drug levels, in: Current therapy in epilepsy, Acta Ncurologica Scandinavia, 97 (Suppl.), 1983, pp.91-114.

Korpinen L., Pietilä T., Peltola J., Nissilä M., Keränen T., Tuovinen T., Falck B., Petránek E.S., Frey H., Evaluation of epilepsy expert, a decision support system, Computer Methods and Programs in Biomedicine. 45. 1994, pp.223-231. 
Lei, J. van der, Experience from computer based records for computer assisted decision making. Methods of Information in Medicine, 32, 1993, pp. 14-15

Meinardi H., Epileptology, military art of healing or science. Inaugural address, Catholic University of Nijmegen, 22 Februari 1985.

Meyer J.W.A. Knowledge, attitude and practice in anti-epileptic drug monitoring, thesis, Nijmegen, 1991.

Musen M., Architectures for architects, Methods of Information in Medicine, 32, 1993, pp. 1213.

Nykänen P. Issues on the evaluation of computer-based support to clinical decision making, Report of the SYDPOL-working group, Institute of informatics, Oslo University, Research report 127, 1990.

O'Keefe R.M., Balci O., Smith E.P., Validating expert systems performance. IEEE Expert, Winter, 1987, pp. $81-89$.

O'Keefe R.M. The evaluation of decision-aiding systems, guidelines and methods, Information and Management, 17, 1989, pp.217-226.

O'Leary D., O'Keefe R. Verifying and validating expert systems, Tutorial at the 11 th IJAI-89, Detroit USA, 1989.

O'Moore R., Clarke K, Smeets R., Brender J., Nykänen P., McNair P., Grimson J., Barber B.: Items of Relevance for the Evaluation of Knowledge Based / Expert Systems and Infuence from Domain Characteristics. KAVAS (AJ021) Report EM 1.l., Trinity College, Dublin, 1990.

Paterson-Brown S., Vipond M.N., Madern aids to clinical decision making in the acute abdomen, British Journal for Surgery, 77, 1990, pp. 13-18.

Perreault L., Wiederhold G., System design and evaluation. In: Medical Informatics. Computer applications in healih care (Shortliffe, Perreault, Wiederhold and Fagan, Eds.), Addison-Wesley Publishing Company, Reading, Mass. 1990.

Peterson G.M., Computer-assisted drug therapy for epilepsy, Drig Intellingence and Clinical Pharmacy, 1983, 17, pp.123-125.

Principe J.C. Smith J.R., Microcomputer-based system for the detection and quantification of petit mal epilepsy, Computers in Biology and Medicine, 12, 1982, pp.87-95.

Reynolds E.H., Do anticonvulsants alter the natural course of epilepsy? Treatment should be started as early as possible, British Medical Journal, 1995, 310. pp. 176.

Rimmer E.M., Richons A., Clinical pharmacology and medical treatment. In: A textbook of upilepsy, (Laidlaw, Richens and Oxley, Eds.), 3rd. edition, Churchill Livingstone, Edinburgh, 1988, pp. 421-483.

Rossi-Mori, A., Pisanelli, D.M., Ricci, F.L. Evaluation stages and design steps for knowledgebase systems in medicine. Medical Informatics, vol. 15, no.3, p.191-204, 1990.

Spiegelhalter D.J. Evaluation of clinical decision-aids with an application to a system for dyspepsia, Statistics in Medicine. 2, 1983, pp.207-216.

Sullivan F., Mitchell E., Has general practitioner computing made a difference to patient care? A systematic review of published reports, British Medical Joumal, 311, 1995, pp.848-852.

Sutton, G.C., Computer-aided diagnosis a review, British Journal for Surgery, 76, 1989, p.8285 .

Ward C.D.. Decision making in refractory epilepsy. an analysis based on computer simulation, Journal ut Neurology. Neurosurgery and Psychiatry, 1989, 49, pp: 781-784. 
Wyatt J., Spiegelhalter, D. Evaluating medical expert systems: what to test and how? Medical Informatics, 15, 1990, pp.205-217. 


\section{Epilepsy}

\subsection{Introduction}

In this chapter both a scientific and a practical view on epilepsy are presented. The scientific part starts with defining the disease and provides a historical overview of various classifications. It shows the impact of technological and pharmacological research on the development of definitions and classifications. Furthermore, epidemiological data are presented. The prognosis and the implications of epilepsy for daily life of the patient will be addressed. Next the view is shifted towards daily practice. The problems the physician encounters when trying to relieve the patient from his seizures by using anti-epileptic drugs (AEDs) are discussed. After the diagnosis is established, a trade-off between the pros and the cons of drug treatment has to be made, given the specific situation of the patient. lssues such as when to start the drug treatment, which drug to select and what initial dose to prescribe will be discussed. As seizures may recur the start of drug treatment for epilepsy implies a longterm commitment to taking medication. Reasons for recurrence of seizures will be addressed. Furthermore the role of anti-epileptic drug monitoring in the treatment process will be discussed. Like in other diseases requiring long-term prophylactic drug treatment such as diabetes or hypertension non-compliance may occur. Causes of non-compliance will be mentioned. Many patients will show an improvement of their seizures. In this case drug withdrawal will be suggested by the patient or the doctor. Aspects of drug withdrawal and the risks of relapse will be described.

\subsection{Description of epilepsy}

Epilepsy is a paroxysmal disturbance of the functioning of the central nervous system. This disturbance is recurrent, stereotype in character and associated with spontaneous, excessive, synchronous and self-limiting neuronal discharge (Aird et al. 1984). It manifests itself in clinically detectable events, i.e. seizures which are perceptible for the subject and/or an observer. Thus, an epileptiform discharge in the EEG registration without a clinical manifestation is not called a seizure. The clinical detectability of an event may change and depends on the tests applied for its registration. For example, a 'bed-side' registration may not detect repeated cognitive impairment during a day while an advanced psychological test can register it 


\subsection{Classifications}

The classification of seizures and epileptic syndromes has changed over the years. This was caused partially by technical and pharmaceutical developments as a historical overview indicates.

\subsubsection{Historical overview}

The early classification of epilepsies was based on the aetiology, symptomatology or anatomy. The aetiology divided the epilepsies into two groups: those which could be attributed to gross cerebral lesions (symptomatic) and those without any identifiable cause (idiopathic) (Laidlaw et al. 1988)

The clinical findings regarding seizures were the basis for differentiation in classifications based on the symptomatology. It was not until 1946 that one could prescribe a drug treatment on the basis of the type of seizure present. At that time trimethadion was discovered. The drug turned out to be most effective against absence seizures (2-15 seconds disturbance or loss of higher psychic capabilities). This finding led to the conclusion that the drug treatment could depend on the type of seizure patterns and urged the need for a better classification of seizures (Cereghino, 1989).

The development of EEG-registration techniques in the late forties and the recognition of typical EEG patterns introduced another factor that could be used in the classification of epilepsies. Classifications of epilepsy based on aetiology (i.e. idiopathic or symptomatic) alone were considered to be inadequate for clinical applications at that time. The use of an EEG registration was required. This technological development resulted in 1954 in a new classification of the epilepsies into centre-encephalic and focal or cortical epilepsies (Dam and Kiørboe, 1982).

Table 2.3-I: International classification of epileptic seizures

\begin{tabular}{|c|c|c|}
\hline Partial scizures & Generalised seizures & Unclassified epileptic seizures \\
\hline A. Simple partial seizures & A. I. Absence seizures & (inadequate or incomplete \\
\hline 1. with molor syinploms & 2. Atypical absence seizures & data) \\
\hline 2. with somatosensory or & B. Myoclonic seizures & \\
\hline special sensory symptorns & C. Clonic seizures & \\
\hline 3. with autonomic symptoms & D. Tonic seizures & \\
\hline 4. with psychic symptoms & E. Tonic-clonic seizures & \\
\hline & F. Atonic seizures & \\
\hline
\end{tabular}

B. Complex partial scizures

Beginning as simple partial seizures and progressing to impainnent of consciousness

with no other features

with features as in A. $1-4$

with automatisnis

With impainment of consciousness at onset

with no other features

with features as in A. 14

with automatisms 
In 1969 a consensus classification for the epileptic seizures was accepted by the International League Against Epilepsy (ILAE) (Gastaut, 1970). A distinction was made between seizures starting locally and seizures being generalised from the onset. The epileptic seizures were categorised based on factors such as clinical features, EEG findings, age at onset, anatomic basis and the aetiology.

Further modification of the classification of epileptic seizures took place in 1981 when the attributes for classification were reduced to clinical manifestations (preferably recorded on videotape) and related EEG findings. This classification was accepted by the ILAE (Table 2.31). The eliminated factors such as aetiology, age at onset and anatomy were taken into account in the classification of epilepsies.

Besides the classification of seizures also a classification of the epilepsies was proposed (Merlis, 1970). The classification of epilepsies distinguished between generalised and partial epilepsies. Clinical characteristics such as aetiology, seizures, neurological status and age of onset were also used for classifying epilepsies. Still much overlap was present in the attributes used for classifying the seizures and epilepsies. In 1985 a new classification for epilepsies was proposed but it was not accepted until 1989 (Table 2.4-II). This classification retains the distinction of epilepsies in generalised and partial (localisation related) categories, based on the localisation of the onset of the seizures, on the cause (both idiopathic or symptomatic) and the age at onset. Furthermore two new categories were added:

- Epilepsies and syndromes of which it is not clear whether they are focal or generalised

- A category of special syndromes which is used for seizures occurring in special circumstances such as fever, intoxication or use of drugs (Commission on Classification and Terminology of the ILAE, 1989).

\subsubsection{Importance of different classifications}

Selection of the drug treatment can be based on the diagnosed seizure type and/or the type of epilepsy. The role of these detailed classification schemes in patient management is, however, rather limited. The seizure classification is mainly based on clinical findings and to a lesser extent connected with the neurophysiological and chemical basis of the convulsions. In fact the classification scheme is more detailed than the knowledge about basic mechanisms of available anti-epileptic drugs (Dichter 1994, Macdonald and Kelly 1994, Montouris 1995, Brodie and Dichter 1996).

The classification of the epilepsies includes factors such as aetiology and natural history which allows for a more complete description of the patient enabling an assessment of the prognosis. Although there are special types of epilepsy which require a specific drug treatment the classification of epilepsies is more detailed then necessary for drug treatment

More recently, new definitions of epilepsy and seizures and seizure type classification have been proposed to bridge the gap between research and patient management (Commission on Epidemiology and Prognosis of the ILAE, 1993)

\subsection{Epidemiology}

There are two main problems in obtaining statistics on epilepsy. The first problem is related to the definition of the disease. The second problem is caused by the sources from which data are gathered 
Table 2.4-II: International classification of epilepsics, epilcptic syndromes and related seizure disorders (ICE)

\begin{tabular}{|c|c|}
\hline Localisation related (focal, local, partial) & Generalised \\
\hline $\begin{array}{l}\text { Idiopathic (Primary) } \\
\text { Benign childhood epilepsy with centre-temporal spike } \\
\text { Childhood epilepsy with occipital paroxysins } \\
\text { Primary reading epilepsy }\end{array}$ & $\begin{array}{l}\text { Idiopathic (primary) } \\
\text { Benign neonatal farnilial convulsions } \\
\text { Benign neonatal convulsions } \\
\text { Benign myoclonic epilepsy in infancy } \\
\text { Childhood absence epilepsy (pyknolepsy) } \\
\text { Juvenile absence epilepsy } \\
\text { Juvenile myoclonic epilepsy (impulsive petit mal) } \\
\text { Epilepsies with grand mal seizures (GTCS) on awakening } \\
\text { Other generalised idiopathic epilepsies } \\
\text { Epilepsies with seizures precipitated by specific modes of } \\
\quad \text { activation }\end{array}$ \\
\hline $\begin{array}{l}\text { Symptomatic (secondary) } \\
\text { Temporal, frontal, parietal and occipital lobe epilepsies } \\
\text { Chronic progressive epilepsia partialis continua of } \\
\text { childhood } \\
\text { Syndrones characterised by seizures with specilic modes } \\
\text { of precipitation }\end{array}$ & $\begin{array}{l}\text { Cryptogenic or synptomatic } \\
\text { West syndrome (infantile spasins, Blitz-Nick-Salaam } \\
\text { Krämpfe) } \\
\text { Lennox-Gastaut syndrome } \\
\text { Epilepsy with myoclonic-astatic seizures } \\
\text { Epilepsy with myoclonic absences } \\
\text { Symptomatic } \\
\text { Non-specific aetiology } \\
\text { Early myoclonic encephalopathy } \\
\text { Early infintile epileptic encephalopathy } \\
\text { with suppression burst } \\
\text { Other symptomatic generalised } \\
\text { epilepsies } \\
\text { Specific syndromes } \\
\text { Epileptic scizures may complicate many } \\
\text { disease states }\end{array}$ \\
\hline \multicolumn{2}{|l|}{$\begin{array}{l}\text { Defined by seizure type, clinical features, aetiology, } \\
\text { anatomical localisation }\end{array}$} \\
\hline $\begin{array}{l}\text { Undetermined epilepsies } \\
\text { With both generalised and focal scizures } \\
\text { With unequivocal generalised or focal features }\end{array}$ & \\
\hline $\begin{array}{l}\text { Spccial syndromes } \\
\text { Situation related seizures (Gelegenheitsanfälle) } \\
\text { Febrile convulsions } \\
\text { Isolated seizures or isolated status epilepticus }\end{array}$ & \\
\hline $\begin{array}{l}\text { Seizures occurring only when there is an acute or } \\
\text { nonketotic hyperglycaemia }\end{array}$ & xic event due to faclors such as alcohol, drugs, eclampsia, \\
\hline
\end{tabular}




\subsubsection{Problems}

The translation of the definition of epilepsy provided by Gastaut and Kugler (1976) and used by the WHO is:

\section{'A chronic brain disorder of various aetiologies characterised by recurrent seizures dive to excessive discharge of cerebral neurones [...]. Single or occasional epileptic seizures as well as those occurring during an acute illness should not be classified as epilepsy'.}

The problem is that this definition is not operational enough. The definition excludes the use of the term epilepsy for seizures provoked by disorders primarily not involving the brain. The International Classification of the Epilepsies does include these as special syndromes. The term 'chronic' is ambiguous. It does not state the period during which the symptoms must be present before one can decide whether the patient really has epilepsy. This means that patients who suffer from several seizures per year as well as those with one seizure every two years can be included as epileptic patients in epidemiological studies. Whether or not seizures should still be manifest to be regarded as epilepsy not withstanding medication is not clear from this definition. It is also not clear when patients are considered to be cured.

The second part of the definition does exclude seizures directly related to a disease. Examples of such seizures are febrile convulsions and sporadic seizures caused by e.g. alcohol withdrawal. However, others claim that there exists insufficient ground to exclude occasional seizures from the diagnosis (Hopkins, 1987).

The ascertainment of epileptic cases for epidemiological studies may be a problem (Aird et al. 1984). The use of medical records results in an underrepresentation of the milder and nonactive epilepsies as well as of undiagnosed and untreated cases, as some patients may not ask for a medical evaluation of their symptoms (Haerer et al. 1986). The severity of the seizures, the social labelling because of the disease, the educational level or the socio-economic status and consequences may influence the decision to consult a health care professional. Field studies suggest that some 4 to $25 \%$ of the patient with epilepsy were never treated for their seizures (Haerer et al. 1986, Zielinski 1988, Keränen and Riekkinen 1993). The range in percentages may be attributed to different case finding methods and diagnostic criteria.

Patient denial also influences epidemiological data obtained from medical records. In an Italian study denial of the disease was reported in $31 \%$ of a patient population (Maremmani et al. 1991).

Data can be obtained from sources other than the medical record, e.g. death certificates and insurance company data. These alternatives have their own biases. Death certificates are generally available but incomplete in reporting epilepsy. Data obtained from insurance companies mainly present a selected population, which may exclude e.g. unemployed people or people with a low economic status. Additional biases may exist due to patients who do not reveal their disease to an insurance company to prevent exclusion or higher financial contributions.

\subsubsection{Prevalence and incidence}

The number of patients per 1000 persons in a given population at a given time is called the prevalence of the disease. The influence of how epilepsy is defined on prevalence data has been demonstrated in two studies concerning 6000 patients from a general practice (Goodridge and Shorvon 1983a, Cockerell et al. 1995a). 
Table 2.4-I: Prevalence of epilepsy in Europe and United Statcs

\begin{tabular}{|c|c|c|c|c|c|c|}
\hline Country & First author & Definition & Study & Sources" & Age & Prev** \\
\hline \multirow[t]{3}{*}{ Denumark } & Juul- Jensen & epilepsy ${ }^{\prime}$ & retrospective & $\mathrm{MH} / \mathrm{II} / \mathrm{DR}$ & & $12.7^{2}$ \\
\hline & & & 37 ytars & & & \\
\hline & Wagner (1983) & active epilepsy ${ }^{3}$ & retrospective ${ }^{4}$ & $\begin{array}{l}\text { MH/EH/DPP } \\
\text { GP }\end{array}$ & $16-66$ & 4.32 \\
\hline Finland & Keränen (1989) & active epilepsy & $\begin{array}{l}\text { retrospective } \\
19 \text { years }\end{array}$ & $\begin{array}{l}\mathrm{MH} / \mathrm{Ps} / \mathrm{EH} \\
\mathrm{MMR} / \mathrm{HC} / \mathrm{NH} \\
\mathrm{EL} / \mathrm{PR}\end{array}$ & $>15$ & 6.29 \\
\hline \multirow[t]{2}{*}{ Italy } & Granieri (1983) & active epilepsy & $\begin{array}{l}\text { retrospective } \\
18 \text { years }\end{array}$ & $\begin{array}{l}\text { MH/ND/NS } \\
\text { PD/EL/LD/DPP } \\
\mathrm{Ch} / \mathrm{T} / \mathrm{SW} \\
\mathrm{MeH}\end{array}$ & all & $(6.4)^{5}$ \\
\hline & Mareminani (1991) & active epilepsy & retrospective & $\begin{array}{l}\text { H/LD/PR/EHn } \\
\text { SD/SW }\end{array}$ & all & $\begin{array}{l}5.1 \\
(5.2)^{5}\end{array}$ \\
\hline \multirow[t]{2}{*}{ Norway } & De Gruaf (1974) & epilepsy & retrospective & ND/EL/IMR & all & 3.5 \\
\hline & & & 5 years & & & \\
\hline Sweden & Forsgren (1992) & active epilepsy & retrospective & ND/NS/M/Ps & $17^{x}$ & 5.5 \\
\hline & & & 13 years & $\begin{array}{l}\text { GD/EL/SLR } \\
\text { DPP/DN }\end{array}$ & & \\
\hline \multirow{4}{*}{$\begin{array}{l}\text { The Nether- } \\
\text { lands }\end{array}$} & Bongers (1976) & epilepsy & retrospective & GP & all & 2.9 \\
\hline & Rutgers (1984) & $\begin{array}{l}\text { active epilcpsy } \\
\text { last } 2 \text { yeatss }\end{array}$ & prospective & GP & $15-66$ & 4.0 \\
\hline & Crobach (1988) & active epilepsy & retrospective & GP/Ch & all & 3.3 \\
\hline & Metsemakers (1994) & epilepsy ${ }^{7}$ & retrospective & GP & all & 8.5 \\
\hline \multirow[t]{3}{*}{$\begin{array}{l}\text { United King- } \\
\text { dom }\end{array}$} & Crombie (1960) & $\begin{array}{l}\text { active epilepsy } \\
\text { last } 2 \text { years }\end{array}$ & $\begin{array}{l}\text { retrospeclive } \\
3 \text { years }\end{array}$ & GP & all & 4.19 \\
\hline & Goodridge (1983a) & $\begin{array}{l}\text { active epilepsy } \\
\text { last } 2 \text { years }\end{array}$ & $\begin{array}{l}\text { retrospective } \\
35 \text { years }\end{array}$ & GP & all & 5.3 \\
\hline & Cockerell (1995a) & $\begin{array}{l}\text { active epilepsy } \\
\text { last } 2 \text { years }\end{array}$ & $\begin{array}{l}\text { retrospective } \\
10 \text { years }\end{array}$ & GP & all & 4.3 \\
\hline \multirow[t]{3}{*}{ Uniled States } & Hauser $(1975)$ & active epilepsy & $\begin{array}{l}\text { retrospective } \\
25 \text { yuirs }\end{array}$ & HIS/MH & all & 5.7 \\
\hline & Haerer (1986) & active epilepsyn & retrospective & $\begin{array}{l}\text { door-10-door } \\
\text { MI LNH }\end{array}$ & all & 6.78 \\
\hline & Hauser (1991) & uctive epilepsy & $\begin{array}{l}\text { retrospective } \\
40 \text { years }\end{array}$ & $\mathrm{H} \amalg \mathrm{S} / \mathrm{MH}$ & all & 6.8 \\
\hline
\end{tabular}

- $\mathrm{Ch}=$ Chemist, $\mathrm{DR}=$ Death register, EL=EEG-lab, EH=Epilepsy hospilal, H=Hospital, HIS=Hospital information system, $\mathrm{MH}=$ Multiple hospitals, MeH=Mental hospital, $\mathrm{HC}=$ Health centre, $\mathrm{LD}=\mathrm{Local}$ doctors, $\mathrm{ND}=\mathrm{New}$ ology dept, $\mathrm{NS}=$ Neurosurgery dept., $\mathbb{M}=\mathrm{Dept.}$ Internal Medicine, $\mathrm{IR}=$ Insurance register, $S \mathbb{R}=\mathrm{Social}$ insurance register, $\mathrm{SD}=\mathrm{Sch} \infty \mathrm{l}$ doctors, $S W=$ Social workers, $P D=$ Pediatric Dept., $G D=$ Geriatrics Dept., GP=General practitioner, PSD=Psychiatry dept, $\mathrm{DPP}=\mathrm{Doctors}$ with private practice. $\mathrm{DN}=$ District uurses, $\mathrm{DMR}=$ Inst. mentally retarded, $\mathrm{NH}=$ Nursing homes, $\mathrm{PR}=\mathrm{Pharmacy}$ register, $T=$ Teacher,

** per 1000 population

(1) Epilepsy without exclusion of patients seizure free for more than 5 years and not on anti-epileptic drug treatment, (2) Cumulative incidence, (3) Epilepsy $\geq 2$ spontaneous seizures excluding patients with controlled seizures and not receiving treatunent, (4) Registration depending on setting. $1-18$ years, (5) Prevalence corrected toward the Italian population, (6) On AED and having $\geq 1$ seizure in 3 years or not on AED and having $\geq 1$ seizure within last year, (7) active and inactive epilepsy. 
The prevalence of epilepsy excluding febrile seizures but including cases such as single, recurrent, active and inactive epilepsy was 20.3 and 21 in the 1983 and 1993 studies respectively. This number dropped to 17.0 by excluding patients with single seizures. An even lower prevalence - 10.5 - was found by including only those patients receiving anti-epileptic treatment and those having active epilepsy defined as at least one seizure within the last 24 months. Prevalence further decreased to 5.3 in 1983 and to 4.3 in 1993 by including only patients with active epilepsy. In the USA improved case ascertainment strategies resulted in different prevalence rates in the same population studied (Hauser and Kurland 1975, Hauser et al. 1991). The use of broad inclusion criteria - not excluding patients free from seizures for more than 5 years and including patients not on anti-epileptic drug treatment - caused the high prevalence rate of the Danish study (Table 2.4-I) (Juul-Jensen and Foldspang, 1983). The minimal prevalence rate of convulsive disorders was estimated to be $4-6 / 1000$ (Cereghino 1974, Rutgers 1984).

The number of new cases within 1 year per 100000 persons is called the incidence of a disease. It describes the rate with which new cases of the disease occur. The incidence rates suffer the same problems as the prevalence. The classic study of incidence is from Rochester, Minnesota. In this study it was shown that the incidence of recurrent seizures was 44 patients per 100000 persons per year over a 50 year period (Hauser et al. 1993). Similar results with average annual incidence rates for recurrent seizures in between 30 and 50 were reported previously (Hauser 1978, Granieri et al. 1983. Zielinski 1988, Keränen et al. 1989, Forsgren 1990). The incidence increased to 61 per year if cases with single seizures are included (Hauser et al. 1993). Inclusion of febrile seizures leads to an even higher incidence, 115 per year (Hauser, 1978)

Incidence rates vary with age. The incidence rate is highest during the first decade, after which it decreases. An increase in the number of new cases is observed after the sixth decade (Zielinski 1974, Lühdorf et al. 1986, Zielinski 1988, Hauser et al. 1991, Hauser 1992). While the incidence for the population was 44 , the highest incidence, 139, was observed in persons over 75 years of age (Hauser et al. 1993). The increase in the number of tumours and cerebrovascular diseases has been suggested as a possible cause. Another explanation for the increase might be the higher level of case ascertainment for elderly people or more extensive medical care for this group (Schoenberg 1983, Hauser et al. 1993)

The incidence rates for children and elderly persons have been compared over the years. These measurements show that the incidence for children decreases while the incidence rate for the elderly increases (Hauser et al. 1993, Cockerell et al. 1995a). These changes may have been caused by improved perinatal care and increased medical attention for the elderly.

\subsection{Aetiology}

Determining the type of seizure and the type of epilepsy is only half of the diagnostic process. Establishing the aetiology is the other. Aird et al (1984) use both causative and underlying neurochemical and neurophysiological factors to describe the aetiology. These factors may suggest possibilities for the treatment of seizures. The main causative factors for epilepsy are genetic and birth defects, infections, toxic mechanisms, traumata, circulatory causes (e.g. haemorrhage, thrombosis), metabolic and nutritional disorders, neoplasms, heredofamilial and degenerative diseases. The neurochemical description of etiologic factors for epilepsy include metabolic derangements as e.g. hypoxia, hypoglycaemia, electrolyte and acid-base imbalances, vitamin deficiencies, changes in excitatory and inhibitory neurotransmitters, endocrine 
dysfunctions, endogenous toxic states and hyperpyrexia. Most epidemiological studies reporting on aetiology, however, describe only the causative factors. Another approach is to distinguish between cause - a more or less steady-state background factor - and precipitating factors which are short term factors which may cause seizures in susceptible people (Hopkins, 1987).

Table 2.5-1: Ethiological factors in epilepsy

\begin{tabular}{llllllllll}
\hline First author & $\begin{array}{l}\text { De Graal } \\
(1974)\end{array}$ & $\begin{array}{l}\text { Bergamini } \\
(1977)\end{array}$ & $\begin{array}{l}\text { Hauser } \\
(1978)\end{array}$ & $\begin{array}{l}\text { Granieri } \\
(1983)\end{array}$ & $\begin{array}{l}\text { Cowan } \\
(1989)\end{array}$ & $\begin{array}{l}\text { Mcinardi } \\
(1990)\end{array}$ & $\begin{array}{l}\text { Sander } \\
(199)\end{array}$ & $\begin{array}{l}\text { Hauser } \\
(1991)\end{array}$ & $\begin{array}{l}\text { Forsgren } \\
(1992)\end{array}$ \\
\hline Age & $>15 y$ & $1 \mathrm{~m}-21 \mathrm{y}$ & all & all & $<19$ y & all & all & all & $>16$ y \\
Unknown & $68 \%$ & $56 \%$ & $64 \%$ & $60.9 \%$ & $69 \%$ & $28.8 \%$ & $61 \%$ & $76 \%$ & $64.8 \%$ \\
Cer.vase. & $4 \%$ & nav & $5 \%$ & $2.9 \%$ & 3 & $1.9 \%$ & $15 \%$ & $6 \%$ & $11.8 \%$ \\
Cer.tumor & $2 \%$ & nav & $4 \%$ & $1.4 \%$ & 3 & $3.5 \%$ & $6 \%$ & $2 \%$ & $4.7 \%$ \\
Alc./loxic & $3 \%$ & excl. & excl. & nav & $2 \%$ & nav & $6 \%$ & excl. & excl. \\
Post-traum. & $9 \%$ & $12.2 \%$ & $5 \%$ & $6.8 \%$ & $4 \%$ & $10.4 \%$ & $3 \%$ & $5 \%$ & $7.2 \%$ \\
Cong./Gen. & $2 \%$ & $1.9 \%$ & $4 \%$ & $1.1 \%$ & $3 \%$ & $3.2 \%$ & 4 & $5 \%$ & $2.6 \%$ \\
Lufections & $2 \%$ & $11.9 \%$ & $3 \%$ & $3.7 \%$ & $3 \%$ & $5.1 \%$ & $2 \%$ & $4 \%$ & $3.5 \%$ \\
Perinatal & $9 \%$ & $17.8 \%$ & nav & $23.7 \%$ & $7 \%$ & $40.2 \%$ & 4 & nav & $5.4 \%$ \\
Febr.conv. & nav & excl. & nav & nav & 3 & $6.9 \%$ & 4 & nav & nav \\
Other cause & nav & nav & $15 \%$ & nav & 3 & nav & $7 \%$ & $1 \%$ & nav \\
\hline
\end{tabular}

(1) data from tertiary care centre, (2) nav, (3) other and multiple causes $12 \%$, (4) this included congenital perinatal and febrile convuisions, (5)history of febrile convulsions

Studies indicate that in $56-76 \%$ of the patients the aetiology of their epilepsy is unknown (Table 2.5-1). Part of this depends on the assessment of a genetic basis for idiopathic epilepsies. As multiple genes may be involved a family history will underestimate genetic causes. Differences in case finding strategies are also important. For example, a high rate of perinatal causes reported by Granieri et al. (1983) can be explained by the fact that this aetiology was more intensely looked for as there was an urgency in improving the prenatal and perinatal care in the region. The study conducted by Meinardi and Pachlato (1985) shows fewer unknowns and a higher percentage of perinatal factors in a population of a special centre for epilepsy.

The aetiology of epilepsy varies with the age of the patient (Janz 1969, Sander et al. 1990, Hauser 1992). In the neonatal period birth-injuries - haemorrhage and anoxia - may cause epilepsy. Also congenital abnormalities, metabolic disorders and infections can induce epileptic seizures. In early childhood febrile seizures, trauma infection, cerebral degeneration, poisons and metabolic defects may cause epilepsy. In childhood and adolescence idiopathic (primary) epilepsy may manifest itself. In addition to this, trauma, infections and cerebral degeneration can induce epilepsy during this period. It has been reported that in $67.6 \%$ of the patients younger than 15 years the cause of the epilepsy was unknown, while in $20 \%$ a congenital defect, in $4.7 \%$ a trauma and in $4 \%$ an infection was identified as the cause of their disease (Hauser, 1992). In early adult life trauma, tumour or infection may cause epilepsy while juvenile idiopathic epilepsies also occur. Birh injuries and cerebral degeneration can still play a role as a cause of epilepsy in this period 
During late adult life vascular disease, trauma, tumour and cerebral degeneration are the most frequent causes for epilepsy (Marsden and Reynolds, 1988). Also alcohol abuse has been mentioned as a frequent cause of epilepsy. In a study on late-onset epilepsy (starting after the age of 25 years) made up a group of one fourth of all patients, while the cause was unknown in $38 \%$. In $16 \%$ of the patients a brain tumour was the cause, cerebrovascular infarctions were registered in $14 \%$ of the patients. Head trauma accounted for $4 \%$ of the cases and miscellaneous causes such as angioma, brain abscess or leukoencephalopathy accounted for $5 \%$ of the patients (Mouritzen Dam et al. 1985). In a field study in elderly over 65 years the percentage of cases with an unknown cause was $48.9 \%$, while $32.4 \%$ had a vascular cause. A degenerative cause was reported in $11.5 \%$ and neoplasms were reported in $2.7 \%$ of these cases (Hauser, 1992). Others reported from a clinical setting that in patients over 60 years cerebrovascular disease was the cause in $32 \%$, tumours in $14 \%$ while in $25 \%$ the cause was unknown (Lühdorf et al. 1986).

\subsection{Prognosis}

There are four possible courses for epilepsy:

- Seizures remit spontaneously and no treatment is required

- Seizures are controlled with drug treatment and the medication can successfully be withdrawn

- Seizures are controlled but anti-epileptic drug treatment must be continued

- Seizures continue even when on anti-epileptic drug treatment

While problems may occur in all four groups, drug related and/or psychosocial problems can be expected, particularly in the third and the fourth category. As these groups require a lifelong treatment, the etiological classification of the epilepsy may change e.g. due to a gradual manifestation of an oncologic cause. This means that in these patients the correctness of the diagnosis has to be periodically reviewed. A sudden change in effectivenuss of the treatment due to alterations in the metabolism of the drug, concomitant diseases or comedication may occur. Drug toxicity may develop gradually, especially with multiple drugs. Besides this, the compliance can change because of side-effects of the drugs or absence of seizures or due to avoiding the daily confrontation with the disease. Such a change in compliance may lead to recurrence of seizures. Also an irregular life-style may alter the effectiveness of the treatment. Patients in both these categories will need attention from the physician on a regular basis.

\subsubsection{Remission}

The idea that epilepsy is a chronic condition especially if left untreated - seizures beget seizures - has been stated already in the last decades of the $19^{\text {th }}$ century (Gowers, 1885 , reprinted in 1994). This idea has lasted for over 80 years. However, this view is gradually changing. Population studies indicate that $4.1 \%$ to $26 \%$ of the patients with epilepsy do not receive anti-epileptic drug treatment (Zielinski 1974, Goodridge and Shorvon 1983b, Haerer et al. 1986, Keränen and Rjekkinen 1993, Cockerell et al. 1995a). In some of these patients epilepsy remitted spontaneously. It is however difficult to be certain of the diagnosis of epilepsy in these patients who reported seizures in the past but on index day had been free from seizures for several years. The number of patients reported to be seizure free for at least 2 years at 10 and 20 years after onset of the epilepsy were $42 \%$ and $52 \%$ respectively 
(Keränen and Riekkinen, 1993). Some 30\% were seizure free for more than 5 years (Zielinski, 1974). Comparison of incidence and prevalence rates indicates that epilepsy lasts 13 years on average. More recent studies in developing countries also suggest that epilepsy may remit without drug treatment (Sander, 1993).

In the traditional view on epilepsy the prognosis was considered poor with only $20 \%$ going into a long-term remission. In the last two decades studies on remission of seizures show that $35-90 \%$ of patients can achieve a remission of 2 years during some period within 2 to 10 years after starting drug treatment (Shorvon and Reynolds 1982, Elwes et al. 1984, Collaborative Group for Study of Epilepsy 1992). Remission of seizures lasting at least 5 years were reported in $61 \%$ of newly diagnosed patients 10 years after the diagnosis. The chance of having achieved a 5 year remission within 10 and 20 years was $65 \%$ and $76 \%$ respectively (Annegers et al. 1979). A similar result - 68\% seizure free for 5 years or more during nine years of follow-up - was found in a national general practitioners study (Cockerell et al. 1995b).

This means that many patients tend to go into a long-term remission during follow-up. At 20 years after diagnosis $70 \%$ of the patients were in remission (Annegers et al. 1979)

Part of the patients with a remission need medication continuously as the anti-epileptic drugs only suppress the occurrence of seizures instead of curing the disease. While $70 \%$ of the patients were in remission some $50 \%$ were seizure free for 5 years and did not take anticonvulsive medication 20 years after the initial diagnosis, while $20 \%$ of the patients continued to take anti-epileptic drugs although they were free for at least 5 years without seizures (Annegers et al. 1979). Others report that 13\% of the patients which were at least 3 years seizure free after 9 years of follow-up were still taking anti-epileptic drugs (Cockerell et al. 1995b).

Table 2.6-I: Remission rates of scizures in community and hospital based studies.

\begin{tabular}{|c|c|c|c|c|c|c|}
\hline First author & $\begin{array}{l}\text { Annegers } \\
(1979) \\
N=618\end{array}$ & $\begin{array}{l}\text { Elwes } \\
(1984) \\
N=106\end{array}$ & $\begin{array}{l}\text { Zielinski } \\
(1988) \\
N=98\end{array}$ & $\begin{array}{l}\text { CGSE }^{\prime} \\
(1992) \\
N=280\end{array}$ & $\begin{array}{l}\text { Keränen }^{2} \\
(1993) \\
N=33\end{array}$ & $\begin{array}{l}\text { Cockerell } \\
(1995 b) \\
N=564\end{array}$ \\
\hline Study & retrospective & prospective & prospective & prospective & retrospective & prospective \\
\hline Setting & $\begin{array}{l}\text { community } \\
\text { based }\end{array}$ & neur.clin. & $\begin{array}{l}\text { community } \\
\text { based }\end{array}$ & hospital & hospital & $\begin{array}{l}\text { community } \\
\text { based }\end{array}$ \\
\hline $\begin{array}{l}\text { Follow-up } \\
\text { duration }\end{array}$ & $\begin{array}{l}328>10 \text { years } \\
141>20 \text { years }\end{array}$ & $\begin{array}{l}\text { median } 66 \mathrm{mths} \\
\text { (range 6-96) }\end{array}$ & 5 years & $\begin{array}{l}\text { median } 48 \text { mths } \\
\text { (range } 1-87 \text { ) }\end{array}$ & $>2$ years & median 7.1 ys \\
\hline Remission & $\geq 5$ years & $\geq 2$ years & $\geq 2$ years & $\begin{array}{l}\geq 2 \text { and }<3 \\
\text { years }\end{array}$ & $\geq 2$ years & $\begin{array}{l}>3 \text { years } \\
>5 \text { years }\end{array}$ \\
\hline \multirow[t]{2}{*}{$\begin{array}{l}\text { \%opatients } \\
\text { achicving } \\
\text { remission }\end{array}$} & $\begin{array}{l}65 \% \text { at } 10 \text { ys } \\
\text { f.u }{ }^{3} \\
76 \% \text { at } 20 \text { ys } \\
\text { f.u. }{ }^{3}\end{array}$ & $\begin{array}{l}35 \% \text { at } 2 \text { ys } \\
6 u^{3} \\
57 \% \text { at } 3 \text { ys } \\
\text { fu? }\end{array}$ & & $\begin{array}{l}71 \% \text { at } 3 \text { ys } \\
\int .{ }^{3} \\
90 \% \text { at } 5 \text { ys } \\
\text { f.u. }{ }^{3}\end{array}$ & & $\begin{array}{l}68 \% \text { at } 9 \text { ys } \\
\text { f.u. in } 3 \text { year } \\
\text { remission }\end{array}$ \\
\hline & $\begin{array}{l}61 \% \text { at } 10 \text { ys } \\
\text { f.u. } \\
70 \% \text { at } 20 \text { ys } \\
\text { f.u. }\end{array}$ & $\begin{array}{l}79 \% \text { al } 5 \text { ys } \\
\text { f.u }{ }^{3} \\
82 \% \text { at } 8 \text { ys } \\
\text { r.u. } \\
48 \% \text { tree } 2 y^{4} s^{3}\end{array}$ & $\begin{array}{l}6.3 \% \text { at } 5 \text { ys } \\
\text { f.u. }\end{array}$ & & $\begin{array}{l}42 \% \text { at } 10 \text { ys } \\
\text { fu. }{ }^{4} \\
52 \% \text { at } 20 \text { ys } \\
\text { f. }{ }^{4}\end{array}$ & $\begin{array}{l}54 \% \text { at } 9 \text { ys } \\
\text { f.u. in } 5 \text { year } \\
\text { remission }\end{array}$ \\
\hline
\end{tabular}

(1) Collaborative Group for Study of Epilepsy, (2) Untreated epilepsy patients, (3) Cumulative percentage, not considering the continuation of the remission until end of study, (4) Percentage of seizure free patients at moment of follow-up (f.u.). 
It is estimated that in $20-30 \%$ of the patients seizures will continue despite anti-epileptic drug treatment (Annegers et al. 1979, Goodridge and Shorvon 1983b, Shorvon 1991, Cockerell et al. 1995a). Eight percent of the patients does not achieve a seizure free period of one year during 8 years of follow-up. A two year seizure free period was not reached by $18 \%$ of the patients (Elwes et al. 1984). Nevertheless, even those patients who are not in remission 5 years after diagnosis may still have a chance of $33 \%$ of going into a remission during the next 10 years (Annegers et al. 1979).

Differences in remission rates may be attributed to case ascertainment, e.g. hospital based studies contain an overrepresentation of the chronic uncontrolled patients, with newly diagnosed patients and patients in remission are underrepresented. Furthermore, differences in duration of follow-up or discrepancies in the definition of the term remission may account for the differences in the percentages of people who are seizure free (Shorvon, 1984).

Also the definition of epilepsy is important for assessing the remission rates. Several studies show a higher remission rate when patients with possible epilepsy are included in the study (Reynolds 1987, Hart et al. 1990, Collaborative Group for Study of Epilepsy 1992, Cockerell et al. 1995a,b). All studies presented in Table 2.6-I include patients with more than one seizure.

\subsubsection{Problems in establishing prognostic factors}

Although some studies suggest that epilepsy may remit spontaneously most studies appear to support an observation made by Gowers that epilepsy is a process which in case it is not treated early may lead to a chronic state of seizures which are more difficult to treat later on (Elwes et al. 1988, Reynolds 1988, Elwes and Reynolds 1990, Reynolds 1990). In this view factors influencing the outcome of the disease are important.

Studies concerning the prognosis of epilepsy are difficult to assess as their results depend on the medical setting where the data are obtained. In contrast with studies in a clinical setting one may expect higher remission rates of epilepsy in community based studies, given the fact that some of the patients do not visit their physician because the seizures do not worry them (Zielinski 1974, Haerer et al. 1986, Keränen and Riekkinen 1993).

If studies on epilepsy are undertaken with patients who had only one seizure, patients and doctor's delay may introduce a bias towards less severely diseased patients. The longer the delay the higher the probability that the patient suffered another seizure and is not included in the study.

\subsubsection{Prognostic factors}

In the literature various conditions are mentioned that suggest a poor prognosis (Annegers et al. 1979, Shorvon and Reynolds 1982, Elwes et al. 1984, Shorvon 1984, Beghi and Tognoni 1988, van Donselaar 1990, Collaborative Group for Study of Epilepsy (CGSE) 1992):

- A high frequency ( $\geq 2$ per month) of tonic-clonic seizures before treatment

- More than one seizure in the first year of treatment or a relapse early during treatment

- Multiple types of seizures or partial seizures

- Additional neurologic, psychiatric or social handicaps as well as a family history of epilepsy 
Table 2.6-II: Prognostic factors for the course of epilepsy.

\begin{tabular}{|c|c|c|c|c|c|c|}
\hline First author & $\begin{array}{l}\text { Annegers } \\
(1979) \\
N=618\end{array}$ & $\begin{array}{l}\text { Shorvon } \\
(1982) \\
N=94\end{array}$ & $\begin{array}{l}\text { l:lwes } \\
(1984) \\
N=106\end{array}$ & $\begin{array}{l}\text { Hopkins } \\
(1988 \text { ) } \\
N=408\end{array}$ & $\begin{array}{l}\text { CGSE } \\
(1988) \\
N=283\end{array}$ & $\begin{array}{l}\text { v. Donselaar } \\
(1990) \\
N=151\end{array}$ \\
\hline Study & retrospective & prospective & prospective & prospective & prospective & prospective \\
\hline Setting & $\begin{array}{l}\text { community } \\
\text { based }\end{array}$ & neur.clinic & neur clinic & $\begin{array}{l}\text { in/out } \\
\text { private pat }\end{array}$ & multicentre & hospital \\
\hline $\begin{array}{l}\text { Type of } \\
\text { patients }\end{array}$ & new referrals & $\begin{array}{l}\text { new referrals } \\
6-77 \text { years } \\
\mu=23\end{array}$ & $\begin{array}{l}\text { new referrals } \\
6-77 \text { years } \\
\mu=23\end{array}$ & $\begin{array}{l}\text { new referrals } \\
>16 \text { years }\end{array}$ & $\begin{array}{l}\text { new referrals } \\
2-81 \text { years } \\
\mu=19\end{array}$ & $\begin{array}{l}\text { new referrals } \\
15-85 \text { years } \\
\mu=38\end{array}$ \\
\hline $\begin{array}{l}\text { Moment of } \\
\text { inclusion }\end{array}$ & $>2$ seizures & $>2$ seizures & $>2$ seizures & $I^{\text {st }}$ seizure & $\begin{array}{l}\text { start treat- } \\
\text { ment }\end{array}$ & $1^{\text {st }}$ seizure \\
\hline $\begin{array}{l}\text { Therapy } \\
\text { before } 2^{\text {nd }} \\
\text { seizure }\end{array}$ & included & excluded & excluded & included & excluded & excluded \\
\hline Follow-up & $\begin{array}{l}328>10 \text { years } \\
141>20 \text { years }\end{array}$ & 13-33 months & $6-96$ months & 36 months & 2.40 months & 12-24months \\
\hline Age & & not related & & not related & & \\
\hline Sex & not related & not related & & & & \\
\hline $\begin{array}{l}\text { Seizure } \\
\text { type }\end{array}$ & related & & $\begin{array}{l}\text { partial seizu- } \\
\text { res related }\end{array}$ & not related & $\begin{array}{l}\text { multiple sei- } \\
\text { zures related }\end{array}$ & $\begin{array}{l}\text { multiple sei- } \\
\text { zures related }\end{array}$ \\
\hline $\begin{array}{l}\text { Seizures } \\
\text { before/anter } \\
\text { therapy }\end{array}$ & & related & related & & related & \\
\hline $\begin{array}{l}\text { Age at on- } \\
\text { set }\end{array}$ & related & not related & not related & not related & & not related \\
\hline Histories & & not related & $\begin{array}{l}\text { family history } \\
\text { related }\end{array}$ & not related & & not related \\
\hline Time of day & & & not related & related & & related \\
\hline Handicap & related & related & related & & & \\
\hline EEG & & not related & not related & not related & & related \\
\hline
\end{tabular}

Factors not related to the prognosis or factors about which disagreement with respect to their relation to the prognosis of epilepsy exists in the literature (Table 2.6-II) are (Annegers et al. 1979, Shorvon and Reynolds 1982, Elwes et al. 1984, Hopkins et al. 1988, van Donselaar 1990):

- EEG findings after a first idiopathic seizure. Some found a relation between (pretreatment) EEG findings and seizure recurrence. Others, however, did not find such a relation.

- The importance of the time during the day at which the seizure occurs. Some researchers did report a relation between time of the day and the risk of recurrence others deny a prognostic value for this factor. 
- Factors such as gender, age or age at onset. Some report a relation between age at onset and the probability of going into remission. Especially younger patients with idiopathic epilepsy were likely to become seizure free. Such a relation was not observed by others.

\subsection{Some implications of epilepsy}

Epilepsy may have various consequences in daily life. People may experience difficulties in finding and keeping a job, their drivers licence may be withdrawn or they have to find alternative leisure activities.

\subsubsection{Employment}

Difficulties with employment is one of the problems most frequently reported by patients (Thompson and Oxley 1988, Chaplin et al. 1992). The problem seems to be of less relevance during periods of full employment (Crombie et al. 1960). However, during a period of high unemployment it has been shown that $46 \%$ of the potentially economically active patients were unemployed compared with $19 \%$ of a control population (Elwes et al. 1991). The employers as well as colleagues (even within health care environments) have an exaggerated fear of accident-proneness and decrease of productivity (Aspinal 1986, Betts 1986). Contrary to the expectations of employers, sickness absence, injuries and accident frequencies and productivity are about the same for adults with and without epilepsy (Rutgers 1984, Lechtenberg 1985, Dick 1986). Although only some jobs have been affected by statutory barriers, safety concerns were mentioned as the main reason for not hiring people with epilepsy over the years (Craig and Oxley 1986, Hicks and Hicks 1991)

Guidelines have been suggested for employing people with epilepsy. One of the main aspects is an understanding of the basic facts about epilepsy. An applicant with epilepsy should be assessed on an individual basis. The main factors which should be taken into account are the type of epilepsy and its control, associated disorders, characteristics of the individual and suitability for the job (Espir and Floyd, 1986). Job suitability should normally be assumed if a person with epilepsy has the right qualifications and experience (Employment Commission of the International Bureau for Epilepsy, 1989).

\subsubsection{Driving}

Local employment opportunities and public transportation are important factors for the employability of the person with epilepsy as they may experience a restriction of driving privileges (Craig and Oxley, 1988). Several studies have been conducted on the risk of car accidents for epilepsy patients (Parsons 1986, Taylor 1986, Gastaut and Zifkin 1987, Hansotia and Broste 1991). Epilepsy has been mentioned as one of the major causes of collapse behind the steering wheel leading to an accident (Taylor 1986). Statistics show that licensed drivers with epilepsy have only a slightly increased risk of traffic accidents, similar to patients with diabetes (Hansotia and Broste 1991, 1993). Reports on casualties and damage are not consistent (van der Lugt 1972, Gastaut and Zifkin 1987). Whether seizures played a role in accidents may be difficult to establish (Hansotia and Broste 1993). There seems to be a consensus that persons with epilepsy, when licensed to drive, are not at any higher risk of 
having accidents (Commission on Epilepsy, Risk and Insurance, International Bureau for Epilepsy, 1993).

Regulations for holding a drivers licence vary between countries (Aird et al. 1984, Rutgers 1984, Fisher et al. 1994, Krumholz 1994, Shorvon 1995). Some countries require complete freedom from seizures for a period of two years, others require one year. In some countries limited licenses for driving passenger cars are issued for short distance travelling at low density motorways for those people who are dependent on driving for their employment. While restrictions with respect to driving passenger cars may become less strict during the years changes in the limitations for driving heavier and more dangerous vehicles or commercial driving are not expected.

Because of the restrictions, difficulties and costs of obtaining an automobile liability insurance some people with epilepsy do not report their epilepsy to the licensing bureau or drive a car although they are not allowed to do so (van der Lugt 1972, Rutgers 1984). The omission to report having seizures raises the question of who is responsible for reporting the seizures. If physicians were required by law to report the seizure status of their patients to the licensing bureau or when patients have to report their seizure status this would result in illegal driving in $33 \%$ and $53 \%$ of the patients, respectively (Salinsky et al. 1992). In both cases a high rate of non-compliance would be observed, which stresses the importance of having patients educated by their physicians.

\subsubsection{Leisure}

Adverse effects on leisure activities is reported to be a problem by almost one third of the patients ((haplin et al. 1992). Several activities may induce a risk for epilepsy patients when no precautions are taken.

Sports in which head injuries may occur are generally not advisable for people without precautions such as wearing riding caps in horse riding. Of all water sports diving is not advised for persons with recurrent seizures, while the risk of swimming can largely be reduced through a 'buddy system' (Craig and Oxley, 1988). The general approach for people with epilepsy who wish to start some kind of sport activity should be an objective individual assessment whether the activity is feasible given the risk of a seizure.

In about $3 \%$ of new epilepsy patients in the age of 7 to 19 years the first seizures may have been triggered by electronic screen games (Quirk et al. 1995). Television and other visual display units can be considered to be a risk when viewed from a distance less than two meters for a patient in which seizures occur spontaneously and/or are induced by flickering lights or by patterns. Seizures can be prevented easily through covering one eye or the use of polarised glasses and display screens (Harding, 1986)

Although it is generally bulieved that excessive alcohol intake can increase the seizure frequency it is difficult to attribute this increase to alcohol only (Hauser et al. 1988). Continuous alcohol abuse may be associated with poor compliance and disregardance of medication instructions (Chan, 1985). It has been shown that moderate drinking (2-3 glasses/day) did not result in an increase in epileptic seizure frequency. Furthermore, alcohol did not result in a change in plasma drug levels in drugs such as carbamazepine (CBZ), phenobarbiton (PB), phenytoin (PHT) while differences in valproate (VPA) plasma drug levels could not be attributed to alcohol intake (Höppener, 1981). Therefore, a prohibition of alcohol 
for epileptic patients can not be justified, although reservations may exist in those patients who do not comply with the advice provided, have a history of alcohol abuse or have been shown to be sensitive for alcohol.

\subsection{General aspects of anti-epileptic drug treatment}

The occurrence of a seizure will have great impact on the patient as well as on his family. The main objective of drug treatment will be to prevent the occurrence of further fits. The decision to start drug treatment however has consequences for the patient. In order to ensure effective drug treatment these consequences will necessitate an assessment of the pros and cons of drug treatment.

\subsubsection{Reasons for drug treatment}

Justifications for starting pharmacological treatment of epilepsy are its medical and social consequences. As said before, it is commonly assumed that a seizure may increase the chance of the occurrence of additional seizures. This process of 'Bahnung' may eventually lead to recurrent seizures which are difficult to treat (Shorvon and Reynolds 1982, Elwes et al. 1984, Reynolds 1987). Brain damage may develop either due to falling caused by the changes in postural tonus or because of development of neural overactivity inducing a generalised tonicclonic seizure. Status epilepticus is the occurrence of one or multiple seizures for at least 30 minutes during which period the patient is not able to regain normal consciousness (Gastaut and Kugler, 1976). Status epilepticus is accompanied by hypoxia, hypoglycaemia, acidosis and hypotension. Generalised convulsive status epilepticus is a medical emergency, with a mortality rate of about $10 \%$ to $37 \%$ (Aird et al. 1984. Scholtes et al. 1993). Considering these consequences and risks the treatment perspectives become important. As discussed earlier epilepsy has a rather good prognosis with many patients going into remission early during treatment.

\subsubsection{Reasons for refraining from drug treatment}

Being on drug treatment will have consequences of a medical, psychological and/or economical nature for the patient. These consequences may be a reason for not starting drug treatment at all.

From the medical point of view the uncertainty about the fact that the patient has epilepsy or about the specific type of epilepsy can be a reason for refraining from drug treatment. If it is not completely certain that the diagnosis is epilepsy or the type of epilepsy is not well established the best course is to wait and let time decide (Chadwick and Reynolds, 1985). An erroneous diagnosis can cause either refractory epilepsy or the intake of anti-epileptic drugs for nonepileptic paroxysmal attacks of altered awareness. Also the frequency and/or the severity of seizures are important in deciding whether or not to start drug treatment. The impairment caused by the disease should balance or outweigh the impairment caused by the drug treatment. Long-term prophylactic treatment should not be started when seizures appear with years in between, when seizures are mild with negligible psychosocial consequences or when seizures are caused by factors which can be avoided (Jøhannessen et al. 1990).

The attitude of patients towards the drug treatment is important as well. If compliance is not to be expected one should not prescribe drug treatment. One of the reasons is that the 
occurrence of withdrawal seizures due to sudden discontinuation presents considerable risk (Shorvon, 1987). Some state that drug treatment should not be started in alcohol or drug abusers as compliance is expected to be poor in such situations (Jøhannessen et al. 1990).

Side-effects of the drug treatment will probably not outweigh the burden of few, little disabling seizures. The drug treatment may interact with co-medication such as antibiotics, anticoagulants or contraceptives. Besides this, psychological factors can be important. The recurrent intake of medication reminds the patient, the family and colleagues about the fact that the patient is suffering from a chronic disease

Although an early publication of Sonnen (1979) stated that the financial consequences of the treatment of epilepsy are of relevance only in countries such as the United States and India, recent changes in the health care systems cause this factor to be of interest in other countries as well. Perhaps this aspect should be considered to be one of the side-effects of drug treatment (Meinardi, personal communication).

\subsubsection{Goals in anti-epileptic drug treatment}

Anti-epileptic dnug treatment can serve several purposes. The perspective of removing the symptoms and curing the disease is what the physicians aim at, although it is not clear whether the anti-epileptic drugs actually cure the disease. A second goal for anticonvulsive treatment can be the prevention of epilepsy development. This may be achieved through intermittent treatment of fibrile convulsions or temporary treatment after neurosurgical interventions or head trauma. Thirdly, the establishment of a diagnosis ex juvantibus may be the intention of the treatment in those cases in which epilepsy is suspected but can not be diagnosed with certainty

A guideline for approaching the patient is the assumption that the patient wants to get well (Porter, 1984). This sets the goal for the physician: control the seizures and if possible cure the disease. Long-term treatment with anti-epileptic drugs is the normal procedure for the patient who is diagnosed as having epilepsy and experiences seizures which are medically and/or socially disabling. The initiation of the anti-epileptic drug treatment implies that the patient will have to take the drug for several years in a daily regular fashion. Compassionate doctors and responsible patients are necessary for reliable drug intake (Feuerstein et al. 1988).

When the physician faces a patient suffering from a generalised convulsive status epilepticus it is obvious that intervention is necessary. The occurrence of a convulsive status epilepticus is life threatening (Porter 1984, Swartz and Delgado-Escueta 1987. Brodie 1990). Besides identification of the type of status the treatment consists of the correction of causes and precipitating factors.

Initiation of prophylactic anticonvulsive treatment has the goal to prevent the development of epilepsy. One of the diseases which may predispose for developing epilepsy are febrile convulsions. Febrile convulsions are seizures which occur in combination with a febrile disease. They occur mostly in children between the age of 6 months and 5 years. Febrile convulsions may recur: about one third of the patients who had two seizures will have more episodes. About $2 \%$ to $7 \%$ of patients with febrile seizures developed epilepsy (Annegers et al. 1987, Wallace 1987). The prophylactic treatment of febrile seizures with PB or Na-VPA is possible but side-effects such as behavioural disorders, learning disability and hepatotoxicity may occur. Intermittent treatment is another option. Rectal diazepam can be administered by parents to arrest febrile convulsions. 
Prophylactic anti-epileptic drug treatment can be considered also in patients having suffered from head injury or neurosurgery. The number of people in the community suffering from a head injury is large. Each year in the United States some 500.000 people have brain injuries caused by cranial trauma (Hauser and Hesdorffer, 1990). In about 5\% of the head injured patients early seizures occur after the trauma. Phenytoin has been shown to be beneficial for this type of seizures (Temkin et al. 1990). The benefit of other anti-epileptic drugs is not clear. Therefore, also in these patients before initiating anti-epileptic drug treatment one has to weigh the high level of non-compliance and the risk of adverse drug reactions against the uncertain benefit of the treatment of patients with a small risk of actually developing epilepsy (Rimmer and Richens, 1988)

Similar problems arise in the prophylactic treatment of patients who had neurosurgery (Rimmer and Richens 1988, Jennett 1987). Here also a delay in treatment until seizures occur seems more appropriate as little benefit, non-compliance and problems in establishing the moment of withdrawal can be expected when anti-epileptic drug treatment is initiated (Chadwick and Reynolds 1985, Foy et al. 1992)

Finally there is the diagnostic approach. If the diagnosis whether a seizure is epileptic or not can not be established a temporary treatment with an anti-epileptic drug can be used as a diagnostic test. However there is doubt about this procedure. Literature indicates that there is only little room for the diagnosis ex juvantibus (Chadwick and Reynolds 1985, Porter 1984, 1989, Shorvon 1987)

\subsection{Initiation of treatment}

Starting anti-epileptic drug treatment has as a consequence daily intake of anticonvulsive medication for at least two years. As patients perceive this as a cumbersome task, commencing drug treatment also neccesitates to address its withdrawal

\subsubsection{Starting the drug treatment}

The moment drug treatment should be started is not well defined (Rimmer and Richens, 1988). The statement that a patient should have recurrent seizures within a short interval does not specify the duration of this period, which leads to variation in practice. Many physicians would consider a period of one year (Overweg et al. 1981, Rimmer and Richens 1988, Chadwick and Reynolds 1985, Chadwick 1994a). Others would only commence if the recurrence of a seizure takes place within a period of half a year (Sonnen, 1979). Some even start treatment after a first seizure. In a study of 88 patients, with an age of $15-64$ years, it was shown that $31 \%$ of the patients received anti-epileptic drug treatment after their first seizure, while in 2 patients treatment was started to prevent epilepsy (Rutgers, 1984). Van Donselaar (1990) showed that EEG abnormalities after a first idiopathic seizure provided a criterion for selecting patients with a high risk of recurrence, for which drug treatment might be started right away.

The detectability of a seizure may be an important aspect in considerations to commence treatment. Generalised tonic-clonic seizures can hardly be overlooked while others go undetected more easily. This may be the reason why the physician starts treatment earlier when diagnosing tonic-clonic seizures (Porter, 1989). It has been shown that the median number of tonic-clonic seizures before treatment was 3 , while the median number of partial seizures was 20 (Shorvon and Reynolds, 1982). 
Another reason to start the treatment as soon as possible is that early treatment may have been prevented due to patient and/or doctor delay. It has been shown that one third of the patients who experienced a first seizure did not visit a doctor immediately. Within this group $52 \%$ visited their family physician within one year. Half of these patients visited the physician because of a second seizure, while others visited their physician after more than two seizures (Rutgers, 1984). Doctors delay was encountered in $46 \%$ of the patients, mainly in patients who were younger than 20 years at the moment of onset. Some $16 \%$ of the patients had both patient and doctor delay.

It has been shown that in $37 \%$ up to $80 \%$ of the patients with a first seizure a second seizure occurs within one year (Goodridge and Shorvon 1983b, Elwes et al. 1985, Hopkins et al. 1988, Sander et al. 1990). Some suggest early treatment (Elwes et al. 1988, Chandra 1992, Reynolds 1995). However, starting treatment immediately, based on the finding of only one seizure is still questionable. The physician has to establish a correct diagnosis which may be difficult especially if this single event is described incompletely. Furthermore it may be difficult to differentiate between epileptic and pseudo-seizures. Additional studies are needed to answer the question whether treatment after the first seizure prevents chronic epilepsy (First Seizure Trial Group 1993, Chadwick 1995)

Table 2.9-I: Preferred drugs for treatment of specific types of epilepsy. The first and second choice medication is indicated by the number.

\begin{tabular}{|c|c|c|c|c|}
\hline \multirow[b]{2}{*}{$\begin{array}{l}\text { First } \\
\text { authors }\end{array}$} & \multicolumn{4}{|c|}{ Type of epilepsy } \\
\hline & gen.idiopathic & gen.symptoinatic & partial epilepsy & unclassified \\
\hline $\begin{array}{l}\text { Dam } \\
(1982)\end{array}$ & $\begin{array}{l}\text { CBZ,PHT,CIZ, } \\
\text { PB,ETT,PRM, } \\
\text { VPAACZA }\end{array}$ & $\begin{array}{l}\text { SGTC: CBZ,PHT, } \\
\text { CLZ,PB,ETT, } \\
\text { PRM, VPA, } \\
\text { ACZA }\end{array}$ & $\begin{aligned} \text { PEES: } & \text { CBZ,PHT,CLZ, } \\
& \text { PB,ETT,PRM, } \\
& \text { VPA,ACZA } \\
\text { PECS: } & \text { CBZ,PHT, } \\
\text { CLZ,ETT, } & \text { PRM,VPA }\end{aligned}$ & \\
\hline $\begin{array}{l}\text { Meinardi } \\
(1983)\end{array}$ & VPA,PB,ACZA & $\begin{array}{l}\text { VPA,CBZ, PHT,BZP, } \\
\text { ACTH }\end{array}$ & $\begin{array}{l}\text { SP: CBZ,PHT, VPA, } \\
\text { BZP } \\
\text { CP.CBZ,PHT,CLZ, } \\
\text { PRM,VPA }\end{array}$ & \\
\hline $\begin{array}{l}\text { Chadwick } \\
\text { (1990) }\end{array}$ & $\begin{array}{l}\text { SA: I VPA.ESM, } 2 \text { BZP } \\
\text { JME: I VPA } 2 \text { PB } \\
\text { ATC: I VPA } 2 \text { CBZ.PHT }\end{array}$ & $\begin{array}{l}1 \text { VPA,BZP } \\
2 \text { CBZ,PHT,PB }\end{array}$ & $\begin{array}{l}1 \text { CBL.VPA } \\
2 \text { PHT.PB }\end{array}$ & $\begin{array}{l}1 \mathrm{CBZ}, \mathrm{VPA} \\
2 \mathrm{PHT}, \mathrm{PB}\end{array}$ \\
\hline $\begin{array}{l}\text { Meinardi } \\
(1993)\end{array}$ & VPA & VPA & $\begin{array}{l}\text { I CBZ } \\
2 \mathrm{PHT}\end{array}$ & \\
\hline $\begin{array}{l}\text { Chadwick } \\
\text { (1994b) }\end{array}$ & $\begin{aligned} \text { SA: } & 1 \text { VPA.ESM } \\
& 2 \text { BZP.LTG } \\
\text { JME: } & 1 \text { VPA } \\
& 2 \text { PB,LTG } \\
\text { ATC: } & 1 \text { VPA. } \\
& 2 \text { CBZ,PHT,LTG }\end{aligned}$ & $\begin{array}{l}\text { I VPA.BBZI' } \\
2 \text { CI37.I'IT,PB }\end{array}$ & $\begin{array}{l}\text { l CBZ,VPA } \\
2 \text { PHI.PB.VGB }\end{array}$ & $\begin{array}{l}1 \mathrm{VPA} \\
2 \mathrm{CBZ} . \mathrm{PHT}\end{array}$ \\
\hline
\end{tabular}

SA: simple absences, MM: juvenile myoclonic epilepsy, ATC: awakening tonic-clonic seizures, SGTC: secondary generalised tonic clonic. PEES: partial epilepsy elementary symptomatology. PECS: partial epilepsy complex symptomatology. SP: simple partial, CP: complex partial

CBZ; Carbamazepine, PHT: Phenytoin. PB: Phenobarbiton, PRM: Primidon, VPA: Valproate, ESM: Ethosuximide, CLZ: Clonazepun, ETT: Ethotoin. ACZA: Acetizolamide, ACTH: Adrenocorticotropic honnone, BZP: Benzodiazepines, LTG: Lamotrigine VGB: Vigabatrine 


\subsubsection{Drug selection}

The classifications of epilepsies and seizures provide a means for communication. There is no consensus on whether the choice of the applicable drug should be based on the type of the epilepsy or on the type of seizure (Dam et al. 1985, Meinardi 1993). Literature shows that the selection of a drug may be based on the type of epilepsy, the type of seizures or both (Table 2.9-I and Table 2.9-II).

Table 2.9-II: Preferred drugs for treatment of specific seizure types.

\begin{tabular}{|c|c|c|c|c|}
\hline \multirow[b]{2}{*}{ First author } & \multicolumn{4}{|c|}{ Seizure type } \\
\hline & absences & tonic-clonic (GE) & partial & SGTC \\
\hline Jeavons (1977) & $\mathrm{VPA} \rightarrow \mathrm{ESM}$ & $\mathrm{VPA}, \mathrm{CBZ} \rightarrow \mathrm{PHT}$ & $\begin{array}{l}\text { SP: CBZ, VPA } \rightarrow \text { CLP } \\
\text { CP: CBZ } \rightarrow \text { VPA,PHT }\end{array}$ & $\mathrm{VPA}, \mathrm{CBZ} \rightarrow \mathrm{PHT}$ \\
\hline Reynolds (1981) & ESM,CLP,VPA & $\begin{array}{l}\text { PB,PHT,PRM, } \\
\text { CBZ,CLP,VPA }\end{array}$ & $\begin{array}{l}\text { PB,PHT,PRM, } \\
\text { CBZ,CLP,VPA }\end{array}$ & $\begin{array}{l}\text { PB,PHT,PRM, } \\
\text { CBZ,CLP,VPA }\end{array}$ \\
\hline Höppener (1983) & $\begin{array}{l}\text { I VPA,ESM } \\
2 \mathrm{MSM}\end{array}$ & $\begin{array}{l}\text { I CBZ,VPA } \\
2 \text { PB,PRM }\end{array}$ & $\begin{array}{l}\text { I CBZ,PHT } \\
2 \text { VPA,PB,PRM, } \\
\text { CLP,SUL,ACTA, } \\
\text { PCM }\end{array}$ & \\
\hline Shorvon (1985) & ESM,VPA & $\begin{array}{l}\text { CBZ, PHT,PB/PRM, } \\
\text { VPA }\end{array}$ & CBZ,PHT,PB,PRM & CBZ,PHT,PB,PRM \\
\hline Mattson (1989) & ESM,VPA & $\begin{array}{l}\text { I VPA } \\
2 \text { CBZ,PHT,PB,PRM }\end{array}$ & $\begin{array}{l}\text { CBZ,PHT, PB, PRM, } \\
\text { VPA? }\end{array}$ & CBZ,PHT,PB,PRM \\
\hline $\begin{array}{l}\text { Johiunessen } \\
(1990)\end{array}$ & I ESM,VPA & $\begin{array}{l}\text { I CBZ } \\
2 \mathrm{VPA} \\
3 \text { CLP,CLB,PB,PHT }\end{array}$ & $\begin{array}{l}1 \mathrm{CBZ} \\
2 \text { PHT.VPA } \\
3 \text { CL.P }\end{array}$ & $\begin{array}{l}1 \mathrm{CBZ} \\
2 \mathrm{PHT}, \mathrm{VPA} \\
3 \mathrm{CLP}\end{array}$ \\
\hline Scheurer (1990) & $\mathrm{ESM} \rightarrow \mathrm{VPA}$ & $\mathrm{VPA} \rightarrow \mathrm{CBZ}, \mathrm{PHT}, \mathrm{PB}$ & CBZ,PITT,PRM,PB & $\begin{array}{l}\text { CBZ,PHT, PRM,PB, } \\
\text { VPA? }\end{array}$ \\
\hline Mattson (1992) & VPA & CBZ, VPA & $\mathrm{CBZ}$ & $\mathrm{CBZ}$ \\
\hline Wilder (1995) & $\begin{array}{l}\text { ESM,VPA, } \\
\text { LTG,FMT }\end{array}$ & $\begin{array}{l}\text { PHT,CBZ, VPA,GBP, } \\
\text { LTG,FMT }\end{array}$ & $\begin{array}{l}\text { PHT,CHZ,GBP, } \\
\text { LTG,IMT }\end{array}$ & $\begin{array}{l}\text { PHT,CBZ,VPA, } \\
\text { GBP,ITS,FMT }\end{array}$ \\
\hline Brodie (1996) & VPA.ESM & $\begin{array}{l}\text { CBZ,PHT,VPA,PB, } \\
\text { PRM,CLP }\end{array}$ & $\begin{array}{l}\text { CBZ,PHT, VPA,PB, } \\
\text { PRM }\end{array}$ & $\begin{array}{l}\text { CBZ,PHT, VPA,PB, } \\
\text { PRM,CLP }\end{array}$ \\
\hline
\end{tabular}

GE: generalised epilepsy, SGTC: secondary generalised tonic clonic, SP: simple partial, CP: complex partial, a $\rightarrow$ b,c: a followed by $b$ or $\mathrm{c}$

CBZ: Carbamazepine, PHT: Phenytoin, PB: Phenobarbital, PRM: Primidon, VPA: Valproate, ESM: Ethosuximide, MSM Mesuximide, CZP: Clorazepate, CLP: Clonazepan, SUL: Sultiam, ACTA: Acetazolamide, PCM: Phenacemide, CLB: Clobazam, LTG: Lamotrigine, GBP: Gahapentin, FMT: Felbanate

Some use the type of epilepsy for the selection of the drug of first choice. They recommend the use of VPA for idiopathic generalised and generalised symptomatic epilepsy and CBZ for partial epilepsy (Meinardi et al. 1983, Chadwick 1990, Meinardi 1993, Chadwick 1994a,b). Another example is juvenile myoclonic epilepsy which responds only to VPA. Others prefer CBZ for idiopathic generalised and partial epilepsy and differentiate between different types of partial epilepsy (Dam and Kiørboe 1982).

Others prefer the use of the seizure diagnosis to determine the appropriate therapy (Reynolds and Shorvon 1981, Höppener and van der Lugt 1983, Porter 1984, Shorvon 1987, Jøhannessen 1990, Shorvon 1991, Brodie and Dichter 1996). 
Although the seizure type can be used as selection criterion it has been emphasised that also aspects of the type of epilepsy are of importance in making a decision (Dreifuss, 1990). Other investigators state that the seizure type enables to establish a relation between the physiologi$\mathrm{cal}$ and neurochemical factors of seizures and their response to drug treatment. Nevertheless, they advocate a pragmatic approach in the use of classifications for drug selection. Thus, the use of a classification of seizure types or epilepsies should be performed as far as this is relevant for the selection of the appropriate therapy (Aird et al. 1984).

\subsubsection{Monotherapy or polypharmacy}

Until the 1970s the traditional approach to treatment of epilepsy was twofold. In the first approach treatment with one drug was started and gradually the dose was increased until the seizures were controlled or toxic side-effects developed. In the second approach two drugs were used in the hope of a synergistic effect or under the assumption that the toxicity of two drugs in a lower dose would be less than the toxicity of one drug at a higher dose. In both approaches the dosage was changed or other drugs were added if seizures were not controlled. During the seventies a change in the view on prescribing anti-epileptic drugs developed. Studies on the effectiveness of polypharmacy indicated that it is unnecessary in newly diagnosed patients (Reynolds et al. 1976, Shorvon and Reynolds 1977, Shorvon et al. 1978, 1980, Reynolds and Shorvon 1981). The general principle that evolved was that in newly diagnosed patients combination therapy should be tried only when monotherapy with several drugs failed (Dam and Kiørboe 1982, Rowan 1983, Höppener and van der Lugt 1983, Aird et al. 1984, Shorvon 1987. Rimmer and Richens 1988, Porter 1989, Jøhannessen et al. 1990. Scheuer and Pedley 1990)

Reynolds (1980) discusses two studies that suggest that polypharmacy may lead to an increased risk of seizures. Side-effects decreased when fewer drugs were prescribed. However, Lammers et al. (1995) have suggested that this may be due to the decrease of drugload and is not caused by the reduction in number of drugs. As was discussed by Höppener and van der Lugt (1983) the introduction of a second drug may cause changes in antiepileptic drug levels of the newly introduced as well as of the used drug. These changes vary over patients. An additional problem concerning side-effects in polytherapy is the determination of the drug responsible for the adverse reaction. The compliance of the patient may be lower when using polytherapy which may result in incomplete seizure control. Furthermore, the costs of the therapy may be lower if one or more of the prescribed drugs is withdrawn.

However, some patients can benefit from polytherapy. It has been reported that in $11 \%$ of the patients, that were given polytherapy because monotherapy failed, complete seizure control was achieved. A reduction in the seizure frequency was observed in $40 \%$ (Mattson et al. 1985). It has been suggested that a systematic change of drugs is necessary using the most effective drug as a background regimen while the other individual drugs should be added and tested sequentially (Shorvon, 1991)

Insight in drug mechanisms is growing. Anti-epileptic drugs seem to decrease membrane excitability by interacting with neurotransmitter receptors and ion channels (Macdonald and Kelly 1994, Montouris 1995, Brodie and Dichter 1996). More recently new drugs VGB, LTG and GBP have been introduced which can be used as add-on therapy (Wilder, 1995). Combination therapy may become more common as drugs with synergistic effects become available (Chadwick, 1994b). 


\subsubsection{Efficacy of drugs}

All new anti-epileptic drugs are subjected to efficacy trials. Also comparative studies of the relative efficacy of major anti-epileptic drugs have been published. Table 2.9-III lists the studies concerned with CBZ, PHT, VPA, PRM and PB. The assessment of studies on the efficacy of anticonvulsive drugs is difficult because of differences in the studied populations, differences in study design, the small number of patients involved in the studies, differences in outcome measures and short duration of follow-up. The paroxysmal character of the epilepsy makes the duration of follow-up an important aspect. Furthermore, a short follow-up period may result in a bias in favour of drugs for which tolerance can be developed during long-term intake. Because of these problems it is not possible to draw definite conclusions about differences in efficacy of anticonvulsives using results from randomised controlled comparison studies of anti-epileptic drugs (Gram et al. 1982, Chadwick and Turnbull 1985, Beghi et al. 1986).

More recently larger drug trials have been conducted. Mattson et al. (1992) studied the efficacy of CBZ and VPA in a randomised double-blind trial in patients with complex partial seizures and secondary generalised tonic-clonic seizures. They did not find a difference between the two drugs in controlling the tonic-clonic seizures. However, for the complex partial seizures CBZ showed a better control and fewer long term side-effects. They conclude that $\mathrm{CBZ}$ should be used as a drug of first choice for partial epilepsy because about $50 \%$ of the patients with partial complex seizures do also suffer from generalised seizures. The higher number of side-effects for VPA however, may have been caused by an upper range plasma drug level which was higher than is usual in clinical practice (Reynolds et al 1993). Others report that $\mathrm{CBZ}$ and VPA are equally effective while a higher withdrawal rate because of sideeffects was observed for CBZ (Richens et al. 1994, Heller 1995).

\subsubsection{Dose of drugs}

Once a drug has been selected the intention is to control seizures without the development of toxic side-effects. The consensus is that the anti-epileptic drugs can be used more effectively with the assistance of serum level monitoring (Richens and Ahmad 1975. Shorvon et al. 1978, Reynolds 1980, Rimmer and Richens 1988, Commission on Anti-epileptic Drugs of the ILAE, 1993). Considerations about efficacy, toxicity and the plasma drug levels resulted in the concept of therapeutic or optimal range. Within this range seizure control is expected to be optimal while the toxic effects are expected to be minimal

Another important aspect of the therapeutic range is the definition of an upper level above which additional control of seizures is unlikely and toxic side-effects can be expected. Unfortunately, the therapeutic range can not be used to predict the efficacy of the treatment for an individual patient (Shorvon 1987, Lammers 1994). Nevertheless these ranges, which are based on averages for large populations, can also be used for the individual case at onset of therapy. One should be aware that differences in opinion on the appropriate plasma drug levels exist (Table 2.9-IV) 
Table 2.9-IV: Overview of therapeutic ranges in $\mathrm{mg} / \mathrm{l}$ presented in the literature

\begin{tabular}{lllllll}
\hline First author & CBZ & PHT & PB & PRM & VPA & ESM \\
\hline Jeavons (1977) & $4-10$ & $10-25$ & $15-40$ & $n a v^{1}$ & $60-100$ & $40-120$ \\
Schobben (1979) & $4-8$ & $7-12$ & $20-35$ & $7-10$ & $50-80$ & $40-80$ \\
Sonnen (1979) & $5-10$ & $10-20$ & $15-40$ & $4-12$ & $40-80$ \\
Reynolds (1980) & $4-10$ & $10-20$ & $15-40$ & $15-40^{2}$ & $50-100$ & $40-80$ \\
Daun (1982) & $4-10$ & $10-20$ & $15-30$ & nav & nav & $40-90$ \\
Höpjwener (1983) & $4-10$ & $10-20$ & $15-40$ & nav & $?$ & $40-120$ \\
Rowan (1983) & $8-12$ & $15-20$ & $15-40$ & $5-8$ & $?$ & $60-100$ \\
Overweg (1984) & $3-12$ & $10-20$ & $10-40$ & $4-12$ & $30-120$ & $40-120$ \\
Shorvon (1987) & $3.5-11.5$ & $7-20$ & $9-40$ & $9-40^{3}$ & nav. & $40-100$ \\
Rimmer (1988) & $5-10$ & $10-20$ & $15-40$ & $15-40^{1}$ & $50-100$ & $50-100$ \\
Porter (1989) & $4-10$ & $10-20$ & $10-40$ & $5-15$ & $50-100$ & $50-100$ \\
& $7^{3}>8^{4}$ & $18^{3}>20^{4}$ & $35^{3}>40^{4}$ & $10^{3}>12^{4}$ & $80^{3}>100^{4}$ & $80^{3}>100^{4}$ \\
Scheuer (1990) & $8-12$ & $10-20$ & $15-35$ & $5-12$ & $50-120$ & $40-100$ \\
Meijer (1991) & $3-7$ & $10-20$ & $20-40$ & nav & $50-90$ & $50-90$ \\
\hline
\end{tabular}

CBZ: Carbamazepine, PHT: Phenytoin, PB: Phenobarbital, PRM: Primidon, VPA: Valproate, ESM: Ethosuximide (1)nav: not available. (2)Therapeutic range of phenobarbital is used for primidon, (3)Level to be reached in refractory seinures, (4)Toxic level

Some authors do not present the therapeutic range values for Na-VPA because the significance of the drug monitoring for this drug is unknown. As large changes in VPA serum level during the day occur it is not possible to correlate side-effects and serum concentration for this drug (Höppener and van der Lugt, 1983). Differences also exist in the presented optimal serum levels for PRM. Some use the real PRM serum values for establishing the therapeutic range of the drug (Sonnen 1979, Rowan 1983, Overweg 1984, Scheuer and Pedley 1990). Others present the values of PB for PRM because PB is produced during the metabolisation of PRM (Reynolds 1980, Shorvon 1987, Rimmer and Richens 1988, Porter 1989).

Also for carbamazepine the therapeutic ranges vary in the literature. Some found mean blood drug levels of 4.7 to $6.5 \mathrm{mg} / \mathrm{l}$ for carbamazepine in successfully treated patients, while therapy failure was observed at a mean plasma level of $9.8 \mathrm{mg} / \mathrm{l}$ (Ramsay et al 1983). Others use a range of 3-12 mg/l (Overweg 1984, Shorvon 1987), while again others state that the therapeutic range starts at $8 \mathrm{mg} /$ (Rowan 1983, Scheuer and Pedley 1990). It has been mentioned that drug toxicity can be expected starting from $8 \mathrm{mg} / \mathrm{l}$ for this drug (Porter 1989)

A similar variation in therapeutic ranges was observed for PHT, PB and ISM. Lower therapeutic levels are quoted, respectively from $7-15,9-20$ and $40-60 \mathrm{mg} /$, while upper therapeutic levels vary from $12-25,30-40$ and $80-120 \mathrm{mg} /$.

The differences in the patient population or the treatment strategy as well as the possibility that the therapeutic ranges are determined more by the desired anti-epileptic effect than by the appearing side-effects may be the cause for the variation in therapeutic ranges

Besides the interindividual variability in therapeutic values it has been suggested that intra individual variability in therapeutic AED serum levels exist. Schmidt and Janz (1980) showed that the initial therapeutic concentration of the drugs PHT and PB for controlling seizures was 
Table 2.9-IV: Overview of therapeutic ranges in $\mathrm{mg} / \mathrm{l}$ presented in the literature

\begin{tabular}{lllllll}
\hline First author & CBZ & PHT & PB & PRM & VPA & ESM \\
\hline Jeavons (1977) & $4-10$ & $10-25$ & $15-40$ & $n a v^{1}$ & $60-100$ & $40-120$ \\
Schobben (1979) & $4-8$ & $7-12$ & $20-35$ & $7-10$ & $50-80$ & $40-80$ \\
Sonnen (1979) & $5-10$ & $10-20$ & $15-40$ & $4-12$ & $40-80$ \\
Reynolds (1980) & $4-10$ & $10-20$ & $15-40$ & $15-40^{2}$ & $50-100$ & $40-80$ \\
Daun (1982) & $4-10$ & $10-20$ & $15-30$ & nav & nav & $40-90$ \\
Höpjwener (1983) & $4-10$ & $10-20$ & $15-40$ & nav & $?$ & $40-120$ \\
Rowan (1983) & $8-12$ & $15-20$ & $15-40$ & $5-8$ & $?$ & $60-100$ \\
Overweg (1984) & $3-12$ & $10-20$ & $10-40$ & $4-12$ & $30-120$ & $40-120$ \\
Shorvon (1987) & $3.5-11.5$ & $7-20$ & $9-40$ & $9-40^{3}$ & nav. & $40-100$ \\
Rimmer (1988) & $5-10$ & $10-20$ & $15-40$ & $15-40^{1}$ & $50-100$ & $50-100$ \\
Porter (1989) & $4-10$ & $10-20$ & $10-40$ & $5-15$ & $50-100$ & $50-100$ \\
& $7^{3}>8^{4}$ & $18^{3}>20^{4}$ & $35^{3}>40^{4}$ & $10^{3}>12^{4}$ & $80^{3}>100^{4}$ & $80^{3}>100^{4}$ \\
Scheuer (1990) & $8-12$ & $10-20$ & $15-35$ & $5-12$ & $50-120$ & $40-100$ \\
Meijer (1991) & $3-7$ & $10-20$ & $20-40$ & nav & $50-90$ & $50-90$ \\
\hline
\end{tabular}

CBZ: Carbamazepine, PHT: Phenytoin, PB: Phenobarbital, PRM: Primidon, VPA: Valproate, ESM: Ethosuximide (1)nav: not available. (2)Therapeutic range of phenobarbital is used for primidon, (3)Level to be reached in refractory seinures, (4)Toxic level

Some authors do not present the therapeutic range values for Na-VPA because the significance of the drug monitoring for this drug is unknown. As large changes in VPA serum level during the day occur it is not possible to correlate side-effects and serum concentration for this drug (Höppener and van der Lugt, 1983). Differences also exist in the presented optimal serum levels for PRM. Some use the real PRM serum values for establishing the therapeutic range of the drug (Sonnen 1979, Rowan 1983, Overweg 1984, Scheuer and Pedley 1990). Others present the values of PB for PRM because PB is produced during the metabolisation of PRM (Reynolds 1980, Shorvon 1987, Rimmer and Richens 1988, Porter 1989).

Also for carbamazepine the therapeutic ranges vary in the literature. Some found mean blood drug levels of 4.7 to $6.5 \mathrm{mg} / \mathrm{l}$ for carbamazepine in successfully treated patients, while therapy failure was observed at a mean plasma level of $9.8 \mathrm{mg} / \mathrm{l}$ (Ramsay et al 1983). Others use a range of 3-12 mg/l (Overweg 1984, Shorvon 1987), while again others state that the therapeutic range starts at $8 \mathrm{mg} /$ (Rowan 1983, Scheuer and Pedley 1990). It has been mentioned that drug toxicity can be expected starting from $8 \mathrm{mg} / \mathrm{l}$ for this drug (Porter 1989)

A similar variation in therapeutic ranges was observed for PHT, PB and ISM. Lower therapeutic levels are quoted, respectively from $7-15,9-20$ and $40-60 \mathrm{mg} /$, while upper therapeutic levels vary from $12-25,30-40$ and $80-120 \mathrm{mg} /$.

The differences in the patient population or the treatment strategy as well as the possibility that the therapeutic ranges are determined more by the desired anti-epileptic effect than by the appearing side-effects may be the cause for the variation in therapeutic ranges

Besides the interindividual variability in therapeutic values it has been suggested that intra individual variability in therapeutic AED serum levels exist. Schmidt and Janz (1980) showed that the initial therapeutic concentration of the drugs PHT and PB for controlling seizures was 
higher than the AED serum therapeutic concentration measured during the maintenance period of the treatment. In a study on withdrawal of anti-epileptic medication in 1013 patients seizure free for at least 2 years, it was found that $30 \%, 76 \%, 67 \%$ and $51 \%$ of the patients using $\mathrm{CBZ}$, $\mathrm{PB}, \mathrm{PHT}$ or VPA, respectively, had AED serum levels in the subtherapeutic range (Medical Research Council AED Withdrawal Study Group, 1991). These differences in therapeutic levels have been attributed to differences in the activity of the epileptic process.

Also the recommendations on the normal daily doses diverge (Table 2.9-V). For establishing the daily dose some use the body weight of the patient (Dam and Kiørboe 1982, de Krom and van der Lugt 1982, Overweg 1984). Others use a more global approach with a predefined amount of milligrams per day (Sonnen 1979, Aird et al. 1984, Lechtenberg 1985, Shorvon 1987, Rimmer and Richens 1988, Brodie and Dichter 1996).

Table 2.9-V: Normal daily dose of anti-epileptic drugs. In the upper part of the table the daily dose is presented in $\mathrm{mg}$. The lower part of the table presents the daily dose of the $\mathrm{drug}$ in $\mathrm{mg} / \mathrm{kg}$ body weight.

\begin{tabular}{llllllll}
\hline & First author & CBZ & PHT & PB & PRM & VPA & ESM \\
\hline \multirow{4}{*}{ daily } & Sonnen (1979) & $400-1600$ & $250-500$ & $100-200$ & $500-1000$ & $900-2400$ & $500-1500$ \\
& Aird (1984) & $600-1200$ & $100-500$ & $100-300$ & $250-1000$ & $1500-3000$ & $500-1500$ \\
dose & Lechtenberg (1985) & $600-1200$ & $300-400$ & $100-150$ & $750-1500$ & $1000-3000$ & $750-2000$ \\
& Shorvon (1987) & $400-1800$ & $200-500$ & $60-180$ & $250-1000$ & $600-2500$ & $500-1500$ \\
& Rimmer (1988) & $400-1800$ & $150-600$ & $30-240$ & $250-1500$ & $600-3000$ & $500-1500$ \\
& Brodic (1996) & $400-2000$ & $100-700$ & $60-240$ & $250-1500$ & $500-3000$ & $500-2000$ \\
\hline \multirow{4}{*}{$\mathrm{mg} / \mathrm{kg}$} & Daun (1982) & $10-20$ & 5 & $1.5-3.0$ & $12-20$ & $900-1800$ & $20-40$ \\
& de Krom (1982) & $8-12$ & $5-7$ & $2-5$ & nav & $20-30$ & $8-12$ \\
& Ovcrweg (11984) & $15-20$ & 5 & $3-5$ & 10 & 20 & $15-20$ \\
\hline
\end{tabular}

CBZ: Carbanazepine, PHT: Phenyoin, PB: Phenobarbital, PRM: Primidon, VPA: Valproate, ESM: Ethosuximide (1) Maily dose in mg

The relation between dose and seizure control is only statistical. The physician has to decide for the individual patient whether a high dose or not is needed to achieve results immediately (Porter, 1989). Some state that AED serum levels should be in the upper part of the therapeutic range (Rowan 1983). Others found that up to one third of the patients will achieve control of seizures with a level below the therapeutic range (Reynolds et al. 1976, Shorvon et al. 1978, 1980). Control was achieved with 200-300 mg PHT per day or 200-400 mg CBZ per day. Seizure control has been observed even with a low starting dose of e.g. $100 \mathrm{mg}$ PHT or 100-200 mg CBZ (Reynolds 1980. Shorvon et al. 1978). An approach for drug treatment is to start treatment with a low dose and if no effect is observed increase the dose to try to reach the therapeutic range (Höppener and van der Lugt 1983, Shorvon 1985, Chadwick 1990). Another approach is to use the middle of the target range (Mattson et al. 1985, 1992). To assure compliance it is preferable not to increase the dose to a level where toxicity may be expected in spite of recent publications which advocate a return to pre drug level monitoring strategies. 


\subsection{Maintenance}

After drug treatment has been initiated it is necessary to see the patient on a regular basis. During these visits the seizures may remit after a short time of drug treatment, show some response to drugs with a tendency to relapse or turn out to be intractable in which remission is unusual. Furthermore, the correctness of the diagnosis and the development of side-effects have to be assessed. During each of these visits it is advisable to check compliance to support and encourage the patient to comply with the treatment as to improve or maintain seizure control.

\subsubsection{Recurrence of seizures}

After having achieved a remission a relapse may occur. The probability of a relapse during the first 5,10 and 20 years after entering remission is $8 \%, 15 \%$ and $24 \%$, respectively (Annegers et al. 1979). This estimate includes patients on and off anti-epileptic drug treatment.

Recurrence of seizures can be caused by misdiagnosis. Jeavons (1977) stated that 270 of the 1172 patients at his epilepsy clinic did not have epilepsy. Fifty percent of the adults in the nonepilepsy group were misdiagnosed because of syncope. Second to this there were misdiagnoses due to psychiatric disorders. The choice of an ineffective drug may be another reason for occurrence of seizures during treatment. Even when a first choice drug is prescribed it is not necessarily the most effective drug for a specific patient. Suboptimal serum levels can also be a cause of the continuation of seizures

Non-compliance is another reason for continuation of seizures or their reoccurrence after a seizure free period. If complete control of seizures has been achieved the compliance may decrease because of absence of seizures (Feuerstein et al. 1988). Although non-compliance can be the cause of a relapse, the correctness of the diagnosis should be evaluated continuously as a unrecognised concomitant neurological disease may develop.

Finally, there is a group of patients in whom complete seizure control cannot be established although plasma drug levels in the therapeutic range have been reached. Drug resistant epilepsy which is only partly responsive to drug treatment is present in about $20 \%$ of the patients in the UK. Another $20 \%$ of the patients have epilepsy which is completely unresponsive to drug treatment (Shorvon, 1991).

\subsubsection{Drug monitoring}

Plasma drug levels together with clinical findings can be used to determine the effectiveness of the drug treatment and provide a basis for adjustment of the dose of the drug. The practical use of plasma drug levels has been studied amongst others by Beardsley et al. (1983) and Meijer (1991). Beardsley et al. showed in a retrospective study of 164 adult epileptic patients that prescribers did not use the blood levels properly in $17 \%$ of the therapeutic decisions. If they did use the plasma drug levels correctly an improvement in the control of the seizures occurred in $51 \%$ of the patients. Inappropriate decisions caused an improvement in seizure frequency in only $5 \%$ of the patient, while in $32 \%$ of the patients with an inappropriate decision the seizure frequency worsened

Meijer (1991) studied the decisions of 16 physicians. From a retrospective analysis of 1006 therapeutic decisions in 64 patient records he concluded that approximately $66 \%$ of the decisions to adapt the dosage of phenytoin and carbamazepine, and $80 \%$ of the decisions on 
changes in dosage of valproate were done in agreement with the serum concentration and clinical signs. Confronting the physicians with plasma drug levels in the subtherapeutic and toxic range led also to variance in the treatment strategy. Although some differences occurred in cases with subtherapeutic levels, most of the variance in decisions was observed for cases with plasma drug levels in the toxic range.

Anti-epileptic drug monitoring may help in discovering the reason for therapy failure. Changes in seizure control can be attributed to e.g. intra individual variation in pharmacokinetic parameters as a consequence of intermittent disease or to the effects of co-medication on plasma drug levels (Höppener and van der Lugt 1983, Scheuer and Pedley 1990). Drug monitoring can facilitate the diagnosis of symptoms possibly caused by drug toxicity and can help to predict whether a change in the drug dose is likely to result in toxic side-effects (Milano Collaborative Group for Studies on Epilepsy, 1980).

Anti-epileptic drug levels should be used as a guide rather than a rigid goal for the optimal use of the drug. The observed levels depend on the drug half-life and can be affected by the interval between the time of drug intake and the moment of blood sampling. Furthermore, inter-individual differences in optimal levels as well as intra individual variation may occur. It has been shown that patients who are seizure free with levels in the sub therapeutic range do not always benefit from an increase in the dose leading to a therapeutic range level (Woo et al. 1988). Thus, clinical judgement should not be substituted by the therapeutic ranges.

Warnings against the overuse of AED monitoring have been published, as a strict relation between serum levels and efficacy and/or toxicity is not available for an individual patient (Chadwick, 1987). Already in the '70s a set of indications for drug monitoring levels was presented (Kutt and Penry, 1974). More recently, guidelines for therapeutic monitoring were presented which amongst other things state that anti-epileptic drug levels should be obtained after:

- Therapy is initiated

- After a change of dosage and/or drug

- In case of poor seizure control or recurrence of seizures which were well controlled previously

Signs or symptoms of intoxication and a routine control of blood levels once or twice a year are also reasons for monitoring (Commission on Anti-epileptic drugs of the ILAE, 1993).

\subsubsection{Change in dosage or drug}

Reasons for changing drug dosage are incomplete control of seizures and the occurrence of side-effects

The appropriate dose for the individual patient preventing the continuation of the seizures can not be determined a priori (Lammers, 1994). In case of persisting seizures the dose will be increased or the drug will be changed depending on the clinical findings. However, frequent changes in drugs or dosages should be avoided (Rowan, 1983). Although the intention is to achieve complete seizure control it is not clear when the dose should be increased: after a reduction in seizure frequency of less than $50 \%$ or less than $90 \%$ or in case any seizure occurs after a steady state has been reached or only after the second seizure.

One should use sufficient time to evaluate therapeutic efficacy. This means at least that the plasma drug level should have reached a steady state before conclusions about the effectiveness of the drug can be drawn. The time period needed to evaluate the effect of treatment on 
the seizure frequency is rarely mentioned in the literature. It has been suggested that a drug should be tried for a period that would be expected to cover three to five seizures or clusters of seizures, or for at least two months whichever period is the longest (Shorvon, 1991). In this period the drug should be provided in a sufficient dose to reach the upper part of the therapeutic range, confirmed by results from repeated drug monitoring.

\subsubsection{Compliance}

Compliance is the extent to which a patient adheres to a given medical and/or health advice. It concerns the drug intake as well as the keeping of therapeutic appointments (Mattson et al. 1988). In general it is estimated that one third to one half of the patients on chronic medication do not follow the medical advice about the prescription as presented by the physician (Schmidt and Leppik, 1988). Reasons for non-compliance are the loss of individual freedom caused by the need of a regular drug intake, the required changes in life-style or loss of independence because of control by the relatives. Disagreement on the goals for treatment and discordance on the value of the elimination of unhealthy conditions can cause non-compliance as well. Worry about one's health can be caused by a high seizure frequency. Contrary to this, persons with few seizures are more lax about their condition and treatment (Peterson et al. 1982). When a patient disapproves the duration of the treatment the treatment may be rejected. Furthermore, patients and doctors can have a different perception of the risks posed by the disease and the tolerance of the drug dosage. Patients weigh the consequences of compliance against the threat and consequences of having seizures. This process of weighing can result in non-compliance.

A deliberate change of a treatment by the patient herself is called the self-regulation or selfmanagement of the patient. It stresses the fact that it is the patient who has control over the drug intake. Self-regulation also includes the option that patients can take on their own initiative more drugs than prescribed (Thorbecke, 1988).

Contrary to a deliberate change, non-compliance may be due to inadvertency i.e despite the understanding, the acceptance and the desire to adhere to the treatment. This includes noncompliance due to changes in daily pattern during weekends or holidays, or simply forgetting because of absence of a routine for intake of tablets (Höppener and van der Lugt, 1983).

Some suggest that there is no significant difference in seizure control in patients who comply and those who are non-compliant (Schmidt et al. 1988). However, it has been shown that improved seizure control can be expected if compliance is monitored closely (Wannamaker et al. 1980). This may be accomplished by the measurement of anti-epileptic drug levels and patient interview (Leppik, 1988). The reports of a patient may well be a minimum estimate of non-compliance (Trostle, 1988). The additional use of special medication containers and self recording of seizures and drug intake can be easily implemented and may enable a more complete view on compliance. Furthermore, good compliance may be achieved by convenient regimen characteristics such as simplicity, individualisation and low intake frequency (Peterson et al. 1984). Besides this, a good partnership between patient and physician is considered to be of great importance to achieve successful treatment (Green and Simons-Morton 1988, Leppik and Schmidt 1988). According to patients sufficient time, presentation of consistent clinical information and acknowledgement of the role of the patient in controlling the disease will enhance compliance (Feuerstein et al 1988) 


\subsection{Withdrawal}

Many patients will enter a period of prolonged remission. The question of tapering drug treatment will become more relevant to a patient the longer the duration of the seizure-free period gets.

\subsubsection{Reasons for withdrawal}

Among the reasons for a reduction or discontinuation of anti-epileptic drug treatment are factors such as association of the drugs with idiosyncratic and dose related adverse reactions, risks of chronic toxicity and teratogenicity of the drug. Furthermore, the removal of the stigma of epilepsy can be a reason for patients to discuss the option to stop drug treatment (Chadwick, 1988). Successful withdrawal of anti-epileptic treatment can be an advantage when applying for a job and at medical fitness examinations (van Heycop ten Ham, 1980). It is known that many patients stop drug treatment without informing their physician (Zielinski 1974, Goodridge and Shorvon 1983b).

Table 2.11-I: Relapse rates Juring and after withdrawal of anti-epileptic drugs

\begin{tabular}{|c|c|c|c|c|c|c|c|c|}
\hline First author & Study & Sel* & $N$ & $\mathrm{SF}^{* *}$ & Relapses & Taper & Withdrawn & F.U.** \\
\hline $\begin{array}{l}\text { Juul-Jensen } \\
(1964) \\
(19+i 8)\end{array}$ & $\begin{array}{l}\text { prospect } \\
\text { retrospecl }\end{array}$ & $\begin{array}{l}\mathrm{EC} \\
\mathrm{EC}\end{array}$ & $\begin{array}{l}200 \\
196\end{array}$ & $\begin{array}{l}\geq 2 y s \\
\geq 2 y s\end{array}$ & $\begin{array}{l}70(35 \%) \\
79(40 \%)\end{array}$ & $\begin{array}{l}20(28 \%) \\
20(25 \%)\end{array}$ & $\begin{array}{l}50(72 \%) \\
59(75 \%)\end{array}$ & $\begin{array}{l}m=24 \\
m=60\end{array}$ \\
\hline $\begin{array}{l}\text { Oller-Daurella } \\
(1076) \\
(1977) \\
(1987)\end{array}$ & $\begin{array}{l}\text { retrospect } \\
\text { retrospect } \\
\text { retrospect }\end{array}$ & $\begin{array}{l}\text { nav } \\
\text { nav } \\
\text { nav }\end{array}$ & $\begin{array}{l}356 \\
522 \\
608\end{array}$ & $\begin{array}{l}\geq 5 \text { ys } \\
\geq 5 \text { ys } \\
\geq 5 \text { ys }\end{array}$ & & $\begin{array}{l}\text { nav } \\
\text { nav } \\
\text { nav } \\
\text { nav }\end{array}$ & $\begin{array}{l}29 / 138(21 \%) \\
82 / 241(30 \%) \\
119 / 474(24 \%) \\
25 / 134(19 \%)\end{array}$ & $\begin{array}{l}(6-240) \\
m=120 \\
m=180 \\
m=55\end{array}$ \\
\hline $\begin{array}{l}\text { van Hevcop ten } \\
\text { Ham }(1980)\end{array}$ & prospect & $\mathrm{EC}$ & 156 & $\geq 5$ ys & $49(31 \%)$ & $28(57 \%)$ & $21(43 \%)$ & $\geq 24$ \\
\hline $\begin{array}{l}\text { Overweg } \\
\text { (1981) }\end{array}$ & prospect & $\mathrm{EC}$ & 46 & $\geq 3 \mathrm{ys}$ & $29(63 \%)$ & 21 & 8 & $(9-19)$ \\
\hline $\begin{array}{l}\text { Bouma } \\
(1987)\end{array}$ & prospecl & $D C N$ & 116 & $2 y s$ & $26(22 \%)$ & mav & nav & $\begin{array}{l}m=51 \\
(9-120)\end{array}$ \\
\hline $\begin{array}{l}\text { Arts' } \\
(1988)\end{array}$ & prospecl & NDOC & 146 & $\geq 2 y \mathrm{~s}$ & $37(25 \%)$ & $7(19 \%)$ & $30(81 \%)$ & $\begin{array}{l}m=52 \\
(16-113)\end{array}$ \\
\hline $\begin{array}{l}\text { Callaghan } \\
\text { (1988) }\end{array}$ & prospecl & nav & 92 & $2 y s$ & $31(34 \%)$ & $13(45 \%)$ & $18(55 \%)$ & $\begin{array}{l}m=35 \\
(6-62)\end{array}$ \\
\hline $\begin{array}{l}\text { Med.Res.C. } \\
\text { AED.Withdr. } \\
\text { (1991) }\end{array}$ & $\begin{array}{l}\text { rundom. } \\
\text { prospect }\end{array}$ & $\mathrm{MC}$ & 1013 & $\geq 2$ & $\begin{array}{l}221 / 510 \\
(43 \%)\end{array}$ & $\begin{array}{l}107 / 221 \\
(48 \%)\end{array}$ & $\begin{array}{l}|14 / 22| \\
(52 \%)\end{array}$ & $\begin{array}{l}\text { med }=27 \\
(17,42)^{3}\end{array}$ \\
\hline
\end{tabular}

- Selting: EC: Epilepsy Clin., NDOC: Neurology Dept. Outpatient Clin., DCN: Devision of Child Neurology, $\mathrm{MC}$ multiple centres

* Seizure-free period in years

**Follow-up period in months

(1) nav: not available,

(2) Study contains only children,

(3) Definition of epilepsy includes patients with $\geq 2$ afebrile or $\geq 3$ atypical febrile seizures

(4) Median and $25^{\text {th }}$ and $75^{\text {th }}$ percentiles 
Social factors are a main issue for patients deciding not to taper drug truatment. An example of this is the obtaining of the drivers license which may cause a patient to refuse withdrawal of drug treatment (van Heycop ten Ham, 1980). Other patients may refuse withdiawal hecause they fear the consequences of a seizure at work (Pedley, 1988). Because of these perceived risks an analysis of costs and benefits should be made by the physician and the patient before starting an attempt to taper the drug treatment .

The interpretation of studies regarding relapse during and after withdrawal is diflicult. Otten information on the duration of the remission period before commencement of the withdrawal, the seizure types and syndromes, the time taken for the drug withdrawal and diagnostic criteria for recurrent seizures as well as the follow-up period is missing (Chadwick 1988, Pedley 1988). Papers that do mention these data often cannot be compared because of differences in populations studied (Table 2. I 1-1).

Patient characteristics that influence the decision to withdraw anti-epileptic drugs in a positive or negative way have been published (Chadwick and Reynolds 1985, Dean and Penry 1989). One of the factors in favour is the diagnosis of primary generalised epilepsy. It has been shown that absence seizures recur only in $5-20 \%$. Primary generalised tonic-clonic seizures have a recurrence rate of 20-33\% (Janz and Sommer-Burkhardt 1976, Oller-Daurella and Oller 1987, Callaghan et al. 1988). Patients with partial epilepsy show a higher relapse rate; this may be due to the inclusion of symptomatic seizures (Annergers et al. 1979).

Contradictory findings have been reported about the predictive value of the age at onset of epilepsy for the outcome of the withdrawal of the drugs (Janz and Sommer-Burkhardt 1976, Oller-Daurella and Oller 1987, Berg and Shinnar, 1994).

The relapse rate after drug tapering in children has been observed to be somewhat lower than in adult patients, $31 \%$ and $35 \%$ respectively (Callaghan et al. 1988). A literature survey of studies regarding adults and children indicates that the age may be important, especially in conjunction with other factors such as duration, severity and frequency of seizures before treatment (Dean and Penry, 1989). In another survey the opposite is stated, based mainly on studies in children (Hauser and Hesdorffer, 1990). However, more recently it has been shown that children have a lower risk than adults for recurrence of seizures during the first two years after withdrawal (Medical Research Council Anti-epileptic Drug Withdrawal Study Group, 1993)

The number of seizure-free years before withdrawal has been reported as a determinant for the success of withdrawal. Without mentioning the exact data some suggest that there does not seem to be much difference between the relapse rates of patients who are 5 years seizure free and those that are 10 years seizure free (Oller-Daurella et al. 1977). It has been shown that there does not seem to be a difference in recurrence rate after withdrawal for children treated for 2, 3, 4 or 5 years (Arts et al. 1988).

Recommendations on the duration of the period during which the drug is withdrawn vary from four weeks up to several years (Oller-Daurella et al. 1977, van Heycop ten Ham 1980, Overweg et al. 1981, Bouma et al. 1987, Arts et al. 1988, Callaghan et al. 1988). A comparison of seizure recurrence between drug tapering periods of 6 weeks and 9 months did not show a significant difference in risk of recurrent seizures (Tennison et al. 1989, 1994). A duration of at least three month has been advocated, however mainly on basis of data in children. 


\subsubsection{Moment of relapse}

Most of the studies indicate that the number of patients who relapse during the tapering of drug treatment is between $19 \%$ and $57 \%$ (Table 2.11-1). A randomised multicenter trial showed that $52 \%$ of the patients will experience a relapse after withdrawal of drugs (Medical Research Council AED Withdrawal Study Group, 1991). Other studies report that $43 \%$ up to $81 \%$ of the patients had a seizure after the withdrawal was completed. However, three Spanish studies indicate a lower rate of relapses after drug withdrawal. Only 19-30\% of the patients had seizures after discontinuing the treatment. A reason for this difference may be the longer seizure free period ( $\geq 5$ years) before patients were admitted to the tapering. Furthermore, the long period used for drug tapering - for most of the patients up to a year and some even longer - may have had an selective effect on the patients reaching drug withdrawal (OllerDaurella et al 1976, 1977, Oller-Daurela and Oller 1987).

Most of the patients in which the drug is withdrawn relapsed during the first year after the treatment was discontinued (Juul-Jensen 1968, Oller-Daurella et al. 1976, Janz and SommerBurkhardt 1976, Thurston et al. 1972, 1982, Shinnar et al. 1985, Bouma et al. 1987, Callaghan et al. 1988, Medical Research Council Anti-epileptic Drug Withdrawal Study Group, 1991). Most of the relapses occur even earlier after withdrawal, mainly during the first six months.

Recommencing the anti-epileptic treatment after a relapse during drug tapering or after withdrawal results in control of the seizures in many but not all of the patients. In a group of 31 patients who relapsed and where reinstalled on treatment 20 patients $(64.5 \%)$ became seizure free again for 4-50 months, while seven patients (22.5\%) continued to have seizures (Callaghan et al 1988). A study in children showed that one third of the patients whose drug treatment was reintroduced, remained seizure-free after a second withdrawal period, while $50 \%$ were seizure-free for at least six months. In $17 \%$ of the patients seizures were difficult to control (Arts et al. 1988).

\subsection{Summary and conclusion}

Although studies on epilepsy suffer from differences in terminology and case finding strategies they indicate a prevalence rate of $0.4-0.6 \%$. The incidence rate ranges from $0.03-0.05 \%$. The main part of community studies indicate that $60-75 \%$ of the patients have an epilepsy of unknown aetiology.

One of the first principles for the management of epilepsy is to establish the diagnosis as precisely as possible. Because of the paroxysmal character of epilepsy the diagnosis may be difficult to establish. Other diseases such as diabetes, cardiac disease or syncopes can mimic the epileptic seizures. Epilepsy should be treated as early as possible to prevent a chronic state of seizures which may be difficult to treat. The consequences of late treatment can conflict with the time necessary for establishing the correct diagnosis. An incorrect diagnosis can result either in long term anticonvulsive drug intake for non-epileptic causes or in the development of refractory epilepsy. Thus, if the diagnosis is not certain the physician should let time make things clear.

Epilepsy can be treated through regular daily intake of medication. A two year remission of the disease within three years after onset of treatment has been reported in $50-70 \%$ of the patients, while up to $90 \%$ of the patient may show a remission after more than five years. However, patients with additional neurologic, psychiatric or social handicaps seem to have a 
poor prognosis for achieving a remission of seizures. Although insight in drug mechanisms is growing the question whether the disease is cured or seizures are suppressed has not been answered unequivocally

Deciding to start drug treatment implies that the patient will be on anticonvulsants for at least two years. Reasons for the treatment should be sufficiently explained to the patient and his family. Before the treatment is started the physician should educate the patient on the different aspects of the disease, the reason for drug intake, the duration of the treatment, the prognosis and the possibility of side-effects. While a chronic disease such as epilepsy is treated problems of a medical, psychological, social and economical nature may occur. Thus, whether or not to start treatment requires an assessment of the pros and cons of the disease and the cure.

The treatment should be based on a correct diagnosis. There is hardly any room for an ex juvantibus approach to establish the correct diagnosis. There is no consensus whether the selection of the anti-epileptic drug should be based on the seizure type or the type of epilepsy. Monotherapy is preferred above polypharmacy when starting treatment. However, in the future new drugs may make polytherapy a viable option again. The literature shows little consensus regarding the drugs which should be applied for the different types of seizures or epilepsy. Small differences exist in the effectiveness of the drugs for treatment of the different types of epilepsy. Therefore the selection of drugs is based on other aspects such as occurrence of side effects. Also little consensus exists in the literature on the appropriate daily dose and the required anti-epileptic serum levels

The differences in selection of drugs, appropriate doses and required anti-epileptic drug serum levels make the treatment of epilepsy to a large extent based on trial and error. Regular follow-up contacts enable the physician to monitor the effect of the treatment. Although physicians seem to agree on the goal of drug treatment, $20-30 \%$ of the treatment decisions are questionable.

Withdrawal of drug treatment can be started after a seizure free period of two years. There is no consensus on the duration of the tapering period. In the literature the duration varies from several weeks up to many years. In those patients who start drug withdrawal some 20$50 \%$ will suffer a relapse before the drugs are stopped completely. Most of the seizures reoccur early after withdrawal, mainly during the first 6 months. Reintroducing drug treatment will be successful in about $65 \%$ of these patients. The rest of the patients have seizures which are difficult to control.

Patients suffering from seizures will worry about the cause of the attacks. Having been confronted with the diagnosis the patient may experience negative emotions in contacis with other people. Furthermore, patients may loose their driving license and will be afraid about further impacts of the disease on their social life. Commencing anti-epileptic drug treatment will have consequences for the patient (e.g. daily drug intake for at least two to three years). Having been healthy and suddenly being confronted with a disease with paroxysmal symptoms will make the patients tend to deny the illness and keep carrying on their life as they did before the diagnosis. It is generally accepted that at least one third of the patients do not follow the prescription presented by the physician. Part of this non-compliance may be caused unintentionally. However, self-regulation of the treatment is also an important cause. The patient will resent the medical profession if the physician does not seem to really listen to his problems or does not support him. Absence of a good patient-doctor contact will cause 
compliance to decrease. Issues such as accomplishment of the same goals by the patient and his physician and acknowledgement of the role of the patient in the treatment together with close monitoring of the patient during remissions can improve the compliance of the patient.

Drug treatment is only part of the management of epilepsy. Guidance of patients as well as those who make treatment decisions that influence the lives of the patients is also part of the proper management.

\subsection{References}

Aird R.B., Masland R.L., Woodbury D.M., The epilepsies a critical review, Raven Press, New York, 1984.

Annegers J.F., Hauser A., Elveback L.R., Remission of seizures and relapse in patients with epilepsy, Epilepsia, 20, 1979, pp. 729-737.

Annegers J.F., Hauser A., Shirts S.B., Kurland L.T., The risk of epilepsy following febrile convulsions, The New England Journal of Medicine, 316, 1987, pp.493-498.

Arts W.F.M., Visser L.H., Loonen M.C.B., Tjiam A.T., Stroink H., Stuurman P.M., Poortvliet D.C.J., Follow-up of 146 children with epilepsy after withdrawal of anti-epileptic therapy, Epilepsia, 29, 1988, pp. 244-250.

Aspinal A., The real obstacles. In: Epilepsy and employment (Edwards, Espir and Oxley, Eds.), Royal Society of Medicine Scrvices Limited, London, 1986, pp. 19-20.

Beardsley R.S., Freeman J.M., Appel F.A.. Anticonvulsant serum levels are useful only if the physician appropriately uses them: an assessment of the impact of providing serum level data to physicians, Epilepsia, 24, 1983, pp. 330-335.

Beghi E., Di Mascio R., Tognoni G., Drug treatment of epilepsy, outlines, criticism and perspectives, Drugs, 31, 1986, pp. 249-265.

Beghi E., Tognoni G., Prognosis of epilepsy in newly referred patients: A multicenter prospective study, Epilepsia, 29, 3, 1988, pp. 236-243.

Berg A.T., Shinnar S., Relapse following discontinuation of anti-epileptic drugs: a meta-analysis, Neurology, 44, 1994, pp.601-608.

Bergamini L., Bergamasco B., Benna P., Gilli M., Acquired etiological factors in 1.785 epileptic subjects: clinical anamnestic research, Epilcpsia, 18, 1977, pp. 437-444.

Betts T., Employment of people with epilepsy within the National Health Service. In: Epilepsy and employment (Edwards, Espir and Oxley, Eds.), Royal Society of Medicine Services Limited, London, 1986, pp. 59-66.

Bongers E., Coppoolse J., Meinardi H., Posthume E., Zijl C. van. A survey of epilepsy in Zeeland The Netherlands, Instituut voor Epilepsiebestrijding Meer en Bosch - De Cruquiushoeve, Heemstede, 1976.

Bouma P.A.D., Peters A.C.B., Arts R.J.H.M., Stijnen Th, Rossum J. van, Discontinuation of antiepileptic therapy: a prospective study in children, Journal of Neurology. Neurosurgery and Psychiatry, 50, 1987, pp. 1579-1583.

Brodie M J. Status epilepticus in adults. The Lancet, 336, 1990, pp. 551-552

Brodic M.J.. Dichter M.A. Anti-cpileptic drugs, The New England Journal of Medicine, 334, 1996, pp. $168-175$

Callaghan N. Garrett A., Goggin T., Withdrawal of anticonvulsant drugs in patients free of seizures for two years, The New England Journal of Midicimi, 318, 1988, pp. 942-946. 
Cereghino J.J, Epidcmiology of epilepsy, Public Health Reviews. 3, 1974, pp. 91-100.

Cereghino J.J., Brock J.T., Meter J.C. van, Penry J.K., Sinith L.D., White B.G., Carbamazepine for epilepsy, a controlled prospective evaluation, Neurology, 1974, pp. 401-410.

Cereghino J.J., Introduction in anti-epileptic drugs. In: Anti-epileptic drugs, (Levy, Mattson, Meldrum, Penry and Dreifuss, Eds.), $3^{\text {rd }}$ edition, Raven Press, New York, 1989, pp. 23-27.

Chadwick D.W., Overuse of monitoring of blood concentrations of anti-epileptic drugs, British Medical Journal, 294, 1987, pp. 723-724.

Chadwick D., Drug withdrawal and epilepsy, when and how ? Drugs, 35, 1988, pp. 579-583.

Chadwick D., Diagnosis of epilepsy, The Lancet, 336, 1990, pp. 291-296.

Chadwick D., Epilepsy after first scizures: risks and implications, Journal for Neurology, Neurosurgery and Psychiatry, 54, 1991, pp. 385-387.

Chadwick D., Epilepsy, Journal of Neurology, Neurosurgery and Psychiatry, 57, 1994a, pp.264-277.

Chadwick D., Standard approach to anti-epileptic drug tre:atment in the United Kingdom, Epilepsia, 35 (Suppl.4), 1994b, s3-s10

Chadwick D., Case for early treatment is not established, British Midical Journal, 310, 1995, pp.177178.

Chadwick D., Reynolds E.H., When do epileptic patients need treatment? Starting and stopping medication, Brilish Medical Journal, 290, 1985, pp. 1885-1888.

Chadwick D., Tumbull D.M. The comparative efficacy of anti-epileptic drugs for partial and tonicclonic seizures, Journal of Neurology. Neurosurgery and Psychiatry, 48, 1985, pp. 1073-1077.

Chan A.W.K., Alcoholism and epilepsy, Epilepsia, 26, 1985, pp. 323-333.

Chandra B., First seizure in adults: to treat or not to treat, Clinical Neurology and Neurosurgery, 94 (Suppl.), 1992, s61-s63.

Chaplin J.E., Yepez Lasso R., Shorvon S.D., Floyd M., National general practice study of epilepsy: the social and psychological effects of the recent diagnosis of epilepsy, British Medical Journal, 304, 1992, pp. 1416-1418

Cockerell O.C., Eckle 1., Goodridge D.M., Sander J.W., Shorvon S.D., Epilepsy in a population of 6000 re-examined: secular trends in first attendance rates, prevalence, and prognosis, Journal for Neurology, Neurosurgery and Psychiatry, 58, 1995a, pp. 570-576

Cockerell O.C, Johnson A.L., Sander J.W., Hart Y., Shorvon S., Remission of epilcpsy. results from the National Gencral Practice Study of Epilepsy, The Lancet, 346, 1995b, pp. 14(1)-144

Collaborative Group for Study of Epilepsy. Prognosis of epilepsy in newly refurred patients: a multicenter prospective study of the effects of monotherapy on the long-term course of epilepsy. Epilepsia, 33, 1992, pp. 45-51

Commission on Anti-epileptic Drugs of the International League Against Epilepsy, Guidelines for therapeutic monitoring on anti-epileptic drugs, Epilepsia, 34, 4, 1993, pp. 585-587

Commission on Classification and Terminology of the Intemational League Against Epilepsy, Proposal for revised classification of epilepsies and cpileptic syndromes. Fipilepsia, 30, 1989, pp. 389-399.

Commission on Epidemiology and Prognosis of the International Lungue Against Epilepsy, Guidelines for epidemiologic studies on epilepsy, Epilepsia, 34, 4, 1993, pp. 592-596.

Commission on Epilepsy, Risk and Insurance of the Intcmational Bureau for Epilepsy, Workshop on epilepsy, risks and insurance, Epilcpsia, 34, 4, 1993, pp. 590-591.

Cowan L.D., Bodensteiner J.B., Leviton A., Doherty L., Prevalence of the epilepsies in children and adolescents, Epilepsia, 30, 1989, pp. 94-106. 
Craig, A., Oxley J., Statutory and non-statutory barriers to the employment of people with epilepsy. In: Epilepsy and employment (Edwards, Espir and Oxley, Eds.), Royal Society of Medicine Services Limited, London, 1986, pp. 21-31.

Craig A., Oxley J., Social aspects of epilepsy. In: A texlbook of epilepsy (Laidlaw, Richens and Oxley, Eds.), Churchill Livingstone, Edinburgh, 1988, pp. 566-610.

Crobach M.J., Niezink G.M., Leden J. van der, Springer M.P., Epilepsie en huisarts: toeval of hoofdzaak? Nederlands Tijdschrift voor de Geneeskunde, 132, 1988, pp. 1888-1892.

Crombie D.L., Cross K.W., Fry J., Pinsent R.J.F.H., Watts C.A.H., A survey of epilepsy in general practice, British Medical Journal, 1960, pp. 416-422.

Dam M., Gram L., Pedersen B., Orum H., Modern approach to anti-epileptic arug treatment, improving quality of life. The Danish Epilepsy Society, Kobenhavn, 1985.

Dam M., Kiorboe E., Epilepsy, diagnosis and treatment, Scriptor, Copenhagen, 1982.

Dean J.C., Penry J.K., Gencral principles, discontinuation of anti-epileptic drugs. In: Anti-epileptic drugs, (Levy, Mattson, Meldrum. Penry and Drcifuss, Eds.), $3^{\text {rd }}$ edition, Raven Press, New York, 1989, pp. 133-142.

Dichter M.A., Emerging insights into the mechanisms of epilepsy: implications for new anti-epileptic drug development, Epilepsia.35 (Suppl.4), 1994, s5!-s57.

Dick D., Epilepsy in the British steel industry. In: Epilepsy and employment (Edwards, Espir and Oxley, Eds.), Royal Society of Medicine Services Limited, London, 1986, pp. 49-52.

Donselaar C. van, Idiopathic first seizure in adull life, thesis, Rotterdam, 1990.

Drcifuss F.E., The cpilepsies: clinical implications of the international classification, Epilepsia, 31 (Suppl), 1990, s3-s!0

Elwes R.D.C., Chesterman P., Rcynolds E.H., Prognosis after a first untreated tonic-clonic seizure, The Lancet, okt., 1985, pp. 752-753.

Elwes R.D., Johnson C.B., Shorvon S.D., Reynolds E.H., The prognosis for seizure control in newly diagnosed epilepsy, The New England Journal of Medicine, October 1984, pp. 944-947.

Elwes R.D.C., Johnson A.L., Reynolds E.H., The course of untreated epilepsy, British Medical Journal, 297, 1988, pp. 948-297

Elwes R.D.C., Marshall J. Beattic A., Newman P.K., Epilepsy and employment. A community based survey in an area of high uncmployment. Journal of Neurology. Neurosurgery. and Psychiatry, 54, 1991, pp. 200-203

Elwes R.D.C., Reynolds E.H., Should people be treated after a first scizure?, Archives of Neurology, 45, 1988, pp.490-491

Elwes R.D.C., Reynolds E.H., The early prognosis of epilepsy, In: Comprehensive Epileptology (Dam and Gram, Eds.), Ravens Press, New York, 1990, pp. 715-727.

Employment Commission of the Intemational Bureau for Epilepsy, Employing people with epilepsy: principles for good practice, Epilepsia, 30, 1989, pp. 4II-4I2.

Espir M, Floyd M., Epilepsy and recruitment, In: Epilepsy and employment (Edwards, Espir and Oxley, Eds.), Royal Seciety of Medicine Services Limited, London, 1986, pp. 39-46.

Feuerstein M., Licb-Jückstock V., Schnaus H., Springmann E., Weber B., Wunderlich M., Compliance, a joint effort of the patient and his doctor, Epilepsy Research. Suppl. I, 1988, s5 Is56.

First Seizure Trial Group. Randomised clinical trial on the efficacy of anti-epileptic drugs in reducing the risk of relapse after a first unprovoked tonic-clonic seizure, Neurology, 43, 1993, pp 478-483 
Fisher R.S., Parsonage M., Beaussart M., Bladin P., Masland R., Sonnen A.E.H., Rémillard G., Epilepsy and Driving: an intemational perspective, Epilepsia, 35, 3, 1994, pp. 675-684.

Forsgren L., Prospcctive incidence study and clinical characterization of seizures in newly referred adults, Epilepsia, 31, 1990, pp.292-301.

Forsgren L., Prevalence of epilepsy in adults in northern Siveden, Epilepsia, 33, 1992, pp. 450-458.

Foy P.M., Chadwick D.W., Rajgopalan N., Johnson A.L., Shaw M.D.M., Do prophylactic anticonvulsant drugs alter the pattern of seizures after craniotomy, Journal of Neurologv. Neurosurgery and Psychiairy, 55, 1992, pp.753-757.

Gastaut H., Clinical and encephalographic classification of epilcptic seizures, Epilepsia, 11, 1970, pp. 102-113.

Gastaut H., Kugler J., Wörferbuch der Epilepsie, Hippokrates Verlag GmbH, Stuttgart, 1976.

Gastaut H., Zifkin B.G., The risk of automobile accidents with seizures occurring while driving: relation to seizure type, Neurology, 37, 1987, pp.1613-1616.

Goodridge D.M., Shorvon S.D., Epileptic seizures in a population of 6000, I: Demography, diagnosis and classification, and hospital services, Brilish Medical Journal, 287, I983a, pp. 64 I-644.

Goodridge D.M., Shorvon S.D., Epileptic seizures in a population of 6000, II: Treatment and prognosis, British Medical Journal, 287, 1983b, pp. 645-647.

Gowers W.R., Epilepsy and other chronic convulsive diseases; their causes, symptoms and treatment, reprint of the first American edition 1885, Arts en Boeve, Nijmegen, 1994, pp.200.

Graaf A.S. de, Epidemiological aspects of epilcpsy in northern Norway, Epilepsia, 15, 1974, pp. 291299.

Gram L., Draclumann Bentsen K., Pamas J., Flachs H, Controlled trials in epilcpsy: a review, Epilepsia, 23, 1982, pp. 491-519.

Gram L., Wulff K., Rasmussen K.E., Flachs H., Würtz-Jorgensen A., Sommerbeck K.W., Lohren V., Valproate sodium: a controlled clinical trial including monitoring of drug levels, Epilepsia, 18, 1977, pp. 141-148

Granieri E., Rosati G., Tola R., Pavoni M., Paolino E., Pinna L., Monetti V., A descriptive study of epilepsy in the district of Copparo, Italy, 1964-1978, Epilepsia, 24, 1983, pp. 504-514.

Green L.W., Simons-Morton D.G., Denial, delay and disappointment: discovering and overcoming the causes of drug errors and missed appointments. Epilepsy Research, Suppl. 1, 1988, s7-s22.

Haerer A.F., Anderson D.W., Schoenberg B.S., Prevalunce and clinical features of epilepsy in a biracial United States population, Epilepsia, 27, 1986, pp. 66-75

Hansotia P., Broste S.K. The effect of epilepsy and diabetes mellitus on the risk of automobile accidents, The New England Journal of Medicine, 324, 1991, pp.22-26.

Hansotia P., Broste S.K., Epilepsy and traffic safety, Epilepsia, 34, 1993, pp. 852-858.

Harding G., Photosensitive epilepsy and employment. In: Epilepsy and employment (Edwards, Espir and Oxley, Eds.), Royal Society of Medicine Services Limited, London, 1986, pp. 75-87

Hart Y.M., Sander J.W.A.S., Johnson A.L., Shorvon S.D., National General Practice Study of Epilepsy: recurrence after a first seizure. The Lancet, 336, 1990, pp. 127/-1274.

Hauser A.W., Epidemiology of epilepsy. In: Advances in Neurology, vol. 19, (Schoenberg ed.), Raven Press, New York, 1978, pp. 313-339

Hauser A.W., Prevention of post-traumatic epilepsy, The New England Journal of Medicine, 323, 1990, pp. 540-541.

Hauser A.W., Seizure disorders: the changes with age, Epilepsia, 33 (Suppl.4), 1992, s6-s 14. 
Hauser A.W., Annegers J.F., Kurland L.T., Prevalence of epilepsy in Rochester, Minnesota: 19401980, Epilepsia, 32, 1991, pp. 429-445.

Hauser A.W., Annegers J.F., Kurland L.T., Incidence of epilepsy and unprovoked seizures in Rochester, Minnesota: 1935-1984, Epilepsia, 34, 1993, pp.453-468.

Hauser A.W., Hesdorffer D.C., Prognosis. In: Epilepsy, frequency, causes, and consequences, (Hauser and Hessdorffer, Eds.), Epilcpsy foundation of America, Maryland, 1990, pp. 197-243.

Hauser A.W., Kurland L.T., The epidemiology of cpilepsy in Rochester, Minnesota, 1935 through 1967, Epilepsia, 16, 1975, pp. 1-66.

Hauser A.W., Ng S.K.C., Brust J.C.M., Alcohol, seizures and epilepsy, Epilepsia, 29 (Suppl.2), 1988, s66-s78

Heller A.J., Chesterman P., Elwes R.D., Crawford P., Chadwick D., Johnson A.L., Reynolds E.H., Phenobarbitone, phenytoin, carbamazepine, or sodium valproate for newly diagnosed adult epilepsy: a randomised comparative monotherapy trial, Journal of Neurology, Neurosurgery and Psychiatry, 38, 1995, pp.44-50.

Heycop ten Ham M.W. van, Complete recovery from epilepsy ? Discontinuation of antiepileptics after five or more seizure-free years, Huisarts en Wetenschap, 23, 1980, pp. 309-311.

Hicks R.A., Hicks M.J., Attitudes of major employers toward the employment of people with epilepsy: a 30-year study, Epilepsia, 32, 1991, pp. 86-88.

Hopkins A., Definition and epidemiology of epilepsy. In: Epilepsy, (Hopkins, Ed.), Chapman and Hall, London, 1987, pp. 1-18

Hopkins A., Garman A., Clarke C., The first seizure in adult life, the value of clinical features, Electroencephalography, and computerised tomographic scanning in the prediction of seizure recurrence, The Lancet, April 2. 1988, pp. 721-726.

Höppener R.J.E.A., Epilepsy and alcohol, thesis, Maastricht, 1981

Höppener R.J.E.A., Lugt P.J.M. van der, De medicamenteuze behandeling bij epilepsie, Pharmaceulisch Weekblad, 118, 1983, pp. 290-296.

Janz D., Die Epilepsien. Spezielle Pathologie und Therapie, Thieme, Stuttgart; 1969.

Janz D., Sommer-Burkhardt E.M., Discontinuation of anti-epilcptic drugs in patients with epilepsy who have been seizure-free for more than two years. In: Epileptology. Proc of the $7^{\text {th }}$ International Symposium on Epilepsy, (Janz ed.), Thieme-Verlag, Stuttgart, 1976, pp. 228-234.

Jeavons P.M. Choice of drug therapy in epilepsy, The Practitioner, 219, 1977, pp. 542-556.

Jennett B., Epilepsy after head injury and intracranial surgery, In: Epilepsy, (Hopkins, Ed.), Chapman and Hall, London, 1987, pp. 40I-4I2.

Johannessen S.1., Loyning Y., Munthe-Kaas A.W., General Aspects. In: Comprehensive Epileptology (Dam and Gram, Eds.), Ravens Press, New York, 1990, pp. 505-524.

Juul-Jensen $P$., Frequency of recurrence after discontinuance of anticonvulsant therapy in patients with epileptic seizures, Epilepsia, 5, 1964, pp. 352-363

Juul-Jensen $\mathrm{P}$., Frequency of recurrence after discontinuance of anticonvulsant therapy in patients with epileptic seizures, a new follow-up study after 5 years, Epilepsia, 9, 1968, pp. 11-16.

Juul-Jensen P., Foldspang A., Natural history of epileptic seizures, Epilepsia, 24, 1983, pp. 297-312.

Keränen T., Riekkinen P.J., Remission of seizures in untreated epilepsy, British Medical Journal, 307, 1993 , pp. 483.

Keränen T., Riekkinen P.J., Sillanpää M., Incidence and prevalence of epilepsy in adults in eastern Finland, Epilepsia, 30, 1989, pp. 413-421. 
Kosteljanetz M., Christiansen J., Mouritzen Dam A., Stengaard Hansen B., Blatt Lyon B., Pedersen H., Dam M., Carbamazepine vs phenytoin, a controlled clinical trial in focal motor and generalised epilepsy, Archives of Neurology, 36, 1979, pp. 22-24.

Krom M.C.T.F.M. de, Lugt P.J.M. van der, Anti-epileptica 2, Geneesmiddelen Bulletin, 16, 1982, pp. 9-12.

Krumholz A., Driving and epilepsy: a historical perspective and review of current regulations, Epilepsia, 35, 3, 1994, pp. 668-674.

Kutt H., Penry K., Usefulness of blood levels of anti-epileptic drugs, Archives of Neurology, 31, 1974, pp. 283-288.

Laidlaw J., Richens A., Oxley J., Parsonage M., Porter R.J., Classification of epileptic seizures. In: $A$ texibook of epilepsy (Laidlaw, Richens and Oxley, Eds.), Churchill Livingstone, Edinburgh, 1988, pp. $1-20$.

Lammers M.W., Clinimetrics in epileptology, thesis, Nijmegen, 1994

Lammers M.W., Hekster Y.A., Keizer A., Meinardi H., Renier W. O., Monotherapy or polytherapy for epilepsy revisited a quantitative assessment, Epilepsia, 36, 1995, pp.440-446.

Lechtenberg R., The diagnosis and treatment of epilepsy, Macmillan Publishing Company, New York, 1985

Leppik I.E., Compliance during treatment of epilepsy, Epilepsia, 29 (Suppl.), 1988, s79-s84.

Leppik I.E., Schmidt D. Consensus statement on compliance in epilepsy, Epilepsy Research, Suppl.1, 1988, s $179-\mathrm{s} 182$

Lugt P.J.M. van der, Epilepsie en verkeer. De medische geschiktheid van epilepsiepatienten tor deelname aan gemotoriseerd wegverkeer, thesis, Rotterdam, 1972.

Lugt P.J.M. van der, Epilepsie, een ratjetoe van problemen,. In: Man en Paard, Liber Amoricum tgv. het Afscheid van prof. A.Staal. 1990, pp. 125-131

Lühdorf K., Jensen L.K., Plesner A.M., Actiology of seizures in the elderly, Epilepsia, 27, 1986 pp $458-463$

Macdonald R.L., Kelly K.M., Mechanisms of action of currently prescribed and newly developed antiepileptic drugs, Epilepsia, 35 (Suppl 4), 1994, s4l-s50.

Maremmani C., Rossi G., Bonucceli U., Murri L.. Descriptive epidemiologic study of epilepsy syndromes in a district of northwest Tuscany, Italy, Epilepsia, 32, 1991, pp. 294-298.

Marsden C.D., Reynolds E.H. Seizures in adults. In: A lextbook of epilepsy (Laidlaw, Richens and Oxley, Eds.), Churchill Livingstone, Edinburgh, 1988, pp. 144-182

Mattson R.H., General principles, selection of anti-epileptic drug therapy, In: Anti-epileptic drugs, (Levy, Mattson, Meldrum, Penry and Drcifuss, Eds.), $3^{\text {rd }}$ edition, Raven Press, New York, 1989, pp. 103-115

Mattson R.H., Cramer J.A., Collins J.F., VA Epilepsy Co-operative Study Group, Aspects of compliance; taking drugs and keeping clinic appointments. Epilepsy Research, Suppl.1, 1988, sll1-sl17.

Mattson R.H., Cramer J.A., Collins J.F., and the Dept. of Veterans Affairs Epilepsy Co-ciperative Study No. 264 Group, A comparison of valproate with carbamazepine for the treatment of complex partial seizures and secondarily generalised tonic-clonic seizures in adults, The New England Journal of Medicine, 327, 1992, pp. 765-771.

Mattson R.H., Cramer J.A., Collins J.F., Smith D.B., Delgado-Escueta A.V., Comparison of carbamazepine, phenobarbital, phenytoin, and primidone in partial and secondarily generalized tonic-clonic seizures, The New England Journal of Medicine, 313, 1985, pp. 145-151. 
Medical Research Council Anti-epilcptic Drug Withdrawal Study Group, Randomised study of antiepileptic drug withdrawal in patients in remission, The Lancet, 337, 1991, pp. 1175-1180.

Medical Research Council Anti-epileptic Drug Withdrawal Study Group, Prognostic index for recurrence of seizures after remission of epilepsy, British Medical Journal, 306, 1993, pp.13741378.

Meijer J.W A., Knowledge, attitude and practice in anti-epileptic drug monitoring, thesis, Nijmegen, 1991.

Meinardi H., Binnie C.D., Goedhart D., Meijer J.W.A., Developments in pharmacotherapy of epilepsy. In: Epilepsy, an update on research and therapy, Alan R Liss Inc, New York, 1983, pp. 249-265.

Meinardi H., Pachlatko C., Special centres for epilcpsy, In: Comprehensive Epileptology (Dam and Gram, Eds.), Ravens Press, New York, pp. 769-779.

Meinardi H., Epilepsie compendium, Katholieke Universiteit Nijmegen, februari 1993.

Merlis J.K., Proposal for an International Classification of the Epilepsies, Epilepsia, 11, 1970, pp. 114119.

Metsemakers J.F.M. Unlocking patients' records in general practice for research, medical education and quality assurance: the Registration Network Family Practices, thesis, Maastricht, 1994.

Milano Collaborative Group For Studies On Epilepsy, Long-term intensive monitoring in difficult patient. Preliminary results of 16 months of observations-usefulness and limitations. In: Antiepileptic therapy advances in drug monitoring, (Johannessen, Pippenger, Schmidt, Morselli, Richens and Meinardi, Eds.), Ravens Prcss, New York, 1980

Montouris G.D. Practical insights and clinical experience with combinations of the new anti-epileptic drugs, Neurology, 45 (Suppl.2), 1995, s25-s28.

Mouritzen Dam A., Fuglsang-Fredriksen A., Svarre-Olsen U., Dam M., Late-onset epilepsy: aetiologies, types of seizures and value of clinical investigation, EEG, and computerized tomography scan, Epilepsia, 26, 1985, pp. 227-231

Oller-Daurella L., Oller F.V.L., Suppression of anti-epileptic treatment, European Neurology, 27, 1987, pp. 106-113.

Oller-Daurella L., Pamies R., Oller L.F.V., Reduction or discontinuance of anti-epileptic drugs in patients seizure-free for more than 5 years. In: Epileprology. Proc. of the $7^{\text {th }}$ International Symposium on Epilepsy, (Janz ed.). Thieme-Verlag, Stuttgart, 1976, pp. 218-227.

Oller-Daurella L., Oller L.F.V., Pamies R., Clinical, therapeutic and social status of epilepsy patients without seizures for more than five years, in: Epilepsy. The $8^{\text {th }}$ International Symposium, (Penry Ed.), Raven Press, New York, 1977, pp. 69-75

Overweg J., Epilepsie; prognose en bchandeling, Nederlands Tijdschrift voor de Geneeskunde, 128, 1984, pp. 1710-1716.

Overweg J., Withdrawal of anti-epileptic drugs in seizure-free adult patients, prediction of outcome, thesis, Amsterdam 1985, Rodopki.

Overweg J., Rowan A.J., Binnic C.D., Oosting J., Nagelkerke N.J.D., Prediction of seizure recurrence after withdrawal of anti-epileptic drugs, In: Advances in Epileptology: $12^{\text {th }}$ International Symposium, (Dam, Gram and Penry, Eds.), Raven Press, New York, 1981, pp. 503-508.

Parsons M., Fits and other causes of loss of consciousness while driving, Quarterly Journal of Medicine, New Series. 58, 1986, pp.295-303.

Pedley T.A., Discontinuing anti-epileptic drugs, New England Journal of Medicine, 318, 1988, pp. 982-984.

Pellock J.M., Standard approach to anti-epileptic drug treatment in the United States, Epilepsia, 35 (Suppl.4), 1994. s11-s18. 
Peterson G.M., McLean S., Millingen K.S., Determinants of patient compliance with anticonvulsant therapy, Epilepsia, 23, 1982, pp. 607-613

Peterson G.M., McLean S., Millingen K.S., A randomised trial of strategies to improve patient compliance with anticonvulsant therapy, Epilepsia, 25, 1984, pp. 412-417.

Porter R.J., Epilepsy, 100 elementary principles, W.B. Saunders Company Ltd., London, 1984.

Porter R.J. General principles how to usc anti-epileptic drugs. In: Anti-epileptic drugs, (Levy, Mattson, Meldrum, Penry and Dreifuss, Eds.), $3^{\text {rd }}$ edition, Raven Press, New York, 1989, pp. 117-131.

Quirk J.A., Fish D.R., Smith S., Sander J.W., Shorvon S.D., Allen P.J., First seizures associated with playing electronic screen games: a community based study, Annals of Neurology, 37, 1995 , pp.733-737.

Ramsay R.E., Wilder B.J., Berger J.R., Bruni J., A double blind study comparing carbamazepine with phenytoin as initial seizure therapy in adults, Neurology, 1983, pp. 904-910.

Reynolds E.H., Serum levels of anticonvulsant drugs; interpretation and clinical value, Pharmacology and Therapeutics, 8, 1980, pp. 217-235.

Reynolds E.H., Early treatment and prognosis of epilcpsy, Epilepsia, 28, 1987, pp. 97-106.

Reynolds E.H., The prevention of chronic epilepsy, Epilepsia, 29 (Suppl.1), 1988, s25-s28.

Reynolds E.H., Changing view of prognosis of epilepsy, British Medical Journal, 301, 1990, pp. $1112-1114$.

Reynolds E.H, Do anticonvulsants alter the natural course of epilepsy? Treatment should be started as carly as possible, British Medical Journal. 310, 1995, pp. 176.

Reynolds E.H., Chadwick D., Galbraith A.W., One drug (phenytoin) in the treatment of epilepsy, The Lancet, May, 1976, pp. 923-926.

Reynolds E.H., Heller A.J., Chadwick D., Valproate versus carbamazepine for seizures, The New England Journal of Medicine, 328, 1993, pp. 207-208.

Reynolds E.H., Shorvon S.D., Monotherapy or polypharmacy for epilepsy?, Epilepsia, 22, 1981, pp. $1-10$.

Richens A., Ahmad S., Controlled trial of sodium valproate in severe epilepsy, British Medical Journal, 1975, pp. 255-256.

Richens A., Davidson D.L.W., Cartlidge N.E.F., Easter J., EPITEG Collaborative Group, a multicentre comparative trial of sodium valproate and carbamazepine in adult onset epilepsy, Journal of Neurology, Neurosurgery and Psychiatry, 57, 1994, pp.682-687.

Rimmer E.M., Richens A., Clinical pharmacology and medical treatment. In: A textbook of epilepsy (Laidlaw, Richens and Oxley, Eds.). Churchill Livingstone, Edinburgh, 1988, pp. 421-483.

Rodin E.A., Rim C.S., Rennick P.M., The effects of carbamazepine on patients with psychomotor epilepsy: Results of a double blind study, Epilcpsia, 15, 1974, pp. 547-56I.

Rowan A.J., Diagnosis and treatment of epilepsy, Hospital and Community Psychiarry, 34, 1983, pp. 540-547.

Rutgers M.J., Geneeskundige en maatschappelijke aspecten van de zorg voor epilcpsiepatienten in Nederland, thesis, Rotterdam, 1984.

Salinsky M.C., Wegener K., Sinnema F., Epilepsy, driving laws and patient disclosure to physicians, Epilepsia, 33, 1992, pp 469-472.

Sander J.W.A.S., Some aspects of prognosis in the epilepsics: a revicw, Epilepsia, 34. 1993. pp. 10071016 
Sander J.W.A.S., Hart Y.M., Johnson A.L., Shorvon S.D., National General Practice Study of Epilcpsy: newly diagnosed epilcptic seizures in a general population, The Lancet, 336, 1990, pp. 1267-1271.

Scheuer M.L., Pedley T.A., The evaluation and treatment of seizures, The New England Journal of Medicine, 323, 1990, pp. 1468-1474.

Schmidt D. Janz D. Therapeutic plasma concentrations of phenytoin and phenobarbitone. In: Antiepileptic therupy advances in dnig moniloring. (Johannessen, Pippenger, Schmidt, Morselli, Richens and Meinardi, Eds.), Ravens Press, New York, 1980.

Schmidt D., Leppik I.E., Compliance in epilepsy: introduction, Epilepsy Research, Suppl.1, 1988, s3s4.

Schmidt D., Reininghaus R., Winkel R., Relevance of poor compliance for scizure control, Epilepsy Research, Suppl. 1, 1988, s141-s146.

Schobben A.F.A M., Pharmacokinetics and therapeutics in epilepsy, thesis, Nijmegen, 1979, pp.216.

Schoenberg B.S., Epideniological aspects of epilepsy. In: Epilepsy, an update on research and therapy, Alan R.Liss Inc, New York, 1983, pp. 331-343.

Scholtes F.B., Renier W.O., Meinardi H., Generalised convulsive status epilepticus, pathophysiology and treatment, Pharmacyworld and Treatment, 1993, 15, pp. 17-28

Shinnar S., Vining E., Mellits E.D., Discontinuing anti-epileptic medication in children with epilepsy after two years without seizures. The New Lingland Journal of Medicine. 313, 1985, pp.976-980.

Shinnar S., Kang H., Bcrg A.T., Goldensohn E.S., Hauser W.A., Moshé S.L., EEG abnormaties in children with a first unprovoked seizure, Epilepsia, 35, 1994, pp. 471-476.

Shorvon S.D., Reynolds E.H., Unnccussary polypharmacy for epilepsy, British Medical Journal, I, 1977, pp. 1635-1637

Shorvon S.D., Chadwick D., Galbraith A.W., Reynolds E.H., One drug for epilepsy, British Medical Journal, I, 1978. pp. 474-476.

Shorvon S.D., Galbraith A.W., Laundy M., Vydelingum L., Reynolds E.H., Monotherapy for epilepsy. In: Anti-epileptic therapy advances in drug monitoring, (Johannessen, Pippenger, Schmidt, Morselli, Richens and Meinardi, Eds.), Ravens Press, New York, 1980, pp. 213-220.

Shorvon S.D., Reynolds E.H., Early prognosis of epilcpsy, British Medical Journal, 285, 1982, pp. 1699-1701.

Shorvon S.D., The temporal aspects of prognosis in epilepsy, Journal of Neurology. Neurosurgery and Psychiatry, 47, 1984, pp. 1157-1165.

Shorvon S.D. The role of single drug and combination drug therapy in the treatment of epilepsy, in: Madern approach to anti-epileptic drug treatment, (Dam, Gram, and Pedersen, Eds.), The Danish Epilepsy Society, Jorgen Jensens Bogtrykkeri, Kobenhavn, 1985

Shorvon S.D., The treatment of epilepsy by drugs. In: Epilepsy, (Hopkins, Ed.), Chapman and Hall, London, 1987, pp. 229-282

Shorvon S.D., The coursc of untreated epilepsy, British Medical Journal, 297, 1988, pp. 1405.

Shorvon S.D., Epidemiology, classification, natural history and genetics of epilepsy, The Lancet, 336, 1990, pp. 93-96.

Shorvon S.D.. Medical assessment and treatment of chronic epilepsy, all patients should have a long term plan of treatment. British Medical Journal. 302, 1991, pp. 363-366.

Shorvon S.D. Epilepsy and driving. British regulations have recently been eased British Medical Jourmal. 310, Ling, pp.885-886. 
Simonsen N., Zander Olsen P., Kühl V., Lund M. Wendelboe J., A comparative controlled study between carbamazepine and diphenylhydantoin in psychomotor epilepsy, Epilepsia, 17, 1976, pp 169-176.

Sonnen A.E.H., De medicamenteuze behandeling van epilepsic, Nederlands Tijdschrift voor de (ienceskunde, 123, 1979, pp. 485-490.

Swartz B.E., Delgado-Escueta A.V., The management of status epilepticus, In: Epilepsy, (Hopkins, Ed.), Chapman and Hall, London, 1987, pp. 417-442.

Taylor J., Driving as an occupation. In: Epilepsy and employment (Edwards, Espir and Oxley, Eds.), Royal Socicty of Medicine Services Limited, London, 1986, pp. 89-92.

Temkin N.R., Dikmen S.S.,Wilensky A.J., Keihm J., Chabal S., Winn H.R, A randomised doubleblind study of phenytoin for the prevention of post-traumatic seizures, The New England Journal of Medicine, 323, 1990, pp. 497-502.

Tennison M.B., Greenwood R.S., Lewis D.V., Benoit S.E., Rate of taper of anti-epileptic drugs and the risk of seizure recurrence, Annals of Neurology, 26, 1989, pp. 439.

Tennison M., Greenwood R. Lewis D., Thorn M., Discontinuing anti-epileptic drugs in children with epilepsy, The New England Journal of Medicine, 330, 20, 1994, pp. 1407-1410.

Thompson P.J., Oxley J. Socioeconomic accompaniments of severe epilepsy, Epilepsia, 29 (Suppl.1), 1988, pp. s9-s।8.

Thorbecke R., Measurement of compliance through patient interviews, Epilepsy Research, Suppl.1, 1988, pp. 79-83.

Thurston J.H., Thurston D.L.. O'Leary J.. Follow-up study of 148 cases in which therapy had been suspended after prolonged anticonvulsant control. The New England Journal of Medicine, 286, 1972, pp. 169-174.

Thurston J.H., Thurston D.L., Hixon B.B., Keller A.J., Additional follow-up of I48 children 15 to 23 years after withdrawal of anticonvulsant therapy, The New England Journal of Medicine, 306, 1982, pp. 831-836.

Trostle J., Doctors orders and patients' sclf-interest: two views of medication usage?, Epilepsy. Research, Suppl. I, 1988, s57-s69.

Troupin A., Moretti Ojemann L., Halpem L.. Dodrill C., Wilkus R., Friel P., Feigl P., Carbamazcpinea double blind comparison with phenytoin, Neurology, 1977, pp. 511-519.

Tumbull D.M., Rawlins M.D.. Weightman D.. Chadwick D.W., A comparison of phenytoin and valproate in previously untrcatcd adult epileptic patients, Journal of Neurology. Neurosurgery and Psychiatry, 45, 1982, pp. 55-59.

Wagner A.L., A clinical and epidemiological study of adult patients with epilepsy, Acta Neurologica Scandinavia, 94 (Suppl), 1983, s63-s72.

Wallace S.J., Febrile convulsions. In: Epilepsy, (Hopkins, Ed.), Chapman and Hall, London, pp. 443467.

Wannamaker B.B., Morton W.A., Gross A.J., Saunders S., Improvement in the anti-epileptic drug levels following reduction of intervals between clinic visits, lipilepsia, 21, 1980, pp. 155-162.

Wilder B.J., Ramsay R.E., Murphy J.V., Karas B.J., Marquardt K., Hammond E.J., Comparison of valproic acid and phenytoin in newly diagnosed tonic-clonic seizures, Neurology, 33, 1983, pp. 1474-1476.

Wilder B.J., The treatment of epilepsy: an overvicw of clinical practices, Neurology, 45 (Suppl.2), 1995, s7-sll. 
Woo E., Chan Y.M., Yu Y.L., Chan Y.W., Huang C.Y., If a well-stabilised epileptic patient has a subtherapeutic anti-epileptic drug level, should the dose be increased? A randomised prospective study, Epilepsia, 29, 1988, pp. 129-139.

Zielinski J.J., Epileptics not in treatment, Epilepsia, 15, 1974, pp. 203-210.

Zielinski J.J., Epidemiology. In: A textbook of epilepsy (Laidlaw, Richens and Oxley, Eds.), Churchill Livingstone, Edinburgh, 1988, pp. $21-48$. 
CHAPTER 3

\section{System development}

\subsection{Introduction}

Rather than using comparatively domain independent methods such as mathematics or statistics, some decision support systems (DSSs) use a model of the domain based on a symbolic representation of expertise to solve problems. Such DSSs are called knowledge based systems (KBSs). Development of a KBS is a type of task which requires an approach different from conventional system development. In this chapter we will discuss different approaches for KBS development.

KADS - Knowledge Acquisition, Documentation and Structuring - is a representative of structured development methodologies (Breuker and Wielinga 1984a). Besides the provision of socalled elicitation techniques for making knowledge explicit, KADS provides a set of domain independent models which enables analysis of the extracted knowledge. An instantiation of these models is the conceptual model: a description of the problem solving process that may be used for implementation. At the end of this chapter we will present two models which describe different parts of the problem solving process with respect to the drug treatment of epilepsy. Both were developed using the KADS methodology.

\subsection{Methods for KBS development}

A KBS is a model of a problem solving task an expert performs (Jackson 1986, Waterman 1986). This is in contrast with conventional systems which focus on data processing and information exchange tasks as organised around forms (Weitzel and Kerschberg, 1989). During the development of a KBS one has to analyse and model the cognitive processes of e.g. interpreting patient data and applying medical knowledge (Hayes-Roth et al 1983). The cognitive processes are less circumscript than the data processing and information exchange tasks. Building a KBS therefore requires different methods than used in conventional software engineering.

The knowledge applied by neurologists consists of facts and heuristics. This knowledge has to be elicited and encoded, i.e. represented in a symbolic way and it has to be stored in a socalled knowledge base. The process of obtaining the knowledge from the expert and representing it in such a way that it can be formalised in a computer program is called knowledge acquisition. The knowledge acquisition process consists of two separate activities: elicitation and analysis of knowledge. Knowledge acquisition is generally recognised as the main bottleneck in the development of KBSs (Hayes-Roth et al. 1983, Buchanan and Shortliffe, 1984) 
Schreiber and Wielinga (1993) present two views on KBS development:

- The traditional tramsfer view in which the knowledge acquisition process is considered as a process of extracting the knowledge from a human expert and transferring the knowledge into the KBS. An example of this approach is development by prototyping (see below). This approach is often constrained by the representation formalism that is available.

- The modelling view in which the knowledge acquisition process is separated from the implementation of the knowledge. An example of this approach is KADS.

These two views will be described in the following sections.

\subsubsection{Prototyping}

Because it is not always clear what a system should do, users may frequently change previously defined requirements (Wellbank, 1983). Prototyping is used to help the user analyse his ideas on the problem at hand, the functionality of the system and the human-computer interface. In prototyping a system develops in an iterative way. The prototype system is used as a device for interviewing the domain expert on issues relevant for a proper performance of the intended system such as data entry, output of results and response time. Thus, prototyping clarifies aspects of the system's functionality by enabling direct feedback by the user on an implementation of a part of the system (Diaper, 1989). Prototyping can also support the establishment of the necessary co-operative atmosphere between the expert providing the knowledge and the knowledge engineer (Heng, 1987). There are three categories of prototyping: experimental, evolutionary and incremental.

Experimental prototyping is used to show the user different aspects of functionality and to clarify the problem at hand. After a short problem identification phase a model is built. This prototype exhibits limited functional capabilities, low reliability and possibly inefficient performance. The prototype is assessed with the objective to clarify the user requirements and to determine additional requirements. After the assessment the model is discarded. A synonym for this technique is throw-away prototyping (van Dijk et al. 1988).

When the user requirements are available, they may be incomplete or may still be subject to change. Evolutionary protolyping allows a system to evolve according to changing user needs. The process consists of a sequence of prototypes which (hopefully) converge into the final system or parts of it (van Dijk et al. 1988, Tate 1990)

Users may also be able to provide a fairly complete description of the functionality of the system. Clarification of requirements may be necessary on subparts only. In incremental prototyping the complete system design is specified and subtasks are identified. Next these subtasks are implemented, assessed and modified according to the user feedback. In contrast to the evolutionary approach, in incremental prototyping the overall design remains in principle unaltered (Stephens and Bates, 1990)

Experience with prototyping has revealed several disadvantages:

- The task to be performed by the system may change. Major design decisions are made in an early phase of development. In case of incorrect decisions the prototype may not be useful for the next iteration. 
- Maintainability of the evolving system may become a problem (Mayhew and Dearnley, 1990). Although a prototype knowledge base may do well, it may not be possible to scale it up to the full size required.

- Since (part of) the prototype should be realistic the user may be misled and take the prototype for the final system. Strong user reluctance to throw away a prototype that was designed to be discarded has been reported (Stephens and Bates, 1990).

\subsubsection{Structured development}

Prototyping is a kind of code-and-fix oriented approach of system development. In a more rigid approach one uses a predefined framework describing how to analyse the domain and what design issues to take into account. This may facilitate a more comprehensive view on the domain.

\section{Slage based approaches}

Stage based or life-cycle approaches to KBS development divide the development process into separate phases. For each of these phases a set of activities is specified. Different life-cycle models have been presented such as the waterfall model (Royce, 1987), the Hayes-Roth feedback model (Hayes-Roth et al. 1983) and the spiral model (Boehm, 1988). The application of these stage based approaches can be difficult: what the intermediate results of each of the stages should include is only vaguely presented by the authors. Also techniques and tools for supporting the different phases are usually lacking (Fen and Veren, 1991).

A more detailed approach for a stage based development of KBSs is the Pragmatic Knowledge Acquisition Methodology (PKAM) (Grover, 1983). It consists of three phases in which a domain is defined, fundamental knowledge is acquired and the obtained knowledge is improved. This methodology mentions a set of issues to be addressed in each of the phases and contains a number of techniques for knowledge elicitation. Studies on the utility of this approach however are lacking.

\section{KADS}

A more comprehensive methodology is KADS (Knowledge Acquisition, Documentation and Structuring, Breuker and Wielinga 1984a). It describes procedures and documentation, as well as tools and methods for KBS development. KADS emphasises the pre-implementation phases. An extensive analysis of the knowledge is advocated before system design and implementation take place.

The analysis phase in the most recent version of KADS consists of two streams: the external and the internal stream (Breuker and Wielinga 1984ab, Breuker and Wielinga 1987, Breuker et al. 1987, Breuker and Winkels, 1989). In the external stream the analysis of requirements and constraints concerning the system takes place. The internal stream describes the analysis of expertise. The methodology of the external stream is hardly documented. The analysis of the expertise was considered most important (Hickman et al. 1989, Schreiber et al. 1993). Analysis of expertise on different levels of abstraction has to be performed:

- Linguistic analysis, the identification of domain concepts in verbal data

- Conceptual analysis; the integration of individual concepts through formalised description(s), e g. compound concepts, relations and models

- Epistemological analysis; the definition of structural properties of the conceptual knowledge 
- Logical analysis; the definition of the formalisms with which the knowledge and inference making is expressed

- Implementation analysis

The level of abstraction increases from the linguistic to the epistemological level and then decreases again. The most important description of expertise results from the epistemological analysis

In KADS the epistemological description of expertise consists of four layers each describing a different category of knowledge: domain, inference, task and strategic knowledge. The domain layer contains the concepts and rules of the domain without an indication of when and how they can be applied. The inference layer depicts the relation between groups of concepts and inference procedures without an indication of the sequence of their application. This sequence of invocation of the inference procedures is described at the task layer. As the problem solving process may consist of multiple tasks, each of these tasks and the procedure for their invocation is described at the strategic layer

KADS provides a set of tools for knowledge elicitation as well as two facilities that support the analysis of expertise at the epistemological level: a conceptual modelling language and interpretation models. The conceptual modelling language provides a set of primitives for bottom-up development and refinement of the inference layer. These primitives consist of so called metaclasses and knowledge sources. Metaclasses are descriptions of domain concepts in abstract terms, indicating their roles in the reasoning process. The knowledge sources contain the inference procedures. They can be viewed as abstract operations on the metaclasses (Breuker and Wielinga, 1987)

To support the description at the inference layer, interpretation models have been developed. An interpretation model is a domain independent description of a specific type of problem solving task (Wielinga and Breuker, 1987). It provides a structure for analysing the verbalised data. The use of interpretation models resembles the idea of generic tasks: a limited set of general problem solving approaches. These generic tasks describe structures for problem solving which can be instantiated for a specific domain. Brown and Chandrasekaran (1989) define generic tasks as an elementary combination of a problem, a representation and an inference strategy. In contrast, the interpretation models in KADS do not at all address issues related to implementation such as representation aspects. The descriptions of tasks and inference structures in KADS are comparable with data-flow diagrams in conventional software engineering (Schreiber and Wielinga, 1993)

Proponents of structured development advocate an extensive analysis of the domain before commencing the implementation of a DSS. Such an analysis clarifies the view on the domain for which a DSS is developed. Due to the separation of analysis of expertise and its implementation, design decisions can be postponed until a more complete view on the domain and tasks has been obtained. It is assumed that analysis of expertise before implementation assures a more complete description of the expertise and thus a more efficient implementation can be expected (Schreiber et al. 1993) 


\subsubsection{Selection of the development method}

The acceptance of a KBS will depend both on the quality of the advice provided and on the functionality of the system. Although both aspects are important, confidence in the advice is considered a primary issue of concern (O'Keefe et al. 1987, O'Keefe 1989, O'Moore et al. 1990, Wyatt 1991). In prototyping the implementation starts with only a part of the knowledge. This approach makes a less optimal implementation more likely. Incremental improvement of implemented knowledge can cause inconsistencies particularly in complex systems (Nguyen et al. 1985). Given these problems a structured approach for the analysis of expertise in the domain of anti-epileptic drug treatment was considered more appropriate. As KADS provides the most comprehensive approach for structured development of KBSs it was selected as the approach for analysis of expertise in our work.

Prototyping is useful within KADS when it is used as a technique to support analysis and design activities (Nobis and Siedka-Bauer 1988, Tansley and Hayball, 1993). It provides the expert with insight in the procedures which are followed by the system (Hickman et al. 1989). The modelling of expertise in our work was alternated with an implementation of the obtained knowledge to analyse and demonstrate the interaction of the different layers of the model of expertise.

\subsection{Knowledge elicitation}

The process of obtaining knowledge from a human expert is called knowledge elicitation. The extracted knowledge may be modelled, i.e interpreted using some conceptual framework. Although elicitation and modelling are different activities, they are closely related and may direct each other's focus to specific parts of the knowledge.

Indirect techniques such as card sorting, multi-dimensional scaling, cluster analysis, general weighted networks and repertory grids provide a low level approach to knowledge elicitation (Wellbank 1983, Cooke and McDonald 1986, 1987, Burton et al. 1987, Wright and Ayton 1987, Shaw 1988). They enable the exploration of the domain by creating maps displaying the structure of the concepts in the domain. This structure is often derived from a (complete) set of perceived distances between pairs of concepts. The usability of the result depends on the completeness of the set of concepts used for obtaining the distance measures. If the set is not complete bias may result. This will show up in an erroneous description of the structure of domain concepts. How the set of concepts shall (or can) be obtained is not defined. One could question whether the development of a KBS is appropriate when the knowledge of a domain is so little developed that the structure of the domain still has to be identified by these techniques.

Apart from the card sorting technique all of the indirect techniques require the use of a kind of distance matrix in which all possible pairs of concepts are presented. Filling in this matrix is a tedious procedure. The expert may not be convinced of the necessity of such an exercise. Furthermore, the use of indirect techniques will not reveal the actual usage of concepts in the problem solving process (Hoffman, 1987).

Focused interviews, structured interviews, introspection, dialogues, protocol analysis, interruption analysis and questionnaires are direct techniques for knowledge elicitation (Reitman Olsen and Rueter, 1987). With these techniques, the role of concepts and the 
structurs: of the problem solving is derived directly from the verbal descriptions (Breuker and Wielinga 1984b).

A critical issue of direct techniques is that a successful application depends on the observer's skills for interviewing the expert. These techniques are also time consuming. However, most of the burden is on the interviewer, not on the domain expert. The interviewer has to transcribe the recordings of the interviews and to analyse the information obtained. Direct techniques are applied most often (Cullen and Bryman, 1988).

There is no formal methodology for knowledge elicitation (Wellbank 1983, Shadbolt and Burton 1989). The applicability of a knowledge elicitation technique may differ across domains. The suitability of a technique may depend on the personality (introvert/extrovert) and cognitive style (field dependent/independent) of the expert (Burton et al. 1988).

We only used direct techniques for knowledge elicitation. They allow the expert to express his knowledge in a natural way. There was no need to establish a structure of concepts in the domain as this knowledge was available. Our main interest was to obtain insight in how the knowledge is applied when performing tasks in the management of epilepsy patients.

\subsection{Models of anti-epileptic drug treatment}

The interviews and discussions with the expert and the literature review demonstrated that the treatment of epilepsy has different phases

- During the first phase the objective is to obtain enough information to establish a diagnosis. More than one patient-physician contact may be needed.

- The second phase starts when the diagnosis is established. Here the treatment is discussed and selected.

- The third phase consists of the evaluation of the treatment. The drug treatment may be adjusted depending on the symptomatology.

The model of expertise is based on an analysis of this phased approach to the treatment of epilepsy. We focused on the knowledge concerning the initiation and modification of the treatment.

\subsection{Strategic layer}

The management of a chronic disease is composed of a set of procedures for the diagnosis, the initiation of a therapy, the monitoring of the effect of the therapy and if possible the withdrawal of the treatment (Figure 3.5-1). For epilepsy the strategy - followed after the establishment of the diagnosis - can be described as follows. A drug therapy will be initiated with the intention to relief the patient from the seizures. The effectiveness of the drug treatment is checked by monitoring the seizure frequency and the well-being of the patient. The effectiveness of a drug may vary among patients. If the drug treatment proves ineffective the treatment is adjusted (a change in the drug dose or the initiation of another anti-epileptic drug). The effect of the adjustment is monitored. The adjustment of the treatment will end when seizures are controlled i.e. they do not occur anymore. When control of seizures has been established the monitoring of the patient continues for at least two years. After this period tapering of the drug treatment may be started. The results of changes in therapy will again be monitored. If seizures remain controlled the reduction of drug treatment will continue. 


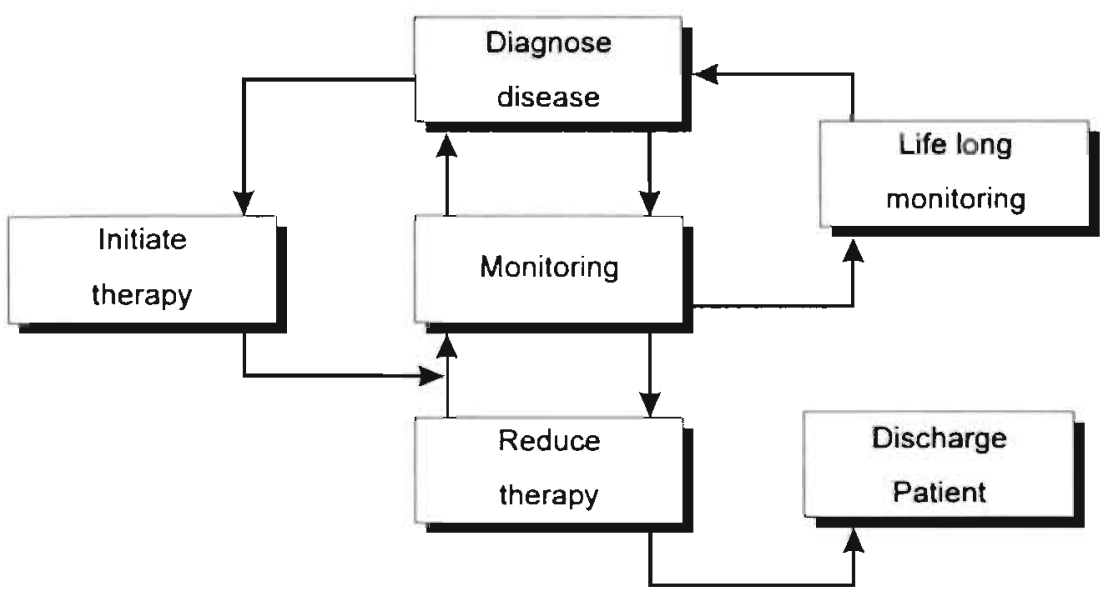

Figure 3.5-I: Strategy for management of epilepsy.

In case drug treatment is ineffective to control seizures completely or when tapering causes reoccurrence of seizures, life-long monitoring may be necessary: an approach in which adjustments of the treatment are made based on balancing the consequences of seizures and sideeffects.

\subsection{Inference structures}

The problem solving structures of both the initiation part and the monitoring part of the treatment were analysed. The analysis of the monitoring part of the treatment was guided by the KADS interpretation models for monitoring and assessment. Due to the lack of appropriate interpretation models for the process of initiating the treatment, we had to construct such a model bottom-up.

\subsubsection{Initiation of drug treatment}

The process for initiation of anti-epileptic drug treatment is decomposed into the subtasks of:

- Selecting the anti-epileptic drug

- Selecting the drug dose and

- Calculating the number of tablets and the intake scheme

For each of these subtasks the relevant domain concepts were extracted from the transcripts of the interviews and dialogues.

The epistemological analysis resulted in an identification of the various roles concepts have in the problem solving process (e.g. the group of main anti-epileptic drugs is used as a set of 
hypotheses from which the most appropriate can be selected; drug side-effects are used as constraints in this selection process). Concepts which have a similar role in the problem solving process are grouped in so-called metaclasses'.

Table 3.6-I: Description of the metaclasses used in the inference model for initiation of anti-epileptic drug treatment.

\begin{tabular}{|c|c|c|}
\hline METACLASS & CONCEPTS & ROL.E \\
\hline SKFLETAL MODEL & prescription data & template of a prescription \\
\hline COMPONENT & $\begin{array}{l}\text { generic drugname } \\
\text { brandname } \\
\text { dose of tablets } \\
\text { number of tablets } \\
\text { intake schemes }\end{array}$ & concepts to be assigned a value \\
\hline RESULT PART & $\begin{array}{l}\text { generic drugname } \\
\text { brundname } \\
\text { dose of tablets } \\
\text { number of tablets } \\
\text { intake schemes }\end{array}$ & instantiated components \\
\hline MODEL & prescription data & suggested drug trealment \\
\hline CASE DESCRIPTION & patient data & portrayal of diseased person \\
\hline HYPOTHESES & anti-epileptic drugs data & $\begin{array}{l}\text { providing options for selection of brand } \\
\text { providing options for selection of daily dose } \\
\text { providing options for selection of dose of tablets }\end{array}$ \\
\hline \multirow[t]{4}{*}{ CONSTRAINTS } & $\begin{array}{l}\text { type of epilepsy } \\
\text { type of seizures } \\
\text { co-medication } \\
\text { drug side-effects } \\
\text { ineflective drugs }\end{array}$ & restrictions for selection of drug \\
\hline & $\begin{array}{l}\text { weight } \\
\text { laboratory results }\end{array}$ & restrictious for selection of daily dose \\
\hline & $\begin{array}{l}\text { generic drugname } \\
\text { brandname } \\
\text { dose of tablets }\end{array}$ & $\begin{array}{l}\text { restrictions for selection of brand } \\
\text { restrictions for selection of dose of tablets } \\
\text { restrictions for assembling of intake scheme }\end{array}$ \\
\hline & $\begin{array}{l}\text { number of tablets } \\
\text { return date }\end{array}$ & $\begin{array}{l}\text { restriclions for assimbling of intake scheme/selection of } \\
\text { total number of tablets }\end{array}$ \\
\hline ALTERNATTVES & $\begin{array}{l}\text { optional generic drug } \\
\text { optional brandname } \\
\text { optional dose of tablets } \\
\text { optional number of lablets } \\
\text { optional intake scheme }\end{array}$ & choices for prescription \\
\hline
\end{tabular}

'The typographic conventions used in the description of inference structures are as follows:

- Tasks are printed in italicised capitals (OBTAIN_DRUGNAME)

- Metaclasses are printed in capitals (c.g. METACLASS)

- Knowledge sources are printed in sinall italicised letters (e.g. knowledge source)

- Individual concepts are printed in small bold capitals (e.g. CONCEPT)

- Values of concepts are printed in sinall capitals (c.g. Valuie)

We use the notation (CONCEPT VALUE) to indicate a concept and its value. 
The metaclasses used in the inference model for initiation of therapy are presented in Table 3.6-I. As concepts may have more than one role in the problem solving process, they can occur in different metaclasses. This also means that a concept (e.g. CARBAMAzEPINE) may occur as a value e.g. in (PRESCRIBED-DRUG CARBAMAZEPINE).

A second part of the elicited knowledge consists of rules, each describing a condition which leads to an assertion of a new fact or an action like the commencement of a task. In our case they are grouped by so called knowledge sources, a set of rules dealing with a specific inference. In an abstract sense a knowledge source is an inference process which instantiates concepts in the output metaclass given instantiated concepts in the input metaclass(es). The inference layer for the initiation of drug treatment is presented in Figure 3.6-I. Metaclasses are represented by rectangles and knowledge sources by ovals. An example of a knowledge source is assign-value which gives a concept in the metaclass RESULT PART a value (e.g. GENERIC DRUGNAME the value CARBAMAZEPINE) based on instantiated concepts in the metaclasses COMPONENT and ALTERNATIVES.

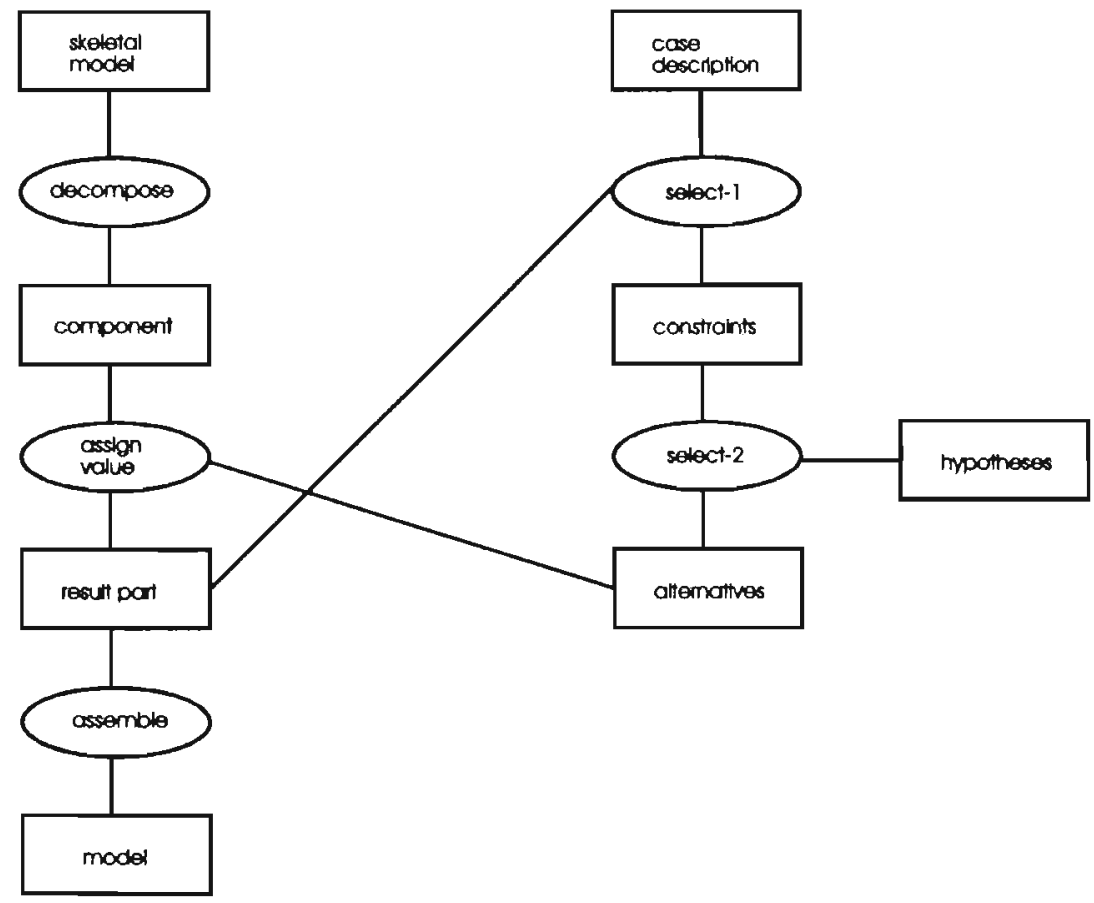

Figure 3.6-I: Inference layer for the initiation of anti-epileptic drug treatment, depicting the metaclasses (rectangles) and knowledge sources (ovals). 
The knowledge sources in Figure 3.6-I are composite ones. By decomposing these knowledge sources into primitive knowledge sources a more detailed view on the inference process can be obtained. Addressing the knowledge sources of the inference layer keeps the knowledge elicitation process focused.

The task structure for the initiation of anti-epileptic drug treatment is presented in Figure 3.6-II. In the task structure, the sequence of application of the knowledge sources is defined as well as the involved metaclasses. In the following the task structure for initiation of a therapy will be discussed and illustrated by the inference model of this task.

Figure 3.6-II: Task layer describing the control structure for the initiation of an anti-epileptic drug treatment.
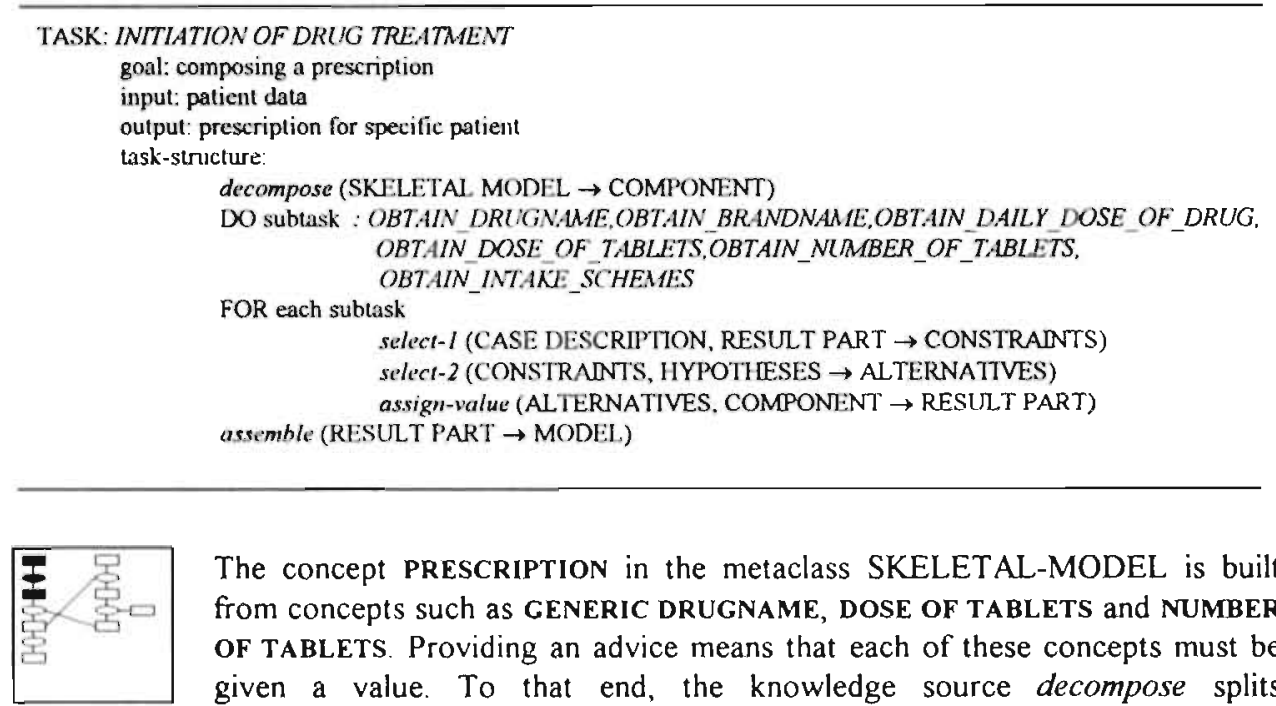

The concept PRESCRIPTION in the metaclass SKELETAL-MODEL is built from concepts such as GENERIC DRUGNAME, DOSE OF TABLETS and NUMBER OF TABLETS. Providing an advice means that each of these concepts must be given a value. To that end, the knowledge source decompose splits PRESCRIPTION into its constituent concepts. These concepts are all contained in the metaclass COMPONENT. ${ }^{2}$

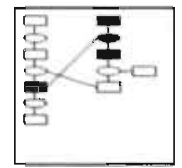

Next several subtasks are to be performed in sequence. As an example we describe the subtask OBTAIN DRUGNAME. This subtask assigns a specific drugname to the concept GENERIC DRUGNAME of the metaclass COMPONENT. To achieve this, information about the patient is extracted from the metaclass CASE DESCRIPTION. For example, the concept TYPE-OFEPILEPSY together with its value PARTIAL EPILEPSY is singled out by the knowledge source select-1. This concept is placed into the metaclass CONSTRAINTS, containing instantiated concepts which narrow down the choice of the drug. Note that the instantiated concepts in RESULT PART can influence the selection

${ }^{2}$ Black rectangles and ovals identify the metaclasses and knowledge sources discussed in the adjacent text. 


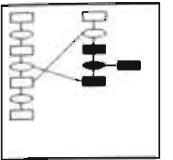

The metaclass HYPOTHESES contains data on the major anti-epileptic drugs. A set of first choice anti-epileptic drugs (CARBAMAZEPINE, PHENYTONN) is derived by select-2 using the information from CONSTRAINTS. This set of drugs becomes the value of the concept OPTIONAL GENERIC DRUGS in the metaclass ALTERNATIVES.

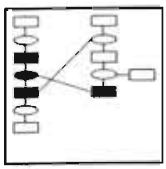

From this set of alternatives a drug (e.g. CARBAMAZEPINE) is selected. The knowledge source assign-value adds this piece of information to the GENERIC DRUGNAME in the metaclass RESULT PART.

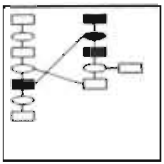

The process is repeated for the other subtasks which each add values to concepts in the metaclass COMPONENT, after obtaining information stored in CASE DESCRIPTION as well as from the intermediate conclusions from metaclass RESULT PART. For example, in the subtask OBTAIN DAILY DO$S E \_O F \_D R U G$, select-I takes the anti-epileptic drug from the GENERIC DRUGNAME in RESULT PART as well as additional restrictive information from the CASE DESCRIPTION such as the LABORATORY-RESULTS and WEIGHT. This information is stored in CONSTRANTS.

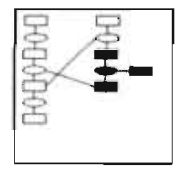

Next, information on DRUG DOSE IN MG/KG, MAXIMUM DOSE and AVAILABLE DOSES of TABLETS is obtained from the drug data in HYPOTHESES. This information is used by the composite knowledge source select-2 to obtain a drug dose for the patient. The dose is adjusted according to the MAXIMUM DOSE, the AVAILABLE DOSE OF TABLETS and the MINIMUM INTAKE FREQUENCY. The calculated dose serves as input for the calculation of values of the concepts OPTIONAL DOSE OF TABLETS and OPTIONAL NUMBER OF TABLETS in ALTERNATIVES.

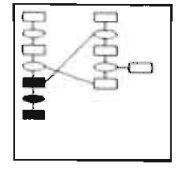

In this way each of the concepts in COMPONENT is assigned a value which is considered most appropriate given the status of the patient and the available medication. When the concepts belonging to the intake scheme are assigned a value, the individual concepts in RESULT PART are restructured by knowledge source assemble into the concept PRESCRIPTION in the metaclass MODEL.

\subsubsection{Monitoring drug treatment}

The KADS library has two interpretation models that are useful for modelling the monitoring of drug treatment: monitoring and assessment. In a monitoring task a comparison of individual data against a norm is made, followed by a classification of the difference, taking into account information from previous patient contacts In the assessment task changes in a group of concepts instead of in a single concept are classified. Table 3.6-III gives an example of the concepts contained by metaclasses in the monitoring process. 
Table 3.6-III: Examples of concepts contained by metaclasses defined in the monitoring process.

\begin{tabular}{|c|c|c|}
\hline METACLASS & CONCEPTS & ROLE \\
\hline CASE DESCRIPTION & patient data & description of cument state of the patient \\
\hline SELECTION CRITERLA & pathophysiological model & description of disease \\
\hline SYSTEM MODEL & physiological model & description of state of a healthy person \\
\hline VARLABLE SET & disease concepts & symptoms related to the patient \\
\hline PARAMETER SET & physiological data of healthy person & symptoms in case not diseased \\
\hline VARIABLE VALUE & $\begin{array}{l}\text { type of epilepsy } \\
\text { type of seizure } \\
\text { frequency of seizures etc. }\end{array}$ & values of symptoms when diseased \\
\hline PARAMETER VALUE & values of physiologic data & values of symptoms when not diseased \\
\hline DIFFERENCE & $\begin{array}{l}\text { presence of epilepsy } \\
\text { presence of seizure } \\
\text { presence of complaints etc. }\end{array}$ & $\begin{array}{l}\text { conclusions on symptoms relevant when } \\
\text { diseased }\end{array}$ \\
\hline HISTORICAL DATA & data from previous contacts & points of reference \\
\hline DISCREPANCY CLASSES & essential data on the patient & course of the disease \\
\hline MODEL SET & prototypical patient descriptions & profiles of standard patients \\
\hline NORM & $\begin{array}{l}\text { standard course of epilepsy } \\
\text { standard course of complaints } \\
\text { standard treaunent procedures etc. }\end{array}$ & $\begin{array}{l}\text { standard values of disease concepts and } \\
\text { treatment strategies }\end{array}$ \\
\hline DECISION CLASS & treatunent procedure & adjustment of treatment \\
\hline
\end{tabular}

Figure 3.6-111: Task structure of the anti-epileptic dng monitoring process.

TASK: MONITORING model-driven

goal: obtain conclusion on progress of disease

input: INITIATION OF DRLIG TREATMENT (MODEL)

output: DISCREPANCY CLASSES

task structure:

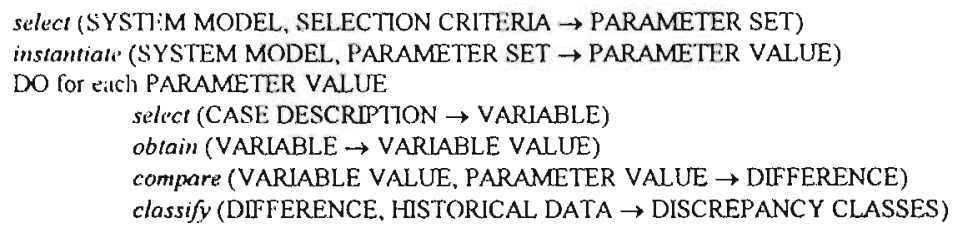

TASK : ASSESSMENT

goal: obtain a treatment procedure input MONITORING (DISCREPANCY CLASSES)

output: DECISION CLASS

task structure

specify (MODEL SET $\rightarrow$ NORMA)

match (DISCREPANCY CLASSES. NORM $\rightarrow$ DECISION CLASS) 


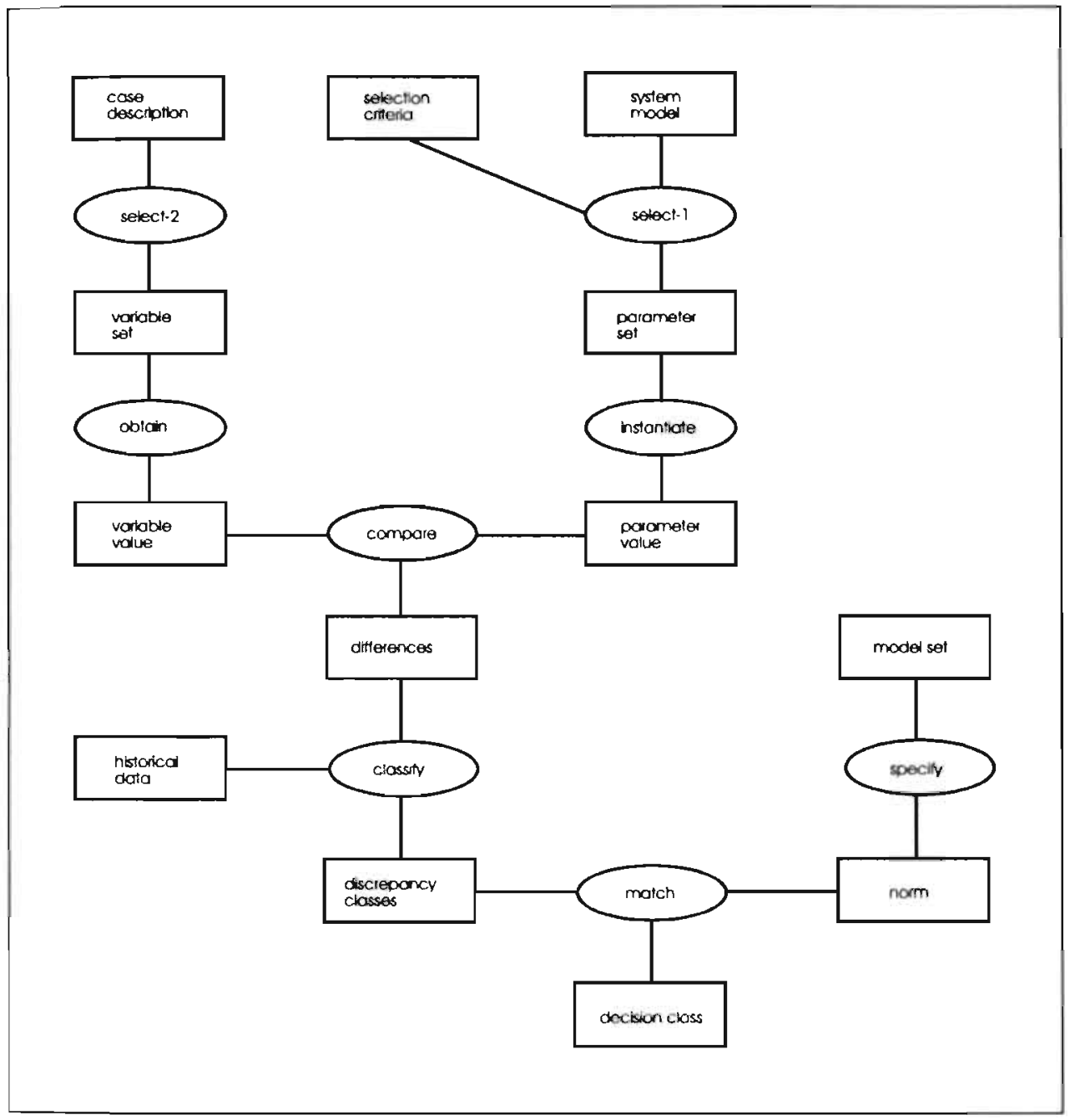

Figure 3.6-rV: Inference model for monitoring drug treatment of epilepsy.

From interviews and on-the-job-recordings it became clear that both a data-driven approach and a model-driven approach to monitoring may be applied. In a data-driven approach it is the patient who takes the initiative by starting to provide information on the effectiveness of the treatment. In the model-driven approach it is the physician who starts asking questions. In both approaches an identical set of issues - but not necessarily in the same sequence - is addressed for analysing the effectiveness of the treatment. In the following discussion the model-driven approach is used since this approach is more structured (see Figure 3.6-III for the task description). In Figure 3.6-IV the inference model of monitoring the drug treatment is presented. 
The interpretation models for the monitoring and assessment tasks are connected through metaclass DISCREPANCY CI.ASSES which contains the concepts that describe the course of the disease in the patient. The data from the concepts in this metaclass are matched with a set of profiles characterising possible courses of the disease. The prototypical profile matching the description of the disease course in the patient under consideration provides information for further treatment.

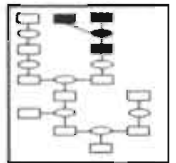

The concepts needed for monitoring the treatment process such as SEIZUREOCCURRENCE, TYPE-OF-SEIZURES, NUMBER-OF-SEIZURES, TYPE-OFEPILEPSY, COMPLAINTS, SIDE-EFFECTS, and COMPLIANCE are contained in the metaclass SELECTION CRITERIA. The SYSTEM MODEL represents the goal of the treatment e.g. the state of a healthy person. The knowledge source select $l$ singles out the relevant concepts from the metaclass SELECTION CRITERIA and obtains the values for these concepts from the SYSTEM MODEL. This piece of information is placed into the metaclass PARAMETER SET. For example, the metaclass PARAMETER SET would contain (SEIZURE-OCCURRENCE NO) for a healthy person.

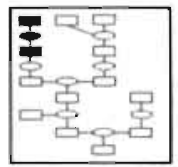

Next the corresponding concepts are singled out by select-2 from the metaclass CASE DESCRIPTION, which contains curTent information on the patient. As an example this could be (SEIZURE-OCCURRENCE YIS). This information is put into the VARIABLE SET

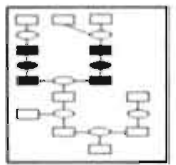

To compare these values, they are retrieved from the metaclasses VARIABLE SET and PARAMETER SET by respectively ubtuin and instantiate. The values are stored into the metaclass VARIABLE VALUE and PARAMETER VALUE respectively.

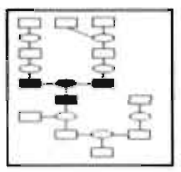

In our example the value of the concept SEIZURE-OCCURRENCE in the patient is different from the value in the physiological model. The conclusion of this comparison is denoted by the concept PRESENCE-OF-SEIZURES with the value PRESENT stored in the metaclass DIFFERENCES

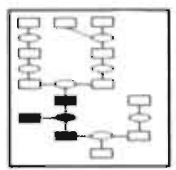

Information on the issue at stake may be compared with the values recorded during previous contacts listed in the metaclass HISTORICAL DATA. The result of the comparisons is contained by the metaclass DISCREPANCY CLASSES. In this case, if the value for PRESENCE-OF-SEIZURES in HISTORICAL DATA is ABSENT, the concept SEIZURE-COURSE in DISCREPANCY CLASSES receives the value INCREASED

By repeating this process for all concepts in metaclass SELECTION CRITERIA, all relevant aspects will be taken from the patient information and compared with historical data.

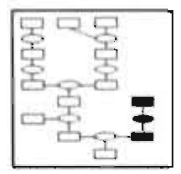

The 'status presence' of disease related items as stored in DISCREPANCY CLASSES is matched with similar items in a set of prototypical cases for which the treatment decision is known (Table 3.6-IV). The MODEL SET contains a description of these prototypical cases. From this metaclass concepts are derived and placed into the metaclass NORM by the knowledge source specify. 
Table 3.6-IV: An example of a description of a case as contained by the metaclass DISCREPANCY CLASSES and a prototypical case as contained by the metaclass NORM.

\begin{tabular}{ll}
\hline Abstract case description (DISCREPANCY CLASSES) & Prototypical case description (NORM) \\
\hline SEIZURE-COURSE INCREASED & SEIZURE-COURSE INCREASED \\
MINOR-SEIZURES ABSENT & MINOR-SEIZURES ABSENT \\
MAJOR-SEIZURES-FREQUENCY INCREASED & MAJOR-SEIZURES-FREQUENCY INCREASED \\
COMPLIANCE AS PRESCRIBED & COMPLIANCE AS PRESCRIBED \\
COMPLAINTS ABSENT & COMPLAINTS ABSENT \\
PLASMA-DRUG-LEVEL LOW THERAPEUTIC & PLASMA-DRUG-LEVEL LOW THERAPEUTIC \\
& TREATMENT-PROCEDURE INCREASEDOSE \\
\hline
\end{tabular}

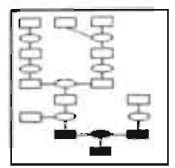

Each of the concepts in DISCREPANCY CLASSES is compared with the corresponding concept in NORM. A set of concepts in DISCREPANCY CLASSES equal to those of the prototypical patient leads to the storing of the concept TREATMENT-PROCEDURE together with its value from the prototypical case in DECISION CLASS. For example, the prototypical case description that matches the description in DISCREPANCY CLASSES contains among other concepts TREATMENT-PROCEDURE with the value INCREASE DOSE. This concept which indicates a further decision with respect to the treatment, is stored by the knowledge source match in the metaclass DECISION CLASS.

Two different types of decision classes were identified. While some decisions can be considered to be relevant only for the present contact, other treatment decisions could lead to treatment changes that have implications for future contacts. For example, switching the type of anti-epileptic drug or the decision to withdraw a drug may require a staged approach. In these procedures, the change in the treatment is stretched out over more than one contact thereby necessitating a monitoring of the consequences of the previous change.

\subsubsection{Additional treatment phases}

At the strategic level two additional phases were mentioned: the life-long monitoring phase and the phase of tapering drug treatment. Both phases are related to the monitoring and assessment tasks. The main difference between monitoring and life-long monitoring is a change in the system model. This is caused by a change in the goal of the drug treatment. Since in monitoring the goal is 'to cure' the patient by controlling the seizures, a physiological model is applied to compare disease concepts with. In life-long monitoring - with the exception of patients with well controlled seizures at a non-toxic level of medication who had recurrence of seizures and for whom therefore withdrawal of medication is no option (or who are known to get reoccurrence of seizures while tapering off the medication) - a balance between 'suppression of seizure and occurrence of side-effects' is required as curing the disease is less likely. Therefore the model is changed into a pathophysiological model of the patient. The withdrawal phase may also require a change in the system model. New seizures which may occur are due to withdrawing the drug instead of due to an ineffective treatment. 


\subsection{Implementation}

The two above described conceptual models were implemented in an experimental system using mul.ISPTM ${ }^{3}$. A layered structure separating the static parts of the knowledge from the procedural parts is used. The static knowledge of the domain layer is contained in frames. These are descriptions of concepts by means of their characteristics, so-called attributes and values. For example, the concept prescription has the attributes drugname, number of tablets and dose of tablets which may have the values carbamazepine, 2 and $200 \mathrm{mg}$, respectively. The domain concepts are arranged into three groups: information about medication, information about epilepsy and information about the patient.

Most of the heuristic knowledge expressed by the expert was captured in IF-THEN rules, which relate a condition to a specific action. Rules which are related to a specific section of the problem solving process are combined in rule sets. In this way the activation of only the relevant part of the knowledge base is possible. In the initiation part 14 of these rule sets with in total 66 rules were used for establishing a drug treatment. The monitoring part contains 965 rules which were combined into 59 rule sets. By using the frame and rule structures the composition of the knowledge as expressed by the expert was preserved in a familiar way. An assessment of the frames could be accomplished easily

Another part of the knowledge concerns the procedural aspects of the problem solving process. This is the task layer of the models described above. This knowledge is separated from the declarative part, but makes use of the information derived from the frames and rules.

A third part of the system is the user interface. The procedure driving the menus is described at the task layer. While in the initiation part we implemented some explanation on why a specific drug is used, in the monitoring part we can only provide a trace of the rules used for obtaining information about the reasoning process of the system.

In the initiation part as well as in the monitoring part a forward-chaining mechanism was used for reasoning. A simple conflict resolution scheme was implemented. The first rule of a set of rules of which the condition part matches the current information is identified. Its conclusion part is executed (an assertion added or a task executed). Next the rule is removed from the set and the process is repeated until the rule set is empty or none of the remaining rules fires.

\subsection{Discussion}

KADS has been presented by its developers as an approach for structured development of KBSs. In the original version of KADS the focus is on the analysis of cognitive processes. The main issue is the analysis of expertise which results in the definition of a model of expertise. This model of expertise is considered a high level specification of the expertise which is required for building the system (Breuker and Wielinga, 1987). This more cognitive oriented approach however widens the gap with implementing a working model. In a later version of KADS less emphasis is put on describing the expertise; a more pragmatic, implementation oriented view has been adopted. The model of expertise is seen as a model which is not a cognitive model of the expert but a specification of the system to be constructed by analysing the expert's problem solving behaviour (Schreiber et al. 1993).

\footnotetext{
3 muLISP is a trademark of Software House, Inc. 3660 Waialae Avenue. Honolulu, Hawaii 96816-3236.
} 
This change in view towards implementation can also be recognised in the changes in the contents of the conceptual model. In the early reports on KADS the conceptual model is a representation of the expertise, contained in a four layer model (Breuker et al. 1987, Schreiber et al. 1988). In the later version, a model of co-operation was added to the conceptual model. This model describes the system-user interaction; tasks to be accomplished by the user and by the system are indicated taking the competence of both agents into account (Breuker and de Greef, 1993). A model of co-operation and particularly a model of communication have been incorporated in the analysis phase (de Greef and Breuker 1992, Tansley and Hayball, 1993).

A third consequence resulting from this shift in attention towards implementation is a change in the project management approach. The early version of KADS is based on the waterfall model for conventional software design. In later reports on KADS the general structure as well as the life-cycle model were modified. A new life-cycle model $\mathrm{CONCH}$ (Client Oriented Normative Control Hierarchy) was introduced, based on the spiral life-cycle model (Boehm 1988, Taylor et al. 1989). A requirements analysis phase is now included. Guidelines and techniques for obtaining these requirements are absent, however (Hickman et al. 1989). More recently, a more detailed description of the processes in the analysis phase has been presented as well as a set of templates for modelling the organisational environment of the KBS (Tansley and Hayball 1993, Schreiber et al. 1994).

Although KADS advocates the use of models for the analysis of expertise, few guidelines with respect to the use and development of models are provided. The use of interpretation models is essential as they are a means for a top-down analysis of the elicited knowledge. Selection and application of these models, however, appears to be difficult. Some of the interpretation models have a very fine granularity. Other models, on the contrary, are less detailed and need extensive refinement. The interpretation models for assessment and monitoring could be used without significant refinement. This seems to be in agreement with the findings of others (Thiemann, 1989).

In case the model library has no interpretation model for the problem at hand, it may be constructed bottom-up. Development of an interpretation model for structuring the verbalised knowledge is supported by the conceptual modelling language. However, the set of primitives presented is only a tentative list of metaclasses and knowledge sources. An extension of the list is necessary, as has been recognised in the literature as well (Linster and Musen 1992, Woodward 1992).

Structured development improves the degree of completeness of the view on the domain but postpones the implementation process. In the knowledge acquisition phase, ideas verbalised by the expert have to be analysed, evaluated and refined. This process requires a spiral life-cycle approach, which enables an incremental evolution of a model of expertise. However, KADS does not offer tools to monitor this incremental process of modelling. Reviewing of intermediate results will require the interaction with a domain expert. In this process the model of expertise is of limited value as it is an abstract representation of his knowledge. Although the inference model will be important during the interaction with the expert, the terminology knowledge sources and metaclasses - as well as the names of the individual knowledge sources and metaclasses have a meaning of their own which is difficult to map on the concepts used by the expert to discuss the domain and his expertise. Also the interaction of the layers of a model will be difficult to comprehend. An extensive translation towards a detailed, less abstract model or an implementation of the model is required. 
As is the case with other approaches for software development KADS provides little support with respect to the evaluation of the quality of models. Work on metrication has taken place in KADS: process related and product related metrics, addressing issues such as duration and milestones, have been developed. A set of primitives which may be used for estimating costs has been defined (Readdie et al. 1989). Also in the new versions of KADS the evaluation of models of expertise needs further consideration. The iterative approach which enables the critiquing of evolving models - as in prototyping - should be included since this leads to a reduction of the risk of building an unsuitable system.

\subsection{References}

Bochm B.W., A spiral model of software development and enhancement, Computer, 3, 1988, pp. 6I72 .

Breuker J., de Greef P., Modelling system-user co-operation in KADS. In: KADS a principled approach to knowledge-based system development, (Schreiber, Wielinga, and Breuker, Eds.) Academic Press, London, 1993, pp. 47-70.

Breuker J., Wielinga B., van Someren M., de Hoog R., Schreiber G., de Greef P., Bredeweg B., Wielemaker J., Billeaut J.P., Davoodi M., Hayward S., Model driven knowledge acquisition, interpretation models, Deliverable task Al, Esprit Project 1098, Dept. Social Science Informatics, University of Amsterdam, 1987.

Breuker J., Wielinga B., Use of models in the interpretation of verbal data. In: Knowledge elicitation for experl systems, a handbook, (Kidd, Ed.), Plenum Press. New York, 1987.

Breuker J, Wiclinga B., Model-driven knowledge acquisition. interpretation models, Deliverable task Al Esprit Project 1098, Dept. Social Science Informatics, University of Amsterdam, 1984a.

Breuker J., Wielinga B., Techniques for knowledge elicitation and analysis, Report 1.5, Dept. Social Science Informatics, University of Amsterdam, 1984b.

Brcuker J., Winkels R., The use of the KADS methodology in designing an intelligent teaching system for diagnosis in physiotherapy. In: Lecture Noles in Medical Informatics 47, Knowledge based systems in medicine, (Talmon and Fox, Eds.), Springer-Verlag. Berlin, 1989, pp. 3-27.

Brown D.C., Chandrasekaran B, Design problem solving, knowledge structures and control strategies, Pitman Publishing, London, 1989.

Buchanan B.G., Shortliffe E.H., Rule-based expert systems, Addison-Wesley, Reading, Massachusetts, 1984

Burton A.M., Shadbolt N.R., Hedgecock A.P., Rugg G., A formal evaluation of knowledge elicitation techniques for expert systems: domain I. In: Research and development in expert systems $\mathrm{W}$, (Moralee, Ed), Cambridge University Press, 1987, pp. 136-145.

Burton A.M., Shadbolt N.R., Rugg G., Hedgecock A.P., Knowledge elicitation techniques in classification domains. In: Proceedings of ICAI-88, (Kodratoff, Ed), Pittman Publishing, London, 1988, pp. 85-90.

Cooke N.M., McDonald J.E., A formal methodology for acquiring and representing expert knowledge, Proceedings of the IEEE, 74, 1986, pp. 1422-1430. 
Cooke N.M., McDonald J.E., The application of psychological scaling techniques to knowledge based s elicitation for knowledge-based systems, International Journal of Man-Machine Studies, 26, 1987. pp.533-550

Cullen J., Bryman A., The knowledge acquisition bottleneck: time for reassessment?, Expert Systems, $5,1988, p p .216-225$

De Greef, P., Breuker J., Analysing system-user cooperation, Knowledge Acquisition, 4, 1992, pp. 89108 .

Diaper D., Designing expert systems, from Dan to Bursheba. In: Knowledge Elicitation, principles, techniques and applications, (Diaper, Ed), Ellis Horwood Limited, Chichester, 1989, pp. 17-46.

Dijk. J.E.M. van, Hilgevoord F.G., Jacques M.T., Otten G.A.M., Sittig A.C., Talmon J.L., Drie methoden voor kennis verwerving, Kennissystemen, 2, 1988, pp. 20-32.

Fen J.A., Veren L.C., Expert system development methodologics in theory and practice. In: Proceedings of the IEEE/ACM Conference on Developing and Managing Expert System Programs, Washington D.C., Sept. 1991, (Fcinstein, Ed), IEEE Computer Society Press, New York, 1991.

Grover M., A pragmatic knowledge acquisition methodology, TRW Defence Systems Group, Redondo Beach, Califomia, 1983.

Hayes-Roth F., Waterman D.A, Lenat D., Buiding expert systems, Addison-Wesley, Reading, Massachusetts. 1983.

Heng M.S.H., Why evolutionary development of expert systems appears to work, Future Generation Computer Systems, 3. 1987, pp. 103-109

Hickman F.R., Killin J.L., Land L., Mulhall T., Poner D. Taylor R.M., Analysis for knowledge-based systems, a practical guide to the KADS methodology, Ellis Horwood Limited, Chichester, 1989.

Hoffman R.R., The problem of extracting knowledge from experts from the perspective of experimental psychology, Artificial Intelligence Magazine. 2, 1987, pp. 53-67.

Jackson P., Introduction to expert systems, International Computer Science Series, Addison-Wesley Publishing Company, Reading, Massachusetts, 1986

Linster M., Musen M.A.. Use of KADS to create a conceptual model of the ONCOCIN task, Knowledge Acquisition, 4, 1992, pp. 55-87.

Mayhew P.J., Dearnley P.A., Organization and management of systems prototyping Information and Sofrware Technology, 32, 1990, pp. 245-252.

Nobis R., Siedka-Bauer H.G.. Prototyping and the LCM, Deliverable G13, NTE-GI3-PR-00I, ESPRIT P1098. NeuTech Entwicklungsgesellschaft mbH \& Co.KG, München, 1988.

Nguyen T., Perkins W.A., Laffey T.J., Checking an cxpert system's knowledge base for consistency and completeness. In: Proceedings of the I.JCAI. 1985, Los Angeles, pp. 375-378

O'Keefe R.M., Balci O., Smith E.P., Validating expert system performance, IEEE Expert, 4, 1987 , pp. $81-90$

O'Keefe R.M., The evaluation of decision-aiding systems: guidelines and methods, Informution and Management, 17, 1989, pp 217-226.

O’Moore R., Clarke K., Smeets R., Brender J., Nykänen P., McNair P., Grimson J., Barbeir B., Items of relevance for the evaluation of knowledge based expert systems and influence from domain characteristics, Deliverable EM-I.I, KAVAS (A I02I). Trinity College, Dublin, 1990.

Readdie M., Streng K.H., Wermser D., KADS metrication, Deliverable G10, SD-G10-R-001, ESPRIT PI098, SD Europe Ltd \& NeuTech Entwicklungsgesellschaft mbH \& Co.KG, München, 1989.

Reitman Olsen J., Rueter H.H., Extracting expertise from experts: methods for knowledge acquisition, Expert Systems, 4, 1987, pp. 152-168. 
Royce W.W., Managing the development of large software systems: concepts and techniques, Proceedings of the $9^{t h}$ International Conference on Sofiware Engineering, Computer Society Press, New York, 1987, pp. 121-125

Schreiber G., Wielinga B., KADS and conventional software engineering. In: KADS a principled approach to KBS development, (Scheiber, Wielinga and Breuker, Eds), Academic Press, London, 1993, pp. 151-165.

Schreiber G., Wielinga B., Breuker J., Introduction and overview on KADS, In: KADS a principled approach to KBS development, (Scheiber, Wielinga and Breuker, Eds), Academic Press, London, 1993, pp. 1-17.

Schreiber G., Wielinga B., Hoog R. de, Akkermans H., Velde W. van de, CommonKADS: a comprehensive methodology for KBS development, IEEE Expert, 12, 1994, pp.28-36.

Schreiber G., Breuker J., Bredeweg B., Wielinga B., Modelling in KBS development, paper presented at the Second Europcan Knowledge Acquisition Workshop '88, Bonn, June 1988.

Shadbolt N., Burton M., Knowledge elicitation. In: Evaluation of human work: practical ergonomics methodology, (Wilson and Corlett. Eds), Taylor and Francis, 1989.

Shaw M.L.G., Knowledge acquisition techniques for knowledge-based systems. In: Proceedings of the ( SC' 'Sl 88, Edmonton, 1988

Stephens M.A., Batıs P.E., Requirements engineering by prototyping: experiences in development of estimating system, Information and Software Technology, 32, 1990, pp. 253-257.

Tate G., Prototyping: helping to build the right software, Information and Software Technology, 32, 1990, pp. 237-244

Tanslcy D.S.W. Hayball C.C., Knowledge-based systems analysis and design: a KADS developer's handhook, Prentic: Hall, New York, 1993.

Taylor R.M., Porter D. Hickman F., Streng K.H., Tansley S., Dorbes G., System evolution, principles and methods (the life-cycle model). ESPRIT project 1098, deliverable Task G9, The KnowledgeBased Systems Centre of Touche Ross Management Consultants, London, 1989

Thiemann J., Wielinga over KADS: weglaten vierde niveau niet verwonderlijk, Kennissystemen, 3, 1989, pp. 3-5

Waterman D.A., A guide to expert systems, Addison-Wesley Publishing Company, Reading, Massachusetts, 1986.

Weitzel J.R., Kerschberg L., Developing knowledge-based systems: reorganizing the system development life cycle, Communications of the $A C M, 32,1989$. pp. 482-488.

Wellbank M., A review of knowledge acquisition techniques for expert systems, British Telecom Research, Martlesham Heath. 1983.

Wielinga B.J., Breuker J.A., Models of expertise. In: Advances in Artificial Intelligence 2, (du Boulay, Hogg, and Stecls. Eds.), Elsevier Science Publishers B.V., Amsterdam, 1987, pp. 497-509.

Woodward J.B., Developing K-ONCOCIN: a case study in the cognitive process of knowledge engineers, Knowledge Acquisition, 4, 1992, pp 237-258.

Wright G. Ayton P., Eliciting and modelling expert knowledge, Decision Support Systems, 3, 1987, pp. $13-26$ 


\section{Reference development}

\subsection{Method for system evaluation}

Evaluation $^{1}$ is generally considered as an essential part in the development of a DSS. Several methodologies have been proposed (Carlson 1974, Spiegelhalter 1983, Green and Keyes 1987, Lundsgaard 1987, Rushby 1988, O’Keefe et al. 1987, O'Keefe 1989, O'Leary and O'Keefe 1989, Rossi-Mori and Ricci 1988, Rossi-Mori et al. 1990, Hollnagel 1989, Preece 1990, Wyatt and Spiegelhalter 1990, O'Moore et al. 1990ab, Rector et al. 1990, Smeets and Talmon 1990, Engelbrecht et al. 1991, Sørgaard 1991, Bankowitz te al. 1992, Clarke et al. 1991, 1994) but none has been accepted as a standard. Usually validation ${ }^{2}$ of a DSS is considered the comerstone of the evaluation process as it addresses among other things the quality of the implemented knowledge. In our case correctness of the output of the system is the issue of interest.

The performance of the system and the neurologist(s) can be expressed by several quality concepts of which correctness and agreement are considered the most important. The correctness metric requires a reference with which the advice of the neurologists and the system can be compared. Correctness then is the percentage of cases which are assigned the reference value for an aspect of the treatment by either the system or a neurologist. Agreement is determined by comparing the treatment proposals of two or more observers without having recourse to a reference. The agreement beyond chance can be expressed by the kappa statistic.

The literature on anti-epileptic drug treatment can be used only as a guideline to assess the correctness of the advice of a system since the recommendations are too general. To assess the correctness of the prescription of an anti-epileptic drug treatment based on data of an individual patient, a reference for each case has to be available. The absence of detailed guidelines for the treatment of an individual patient necessitates consensus about what constitutes good treatment. Human expert(s) must be involved in the development of such a reference. To obtain a generally accepted opinion multiple experts have to be involved. To cope with multiple opinions a method for consensus formation is required.

\footnotetext{
'In general the definitions of ISO/IEC 9126: 1991 (E) are adhered to. The term evaluation incorporates the evaluation preparation - defined as metric selection, rating level definition and assessinent criteria definition - as well as the evaluation procedure i.e. the measurement, rating and assessment part of the evaluation procedure model.

2 Validation is a phase in the evaluation process which is focused on the correctness of the output of the system. It is concemed with the question whether the right system was built. It has to show that the program satisfies the user requirements and is considered to be a 'live' activity in which the software is tested.
} 
such records. In this process the case descriptions were evaluated on their clarity, fidelity and usability to assure that they contained sufficient information to enable diagnosis and treatment decision making. First we will describe the design of our 60 test cases in more detail. Secondly, it is described how from these 60 cases two reference sets for performance evaluation of the system were constructed.

\subsection{Test case design}

Written case descriptions were used in our studies. Although this approach does not mimic the daily patient-doctor contacts, physicians are used to paper cases when discussing and evaluating patients. Hence it is expected that an experimental set-up based on such paper cases will not introduce large biases. Also the fact that during a patient-doctor contact at the outpatient clinic, symptoms caused by an epileptic seizure are normally not observed but communicated verbally makes a written case description sufficiently similar to a patient-doctor contact. Therefore it is safe to assume that relevant information can be presented in writing (as if it were told by an eyewitness), without causing too much deformation of the problem solving process.

\subsubsection{Patient selection}

Patient records formed the basis for our paper cases. Epilepsy patients visiting the outpatient neurology clinic of the University Hospital of Maastricht who were 16 years or older were asked for consent to use anonymously their patient record. In total 70 patients were approached during a period of four months. Patients on anti-epileptic drug medication as well as patients off medication were included. Sixty-six patients gave their permission. Based on the availability of diagnostic information in the patient record 60 cases were finally used for our study.

Information normally gathered during the first few visits to a neurologist such as data concerning the admission of the patient, the patient history and hetero anamnestic information was extracted from the patient record. The family history, the tractus anamneses, psychosocial circumstances, the use of medication, intoxications and data from the physical examination, laboratory and other investigations such as EEG and CT-scans were included as well.

\subsubsection{Validity of paper cases}

The content of the paper cases was first assessed by two neurologists with more than 20 years of practical experience in epilepsy. One of them was professor and head of the Department of Neurology at the University Hospital Maastricht. The other a professor in epileptology and head of a special centre for epilepsy.

The paper cases were presented together with the original diagnosis. The assessors were asked to rate several aspects of the case on a five point scale.

\section{Clarity}

Clarity deals with how the case is presented: can the data be interpreted and is the information sufficient and not contradictory.

In 50 out of our 60 cases $(83.3 \%)$ both neurologists considered the case description to be clear. In five cases both stated that the case description was not clear. 
In the remaining five cases one of the participants did not provide a judgement, due to absence of relevant information or contradictions in the presented information. In three of these cases the other neurologist stated that the description was clear.

\section{Fidelity}

Fidelity deals with whether the case description could come from a real patient.

In fifty-three case descriptions both neurologists considered the case description realistic In four cases at least one neurologist disagreed with the statement that the case had a sufficient fidelity.

\section{Usability}

Usability deals with how well the case description is suited for our purpose. In 47 cases both experts agreed with the statement that the case was usable. In five cases at least one of the participants disagreed with the statement, while in eight cases both participants considered the case not usable.

On the basis of these results 13 case descriptions were refined taking the comments of the experts into account. The remarks mainly concerned unclear symptomatology of seizures and the lack of EEG-findings.

\section{Check on the usability}

After this refinement all 60 cases were assessed on their practical usability through the establishment of a diagnosis and prescription of a therapy by a second neurologist (Neurologist II) from the Neurology Department of the University Hospital in Maastricht.

Neurologist II diagnosed 51 cases as a specific type of epilepsy while in three cases the type of epilepsy was not certain. In six cases Neurologist II felt he needed additional information from a sleep-EEG and refrained from diagnosis.

In 50 of the 51 cases in which a type of epilepsy was diagnosed Neurologist II wrote a prescription. In one case he established a diagnosis but postponed drug treatment to enable registration of the EEG prior to medication.

For the three cases in which the type of epilepsy was uncertain no prescription was provided In the six cases in which Neurologist II requested a sleep-EEG, this information could not be provided and the cases were included unchanged in the set of test cases. The three cases in which the type of epilepsy was uncertain were not modified, as a treatment can be based on such a diagnosis.

\subsubsection{Description of the test cases}

The 60 cases concerned 34 male $(56.6 \%)$ and 26 female patients (43.3\%). The age of the patients ranged from 16 to 72 years. Forty-five percent of the patients was less than 30 years old, $36.6 \%$ was between 30 and 50 years of age while $18.4 \%$ was older than 50 years. Compared with Northern European (adult population) studies of active epilepsy our population is younger (Table 4.2-1). The age distribution resembles closer the adult population of an epilepsy centre (Meinardi 1990), which reflects the selection of problem cases from the Maastricht cohort. 
Table 4.2-I: Age distribution of the cases in our study compared with Northern European adult studies.

\begin{tabular}{llllllll}
\hline $\begin{array}{l}\text { Age } \\
\text { distribution }\end{array}$ & $\begin{array}{l}\text { Our study } \\
\mathrm{n}=60\end{array}$ & $\begin{array}{l}\text { Granf } \\
(1974) \\
\mathrm{n}=489\end{array}$ & $\begin{array}{l}\text { Wagner } \\
(1983) \\
\mathrm{n}=2572\end{array}$ & $\begin{array}{l}\text { Kerainen } \\
(198.9) \\
\mathrm{n}=1233\end{array}$ & $\begin{array}{l}\text { Sander } \\
(1990) \\
\mathrm{n}=425\end{array}$ & $\begin{array}{l}\text { Forsgren } \\
(1992) \\
\mathrm{n}=713\end{array}$ & $\begin{array}{l}\text { Meinardi* } \\
(1990) \\
\mathrm{n}=318\end{array}$ \\
\hline $15-19$ & 18 & 19 & 31.4 & 8.9 & 15.5 & 6.2 & 11.6 \\
$20-29$ & 22 & 30 & & 22.4 & 17.4 & 18.1 & 31.4 \\
$30-39$ & 20 & 13.7 & 34.0 & 18.0 & 14.6 & 21.3 & 17.6 \\
$40-49$ & 22 & 11.9 & & 16.6 & 8.2 & 15.4 & 15.1 \\
$50-59$ & 10 & 11.5 & 34.6 & 16.6 & 12.2 & 15.6 & 8.2 \\
$60-69$ & 8 & 8.2 & & 11.7 & 14.8 & 15.4 & 5.0 \\
$70-79$ & 2 & 5.7 & & 6.3 & 10.1 & 8.0 & \\
$>=80$ & & & & & 7.1 & & \\
\hline
\end{tabular}

Age distribution used: 16-20,21-30 etc

The following types of epilepsy (as validated by our two experts) were present:

- 28 cases $(47 \%)$ had partial epilepsy with secondary generalised partial seizures

- 16 cases (27\%) had primary (idiopathic) generalised epilepsy

- 9 cases $(15 \%)$ had partial epilepsy with complex partial seizures

- 2 cases $(3 \%)$ had partial epilepsy with simple partial seizures

- 5 cases $(8 \%)$ were diagnosed as partial epilepsy with complex partial seizures and partial epilepsy with secondary generalised partial seizures.

Using the classification of the International League Against Epilepsy 44 cases (73.3\%) had partial epilepsy while 16 cases (26.7\%) suffered from (idiopathic) generalised epilepsy.

Table 4.2-11: Distribution (in percentages) of epilepsies in diffenent populations as reported by Overweg (1985). For generalised epilepsy the percentage of cases with idiopathic generalised cpilepsy are presented as well.

\begin{tabular}{|c|c|c|c|c|}
\hline Type of epilepsy & study 1 & study 2 & study 3 & our study \\
\hline Generalised epilepsy & 45.8 & 23.0 & 18.2 & 26.7 \\
\hline Idiopathic & 4.3 .7 & 21.2 & 16.6 & 26.7 \\
\hline Other & 2.1 & 1.8 & 1.6 & 0.0 \\
\hline Partial epilepsy & 52.1 & 73.5 & 63.3 & 73.3 \\
\hline Unclassifiable & 1.7 & 3.4 & 18.4 & 0.0 \\
\hline
\end{tabular}

Overweg (1985) describes three studies which classify epilepsies according to the international classification of epilepsies of Gastaut (Table 4.2-I1). The first study describes the occurrence of epilepsies in a population referred to a single general hospital by primary care physicians. The two other studies describe patient populations in epilepsy centres.

Idiopathic generalised epilepsy occurred in respectively $16.6 \%$ and $21.2 \%$ of the patients of 15 years of age and older coming from epilepsy centres, while in the general hospital this occurred in $43.7 \%$ of the patients. The number of patients with partial epilepsies ranged from $63.3 \%$ up to 
$73.5 \%$. Also with respect to epilepsy syndromes our population resembles the more selected populations of epilepsy centres.

\subsection{Reference development}

Since no objective standard is available for assessing the system, the system was compared with the neurologists. Reference sets were created to obtain sets of cases with which the agreement between neurologists and the system could be assessed. In this section the procedure of constructing reference sets is presented. Two different reference sets were constructed: a diagnostic reference set containing cases for which at least two out of three neurologists agreed about the diagnosis and a treatment reference set for which both a majority agreement with respect to the diagnosis and treatment was obtained.

\subsubsection{Method for consensus formation}

In general two different approaches exist to reduce diversity in opinions among a group of individuals:

- Nominal techniques: a review of anonymous arguments/solutions

- Interactive techniques: a review of onymous arguments/solutions

Social and emotional factors which occur during an interactive group process may influence the way in which an agreement is reached. For example, dominance of some partners in the discussion, the tendency to embark quickly on a solution and the tendency to avoid conflicts, may induce nonoptimal solutions (van der Ven and Delbecq 1971, Delbecq et al. 1975). To avoid these problems, nominal techniques - the Nominal Group Technique (NGT) and the Delphi technique - were developed. In these approaches it remains unknown for the other participants who provided an opinion or solution. This anonymity of views makes it easier to discuss the various options without any prejudice. Nominal techniques have been applied successfully to establish objectives and standards (Loughlin and Moore 1979, Romm and Hulka 1979, Farrel and Scherer 1983, Quaglini et al. 1988, Kors et al. 1989, Kors and van Bemmel 1990), to explore risks and costs and to forecast events (Goodale and Gander 1976, Hill and Goodale 1981, Barret-Connor 1984, Arevalo and Washington 1988). The use of a modified Delphi approach has been shown to be valuable for developing references (Willems et al. 1985).

The NGT and the Delphi technique both consist of three steps: generating and pooling of ideas, feedback and evaluation. In the feedback round of the NGT, participants are expected to explain and discuss different views. In the Delphi procedure statistical information on the generated ideas is provided as feedback to the participants. In the third round participants still present their possibly revised opinions in an anonymous way (Delbecq and van der Ven 1975 , Linstone and Turoff 1975)

In our study we used a Delphi procedure in combination with a consensus meeting to obtain a reference for each case. The Delphi round was used to reduce the variation in prescribing. After the Delphi round the anonymous prescriptions were assessed by a peer review. The annotated prescriptions were discussed in the consensus meeting to reach a reference treatment. 


\subsubsection{Reference development/Acceptance testing}

Complete agreement among neurologists on diagnosis and treatment is expected difficult to achieve given the various opinions in the literature. A majority agreement among the participants was therefore considered to be sufficient for establishing a reference.

Although a neurologist may provide a prescription that is not equal to the reference it may still be considered an acceptable treatment. To assess the acceptability of the (possibly non-reference) prescriptions independent experts are needed to judge the quality of the prescriptions. To avoid to have to rely on the opinion of one single neurologist, each prescription was assessed by two experts

Table 4.3-I: Prescribing (P) and acceptability testing (A) scheme.

\begin{tabular}{cccccc}
\hline Data & Participant & & & & \\
set & A & B & C & D & E \\
\hline I & P & P & P & A & A \\
II & P & P & A & P & A \\
II & P & P & A & A & P \\
IV & P & A & P & P & A \\
V & P & A & P & A & P \\
VI & P & A & A & P & P \\
VII & A & P & P & P & A \\
VIII & A & P & P & A & P \\
IX & A & P & A & P & P \\
X & A & A & P & P & P \\
\hline
\end{tabular}

We designed a scheme for the reference development and acceptability testing procedure with the following properties (Table 4.3-1):

- each participant assessed the acceptability of prescriptions of cases for which he did not prescribe,

- each participant provided prescriptions for the same number of cases,

- each participant assessed the acceptability of the same number of prescriptions,

- each pair of participants was given the same number of cases to provide prescriptions,

- each pair of participants assessed the acceptability of the same number of prescriptions.

The 60 cases were divided into ten sets of six cases in such a way that each neurologist encountered approximately the same case mix (Table 4.3-11).

\subsubsection{Participating neurologists}

Five neurologists were asked to participate in this part of the study. The neurologists were working in neurology departments in five different general hospitals. Their clinical experience in neurology ranged from two to more than 20 years. Two of the participants received their training in neurology from the same supervisor. None of the participants was trained by the 
expert involved in the development of the system. All five neurologists were practising physicians, four as general neurologists, one with more emphasis on epilepsy care. Three participants saw an epilepsy patient every 1-3 days, 2 participants saw at least one epilepsy patient every day. Before the consensus meeting part of our study, one of the neurologists dropped out. In the follow-up part (see chapter 5) he was replaced by a colleague from the same hospital, having an equivalent number of years of clinical experience.

Table 4.3-II: Distribution of diagnoses presented to each participant for prescribing drug treatment.

\begin{tabular}{lrrrrr}
\hline Diagnosis* & N II & N IV & N V & N VI & N VII \\
\hline PEPCS & 6 & 6 & 5 & 5 & 5 \\
PESGPS & 17 & 17 & 17 & 16 & 17 \\
PEPCS+PESGPS & 3 & 4 & 3 & 2 & 3 \\
PESPS & 1 & 1 & 1 & 2 & 1 \\
IGE & 9 & 8 & 10 & 11 & 10 \\
Total & 36 & 36 & 36 & 36 & 36 \\
\hline
\end{tabular}

* PEPCS: partial epilepsy with complex parial seizures. PESGPS: partial epilcpsy with secondary generalised partial seizures, PESPS: partial epilepsy with simple partial seizurcs, IGE: primary (idiopathic) generalised epilepsy.

\subsubsection{Reference development process}

Since the diagnosis may determine the use of specific anti-epileptic drugs a majority agreement on the diagnosis has to be obtained before prescriptions can be compared. The cases for which no majority agreement (two out of three neurologists) on the general diagnosis (partial or generalised epilepsy) was reached were sent back to the neurologists. The various diagnoses established on such a case were given as feedback.

Cases for which the majority of the neurologists agreed on the diagnosis $(3 / 3$ or $2 / 3)$ but for which no majority agreement on the generic drug was obtained were retumed for review as well. Feedback was provided about the different drugs prescribed for the case. Furthermore, information on the frequency of suggested treatments in cases with the same general diagnosis, was given. Because the number of cases for which no agreement on the general diagnosis could be established was rather small, the reviewing of the diagnoses and the review of the generic drug were done simultaneously

After agreement was obtained on the generic drug, the prescribed daily doses were reviewed. For each drug the 5 and 95 percentiles of the daily dose in $\mathrm{mg} / \mathrm{kg}$ body weight were used to keep the number of cases to be reviewed within acceptable limits.

Prescriptions with a drug dose outside this range not caused by rounding off dosages towards an integer number of tablets were classified as outliers and returned for review

\subsection{Statistical analysis}

The $\chi^{2}$ test is used for analysing frequencies of cases in specific categories. It tests whether there is a significant difference between an observed number of cases in the categories and an expected number of cases based on the null hypothesis. The $\chi^{2}$ will be small if the observed and expected frequencies of cases are close to each other (Siegel and Castellan, 1988). 
The McNemar $\chi^{2}$ test is used for the analysis of 'before and after' designs in which the subjects are used as their own controls. The measurements should be made on an nominal or ordinal scale. The number of changes which occur are of importance. The test indicates whether the observed changes are significant (Siegel and Castellan, 1988).

For interpreting the significance of the results from the paired experiments on the acceptabilty scores of the prescriptions for a case the paired Student T-test is used. The differences were expected to be normally distributed. The degrees of freedom is $\mathrm{N}-1$, in which $\mathrm{N}$ is the number of pairs (Hayes, 1980).

The kappa statistic was used for obtaining information on the agreement beyond chance. This statistic was calculated for agreement among pairs of observers as well as for the agreement between one observer and a group (Schouten, 1985).

\subsection{Results}

In this paragraph the results are described of the agreement on the diagnosis and treatment before and after feedback. The agreement of the neurologists with the diagnostic and treatment reference is presented. Furthermore, the agreement between the diagnostic and consensus reference is described.

\subsubsection{Agreement on the diagnosis, generic drug and daily dose}

The degree of agreement among the neurologists on the diagnosis - both at a detailed and a general level - was established. In Table 4.5-I the different diagnostic categories at the general and detailed level are presented. This categorisation is based on the ILAE diagnostic classification (Commission on Classification and Terminology of the ILAE, 1989).

Table 4.5-I: General and detailed diagnoses

\begin{tabular}{ll}
\hline General type of epilepsy & Detailed type of epilepsy \\
\hline Generalised epilepsy & $\begin{array}{l}\text { Primary (idiopathic) generalised epilepsy } \\
\text { Myoclonic epilepsy } \\
\text { Lennox-Gastaut syndrome } \\
\text { West syndrome } \\
\text { Combinations }\end{array}$ \\
Partial epilepsy & $\begin{array}{l}\text { Partial epilepsy with complex partial seizures } \\
\text { Partial epilepsy with simple partial seizures } \\
\text { Partial epilepsy with secondary generalised seizures } \\
\text { Combinations }\end{array}$ \\
Combination & Combination of generalised and partial epilepsy \\
Other & Other suggestions \\
& $\begin{array}{l}\text { Withdrawal seizures } \\
\text { Multifocal epilepsy }\end{array}$ \\
Unknown & Diagnosis unknown or uncertain \\
& Diagnosis unknown or uncertain in combination with any of the above
\end{tabular}


Table 4.5-II presents the degree of agreement on the diagnosis and the prescribed generic drug before feedback. The table is divided into two sections. In the upper part of the table a breakdown of the cases is given according to the agreement with respect to the general and the detailed diagnosis.

Since for each case three neurologists provided a diagnosis and a prescription three $(3 / 3)$ or two neurologists $(2 / 3)$ can give the same diagnosis, or each neurologist may provide a different diagnosis (no). The third row of the upper part of the table presents the number of cases for each level of agreement.

The level of agreement between the three neurologists concerning the medication (stratified with respect to the degree of agreement on the diagnosis) is given in the lower part of the table (Therapy).

Total agreement with respect to the diagnosis (3/3) does not always lead to a total agreement with respect to the medication. Partial agreement with respect to the diagnosis $(2 / 3)$ can still result in total agreement with respect to the treatment, as the third neurologist who disagreed on the diagnosis may still prescribe the same generic drug. Of the 20 cases in which two out of three neurologists agreed on the general diagnosis, all three neurologists agreed about the generic drug in four cases. In 12 of these cases two out of three agreed on the treatment. In ten of these latter cases the pair of neurologists who agreed on the diagnosis also agreed on the generic drug.

Table 4.5-II: Agrecment on the general and detailed diagnosis and the type of generic drug before feedback.

\begin{tabular}{|c|c|c|c|c|c|c|c|c|}
\hline Diagnosis & \multicolumn{4}{|c|}{ general } & \multicolumn{4}{|c|}{ detailed } \\
\hline Agreement on type of epilepsy & $3 / 3$ & \multicolumn{2}{|c|}{$2 / 3$} & no & $3 / 3$ & \multicolumn{2}{|c|}{$2 / 3$} & no \\
\hline Number of cases & 36 & \multicolumn{2}{|c|}{20} & 4 & 20 & \multicolumn{2}{|c|}{26} & 14 \\
\hline Participants & All & Al! & $\begin{array}{l}\text { Diagn } \\
\text { agree }\end{array}$ & All & All & All & $\begin{array}{c}\text { Diagn } \\
\text { agree }\end{array}$ & All \\
\hline Therapy & & & & & & & & \\
\hline Agreement $3 / 3$ & 12 & 4 & 11.a. & 0 & 7 & 6 & ก.a. & 3 \\
\hline Agreement $2 / 3$ & 19 & 12 & 14 & 3 & 10 & 14 & 15 & 10 \\
\hline No ägreement & 5 & 4 & 6 & 1 & 3 & 6 & I! & l \\
\hline
\end{tabular}

Agreement on the diagnosis and the generic drug after feedhack

After the second round the number of cases with a majority agreement on the general diagnosis slightly increased (Table 4.5-III). In 58 out of 60 cases at least two out of three participants agreed on the general diagnosis. Also the agreement with respect to the therapy increased. A majority agreement on both the general diagnosis and the generic drug was reached in 55 out of 60 cases. 
Table 4.5-III: Agreement after foedluack.

\begin{tabular}{|c|c|c|c|c|c|c|c|c|}
\hline Diagnosis & \multicolumn{4}{|c|}{ general } & \multicolumn{4}{|c|}{ detailed } \\
\hline Agreement on type of epilepsy & $3 / 3$ & & & no & $3 / 3$ & & & no \\
\hline Number of cases & 36 & & & 2 & 20 & & & 13 \\
\hline Participants & All & All & $\begin{array}{l}\text { Dingn } \\
\text { agree }\end{array}$ & All & All & All & $\begin{array}{l}\text { Diagn } \\
\text { agree }\end{array}$ & All \\
\hline $\begin{array}{l}\text { Therapy } \\
\text { Agreement } 3 / 3\end{array}$ & 13 & 6 & n.a. & 0 & 8 & 8 & п.а. & 3 \\
\hline Agreement $2 / 3$ & 23 & 14 & 19 & I & 12 & 17 & 19 & 9 \\
\hline No agreement & 0 & 2 & 3 & 1 & 0 & 2 & 8 & 1 \\
\hline
\end{tabular}

For the detailed diagnosis level a majority agreement on both the diagnosis and treatment was reached in $65 \%$ of the cases as compared to $53 \%$ in the first round.

For the general diagnosis level after the first round there were $15(25.0 \%)$ cases with no agreement on either the general diagnosis or generic drug or both. This number decreased to $5(8.3 \%)$ cases after feedback (McNemar $\chi^{2}=8.1 \mathrm{p}<0.01$ ). In three cases disagreement remained with respect to the prescribed generic drug despite the fact that two neurologists agreed on the general type of epilepsy. In two other cases the participants disagreed on the general diagnosis. For the detailed diagnosis in 28 cases $(47 \%$ ) a majority agreement on either the general diagnosis, the generic drug or both was not reached initially. After feedback for 21 cases $(35 \%)$ a majority agreement was not reached.

Agreement on the drug dosage

The upper 95 percentile and the lower 5 percentile $\mathrm{mg} / \mathrm{kg}$ dosage of the drug were calculated for CBZ, VPA and PHT from the cases in which those drugs were prescribed in the second round. These dosages were then used to calculate the maximally and minimally acceptable dose for each case. In 10 cases the prescribed dose was outside these margins. In 7 of these cases the higher or lower dose could be attributed to rounding towards an integer number of tablets. In 3 cases a dose of CBZ was prescribed which was outside the obtained ranges. Review of these cases did not lead to a change in the prescribed dose.

\subsubsection{Description of the reference sets}

Diagnostic reference set

Whether an anti-epileptic drug has to be selected on the basis of a detailed diagnosis or whether a global description of the type of epilepsy is sufficient is not clear from the literature (chapter 2). Therefore, it was decided to use the set of 47 cases where the majority of the neurologists agreed about the detailed diagnosis for the assessment of the system.

The 47 cases in which the majority of the neurologists agreed about the detailed diagnosis is called the diagnosic reference sel. Although each neurologist saw 36 cases the reference cases are not equally divided over the individual neurologists. Table 4.5-IV gives the number of cases which each individual neurologist agreed with the detailed diagnosis for. 
Table 4.5-IV: Number of cases from the diagnostic reference set seen by each neurologist.

\begin{tabular}{|c|c|c|c|c|c|}
\hline Neurologist & III $(\mathrm{n}=36)$ & IV $(n=.36)$ & $V(n=36)$ & $V I(n=36)$ & VU $(n=36)$ \\
\hline Agreement with ref. & 23 & 25 & 21 & 22 & 23 \\
\hline detailed diagnosis & $64 \%$ & $69 \%$ & $58 \%$ & $61 \%$ & $64 \%$ \\
\hline
\end{tabular}

The reference cases were mainly diagnosed as partial epilepsies $(64 \%)$, while $30 \%$ of the cases were diagnosed as primary (idiopathic) generalised epilepsy. Furthermore, twice the neurologists agreed that the type of epilepsy was uncertain or unknown. Once a combination of partial and generalised epilepsy was diagnosed.

One hundred and fourteen prescriptions were generated for the 47 cases by the neurologists that agreed on the detailed diagnosis. In $51.8 \%, 24.6 \%$ and $10.5 \%$ of these prescriptions the selected drug was CBZ, VPA and PHT respectively. Eleven times a neurologist decided not to initiate anti-epileptic drug treatment. For the 47 cases a majority treatment was defined (if it existed) consisting of the opinions of those neurologists which agreed about the detailed diagnosis (Table 4.5-V)

Of the cases diagnosed as a kind of partial epilepsy $63 \%$ received a prescription of $\mathrm{CBZ}$, while in 10\% PHT was the drug prescribed by the majority. In seven cases (23\%) a majority agreement about the treatment was not obtained (No ref) despite the fact that the neurologists agreed about the diagnosis. In one case the reference was not to start drug treatment No drug)

The drug for cases diagnosed as a primary (idiopathic) generalised epilepsy was mainly VPA $(71 \%)$. CBZ was the treatment of the majority in $10 \%$ of these cases. In one case a majority treatment was not obtained.

For the combination of partial and generalised epilepsy $\mathrm{CBZ}$ was used by the majority. For the two cases in which the diagnosis was not certain anti-epileptic drug treatment was not initiated.

Table 4.5-V: Detailed diagnoses and treatment in the diagnostic reference set.

\begin{tabular}{|c|c|c|c|c|c|c|c|c|}
\hline Diagnosis & & Cuses & CBZ & VPA & PHT & No ref & No drug & $\%$ \\
\hline \multirow{4}{*}{$\begin{array}{l}\text { Partial } \\
\text { epilepsy }\end{array}$} & PECPS & 6 & 4 & & & 2 & & 13 \\
\hline & PESGS & 10 & 6 & & 2 & 2 & & 21 \\
\hline & PECPS+PI:SGS & 11 & 8 & & & 2 & 1 & 23 \\
\hline & PESIS & 3 & 1 & & 1 & I & & 6 \\
\hline $\begin{array}{l}\text { Gencralised } \\
\text { epilepsy }\end{array}$ & IGE & 14 & 3 & 10 & & 1 & & 30 \\
\hline Combi & IGIT+PECPS & 1 & 1 & & & & & 2 \\
\hline Unknown & & 2 & & & & & 2 & 4 \\
\hline Total & & 47 & 23 & 10 & 3 & 8 & 3 & 100 \\
\hline
\end{tabular}




\section{Treatment reference set}

In 39 out of the 47 cases a majority agreement on the prescribed treatment was reached. This subset of cases is called the trealmenl reference set.

Cases in which two neurologists agreed about the detailed diagnosis but disagreed about the prescribed drug were excluded, even if the third neurologist who disagreed about the diagnosis agreed on the prescribed drug.

In Table 4.5-VI the number of cases for which the individual neurologists agreed with the reference are presented. Because the neurologists may disagree with the diagnosis or with the prescribed drug the number of cases per neurologist differs.

Table 4.5-VI: Number of cases in which an individual ncurologist agrees with the treatment reference. The number of cases seen by each neurologist and belonging to the diagnostic reference set is indicated by $n$.

\begin{tabular}{llllll}
\hline Neurologist & III $(n=23)$ & IV $(n=25)$ & V $(n=21)$ & V $(n=22)$ & VI $(n=23)$ \\
\hline Agreement with reference drug & 18 & 20 & 14 & 16 & 18 \\
& $78.3 \%$ & $80.0 \%$ & $66.6 \%$ & $72.7 \%$ & $78.3 \%$ \\
\hline
\end{tabular}

Overall, in 18 of the 47 cases (38\%) the majority of the neurologists agreed on the detailed diagnosis as well as on the generic drug and daily dose. In three of these cases no drug treatment was started. In the remaining 15 cases a majority agreement on the generic drug as well as on the daily dose was observed

In 13 of the 14 cases diagnosed as primary (idiopathic) generalised epilepsy, the majority agreed about the drug. In eight of these 13 cases (57\%) at least two neurologists prescribed the same daily dose.

In 22 of the 30 cases diagnosed as a type of partial epilepsy the neurologists agreed about the prescribed drug. In six of these 22 cases $(27 \%)$ the majority agreed about the daily dose. This concerned five cases for which $\mathrm{CBZ}$ and one case for which PHT was prescribed. The neurologists also agreed on the prescribed drug and daily dose for the one case diagnosed as a combination of partial and generalised epilepsy.

\section{Consensus meeting reference}

After the three Delphi rounds the neurologists still varied with respect to the prescribed drug and the daily dose for a specific case. Therefore a consensus meeting was organised. The cases of the diagnostic reference set were discussed. Instead of a reference for an individual case in this consensus meeting guidelines were developed on how to approach a patient with epilepsy in general. A detailed outline of these guidelines is presented in chapter 5 . 
Table 4.5-VII: Comparison of the diagnostic reference set and the consensus.

\begin{tabular}{|c|c|c|c|c|c|c|}
\hline \multirow{8}{*}{$\begin{array}{l}\text { Diagnostic } \\
\text { reference set } \\
(n=47)\end{array}$} & \multirow[b]{2}{*}{ Drug } & \multicolumn{5}{|c|}{ Consensus drug } \\
\hline & & $\mathrm{CBZ}$ & VPA & PHT & No drug & Total \\
\hline & $\mathrm{CBZ}$ & 18 & 2 & 3 & & 23 \\
\hline & VPA & & 10 & & & 10 \\
\hline & $\mathrm{PHT}$ & 3 & & & & 3 \\
\hline & No drug & 1 & & & 2 & 3 \\
\hline & No ret & 4 & 2 & 2 & & 8 \\
\hline & Tota! & 26 & 14 & 5 & 2 & 47 \\
\hline
\end{tabular}

The guidelines defined in the consensus meeting were applied to the diagnostic reference set. Of the 34 cases with partial epilepsy, $26(84 \%)$ should receive CBZ while five cases ( $16 \%$ ) needed PHT according to the consensus (Table 4.5-VII). All 14 cases diagnosed as primary (idiopathic) generalised epilepsy received VPA. For cases in which the type of epilepsy was unknown or uncertain drug treatment was not initiated

In 30 cases (64\%) the majority drug was in accordance with the consensus. Cases in which the reference drug was PHT should have received $\mathrm{CBZ}$ according to the consensus. The case for which treatment was not started although the diagnosis was established should have been treated with CBZ. Using the guidelines a treatment could be defined for those cases for which no reference was obtained by means of the Delphi procedure.

\subsection{References}

Arevalo J.A., Washington E., Cost-effectiveness of the prenatal screening and immunization for hepatitis B virus, Journal of the American Medical Association, 259, 1988, pp 365-369.

Bankowitz R.A., Lave J R., McNcil M.A.. A method for assessing the impact of a computer based decision support system on health care outcomes, Methods of Information in Medicine, 31, 1992, pp.3-11.

Barret-Connor E., Natural history of intestinal parasites in asymptomatic adults, The Journal of Family Practitioners, 19, 1984, pp.635-639.

Carlson E.D., Evaluating the impact of information systems, Management Informatics, 3, 1974, pp.5767.

Clarke K., O'Moore R., Smects R., Talmon J., Brender J., McNair P., Nykänen P., Grimson J., Barber B., A methodology for evaluation of knowledge-based systems. In: Lecrure notes in Medical Informatics 45, Proceedings MIE 91, (Adlassnig, Graber, Bengtsson and Hansen, Eds.), SpringerVerlag, Berlin, 1991, pp. 361-365.

Clarke K., O`Moore R., Smects R., Talmon J., Brender J., McNair P., Nykänen P., Grimson J., Barber B., Methodology for evaluation of knowledge-bascd systems in medicine, Artificial Intelligence in Medicine, 6, 1994, pp.107-121.

Commission on Classification and Terminology of the Intemational League Against Epilepsy, Proposal for revised classification of epilepsies and epileptic syndromes, Epilepsia, 30, 1989, pp. 389-399.

Delbecq A.L., Ven A.H. van der, Gustafson D.H., Group lechniques for program planning: a guide 10 nominal group and Delphi processes, Scott Foresman, Glenvicw IL, 1975. 
Engelbrecht R., Fitter M., Rector A., Requirements for a medical workstation using user-centered design. In: Lecture notes in Medical Informatics 45. Proceedings MIE 91 . (Adlassnig, Graber, Bengtsson and Hansen. Eds.), Springer-Verlag, Berlin, 1991, pp. 140-144.

Farrel P., Scherer K., The Delphi technique as a method for selecting criteria to evaluate nursing care, Nursing Papers, 15, 1983, pp.51-60.

Forsgren L., Prevalence of epilepsy in adults in northem Sweden, Epilepsia, 33, 1992, pp. 450-458

Goodale F., Gander G.W., The future of pathology: a Delphi study by pathology dcpartment chairmen, Journal of Medical Education, 51, 1976, pp.897-903

Graaf A.S de, Epidemiological aspects of epilepsy in northem Norway, Epilepsia, 15, 1974, pp. 291 299.

Green C.J., Keyes M.M., Verification and validation of expert systems. In: Proceedings of the Western Conference on Expert Systems, IEEE Computer Society Press, New York, 1987, pp.38-43.

Hayes W.L., Statistics for the social sciences, $2^{\text {nd }}$ edition, Holt Rineman and Winston, London, 1980.

Hill R.B., Goodale F. The Delphi predictions of pathology chaimen; a six-year retrospective view, Journal of Medical Education, 56, 1981, pp.537-546.

Hollnagel E., Evaluation of expert systems. In: Topics in expert system design, eds. Guida G., Tasso C., Elsevier Science Publishers B.V. (North-Holland), Amsterdam, 1989, pp.377-416

Kors J., Bemmel J.H. van, The Delphi method: a review of its applications in medicine. In: Proceedings MEDINFO'89, (Eds.)Beijing Singapore, 1989, pp. 156-160.

Kors J., Sittig A.C.. Bemmel J.H. van, The Delphi method to validate diagnostic knowledge in computerized HCG interpretation. Methods of Informalion in Medicine. 29. 1990, pp.44-50.

Keränen T., Riekkinen P.J., Sillanpää M., Incidence and prevalence of epilepsy in adults in eastem Finland, Epilepsia, 30, 1989, pp.413-421

Linstone H.A., Turoff M., The Delphi method: techniques and applications, Reading, Mass. AddisonWesley, 1975.

Loughlin K.G., Moore L.F., Using Delphi to achieve congruent objectives and activities in a pediatrics department, Journal of Medical Education, 54, 1979, pp.101-106.

Lundsgaard H.P., Evaluating medical expert systems, Social Science in Medicine. 24, 1987, pp. 805819

Meinardi H., Pachlatko C.. Special centers for epilepsy. In: Comprehensive lipileptology, (Dam and Gram, Eds.), Raven Press, New York, 1990, pp. 769-779

O'Keefe R.M., Balci O., Smith E.P., Validating expert systems performance, IEEE Expert, 4, 1987, pp. 81-89.

O'Keefe R.M., The evaluation of decision-aiding systems: guidelines and methods, Information and Management, 17, 1989, pp.217-226.

O'Leary D., O'Keefe R.M. Verifying and validating expert systems. Tulorial at the IJAI-89, Detroit, 1989.

O'Moore R., Clarke K., Smeets R., Brender J., Nykänen P., McNair P., Grimson J., Barber B., ltems of relevance for the evaluation of knowledge basedlexpert systems and the influence of domain characteristics, KAVAS (A 1021), Report EM-1.1, Trinity College, Dublin, 1990

O'Moore R., Clarke K., Brender J., McNair P., Nykänen P., Smeets R., Talmon J., Grimson J., Barber B., Methodology for evaluation of knowledge based systems, KAVAS (AI021), Report EM-1.2, Trinity College, Dublin, 1990.

Ovenweg J., Withdrawal of anti-epileptic drigs in seizure-free adult patients. prediction of outcome, thesis, Amsterdam, 1985 
Preece A.D., Towards a methodology for evaluating expert systems, Expert Systems, 7, 1990, pp.215223

Quaglini S., Stefanelli M., Barosi G., Benzuini A., A performance evaluation of the expert system ANEMIA, Computers and Biomedical Research, 21, 1988, pp.307-323.

Rector A., Engelbrecht R., Diedrich T., Fieschi M., Fitter M., Moser W., Schneider J., Report on validation and verification requirements and operational criteria, Eurodiabeta (AIM 1019), Deliverable I2, DG-XIII of the Commission of the EC, Brussels, 1990.

Romm F.J., Hulka B.S., Developing criteria for quality of care assessment: effect of the Delphi technique, Health Service Research, 14, 1979, pp.309-312.

Rossi-Mori A., Ricci F.L., On the assessment of medical expert systems. In: Expert systems and decision support in medicine. (Rienhoff, Piccollo and Schneider, Eds.), Lecture notes in medical Informatics 36, Springer Verlag, Berlin, 1988, pp. 292-297.

Russi-Mori A., Pisanelli D.M., Ricci F.L., Evaluation stages and design stups for knowledge-base systems in medicine, Medical Informatics. 15, 1990, pp.191-204.

Rushby J., Quality measures and assurance for AI sofiware, NASA contractor report 4187, SRI Intemational, Menlo Park, 1988.

Sander J.W.A.S., Hart Y.M., Johnson A.L., Shorvon S.D., National General Practice Study of Epilepsy: newly diagnosed epileptic seizures in a general population, The Lancet, 336, 1990, pp. 1267-1271.

Siegel S., Castellan N.J., Nonparametric statistics for the behavioral sciences, $2^{\text {nd }}$ edition, McGrawHill Book Company, New York, 1988.

Schouten H.J.A., Statistical Measurement of interobserver agreement, analysis of agreements and disagreements between observers, thesis, Rotterdam, 1985.

Smeets R., Talmon J., General methodology for the assessment of decision support systems. In: A critique of the evaluation methodology, (Nolan, Ed.), KAVAS (AIM 102.1), Report EM-2.I, Hvidovre Hospital, Hvidovre, 1990.

Sorgaard P.. Evaluating expert system prototypes. Artificial Intelligence and Socicty, 5, 1991, pp.317.

Spiegelhalter D., Evaluation of clinical decision aids. with an application to a system for dyspepsia, Statistics in Medicine, 2, 1983, pp 207-216.

Ven A. van der., Delbecq A.L. Nominal versus group processes for committee decision-making effectiveness, Academy of Management Journal, 6, 1971, pp. 203-212.

Wagner A.L., A clinical and epidemiological study of adult patients with epilepsy, Acta Neurologica Scandinavia, 94 (Suppl.). 1983, pp. 63-72

Willems J.L., Amaud P., Bemmel J.H. van, Bourdillon P.J., Degani R, Dennis B. Harms F.M.A., Macfarlane P.W., Mazzocca G., Meyer J., Ritsema van Eck H.J., Robles de Medina E.O., Zywietz C.. Establishment of a reference library for evaluating computer ECG measurement programs, Computers and Biomedical Research, 18, 1985, pp.439-457.

Wyatt J., Spicgellialter D., Evaluating medical expert systems: what to test and how?. Medical Iniormatics, 15, 1990, pp. 205-217. 



\section{Results on initiation of treatment}

\subsection{Introduction}

In this chapter the results of the analysis of the performance of the system in prescribing antiepileptic drugs in the initiation phase are reported. The advice of the system is compared both with the advice of two neurologists from the same department, one of which provided the knowledge implemented in the system and with that of five neurologists from different neurology departments. In the latter case the final prescriptions of the neurologists as obtained via the Delphi procedure are compared with those of the system.

To assess the acceptability of the advice - independent of whether the advice was according to the majority agreement - the prescriptions were reviewed by two neurologists.

A consensus meeting was held to discuss and possibly reduce the variability among the neurologists that remained after the Delphi procedure. While in the Delphi procedure the objective was to obtain a majority decision for each individual case, a more global approach for treatment was defined in the consensus meeting. These guidelines are used as a reference to compare the system and the neurologists with.

\subsection{Study design}

The goal of the study is to establish the adequacy of the advice of the system by comparing it with the advice of neurologists. A staged approach was adopted for the evaluation.

Five different assessments of the advice of the system were made. For each of the 60 cases of our test set, the expert neurologist (Neurologist I) whose knowledge was implemented in the system, provided a diagnosis and a prescription. The advice of the system, based on the detailed diagnosis of the neurologist and the other relevant patient data, was compared with the advice of this neurologist. This assessment provides some insight in the quality of the knowledge acquisition process.

To assess the impact of local differences a second neurologist (Neurologist II) from the same department as Neurologist $\mathrm{l}$ also provided a detailed diagnosis and a prescription for the 60 cases. A comparison was made with the advice of the system using Neurologist II's diagnosis.

In a third experiment the drug and the daily dose proposed by the system are compared with the treatments suggested by the neurologists after the Delphi method. The diagnostic reference set was used for assessing the system's proposed generic drug, the treatment reference set for assessing the suggested daily dose. This comparison provides a more objective measure of the 
performance of the system as these neurologists did not belong to the department of the expert neurologist.

A prescription different from the majority decision may still be considered a good option for treatment. Therefore, the acceptability of the prescriptions for each case in the diagnostic reference set was assessed by two neurologists who did not provide prescriptions for such a case.

To reduce the interobserver variability to determine the system's correctness, discrepancies in prescriptions were discussed during a consensus meeting. In this meeting general guidelines were proposed by the neurologists. From these guidelines a reference prescription was derived for each case in our diagnostic reference set. The advice of the system and the prescriptions of the individual neurologists were compared with this reference.

In the above design the focus of the assessment gradually shifts from an assessment of the coverage of the implemented knowledge and the correctness of the implementation to an assessment and comparison of the system's and neurologists' performance using a set of cases with reference diagnoses and prescriptions

When discussing the results a difference is made between cases and prescriptions. One has to remember that for each case up to three neurologists prescribe. The sets of cases for which individual neurologists provide a prescription vary. Therefore in most analyses the number of prescriptions is used.

\subsection{Comparing the system with Neurologist I and Neurologist II}

In this paragraph the advice of the system is compared with that of Neurologists I and II, using the 60 test cases. The diagnosis established by the neurologist is entered into the system. Since both neurologists independently diagnose the same case, it is possible that a case receives two different diagnoses. These cases are then entered twice into the system and the treatment advice of the neurologist is compared with that of the system using the diagnosis of that neurologist. In addition to the diagnosis, information about seizures and previous successful treatments, side-effects, use of co-medication, liver and kidney function and weight is entered into the system and used in the reasoning process

\subsubsection{Agreement on the generic drug}

Neurologist 1 provided 59 prescriptions. Although he was able to diagnose the type of epilepsy by using the available information, in one case he did not prescribe a drug because he wanted an additional EEG registration to be made before commencing drug treatment. Monotherapy was used in all cases.

Table 5.3-I: Agreement between Neurologist I, Neurologist II and the system.

\begin{tabular}{|c|c|c|c|c|c|c|}
\hline \multirow{2}{*}{$\begin{array}{l}\text { Gencric drug } \\
\text { System }\end{array}$} & \multicolumn{3}{|c|}{ Neurologist I } & \multicolumn{3}{|c|}{ Neurologist II } \\
\hline & No drug & CBZ. & $\mathrm{Na}-\mathrm{VPA}$ & No drug & $\mathrm{CBZ}$ & Na-VPA \\
\hline No drug & 0 & 0 & 0 & 9 & 0 & 0 \\
\hline CBZ & l & 44 & 0 & 0 & 40 & 0 \\
\hline Na-VPA & 0 & 0 & 15 & 1 & 0 & 10 \\
\hline
\end{tabular}

$\mathrm{CBZ}=$ carbanazepine, $\mathrm{Na}-\mathrm{VPA}=$ sodium valproate 
In 59 out of the 60 cases the system prescribed the same generic drug as Neurologist I (Table 5.3-1). Although the detailed diagnosis is entered, the system reasons with the global diagnosis. Also Neurologist I assigns a particular drug on the basis of the global diagnosis. If the diagnosis was partial epilepsy CBZ was proposed. For the cases diagnosed as primary (idiopathic) generalised epilepsy Na-VPA was selected. In the one case in which the neurologist established a diagnosis but did not provide a prescription the system suggested CBZ since the diagnosis was partial epilepsy.

Neurologist II established a diagnosis in 51 cases while a prescription was provided in 50 cases. Monotherapy was used for all of these cases. In six cases Neurologist Il could not establish the diagnosis and in three cases he was not certain about the diagnosis. In these nine cases Neurologist II decided not to prescribe a drug. In one additional case the neurologist decided not to prescribe a drug although he reached the diagnosis primary (idiopathic) generalised epilepsy. The reason for this was that he wanted to have additional EEG data about the patient. Because anti-epileptic drugs can influence the recordings he decided not to prescribe any drugs until the EEG findings were available

In 50 out of 51 cases (Table 5.3-1) the system and Neurologist II agreed on the drug treatment. Also Neurologist II selects a specific drug on the basis of the global diagnosis. The 39 cases diagnosed by Neurologist II as partial epilepsy received CBZ. In the one case diagnosed as primary (idiopathic) generalised epilepsy and partial epilepsy with secondary generalised partial seizures CBZ was also prescribed. In all ten cases diagnosed as primary (idiopathic) generalised epilepsy Na-VPA was prescribed. The system prescribed the same drugs. For the nine cases in which the neurologist did not provide a diagnosis the system did not suggest a treatment. In the one case in which the neurologist established the diagnosis primary (idiopathic) generalised epilepsy but did not prescribe a drug, the system suggested Na-VPA.

\subsubsection{Agreement on tradename}

When prescribing a drug one can use a generic name, leaving the choice of the brand to the pharmacist, or a tradename. Both neurologists used only tradenames. The system was programmed to do likewise.

The neurologists and the system used only one brand for each of the generic drugs. In case of Na-VPA all three selected Depakine* EC. For CBZ all three used the same brand. Neurologist I used Tegretol ${ }^{\text {w }}$, while Neurologist Il used only the controlled release variant, Tegretol ${ }^{\text {in: }} \mathrm{CR}$. The system uses the same formulation as Neurologist $\mathrm{I}$, hence there is a consistent difference with Neurologist II on the formulation of CBZ.

\subsubsection{Agreement on daily dose}

Both formulations for $\mathrm{CBZ}$ contain the same biologically active substance. The drug is available in the same doses of tablets for both formulations. Therefore, one can compare the prescribed daily doses. In 48 of the 59 cases the system prescribed the same daily dose as Neurologist I. Although the system and the Neurologist II completely agreed in 50 cases about the drug, in only 3 cases the system proposed the same daily dose. Table 5.3-II and Table 5.3III present the results comparing the daily doses of CBZ and Na-VPA prescribed by Neurologist I, Neurologist II and the system. 
Table 5.3-II: Daily dose for CBZ suggested by the system and by Neurologists I and IL.

\begin{tabular}{|c|c|c|c|c|c|c|c|c|}
\hline \multirow[t]{2}{*}{ CBZ } & & \multicolumn{7}{|c|}{ Prescribed daily dose (mg) } \\
\hline & & Neuro & 300 & 400 & 500 & 600 & 700 & 800 \\
\hline \multirow{8}{*}{$\begin{array}{l}\text { System's } \\
\text { daily dose }\end{array}$} & 200 & I & 1 & & & & & \\
\hline & & II & & 2 & & & & \\
\hline & 300 & I & 24 & 3 & & & & \\
\hline & & $\amalg$ & & 1 & 15 & 7 & & \\
\hline & 400 & 1 & 3 & 11 & & 1 & & \\
\hline & & Ш & & & & 6 & 6 & 3 \\
\hline & 500 & I & & 1 & & & & \\
\hline & & II & & & & & & \\
\hline
\end{tabular}

Forty-four times Neurologist I provided a prescription for CBZ. The daily dose of CBZ prescribed by Neurologist 1 ranged from 300 to $600 \mathrm{mg}$ per day. The dosages recommended by the system for $\mathrm{CBZ}$ ranged from 200 to $500 \mathrm{mg}$ per day. In 35 of the 44 prescriptions the daily dose suggested by the system was identical. Five times the neurologist prescribed a higher dose than that proposed by the system. Twice a daily dose two thirds of that prescribed by the neurologist was suggested. Four times the system proposed a higher dose than the neurologist

Table 5.3-III: Daily dose for VPA suggested by the system and by Neurologists I and II.

\begin{tabular}{|c|c|c|c|c|c|c|}
\hline \multirow[t]{2}{*}{ VPA } & & \multicolumn{5}{|c|}{ Prescribed daily dose (mg) } \\
\hline & & Neuro & 900 & 1000 & 1200 & 1500 \\
\hline \multirow{4}{*}{$\begin{array}{l}\text { System's } \\
\text { daily dose } \\
\text { (mg) }\end{array}$} & 900 & 1 & 13 & & 1 & \\
\hline & & [1] & 3 & 4 & 2 & \\
\hline & 1050 & 1 & 1 & & & \\
\hline & & Il & & & & 1 \\
\hline
\end{tabular}

Neurologist II approaches the treatment of partial epilepsy differently. The daily dose for CBZ ranges from 400 to $800 \mathrm{mg}$. In all except one case the system suggested only $50-66 \%$ of the daily dose prescribed by the neurologist.

For Na-VPA Neurologist $\mathrm{l}$ used a daily dose between 900 and $1200 \mathrm{mg}$. The system recommended Na-VPA in a daily dose of 900 or $1050 \mathrm{mg}$ per day. Twice the system suggested a daily dose different from that of the neurologist.

Neurologist II again prescribed higher daily doses for the cases diagnosed as primary (idiopathic) generalised epilepsy. The daily doses ranged from 900 to $1500 \mathrm{mg}$ per day. Also the prescription of Na-VPA differed from that of the system although the differences are smaller as compared to the differences for CBZ. In three of the 10 cases a dose less than $90 \%$ of the neurologist's dose was suggested.

\subsection{Comparing the system with an external reference}

In this paragraph the advice of the system is assessed using the cases from the diagnostic and treatment reference sets (see chapter 4 par. 4.2). 


\subsubsection{Agreement about the drug using the diagnostic reference set}

The agreement of the system with the neurologists on the cases of the diagnostic reference set is presented in Table 5.4-]. The system is compared with only those neurologists who agreed on the detailed diagnosis.

Table 5.4-I: Agreement between the system and the neurologists on the prescribed generic drug using the diagnostic reference set.

\begin{tabular}{|c|c|c|c|c|c|c|c|}
\hline \multirow{3}{*}{\multicolumn{2}{|c|}{$\begin{array}{l}\text { Agreement on: } \\
\text { delailed diagnosis } \\
\text { prescribed drug }\end{array}$}} & \multicolumn{4}{|c|}{ Neurologists } & & \\
\hline & & \multicolumn{2}{|c|}{$3 / 3$} & \multicolumn{2}{|c|}{$2 / 3$} & \multirow{2}{*}{\multicolumn{2}{|c|}{ Total }} \\
\hline & & $3 / 3$ & $2 / 3$ & $2 / 2$ & no & & \\
\hline \multirow[b]{2}{*}{ Agreement } & 3 neuso's & 7 & & & & 7 & \multirow[b]{2}{*}{ Agroement } \\
\hline & 2 neuro's & & 8 & 17 & & 25 & \\
\hline system & I neuro & & 3 & & 8 & 11 & \\
\hline \multirow[t]{2}{*}{ with } & 0 neuro's & I & 1 & 2 & 0 & 4 & Disagreement \\
\hline & Total & 8 & 12 & 19 & 8 & 47 & \\
\hline
\end{tabular}

Full agreement on diagnosis

In 8 cases all three neurologists agreed on the detailed diagnosis and the generic drug (Table 5.4-1). The system agreed in 7 of these cases with the generic drug. The diagnosis of the remaining case was partial epilepsy with secondary generalised seizures. While all three neurologists prescribed PHT the system prescribed CBZ.

There were 12 cases in which two out of three neurologists agreed about the generic drug although all agreed about the detailed diagnosis. The system agreed with the majority in 8 of these cases. In three cases the system recommended the drug prescribed by the third neurologist. In one case the system prescribed a drug which was not prescribed by any of the experts. In this case, diagnosed as partial epilepsy with complex partial seizures and partial epilepsy with secondary generalised seizures, two of the neurologists recommended no drug treatment while the third neurologist decided to prescribe Na-VPA. The system prescribed CBZ.

\section{2/3 agreement on diagnosis}

In 27 cases two out of three neurologists agreed on the detailed diagnosis. In 19 cases these two neurologists also agreed on the generic drug. The system prescribed the same drug in 17 cases (Table 5.4-I). The system prescribed Na-VPA for a case diagnosed as primary (idiopathic) generalised epilepsy while both neurologists prescribed CBZ. In another case, diagnosed as partial epilepsy with simple partial seizures the system suggested CBZ while the neurologists both prescribed PHT for this case.

In 32 of the 47 cases, the generic drug suggested by the system was in agreement with the reference drug. In eleven cases the system proposed a generic drug which was prescribed by only one neurologist. In all eleven cases this neurologist prescribed a drug different from that prescribed by his colleague(s) despite agreement on the detailed diagnosis. In four cases the system suggested a drug not prescribed by any of the neurologists. 


\subsubsection{Variability in neurologist's prescriptions}

In 20 cases of the diagnostic reference set all three neurologists established the same detailed diagnosis and reached a majority agreement on the prescribed drug in all of these cases. In the subset of 27 cases in which 2/3 neurologists agreed on the detailed diagnosis $63 \%$ of the cases had a majority agreement on the prescribed drug. As the selection of the drug is mainly based on the diagnosis, we required total agreement about this issue when comparing the proposed drugs of the system with those of the individual neurologists. Therefore the earlier mentioned subset of 20 cases is used for assessment of the generic drug suggested by the system.

Table 5.4-II presents how often each of the individual neurologists prescribed a generic drug agreed upon by the majority of the neurologists in cases of this subset of 20 cases. All except one of the neurologists prescribed the majority drug in more than $80 \%$ of his cases. One neurologist agreed in only $46 \%$ of the cases.

Table 5.4-1I: Generic drug prescribed in accordance with the majority.

\begin{tabular}{|c|c|c|c|c|c|c|c|c|c|c|c|}
\hline \multicolumn{12}{|c|}{ Agreement of participants and system with drug in $N=20$} \\
\hline \multirow[t]{2}{*}{ Participant } & III & \$ & IV & $S$ & $\mathrm{~V}$ & $\$$ & $\mathrm{~V}$ & $\mathrm{~S}$ & V & $\mathrm{S}$ & system \\
\hline & \multicolumn{2}{|c|}{$n=13$} & \multicolumn{2}{|c|}{$\mathrm{n}=16$} & \multicolumn{2}{|c|}{$n=11$} & \multicolumn{2}{|c|}{$\mathrm{n}=\mathrm{ll}$} & \multicolumn{2}{|c|}{$n=9$} & $n=20$ \\
\hline Drug agreed & 11 & 9 & 15 & 10 & 5 & 9 & 9 & 10 & 8 & 6 & 15 \\
\hline$\%$ & 85 & 75 & 24 & 63 & 46 & 82 & 82 & 91 & 89 & 67 & 75 \\
\hline
\end{tabular}

The system agreed on the prescribed drug with at least two neurologists in 15 of these 20 cases. As a majority was established if a neurologist agreed with at least one of his colleagues it was also examined in what percentage the system agreed with at least one neurologist. In 18 cases $(90 \%)$ the system prescribed the same drug as at least one of the prescribing neurologists

The system's advice was compared with that of an individual neurologist on the basis of the cases seen by both. Three neurologists prescribed more in accordance with the majority than the system. This analysis is in favour of the neurologists as they defined the drug used by the majority, while the system did not

\subsubsection{Agreement about the daily dose}

To obtain insight in the adequacy of the suggested dosage the treatment reference set was used. In 32 out of the 39 cases the system prescribed the same drug. These 32 cases were used to evaluate the dosages prescribed. In Table 5.4-111 a breakdown is presented of how many of these cases are seen by each of the neurologists and by the system.

Table 5.4-III: Agreement with the reference daily Jose.

\begin{tabular}{lcccccc}
\hline Neurologist & $3(\mathrm{n}=14)$ & $4(\mathrm{n}=16)$ & $5(\mathrm{n}=13)$ & $6(\mathrm{n}=14)$ & $7(\mathrm{n}=14)$ & System $(\mathrm{n}=32)$ \\
\hline $\begin{array}{l}\text { Cases for daily dose } \\
\text { comparison }\end{array}$ & 6 & 5 & 6 & 5 & 4 & 11 \\
Agreed daily dose & $43 \%$ & $31 \%$ & $46 \%$ & $36 \%$ & $29 \%$ & 32 \\
\hline
\end{tabular}


A majority dosage (at least two neurologists prescribe the same daily dose) could be obtained in 11 cases (34\%). Within this set of 11 cases the system agreed with the majority of the neurologists in 5 cases (46\%). In $20(63 \%)$ out of the 32 cases the system agreed about the daily dose with at least one neurologist.

Table 5.4-IV:Differences in the daily dose of CBZ (in parentheses the dose prescribed by the system is given as a percentage of the dose prescribed by the neurologists). The number of $100 \mathrm{mg}$ tablets presented in the first row indicates the number of tablets the system preseribed too few or too many.

\begin{tabular}{|c|c|c|c|c|c|c|c|}
\hline $\begin{array}{l}\text { Daily dose } \\
\text { neurologist (mg) }\end{array}$ & -5 tabl. & -4 tabl. & -3 tabl. & -2 lathl & -1 tabl. & 0 tabl. & {$[t .(b)$.} \\
\hline 200 & & & & & & & $2(150 \%)$ \\
\hline 300 & & & & & & $2(100 \%)$ & \\
\hline 400 & & & & & $12(75 \%)$ & $6(100 \%)$ & \\
\hline 600 & & & $9(50 \%)$ & $9(66,7 \%)$ & & & \\
\hline 800 & $1(37.5 \%)$ & $3(50 \%)$ & & & & & \\
\hline 900 & $\mathrm{I}(44 \%)$ & & & & & & \\
\hline
\end{tabular}

In Table 5.4-IV and Table 5.4-V the differences in daily dose between the system and the neurologists are presented for the drugs CBZ and Na-VPA using the 32 cases from the treatment reference set. In this analysis we used the doses of the individual prescriptions of the neurologists.

In 20 of the 32 cases in which the system prescribed the same dug as the majority of the neurologists CBZ was prescribed. In total 45 prescriptions were provided for this drug by the neurologists. The daily dose ranged from 200 to $900 \mathrm{mg}$. A daily dose of more than $600 \mathrm{mg}$ is prescribed 5 times $(12 \%)$. One neurologist prescribed more than $800 \mathrm{mg} \mathrm{CBZ}$.

Table 5.4-V: Differences in prescribed daily dose of CBZ between system and 5 neurologists.

\begin{tabular}{lccccc}
\hline $\begin{array}{l}\text { Difference in CBZ } \\
\text { daily dose (mg) }\end{array}$ & N III & N IV & N V & N VI & N VI \\
\hline-500 & 2 & & & 2 & \\
-400 & 5 & 1 & 3 & 1 & \\
-300 & 1 & & 5 & 3 & \\
-200 & & 6 & & 3 & 3 \\
-100 & & 4 & 1 & 1 & 2 \\
0 & & & & & 2 \\
\hline
\end{tabular}

Table 5.4-IV presents the differences in daily dose for CBZ between the neurologists and the system. In $8(18 \%)$ of these 45 prescriptions the system and a neurologist agreed on the daily dose of CBZ. If a difference of 1 tablet of $100 \mathrm{mg}$ is still acceptable the number of acceptable doses of the system increases to $22(49 \%)$. The system agrees with the neurologists in the lower dosage range. The higher the dose prescribed by the neurologists the larger the 
difference becomes. In 14 prescriptions a neurologist recommended twice or more the daily dose prescribed by the system. Table $5.4-\mathrm{V}$ shows the difference in agreement between the individual neurologists and the system. The system tends to prescribe approximately the same daily doses for CBZ as Neurologist IV and VII.

In 10 cases the system prescribed Na-VPA according to the reference (Table 5.4-VI). Agreement between the neurologists and the system on the prescribed dose of (Na-)VPA occurs in 14 out of 22 prescriptions $(64 \%)$. There are 6 prescriptions where the dose suggested by the system is $90 \%$ of the prescribed dose of the neurologist. This difference in doses is caused by a difference in intake scheme: $3 \times 300 \mathrm{mg}$ versus $2 \times 500 \mathrm{mg}$. Including these cases leads to an agreement in 20 out of 22 prescriptions. In 2 prescriptions ( $9 \%$ ) the system recommended a daily dose which was only $60 \%$ the dose prescribed by one of the neurologists.

Table 5.4-VI: Differences in the daily dose of ( $\mathrm{Na}$-)VPA. The percentages present the dose of the system relative to that of the neurologist. The number of tablets presented in the first row indicates the number of $300 \mathrm{mg}$ tablets the system prescribed too few.

\begin{tabular}{llll}
\hline $\begin{array}{l}\text { Daily dose of } \\
\text { neurologist (mg) }\end{array}$ & -2 tabl. & $-1 / 3$ labl. & 0 tabl. \\
\hline 900 & & & $14(100 \%)$ \\
1000 & & $6(90 \%)$ & \\
1500 & $2(60 \%)$ & & \\
\hline
\end{tabular}

\subsection{Review of the prescriptions}

All prescriptions for each of the 47 cases in the diagnostic reference set were assessed by two neurologists who did not prescribe for such a case. The reviewers judged the overall acceptability of each of the prescriptions on a five point scale (excellent, sufficient, neutral, insufficient, poor). For those prescriptions judged as of possibly poor quality - scored as neutral, insufficient or poor acceptability - the various components of a prescription e.g. the generic drug and the dose of tablets were assessed separately.

\subsubsection{Overview of the results for system and individual neurologists}

In total 114 prescriptions from the neurologists and 47 from the system were reviewed. For all prescriptions of the system both reviewers assessed the overall quality, resulting in 94 reviews. For seven prescriptions of the neurologists a reviewer did not provide an overall judgement. So only 221 rather than 228 review reports for the neurologists' prescriptions are available

In Table 5.5-I more than half of the prescriptions of all but one of the neurologists were considered to be of good quality by the reviewers (range 51 to $63 \%$ ). In less than $20 \%$ (range 13 to $19 \%$ ) their prescriptions were considered inadequate. Compared with these four neurologists a lower percentage of prescriptions of the system was considered to be of good quality but also a smaller percentage of prescriptions were considered inadequate. 
Table 5.5-I: Overall judgements by each of the two reviewers on the prescriptions provided by an individual neurologist. At most two judgements per prescription are available.

\begin{tabular}{|c|c|c|c|c|c|c|c|c|c|c|c|c|}
\hline \multirow{2}{*}{$\frac{\text { Participant }}{\text { Judgement }}$} & \multicolumn{2}{|c|}{ m } & \multicolumn{2}{|c|}{ IV } & \multicolumn{2}{|c|}{$\mathrm{V}$} & \multicolumn{2}{|c|}{$\mathrm{Vl}$} & \multicolumn{2}{|c|}{ VII } & \multicolumn{2}{|c|}{ System } \\
\hline & $\mathrm{n}$ & $\%$ & $n$ & $\%$ & $n$ & $\%$ & $\mathrm{n}$ & $\%$ & $\mathrm{n}$ & $\%$ & $\mathbf{n}$ & $\%$ \\
\hline poor' & 6 & 13 & 8 & 16 & 12 & 29 & 8 & 20 & 8 & 18 & 10 & 11 \\
\hline neutral & 14 & 30 & 15 & $3 !$ & 16 & 39 & 7 & 17 & 14 & 31 & 39 & 42 \\
\hline $\operatorname{good}^{2}$ & 26 & 57 & 26 & 53 & 13 & 32 & 26 & 63 & 22 & 51 & 45 & 48 \\
\hline Total judgements & 46 & 100 & 49 & 100 & 41 & 100 & 41 & 100 & 44 & 100 & 94 & 100 \\
\hline Cases diagn.ref.set & 23 & & 25 & & 21 & & 22 & & 23 & & 47 & \\
\hline Missing judgem. & 0 & & I & & I & & 3 & & 2 & & 0 & \\
\hline
\end{tabular}

(1) poor consists of insufficient and poor, (2) good contains the classes: good and excellent of the five point scale.

In 147 of the 157 review reports indicating a poor or neutral overall acceptability assessments of the prescribed drug were presented. For three of the neurologists more than $50 \%$ of these judgements was positive (range $54 \%$ to $70 \%$ ). Also for the system most (67\%) of the assessments indicated that a proper drug was prescribed (Table 5.5-II). For four of the neurologists $17 \%$ to $36 \%$ of the prescribed drugs were considered not appropriate. The system and one of the neurologists suggested an inappropriate drug in less than $5 \%$ of the prescriptions.

Table 5.5-II: Judgements on the generic drug for prescriptions with an overall poor or neutral quality. For each prescriber the obtained number of judgements indicating that the prescribed drug is appropriate, neutral or inappropriate is presented.

\begin{tabular}{|c|c|c|c|c|c|c|c|c|c|c|c|c|}
\hline \multirow{2}{*}{$\begin{array}{l}\text { Participant } \\
\text { Judgement drug }\end{array}$} & \multicolumn{2}{|c|}{ III } & \multicolumn{2}{|c|}{ IV } & \multicolumn{2}{|c|}{ v } & \multicolumn{2}{|c|}{$\mathrm{VI}$} & \multicolumn{2}{|c|}{ VII } & \multicolumn{2}{|c|}{ System } \\
\hline & $\mathrm{n}$ & $\%$ & $n$ & $\%$ & $n$ & $\%$ & 川 & $\%$ & n & $\%$ & $\mathrm{n}$ & $\%$ \\
\hline inappropriate & 3 & 17 & I & 4 & 5 & 21 & 5 & 36 & 4 & 20 & 1 & 2 \\
\hline neutral & 8 & 44 & 6 & 26 & 6 & 25 & 1 & 7 & 7 & 35 & 15 & 31 \\
\hline appropriate & 7 & 39 & 16 & 70 & 13 & 54 & 8 & 57 & 9 & 45 & 32 & 67 \\
\hline Total judgements & 18 & 100 & 23 & 100 & 24 & 100 & 14 & 100 & 20 & 100 & 48 & 100 \\
\hline Poor/neutral judg. & 20 & & 23 & & 28 & & 15 & & 22 & & 49 & \\
\hline Missing & 2 & & 0 & & 4 & & 1 & & 2 & & 1 & \\
\hline
\end{tabular}

For the prescriptions with a neutral or poor overall quality and an appropriate drug, the quality of the prescribed dose was analysed (Table 5.5-III). For one of these prescriptions a judgement was not provided on this issue. The percentage of judgements suggesting an inappropriate dose ranged from $53 \%$ to $60 \%$ for three of the neurologists, while for two neurologists more than $85 \%$ the daily dose was inappropriate. The system suggested an inappropriate dose in $91 \%$ of these prescriptions. Three neurologists tend to prescribe a (too) high rather than a (too) low dose. Most of the dosages prescribed by the system were judged as being too low.

In 21 of the 157 prescriptions which were considered to be of a (possibly) poor quality the judgement could not be attributed to an inadequate generic drug or daily dose but was caused by an inadequate brand, dose of tablets and/or intake scheme. 
Table 5.5-1II: Judgements provided on the daily dose for prescriptions in which the prescribed drug was considered to be appropriate but the overall judgement indicated (possibly) poor quality. For each neurologist the number of obtained judgements indicating a (too) low, neutral and (too) high daily dose is provided.

\begin{tabular}{|c|c|c|c|c|c|c|c|c|c|c|c|c|}
\hline \multirow{2}{*}{$\frac{\text { Participant }}{\text { Judgm. daily dose }}$} & \multicolumn{2}{|c|}{ III } & \multicolumn{2}{|c|}{ IV } & \multicolumn{2}{|c|}{ V } & \multicolumn{2}{|c|}{$\mathrm{VI}$} & \multicolumn{2}{|c|}{ VII } & \multicolumn{2}{|c|}{ System } \\
\hline & $\mathrm{n}$ & $\%$ & $\mathrm{n}$ & $\%$ & $\mathrm{n}$ & $\%$ & $n$ & $\%$ & $\mathrm{n}$ & $\%$ & $\pi$ & $\%$ \\
\hline$(1 \infty)$ low & 0 & 0 & 7 & 47 & 3 & 23 & 2 & 25 & 6 & 67 & 28 & 88 \\
\hline neutral & 1 & 14 & 6 & 40 & 6 & 46 & 3 & 38 & 1 & 11 & 3 & 9 \\
\hline (too) high & 6 & 86 & 2 & 1.3 & 4 & $3 !$ & 3 & 38 & 2 & 22 & 1 & 3 \\
\hline Tolal judgements & 7 & 100 & 15 & 100 & 13 & 100 & 8 & 100 & 9 & 100 & 32 & 100 \\
\hline Judgen.drug appr. & 7 & & 16 & & 13 & & 8 & & 9 & & 32 & \\
\hline Missing & 0 & & I & & 0 & & 0 & & 0 & & 0 & \\
\hline
\end{tabular}

\subsubsection{Comparing overall scores of system and individual neurologists}

For each case two or three neurologists and the system provided a prescription. The score for a prescription of a neurologist can be compared with the score for the prescription of the system on the same case. The difference between the overall acceptability of the prescription of a neurologist and the system gives an indication which of the two prescriptions is favoured. A positive difference indicat "; that the reviewer favours the prescription of the neurologist, a negative difference is in favour of the system. In Table 5.5-IV the difference scores are presented as a function of

Table 5.5-IV: Difference in overall acceptability scores of the reviewer when comparing the scores of the neurologist with the score of the system.

\begin{tabular}{|c|c|c|c|c|c|c|c|c|c|c|c|c|c|c|c|}
\hline \multirow[t]{2}{*}{ Overall score } & \multicolumn{3}{|c|}{ Reviewer 3} & \multicolumn{3}{|c|}{ Reviewer 4} & \multicolumn{3}{|c|}{ Reviewer 5} & \multicolumn{3}{|c|}{ Reviewer 6} & \multicolumn{3}{|c|}{ Reviewer 7} \\
\hline & - & 0 & + & $=$ & 0 & + & . & 0 & + & - & 0 & + & - & 0 & + \\
\hline n3-syst & & & & 2 & 7 & 2 & l & 4 & 5 & 2 & 8 & 3 & 4 & 6 & 2 \\
\hline n4-syst & 3 & 8 & 0 & & & & 1 & 3 & 9 & 2 & $\bar{y}$ & 0 & 2 & 10 & 2 \\
\hline n5-syst & 4 & 4 & 3 & 4 & 4 & 1 & & & & 4 & 4 & 2 & 4 & 5 & 2 \\
\hline n6-syst & 1 & 9 & । & 3 & 3 & 1 & 3 & 1 & 5 & & & & 4 & 3 & 7 \\
\hline n7-syst & 3 & 6 & 4 & 3 & 4 & 2 & 4 & 3 & 3 & 1 & 7 & 4 & & & \\
\hline Total & 11 & 27 & 8 & 12 & 18 & 6 & 9 & 11 & 22 & 9 & 28 & 9 & 14 & 24 & 13 \\
\hline$\%$ & 24 & 59 & 17 & 33 & 50 & 17 & 21 & 26 & 52 & 20 & 60 & 20 & 28 & 47 & 26 \\
\hline
\end{tabular}

\begin{tabular}{lccccccc}
\hline Overall score & \multicolumn{3}{c}{ Total } & \multicolumn{4}{c}{ Percentage } \\
& \multicolumn{1}{c}{0} & \multicolumn{1}{c}{+} & \multicolumn{1}{c}{0} & + \\
\hline nj-syst & 9 & 25 & 12 & 20 & 54 & 26 \\
n4-syst & 8 & 30 & 11 & 16 & 61 & 23 \\
n3-syst & 16 & 17 & 8 & 39 & 42 & 20 \\
n6-syst & 11 & 16 & 14 & 27 & 39 & 34 \\
n7-syst & 11 & 20 & 13 & 24 & 44 & 29 \\
\hline Total & 55 & 108 & 58 & & & \\
$\%$ & & & & 25 & 49 & 26 \\
\hline
\end{tabular}


the reviewing neurologist. Since in total 221 prescriptions of the neurologists were reviewed, 221 differences could be computed

Twenty prescriber-reviewer combinations exist. In 10 of these combinations the system's prescription was favoured above the neurologists' prescription more often than the reverse. In 7 the neurologists prescription was favoured.

It appears that Neurologist $V$ judges the performance of the system much lower than the other neurologists do. In turn Neurologist $V$ is judged less positively by his colleagues than the system. Overall the system's advice is judged as being of comparable quality as the neurologists.

Exclusion of Neurologist $\mathrm{V}$ as reviewer and as prescriber does not change the conclusion that the system's prescriptions are just as acceptable as the neurologists' prescriptions.

\subsubsection{Variability of the reviewers}

The prescriptions for a case have been independently assessed by two reviewers. This allows the study of the variability in the assessments of the reviewers.

Again we determined for each case the difference between the overall scoring of the recipe of the neurologist and the overall scoring of the corresponding recipe of the system: + indicating that the system's recipe had a better score, 0 indicating equal scores and - indicating that the recipe of the system obtained a lower score than the neurologist's recipe. This comparative score is determined for the assessments of both reviewers

Table 5.5-V gives an overview of how often in the 109 prescriptions of the neurologists for which two assessments were available, two judges give a certain comparative score.

In $14(13 \%)$ prescriptions both reviewers disagreed completely on which prescription is of better quality: one reviewer considered the neurologist's recipe better than the system's, the other reviewer did the reverse. For 42 prescriptions (38\%) both reviewers provided the same assessment for the two recipes.

Table 5.5-V: Agreement between reviewers compa-

ring the overall score for the system

with that for the prescription of a

neu rologist.

\begin{tabular}{crrr}
\hline Judge 1 & Judge 2 & No. cases & $\%$ \\
\hline+ & + & 6 & 5.5 \\
+ & 0 & 32 & 29.4 \\
+ & - & 14 & 12.8 \\
0 & 0 & 2 & 24.8 \\
0 & - & 21 & 19.3 \\
- & - & 9 & 8.3 \\
\hline \multicolumn{2}{l}{ Tolal no or cases } & 109 & 100 \\
\hline
\end{tabular}

The prescription of the system can be considered to be of lower quality when one of the reviewers considered a recipe of the neurologist of better quality and the other reviewer says that the neurologist's recipe is equal or better than the system's prescription. This occurs for 
30 prescriptions of the neurologists (28\%). For 38 pairs of prescriptions $(35 \%)$, the system's prescription is considered better by one reviewer and of equal or better quality than the neurologist's recipe by the other reviewer

\subsection{Results of the consensus meeting}

In this section the results of the consensus meeting are presented. The meeting dealt with general principles and not with individual cases although the diagnostic reference set served as background material for the discussion. Only the issues that are related to the diagnosis, selection of drugs and the initial dose are presented. The discussion resulted in a reference against which the prescriptions of the neurologists and of the system can be compared.

\subsubsection{The use of diagnostic classification schemes}

The diagnostic classification schemes as presented in the literature are considered to be more detailed than necessary for daily medical practice. The reliability of a detailed classification of the epilepsy of a patient may be doubtful. Cases admitted for neurosurgery may require a more detailed classification. Also for research purposes a detailed classification is valuable.

\subsubsection{Initiation of drug treatment}

In the literature a diagnosis ex juvantibus approach as well as initiation of anticonvulsive treatment if the diagnosis is not certain are both considered to be inappropriate (Chadwick and Reynolds 1985, Porter 1984, 1989, Shorvon 1987). In our study drug treatment was initiated twice in the 11 instances that a neurologist could not establish a diagnosis. In the consensus meeting and also in the review process drug treatment was considered unacceptable when no diagnosis is established. It was agreed that one should wait and let time decide what the diagnosis is before drug treatment is started.

Although the neurologists are willing to make a trade-off between the burden of antiepileptic drug treatment and the conseguences and the severeness of the seizures, all agreed that drug treatment should be started as soon as the diagnosis is established and two or more seizures have occurred. Also in the review process refraining from drug treatment was considered unacceptable when the diagnosis was established. However, in daily practice the opinion of the patient should be taken into account. If a patient refuses anticonvulsive treatment, despite knowing the consequences and risks, close monitoring of the patient is required to start the treatment immediately in case he changes his opinion.

\subsubsection{Selection of generic drug}

Based on the literature we concluded that at present there exists no consensus on whether the selection of the drug should be based on the type of epilepsy or on the type of seizures. On the basis of the meeting the neurologists agreed that the type of epilepsy should be the basis for the selection of the anticonvulsive treatment. A differentiation between generalised and partial epilepsy was considered to be sufficient for initial drug selection.

The neurologists agreed that if treatment is started in a newly diagnosed patient monotherapy should be used. The advantages of monotherapy are: a clear relation between drug and 
side-effects, a narrow spectrum of interactions and a better compliance. Polytherapy may be used in those patients where monotherapy proved to be ineffective.

Cases where complete seizure control was achieved in the past with polytherapy while monotherapy was not tried or tried inappropriately, may pose a problem in case of a relapse. Also in these patients the use of monotherapy is preferred even when a relapse occurs

For treatment of primary (idiopathic) generalised epilepsy Na-VPA is preferred. The argument that VPA (Convulex ${ }^{(i)}$ ) does not increase the sodium intake did not outweigh the advantages of Depakine ${ }^{\text {in }}$ EC. Even in case of hypertension the use of VPA is not advised nor has hypertension been mentioned as a contraindication for Na-VPA (Meinardi 1993, Shorvon 1995, Brodie and Dichter 1996).

The neurologists preferred the use of CBZ for treatment of partial epilepsy. Little agreement was observed when to apply PHT. The consensus meeting led to the conclusion that PHT should be the drug of first choice only for patients older than sixty years mainly because of side-effects such as gingival hyperplasia and coarsing of features in younger patients. PHT is a time honoured drug. The therapeutic range can be reached quickly and its use is easy due to the long half-life. Furthermore, the use of PHT for older patients has the advantage that a change in therapy is not necessary in case of surgery as a formulation for parenteral administration is available

\subsubsection{Generic drug or brand, formulation}

All neurologists preferred the use of the brand when prescribing a drug. The advantages mentioned were:

- The quality of a brand is fairly constant. The components as well as the production process have met criteria of quality control

- Variability in drug effectiveness is reduced. The occurrence of seizures can be attributed to a number of known factors.

A special formulation of the drug may prevent the occurrence of side-effects. Fluctuation in the plasma drug level and the occurrence of seizures at a particular time during the day, for example only in the early morning, may be important factors determining the selection of a special formulation of the drug. Furthermore, the compliance of the patient influences the selection of the formulation.

The neurologists agreed that Na-VPA may be prescribed as an uncoated formulation as far as the effectiveness of the drug is concerned. However, they prefer the enteric coated (EC) formulation because gastric distress occurs less often. Besides the $\mathrm{EC}$ formulation the Chrono formulation may be prescribed. This formulation has the advantage of less fluctuation in the plasma drug level while the peak level is reached faster. Nevertheless the EC formulation was preferred, using $300 \mathrm{mg}$ tablets in case the drug is taken three times a day or $500 \mathrm{mg}$ tablets if the drug is taken twice a day

The short half-life of CBZ together with the fluctuations in the plasma drug level require either a frequent drug intake or a controlled release (CR) formulation for this drug. All participants agreed on the use of the CR formulation when $C B Z$ is prescribed. The reference dose of the tablets is considered to be $200 \mathrm{mg}$. Tablets of $400 \mathrm{mg}$ should only be used in case a daily dose of $800 \mathrm{mg}$ or more is prescribed. The use of tablets of $400 \mathrm{mg}$ when a daily dose of less than $800 \mathrm{mg}$ is prescribed is considered to be inappropriate. 


\subsubsection{Daily dose of the drug}

In chapter 2 it has been shown that different opinions exist in the literature about the appropriate daily dose for Na-VPA, CBZ and PHT. Apart from the weight of the patient, which is according to our neurologists the basis for the determination of the daily dose, other factors such as stability of the plasma drug level, the risk of adverse drug reactions, the wish of the patient or the temperament of the neurologist, may influence the prescribed dose.

Although a 'middle of the target range' approach may be applied in selecting the daily dose, all participants agreed that in newly diagnosed patients one should start with a low daily dose and gradually increase the dose based on clinical findings. This is in contrast with the prescriptions they provided in which also a high initial dose occurred.

The ranges of the daily doses for the different drugs used by the neurologists were 900 to $1500 \mathrm{mg}$ for Na-VPA, 200 to $900 \mathrm{mg}$ for CBZ and 150 to $300 \mathrm{mg}$ for PHT. During the discussion the neurologists reached the conclusion that the prescribed daily doses were rather high. They agreed that if:

- Na-VPA or VPA was prescribed one should start with a dose of 900 to $1000 \mathrm{mg}$ per day, depending on the intake frequency

- $\mathrm{CBZ}$ is prescribed and the weight of the patient was less than $80 \mathrm{~kg} 400 \mathrm{mg}$ per day is sufficient. If the weight of the patient is higher than $80 \mathrm{~kg}$ the initial daily dose of the patient should be $600 \mathrm{mg}$

- PHT is selected as the drug for treatment and the weight is less than $80 \mathrm{~kg}$ a daily dose of $200 \mathrm{mg}$ PHT should be prescribed. In case the weight of the patient is higher than $80 \mathrm{~kg}$ they preferred a daily dose of $300 \mathrm{mg}$

One of the reasons for the relatively low daily dose of $\mathrm{CBZ}$ was the risk of adverse drug reactions. Side-effects may have consequences for the compliance. $\mathrm{CBZ}$ strongly induces its own catabolism. Therefore the initial daily dose has to be lower than the daily dose required eventually. The risk of having another seizure was not sufficient for the neurologists to initiate treatment with a higher daily dose. The kindling effect of additional seizures was considered not proven. Nevertheless, they agreed that sufficient prevention against seizures should be guaranteed. In addition to this, the wish of the patient should be taken into account when determining the daily dose. Some of the patients oppose drug treatment and do not like to be reminded several times a day that they are suffering from epilepsy. Others are afraid of having additional seizures and prefer a high dose to suppress further seizures.

\subsubsection{Intake frequency}

To be able to comply. patients need an intake scheme that is easy to handle. Therefore, the neurologists should try to keep the number of intakes per day as low as possible. The drug half-life as well as the formulation are important determinants for establishing the intake frequency. Practical consequences of drug intake have to be considered. While some neurologists combine the intake of the drug with meals, others stated that most patients tend to forget the intake during lunch because of the social consequences. The neurologists agreed that a drug intake of twice a day is most optimal as far as patient compliance is concerned 


\subsubsection{Gradual increase of the dose}

Dose related side-effects can be prevented through a gradual increase of the daily dose. The use of a gradual increase procedure is considered relevant for $\mathrm{CBZ}$. The half-life of $\mathrm{CBZ}$ when starting the intake is about 16 hours while long-term use of the drug decreases the half-life to 8 hours. Side-effects of each dosage can be assessed in a steady state which is reached after five times the half-life after the increase. Theoretically one can increase the dose on the fifth day. However, the neurologists prefer to increase the dose once a week. A more complicated intake scheme may hamper patient compliance. In case of $400 \mathrm{mg}$ the final dose will be reached in the second week, while with $600 \mathrm{mg}$ the final dose will be reached in the third week. The neurologists agreed that reaching an intake of 400 or $600 \mathrm{mg}$ within one week is not appropriate.

\subsection{Comparing the system and the neurologists with the consensus meeting reference}

Based on the guidelines obtained in the consensus meeting, a consensus treatment was established for cases from the diagnostic reference set. The prescriptions of the neurologists as well as the prescriptions of the system were compared with this consensus reference treatment. For the neurologists the number of prescriptions in accordance with the reference was established. The system was compared with the individual neurologists using the same set of cases.

\subsubsection{Agreement on the generic drug}

The group of neurologists provided 114 treatments. Five different drugs were used by the neurologists, whereas in the consensus reference only three drugs were used. The system used only two drugs for treatment (Table 5.7-1).

Anti-epileptic drug therapy was initiated 103 times by the neurologists. Eleven times drug treatment was not started. Seven times this was not according to the consensus meeting reference as this reference stated that drug treatment should be started when the diagnosis is established

Table 5.7-I:Agreement between the group of neurologists and the system with the reference developed during the consensus mecting.

\begin{tabular}{l|rrrrrrr|rrrrr}
\hline $\begin{array}{l}\text { Reference } \\
\text { Treatunent }\end{array}$ & \multicolumn{7}{|c|}{ Neurologists } & \multicolumn{5}{c}{ System } \\
\hline & No & CBZ & PB & PHIT & VF'A & OCI3 & Tolal & No & CBZ & PHT & VPA & Total \\
No & 4 & 0 & 0 & 0 & 0 & 0 & 4 & 2 & 0 & 0 & 0 & 2 \\
CBZ & 6 & 42 & 0 & 9 & 2 & 3 & 62 & 0 & 26 & 0 & 0 & 26 \\
PHT & 0 & 9 & 0 & 2 & 1 & 0 & 12 & 0 & 5 & 0 & 0 & 5 \\
VPA & 1 & 8 & 1 & 1 & 25 & 0 & 36 & 0 & 0 & 0 & 14 & 14 \\
\hline & 11 & 59 & 1 & 12 & 28 & 3 & 114 & 2 & 31 & 0 & 14 & 47 \\
\hline
\end{tabular}

In 73 of the 114 prescriptions (64\%) the selected drug of a neurologist was in accordance with the consensus reference. In two cases two neurologists decided that the type of epilepsy was unknown. In these cases both neurologists did not start drug treatment. 
The system suggested in 42 of the 47 cases (89\%) a drug according to the reference. In 2 cases drug treatment was not started because the type of epilepsy was not certain.

All neurologists prescribed a drug in accordance with the reference in more than half of the cases (range $52 \%$ to $72 \%$ ). The system compared with the individual neurologists suggested a drug according to the consensus consistently more often (range $88 \%$ to $91 \%$ )(Table $5.7-\Pi$ ).

The system suggested CBZ according to the reference consistently more often than the individual neurologist, while the proposal to use VPA was consistently at least as good as that of a neurologist. From the consensus meeting it was concluded that PHT should be used as well. This drug was not recommended by the system. Before the consensus meeting the neurologists disagreed more about the prescription of PHT than about the use of CBZ and VPA. Only twice (17\%) a neurologist used the drug in accordance with the reference, compared to $68 \%$ for $\mathrm{CBZ}$ and $69 \%$ for VPA.

Table 5.7-II: Number of cases for which the system and the individual neurologist recommended the same prescription as the reference.

\begin{tabular}{lllllllllll}
\hline Participant & III & Syst & IV & Syst & $V$ & Syst & V & Syst & VI & Syst \\
\hline Cases & $\mathrm{n}=23$ & $\mathrm{n}=23$ & $\mathrm{n}=25$ & $\mathrm{n}=25$ & $\mathrm{n}=21$ & $\mathrm{n}=21$ & $\mathrm{n}=22$ & $\mathrm{n}=22$ & $\mathrm{n}=23$ & $\mathrm{n}=23$ \\
Correct & 15 & 21 & 18 & 22 & 11 & 19 & 13 & 20 & 16 & 21 \\
Total \% & 65 & 91 & 72 & 88 & 52 & 91 & 59 & 91 & 70 & 91 \\
\hline
\end{tabular}

\subsubsection{Agreement on the daily dose}

For those cases in which the detailed diagnosis established by the neurologist was in agreement with the reference diagnosis and the prescribed drug and the reference drug were the same, the daily doses were compared. In Table 5.7-III prescribed daily doses are expressed as percentages of the reference dose. Since in the case of VPA a range was considered appropriate, doses between $100 \%$ (equivalent to $900 \mathrm{mg}$ ) and $111 \%$ (equivalent to $1000 \mathrm{mg}$ ) are appropriate. For $\mathrm{CBZ}$ the daily dose depends on the weight of the patient.

\section{Total set of prescriptions}

If the neurologists prescribe a drug according to the reference they achieve a better agreement with the reference daily dose than the system (57\% vs $37 \%$ of the prescriptions had the same dosage). When VPA is prescribed little difference between the neurologists and the system was observed $(88 \%$ and $94 \%$ of the prescriptions had the same daily dose as the reference, respectively). The prescribed daily dose of the neurologists ranged from $100 \%$ to $167 \%$ of the reference dose. The system suggested a daily dose ranging from $100 \%$ to $117 \%$ of the reference (Table 5.7-III).

When $\mathrm{CBZ}$ is prescribed - 42 and 62 times by the neurologists and the system respectively the system suggested only twice (3\%) a daily dose equal to that of the consensus, while in $36 \%$ of the prescriptions of the neurologists the dose was according to the reference. In $21 \%$ of the prescriptions the neurologists prescribed a lower daily dose (ranging from $50 \%$ to $75 \%$ of the reference dose), in $43 \%$ the neurologists prescribed a higher daily dose than the reference (ranging from $133 \%$ to $225 \%$ of the reference daily dose). The dosages suggested by the system were lower than the reference in $97 \%$ of the prescriptions (the daily dose ranged from $67 \%$ to $75 \%$ of the reference dose) 
Table 5.7-III: Variations in daily dose for those cases for which the consensus treatment and the detailed diagnosis are agreed upon by the participant.

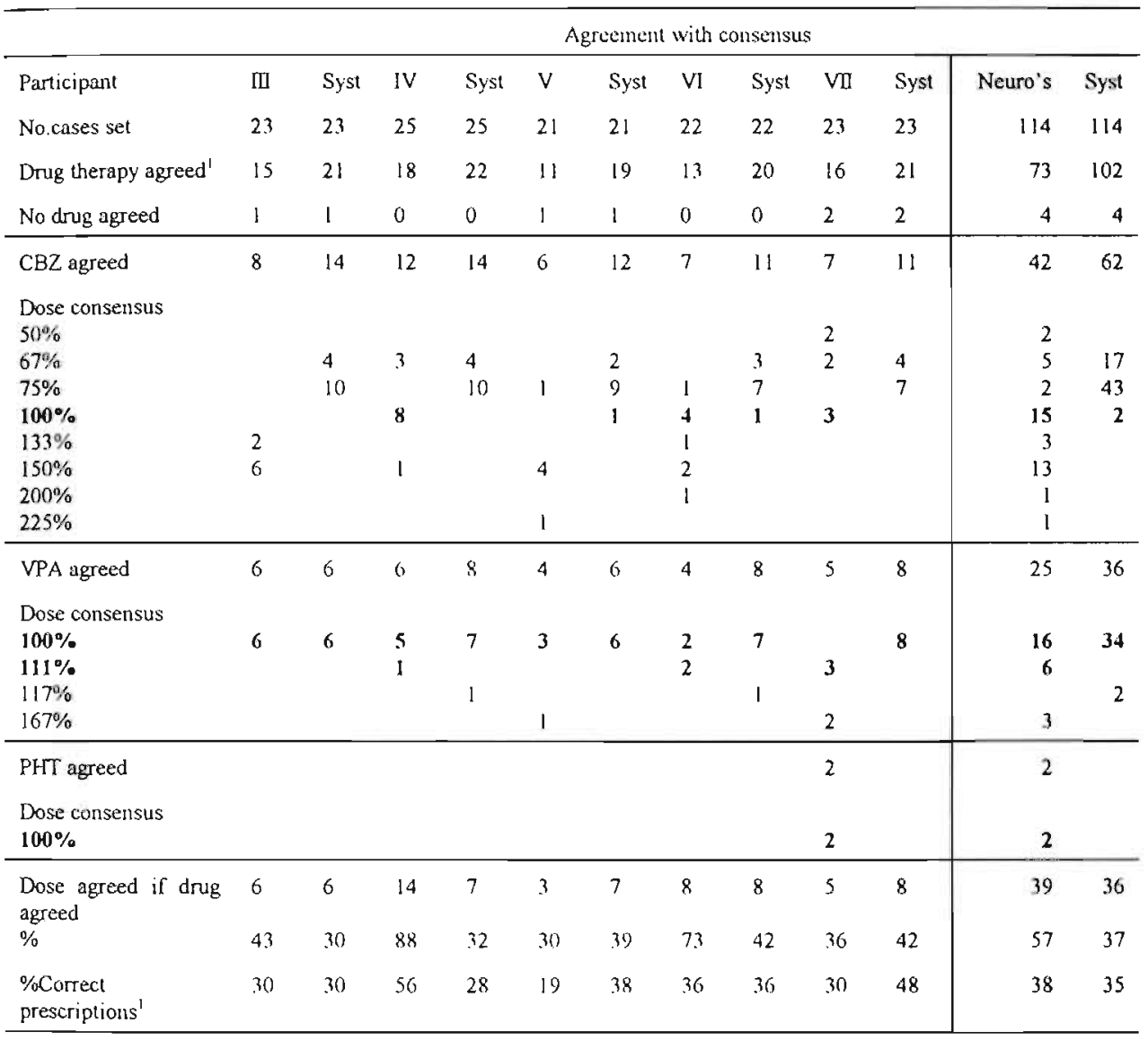

${ }^{1}$ This includes also the cuses for which the reference treatment was to provide no drug treatunent

Agreement in the sets of the individual nenrologist

In those cases in which the individual neurologist prescribes $\mathrm{CBZ}$ in agreement with the reference a daily dose according to the reference was suggested in 0 to $75 \%$ of the prescriptions. The system agreed in $10 \%$ or less for each of the sets seen by an individual neurologist. The system agreed with the reference in less cases than four of the neurologists.

Three neurologists prescribed VPA in a daily dose equal to the reference. Also the system prescribed VPA equal to the reference daily dose in three sets of the individual neurologist.

Agreement on drug and daily dose

All but one of the neurologists provided a prescription (drug and daily dose) according to the reference in less than $40 \%(19 \%$ to $36 \%)$. The other neurologist agreed with the reference in $56 \%$. The system provided a prescription according to the reference in $28 \%$ to $48 \%$ of the cases (for the various sets of cases common with the individual neurologists). Except for one 
of the neurologists the system consistently suggested an equal or higher number of prescriptions in accordance with the reference mainly because of a better agreement on VPA.

\subsection{References}

Chadwick D., Reynolds E.H., When do epileptic putients need treatment? Starting and stopping medication. British Medical Journal, 290. 1985, pp. 1885-1888.

Meinardi H., Epilepsie compendium, Katholicke Universiteit Nijmegen, 1993.

Porter R.J., Epilepsy, 100 elemenlary principles, W.B. Saunders Company Ltd., London, 1984.

Porter R.J. General principles how to use anti-epileptic drugs. In: Anti-epileptic drugs, $3^{\text {rd }}$ edition, (Levy, Mattson, Meldrum, Penry and F.E.Dreifuss, Eds.), Raven Press Ltd., New York, 1989, pp. 117-131.

Shorvon S.D., The trcatment of cpilepsy by drugs. In: Epilepsy. (Hopkins, Ed.), Chapman and Hall, London, 1987, pp. 229-282. 


\section{Results on follow-up of treatment}

\subsection{Introduction}

After a treatment is initiated its effectiveness is assessed during the next patient-doctor contacts; the follow-up. Based on this assessment a new therapy can be prescribed that may be different from the initial treatment. This chapter describes the results of our study concerning this second phase of anti-epileptic drug treatment.

First the treatment proposals provided by the neurologists based on 'paper cases' are compared with the advice provided by our system. The question to be answered is whether the treatments provided by the system are similar to the treatments provided by the neurologists. Several aspects of the treatment suggestions can be studied. We restrict ourselves to the global decisions to adjust the treatment: a continuation of the prescribed treatment ('Equal'), an adjustment of the daily dose of the drug ('Decrease' or 'Increase'), a switch to another antiepileptic drug ('Change') or adding an additional drug to the prescribed treatment ('Extra').

Since no objective standard exists for treatment of epilepsy we use the majority decision of a group of neurologists as the reference. To obtain insight in the variability of treatment decisions and to obtain information about possible alternatives without being dependent on the opinion of a single participant five neurologists were involved in defining a reference treatment for each case. Although consensus development by using a Delphi procedure has been considered, the time investment was deemed to be considerable. Therefore the majority decision among five neurologists was used for obtaining the reference treatment for each case. Comparing the correctness of the neurologists' treatment proposals with the system's output using this reference is disadvantageous for the system. Each of the neurologists has contributed to the reference and needs only to be in agreement with two other neurologists to have a correct proposal. The system needs to be in agreement with three neurologists to be correct. Therefore a second reference is defined by taking the majority of the treatment proposals of the system and the neurologists.

The adequateness of the system is evaluated in the following ways

- Comparison of the agreement between the system and neurologist( $s$ ). This analysis consists of two parts. Firstly the agreement between the system and the individual neurologists as well as among the individual neurologists was determined to establish the position of the system among the neurologists. In this study 265 cases (set 0) are used.

In the second analysis the prescriptions of each neurologist and the system are assessed by comparing them with prescriptions of the other four neurologists. Set 0 is used for this analysis. 
- Evaluation of the system and individual neurologists using the majority decision of the neurologists as the reference. This assessment provides information on how the neurologists and the system agree with a clinical reference. A reference was obtained in 219 of the 265 cases (set I).

- Evaluating the system and the neurologists using the majority decision of the neurologists and the system as the reference. In this study the set of 189 out of 265 cases for which such a reference was established was used (set II)

It is reasonable to assume that case characteristics determine which decision has to be taken. The fact that a majority decision is found in only 219 cases is, however, an indication that individual variation exists. In the second part of this chapter another type of analysis of the follow-ups is presented. Using a non parametric partitioning algorithm, a decision tree has been created based upon the case characteristics. In the ideal situation each terminal or endnode identifies cases of a certain (therapeutic) category. There may be several end nodes which point to the same category. The path from the root to the end-node contains the criteria to reach such an end-node. Four aspects of the derived tree are discussed

- The actual pureness of end-nodes in the decision tree

- The existence of cases with the same combination of patient characteristics that lead to an end-node, for which no reference treatment could be established

- The decision profiles within a impure end-node

- The decisions taken by the system for the cases of an end-node

Based upon this analysis we deduced a set of guidelines to be applied in the assessment of the initiated treatment that lead to specific treatment decisions.

\subsection{Design of test cases}

Like in our study on initiation of the drug treatment, paper cases were used. Information about the first follow-up after treatment initiation was added to the information in the paper cases of our previous study.

From patient records and interviews with neurologists it was determined which information is relevant for evaluating a patient who returns for an assessment of the prescribed treatment. Five characteristics were used to describe the course of the disease: the frequency of the seizures of various types, the compliance, the (kind of) complaints and the plasma drug level of the anti-epileptic drug. As in seizure severity scales the terminology (major and minor seizures) used for the seizure types is related to the severity of the seizures (Duncan and Sander 1991, Cramer 1993).

The following descriptors were used for these characteristics:

- The seizures (both major and minor) may be controlled (absent), decreased (the number of seizures decreased $\geq 50 \%$ ), equal (the number of seizures is unchanged, increased or decreased $<50 \%$ ) or not applicable (for a seizure type which was not present)

- There may be either a good compliance or non-compliance

- There can either be no complaints, dose related or idiosyncratic complaints

- The range of the plasma drug level can be either sub therapeutic, low therapeutic, therapeutic, high therapeutic or toxic 
In total 480 different combinations of these characteristics are possible.

We included - where relevant - other information related to the awareness of the patient, the occurrence of warnings, falling, incontinence, automatisms, convulsion, duration of the seizures and the duration before the patient is able to resume the activity he was pursuing before the seizure. The cause for non-compliance is presented where relevant. Furthermore, in case of complaints a description of the complaint together with information about the start and circumstances was presented. The prescription for the initiation of treatment was presented as well, including the brand of the medication, formulation, dose, number of tablets and number of intakes.

Apart from the detailed descriptors as discussed above a more global classification of the change in seizure frequency is used also (Table 6.2-I). In cases with several seizure types for which only one type of seizures was controlled or decreased in frequency, the reduction was still considered to be significant. Both case types were grouped into the class 'seizures decreased'.

Table 6.2-I: Overview of the seizure frequencies and the used terminology.

\begin{tabular}{|l|l|l|l|}
\hline Major seizures & Minor seizures & Litennediate & Global \\
\hline $\begin{array}{l}\text { equal } \\
\text { equal }\end{array}$ & $\begin{array}{l}\text { n.a. } \\
\text { equal } \\
\text { equal }\end{array}$ & uncontrolled & equal \\
\hline $\begin{array}{l}\text { decreased } \\
\text { n.a. } \\
\text { decreased } \\
\text { decreased } \\
\text { equal } \\
\text { equal } \\
\text { controlled }\end{array}$ & $\begin{array}{l}\text { n.a. } \\
\text { decreased } \\
\text { decreased } \\
\text { equal } \\
\text { decreased } \\
\text { controlled } \\
\text { equal }\end{array}$ & hurdly controlled & decreased \\
\cline { 1 - 2 } $\begin{array}{l}\text { controlled } \\
\text { decreased }\end{array}$ & $\begin{array}{l}\text { decreased } \\
\text { controlled }\end{array}$ & alinost controlled & \\
\hline $\begin{array}{l}\text { controlled } \\
\text { n.a. } \\
\text { controlled }\end{array}$ & $\begin{array}{l}\text { n.a. } \\
\text { controlled } \\
\text { controlled }\end{array}$ & controlled & absent \\
\hline
\end{tabular}

Of the 480 different case descriptions that can be constructed from the case characteristics, 270 follow-up contacts were actually used by:

- Reducing the number of cases with complete seizure control. Only few cases with complete seizure control were included as these cases usually do not entail difficult decisions

- Reducing the number of cases with only one type of seizures. Cases with two different types of seizures were considered of greater interest with regard to careful decision making

- Eliminating less realistic combinations of characteristics

\subsection{Description of the cases}

The 270 cases used in this study were derived from a subset of 43 case descriptions from the diagnostic test set of our study on the initiation of therapy. Each of these cases was the basis 
for one or more cases in the follow-up study. From 22 cases one to five different follow-up contacts were derived; 16 cases were the basis for six to ten different follow-ups. From five cases more than 10 follow-ups were created. The majority of the 43 cases had partial epilepsy. This selection was deliberate as this type of epilepsy is the most difficult to treat and has a larger variety of expressions of seizures (Elwes and Reynolds, 1990).

In 267 of the 270 cases all five neurologists provided a prescription. In the remaining three cases only four neurologists gave a prescription. In two of the 267 cases the system did not present a prescription. So 265 cases remained for comparison.

In this set of 265 cases 238 cases $(89.8 \%$ ) had a diagnosis of partial epilepsy, 12 cases (4.5\%) had idiopathic generalised epilepsy and 15 cases $(5.7 \%)$ had a combination of generalised and partial epilepsy. Three different drugs were prescribed in the 265 cases. CBZ was used in 219 cases $(82.6 \%)$, PHT was used in 36 cases (13.6\%) and Na-VPA was used in 10 cases $(3.8 \%)$.

Table 6.3-I: Distribution of the main characteristics of the paper cases used.

\begin{tabular}{|c|c|c|c|c|c|c|c|c|c|c|}
\hline \multirow{4}{*}{$\begin{array}{l}\text { Chancteristics } \\
\text { Compliance } \\
\text { Complaints } \\
\text { Seizures }\end{array}$} & \multicolumn{10}{|c|}{ Values and frequencies } \\
\hline & \multicolumn{4}{|c|}{ Compliant } & \multicolumn{4}{|c|}{ Non-compliance } & \multirow{2}{*}{\multicolumn{2}{|c|}{ Total }} \\
\hline & \multicolumn{2}{|c|}{ Absent } & \multicolumn{2}{|c|}{ Presient } & \multicolumn{2}{|c|}{ Absent } & \multicolumn{2}{|c|}{ Present } & & \\
\hline & $n$ & $\%$ & n & $\%$ & 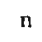 & $\%$ & $n$ & $\%$ & $\mathbf{n}$ & $\%$ \\
\hline controlled & 7 & 2.6 & 17 & 6.4 & 5 & 1.9 & II & 4.2 & 40 & 15.1 \\
\hline almost controlled & 8 & 3.0 & 15 & 5.7 & 6 & 2.3 & 13 & 4.9 & 42 & 15.8 \\
\hline hardly controlled & 20 & 7.6 & 39 & 14.7 & 22 & 8.3 & 34 & 12.8 & 115 & 43.4 \\
\hline uncontrolled & 11 & 4.2 & 18 & 6.8 & 22 & 8.3 & 17 & 6.4 & 68 & 25.7 \\
\hline Total & 46 & 17.4 & 89 & 33.6 & 55 & 20.8 & 75 & 28.3 & 265 & 100 \\
\hline
\end{tabular}

The set of cases is an enriched sample of what one would expect in daily clinical practice. Relatively more cases with (multiple) problems were included (e.g. cases in which seizures were not controlled by the initial treatment and/or cases with side-effects). In our set $95 \%$ of the cases had seizures which were not controlled (Table 6.3-I). This should be contrasted with a clinical setting where about one third of the patients may be controlled by the initial drug treatment (Elwes et al 1984, Beghi and Tognoni 1988,van Donselaar 1990, Collaborative Group for the Study of Epilepsy 1992). Hence it is to be expected that in our study the disagreement between neurologists concerning a specific treatment is larger than that found in daily practice.

\subsubsection{Description of reference set I}

In 219 of the 265 cases the majority of the five neurologists agreed about a specific global treatment decision. In Table 6.3-11 the distribution of the 219 cases with respect to the main characteristics is presented. Seizures were not completely controlled in the majority of these cases $(83.1 \%$ ). In more than half of the cases $(59.8 \%$ ) complaints were present. A combination of incompletely controlled seizures, presence of complaints and non-compliance is observed in $23.3 \%$ of the cases. 
Table 6.3-II: Overview of reference set I.

\begin{tabular}{|c|c|c|c|c|c|c|c|c|c|c|}
\hline \multirow{3}{*}{$\begin{array}{l}\text { Characteristics } \\
\text { Compliance } \\
\text { Complaints }\end{array}$} & \multicolumn{10}{|c|}{ Values and frequencies } \\
\hline & \multicolumn{4}{|c|}{ Compliant } & \multicolumn{4}{|c|}{ Non-compliant } & & \\
\hline & \multicolumn{2}{|c|}{ Absent } & \multicolumn{2}{|c|}{ Present } & \multicolumn{2}{|c|}{ Absent } & \multicolumn{2}{|c|}{ Present } & \multicolumn{2}{|c|}{ Total } \\
\hline Seizures & $\mathrm{n}$ & $\%$ & $\mathrm{n}$ & $\%$ & $\mathrm{n}$ & $\%$ & $\mathrm{n}$ & $\%$ & $\mathrm{n}$ & $\%$ \\
\hline controlled & 7 & 3.2 & 14 & 6.4 & 5 & 2.3 & 11 & 5.0 & 37 & 16.9 \\
\hline a most controlled & 8 & 3.7 & 11 & 5.0 & 5 & 2.3 & 7 & 3.2 & 31 & 14.2 \\
\hline hardly controlled & 16 & 7.3 & 28 & 12.8 & 19 & 8.7 & 27 & 12.3 & 90 & 41,1 \\
\hline uncontrolled & 10 & 4.6 & 16 & 7.3 & 18 & 8.2 & 17 & 7.8 & 61 & 27.9 \\
\hline Total & 41 & 18.7 & 69 & 31.5 & 47 & 21.5 & 62 & 28.3 & 219 & 100 \\
\hline
\end{tabular}

\section{Agreement profiles}

The degree of agreement among the neurologists may vary from case to case. The degree of agreement is indicated by means of agreement profiles. Each entry in the profile indicates the number of neurologists that agree about a treatment decision. For example a 3-2 profile indicates a majority of 3 neurologists while the other two neurologists disagreed with the majority but agreed among themselves.

Table 6.3-MI: Agreement profiles in the group of five neurologists.

\begin{tabular}{lrrrr|rrr}
\hline & \multicolumn{3}{c|}{ Reference set } & \multicolumn{3}{c}{ No reference } \\
\hline Profile & 5 & \multicolumn{1}{c}{$4-1$} & $3-2$ & $3-1-1$ & $2-2-1$ & $2-1-1-1$ & $1-1-1-1-1$ \\
Cases & 58 & 75 & 52 & 34 & 33 & 13 & 0 \\
$\%$ & 21.9 & 28.3 & 19.6 & 12.8 & 12.5 & 4.9 & 0 \\
\hline \multicolumn{3}{c}{ Total } & \multicolumn{3}{c}{$\mathrm{n}=219,82.6 \%$} & & \multicolumn{3}{c}{$\mathrm{n}=46.17 .4 \%$} \\
\hline
\end{tabular}

Table 6.3-IIl shows the distribution of the agreement profiles in the 265 cases. In 219 cases $(82.6 \%)$ a majority decision of the neurologists could be established. In 58 out of 265 cases all five neurologists completely agree on how to treat the patient.

\subsubsection{Description of reference set II}

In 189 of the 265 cases (71.3\%) a majority of at least four out of the six participants - the five neurologists and the system - agreed on the treatment decision. Table 6.3-IV presents the main characteristics of these 189 cases. When this set is compared with the set of 219 cases, minor changes are observed in the frequency of cases with seizures controlled and seizures uncontrolled. The system proposes an approach different from the majority of neurologists mainly in cases with.

- seizures almost controlled

- seizures hardly controlled and complaints

- seizures uncontrolled, complaints absent and non-compliance

- seizures controlled, complaints present and good compliance 
Table 6.3-IV: Overview of reference set II.

\begin{tabular}{|c|c|c|c|c|c|c|c|c|c|c|}
\hline \multirow{4}{*}{$\begin{array}{l}\text { Characteristics } \\
\text { Compliant } \\
\text { Complaints } \\
\text { Seizures }\end{array}$} & \multicolumn{10}{|c|}{ Values and frequencies } \\
\hline & \multicolumn{4}{|c|}{ Compliaut } & \multicolumn{6}{|c|}{ Non-compliant } \\
\hline & \multicolumn{2}{|c|}{ Absent } & \multicolumn{2}{|c|}{ Present } & \multicolumn{2}{|c|}{ Absent } & \multicolumn{2}{|c|}{ Present } & \multicolumn{2}{|c|}{ Total } \\
\hline & $\mathrm{n}$ & $\%$ & $\mathrm{n}$ & $\%$ & n & $\%$ & $\mathrm{n}$ & $\%$ & $\mathrm{n}$ & $\%$ \\
\hline controlled & 7 & 3.8 & 11 & 5.8 & 5 & 2.7 & 11 & 5.8 & 34 & 18.0 \\
\hline almost controlled & 5 & 2.7 & 10 & 5.3 & 5 & 2.7 & 5 & 2.7 & 25 & 13,2 \\
\hline hardly controlled & 15 & 7.9 & 22 & 11.6 & 17 & 9.0 & 21 & 11.1 & 75 & 39.7 \\
\hline uncontrolled & 9 & 4.8 & 16 & 8.5 & 13 & 6.9 & 17 & 9.0 & 55 & 29.1 \\
\hline Total & 36 & 19.1 & 59 & 31.2 & 40 & 21.2 & 54 & 28.6 & 189 & 100 \\
\hline
\end{tabular}

\section{Agreement profiles in reference cases}

The agreement profiles based on all six participants are shown in Table 6.3-V. Inclusion of the system in the judgement procedure yields reference cases in which either at least four neurologists or three neurologists and the system agree about the treatment approach.

Table 6.3-V: Comparison of the agreement in the group of five neurologists with that of the system and the group consisting only of five neurologists.

\begin{tabular}{|c|c|c|c|c|c|c|c|c|c|c|c|c|}
\hline \multirow{2}{*}{\multicolumn{2}{|c|}{$\begin{array}{l}\text { Agrecment among } \\
\text { five neurologists }\end{array}$}} & \multicolumn{11}{|c|}{ five neurologists and the system } \\
\hline & & \multicolumn{4}{|c|}{ Reference $\mathrm{N}=189.713 \%$} & \multicolumn{7}{|c|}{ No reference $\mathrm{N}=76,28.8 \%$} \\
\hline & Profile & 6 & $5-1$ & $4-2$ & $4-1-1$ & $3-3$ & $3-2-1$ & $3-1-1-1$ & $2-2-2$ & $2-2-1-1$ & $2-1-1-1-1$ & To \\
\hline Ref & 5 & 54 & 4 & & & & & & & & & 58 \\
\hline $\mathrm{N}=219$ & 4.1 & & 61 & 9 & 5 & & & & & & & 75 \\
\hline \multirow[t]{2}{*}{$82.6 \%$} & $3-2$ & & & 34 & & 16 & 2 & & & & & 52 \\
\hline & $3-1-1$ & & & & 22 & & 12 & & & & & 34 \\
\hline No ref & $2-2-1$ & & & & & & 26 & & 5 & 2 & & 37 \\
\hline $\begin{array}{l}N=46 \\
17.4 \%\end{array}$ & $2-1-1-1$ & & & & & & & 5 & & 7 & 1 & 13 \\
\hline \multicolumn{2}{|l|}{ Total } & 54 & 65 & 43 & 27 & 16 & 40 & 5 & 5 & 9 & 1 & 268 \\
\hline
\end{tabular}

In 54 of the 265 cases $(20.4 \%)$ the system completely agreed with the five neurologists (profile 6) In $\mathbf{3} 33$ cases the five neurologists had a 5 or 4 -1 agreement profile. When only the neurologists were used as a reference in 86 cases three out of five neurologists agreed about the treatment decision (profiles 3-2 and 3-1-1) Including the system resulted in the definition of a reference in 56 of these cases (profiles 4-2 and 4-1-1). In $16(13.5 \%)$ of the remaining 30 cases the system agreed with the minority of two neurologists. In 12 cases the system proposed a treatment decision equal to that of one of the neurologists.

In 14 of the 265 cases the system suggested a treatment different from any of the treatments suggested by the group of neurologists In nine of these cases there was a reference treatment (5-! and 4-I-! agreement profile), As a 3-3i agreement profile was not sufficient for a case to 
be incorporated as reference case, including the system could not cause cases not in set I to become part of set II. Where a reference had been based on a majority decision of three neurologists the system could either strengthen the agreement or refute it.

\subsubsection{Cases not included in the reference set II}

Reference set II contains 189 cases in which a majority decision was reached. In 76 cases no majority decision could be obtained. Table 6.3-VI gives an overview of the main characteristics of the cases for which a reference treatment could not be obtained. The results can be summarised as:

- In 51 of the 76 cases with no reference a complaint was present, which means that in $31.1 \%$ of the 164 cases in which a complaint was present a reference was not obtained In contrast, in 25 of the 101 cases $(24.8 \%$ ) without a complaint no reference was reached

- In 57 of the cases with no reference, seizures were hardly or almost completely controlled This means that in $36.3 \%$ of the 157 cases in which the anti-epileptic drug treatment seemed to have some but insufficient effect no majority decision could be achieved

- In 17 of the 42 cases $(40.5 \%)$ with seizures which were almost controlled a reference was not obtained.

- In 40 of 115 cases $(34.8 \%$ ) with seizures hardly controlled a reference could not be obtained.

- In 6 of the 40 cases $(15 \%)$ in which seizures were controlled a reference could not be defined. Also for less than one fitth of the cases $(19 \%)$ in which the seizure frequency remained equal to the previous visit a majority agreement could not be obtained

Table 6.3-VI: Description of the cases not included in the reference. In the column ' $\%$ ' the percentage of the total of 76 cases is presented. In the column $\%$ tor the percentage is related to the number of cases in the total test set with the same characteristics.

\begin{tabular}{|c|c|c|c|c|c|c|c|c|c|c|c|c|c|c|c|}
\hline \multirow{3}{*}{$\begin{array}{l}\text { Characteristic } \\
\text { Compliance } \\
\text { Complaints }\end{array}$} & \multicolumn{15}{|c|}{ Values and freyuencies } \\
\hline & \multicolumn{6}{|c|}{ Compliant } & \multicolumn{6}{|c|}{ Non-comp̣tiant } & \multirow{2}{*}{\multicolumn{3}{|c|}{ Total }} \\
\hline & & Ahsent & & & Frese: & & & Abser & & & Presen & & & & \\
\hline Seizures & n & $\%$ & $\% 10 t$ & $n$ & $\%$ & $\because$ tot & n & $\because$ & $\sim \operatorname{tot}$ & n & $\because$ & $*+104$ & $n$ & $\because$ & "stust \\
\hline centrolled & 0 & 0 & a & 6 & 7.9 & 35.1 & 0 & 0 & 0 & 4 & 0 & 0 & 6 & $7 \%$ & 150 \\
\hline almost cont. & 3 & 39 & 37.5 & 5 & 66 & 33.1 & 1 & 1.3 & 167 & $\mathrm{x}$ & 105 & 6.15 & 17 & $2: 4$ & 405 \\
\hline hardly con! & 5 & 6.6 & 25.0 & 17 & 22.4 & 436 & 9 & 66 & 227 & 13 & 171 & $1 \times 2$ & 40 & 826 & $34 x$ \\
\hline uncontr. & 2 & 2.6 & 18.2 & 2 & 2.6 & 111 & 91 & 118 & 409 & 0 & 4 & $\theta$ & 17 & 171 & 191 \\
\hline Totat & 10 & 132 & 217 & 30 & 395 & 337 & 15 & 197 & 273 & $2 !$ & 276 & $2 \times 0$ & 76 & 100 & 100 \\
\hline
\end{tabular}

Agreement profiles in non-reference cases

in $61(80.3 \%)$ of the 76 cases with no reference treatment three out of six participants agree about a specific treatment (profiles $3-3,3-2-1$ and $3-1-1-1$ ) (Table $63-\mathrm{V}$ )

The agreement of each participant with the other participants in these 76 cases is presented in Table 6.3-VII. 'Score' indicates the number of other participants which agree with the participant. 
Table 6.3-VII: Variability in agreement between participants in the 76 cases. The item 'Score' indicates the number of participants which agree with the decision taken by the neurologist or the system.

\begin{tabular}{lrrrrrrrrrrrrr}
\hline & \multicolumn{1}{c}{ NUI } & \multicolumn{3}{c}{ NIV } & \multicolumn{2}{c}{ NV } & \multicolumn{2}{c}{ NVI } & \multicolumn{2}{c}{ NVI } & \multicolumn{2}{c}{ System } \\
\hline Score & n & $\%$ & $\mathrm{n}$ & $\%$ & $\mathrm{n}$ & $\%$ & $\mathrm{n}$ & $\%$ & $\mathrm{n}$ & $\%$ & $\mathrm{n}$ & $\%$ \\
0 & 17 & 22.4 & 12 & 15.8 & 18 & 23.7 & 14 & 18.4 & 11 & 14.5 & 5 & 6.6 \\
1 & 27 & 35.5 & $3 !$ & 40.8 & 15 & 19.7 & 27 & 35.5 & 24 & 31.6 & 24 & 31.6 \\
2 & 32 & 42.1 & 33 & 43.4 & 43 & 56.6 & 35 & 46.1 & 41 & 54.0 & 47 & 61.8 \\
\hline
\end{tabular}

The percentage of cases for which a neurologist prescribed a treatment different from those prescribed by any of the other participants ranges from $14.5 \%$ to $23.7 \%$. The system provided an advice different from those of all neurologist's in only $6.6 \%$ of the cases.

Each of the neurologists agreed with two other participants in $42.1 \%$ to $56.6 \%$ of the cases. The system agreed with two neurologists in $61.8 \%$ of the cases.

\subsubsection{Relevance of characteristics for agreement among neurologists}

Various levels of agreement among the neurologists occur in the cases with a reference treatment (set l). The degree of agreement probably depends on the characteristics of the case. For each of the main features in the case descriptions we determined the level of agreement. The five out of five and four out of five agreement levels are considered to represent high degrees of agreement as changing the opinion of one of the neurologists will not affect the reference treatment.

\section{Seizure froyuency}

In $78 \%$ of the 37 cases in which seizures were completely controlled and in $65.6 \%$ of the 61 cases in which seizures remained equal to the previous visit there is a high degree of agreement on the reference treatment (Table 6.3-VIII). For the cases in which seizures were almost completely controlled a lower $(58.1 \%)$ percentage of cases with a high degree of agreement is observed.

Table 6.3-VII: Distribution of the agreement profiles for the frequencies of the type of seizures.

\begin{tabular}{|c|c|c|c|c|c|c|c|c|c|c|c|c|c|c|}
\hline Characteristic & \multicolumn{9}{|c|}{ Reference treatinent } & \multicolumn{5}{|c|}{ No reference treatmint } \\
\hline Profile & \multicolumn{2}{|c|}{5} & \multicolumn{2}{|c|}{$4-1$} & \multicolumn{2}{|c|}{$3-2$} & \multicolumn{2}{|c|}{$3-1-1$} & Tolal & \multicolumn{2}{|c|}{$2-2-1$} & \multirow{2}{*}{$\begin{array}{l}2-1-1-1 \\
N \%\end{array}$} & \multicolumn{2}{|c|}{ Total } \\
\hline Seizures: & $\mathrm{N}$ & $\%$ & $N$ & $\%$ & $N$ & $\%$ & $N$ & $\%$ & $N \%$ & $\mathrm{~N}$ & $\%$ & & $\mathrm{~N}$ & $\%$ \\
\hline controlled & 17 & 45.6 & 12 & 32.4 & 5 & 13.5 & 3 & 8.1 & 37100 & 3 & 100 & 00 & 3 & 100 \\
\hline almost contr & 8 & 25.8 & 10 & 32.3 & 4 & 12.9 & 9 & 29.0 & 31100 & 5 & 46 & 655 & 11 & 100 \\
\hline hardly contr & 19 & 21.1 & 27 & 30.0 & 32 & 35.6 & 12 & 13.3 & $90 \quad 100$ & 18 & 72 & 728 & 25 & 100 \\
\hline uncontr & 14 & 23.0 & 26 & 42.6 & 11 & 180 & 10 & 16.4 & 61100 & 7 & 100 & 0 & 7 & 100 \\
\hline Total & 58 & 26.5 & 75 & 34.3 & 52 & 23.7 & 34 & 15.5 & 219100 & 33 & 72 & 1328 & 46 & 100 \\
\hline
\end{tabular}


For cases that were hardly controlled the high degree of agreement decreases to $51.1 \%$. This drop in strong agreement can partially be explained by the fact that there seem to be two alternative treatments for certain cases. For example in $35.6 \%$ of the cases in which seizures are hardly controlled the two neurologists who propose a treatment different from the reference agree among themselves (the 3-2 agreement profile). This in contrast with a 3-2 agreement profile in at most $18 \%$ of the other types of seizure frequency.

\section{Compliance}

Compliance appears to have no effect on the degree of agreement among the neurologists (Table 6.3-IX). A high degree of agreement is found in $63.6 \%$ and $57.8 \%$ of the cases with a good compliance and non-compliance, respectively.

Table 6.3-IX: Dist ribution of the agreement profiles for the characteristic 'Compliance'.

\begin{tabular}{|c|c|c|c|c|c|c|c|c|}
\hline \multirow{3}{*}{$\begin{array}{l}\text { Characteristic } \\
\text { Profile } \\
\text { Compliance }\end{array}$} & \multicolumn{5}{|c|}{ Reference trealtnent } & \multicolumn{3}{|c|}{ No reference } \\
\hline & 5 & 4-1 & $3-2$ & $3-1-1$ & Total & $2-2-1$ & $2-1-1-1$ & Total \\
\hline & $N \%$ & $\mathrm{~N} \%$ & $\mathrm{~N} \%$ & $\mathrm{~N} \%$ & $N \quad \%$ & $\mathrm{~N} \%$ & $\mathrm{~N} \%$ & $\mathrm{~N} \%$ \\
\hline $\operatorname{good}$ & $35 \quad 31.8$ & 3531.8 & 2220.0 & $18 \quad 16.4$ & $110 \quad 100$ & 1872 & 728 & 25100 \\
\hline non-compliance & 2321.1 & $40 \quad 36.7$ & 3027.5 & $16 \quad 14.7$ & $109 \quad 100$ & 1571 & 29 & 21100 \\
\hline Total & $58 \quad 26.5$ & $\begin{array}{ll}75 & 34.3\end{array}$ & $52 \quad 23.7$ & $34 \quad 15.5$ & $219 \quad 100$ & 3372 & 1328 & 46100 \\
\hline
\end{tabular}

\section{Complaints}

In $63.7 \%$ of the cases where complaints are absent more than three neurologists agree on the treatment (Table 6.3-X). For cases in which idiosyncratic complaints are present strong agreement occurs in $83.1 \%$ of the cases.

Contrary to this, strong agreement is present in only $38.9 \%$ for cases with dosc related complaints. In $44.4 \%$ of the cases with dose related complaints, there is a $3-2$ agreement pattern, indicating that in these circumstances two alternative treatments may exist.

Table 6.3-X: Distribution of the agreement profiles for the characteristic 'Complaints'.

\begin{tabular}{|c|c|c|c|c|c|c|c|c|c|c|c|c|}
\hline \multirow{3}{*}{$\begin{array}{l}\text { Characteristic } \\
\text { Profile } \\
\text { Complaints }\end{array}$} & \multicolumn{9}{|c|}{ Reference treatınent } & \multicolumn{3}{|c|}{ No reference } \\
\hline & \multicolumn{2}{|r|}{5} & \multicolumn{2}{|c|}{$4-1$} & \multicolumn{2}{|c|}{$3-2$} & \multicolumn{2}{|c|}{$3-1-1$} & Total & $2-2-1$ & $2-1-1-1$ & \multirow{2}{*}{$\begin{array}{l}\text { Total } \\
\text { N } \%\end{array}$} \\
\hline & $N$ & $\%$ & $N$ & $\%$ & $\mathrm{~N}$ & $\%$ & $N$ & $\%$ & $N \%$ & $\mathrm{~N} \%$ & $\mathrm{~N} \%$ & \\
\hline ahsent & 24 & 27.3 & 32 & 36.4 & 17 & 19.3 & 15 & 17.1 & $88 \mathrm{l}(n)$ & 1077 & 323 & 13100 \\
\hline dose related & 6 & 8.3 & 22 & 30.6 & 32 & 44.4 & 12 & 16.6 & 72100 & 2067 & 1033 & 30100 \\
\hline idiosyncratic & 28 & 47.5 & 21 & 35.6 & 3 & 5.1 & 7 & 11.9 & $59 \quad 100$ & 3100 & 00 & 3100 \\
\hline Total & 58 & 26.5 & 75 & 34.3 & 52 & 23.7 & 34 & 15.5 & 219100 & 3372 & 1328 & 46. 100 \\
\hline
\end{tabular}

\section{Plasma drug level}

'Plasma drug level' hardly influences the degree of agreement. Strong agreement is found in more than $55 \%$ of the cases for all plasma drug levels except for levels in the toxic range. Even in the latter strong agreement was found in $50 \%$ of the cases (Table 6.3-XI). 
Table 6.3-XI: Distribution of the agreement profiles for the characteristic 'Plasma drug level'.

\begin{tabular}{|c|c|c|c|c|c|c|c|c|c|c|c|c|}
\hline \multirow{3}{*}{$\begin{array}{l}\text { Characteristic } \\
\text { Profile } \\
\text { Plasma drug level }\end{array}$} & \multicolumn{9}{|c|}{ Reference treatment } & \multicolumn{3}{|c|}{ No reference } \\
\hline & \multicolumn{2}{|c|}{$s$} & \multicolumn{2}{|c|}{$4-1$} & \multicolumn{2}{|c|}{$3-2$} & \multicolumn{2}{|c|}{$3-1-1$} & Total & $2-2-1$ & $2-1-1-1$ & Tola] \\
\hline & $\mathrm{N}$ & $\%$ & $N$ & $\%$ & $\mathrm{~N}$ & $\%$ & $\mathrm{~N}$ & $\%$ & $\mathrm{~N} \%$ & $\mathrm{~N} \%$ & $\mathrm{~N} \%$ & N \% \\
\hline sub ther & 15 & 33.3 & 14 & 31.1 & 12 & 26.7 & 4 & 8.9 & $45 \quad 100$ & 571 & 229 & $7 \quad 100$ \\
\hline low ther & 16 & 36.4 & 14 & 31.8 & 9 & 20.5 & 5 & 11.4 & $44 \quad 100$ & 6100 & 00 & 6100 \\
\hline ther & 11 & 20.8 & 21 & 39.6 & 12 & 22.6 & 9 & 17.0 & $53 \quad 100$ & 770 & 330 & $10 \quad 100$ \\
\hline high ther & 9 & 20.0 & 17 & 37.8 & 9 & 20.0 & 10 & 22.2 & $45 \quad 100$ & 975 & 325 & 12100 \\
\hline toxic & 7 & 21.9 & 9 & 28.1 & 10 & 31.3 & 6 & 18.8 & $32 \quad 100$ & 655 & 546 & 11100 \\
\hline Total & 58 & 26.5 & 75 & 34.3 & 52 & 23.7 & 34 & 15.5 & 219100 & 3372 & 1328 & $46 \quad 100$ \\
\hline
\end{tabular}

\subsubsection{Alternative treatments}

The previous section showed that in more than one third of the cases with hardly controlled seizures as well as in cases with dose related complaints a 3-2 agreement profile was frequently observed. For these two characteristics the alternative treatment is discussed.

\section{Seizure frequency}

In the 32 cases with hardly controlled seizures two neurologists propose an alternative treatment (see Table 6.3-XII). Change of drug is the most common alternative proposal for the reference treatments

Table 6.3-XII: Distribution of the reference treatments and the alternatives for the 32 cases in which a 3-2 agreement pattern was observed.

\begin{tabular}{|c|c|c|c|c|c|c|}
\hline \multirow[t]{2}{*}{ Treatment } & & \multicolumn{5}{|c|}{ Reference treatment } \\
\hline & Decision & Decrease & Equal & Increase & Change & Extra \\
\hline \multirow{4}{*}{$\begin{array}{l}\text { Altenative } \\
\text { tratunent }\end{array}$} & Decrease & & & & d & \\
\hline & Equal & 2 & & & 4 & \\
\hline & Increase & & & & 1 & \\
\hline & Change & 12 & 6 & 1 & & 2 \\
\hline
\end{tabular}

\section{Dose related complaints}

In 32 cases with dose related complaints (Table 6.3-XIII) in most cases (75\%) a change of drug was either the reference treatment or the alternative treatment. With dose related complaints in the history in all cases (9/9) the alternative treatment suggested was a change of drug when the reference decision was to decrease the dose, and also a change of drug in $7 / 12$ cases where the reference was to keep the medication constant. In five cases with dose related complaints where the reference was to continue the treatment, presumably because of seizure related reasons, the alternative was an increase of the dose.

A decision by either the reference or the alternative treatment to increase the dose was rarely observed in combination with an advice to change the drug (3/10) 
Table 6.3-XII: Distribution of the reference treatments and the altematives for the 32 cases with dose related complaints in which a 3-2 agreement pattern was observed.

\begin{tabular}{|c|c|c|c|c|c|c|}
\hline Treatment & & \multicolumn{5}{|c|}{ Reference treatment } \\
\hline \multirow{5}{*}{$\begin{array}{l}\text { Altemative } \\
\text { treatment }\end{array}$} & Decision & Decrease & Equal & Increase & Change & Extra \\
\hline & Decrease & & & & 1 & \\
\hline & Equal & & & 2 & 4 & \\
\hline & Increase & & 5 & & 2 & \\
\hline & Change & 9 & 7 & 1 & & 1 \\
\hline
\end{tabular}

\subsection{Agreement and correctness}

Two of the metrics mainly used when evaluating a DSS are agreement and correctness. The term agreement is used when comparing the advice of the system with that of a neurologist or a group of neurologists. Correctness is used when assessing the advice of the system using a previously defined reference.

\subsubsection{Overview of the decisions in the total set}

Eight different decisions were taken by the neurologists. The decisions to continue the prescribed treatment are grouped into the class 'Equal'. If the daily dose was decreased or increased the decisions are labelled as 'Decrease' and 'Increase'. The class 'Change' contains decisions in which a different anti-epileptic drug was selected for further treatment. This class does not include the addition of a second anti-epileptic drug to the prescribed treatment. The class 'Stop' contains the decision to discontinue the treatment immediately. The class 'Other' contains decisions which differed from adjustment of the treatment, e.g. a referral. Finally, two

Table 6.4-I: Overview of the frequencies of decisions for further treatment of the patient.

\begin{tabular}{|c|c|c|c|c|c|c|c|c|c|c|c|c|c|c|}
\hline \multirow[t]{2}{*}{ Decision } & \multicolumn{2}{|c|}{ Decruase } & \multicolumn{2}{|c|}{ Equal } & \multicolumn{2}{|c|}{ Increase } & \multicolumn{2}{|c|}{ Change } & \multicolumn{2}{|c|}{ Extra } & \multicolumn{2}{|c|}{ Stop } & \multicolumn{2}{|c|}{ Other } \\
\hline & $\mathrm{n}$ & $\%$ & $n$ & $\%$ & $n$ & $\%$ & $\mathrm{n}$ & $\%$ & $n$ & $\%$ & $\mathrm{n}$ & $\%$ & $\mathrm{n}$ & $\%$ \\
\hline III & 50 & 18.9 & 67 & 25.3 & 39 & 14.7 & 96 & 36.2 & 13 & 4.9 & 0 & 0 & 0 & 0 \\
\hline IV & 37 & 14.0 & 113 & 42.6 & 56 & 21.1 & 47 & 17.7 & 9 & 3.4 & 2 & 0.8 & I & 0.4 \\
\hline V & 13 & 4.9 & 30 & 11.3 & 52 & 19.6 & 154 & 58.1 & 14 & 5.3 & 2 & 0.8 & 0 & 0 \\
\hline $\mathrm{VI}$ & 31 & 11.7 & 62 & 23.4 & 65 & 24.5 & 107 & 40.4 & 0 & 0 & 0 & 0 & 0 & 0 \\
\hline VII & 2 & 0.8 & 91 & 34.3 & 34 & 12.8 & 1.35 & 50.9 & 2 & 0.8 & 1 & 0.4 & 0 & 0 \\
\hline System & 11 & 4.2 & 54 & 20.4 & 62 & 2.3 .4 & 136 & 51.3 & 0 & 0 & 2 & 0.8 & 0 & 0 \\
\hline
\end{tabular}

decisions were observed in combination with adding an additional drug to the existing treatment ('Extra'); a continuation of the existing treatment or an alteration of the existing daily dose. Table 6.4-I presents the distribution of the decisions of each of the five neurologists and the system on the 265 cases (in the table the eight types of decisions have been reduced to seven by combining 21 decisions in which an anti-epileptic drug was added to 
the existing treatment and 11 decisions in which both an anti-epileptic drug was added and the original dose was altered into the category 'Extra')

\subsubsection{Differences in treatment approaches}

There are clear differences in the approaches taken by the neurologists. Neurologist IV is the one that most often sticks to the initiated treatment. In $42.6 \%$ of the cases he does not make any adjustment in the dose or the drug. In only $17.7 \%$ he decides to change the treatment. This is in contrast with Neurologist $V$ who predominantly changes the therapy $(58.1 \%$ of the cases) and keeps the drug dose as it was in only a small fraction of the cases.

Also the fraction of cases in which the decision was taken to adjust the dose of the drug shows large variations. While Neurologist III decides to decrease the drug dose in almost one fifth of the cases, Neurologist VII decides to decrease the drug dose in less than $1 \%$.

An increase of the drug dose was decided upon by Neurologist VI in almost a quarter of the cases which is almost twice as often as Neurologist VII.

Adding a second anti-epileptic drug to the existing drug treatment or the immediate stopping of the prescribed drug are not very common decisions. All neurologists decide to switch to polytherapy in less than $6 \%$ of the cases, while the decision to stop the drug is taken by all neurologists in less than $1 \%$ of the cases.

On average, in $25 \%$ of the cases the same treatment was suggested, while in approximately $40 \%$ of the cases the drug was changed. These numbers clearly show that the cases are heavily biased towards cases that are more difficult to treat.

The system proposed to change the drug in more than $50 \%$ of the cases. In less than $5 \%$ of the cases a decrease of the dose of the drug was suggested, while an increase was suggested in almost one fourth of the cases. In $20 \%$ of the cases a continuation of the prescribed treatment was suggested.

Table 6.4-II: $k$-values for the individual neurologists and the system on the total set of 265 cases.

\begin{tabular}{lccccc}
\hline & Neuro II & Neuro IV & Neuro V & Neuro VI & Neuro VI \\
\hline Neuro IV & 0.42 & & & & \\
Neuro V & 0.40 & 0.28 & & & \\
Neuro VI & 0.40 & 0.41 & 0.44 & & \\
Neuro VII & 0.35 & 0.26 & 0.35 & 0.29 & \\
System & 0.42 & 0.35 & 0.56 & 0.46 & 0.40 \\
\hline
\end{tabular}

The agreement beyond what is to be expected by chance alone between two observers can be expressed with the kappa coefficient. $\kappa$-values between 0.21 and 0.40 are considered to indicate fair agreement, those ranging from 0.41 up to 0.60 are considered to denote moderate agreement, while the values between 0.61 and 0.80 and those larger than 0.81 indicate substantial and almost perfect agreement, respectively (Landis and Koch, 1977). 
The $\kappa$-values for the pair wise comparisons of the decisions of neurologists range from 0.26 to 0.44 , while the systen when compared with the individual neurologists has $\mathrm{K}$-values between 0.35 and 0.56 (Table 6.4-II). The agreement of the system with a neurologist is at least as good as the agreement of that neurologist with any of the other neurologists, except for Neurologist IV.

\subsubsection{Agreement between an individual and a group}

The agreement of each individual neurologist with the four remaining neurologists is compared with the agreement of the system with the same group of neurologists. The number of neurologists that took the same decision as the individual neurologist or the system is counted for each case. In Table 6.4-III the distribution of the degree of agreement for each of the neurologists and the system with the other four neurologists is shown for the 265 cases.

The system achieved a significantly higher mean score than all the individual neurologists (paired student t-test, $\mathrm{p}<0.05$ )

Table 6.4-1U: Overview of the scores comparing the decision concerning 265 cases of an individual neurologist with the remaining neurologists. The number of neurologists which agree with the decision for treatment of the individual neurologist is represented by the score.

\begin{tabular}{|c|c|c|c|c|c|c|c|c|c|c|c|c|}
\hline \multirow[b]{2}{*}{ Score } & \multicolumn{2}{|c|}{ N III } & \multicolumn{2}{|c|}{ System } & \multicolumn{2}{|c|}{ NIV } & \multicolumn{2}{|c|}{ System } & \multicolumn{2}{|c|}{$\mathrm{NV}$} & \multicolumn{2}{|c|}{ System } \\
\hline & n & $\%$ & $\mathrm{n}$ & $\%$ & 11 & $\%$ & $\mathrm{n}$ & $\%$ & $n$ & $\%$ & $\mathrm{n}$ & $\%$ \\
\hline 0 & 38 & 14.3 & 18 & 6.8 & 52 & 19.6 & 18 & 6.8 & 43 & 16.2 & 23 & 8.7 \\
\hline 1 & 53 & 20.0 & 42 & 15.8 & 66 & 24.9 & 43 & 16.2 & 42 & 15.8 & 53 & 20.0 \\
\hline 2 & 47 & 17.7 & 62 & 23.4 & 41 & 15.5 & 55 & 20.8 & 60 & 22.6 & 64 & 24.2 \\
\hline 3 & 69 & 26.0 & 84 & 31.7 & 48 & 18.1 & 72 & 27.2 & 62 & 23.4 & 63 & 23.8 \\
\hline 4 & 58 & 21.9 & 59 & 22.3 & 58 & 21.9 & 77 & 29.1 & 58 & 21.9 & 62 & 23.4 \\
\hline \multicolumn{5}{|c|}{$t=3.22 p<0.0005$} & \multicolumn{4}{|c|}{$t=5.96 p<0.0005$} & \multicolumn{4}{|c|}{$t=1.94 p<0.05$} \\
\hline
\end{tabular}

\begin{tabular}{lcccccccc}
\hline & \multicolumn{2}{c}{ Neuro VI } & \multicolumn{2}{c}{ System } & \multicolumn{2}{c}{ Ncuro VII } & \multicolumn{2}{c}{ Systen } \\
\hline Score & $\mathrm{n}$ & $\%$ & $\mathrm{n}$ & $\%$ & $\mathrm{n}$ & $\%$ & $\mathrm{n}$ & $\%$ \\
0 & 37 & 14.0 & 22 & 8.3 & 45 & 17.0 & 22 & 8.3 \\
$\mathrm{I}$ & 47 & 17.7 & 40 & 15.1 & 54 & 20.4 & 48 & 18.1 \\
2 & 55 & 20.8 & 67 & 25.3 & 55 & 20.8 & 61 & 23.0 \\
3 & 68 & 25.7 & 76 & 28.7 & 53 & 20.0 & 61 & 23.0 \\
4 & 58 & 21.9 & 60 & 22.6 & 58 & 21.9 & 73 & 27.5 \\
& & $\mathrm{t}=2.37 \mathrm{p}<0.025$ & & & $1=3.85$ & $0<0.0005$ \\
\hline
\end{tabular}

\section{Complete agreement}

Total agreement among the five neurologists occurs in $21.9 \%$ of the cases. The system takes more decisions which agree with any group of 4 neurologists. In $22.3 \%$ to $29.1 \%$ of the cases, groups of four neurologists agree with the system. The system agrees significantly more with the remaining neurologists than Neurologists IV and VII (McNemar $\chi^{2}=12 p<0.05$, McNemar $\chi^{2}=8.52 \mathrm{p}<0.05$, respectively) 


\section{Majority}

A majority decision was reached by the neurologists in 219 of the 265 cases $(82.6 \%)$. For each neurologist the number of cases in which he agrees with two or more of his colleagues is counted. Also for the system the number of cases in which the system reaches a majority decision with each group of the four remaining neurologists is counted. Each individual neurologist participated in majority agreements in $55.5 \%$ to $68.3 \%$ of the cases, as compared to $71.3 \%$ to $77.4 \%$ when the system replaces the neurologist.

The system achieved a majority agreement (system + two neurologists) significantly more often than four of the individual neurologists (McNemar $\chi^{2}=10.84 p<0.05 ; \chi^{2}=30.45 p<0.05$; $\chi^{2=1.26} \mathrm{NS} ; \chi^{2}=5.96 \mathrm{p}<0.05 ; \chi^{2}=10.74 \mathrm{p}<0.05 ; \mathrm{df}=1$, for Neurologists III to VII, respectively).

\section{Complete disagreement}

A neurologist may take a decision which is not made by any of his colleagues. This occurs in $14.0 \%$ to $19.6 \%$ of the cases for the individual neurologists, whereas for the system this percentage varies between $6.8 \%$ to $8.7 \%$ of the cases. The system had significantly fewer cases with which all neurologists disagreed than each of the neurologists (McNemar $\chi^{2}=8.21$ $p<0.05, \chi^{2}=18.78 p<0.05, \chi^{2}=8.21 p<0.05, \chi^{2}=4.78 p<0.05, \chi^{2}=10.76 p<0.05$ for neurologists III to VII, respectively).

Table 6.4-IV: Agreement of a particular ncurologist and the system with the remaining neurologists, expressed as a kappa value.

\begin{tabular}{lllllllllllll}
\hline \multicolumn{10}{c}{ Assessment of individual neurologist and system by } \\
\hline Group & $4-5-6-7$ & \multicolumn{3}{c}{$3-5-6-7$} & \multicolumn{3}{c}{$3-4-6-7$} & \multicolumn{3}{c}{$3-4-5-7$} & \multicolumn{3}{c}{$3-4-5-6$} \\
Prescriber & N III & Syst & N IV & Syst & N V & Syst & N VI & Syst & N VII & Syst \\
Kappa & 0.39 & 0.44 & 0.34 & 0.46 & 0.36 & 0.43 & 0.39 & 0.46 & 0.35 & 0.47 \\
\hline
\end{tabular}

Agreement beyond chance

The agreement between a particular neurologist and the remaining group of four colleagues can also be expressed by a $\kappa$-value (Schouten, 1985). The $\kappa$-values range from 0.34 to 0.39 (Table 6.3-IV). The agreement of the system with the groups of neurologists ranges from 0.43 to 0.47 and is in all cases higher than the kappa of each individual neurologist.

\subsubsection{Correctness}

In the previous paragraphs the advice of the system was compared with the decisions of individual neurologists. This gave an indication about the capability of the system to make decisions similar to those made by individual neurologists. The correctmess of the decisions of the neurologist and of the system has been assessed using both reference sets.

For set I - the reference based on the neurologists alone - the correctness of the system is somewhat higher than the average correctness of the neurologists $(77.4 \%)$. However, three neurologists score better than the system (Table 6.4-V)

Including the system when establishing the reference increased the correctness for all participants. The system provided a correct advice more often than each individual neurolo- 
gist. This was significantly better for three of the five neurologists $\left(\mathrm{McNemar} \chi^{2}=4.5 \mathrm{p}<0.025\right.$; $\chi^{2}=24.85 \mathrm{p}<0.0005 ; \chi^{2}=0.52 \mathrm{NS} ; \chi^{2}=1.84 \mathrm{NS} ; \chi^{2}=9.13 \mathrm{p}<0.005 ; \mathrm{df}=1$, for neurologists III to VII respectively).

Table 6.4-V: Correctness with the reference of set I (i.e. majority of five neurologists) and set II (i.e. majority of five neurologists and the system).

\begin{tabular}{l|lcccccc}
\hline Set & Participants & N III & N IV & N V & N VI & N VI & System \\
\hline Set I, 3/5 neur. & Cases & 174 & 147 & 180 & 181 & 166 & 171 \\
N=219 & Correciness & 79.5 & 67.1 & 82.2 & 82.6 & 75.8 & 78.1 \\
\hline Set II, 4/6 neur.-syst. & Cases & 155 & 128 & 166 & 161 & 148 & 171 \\
N=189 & Correctness & 82.0 & 67.7 & 87.8 & 85.2 & 78.3 & 90.5 \\
\hline
\end{tabular}

\subsubsection{Variability in agreement in set II}

Since the reference treatment procedure is based on a majority, individual participants may disagree with the reference. In this paragraph the degree of agreement of the individual participants with his colleagues is discussed by.

- Comparing the mean of the scores obtained by each individual participant

- Addressing for each participant the disagreement with the reference treatment

- Studying the complete disagreement of each participant with the rest of the participants Furthermore, for each reference treatment the decisions taken by the group of neurologists and the system are presented.

\section{Overall score}

For the reference cases Table 6.4-VI provides a break-down of the number of participants that agree with a specific neurologist or the system. 'Score' indicates the number of participants a neurologist or the system agrees with. A score of 2 cannot be obtained because the reference contains only the agreement profiles $6,5-1,4-2$ and $4-1-1$. The system achieved a significantly higher score than each individual neurologist does (paired T-test $t=10.68 p<0.05, t=19.62$ $\mathrm{p}<0.05, \mathrm{t}=9.79 \mathrm{p}<0.05, \mathrm{t}=10.11 \mathrm{p}<0.05, \mathrm{t}=15.18 \mathrm{p}<0.05)$.

\section{Disagreement with the reference}

As the reference is defined by at least four participants a score less than 3 indicates disagreement of the participant with the reference. This is observed in 18 cases $(9.5 \%)$ for the system up to 61 cases $(32.3 \%)$ for neurologist IV. The agreement of the system with more than two other participants is better than that of three of the individual neurologists. 
Table 6.4-VI: Variability in the degree of agreement between participants for set II $(n=189)$. The item 'Score' indicates the number of participants which agree with the decision taken by the neurologist or the system.

\begin{tabular}{lrrrrrrrrr}
\hline & \multicolumn{3}{c}{ Neurologist III } & \multicolumn{3}{c}{ Neurologist IV } & \multicolumn{3}{c}{ Neurologist V } \\
\hline Score & $\mathrm{n}$ & $\%$ & cum & $\mathrm{n}$ & $\%$ & cum & $\mathrm{n}$ & $\%$ & cum \\
0 & 17 & 9.0 & 9.0 & 36 & 19.1 & 19.1 & 16 & 8.5 & 8.5 \\
1 & 17 & 9.0 & 18.0 & 25 & 13.2 & 32.3 & 7 & 3.7 & 12.2 \\
3 & 41 & 21.7 & 39.7 & 32 & 16.9 & 49.2 & 55 & 29.1 & 41.3 \\
4 & 60 & 31.7 & 71.4 & 42 & 22.2 & 71.4 & 57 & 30.2 & 71.4 \\
5 & 54 & 28.6 & 100 & 54 & 28.6 & 100 & 54 & 28.6 & 100 \\
Total & 189 & 100 & & 189 & 100 & & 189 & 100 & \\
mean & 3.44 & & & $2.9 \%$ & & & 3.55 & & \\
\hline
\end{tabular}

\begin{tabular}{lrrrrrrrrr}
\hline & \multicolumn{3}{c}{ Neurologist VI } & \multicolumn{4}{c}{ Neurologist VII } & \multicolumn{3}{c}{ System } \\
\hline Score & $\mathrm{n}$ & $\%$ & cum & $\mathrm{n}$ & $\%$ & cum & $\mathrm{n}$ & $\%$ & cum \\
0 & 15 & 7.9 & 7.9 & 26 & 13.8 & 13.8 & 9 & 4.8 & 4.8 \\
$\mathrm{l}$ & 13 & 6.9 & 14.8 & 15 & 7.9 & 21.7 & 9 & 4.8 & 9.5 \\
3 & 48 & 25.4 & 40.2 & 48 & 25.4 & 47.1 & 56 & 29.6 & 39.2 \\
4 & 59 & 31.2 & 71.4 & 46 & 24.3 & 71.4 & 61 & 32.3 & 71.4 \\
5 & 54 & 28.6 & 100 & 54 & 28.6 & 100 & 54 & 28.6 & 100 \\
Total & 189 & 100 & & 189 & 100 & & 189 & 100 & \\
mean & 3.51 & & & 3.24 & & & 3.65 & & \\
\hline
\end{tabular}

\section{('omplete disagreement with the other participants}

The number of cases for which a neurologist disagreed with all other participants (Score 0) ranges from $15(7.9 \%)$ to 36 cases $(19.1 \%)$. In nine cases $(4.8 \%)$ the system proposes a treatment different from any proposal of the neurologists

Table 6.4-VII also gives an overview of the agreement profiles for those cases where the individual participant disagreed completely with the rest of the group. The percentage of cases in which a participant disagreed while all other participants agreed about the treatment decision ranged from $2.7 \%$ to $12.2 \%$ of the cases (three neurologists caused a $5-1$ agreement profile in less than $5 \%$, two neurologists in more than $10 \%$ of the cases). In four cases (2.1\%) the system did not agree with the treatment procedure although all five neurologists did agree completely. Each neurologist as well as the system disagreed completely with the five other participants of which a group of four agreed among themselves (agreement profile 4-1-1) in less than $10 \%$ of the cases 
Table 6.4-VII: Distribution of cases for which the individual participant disagreed completely with the other five participants in cases from set II.

\begin{tabular}{lrrrrrrrrrrrr}
\hline Participant & \multicolumn{2}{c}{$\mathrm{N} \mathrm{WI}$} & \multicolumn{2}{c}{$\mathrm{N} \mathrm{IV}$} & \multicolumn{2}{c}{$\mathrm{N} \mathrm{V}$} & \multicolumn{2}{c}{$\mathrm{N} \mathrm{VI}$} & \multicolumn{2}{c}{$\mathrm{N} \mathrm{VM}$} & \multicolumn{2}{c}{ System } \\
\hline Agreement profile & $\mathrm{n}$ & $\%$ & $\mathrm{n}$ & $\%$ & $\mathrm{n}$ & $\%$ & $\mathrm{n}$ & $\%$ & $\mathrm{n}$ & $\%$ & $\mathrm{n}$ & $\%$ \\
$5-1$ & 5 & 2.7 & 23 & 12.2 & 8 & 4.2 & 6 & 3.2 & 19 & 10.1 & 4 & 2.1 \\
$4-1-1$ & 12 & 6.4 & 13 & 6.9 & 8 & 4.2 & 9 & 4.8 & 7 & 3.7 & 5 & 2.7 \\
Total & 17 & 9.0 & 36 & 19.0 & 16 & 8.5 & 15 & 7.9 & 26 & 13.8 & 9 & 4.8 \\
\hline
\end{tabular}

\section{Difference in treatment decisions}

Four different reference treatments were suggested in the 189 cases. In 10 cases (5.3\%) the dose is decreased, in 38 cases $(20.1 \%)$ the prescribed treatment is continued without any changes, in 41 cases $(21.7 \%)$ the dose is increased and in 100 cases (52.9\%) another drug was selected. The decisions of all neurologists and the system were compared with the reference decision (Table 6.4-VIII). The numbers in the cells on the diagonal (shaded grey) show the agreement of the neurologists and the system with the reference decision. In each cell the following information is provided: the number of decisions, the percentage of these decisions related to the total number of decisions taken ( 945 for the neurologists, 189 for the system) and the percentage of the decisions related to the number of cases in the reference treatment.

Table 6.4-VIn: Overview of all 945 ( $5 \times 189$ ) decisions of the neurologists and the system on the 189 reference cases. Each cell contains information on the number of times a decision is made, as well as the percentage of the total number of decisions and the percentage of the decisions taken (in)correctly. For each cell in the column 'Total' the first row presents the number of times a specific reference decision is made. The second row presents for a specific reference decision the percentage of the total number of decisions (945). The third row presents for the neurologists and the system the percentage of decisions according to the reference.

\begin{tabular}{|c|c|c|c|c|c|c|c|c|c|c|c|c|}
\hline \multirow{3}{*}{$\begin{array}{l}\mathrm{N}=945 \\
\text { Decision } \\
\text { Reference }\end{array}$} & \multicolumn{12}{|c|}{ Participants } \\
\hline & \multicolumn{2}{|c|}{ decrease } & \multicolumn{2}{|c|}{ equal } & \multicolumn{2}{|c|}{ increase } & \multicolumn{2}{|c|}{ change } & \multicolumn{2}{|c|}{ rest } & \multicolumn{2}{|c|}{ Total } \\
\hline & N3-7 & $\mathrm{s}$ & N3-7 & $s$ & N3-7 & $\mathrm{s}$ & N3-7 & $\mathrm{s}$ & N3-7 & $\mathrm{s}$ & N3.7 & $\mathrm{S}$ \\
\hline decrease & 40 & 9 & 5 & 1 & 0 & 0 & 5 & 0 & 0 & 0 & 50 & 10 \\
\hline$\%$ total & 4.2 & 4.8 & 0.5 & 0.5 & 0 & 0 & 0.5 & 0 & 0 & 0 & $s$ & \\
\hline$\%$ (dis)agree & 80.0 & 90.0 & 10.0 & 10.0 & 0 & 0 & 10.0 & 0 & 0 & 0 & 80.0 & 90.0 \\
\hline equal & 4 & 0 & 161 & 31 & 12 & 6 & 11 & 1 & 2 & 0 & 190 & 38 \\
\hline$\%$ total & 0.4 & 0 & 17.0 & 16.4 & 1.3 & 3.2 & 12 & 0.5 & 0.2 & 6 & 20 & \\
\hline$\%$ (dis)agree & 2.1 & 0 & 84.7 & 81.6 & 6.3 & 15.8 & 58 & 2.6 & 1.1 & 0 & 84.7 & 81.6 \\
\hline increase & 0 & 0 & 31 & 0 & 162 & 37 & 8 & 4 & 4 & 0 & 205 & 41 \\
\hline$\%$ total & 0 & 0 & 3.3 & 0 & 17.1 & 19.6 & 0.9 & 2.1 & 0.4 & 0 & 21 & \\
\hline$\%$ (dis)agree & 0 & 0 & 15.1 & 0 & 79.0 & 90.2 & 3.9 & 9.8 & 2.0 & 0 & 79.0 & 90.2 \\
\hline change & 33 & 0 & 44 & 2 & 18 & 2 & 395 & 94 & 10 & 2 & 500 & 100 \\
\hline$\%$ total & 3.5 & 0 & 4.7 & 1.1 & 1.9 & 1.1 & 41.8 & 49.7 & 1.1 & 1.1 & 52 & \\
\hline$\%$ (dis)agree & 6.6 & 0 & 8.8 & 2 & 3.6 & 2.0 & 79.0 & 94.11 & 2.0 & 20 & 79.0 & 94.0 \\
\hline
\end{tabular}


In cases in which the reference treatment was to decrease the drug dose the system made more correct decisions than the neurologists. However, the number of cases in this group is rather small.

The neurologists and the system do not differ much with respect to their correctness for cases in which the reference treatment decision is to continue the existing treatment (equal). For cases with this reference decision almost half $(48.3 \%)$ of the decisions different from the reference were taken by one particular neurologist. In $6(15.8 \%)$ of the 38 cases in which the reference decision was to continue the treatment the system suggested to increase the dose.

The system also made more correct decisions than the neurologists for cases where the reference indicated to increase the drug dose. Of the incorrect decisions proposed by the neurologists $20(46.5 \%)$ were taken by one neurologist. The system decided to change the prescribed drug in $4(9.8 \%)$ of the 41 cases.

The largest difference $(15 \%)$ in correct decisions between the system and the neurologists is found for the reference 'Change'. Again almost half (47.6\%) of the incorrect decisions could be attributed to one particular neurologist.

\subsection{Decision criteria}

It may be expected that characteristics of a case are important for reaching a decision. For each of the main characteristics of a case it was determined whether it could predict the reference treatment. Set I was used for this purpose.

\subsubsection{Treatments indicated by a single attribute}

Only in cases in which idiosyncratic complaints occur there is an almost unanimous decision to change the drug treatment. In only one of the 59 cases the reference treatment was to keep the treatment unchanged. In this case seizures were completely controlled, there existed a good compliance and a high therapeutic plasma drug level was observed (Table 6.5-I)

Table 6.5-I: Breakdown of the reference treatment decisions as a function of the presence of complaints.

\begin{tabular}{lcccccc}
\hline Complaints & \multicolumn{2}{l}{ Absent } & \multicolumn{2}{c}{ Dose relaled } & \multicolumn{2}{l}{ Idiosyncratic } \\
\hline Reftreatment & $\mathrm{N}$ & $\%$ & $\mathrm{~N}$ & $\%$ & $\mathrm{~N}$ & $\%$ \\
decrease & & & 14 & 19.4 & & \\
equal & 34 & 38.6 & 16 & 22.2 & 1 & 1.7 \\
increase & 43 & 48.9 & 1 & 1.4 & & \\
chunge & 10 & 11.4 & 41 & 56.9 & 58 & 98.3 \\
extra & 1 & 1.1 & & & & \\
\hline
\end{tabular}

The system's advice was to change to another drug in 57 of the 59 cases. In the other two cases the system suggested to stop the treatment immediately. In both cases all five neurologists agreed that the drug should be changed and immediate discontinuation of the drug was not deemed necessary. 
As the decision to change the drug when idiosyncratic complaints are present may obscure the possible relation between other case characteristics and treatment decisions, we further analysed the 160 cases without idiosyncratic complaints. In these cases, however, no single characteristic could be identified that leads to a specific treatment decision.

\subsubsection{Treatments ruled out by a single attribute}

Although an attribute may not indicate a specific treatment decision, it may rule out a particular treatment decision. For the 160 reference cases without idiosyncratic complaints, the absence of treatment decisions in the reference is described.

\section{Seizure frequency}

Even when seizures are controlled, one could consider to increase the dose when the plasma drug level is below the therapeutic range. However, when considering cases with completely controlled seizures this decision is not made. Also the decision to change the drug due to e.g. dose related complaints is not made in these cases (Table 6.5-II).

In those cases in which seizures were either almost completely or hardly controlled the dose hardly ever was decreased despite the fact that in more than two thirds of these cases a complaint was present.

The decision to decrease the drug dose is never made when the seizure frequency remains uncontrolled. The presence of complaints in these cases does not lead to a reduction of the dose but mainly to a change of the drug.

The system did not suggest to change or increase the dose in cases in which seizures were completely controlled. For cases in which seizures were still present the system did never decide to decrease the dose of the drug. In the three cases in which the reference treatment was to decrease the drug dose, the system proposed to change to another drug.

Table 6.5-II: Distribution of reference treatments when related to seizure frequency in 160 cases without idiosyncratic complaints.

\begin{tabular}{lcccccccc}
\hline Seizures & \multicolumn{2}{c}{ controlled } & \multicolumn{2}{c}{ almost contr } & \multicolumn{2}{c}{ hardly contr } & \multicolumn{2}{c}{ uncontrolled } \\
\hline Ref treatment & $\mathrm{N}$ & $\%$ & $\mathrm{~N}$ & $\%$ & $\mathrm{~N}$ & $\%$ & $\mathrm{~N}$ & $\%$ \\
decrease & 11 & 36.7 & 2 & 9.5 & 1 & 1.5 & & \\
equal & 19 & 63.3 & 12 & 57.1 & 15 & 22.4 & 4 & 9.5 \\
increase & & 4 & 19.0 & 26 & 38.8 & 14 & 33.3 \\
change & & 3 & 14.3 & 25 & 37.3 & 23 & 54.8 \\
extra & & & & & & 1 & 2.4 \\
\hline
\end{tabular}

\section{Complaints}

When complaints are absent, the decision to decrease the drug dose is never taken by the neurologists or the system.

In only one case with a dose related complaint the neurologists decided to increase the drug dose. The decision profile for this exceptional case was 3-2; two of the neurologists as well as the system decide to change the drug. In none of the cases with a dose related complaint the system proposed to increase the dose. 


\section{Plasma drug level}

For each plasma drug level the four major reference treatments were present. The system applied all but one of the decisions for each of the plasma drug levels. For cases in which the plasma drug level was in the sub therapeutic range the system never decreased the drug dose.

Table 6.5-III: Distribution of the reference treatment decisions when related to the range of the plasma drug level.

\begin{tabular}{lrrrrrrrrrr}
\hline Plasma drug level & \multicolumn{1}{c}{ sub ther. } & \multicolumn{2}{c}{ low ther. } & \multicolumn{2}{c}{ therapeutic } & \multicolumn{2}{c}{ high ther. } & \multicolumn{2}{c}{ toxic } \\
\hline Ref.treatment & $\mathrm{N}$ & $\%$. & $\mathrm{N}$ & $\%$. & $\mathrm{N}$ & $\%$ & $\mathrm{~N}$ & $\%$. & $\mathrm{N}$ & $\%$. \\
decrease & 2 & 5.9 & 1 & 3.2 & 1 & 2.6 & 5 & 16.7 & 5 & 18.5 \\
equal & 10 & 29.4 & 9 & 29.0 & 17 & 44.7 & 8 & 26.7 & 6 & 22.2 \\
increase & 14 & 41.1 & 13 & 41.9 & 13 & 34.2 & 3 & 10.0 & 1 & 3.7 \\
change & 8 & 23.5 & 8 & 25.8 & 7 & 18.4 & 13 & 43.3 & 15 & 55.6 \\
extra & & & & & & & 1 & 3.3 & & \\
\hline
\end{tabular}

\subsection{Treatments indicated by combinations of attributes}

To identify combinations of characteristics which predict the reference treatment an induction algorithm was used to analyse the 219 cases in set 1 . The algorithm iteratively partitions the high-dimensional feature space such that in principle each end-node of the resulting classification tree groups together (almost) only cases with one type of reference treatment. The algorithm has as additional stop criterion that a split has to provide sufficient information. If that is not the case, an impure node is not further splitted (Talmon, 1986).

When we assign to each end-node the treatment decision of the majority of cases in that node the tree has a correctness of $85.4 \%$ on set 1 . In total 14 different end-nodes were created (see figure on next page). In this paragraph first the 'pure' end-nodes, containing cases with the same reference treatment, are discussed. Next the mixed end-nodes, with two different treatments are reviewed.

For the cases in each end-node the treatments suggested by the neurologists and by the system are presented. To assure that there is sufficient support to decide that a combination of case characteristics leads to a specific treatment decision the set of cases for which no majority treatment decision exists was classified according to the criteria described in the decision tree. The assigned treatments were compared with the suggested treatments for these cases. Support for a single treatment decision was considered strong for nodes in which:

- The number of cases from a reference group was relatively large $(\mathrm{N} \geq 5)$

- The number of cases with a treatment other than the main treatment among the cases at an end node is relatively small $(<20 \%)$ and

- The non-reference group that fulfilled the criteria was relatively small when compared with the number of cases from the reference group ( $<20 \%$ of the cases)

Finally the nodes with the largest variation in decisions are discussed briefly. For these nodes little can be concluded about the use of a single treatment given the occurrence of three decisions. 


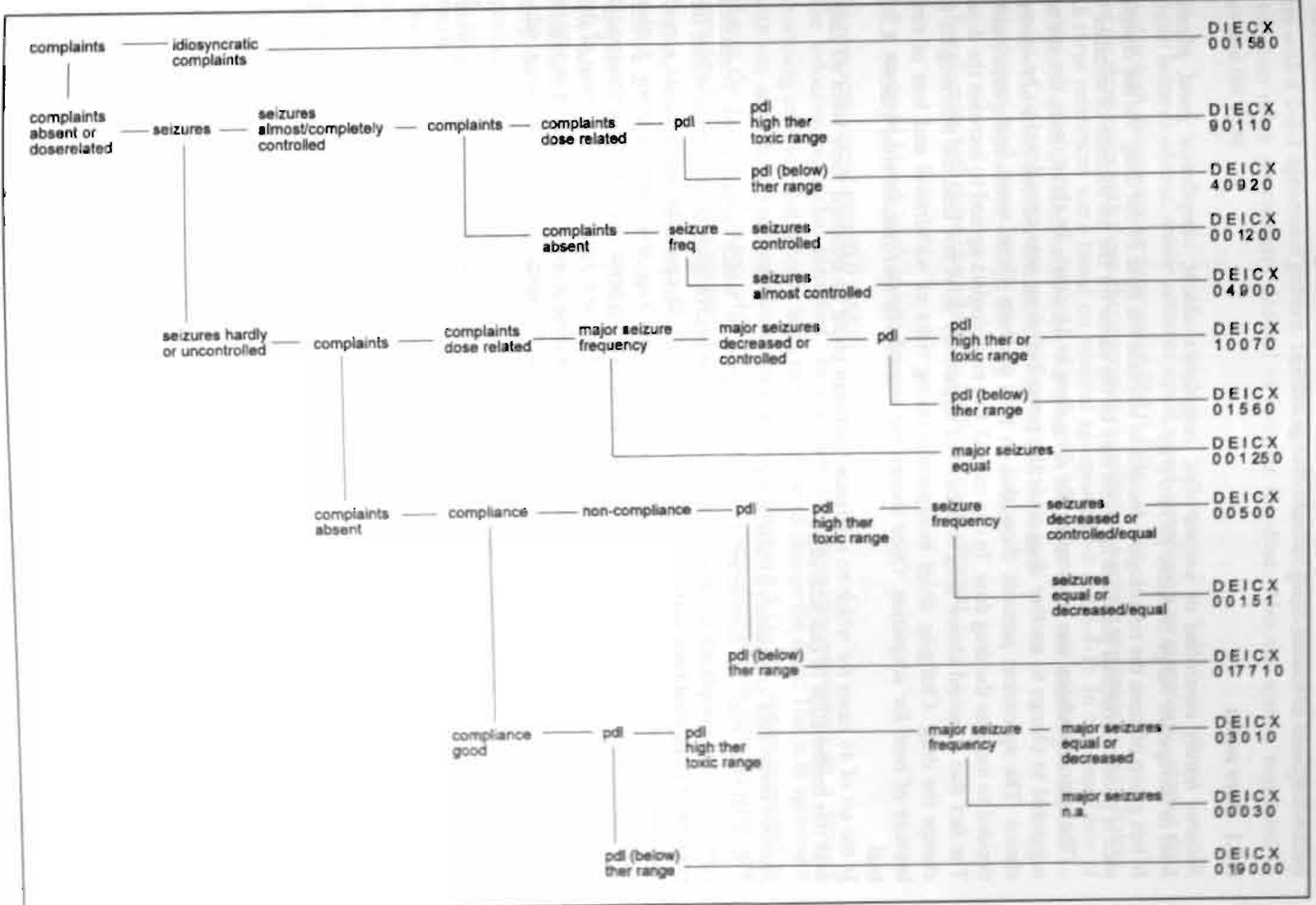


In the following each node is represented by a vector indicating the number of cases with a certain treatment (decrease equal increase change extra).

\subsubsection{Pure nodes}

Seizures hardly controlled or uncontrolled, complaints absent, compliance good, plasma level in therapeutic range or below $(019000)^{1}$

In this group of cases the neurologists decided to increase the dose of the drug. As the compliance is good, suboptimal plasma drug levels may be the cause for the persistence of seizures.

The system's advice was to increase the drug dose in 16 cases. In three cases the system suggested to change to another drug since the prescribed drug was considered to be second choice. The agreement patterns show that in two of these three cases four neurologists decided to increase the drug dose. In one case all five neurologists agreed to increase the dose. The fact that a second choice drug is prescribed apparently does not lead the neurologists to change the drug. Changing drugs incorporates taking risks of withdrawal and loss of time because of need for adaptation. Once chosen the second choice drug should be given a full trial.

In the set of 46 cases for which no reference treatment could be obtained there were no cases with this combination of characteristics.

\section{Seizures controlled, complaints absemt $\left(\begin{array}{lllll}0 & 0 & 12 & 0 & 0\end{array}\right)$}

The treatment may be considered successful in cases in which seizures are controlled completely and complaints are absent. In these cases the neurologists decided to continue the prescribed drug treatment without further changes. In all 12 cases the neurologists agreed completely. The system also decided to continue the drug treatment without further adjustments in all cases. In five of these cases the plasma drug level was below the therapeutic range, while in two cases the plasma drug level was in the toxic range. An adjustment of the drug dose was not suggested. This indicates that plasma drug levels are not applied rigidly to modify the treatment when it is effective in controlling the seizures without inducing sideeffects.

Among the 46 cases without a reference no cases with the same characteristics were observed.

\section{Seizures hardly controlled, non-compliance, plasma drug level high therapeutic or toxic

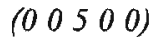

In cases in which seizures were hardly controlled, with non-compliance and a plasma drug level in the high therapeutic or toxic range, the neurologists decided to continue the prescribed treatment. In these five cases the system also decided to continue the prescribed drug treatment.

Non-compliance may explain the persistence of seizures. In these cases it is preferred to improve the compliance as this may lead to improved seizure control.

\footnotetext{
'Decisions taken by the neurologists (Decrease Equal increase Change Extra) are presented in parentheses (D E I C X). In

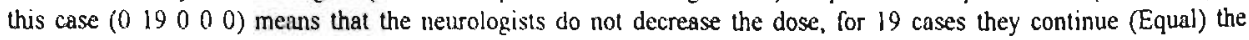
treatment without change, they do not increase, change or add an additional drug.
} 
In the set of cases without a reference treatment the same characteristics were found in one case with a 2-2-1 agreement pattern. In this case two neurologists decided to change the drug while two other neurologists decided to increase the dose. Also the system suggested to increase the dose.

Major seizures absent, minor seizures equal, goad compliance, no complaints, plasma drug level in high therapeutic or toxic range $\left(\begin{array}{lllll}0 & 0 & 0 & 3 & 0\end{array}\right)$

For the three cases with these characteristics the reference suggests to change to another drug. All three references were based on minimal agreement (3-2 and 3-1-1). In two cases two of the neurologists decided to increase the dose. In the other case one neurologist decided to increase the dose, another continued the prescribed treatment.

The system proposed to change the drug in all three cases.

For all cases with these characteristics a reference was obtained

\subsubsection{Nearly pure nodes; two decisions}

\section{Idiosyncratic complaints (0 0 I 580$)$}

The induction algorithm identified the presence of idiosyncratic complaints as a kind of overruling criterion that directly leads to the decision to change the drug. This is in agreement with our analysis of the single case characteristics (section 6.5.1). The system suggested to change the drug in 57 cases, while in two cases the system proposed to stop the treatment immediately as the complaints were very severe.

In the 46 cases of the non reference set, three cases had idiosyncratic complaints. In all three cases a 2-2-1 agreement pattern was present. In two of these cases in which seizures were completely controlled, compliance was good and the plasma drug level was in the sub- and low-therapeutic range two of the neurologists decided to change the drug. An alternative treatment for these cases was proposed by two other neurologists. In one case they decided to stop the prescribed drug without replacement, and in the other case they continued the prescribed drug without adjustments.

In the third case seizures decreased, compliance was good and the plasma drug level was low therapeutic. For this case only one of the neurologists decided to change the prescribed drug. One pair of neurologists decided to increase the drug dose. The other pair continued the treatment without adjustments.

In all three cases the system suggested to change to another drug.

\section{Major seizures equal or minor seizures equal or decreased, and dose related complaints $(001250)$}

In 25 of 26 cases a change of the drug was decided upon. In one case a continuation of the prescribed treatment was the reference. For this case there was a 3-2 agreement profile, in which the two remaining neurologists decided to change the drug

The system used two different approaches for these cases. In 21 cases the system also decided to change the drug, while in 5 cases a continuation of the prescribed treatment was suggested. In all five cases dose related complaints occurred with a plasma drug level below or in the therapeutic range. In two of these cases in which the seizure frequency did not change four of the neurologists decided to change the drug, while the fith chose to continue the 
treatment. In the three remaining cases in which the frequency of minor seizures decreased, a 3-2 decision profile was found, in which continuation of the prescribed treatment was the alternative used by two of the neurologists.

In the set of 46 cases without a reference five cases were found with the same characteristics all of which had a plasma drug level in or above the therapeutic range. In four of the cases (three times 2-2-1 and once a 2-1-1-1 agreement profile) two neurologists agreed to change the drug. In two of these cases two neurologists decided to continue the treatment, while in one case two neurologists decided to decrease the dose. In the remaining case (2-1-1-1) two neurologists decided to continue the prescription.

The system proposed to change the drug in four cases while in one case with a low plasma drug level a continuation of the treatment was suggested, which was also decided upon by two of the neurologists.

While the presence of incompletely or uncontrolled seizures and dose related complaints seems to lead the neurologists towards a change of the drug, the presence of low therapeutic plasma drug levels modifies the decision of the system towards continuation of the treatment.

Major seizures decreased and minor seizures decreased, equal, or absent, or major seizures controlled and minor seizures equal, dose related complaints and plasma level in the high therapeutic or toxic range (1 0070$)$

This node contains eight cases, in seven of which the reference was to change the drug.

A 3-2 decision profile was found in 6 of the cases for which change was the reference. In four of these cases a decrease of the dose was proposed, in one case the dose was decreased and an additional drug was added, in one case a continuation was proposed by the two other neurologists. In one case with seizures hardly controlled, good compliance, dose related complaints and a plasma drug level in the toxic range, a 4-1 agreement profile was observed. In one case with both seizure types decreased, a good compliance and a plasma drug level in the high therapeutic range, three neurologists decided to decrease the dose, while two changed to another drug.

In all 8 cases the system decided to change the drug.

In the set of cases without a reference two cases were found with the same characteristics In both cases two neurologists decided to change the drug while two other neurologists decided to decrease the dose and one neurologist decided to continue the treatment.

In both cases the system decided to change the drug.

Despite some success in controlling the seizures, the presence of dose related complaints and high or toxic range plasma drug levels mainly causes the neurologists to adjust the treatment by changing the drug. As a decrease could lead to further continuation of seizures, the system also suggests to change the drug.

Major seizures equal or major and minor seizures decreased, good compliance, no complaints and plasma drug level in the high therapeutic or toxic range $\left(\begin{array}{lllll}0 & 3 & 0 & 1 & 0\end{array}\right)$

Four cases were found with these characteristics in the set of 219 cases with a reference. In three of these cases the reference was to increase the drug dose (agreement profile 3-1-1 or 41) In one case, with major and minor seizures which remained equal a change of the drug was decided upon by three neurologists, the other two neurologists increased the dose 
In two cases the system decided to increase the dose, while in two cases a change was proposed.

In the 46 cases without a reference, four cases were observed with the same characteristics. In all four cases a 2-2-1 agreement profile was present. In total three different treatment decisions were taken by a pair of neurologists i.e. increase, change or equal.

The system decided to increase the dose in three cases in which seizures were hardly controlled. A change was proposed in a case in which the seizures did not change and the plasma drug level was in the toxic range.

The number of cases in this node is small and an equal number of cases was found in the non reference set. Little more can be concluded than that for these characteristics the neurologists mainly try to achieve complete seizure control by either increasing the dose or changing to another drug.

Seizures almost controlled, complaints absent $(04900)$

A group of 13 cases was identified in which either an increase in the dose or a continuation of the treatment was agreed upon. In 6 cases major seizures were controlled. In five of these the neurologists decided to continue the treatment, although the minor seizures were still incompletely controlled. In one case with a sub therapeutic plasma drug level, the dose was increased. While for cases in which major seizures are controlled the neurologists tend to continue the treatment, for minor seizures the treatment decision is less clear.

The system proposed in all 8 cases with a good compliance to increase the dose. In cases with non-compliance either an increase of the dose -three cases with a (below) therapeutic plasma drug level- or a continuation of the prescribed treatment -two cases with a high therapeutic plasma drug level- was suggested.

In the set of cases without a reference treatment one case was found with the same characteristics. For this case two neurologists agreed about the treatment to continue the treatment. The system also proposed to continue the treatment without adjustments as noncompliance and a toxic range plasma drug level were present.

The system increases the dose if seizures are not completely controlled unless noncompliance and high therapeutic or toxic plasma drug levels are present. The neurologists mainly tend to continue the prescription although seizures are not completely controlled and compliance is good.

\subsubsection{Mixed nodes; three decisions}

In five nodes covering 70 cases three different reference treatment decisions were observed. The system suggested the same treatment as the reference in 45 cases (64\%). In nine cases the system agreed with two neurologists who did not agree with the reference but agreed among themselves on a different treatment (Table 6.6-I). 
Table 6.6-I: Overview of the reference decisions and the advice of the system concerning the 70 cases in the 5 nodes in which three different decisions were observed.

\begin{tabular}{l|lccccc}
\hline Treatment & \multicolumn{6}{|c}{ Reference } \\
\hline \multirow{3}{*}{ System } & Decisions & Decreasc & Equal & Increase & Change & eXtra \\
& Decrease & 9 & 2 & & & \\
& Equal & 2 & 12 & 1 & 4 & \\
& Increase & & 6 & 16 & 3 & 1 \\
& Change & 2 & 3 & 1 & 8 & \\
\hline
\end{tabular}

In two of the five nodes the correctness of the system is more than $70 \%$, in one node more than $60 \%$ was reached, while twice the system achieved a correctness of less than $60 \%$. In all nodes except node $\left(\begin{array}{lllll}9 & 0 & l & 1 & 0\end{array}\right)$ more than $50 \%$ of the reference treatments is based on a minimal agreement pattern (3-2 or 3-1-1). In (901llo) this was $27 \%$.

Only for node $\left(\begin{array}{lllll}9 & 0 & 1 & 1 & 0\end{array}\right)$ one can speak of an indication for a reference treatment (Table 6.6-II). In this node with seizures almost or completely controlled, dose related complaints and a plasma drug level in the high therapeutic or toxic range, 3 cases have a minimal agreement pattern (3-1-1 or 3-2). For 7 of the 9 cases in which both seizure types are completely controlled and compliance is good the system as well as the neurologists decided to decrease the drug dose. No cases with these characteristics were observed in the non-reference group. In the case in which both the neurologists and the system decided to change to another drug, the seizures were almost controlled and a high therapeutic plasma drug level was observed. Four neurologists agreed on this treatment.

Table 6.6-II: Distribution of the reference decision and the treatment decision suggested by the system for node $\left(\begin{array}{lllll}9 & 0 & 1 & 1 & 0\end{array}\right)$

\begin{tabular}{l|lccc}
\hline Treatment & \multicolumn{4}{|c}{ Reference } \\
\hline \multirow{3}{*}{ System } & Decisions & Decrease & Equal & Change \\
& Decrease & 7 & 1 & \\
& Equal & 1 & & \\
& Change & 1 & & 1 \\
\hline
\end{tabular}

The non-reference group of this node contained five cases ( $31 \%$ of the total set) with the same characteristics. All of these cases had one type of seizures controlled while the other decreased

In node ( $\left.\begin{array}{lllll}4 & 0 & 9 & 2 & 0\end{array}\right)$ with cases with seizures almost or completely controlled, dose related complaints and a plasma drug level in or below the therapeutic range, the neurologists as well as the system mainly decide to continue the treatment. The dose was decreased in cases in which compliance was good and complaints were severe. In case of severe complaints and non-compliance a change of the drug was suggested. One single approach for treatment for 
these cases is not indicated. The system and the neurologists agree in $73 \%$ of the cases covering three different decisions.

Table 6.6-III: Decisions of the neurologists and the system for node $(40920)$.

\begin{tabular}{l|lccc}
\hline Treatment & \multicolumn{4}{|c}{ Reference } \\
\hline \multirow{3}{*}{ System } & Decisions & Decrease & Equal & Change \\
& Decrease & 2 & 1 & \\
& Equal & 1 & 8 & 1 \\
& Change & 1 & & 1 \\
\hline
\end{tabular}

Differences in treatment between the system and the neurologists were observed for three nodes $\left(\begin{array}{llll}0 & 17 & 7 & 1\end{array}\right),\left(\begin{array}{lllll}0 & 0 & 5 & 1\end{array}\right)$ and $(01560)$

In node $\left(\begin{array}{llll}0 & 17 & 7 & 1\end{array}\right)$, the neurologists used one treatment decision in $68 \%$ of the cases in which seizures are hardly or uncontrolled, complaints are absent, non-compliance and a plasma drug level in or below the therapeutic range (Table 6.6-IV). The neurologists decided mainly to increase the dose, while in 7 cases the treatment is continued without further adjustments. In $68 \%$ of the cases agreement between the system and the reference was observed. The system adheres to a larger extent to the decision to increase the dose for these cases ( $92 \%$ ).

Table 6.6-IV: Decisions of the neurologists and the system for node $\left(\begin{array}{llllll}0 & 17 & 7 & 1 & 0\end{array}\right)$.

\begin{tabular}{l|lccc}
\hline Treatment & \multicolumn{4}{|c}{ Reference } \\
& Decisions & Equal & Increase & Change \\
System & Equal & 1 & 1 & \\
& Increase & 6 & 16 & 1 \\
\hline
\end{tabular}

In node $\left(\begin{array}{lllll}0 & 0 & 1 & 5 & 1\end{array}\right)$ in which seizures are hardly controlled, non-compliance is present and a plasma drug level in the high therapeutic or toxic range is observed the main reference treatment is a change of the drug. In one case the dose was kept equal, while in another case a second anti-epileptic drug was added. The system did not suggest a specific treatment for the cases at this node. In three cases the system decided to continue the prescription, in three other cases an increase of the dose was proposed. In one case a change of the drug was suggested. Two of these decisions coincide with the reference. 
Table 6.6-V: Overview of the decisions taken by the neurologists and the system on node ( 01560 )

\begin{tabular}{l|lccc}
\hline \multirow{2}{*}{ Treatment } & \multicolumn{4}{c}{ Reference } \\
\hline \multirow{3}{*}{ System } & Decisions & Equal & Increase & Change \\
& Equal & 2 & 1 & 1 \\
& Change & 3 & 5 \\
\hline
\end{tabular}

In node ( $\left.\begin{array}{lllll}0 & 1 & 5 & 6 & 0\end{array}\right)$ in which seizures are hardly controlled, dose related complaints are present and the plasma drug level was in or below the therapeutic range all reference treatments were based on minimal agreement (3-2 and 3-1-1).

The single decision to increase the dose was taken by three of the neurologists, while the other two proposed to change the drug. The system suggests only two different treatments of which nine $(75 \%)$ are a change of the drug. The neurologists however use a continuation of the treatment almost as often as a change of the treatment (Table 6.6-V).

\subsection{References}

Beghi E., Tognoni G., Prognosis of epilepsy in newly referred patients: A multicentre prospective study, Epilepsia, 29, 1988, pp. 236-243.

Collaborative Group for Study of Epilepsy, Prognosis of epilepsy in newly referred patients: a multicintre prospective study of the effects of monotherapy on the long-term course of epilepsy, Epilepvia, 33, 1992, pp. 45-51.

Cramer J.A., A clinimetric approach to assessing quality of life in epilepsy, Epilepsia, 34, 1993, (Suppl.), 4, s8-s13.

Donselaar C. van, Idioparhic first seizure in adult life, thesis, Rotterdam, 1990.

Duncan J.S., Sander J.W.A.S., The Chalfont seizure severity scale. Journal of Neurology, Neurosurgery and Psychiatry, 54, 1991, pp. 873-876.

Elwes R.D., Johnson C.B., Shorvon S.D., Reynolds E.H., The prognosis for seizure control in newly diagnosed epilepsy, The New England Journal of Medicine, 40, 1984, pp. 944-947.

Elwes R.D.C., Reynolds E.H., The early prognosis of epilepsy. In: Comprehensive Epileptology, (Dam and Gram, Eds.), Ravens Press, New York, 1990, pp. 715-727.

Landis J.R., Koch G.G., The measurement of ubserver agreement for categorical data, Biometrics, 1977,33 , pp. 159-174

Schouten H.J.A., Statistical measurement of inlerobserver agreement, analysis of agreements and disagreements between observers, thesis, Rotterdam, 1985.

Talmon J.L., A multiclass nonparametric partitioning algorithm, Pattern Recognition Letters, 1986, 4, pp. 31-38. 


\section{Conclusions and discussion}

\subsection{Introduction}

In this thesis the development and assessment of a computerised model of expert knowledge for prescribing anti-epileptic drugs was described.

The research question investigated was:

- Is it possible to develop a decision support system that provides adequate advice concerning anti-epileptic drug therapy?

Both the initiation of anti-epileptic drug treatment and the adjustment of the treatment during a first follow-up contact were studied.

\subsection{System development}

A structured approach for system development, the KADS methodology, was used At the time we used KADS, its orientation was mainly towards the analysis of the cognitive process. In the more recent literature on KADS, this orientation shifted towards a more pragmatic approach regarding the implementation of models (Schreiber et al. 1993, Tansley and Hayball 1993).

In the early versions of KADS a waterfall-based model for system development was used. Limited attention was paid to project management in these versions. Later, a spiral life-cycle model was introduced for project management (Hickman et al. 1989)

KADS provides little support with respect to the evaluation of the quality of the developed models. The little work on metrication that has taken place was mainly directed towards project management (Readdie et al. 1989). It is the philosophy of KADS that because of the extensive analysis of knowledge there is a better chance that the right implementation is made. However, we recognised that also implementation of the (partial) model of expertise could be of value to support elicitation and analysis. In this context it would be appropriate if KADS would include a reference to a recommended evaluation methodology.

Several problems were encountered with the application of KADS. The use of interpretation models appeared to be difficult. This could party be attributed to differences in granularity 
between models. Furthermore, the model library was incomplete, requiring the design of additional interpretation models. Although development of interpretation models is supported by a conceptual modelling language, this language only provides a tentative list of metaclasses and knowledge sources. Moreover, due to the high level of abstraction of the models they need to be translated before they can be presented to a domain expert.

The goal of our study was the development of a computerised model for prescribing antiepileptic drug treatment. The expertise for such a knowledge-based system may be obtained from a single expert or multiple experts. In our case we decided to use only one expert because of the availability of the extensive literature. It was expected that one neurologist was enough to disambiguate any problems found in the literature and to offer additional information. The plan was to assess and - if necessary to adapt - the developed models using experts that are successively less involved in the development of the system and less aware of the working procedures of the expert who provided the knowledge. In retrospect, we expect that involvement of multiple experts during the development could have reduced some of the evaluation efforts and would have made us aware of some of the observed problems earlier in the development process

The developed models for the initiation and monitoring of anti-epileptic drug treatment were implemented in an experimental system using muLISP ${ }^{\mathrm{rm}}$. The system has a layered structure separating the static parts of the knowledge from the procedural parts. A frame and rule based structure was used. Rules related to a specific section of the problem solving process were combined in rule sets which can be used when a knowledge source is activated. In the initiation part as well as in the monitoring part a forward-chaining mechanism was used for reasoning. Most of the rule sets belong to the assessment task of the monitoring part of drug treatment. The use of the frame and rule structures preserved the structure of the knowledge in a way recognisable to the expert. Therefore, a static assessment was possible.

\subsection{Reference development procedure}

Detailed guidelines for anti-epileptic drug treatment of individual patients are not available. Therefore a reference had to be developed to assess the system's advice. This reference was also used to obtain insight in the variability of the treatment decisions of neurologists. Paper cases based on real patient data were designed and used for the determination of the reference. The ultimate goal was to have a set of cases with for each case an agreed upon diagnosis and an agreed upon prescription. The majority decision among three neurologists - if it existed was taken as reference.

A Delphi-like approach was used to reduce the variability and remove erroneous treatment suggestions of the neurologists. The use of statistical feedback significantly increased the number of cases in which a majority decision on the drug was reached.

Due to the variation in the prescribed daily dose among the neurologists we applied a range of acceptable daily doses to reduce the outliers. Feedback was less successful in this situation because part of the outliers could be attributed to rounding off dosages toward an integer number of tablets and part of the outliers concerned mainly one participant who did not change his prescriptions 
In our initial set of 60 cases the neurologists achieved a majority on the detailed diagnosis in 47 cases (the diagnostic reference set). In 39 of these 47 cases they achieved also a majority decision on the selected drug (the treatment reference set)

A prescription not equal to that of the majority may still be considered an acceptable treatment. Therefore, after the Delphi round the prescriptions were assessed on their acceptability by two independent neurologists.

A consensus meeting was organised to establish objective decision criteria. The annotated prescriptions containing information on the acceptability were discussed in a consensus meeting. For each of the 47 cases in the diagnostic reference set a reference treatment could be obtained on the basis of guidelines. The discussion led to a better agreement among the neurologists with respect to the use of phenytoin.

The Delphi-approach could also have been used to establish a reference for the assessment of the treatment decisions. However, the number of cases needed to investigate the decision process would require too much time from the neurologists to achieve a reference. Instead of this, the decisions of five neurologists on 265 cases are used to obtain two reference subsets: a clinical reference consisting of the majority decision of the five neurologists and a reference consisting of the majority decision of both the neurologists and the system. Whereas the clinical reference indicates the minimal level of agreement of the system with the neurologists as the system was not involved in defining the reference, the second reference enables a more balanced assessment of the neurologists' and the system's performance.

\subsection{Initiating anti-epileptic drug treatment}

For the evaluation of the initiation of drug treatment a staged approach was used. The focus gradually shifts from an assessment of the coverage and correctness of the implemented knowledge to an evaluation of the system's and neurologists' performance using reference diagnoses and prescriptions.

\section{Quality of the knowledge acquisition}

The prescriptions of the neurologist who provided the implemented knowledge and the advice of the system were compared. The 60 paper cases were used. In all but one case the neurologist and the system agreed on the generic drug as well as on the selected brand and formulation.

In $80 \%$ of the prescriptions for carbamazepine the daily dose was identical to that of the neurologist. The system suggested a lower dose for carbamazepine $(66 \%-75 \%$ of the daily dose) in $11 \%$ of the cases and a higher dose (125-133\% of the daily dose) in $9 \%$ of the cases.

When prescribing sodium-valproate the neurologist and the system differed in two of the fifteen cases (13\%). Once a higher dose (116\% of the daily dose) and once a lower dose (75\% of the daily dose) was prescribed.

In one case the neurologist did not provide a prescription (because although a diagnosis was established he wanted additional information from the EEG). This showed that despite the fact that a diagnosis is established, drug treatment need not be started. The system was programmed to initiate a drug treatment when a diagnosis is established.

From this experiment it was concluded that the knowledge acquisition was appropriate. 


\section{Local differences}

The same 60 cases were presented to another neurologist (Neurologist II) from the same department as the expert neurologist.

Neurologist II and the system use the same drugs for treatment of epilepsy. The neurologist did not initiate drug treatment when the diagnosis was unknown. Again in one case the system suggested to start anti-convulsive treatment as a diagnosis was established. The neurologist, however, refrained from drug treatment as he also felt he needed additional information about the EEG

A difference in the formulation as well as in the daily dose was observed for carbamazepine. Neurologist II and the system do not seem to agree as far as the daily dose is concerned. The system suggested $50 \%$ to $75 \%$ of the daily doses for carbamazepine prescribed by the neurologist. With respect to the formulation the neurologist preferred the controlled release variant

The daily dose for sodium-valproate differed less. In three out of 10 cases an identical dose was prescribed, while in 4 cases $90 \%$ of the daily dose of the neurologist was prescribed. This difference in the daily dose of sodium-valproate could be attributed to the use of a different intake scheme. The neurologist preferred a tablet intake twice a day, thereby being able to prescribe tablets of $500 \mathrm{mg}$ instead of $300 \mathrm{mg}$. Still there were three cases in which Neurologist II preferred to start with a higher dose. In these cases, 70-75\% of the dose of the neurologist was suggested by the system.

The experiment showed that even within the same department different approaches exist. Neurologist 1 starts with a relatively low dose which may be increased when treatment proves not to be effective. Neurologist II on the other hand seems to adhere more to a 'middle of the target range' approach, reducing the dose when side-effects occur.

Some investigators suggest that anti-epileptic drugs have a certain specificity for certain types of epilepsy (Porter 1984, Mattson et al. 1988, 1992). However, conclusions about differences in efficacy of anti-epileptic drugs in controlling particular seizure types or epilepsies are difficult to draw, due to differences in patient populations and study designs (Gram et al. 1982, Chadwick and Tumbull 1985, Chadwick 1994ab, Brodie and Dichter, 1996). Both our neurologists consistently used different drugs for the different types of epilepsy: carbamazepine for treatment of partial epilepsy and sodium-valproate for treatment of idiopathic generalised epilepsy

Little difference was expected with respect to the daily dose; both neurologists worked in the same department and agreed when selecting the anti-epileptic drug for treatment. However, the different approaches - start with a low dose and increase when necessary versus start with a high dose and decrease in case of side-effects - suggests differences in the assessment of the risk of occurrence and consequences of seizures and complaints. Differences in treatment policy were also observed in recent literature (Mattson et al. 1992, Reynolds et al. 1993, Heller et al. 1995).

To be able to cope with such differences - given that consensus on this issue not yet exists systems that provide therapeutic advice should be tuneable to different treatment styles. Whether the dosage schemes for a given treatment style are 'hand-coded' or user-adjustable remains an issue for discussion 


\section{Impact of local preferences}

The cases in the diagnostic reference set were used to assess the prescribed drug.

The system prescribed a drug in accordance to this reference in $75 \%$ of the cases (range neurologists $46 \%$ to $94 \%$ ). Since the system was not involved in the establishment of the reference a bias in favour of the neurologists is present as each neurologist

- was allowed to change his prescription in the Delphi procedure

- only had to agree with one of his two colleagues to define a reference.

In $90 \%$ of the cases there was at least one neurologist that prescribed the same generic drug as the system did.

In 32 of the 39 cases of the treatment reference set the system prescribed the same generic drug as the treatment reference. A reference for the daily dose (defined as a dose prescribed by two neurologists who agreed on the prescribed drug) could be established in a subset of 11 cases. The system agreed in 5 of these cases (46\%). In $62.5 \%$ of these cases the system suggested a daily dose identical to that of at least one of the neurologists. The individual neurologists agreed with the reference daily dose in $75 \%$ to $100 \%$ of the cases.

Differences in doses mainly occurred in cases in which carbamazepine was the reference drug. The neurologists seem to adhere more to a 'middle of the target range' approach, whereas the system prescribed according to a 'minimal therapy' principle. The fact that carbamazepine induces its own catabolism, which may necessitate a dose higher than the initial dose for controlling the seizures, may have been a reason for the higher daily dose prescribed by the neurologists. Another reason for the difference may be due to the risks of dose related complaints after an initial dose of carbamazepine. The balancing of the risks of having another seizure and the occurrence of side-effects may cause variations in the prescribed daily dose. The system does not balance these risks explicitly

\section{Judgemen of quality of prescriptions}

The overall acceptability (as judged by two independent reviewers) of the system's advice was compared with the acceptability of the advice of each of the neurologists. Little difference was observed between the overall acceptability of the prescriptions of the system and those of the prescribing neurologists. The system obtained a lower number of judgements indicating a poor acceptability $(11 \%)$ than the neurologists (range 13\% to $29 \%$ ). On the other hand also the number of prescriptions with a good acceptability (48\%) were fewer than those of most of the neurologists (range $32 \%$ to $63 \%$ ).

\section{Judgement on selected drug and daily dose}

It is apparent from the literature that there exists disagreement about the choice of antiepileptic drugs. Also our study showed disagreement about the appropriateness of the prescribed drug. A 'neutral' or 'poor' overall acceptability judgement was given 157 (49.8\%) times. For these prescriptions judgements were also given with respect to the prescribed drug and the daily dose. In 62 (39.5\%) of these reviews the drug selection was judged 'neutral' or 'inappropriate'. For three of the neurologists more than $50 \%$ of the judgements indicated that an appropriate drug was prescribed (range $54 \%$ to $70 \%$ ). Also the system mainly $(67 \%)$ obtained a positive judgement for the suggested drug. Except for one of the neurologists the drug was considered inappropriate in $17 \%$ to $36 \%$. For the remaining neurologist as well as for the system less than $5 \%$ of the judgements indicated that an inappropriate drug was prescribed. 
The system's choice of a specific drug is therefore less often the cause for a low acceptability of the prescriptions than for four of the neurologists.

The doses to control seizures recommended in the literature vary. The initial doses for carbamazepine ranges from 200 to $800 \mathrm{mg}$ whereas the dose for sodium-valproate ranged from $600 \mathrm{mg}$ to $3000 \mathrm{mg}$ (Sonnen 1979, Shorvon et al. 1980, Aird et al. 1984, Lechtenberg 1985, Shorvon 1987, Rimmer and Richens 1988, Brodie and Dichter 1996). Also in our study differences in the daily doses of the prescriptions of the neurologists were observed. For the individual neurologists the percentage of judgements indicating an inappropriate dose ranged from $53 \%$ to $60 \%$ for three of the neurologists, while for two neurologists more than $85 \%$ of the judgements indicated that the daily dose was inappropriate. For the system in $91 \%$ of these judgements the dose was considered inappropriate. All but one of the judgements indicated that the daily dose was (too) low. Overall there is little difference between the acceptability of the advice of the system and that of the neurologists.

It has been recently suggested that the main anti-epileptic drugs differ little with respect to their effectiveness of controlling seizures (Richens et al. 1994, Heller et al. 1995). Nevertheless, the approach in which carbamazepine and phenytoin are the first choice drugs for partial epilepsy and valproate for generalised epilepsy is favoured in daily practice (Chadwick 1994ab, Shorvon 1995, Brodie and Dichter 1996). This was also observed in our study. As far as the proposed drug is concerned the advice of the system appears to be sound

Although doses of anti-epileptic drugs are not predictive for efficacy (Shorvon 1987, Commission on Anti-epileptic Drugs 1993, Lammers 1994) disagreement about the prescribed daily dose in our study turned out to be the main reason for a low acceptance of prescriptions of the system.

\section{Consensus meeting}

To establish an objective reference, differences among the neurologists were discussed during a consensus meeting. It was concluded that the initial daily dose for carbamazepine and sodiumvalproate as prescribed by the neurologists was rather high. Consensus was reached about the fact that the initial daily dose of carbamazepine for patients with a body weight up to $80 \mathrm{~kg}$ should be $400 \mathrm{mg}$, while patients with a higher body weight should receive $600 \mathrm{mg}$ per day. An appropriate initial dose of sodium-valproate was considered to be 900 to $1000 \mathrm{mg}$ depending on whether two or three intakes per day are used. For the system this means an adjustment of its 'minimal effective dose' with respect to carbamazepine, while the system's sodiumvalproate minimal effective dose agreed with the one proposed by the neurologists.

A relation between the drug dose and the efficacy of the drug has not been found so far (I.ammers, 1994). It has been suggested that the policy to achieve therapeutic plasma levels soon after the onset of treatment may induce side-effects (Reynolds et al. 1993, Heller et al. 1995). The adverse drug effects may contribute significantly to decreased patient compliance (Allen 1995). Increasing attention is paid to quality-of-life issues such as behavioural and cognitive effects and the ability to sustain as normal a life as possible while receiving medication (Pellock 1994). These arguments together with reports from the literature which show that the view with respect to the prognosis of epilepsy is changing from a chronic outcome towards a more benign course with a good chance of achieving a remission, lead to the conclusion that there is little room left for a 'middle of the target' policy in drug treatment of epilepsy. The consensus on the daily dose as achieved in our study is near the lower range of 
the daily doses found in the literature, which suggests that also the neurologists in our study agree most with the treatment policy of 'minimal therapy'.

\section{Comparing the proposed drug with the consensus}

The guidelines from the consensus meeting were used for establishing a consensus meeting reference prescription for each case. Comparing prescriptions of the neurologists and the advice of the system with this consensus meeting reference showed that all neurologists prescribed a drug in accordance with the reference in more than half of the cases (range $52 \%$ to $72 \%$ ). The system reached a consistently better agreement (range $88 \%$ to $91 \%$ ) with the reference drug on the subsets of cases seen by the individual neurologists.

The consensus meeting advised that phenytoin should be used as well. This drug was not recommended by the system as a first choice. The neurologists disagreed more with the consensus meeting reference about the prescription of phenytoin than about the use of carbamazepine and valproate. Only twice (17\%) a neurologist used the drug in accordance with the consensus meeting reference, compared to $68 \%$ for carbamazepine and $69 \%$ for valproate.

\section{Comparing the proposed daily dose with the consensus}

If the group of neurologists prescribed a drug according to the consensus meeting reference, they achieved a better agreement with the reference daily dose than when the system agreed with the drug reference. When valproate is prescribed according to the consensus, little difference between the neurologists and the system was observed ( $88 \%$ vs. $94 \%$ ). For carbamazepine the system suggested only twice (3\%) a daily dose equal to that of the reference, while in $36 \%$ of the prescriptions of the neurologists the dose was in accordance with the consensus meeting reference.

\section{Correctness of the prescribed drug and the daily dose}

All but one of the neurologists provided a prescription - taking also the decision "no drug treatment' into account - according to the consensus (drug and daily dose) in less than $40 \%$ (19\% to $36 \%$ ). The other neurologist agreed with the reference in $56 \%$. The system prescribed according to the consensus reference in $28 \%$ to $48 \%$ of the cases (for the various sets of cases common with the individual neurologists), which was equal to or better than most of the neurologists did

Since the dosage prescribed by the system can be easily corrected to agree with the consensus, it can be concluded that the system is suitable for providing advice regarding the initiation of anti-epileptic drug treatment.

\subsection{Follow-up of treatment}

The second evaluation of the system concerned the adjustment of the treatment based on information obtained in the first follow-up contact. The global decisions of the system (continuation, increase or decrease of the daily dose or change of the prescribed drug) were compared with the decisions taken by five neurologists. Three different analyses were performed. 
First, the agreement between the system and the neurologists was studied on a set of 265 cases. The system had a moderate agreement (as measured with the kappa-statistic) with three individual neurologists on the treatment decision. Only two pairs of neurologists showed such a moderate agreement, all other combinations of neurologists had a lower level of agreement.

The system reached a fair agreement beyond chance with each of the subgroups of four neurologists, whereas the individual neurologists only reach a moderate agreement with their group of peers. Also the system takes less decisions with which the neurologists completely disagree.

In a second analysis a clinical reference was developed, being the majority decision of the five neurologists. A reference treatment decision was obtained in 219 cases (set I). Although this reference was positively biased towards the neurologists as they contributed to the reference, the system reached a correctness of $78.1 \%$ for these cases which was comparable with the average correctness of the neurologists $77.4 \%$ (range $67.1 \%$ to $82.6 \%$ )

To correct for the bias a second reference of 189 cases was developed based on the treatment decisions of the neurologists as well as of the system (set II). In this set the system reached a correctness of $90.5 \%$, whereas the average correctness within the group of neurologists was $81.9 \%$ (range $67.7 \%$ to $87.8 \%$ ). The system achieved a significantly higher correctness than three of the neurologists.

Also for the set of 76 cases for which no reference treatment decision could be obtained, the system proposed fewer treatment decisions (5\%) which were different from the decisions taken by any of the neurologists than any of the individual neurologists did (range $8 \%$ to 19\%).

\section{Criteria for adjustment of the therapy}

Set I was used to investigate whether criteria could be derived that are used by the neurologists for certain treatment decisions. With the help of machine learning techniques a decision tree was constructed. The algorithm tries to build a tree such that the end nodes contain sets of cases with a single therapy. Stop criteria may prevent splits that have insufficient predictive value. Hence, impure end nodes may occur. The internal nodes contain criteria indicating which way to proceed in the tree. The tree had 14 end nodes. Support for a treatment decision was considered to be strong for six nodes. From the criteria in the tree that lead to these nodes the following guidelines for adjustment of the treatment were derived:

- In cases in which seizures are completely controlled and complaints are absent the prescribed treatment is continued. In the literature it is mentioned that an increase of the drug dose was a common decision if drug plasma levels were low (Meijer, 1991). In our study, however, as also has been recommended in the literature (Shorvon, 1995), neurologists did not apply serum levels as rigidly in these cases. Also the system decided to continue the treatment without adjustments for these cases.

- In cases with idiosyncratic complaints a change of the drug is appropriate. For the neurologists as well as for the system the presence of idiosyncratic complaints leads to the use of another drug.

- The drug dose is increased when seizures are hardly controlled (the seizure frequency decreased, or one type of seizures remained the same, while the other type decreased or was controlled) or uncontrolled (the frequency of the seizure type(s) did not change), in combination with absence of complaints, a good compliance and a plasma drug level below or in the therapeutic range. It is stated in the literature that often the dose is 
increased without checking the plasma drug level (Meijer 1991). In our study however, neurologists appear to take the plasma drug level as well as information about complaints and compliance into account to decide whether or not to increase the dose. The system reached the same treatment decision in all except three cases in which the prescribed drug was considered to be second choice. Receni studies indicate that as far as controlling the seizures is concerned little difference is observed in effectiveness of the main anti-epileptic drugs (Richens et al 1994, Heller et al. 1995). Given the risks of drug withdrawal and time loss, once a second choice drug has been initiated it should be given a full trial.

- In cases with uncontrolled major seizures and dose related complaints the prescribed drug is changed. In 21 of the 26 cases the system also decides to change the drug. In all five cases dose related complaints occurred at a (below) therapeutic plasma drug level immediately after initiation of the treatment. These complaints may be transient in contrast with those occurring in combination with a high or toxic range plasma dnug level. A continuation of the treatment was proposed by the system and agreed at by at least one of the neurologists.

- The prescribed drug is changed in cases in which some success is achieved in controlling the seizures (either major seizures decreased while minor seizures were not controlled or major seizures controlled and minor seizures remained equal), dose related complaints are present and the plasma drug level is in the high therapeutic or toxic range. These findings are in accordance with the findings of others who considered an increase of the dose in case of a (near) toxic serum concentration to be a dubious decision (Meijer, 1991). The plasma drug level is used as indication whether toxicity develops and additional anticonvulsive action is small (Shorvon, 1995). The system decided to change the drug in all eight cases.

- The drug treatment is continued in cases in which the seizure type(s) decreased or in which one type of seizures was controlled while the other remained equal, with noncompliance and a plasma drug level in the high therapeutic or toxic range

For $39.3 \%$ of the cases in set I criteria leading to a single therapy could not be obtained. The reason could be the way in which the cases were described and analysed, or the existence of alternative treatments. The first reason can largely be rejected as a detailed analysis did not show any discriminative factors not taken into account in the analysis. There is some evidenci that alternative treatments may exist for some subgroups. For some groups of cases a single reference treatment does not seem to exist, although in individual cases a reference treatment was defined by the neurologists

\subsection{General conclusion and future prospects}

The question whether it is possible to provide adequate advice for guiding anti-epileptic drug treatment by means of a computerised model can be answered in the affirmative

1. For the initiation of the therapy and the monitoring of the treatment, the agreement of the system with individual neurologists was in general at least as good as the agreement among the neurologists

2. For the monitoring of the treatment the correctness of the system on a clinical reference was as good as the correctness of the neurologists even though the system was not involved in establishing the reference while the neurologists were.

It was possible to elicit the necessary knowledge from an expert neurologist. 
With respect to the question whether the system suggested an adequate anti-epileptic drug it was concluded that the system proposed an adequate anti-epileptic drug when initiating drug treatment. When comparing the overall acceptability (as judged by two independent reviewers) of the prescriptions there was little difference between the neurologists and the system. The neutral or poor quality overall judgement was in the case of the system due to a (too) low prescribed dose.

It was apparent that even after a two-staged Delphi procedure there existed a relatively large inter-individual variability between the neurologists. A similar variability was observed among the reviewers. Because of this variability in opinions with respect to the way how to initiate the therapy a consensus meeting was organised. When assessing the correctness of the prescriptions (in relation to the reference obtained in the consensus meeting) the system's proposals for initiating anti-epileptic drug treatment were better than those of most of the individual neurologists.

With respect to the follow-up the system appears to be as good as the neurologists in taking treatment decisions. There is a moderate agreement between the treatment proposals of individual neurologists and the system. The system had a consistently better agreement with a group of four neurologists than each other neurologist individually. Comparison of the system's prescriptions with a reference defined by the group of neurologists (clinical reference) showed that the system reached a correctness comparable to that of the individual neurologists Compared with the reference based on the decisions of the neurologist as well as the system, the system reached a better correctness than three of the neurologists.

As far as the criteria for adjustment of the treatment are concerned, six guidelines for adjustment were identified. For each of these guidelines the system seems to apply the same treatment decisions.

The described system is an implementation of the elicited knowledge on anti-epileptic drug treatment rather than a prototype of a system that can be used in clinical practice. It lacks the interface with a clinical information system as well as a user intertace that will meet the requirements of the clinician. Also relevant functionalities for clinical use are lacking. One of the aspects which will require further development is the explanation facility. Although a trace of the rules used for obtaining an advice may be provided, they have to be reworded to enable interpretation of the reasoning process of the system by a neurologist.

Despite these shortcomings our studies indicate that the developed models are fit for integration with other software such as electronic medical records or clinical information systems. The model also would be eminently suited for incorporation into an interactive teaching system for neurological residents. The cases used in the consensus meeting could also be used for this purpose.

Because of the observed inter-individual variability it is advisable to further develop the system such that it can be installed in a neurologist's office initially for testing purposes. In these circumstances the reasons for deviating from the system's advice should be analysed. In this way information can be obtained that will assist in the further development of guidelines for treatment of epilepsy. At the same time such guidelines become available in computerised form to ease their application for individual patients. Affer the system is released for use and knowledge with respect to the treatment is maintained the system can be used by the neurologists for a second opinion. 
Also the system could be extended to incorporate the diagnostic process. It can be expected that future changes in the diagnostic criteria will be less than in the past. The current criteria have been reviewed and established over the years and are generally accepted.

An organisation should volunteer to organise regular consensus meetings in order to keep diagnosis and treatment protocols up to date. This knowledge could be stored in a knowledge base and be distributed among the neurologists.

\subsection{References}

Aird R.B., Masland R.L., Woodbury D.M., The epilepsies a critical review, Raven Press, New York, 1984.

Allen F.H., Entering a new drug era: practical issues in the treatment of epilepsy, Neurology, 45, 1995, (Suppl 2), s5-s6.

Brodie M.J., Dichter M.A., Antiepileptic drugs. The New England Journal of Medicine, 334, 1996, pp.168-175.

Chadwick D., Epilepsy, Journal of Neurology, Neurosurgery and Psychiatry, 57, 1994a, pp.264-277.

Chadwick D., Standard approach to anticpileptic drug treatment in the United Kingdom, Epilepsia, 35 (Suppl.4), 1994b, s3-s 10.

Chadwick D., Tumbull D.M., The comparative efficacy of antiepileptic drugs for partial and tonicclonic seizures, Journal of Neurology, Neurosurgery and Psychiairy, 48, 1985, pp. 1073-1077.

Commission on Antiepileptic Drugs of the International League Against Epilepsy, Guidelines for therapeutic monitoring on antiepileptic drugs, Epilepsia, 34, 4, 1993, pp 585-587.

Dreifuss F.E., Risk benefit analysis in the management of the child with cpilepsy. In: Modern approach 10 antiepileptic drug treatment, improving quality of life, (Dam, Gram, Pcdersen and orum, Eds.), The Danish Epilepsy Society, Kobenhavn, 1985.

Dreifuss F.E, The epilepsies: clinical implications of the international classification, Epilepsia, 31 , (Suppl. 3), 1990, s3-s10.

Gram L., Drachmann Bentsen K., Parnas J., Flachs H., Controlled trials in epilepsy: a review, Epilepsia, 23. 1982, pp. 49I-519.

Heller A.J., Chesteman P., Elwes R.D., Crawford P., Chadwick D., Johnson A.L., Reynolds E.H., Phenobarbitone, phenytoin, carbamazepine, or sodium valproatc for newly diagnosed adult epilcpsy: a randomised comparative monotherapy trial, Journal of Neurology. Neurosurgery and Psychiary, 38,1995 , pp. 44-50.

Hicknan F.R., Killin J.L, Land L., Mulhall T., Porter D., Taylor R.M., Analysis for knowledge-based systems, a practical guide to the KADS methodology, Ellis Horwood Limited, Chichester, 1989.

Lammers M.W., Clinimetrics in epileprology, thesis, Nijmegen, 1994.

Lechtenberg R., The diagnosis and ireatment of epilepsy, Macmillan Publishing Company, New York, 1985.

Mattson R.H., Cramer J.A., Collins J.F., VA Epilepsy Cooperative Study Group, Aspects of compliance; taking drugs and keeping clinic appointments. In: Compliance in Epilepsy (Schmidt and Leppik, Eds.), Elsevier Science Publishers B.V., Amsterdam, 1988, pp. III-117.

Mattson R.H., Cramer J A. Collins J.F., and the Dept. of Veterans Affairs Epilepsy Cooperative Study No 264 Group, A comparison of valproate with carbamazepine for the treatment of complex partial seizures and secondarily generalised tonic-clonic seizures in adults, The New England Journal of Medicine, 327, 1992, pp. 765-771 
Meijer J.W.A., Knowledge, attitude and practice in antiepileptic drug monitoring, thesis, Nijmegen, 1991

Pellock J.M., Standard approach to antiepileptic drug treatment in the United States, Epilepsia, 35(Suppl.4), 1994, s11-s 18.

Porter R.J., Epilepsy, 100 elementary principles, W.B. Saunders Company Ltd., London, 1984.

Principe J.C., Smith J.R., Microcomputer-based system for the detection and quantification of petit mal epilepsy, Computers in Biology and Medicine, 1982, 12, pp. 87-95

Readdie M., Streng K.H., Wermser D, KADS metrication, Deliverable G10. SD-G10-R-001, ESPRT P1098, SD Europe Ltd \& NeuTech Entwicklungsgesellschaft mbH \& Co.KG, 1989.

Reynolds E.H., Heller A.J., Chadwick D., Valproate versus carbamazepine for seizures, The New England Journal of Medicine, 328, 1993, pp. 207-208.

Richens A., Davidson D.L.W., Cartlidge N.E.F., Easter D.J., A multicentre comparitive trial of sodium valproate and carbamazepine in adult onset epilepsy. Journal of Neurology. Neurosurgery and Psychiatry, 1994, 57, pp. 682-687.

Rimmer E.M., Richens A., Clinical phamacology and medical treatment. In: A textbook of epilepsy, (Laidlaw, Richens and Oxley, Eds.), $3^{\text {rd }}$ edition, Churchill Livingstone, Edinburgh, 1988, pp. 421483.

Scheiber G., Wielinga B., Breuker J., KADS a principled approach to KBSS development, Academic Press, London, 1993.

Shorvon S.D., Galbraith A.W.. Laundy M., Vydelingum L., Reynolds E.H., Monotherapy for epilepsy. In: Antiepileptic therapy: advances in drug monitoring. (Johannessen, Pippenger, Schmidt, Morselli, Richens and Meinardi, Eds.). Raven Press, New York, 1980, pp. 213-220.

Shorvon S.D., The treatment of epilepsy by drugs. In: Epilepsy (Hopkins, Ed.), Chapman and Hall, London, 1987, pp. 229-282.

Shorvon S., The drug treatment of epilepsy. In: Epilepsy, (Hopkins, Shorvon and Cascino, Eds.), $2^{\text {nd }}$ edition, Chapman and Hall Medical, London, 1995.

Sonnen A.E.H., De medicamenteuze behandeling van epilepsie, Nedcrlands Tijdschrift voor de Geneeskunde, 123, 1979, pp. 485-490.

Tansley D.S.W., Hayball C.C., Knowledge-based systems analysis and design, a KADS developer's handbook, Prentice Hall, New York, 1993.

Ward C.D., Decision making in refractory epilepsy: an analysis based on computer simulation, Journal of Neurology: Neurosurgery and Psychiatry, 1989, 49, pp. 781-784. 


\section{Summary}

Given the ageing of the population and the expected growth in the number of patients with multiple problems, an increase can be expected in the amount of information that has to be processed when dealing with an individual patient. Simultaneously there is a pressure to practice medicine in a cost-effective way. The clinician who wants to do the best for his patient must try to maximise the expected utility of his actions. The expectations of patients with respect to the capabilities of medicine complicate this process as conflicts may arise between the requests of individual patients and societal needs. Physicians need tools to assist them in mastering the growing amount of increasingly more complex medical knowledge to assure optimal care. Information technology may assist the physician with clinical decision making in daily practice to achieve best medical practice. Also in the domain of epilepsy - a disorder of the brain which manifests itself through brief usually unprovoked stereotypical disturbances of behaviour, emotion, motor function or sensation - supporting the doctor by a 'computer fed by a team of specialists' was considered as an option to improve patient management.

In this thesis the development of a computerised model of the expert knowledge used for prescribing anti-epileptic drugs is described. It was studied whether such a model can provide advice of a good quality. The advice concerns:

1. the initiation of anti-epileptic drug treatment after the diagnosis has been established and

2. the adjustment of the drug treatment after assessment of its effectiveness.

\subsection{The domain of epilepsy}

In chapter 2 a review of the literature with respect to diagnosis and treatment of epilepsy is presented. Problems discussed are related to

- the definition of epilepsy which can be considered as a framework rather than a fixed set of criteria for diagnostic interpretation of symptoms.

- the presence of different approaches for deciding on drug treatment.

- the disagreement on therapeutic ranges of drugs and the usage of plasma drug levels

- the psychological and social consequences of the long-term character of the treatment procedures. 
Although guidelines with respect to diagnosis and treatment of epilepsy are available the actual context for their application is usually missing. Guidelines to enhance the decision making process of the physician in managing a specific patient could therefore be of value.

\subsection{Building a model of expertise}

The KADS structured approach for knowledge based systems development was used. Two inference models describing the initiation and the monitoring of the treatment in patients with epilepsy were developed (Chapter 3 )

At the time we used KADS two main problems were encountered:

- KADS orientation towards the analysis of the cognitive process instead of the implementation of a model of expertise.

- the lack of support for the evaluation of the quality of the models.

Over the years the orientation of KADS has shifted from the analysis of cognitive processes towards a more pragmatic approach for implementation of models. Some work on metrication has also taken place. This work, however, is mainly directed towards project management. Little has been done with respect to the evaluation of models. Integrating KADS with an evaluation methodology could enhance user acceptance of a decision support system.

\subsection{Reference development}

The evaluation of computerised models is a difficult but essential part of the development of a decision support system. Several methodologies have been proposed in the literature. Most methodologies consider validation of a decision support system as the most important part of the evaluation process. The assessment of the correctness of the output of the system is the main issue in this phase. An important problem in the evaluation of decision support systems is the development of a reference for assessment of the system's advice. As described in chapter 2 an objectively correct treatment for an individual patient is not available. This means that a reference for evaluating the advice of the system had to be developed by a group of neurologists.

\section{Reference for the initiation of treatment}

For the initiation of the treatment sixty paper cases were constructed describing the clinical presentation of a patient's first two or three visits (Chapter 4). For each case three neurologists were asked to provide a prescription. By means of a two step Delphi-like approach addressing the diagnosis and the prescribed drug, the variability in prescribing could be reduced as far as the drug was concerned. The feedback round on the daily dose was less successful in reducing the variance in dosage.

Two reference sets were developed, a diagnostic reference set for the assessment of the prescribed drug and a treatment reference set for the assessment of the daily dose.

Because a treatment not equal to that provided by the majority may still be considered an acceptable treatment, the acceptability of the prescriptions was assessed by two independent neurologists after the Delphi round.

The variability among the neurologists made it difficult to obtain a set of cases for which the neurologists agreed about the diagnosis as well as about the treatment. Therefore a consensus 
meeting was organised to obtain more objective decision criteria. For the cases of the diagnostic reference set a consensus reference treatment was obtained on the basis of the guidelines defined in the meeting. The consensus meeting led to a better agreement among the neurologists with respect to the use of phenytoin.

\section{Reference for the follow-up of treatment}

The assessment of the advice in a first follow-up contact also required the use of a majority decision of a group of neurologists as no objective standard existed. Two reference sets were obtained (Chapter 6). A clinical reference consisted of cases about which the majority of the neurologists agreed. A second reference consisted of cases about which a majority decision of both the neurologists and the system could be obtained.

In addition to this more global approach we identified case characteristics, using machine learning techniques, which the neurologists may have used when deciding about the adjustment of the treatment.

\subsection{Evaluation of the initiation of the treatment}

Comparing the system's advice for cases in the diagnostic reference set with the prescriptions of the neurologist showed that the system prescribed an anti-epileptic drug in accordance with the reference as least as often as an individual neurologist (Chapter 5).

The system prescribed a daily dose in accordance to the reference for sodium-valproate. For carbamazepine the system predominantly prescribed a dose lower than the neurologists did. A difference in treatment approach apparently existed. While the neurologists adhered more to a 'middle of the target' approach the system prescribed according to the 'minimal therapy' principle which means that initially a low dose is used which is increased if seizures do not remit.

The acceptability analysis showed little difference in overall acceptability between the prescriptions of the system and the neurologists. The system received a lower number of judgements indicating a poor acceptability than the individual neurologists. On the other hand the number of prescriptions for which a good acceptability was observed was lower than those of most of the neurologists. The system suggested more often an acceptable drug than most of the neurologists. If the acceptance of the suggested prescription of the system was judged low this was mainly due to a too low daily dose.

This evaluation of the system's advice for the reference cases and the assessment of the acceptability provided an indication of the performance of the system but the variability in prescribing among the neurologists did not enable us to make a firm statement about the system's correctness. To reduce the variability a consensus meeting was organised. It appeared that the daily dose initially prescribed by the neurologists was rather high

The system as well as the group of neurologists for sodium-valproate mainly prescribed a daily dose in accordance with the consensus guidelines. For carbamazepine the system prescribed less often a daily dose in accordance with the consensus guidelines than the group of neurologists.

Except for one of the neurologists who prescribed according to the consensus in more than half of the cases, the system suggested an equal or higher number of prescriptions (drug and daily dose) according to the consensus than the other neurologists did. 


\subsection{Evaluation of the follow-up of the treatment}

To assess whether the system takes adequate decisions during monitoring the drug treatment, the global treatment decisions of the system were compared with the decisions of the five neurologists.

The system reached a moderate agreement on the treatment decision - as expressed with the $\kappa$-statistic - with three of the individual neurologists on the treatment decision. Only twice a pair of neurologists showed a moderate agreement, while the other combinations of two neurologists had a lower level of agreement. This shows that the system agreed better with each individual neurologist than any of the neurologist with their colleagues.

Comparing the agreement between the system and a group of four neurologists with that of an individual neurologist with the same four neurologists showed that the system reached a fair agreement, while each of the individual neurologists moderately agreed with their four colleagues.

A clinical reference was developed, based on the treatment decisions of the majority of the five neurologists. For 219 out of 265 cases such a majority decision could be obtained. The system reached a correctness comparable to that of the individual neurologist on this set of cases. This reference was positively biased towards the neurologists, as they contributed to the definition of the reference treatment decision while the system did not. Including the system's treatment decision in the reference creation process led to a subset of 189 cases in which a majority decision could be obtained. Within this subset the system reached a higher correctness than the neurologists. The analysis of the set of 76 cases for which no reference treatment could be obtained showed that the system suggested fewer treatment decisions different from the decisions taken by any of the neurologists, than each of the individual neurologists did.

Case characteristics may be expected to be important for reaching specific treatment decisions. To obtain insight in the decision making process of the group of neurologists a decision tree was constructed with the help of machine learning techniques. The tree consists of branches which describe a path in theory leading to one specific treatment. Each path may use different case characteristics. An end-node presents the number of cases for each different treatment that fulfil the criteria belonging to the specific branch.

An assessment of the criteria for adjustment of the therapy showed that for six branches the end-nodes contained cases of predominantly one treatment. These criteria were taken as guidelines for follow-up of the treatment. The system uses the same treatment decisions for cases described by each of these guidelines

\subsection{General conclusion}

The question whether a computerised model based on expert knowledge can provide quality advice on anti-epileptic drug treatment can be answered in the affirmative. Comparing the system with the diagnostic reference and the consensus reference showed that it provides a correct advice for initiating anti-epileptic drug treatment. Poor acceptability of an advice could be attributed to a low dose. This part of the knowledge base can easily be corrected.

Also for the adjustment of the initiated treatment the system proposed treatment decisions which were in good agreement with those of the individual neurologists as well as with those 
of a group of neurologists. Because of the detected inter-individual differences it is advisable to further develop the system (including a user-friendly user interface) and install it for testing in a neurologist's office to analyse reasons for deviations from the system's advice. When the knowledge base is maintained over time the system's advice can be used as a second opinion or for educational purposes 


\section{Samenvatting}

Door de stijgende leeftijd van de bevolking en de verwachte groei van het aantal patienten met meer dan één gezondheidsprobleem, kan men een toename verwachten van de hoeveelheid informatie die verwerkt moet worden bij het behandelen van een individuele patient. Gelijktijdig wordt er druk uitgeoefend om zo kosten-effectief mogelijk te werken in de gezondheidszorg. De clinicus die het beste voor zijn patient wil bereiken moet trachten de verwachte opbrengst van zijn activiteiten te maximaliseren. De verwachtingen van patienten ten aanzien van de mogelijkheden van de geneeskunde bemoeilijken dit proces doordat er conflicten kunnen optreden tussen wensen van de individuele patient en het maatschappelijke belang. Artsen hebben behoefte aan methoden die helpen bij het verwerken van de groeiende hoeveelheid van steeds complexere medische kennis om optimale zorg te waarborgen. Informatietechnologie kan de arts bij zijn dagelijkse klinische besluitvorming helpen optimale zorg te verlenen. Ook op het gebied van epilepsie - een aandoening van de hersenen welke zich manifesteert door korte, meestal niet geprovoceerde stereotypische verstoringen van gedrag, emotie, motoriek of gevoel - werd ondersteuning van de arts door 'een computer gevoed door een team van specialisten' gezien als mogelijkheid om het beleid met betrekking tot de patient te verbeteren.

In dit proefschrift wordt de ontwikkeling van een geautomatiseerd model van de kennis van een expert met betrekking tot het voorschrijven van anti-epileptische medicatie beschreven. $\mathrm{Er}$ werd onderzocht of een dergelijk model een kwalitatief goed advies kan geven. Dit advies betreft:

1. het starten van de behandeling met anti-epileptica nadat de diagnose gesteld is en

2. de aanpassing van de medicamenteuze behandeling na beoordeling van de effectiviteit.

\section{Epilepsie}

In hoofdstuk 2 wordt een overzicht van de literatuur met betrekking tot diagnostick en behandeling van epilepsie gepresenteerd. Verschillende problemen worden besproken:

- de definitie van epilepsie, die eerder beschouwd kan worden als een raamwerk in plaats van een vastgestelde set van criteria voor diagnostische interpretatie van symptomen

- de verschillende benaderingen met betrekking tot beslissingen over de medicamenteuze behandeling

- het verschil in mening betreffende de therapeutische range van de medicamenten en het gebruik van plasmaspiegels bij de instelling en bewaking van de therapie

- de psychische en sociale consequenties van het lange termijn karakter van de behandelingsprocedure

Ofschoon richtlijnen voor diagnostiek en behandeling van epilepsie beschikbaar zijn, ontbreekt hierin meestal de context voor de toepassing ervan. Ondersteuning van het besluitvormingsproces van de arts middels uitgebreidere richtlijnen betreffende de behandeling van de specifieke patient kunnen van waarde zijn. 


\section{Het bouwen van een kennismodel}

KADS, een gestructureerde benadering voor de ontwikkeling van kennissystemen, werd gebruikt om de kennis over de behandeling van epilepsie te modelleren. Twee inferentie modellen - een voor de initiatie en een voor het bewaken van de medicamenteuze behandeling van patienten met epilepsie - werden ontwikkeld.

Bij het gebruik van KADS werden twee belangrijke problemen geconstateerd:

- de gerichtheid van KADS op het analyseren van het cognitieve proces in plaats van op de implementatie van een kennismodel.

- het ontbreken van ondersteuning voor het evalueren van de kwaliteit van de modellen.

Door de jaren heen is de nadruk van KADS op de analyse van cognitieve processen steeds meer verschoven in de richting van een meer pragmatische benadering voor implementatie van modellen. Verschillende metrieken zijn ontwikkeld. Dit werk was voornamelijk gericht op projectmanagement. Met betrekking tot de evaluatie van modellen heeft weinig onderzoek plaatsgevonden. Integratie van KADS met een evaluatiemethode kan de acceptatie van een besluitvormingondersteunend systeem door de gebruiker verbeteren

\section{Ontwikkeling van referentie sets}

De evaluatie van een implementatie van een kennismodel is een moeilijk maar essentieel onderdeel van de ontwikkeling van een besluitvormingondersteunend systeem. Verschillende methoden zijn beschreven in de literatuur. De meeste methoden beschouwen validatie van het besluitvormingondersteunend systeem als het belangrijkste onderdeel van het evaluatieproces. De beoordeling van de correctheid van de output van het systeem is hierbij het belangrijkste onderwerp. Voor de evaluatie van een besluitvormingondersteunend systeem is de ontwikkeling van een referentie, waarmee het advies van het systeem kan worden beoordeeld, belangrijk. Zoals beschreven in hoofdstuk 2 zijn er geen objectieve criteria voor correcte behandeling van een individuele patient beschikbaar. Dit betekent dat een referentie voor de evaluatie van het systeem ontwikkeld moet worden door een groep van op dit gebied deskundige neurologen

\section{Referentie voor het initieren van de behandeling}

Voor het initierren van de behandeling werden zestig papieren casus ontwikkeld, die elk een klinische presentatie van de eerste twee of drie poliklinische bezoeken van de patient beschrijven (hoofdstuk 4). Voor elk van deze casus werden drie neurologen verzocht een recept te schrijven. Met behulp van een twee stappen Delphi-achtige benadering, die zowel de diagnose als het voorgeschreven medicament betrof, kon de variabiliteit in voorschrijven van het soort medicament worden beperkt. De feedbackronde voor de dagelijkse dosering was minder succesvol in het reduceren van de variabiliteit

Twee referentie sets werden ontwikkeld, een diagnostiek referentie set voor de beoordeling van het voorgeschreven medicament en een therapeutische referentie set die gebruikt werd voor de beoordeling van de dagelijkse dosering.

Omdat een behandeling die niet in overeenstemming is met die voorgeschreven door de meerderheid nog steeds acceptabel kan zijn, werd na de Delphi ronde de aanvaardbaarheid van de recepten beoordeeld door twee onafhankelijke neurologen 
De variabiliteit onder de neurologen maakte het moeilijk een set van casus te verkrijgen met volledige overeenstemming op zowel de diagnose als de behandeling. Daarom werd een consensus bijeenkomst georganiseerd om een objectief criterium te verkrijgen. Voor de casus uit de diagnostiek referentie set werd een consensus referentie behandeling opgesteld op basis van de richtlijnen van de consensus bijeenkomst. De consensus bijeenkomst leidde tot een betere overeenstemming onder de neurologen met betrekking tot het gebruik van met name phenytoine.

\section{Referentie voor de follow-up van de behandeling}

Voor de beoordeling van het advies in een eerste follow-up contact moest een meerderheidsbeslissing van een groep van neurologen gebruikt worden aangezien hiervoor geen objectieve standaard bestaat. Twee referentie sets werden verkregen (hoofdstuk 6). Een klinische referentie set bestaande uit casus waarbij de meerderheid van de neurologen het eens was en een tweede referentie set bestaand uit casus waarbij een meerderheidsbeslissing verkregen werd op basis van zowel de uitspraken van de neurologen en de adviezen van het systeem Verder identificeerden we karakteristieken van de casus, middels machinaal leren, die de neurologen gebruikt kunnen hebben bij het beslissen over het aanpassen van de behandeling.

\section{Evaluatie van de initiatie van de behandeling}

Een vergelijking van de adviezen van het systeem, voor de casus uit de diagnostiek referentie set, met de recepten van de neurologen toonde aan dat het systeem ten minste net zo vaak als een individuele neuroloog anti-epileptica voorschrijft in overeenstemming met de referentie (hoofdstuk 5).

Het systeem adviseert voor natrium-valproaat een dagelijkse dosering in overeenstemming met de referentie. Voor carbamazepine suggereert het systeem vournamelijk een lagere dosering dan de neurologen. Klaarblijkelijk bestond er een verschil in benadering bij de behandeling. Terwijl de neurologen meer aansluiten bij een 'middle of the target' aanpak, adviseerde het systeem meer volgens het 'minimale therapie' principe, hetgeen betekent dat bij aanvang een lage dosering van het medicament wordt geadviseerd die verhoogd wordt indien de toevallen niet afnemen.

De analyse van de aanvaardbaarheid van de medicamenteuze adviezen toonde op globaal niveau weinig verschil in acceptatie tussen de recepten van het systeem en die van de neurologen. Het systeem had een kleiner aantal oordelen met een lage graad van acceptatie dan de individuele neurologen. Echter het aantal recepten met een hoge acceptatie was lager dan dat van de meeste neurologen. Het systeem adviseerde vaker een acceptabel medicament dan de meeste neurologen. Indien de acceptatiegraad van het door het systeem geadviseerde recept laag was werd dit meestal veroorzaakt door een te lage dagelijkse dosering

De evaluatie van de adviezen van het systeem voor de referentie casus en de beoordeling van de aanvaardbaarheid gaven een indruk van de prestatie van het systeem. De variabiliteit onder de neurologen maakte het echter moeilijk een harde uitspraak te doen over de correctheid van het systeem. Om de variabiliteit te verminderen werd een consensus bijeenkomst gehouden. Hierbij kwam naar voren dat de dagelijkse dosering die initieel door de neurologen werd voorgeschreven, vrij hoog was. 
Zowel het systeem als de groep van neurologen schreven voor natrium-valproaat meestal een dagelijkse dosering voor in overeenstemming met de consensus richtlijn. Voor carbamazepine adviseerde het systeem minder vaak een dagelijkse dosering in overeenstemming met de consensus richtlijnen dan de groep neurologen

Het systeem suggereerde een gelijk of hoger aantal recepten (medicament en dagelijkse dosering) in overeenstemming met de consensus dan vrijwel alle neurologen. Slechts een neuroloog schreef in meer dan de helft van de casus een recept in overeenstemming met de consensus voor en was hierbij beter dan het systeem.

\section{Evaluatie van de follow-up van de behandeling}

Om te beoordelen of het systeem adequate beslissingen neemt tijdens het bewaken van de medicamenteuze behandeling, werden de globale beslissingen betreffende de behandeling zoals voorgeschreven door het systeem vergeleken met de beslissingen van de vijf neurologen.

Het systeem bereikte een matige overeenstemming met betrekking tot het behandelingsbesluit - uitgedrukt in de $\mathrm{k}$-waarde - met drie van de individuele neurologen. Slechts twee paren van neurologen hadden een matige overeenstemming; de overige combinaties van twee neurologen hadden een lagere graad van overeenstemming. Het systeem stemde dus betere overeen met elk van de individuele neurologen dan elk van de neurologen met zijn collega's

De vergelijking van de overeenstemming tussen het systeem en een groep van vier neurologen met de overeenstemming tussen een individuele neuroloog en dezelfde groep van vier neurologen, wees uit dat het systeem een vrij goede overeenstemming bereikte, terwijl elk van de individuele neurologen matig overeenstemde met zijn vier collega's

Een klinische referentie werd ontwikkeld, gebaseerd op de behandelingsbesluiten van de meerderheid van de vijf neurologen. Voor 219 van de 265 casus kon een dergelijke meerderheidsbeslissing verkregen worden. Op deze set van casus bereikte het systeem een correctheid vergelijkbaar met die van de individuele neuroloog. Deze referentie heeft echter een bias ten gunste van de neurologen doordat deze laatsten bijdragen aan de definitie van de referentie voor de behandeling, terwijl het systeem dit niet deed. Door het advies van het systeem te betrekken bij de definitie van de referentie behandeling werd voor een subset van 189 casus een meerderheidsoordeel verkregen. Binnen deze subset van casus bereikte het systeem een betere correctheid dan de neurologen. De analyse van de 76 casus waarbij geen referentie verkregen kon worden, toonde aan dat het systeem minder vaak dan elk van de individuele neurologen een behandelingsbesluit adviseerde dat door geen van de neurologen was voorgesteld.

Karakteristieken van een casus kunnen belangrijk zijn voor het bereiken van specifieke behandelingsbesluiten. Om meer inzicht te krijgen in het besluitvormingsproces van de groep van neurologen werd een beslisboom geconstrueerd met behulp van machinaal leren. De boom bestaat uit paden die elk leiden tot een eindknoop waaraan in principe één specifieke behandeling toegewezen kan worden. Elk pad gebruikt verschillende karakteristieken van een casus. Een eindknoop geeft het aantal casus weer voor elk van de verschillende behandelingsbesluiten, dat aan de criteria voldoet behorende bij het specifieke pad, dat naar deze eindknoop leidt. 
Een analyse van de criteria voor aanpassing van de therapie toonde aan dat bij zes paden de eindknopen casus bevatten met voornamelijk één behandelingsbesluit. Deze criteria werden gebruikt als richtlijnen voor de follow-up van de behandeling. Voor elk van deze richtlijnen adviseer het systeem dezelfde behandelingsbesluiten als de groep neurologen

\section{Algemene conclusies}

De vraag of een geautomatiseerd model gebaseerd op kennis van een expert een kwalitatief goed advies voor de medicamenteuze behandeling van epilepsie kan geven kan bevestigend beantwoord worden. Een vergelijking van de adviezen met de diagnostiek referentie en de consensus referentie toonde aan dat het systeem een correct advies geeft voor het initiëren van de behandeling met anti-epileptica. Een lage graad van aanvaardbaarheid van een advies kon worden toegeschreven aan een (te) lage dagelijkse dosering. Dit deel van het kennisbestand kan gemakkelijk aangepast worden.

Ook voor de aanpassing van de geïnitieerde behandeling stelde het systeem behandelingsbesluiten voor die een goede overeenstemming hadden met zowel de individuele neurologen als groepen van neurologen.

Vanwege de waargenomen inter-individuele verschillen is het raadzaam het systeem verder te ontwikkelen (inclusief een gebruikersvriendelijke interface) en het voor verdere tests te installeren in een neurologische polikliniek, om zo de redenen voor het niet volgen van het advies van het systeem te kunnen analyseren. Als het kennisbestand van het systeem onderhouden wordt, kan het gebruikt worden voor een second opinion, of voor onderwijsdoeleinden. 


\section{Tot slot}

Het schrijven van een proefschrift is niet mogelijk zonder de hulp en de steun van anderen. Dit is reeds vele malen geschreven in het dankwoord van een proefschrift. Ook bij het schrijven van dit proefschrift werd het wederom proefondervindelijk vastgesteld. Daarom wil ik hier enkele mensen speciaal bedanken die voor mij van groot belang zijn geweest in de afgelopen jaren.

Wijlen Prof.dr.van der Lugt voor zijn bereidwilligheid tot een reflecterende kijk op het medisch denken en handelen en het stimuleren van een idee van een tweede jaars student geneeskunde.

Prof.dr.ir.Hasman: Beste Arie, het idee medische kennis te standaardiseren vond bij jou gehoor. Bedankt voor je interesse en de deskundige begeleiding met name in de laatste fase van het proefschrift.

Prof.dr.Meinardi: Beste Harry, je interesse, je praktische kijk, je gevoel voor humor en je inzet hebben me vaak verwonderd. Bedankt voor je stimulerende invloed.

Prof dr.Troost: Beste Jaap, bedankt voor je positieve commentaar en de duwtjes in de goede richting

Dr.ir. Talmon: Beste Jan, een speciaal woord van dank geldt jou. Je enorme energie en je flexibiliteit zijn essentieel voor een promovendus.

Drs. Groothuis en drs. Tange: Beste Siebren en Huibert, gelukkig was er ook nog iets anders dan promoveren. Ik wens jullie alle twee veel succes met jullie promotie.

Drs.Kubben, internist. Beste Frank, je belangstelling en vertrouwen is voor menig mens een verademing. Ik wens je veel succes met je promotie.

Ir.Stallenberg: Beste Gerard, je nuchtere kijk op zaken en je gevoel voor humor worden zeer op prijs gesteld. Onze wandelingen waren een welkome afwisseling. Arigato.

Verder wil ik de neurologen die aan het onderzoek hebben meegewerkt bedanken voor het beschikbaar stellen van hun tijd en hun bereidwilligheid om op een andere manier naar medisch handelen te kijken. Dr.H.Schouten en dr. A.van Kesteren wil ik bedanken voor hun statistische adviezen

Dat de laatste loodjes het zwaarst wegen heefl naast enkele goede vrienden met name de fam. moeten ervaren. Mijn dank gaat dan ook uit naar hen wier steun ik heb ontvangen, in het bijzonder naar mijn moeder, voor al haar goede zorgen, mijn vader, voor al zijn inspanningen, en Marjo, Desirée en Vincent voor hun warme belangstelling.

Tot slot wil ik Eline, mijn vrouw, bedanken voor haar steun en bereidheid mijn werk te laten prevaleren boven andere belangrijke zaken. Dankzij jouw laatste zetjes is het gelukt. Het is af. 

Decisions

and Strategies

in Drug Treatment

of Epilepsy

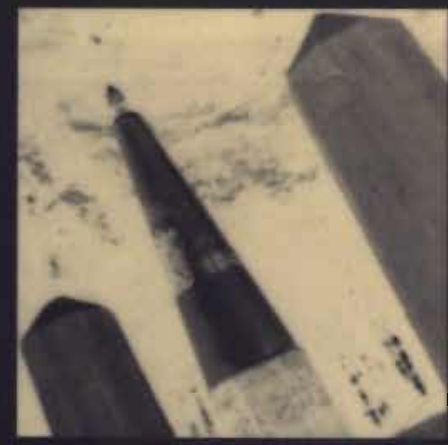

Raymond Smeets 
Decisions and strategies in drug treatment of epilepsy 


\title{
Decisions and strategies in drug treatment of epilepsy
}

\author{
PROEFSCHRIFT
}

ter verkrijging van de graad van doctor aan de Universiteit Maastricht, op gezag van de Rector Magnificus, Prof.mr.M.J.Cohen, volgens het besluit van het College van Dekanen, in het openbaar te verdedigen op

vrijdag 29 november 1996 om 12.00 uur

door

Raymond Pierre Angèle Marie Smeets

geboren te Rothem-Meerssen in 1961 
Promotores:

Co-promotor:

Beoordelingscommissie:
Prof.dr.ir. A. Hasman

Prof.dr. H. Meinardi

Prof.dr. J. Troost

Dr.ir. J.L. Talmon

\author{
Prof.dr. J.A. Knottnerus (voorzitter) \\ Dr. J. van der Lei (Erasmus Universiteit Rotterdam) \\ Prof.dr. P. Pop \\ Dr. W.O. Renier (Katholieke Universiteit Nijmegen) \\ Prof.dr. R.W. Stockbrügger
}

ISBN: $90-9010008-3$

(c) R.P.A.M. Smeets, Maastricht, 1996.

Produktie: Datawyse, Maastricht

Omslag: Consultatie: John van de Poel, Ruud Groothedde.

Foto's: Borders-No Borders, Herbert Rikers, Bunde.

Lay-out: Datawyse, Joop Caris.

Dit proefschrift kwam tot stand in het kader van het instituut ExTra, deeluitmakendvan de landelijke onderzoekschool CaRe (Netherlands School of Primary Care Research), in 1995 erkend door de KNAW.

Het in dit proefschrif gepresenteerde onderzoek werd gesteund door subsidies van het Nationaal Epilepsie Fonds - De Macht van het Kleine en de SWOL.

Met dank aan het Nationaal Epilepsie Fonds - De Macht van het Kleine, Ciba Geigy B.V.. Dumex B.V., Janssen-Cilag B.V., Karwijk Fama B.V., Novo Nordisk Farma B.V., Schering-Plough B.V., Yamanouchi Farma B.V., inzake totstandkoming van dit proefschrift. 


\section{Voor Eline.}





\section{CONTENTS}

\section{CHAPTER 1 INTRODUCTION}

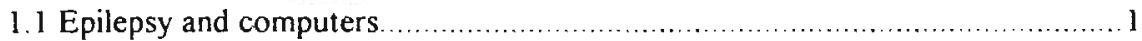

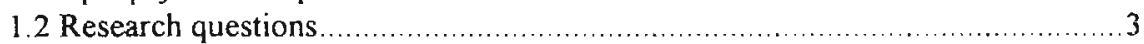

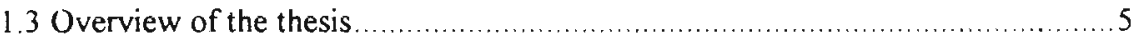

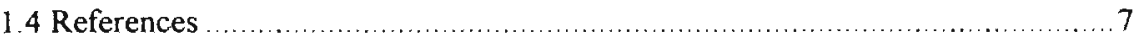

\section{CHAPTER 2 EPILEPSY}

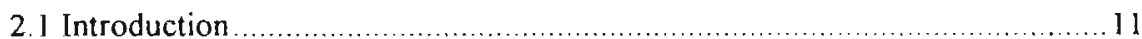

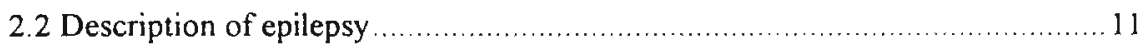

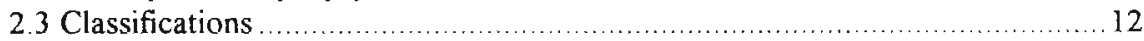

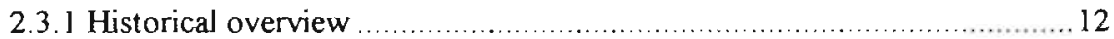

2.3.2 Importance of different classifications ............................................ 13

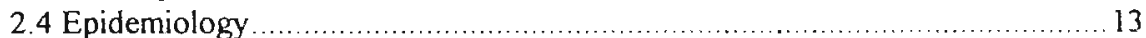

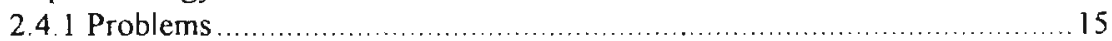

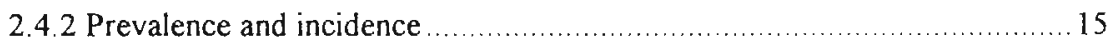

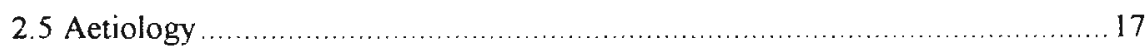

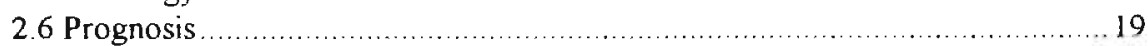

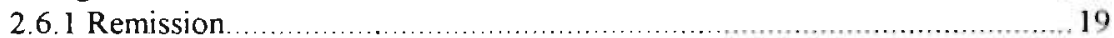

2.6.2 Problems in establishing prognostic factors ................................. 21

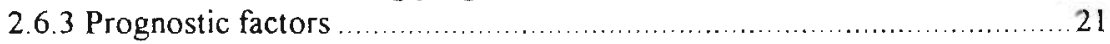

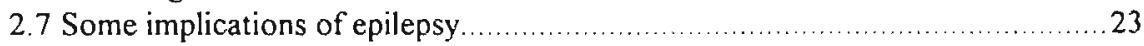

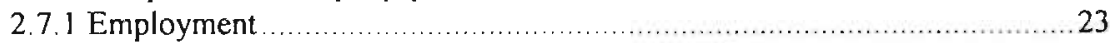

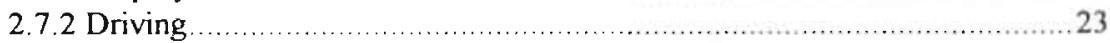

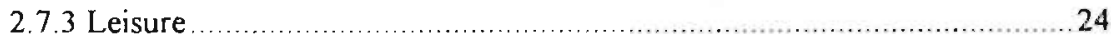

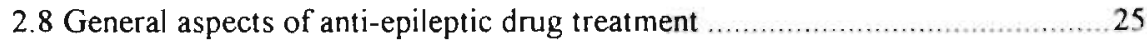

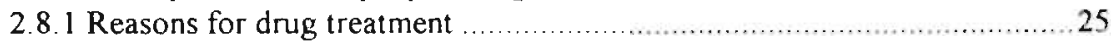

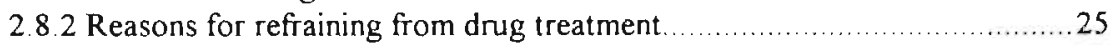

2.8.3 Goals in anti-epileptic drug treatment .....................................26

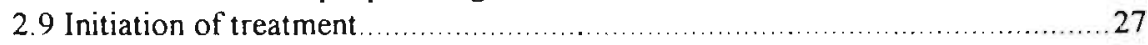

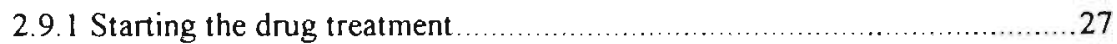

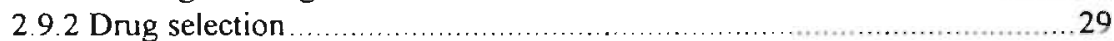

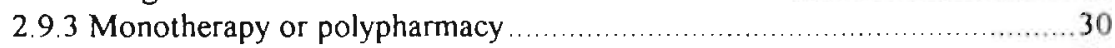

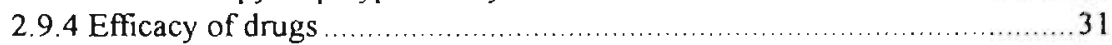

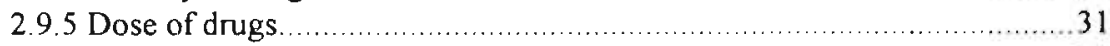

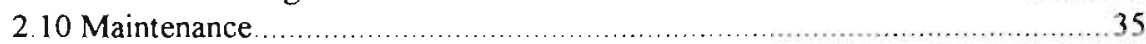

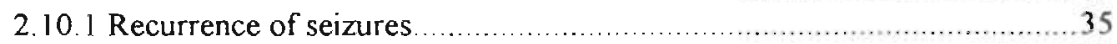

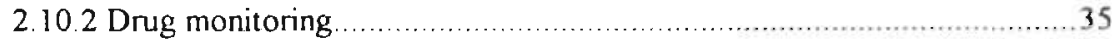

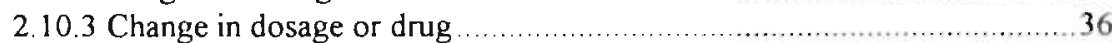

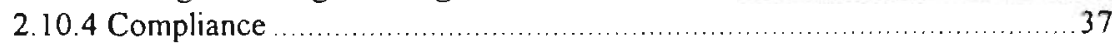

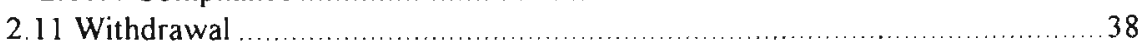

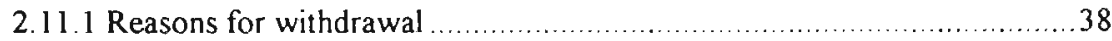

2.11.2 Moment of relapse......................................................... 40

2.12 Summary and conclusion......................................................... 40

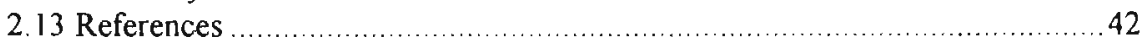




\section{CHAPTER 3 SYSTEM DEVELOPMENT}

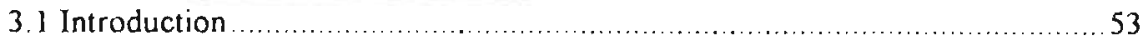

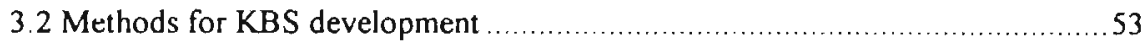

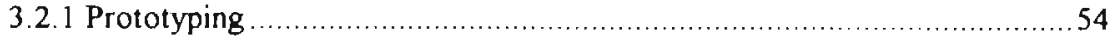

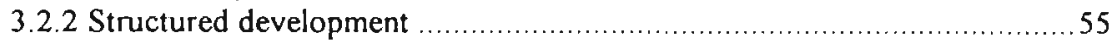

3.2.3 Selection of the development method ........................................ 57

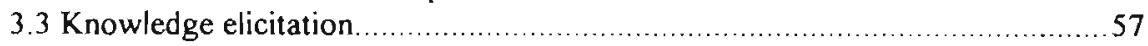

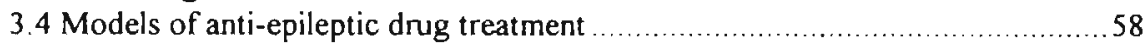

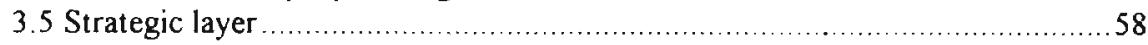

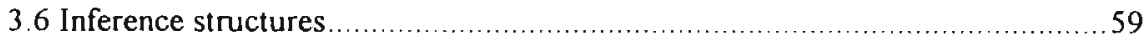

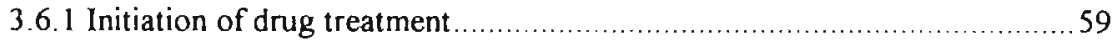

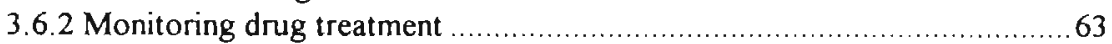

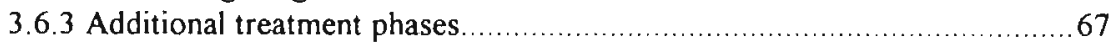

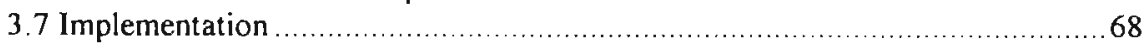

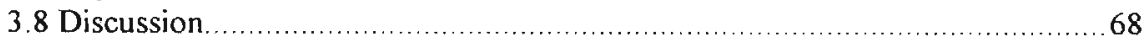

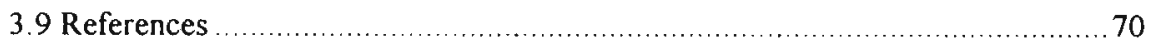

CHAPTER 4 REFERENCE DEVELOPMENT

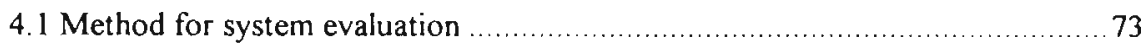

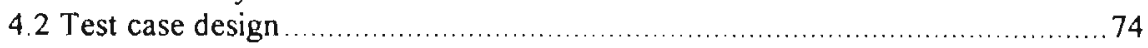

4.2.1 Patient selection .................................................................... 74

4.2.2 Validity of paper cases ............................................................. 74

4,2.3 Description of the test cases ................................................. 75

4.3 Reference development ........................................................... 77

4.3.1 Method for consensus formation .............................................. 77

4.3.2 Reference development/Acceptance testing ...................................78

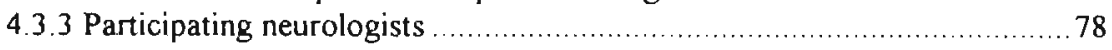

4.3.4 Reference development process ................................................ 79

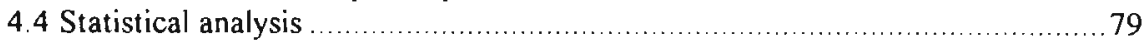

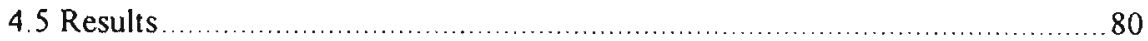

4.5.1 Agreement on the diagnosis, generic drug and daily dose ................... 80

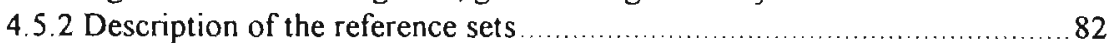

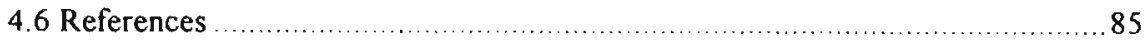

CHAPTER 5 RESULTS ON INITIATION OF TREATMENT

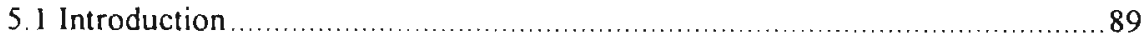

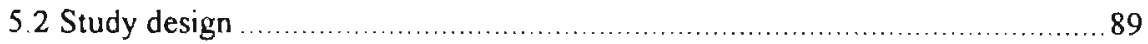

5.3 Comparing the system with Neurologist I and Neurologist II _................... 90

5.3.1 Agreement on the generic drug ................................................. 90

5.3.2 Agreement on tradename ....................................................... 91

5.3 .3 Agreement on daily dose ....................................................... 91

5.4 Comparing the system with an external reference .................................. 92

5.4.1 Agreement about the drug using the diagnostic reference set ................93

5.4 .2 Variability in neurologist's prescriptions ....................................... 94

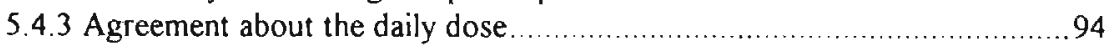

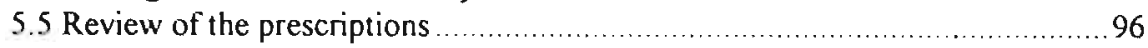


5.5.1 Overview of the results for system and individual neurologists...............96

5.5.2 Comparing overall scores of system and individual neurologists..............98

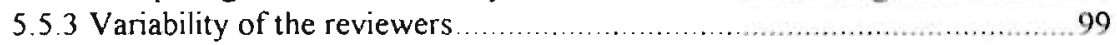

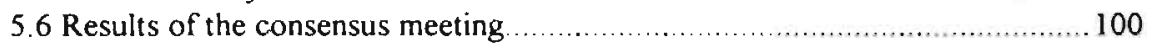

5.6.1 The use of diagnostic classification schemes ............................ 100

5.6 .2 Initiation of drug treatment ..................................................... 100

5.6 .3 Selection of generic drug .................................................... 100

5.6 .4 Generic drug or brand, formulation .......................................... 101

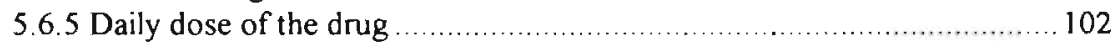

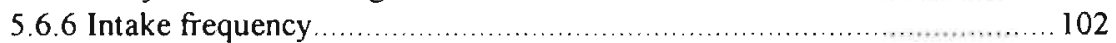

5.6 .7 Gradual increase of the dose ............................................. 103

5.7 Comparing the system and the neurologists with the consensus meeting

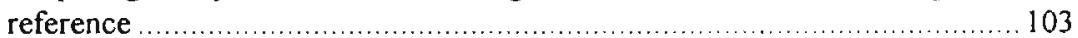

5.7.1 Agreement on the generic drug .............................................. 103

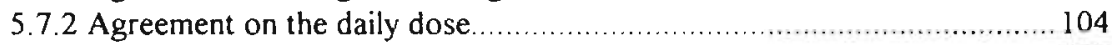

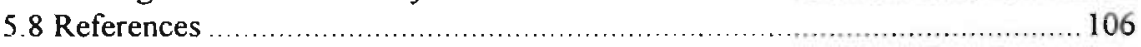

CHAPTER 6 RESULTS ON FOLLOW-UP OF TREATMENT

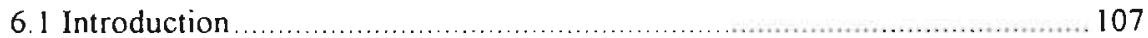

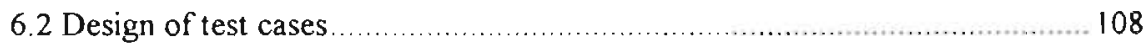

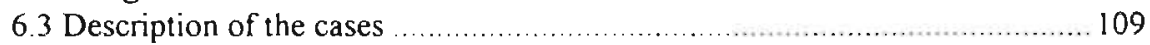

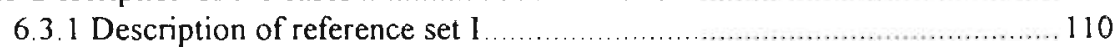

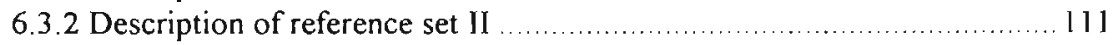

6.3.3 Cases not included in the reference set II .................................. 113

6.3.4 Relevance of characteristics for agreement among neurologists ............. 114

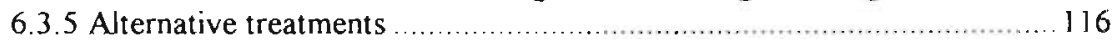

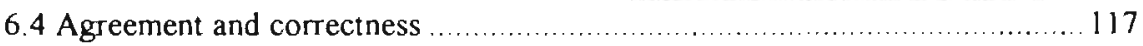

6.4.1 Overview of the decisions in the total set ................................. 117

6.4.2 Differences in treatment approaches ........................................... 118

6.4.3 Agreement between an individual and a group .............................. 119

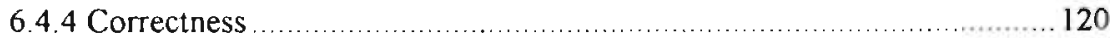

6.4.5 Variability in agreement in set II .......................................... 121

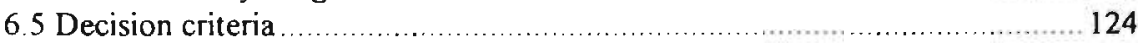

6.5.1 Treatments indicated by a single attribute ................................ 124

6.5.2 Treatments ruled out by a single attribute ................................. 125

6.6 Treatments indicated by combinations of attributes .............................. 126

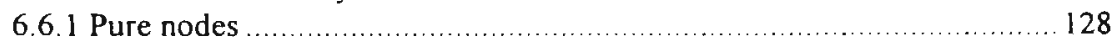

6.6.2 Nearly pure nodes; two decisions ............................................. 129

6.6.3 Mixed nodes; three decisions ................................................ 13 ]

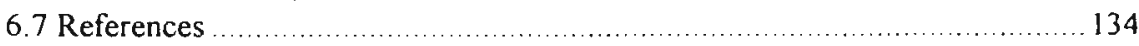


CHAPTER 7 CONCLUSIONS AND DISCUSSION

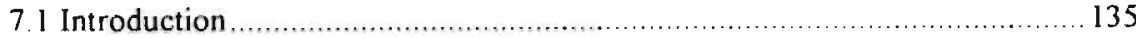

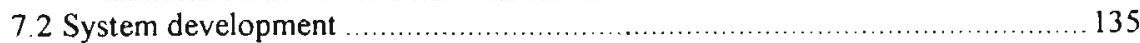

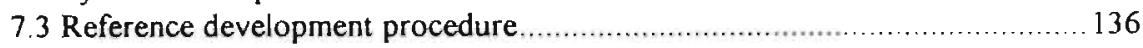

7.4 Initiating anti-epileptic drug treatment ............................................ 137

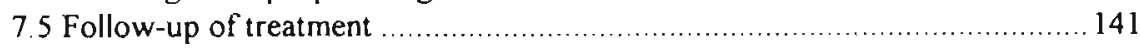

7.6 General conclusion and future prospects ...................................... 143

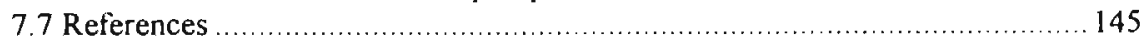

CHAPTER 8 SUMMARY

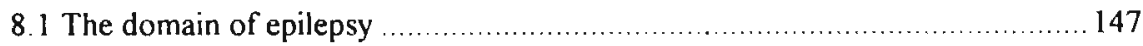

8.2 Building a model of expertise .................................................. 148

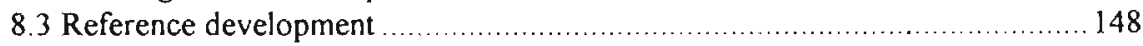

8.4 Evaluation of the initiation of the treatment ...................................... 149

8.5 Evaluation of the follow-up of the treatment ..................................... 150

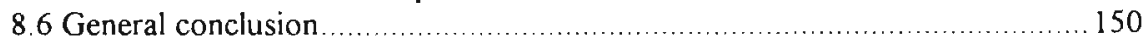

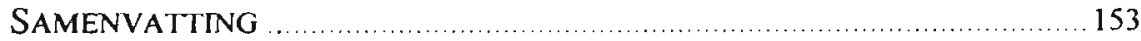

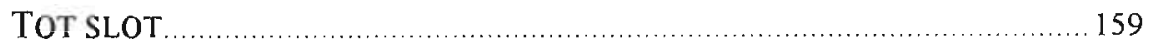

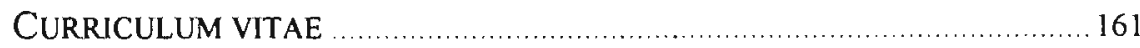




\section{Introduction}

\subsection{Epilepsy and computers}

Epilepsy is a disorder which manifests itself through brief, usually unprovoked stereotypical disturbances of behaviour, emotion, motor function or sensation. The occurrence of these symptoms will have a great impact on the daily life of the patient and his family. Such seizures are normally treated with anti-epileptic drugs. Although doctors may have the same education, it seems that some are more successful than others in the treatment of patients. Part of this difference may be attributed to experience. The use of a 'computer fed by a team of specialists' to support the doctor has been considered an option to improve patient management in the domain of epilepsy (Meinardi, 1985).

Computer programs, either embedded or stand-alone, which are designed to help health professionals in patient management by suggesting or providing patient specific advice or treatment are called decision support systems (DSSs). Evidence, especially the more recent, suggests that this type of computerised decision support can improve physician performance (Sutton 1989, Paterson-Brown and Vipond 1990, Johnston et al. 1994, Sullivan and Mitchell 1995). DSSs analyse and reason about patient data and support the diagnosis, treatment and disease prevention tasks by producing relevant conclusions or by (suggesting) actions for a given problem of a specific patient. This contrasts with clinical information systems and literature reference systems. These latter systems provide information but do not specify how to apply it in the decision making process for the specific problem of an individual patient (Perreault and Wiederhold, 1990).

Taking into account under which circumstances the advice is generated three types of DSSs can be discerned

- Solicited advice systems i.e. systems providing advice upon request of the user

- Unsolicited advice systems i.e systems providing suggestions without a specific request of the user

- Autonomous systems or closed-loop systems i.e. systems undertaking medical actions without having a physician in the loop

Each of these types of systems requires a different involvement from the user. In case of a system providing solicited advice, the physician has to make a request for information and to take a decision on the applicability of the information provided. 
Systems providing unsolicited advice do so on the basis of existing patient data. The user decides whether or not to act on the advice. Autonomous systems both analyse the information and apply the advice for a patient specific problem. The user has to decide whether to apply the system for a specific task.

Studies of the impact of DSSs in various domains have shown that such systems can enhance patient care through (see e.g. Johnston et al. 1994 for a review):

- Improvement of the diagnostic accuracy

- More effective control of the disease by:

a. Having serum levels of therapeutics in accepted ranges more often

b. Earlier achievement of therapeutic doses

c. A reduction of adverse drug reactions

$\mathrm{d}$. Earlier change of ineffective treatments

e. Improvement of uniformity in drug treatment

- Increase in the use of preventive procedures

- Improvement of patient follow-up

In the management of patients with epilepsy three sub-domains can be considered as problem areas for which it may be worthwhile to develop a DSS:

- The diagnostic process

- The decision whether or not to start drug treatment

- Drug treatment

Establishing the correct diagnosis is an essential part in the decision making process. Uncertainty about the fact whether a patient has epilepsy may be a reason for refraining from drug treatment (Chadwick and Reynolds, 1985). Decision support in this domain has been considered. The definitions of disease concepts are not stable or specific enough to enable optimal diagnostic support. For example, recently a new set of definitions has been proposed (Commission on Epidemiology and Prognosis LAE, 1993). Others who developed procedures for diagnostic support encountered problems related to definitions (Principe and Smith 1982, Korpinen et al 1994). Hence a diagnostic decision support system seems less promising.

The decision to start anti-epileptic drug treatment has as a consequence the intake of a drug for at least two years in a regular, daily fashion. The risks of recurrence of seizures has to balance or outweigh the disadvantages of the treatment. In this process of decision making issues such as consequences of the seizures, side-effects, compliance and psycho-social consequences of the drug intake have to be addressed (Dreifuss, 1985). Little guidance was found in the literature on how to incorporate issues such as compliance and psycho-social aspects in the decision making (Chadwick and Reynolds 1985, Jøhannessen et al. 1990). Building decision support for this domain would rely to a large extent on common sense and subtleties that are difficult to formalise. The literature still is not conclusive about the perceived risks of seizures and the moment of commencing treatment (Chadwick 1995, Reynolds 1995).

Once the decision to start treatment has been taken, problems such as the selection of the drug, the determination of an adequate drug dose regime and the adjustment of initiated treatment will have to be addressed. Multiple reasons for adjustment of the treatment can occur (Beardsley et al. 1983, Ward 1989, Meyer 1991, Dichter 1993). 
Insufficient effectiveness of the drug is a common problem. Also side-effects may warrant modification of the treatment. Decision making may be complicated by noncompliance of the patient. More or less formalised but still not uniform procedures for drug treatment are discussed in the literature. Guidance in dosage determination has been provided by models which mainly address the pharmacodynamics of drug treatment (van der Kleijn 1983, Peterson 1983). The domain is relatively stable, as only a few new drugs enter the market. It has been assumed that models which support the physician in treatment may improve patient care (Meinardi 1985, Rimmer and Richens 1988).

It was concluded on the basis of the perceived problems in the three sub-domains that drug treatment was the most suitable domain for decision support. Our study therefore focuses on the elicitation, implementation and assessment of knowledge of treatment procedures for anti-epileptic drugs.

Automated decision support addresses the system of medical practice as a whole including health care professionals and patients (Musen, 1993). However, before one starts to study how a DSS has to be integrated in the system of medical practice it is necessary to establish the efficacy of a DSS. If a DSS has a low efficacy in a domain then one should not put it into routine use. Despite considerable development and evaluation efforts, few DSSs are used in daily practice (Nykänen 1990, O'Moore et al. 1990, Heathfield and Wyatt 1993, de Dombal 1993). Factors hampering the acceptance of DSSs for routine use in medical care may have a technical, economic, organisational, psychological and/or medical origin. It has been suggested that by taking these factors into account the introduction of DSSs in the medical domain may be stimulated (van der Lei, 1993).

A generally accepted evaluation methodology which addresses the above mentioned problem areas is needed. Most existing evaluation methodologies have as a common denominator at least the validation of a system, of which the assessment of the implemented knowledge is considered to be the cornerstone (Spiegelhalter 1983, Green and Keyes 1987, O'Keefe et al. 1987, O'Keefe 1989. O'Leary and O'Keefe 1989, RossiMori et al. 1990, Wyatt and Spiegelhalter 1990, Engelbrecht et al. 1991, Grant et al. 1991, Clarke et al. 1994). We restricted ourselves to this type of assessment.

\subsection{Research questions}

In this thesis the main research question is

"Is it possible to develop decision support that provides adequate advice concerning anti-epileptic drug treatment?"

To answer this question we elicited and analysed the knowledge of an exper neurologist. This knowledge was implemented in a prototype system. The developed system can give advice in two different situations:

- the initiation of the anticonvulsive treatment after the diagnosis has been established and

- the adjustment of the drug treatment after an assessment of its effectiveness. 
The adequacy of the system's advice for initiation of anti-epileptic drug treatment is evaluated by addressing the following questions:

1. Does the system suggest an adequate anti-epileptic drug when initiating drug treatment?

2. Does the system provide an adequate daily dose when initiating drug treatment?

When an objectively correct treatment would be available for each case, such a treatment could serve as reference for answering the above listed questions. In the management of epilepsy there is no such objectively correct treatment (see chapter 2). Therefore we compare the system's advice with the suggestions of a group of neurologists.

For the initiation of drug treatment a Delphi procedure was followed to reduce variability and remove erroneous treatment suggestions of the neurologists. From the set of 60 cases we obtained a diagnostic reference set where the majority of three neurologists agreed on the detailed diagnosis. This diagnostic reference set was reduced in size to a treatment reference set where the majority of the neurologists agreed on the generic drug.

The diagnostic reference set is used for assessment of the proposed generic drug; the treatment reference set for assessment of the proposed daily dose of the drug. For both sets of cases the agreement between the system and individual neurologists was determined. It was decided that the system's advice would be considered adequate when it conforms to the reference as good as or better than the individual neurologists. If the system does not agree with the majority but gave an advice equal to that of one neurologist such an advice can be considered an alternative treatment.

As more than one treatment may be appropriate for a case the acceptability of the prescriptions for cases in the diagnostic reference set was assessed by two independent (non prescribing) neurologists. The question addressed is:

3. What is the overall acceptability of the advice of the system compared with that of the neurologists?

If the prescription was considered of poor quality, the judgement on the appropriateness of the generic drug and the prescribed daily dose was analysed to determine which of these aspects was the reason for the poor quality.

The comparison of the neurologist's prescriptions with the system's advice for the cases in the reference sets and the assessment of the acceptability provided an indication about the performance of the system. However, it did not enable us to make a statement about the correctness of the detailed prescriptions due to the variance in prescribing among the neurologists. To reduce the variability in prescribing among the neurologists we decided to have a consensus meeting in which the prescriptions for each of the cases were discussed. During this discussion global guidelines were agreed upon. These guidelines could be used to determine a reference treatment for each of the cases. The advice of the system as well as the prescriptions of the neurologists are compared with this reference. The question addressed was:

4. What is the correctness of the system's prescriptions compared with the correctness of the neurologists' prescriptions? 
The adjustment of drug treatment is another part of the process for which an advice may be provided by the system. This is evaluated by addressing the question:

5. Does the system take adequate treatment decisions while monitoring drug treatment?

The global decisions to adjust the treatment - i.e. continue, decrease or increase the daily dose or change of drug - were used for comparison.

On the basis of a set of 265 descriptions of follow-up contacts the agreement of the system with each individual neurologist is determined. A comparison between the agreement of an individual neurologist and the remaining group of four neurologists and between the system and this group is made to obtain information about the adequacy of the system.

Based on the 265 follow-up descriptions two reference treatment sets were established to determine the performance of the system. A clinical reference set contained cases for which a majority decision of the five neurologists could be established. The second reference used the majority decision obtained from both the five neurologists and the system. To obtain a more tangible measure, the agreement between the system and the neurologists was expressed as a score, the level of agreement, indicating the number of neurologists which agree or disagree with the system.

To compare the decision making process of the group of neurologists with that of the system a classification tree is constructed based on case characteristics and the treatment decisions. The end-nodes of the tree present sets of cases which share particular case characteristics. The path to an end-node which contains mainly cases with one treatment decision indicates a guideline which may have been applied by the group of neurologists. End-nodes with several treatment decisions indicate categories of cases for which guidelines are difficult to obtain. For each of the major end-nodes the decision making process of the neurologists and the system are compared. The question addressed here is:

6. How do the treatment decisions of the system compare with those of the group of neurologists for the cases that meet the guidelines derived from the neurologists' decisions?

\subsection{Overview of the thesis}

In chapter 2 a review of the literature on epilepsy and its treatment is presented. The chapter is divided into two parts. In the first part a description of epilepsy and an overview of the diagnostic classifications are presented. Studies on prevalence and incidence in the United States and European countries indicate how common epilepsy is. In the second part treatment of epilepsy is discussed including aspects of drug treatment such as initiation, maintenance and withdrawal of drug treatment. This information was used during the design of the system.

Since the literature does not provide enough information about the criteria to be used in the decision making process of drug treatment it was decided also to elicit this knowledge from an expert. The amount of data required for commencing and monitoring the drug treatment makes the use of flow charts unfeasible and calls for 
computer support. Several approaches for knowledge acquisition are reviewed in chapter 3. Models describing the initiation and monitoring part of the treatment process are presented. Problems in analysing expertise using KADS are discussed.

The evaluation of the system focused on the implemented knowledge. As a standard for anti-epileptic dnug treatment was not available, a reference treatment had to be developed for a set of test cases. A Delphi-like approach in combination with a consensus meeting was selected for establishing a reference with which to compare the system. In chapter 4 the construction of the test cases and development of the reference are presented.

Chapter 5 addresses the assessment of the system's advice for initiating anti-epileptic drug treatment. Three analyses are presented in which the advice of the system is compared with the prescriptions of:

- The neurologist who provided the expertise to investigate whether the domain was sufficiently covered during the knowledge elicitation process

- A neurologist from the same department not involved in the development of the system to obtain some information on local differences in treatment

- A group of five neurologists from five different general hospitals in The Netherlands to assess the impact of individual preferences

In each of the experiments the prescribed drug and the daily dose are assessed. A comparison of the performance of individual neurologists and of the system is presented

To be able to determine the correctness of prescriptions a consensus meeting was held to define a treatment reference. The reference determined the required generic drug as well as the daily dose. The advice of the system as well as the prescriptions of the neurologists are compared with this reference.

In chapter 6 the advice of the system is assessed using case descriptions, in which drug treatment has been started, who return for a follow-up. In this chapter the treatment decisions are assessed at a global level: increase, decrease or keep the dose as it is or change the type of drug. The chapter consists of two parts. In the first part the advice of the system is addressed. Three different analyses are presented:

- The degree of agreement between the system and the neurologist(s) is determined to establish how good the system is in relation to the individual neurologist

- A clinical reference based on the majority decision of the group of neurologists was used to assess the correctness of the system and the neurologists

- A comparison using a reference based on the majority decision of both the neurologists and the system provided information on how the system performed when the system is considered as a peer

One may expect that case characteristics determine which treatment decision is taken. The second part of chapter 6 describes the results of an analysis leading to criteria that may have been used by the neurologists in arriving at a treatment proposal. The treatment decisions of the system are compared with them. 
In chapter 7 the final conclusions are presented and discussed. Directions for future research are indicated as well.

\subsection{References}

Beardsley R.S., Freeman J.M., Appel F.A., Anti-convulsant serum levels are useful only if the physician appropriately uses them: an assessment of the impact of providing serum level data to physicians, Epilepsia, 24, 1983, pp. 330-335.

Chadwick D., Reynolds E.H., When do epileptic patients need treatment? Starting and stopping medication, British Medical Journal, 290, 1985, pp. 1885-1888.

Chadwick D., Case for early treatment is not established, British Medical Journal, 1995, 310 , pp.177-178.

Clarke K., O'Moore R., Smeets R., Talmon J., Brender J., McNair P., Nykänen P., Grimson J., Barber B.A., A methodology for evaluation of knowledge-based systems in medicine, Artificial Intelligence in Medicine, 1994, 6, pp. 107-121.

Commission on Epidemiology and Prognosis of the International League Against Epilepsy, Guidelines for epidemiologic studies on epilepsy, Epilepsia, 34, 1993, pp.592-596.

Dichter M.A., The premise, the promise, and the problems with basic research in epilepsy, Epilepsia, 34, 1993, pp.791-799.

Dombal F.T de, Background and current state-of-the-art Technology and Healih Care. European Society for Engineering and Medicine. Vol. I, April 1993, pp. 77-83.

Dreifuss F.E., Risk benefit analysis in the management of the child with epilepsy. In: Modern approach to anti-epileptic drug treatment, improving quality of life, (Dam, Gram, Pedcrsen and Ørum, Eds.), The Danish Epilepsy Society, Kobenhavn, 1985.

Engelbrecht R., Fitter M., Rector A., Requirements for a medical workstation using usercentred design. In: Proceedings MIE '9l (Adlassnig, Graber, Bengtsson and Hansen, Eds.), Lecture Notes in Medical Informatics, vol 45. Springer-Verlag, Berlin, 1991, pp. 140-144.

Grant A., Parker-Jones C., White R., Cramp D, Barreiron A., Mira P., Artal A., Monteror J., Evaluation of knowledge based systems from the user perspective. In: Knowledge-hased systems in Medicine: Methods Applications and Evaluation, (Talmon and Fox, Eds.), Lecture Notes in Mcdical Informatics, vol 47, Springer Verlag, Berlin, 1991, pp. 312-324

Green C.J., Keyes M.M., Verification and validation of expert systems. In: Proceedings Western Conference on expert systems Anaheim, IEEE Computer Socicty Press, New York, 1987, pp. 38-43.

Heathfield H.A., Wyatt J., Philosophies for the design and development of clinical dicision support systems. Methods of Information in Medicine, 32, J993, pp. 1-8

Johannessen S.I., Loyning Y., Munthe-Kaas A.W. General Aspects. In: Comprehensive Epileptology (Dam and Gram, Eds.), Raven Press, New York, 1990, pp 505-524.

Johnston M.E., Langton K.B., Hayncs R.B., Mathicu A., Effects of computer-based clinical decision support systems on clinician performance and patient outcome, a critical appraisal of research, Annals of Internal Medicine, 120, 1994, pp. $135-142$.

Kleyn E. van der, Continuous monitoring of plasma anti-epileptic drug levels, in: Current therapy in epilepsy, Acta Ncurologica Scandinavia, 97 (Suppl.), 1983, pp.91-114.

Korpinen L., Pietilä T., Peltola J., Nissilä M., Keränen T., Tuovinen T., Falck B., Petránek E.S., Frey H., Evaluation of epilepsy expert, a decision support system, Computer Methods and Programs in Biomedicine. 45. 1994, pp.223-231. 
Lei, J. van der, Experience from computer based records for computer assisted decision making. Methods of Information in Medicine, 32, 1993, pp. 14-15

Meinardi H., Epileptology, military art of healing or science. Inaugural address, Catholic University of Nijmegen, 22 Februari 1985.

Meyer J.W.A. Knowledge, attitude and practice in anti-epileptic drug monitoring, thesis, Nijmegen, 1991.

Musen M., Architectures for architects, Methods of Information in Medicine, 32, 1993, pp. 1213.

Nykänen P. Issues on the evaluation of computer-based support to clinical decision making, Report of the SYDPOL-working group, Institute of informatics, Oslo University, Research report 127, 1990.

O'Keefe R.M., Balci O., Smith E.P., Validating expert systems performance. IEEE Expert, Winter, 1987, pp. $81-89$.

O'Keefe R.M. The evaluation of decision-aiding systems, guidelines and methods, Information and Management, 17, 1989, pp.217-226.

O'Leary D., O'Keefe R. Verifying and validating expert systems, Tutorial at the 11 th IJAI-89, Detroit USA, 1989.

O'Moore R., Clarke K, Smeets R., Brender J., Nykänen P., McNair P., Grimson J., Barber B.: Items of Relevance for the Evaluation of Knowledge Based / Expert Systems and Infuence from Domain Characteristics. KAVAS (AJ021) Report EM 1.l., Trinity College, Dublin, 1990.

Paterson-Brown S., Vipond M.N., Madern aids to clinical decision making in the acute abdomen, British Journal for Surgery, 77, 1990, pp. 13-18.

Perreault L., Wiederhold G., System design and evaluation. In: Medical Informatics. Computer applications in healih care (Shortliffe, Perreault, Wiederhold and Fagan, Eds.), Addison-Wesley Publishing Company, Reading, Mass. 1990.

Peterson G.M., Computer-assisted drug therapy for epilepsy, Drig Intellingence and Clinical Pharmacy, 1983, 17, pp.123-125.

Principe J.C. Smith J.R., Microcomputer-based system for the detection and quantification of petit mal epilepsy, Computers in Biology and Medicine, 12, 1982, pp.87-95.

Reynolds E.H., Do anticonvulsants alter the natural course of epilepsy? Treatment should be started as early as possible, British Medical Journal, 1995, 310. pp. 176.

Rimmer E.M., Richons A., Clinical pharmacology and medical treatment. In: A textbook of upilepsy, (Laidlaw, Richens and Oxley, Eds.), 3rd. edition, Churchill Livingstone, Edinburgh, 1988, pp. 421-483.

Rossi-Mori, A., Pisanelli, D.M., Ricci, F.L. Evaluation stages and design steps for knowledgebase systems in medicine. Medical Informatics, vol. 15, no.3, p.191-204, 1990.

Spiegelhalter D.J. Evaluation of clinical decision-aids with an application to a system for dyspepsia, Statistics in Medicine. 2, 1983, pp.207-216.

Sullivan F., Mitchell E., Has general practitioner computing made a difference to patient care? A systematic review of published reports, British Medical Joumal, 311, 1995, pp.848-852.

Sutton, G.C., Computer-aided diagnosis a review, British Journal for Surgery, 76, 1989, p.8285 .

Ward C.D.. Decision making in refractory epilepsy. an analysis based on computer simulation, Journal ut Neurology. Neurosurgery and Psychiatry, 1989, 49, pp: 781-784. 
Wyatt J., Spiegelhalter, D. Evaluating medical expert systems: what to test and how? Medical Informatics, 15, 1990, pp.205-217. 


\section{Epilepsy}

\subsection{Introduction}

In this chapter both a scientific and a practical view on epilepsy are presented. The scientific part starts with defining the disease and provides a historical overview of various classifications. It shows the impact of technological and pharmacological research on the development of definitions and classifications. Furthermore, epidemiological data are presented. The prognosis and the implications of epilepsy for daily life of the patient will be addressed. Next the view is shifted towards daily practice. The problems the physician encounters when trying to relieve the patient from his seizures by using anti-epileptic drugs (AEDs) are discussed. After the diagnosis is established, a trade-off between the pros and the cons of drug treatment has to be made, given the specific situation of the patient. lssues such as when to start the drug treatment, which drug to select and what initial dose to prescribe will be discussed. As seizures may recur the start of drug treatment for epilepsy implies a longterm commitment to taking medication. Reasons for recurrence of seizures will be addressed. Furthermore the role of anti-epileptic drug monitoring in the treatment process will be discussed. Like in other diseases requiring long-term prophylactic drug treatment such as diabetes or hypertension non-compliance may occur. Causes of non-compliance will be mentioned. Many patients will show an improvement of their seizures. In this case drug withdrawal will be suggested by the patient or the doctor. Aspects of drug withdrawal and the risks of relapse will be described.

\subsection{Description of epilepsy}

Epilepsy is a paroxysmal disturbance of the functioning of the central nervous system. This disturbance is recurrent, stereotype in character and associated with spontaneous, excessive, synchronous and self-limiting neuronal discharge (Aird et al. 1984). It manifests itself in clinically detectable events, i.e. seizures which are perceptible for the subject and/or an observer. Thus, an epileptiform discharge in the EEG registration without a clinical manifestation is not called a seizure. The clinical detectability of an event may change and depends on the tests applied for its registration. For example, a 'bed-side' registration may not detect repeated cognitive impairment during a day while an advanced psychological test can register it 


\subsection{Classifications}

The classification of seizures and epileptic syndromes has changed over the years. This was caused partially by technical and pharmaceutical developments as a historical overview indicates.

\subsubsection{Historical overview}

The early classification of epilepsies was based on the aetiology, symptomatology or anatomy. The aetiology divided the epilepsies into two groups: those which could be attributed to gross cerebral lesions (symptomatic) and those without any identifiable cause (idiopathic) (Laidlaw et al. 1988)

The clinical findings regarding seizures were the basis for differentiation in classifications based on the symptomatology. It was not until 1946 that one could prescribe a drug treatment on the basis of the type of seizure present. At that time trimethadion was discovered. The drug turned out to be most effective against absence seizures (2-15 seconds disturbance or loss of higher psychic capabilities). This finding led to the conclusion that the drug treatment could depend on the type of seizure patterns and urged the need for a better classification of seizures (Cereghino, 1989).

The development of EEG-registration techniques in the late forties and the recognition of typical EEG patterns introduced another factor that could be used in the classification of epilepsies. Classifications of epilepsy based on aetiology (i.e. idiopathic or symptomatic) alone were considered to be inadequate for clinical applications at that time. The use of an EEG registration was required. This technological development resulted in 1954 in a new classification of the epilepsies into centre-encephalic and focal or cortical epilepsies (Dam and Kiørboe, 1982).

Table 2.3-I: International classification of epileptic seizures

\begin{tabular}{|c|c|c|}
\hline Partial scizures & Generalised seizures & Unclassified epileptic seizures \\
\hline A. Simple partial seizures & A. I. Absence seizures & (inadequate or incomplete \\
\hline 1. with molor syinploms & 2. Atypical absence seizures & data) \\
\hline 2. with somatosensory or & B. Myoclonic seizures & \\
\hline special sensory symptorns & C. Clonic seizures & \\
\hline 3. with autonomic symptoms & D. Tonic seizures & \\
\hline 4. with psychic symptoms & E. Tonic-clonic seizures & \\
\hline & F. Atonic seizures & \\
\hline
\end{tabular}

B. Complex partial scizures

Beginning as simple partial seizures and progressing to impainnent of consciousness

with no other features

with features as in A. $1-4$

with automatisnis

With impainment of consciousness at onset

with no other features

with features as in A. 14

with automatisms 
In 1969 a consensus classification for the epileptic seizures was accepted by the International League Against Epilepsy (ILAE) (Gastaut, 1970). A distinction was made between seizures starting locally and seizures being generalised from the onset. The epileptic seizures were categorised based on factors such as clinical features, EEG findings, age at onset, anatomic basis and the aetiology.

Further modification of the classification of epileptic seizures took place in 1981 when the attributes for classification were reduced to clinical manifestations (preferably recorded on videotape) and related EEG findings. This classification was accepted by the ILAE (Table 2.31). The eliminated factors such as aetiology, age at onset and anatomy were taken into account in the classification of epilepsies.

Besides the classification of seizures also a classification of the epilepsies was proposed (Merlis, 1970). The classification of epilepsies distinguished between generalised and partial epilepsies. Clinical characteristics such as aetiology, seizures, neurological status and age of onset were also used for classifying epilepsies. Still much overlap was present in the attributes used for classifying the seizures and epilepsies. In 1985 a new classification for epilepsies was proposed but it was not accepted until 1989 (Table 2.4-II). This classification retains the distinction of epilepsies in generalised and partial (localisation related) categories, based on the localisation of the onset of the seizures, on the cause (both idiopathic or symptomatic) and the age at onset. Furthermore two new categories were added:

- Epilepsies and syndromes of which it is not clear whether they are focal or generalised

- A category of special syndromes which is used for seizures occurring in special circumstances such as fever, intoxication or use of drugs (Commission on Classification and Terminology of the ILAE, 1989).

\subsubsection{Importance of different classifications}

Selection of the drug treatment can be based on the diagnosed seizure type and/or the type of epilepsy. The role of these detailed classification schemes in patient management is, however, rather limited. The seizure classification is mainly based on clinical findings and to a lesser extent connected with the neurophysiological and chemical basis of the convulsions. In fact the classification scheme is more detailed than the knowledge about basic mechanisms of available anti-epileptic drugs (Dichter 1994, Macdonald and Kelly 1994, Montouris 1995, Brodie and Dichter 1996).

The classification of the epilepsies includes factors such as aetiology and natural history which allows for a more complete description of the patient enabling an assessment of the prognosis. Although there are special types of epilepsy which require a specific drug treatment the classification of epilepsies is more detailed then necessary for drug treatment

More recently, new definitions of epilepsy and seizures and seizure type classification have been proposed to bridge the gap between research and patient management (Commission on Epidemiology and Prognosis of the ILAE, 1993)

\subsection{Epidemiology}

There are two main problems in obtaining statistics on epilepsy. The first problem is related to the definition of the disease. The second problem is caused by the sources from which data are gathered 
Table 2.4-II: International classification of epilepsics, epilcptic syndromes and related seizure disorders (ICE)

\begin{tabular}{|c|c|}
\hline Localisation related (focal, local, partial) & Generalised \\
\hline $\begin{array}{l}\text { Idiopathic (Primary) } \\
\text { Benign childhood epilepsy with centre-temporal spike } \\
\text { Childhood epilepsy with occipital paroxysins } \\
\text { Primary reading epilepsy }\end{array}$ & $\begin{array}{l}\text { Idiopathic (primary) } \\
\text { Benign neonatal farnilial convulsions } \\
\text { Benign neonatal convulsions } \\
\text { Benign myoclonic epilepsy in infancy } \\
\text { Childhood absence epilepsy (pyknolepsy) } \\
\text { Juvenile absence epilepsy } \\
\text { Juvenile myoclonic epilepsy (impulsive petit mal) } \\
\text { Epilepsies with grand mal seizures (GTCS) on awakening } \\
\text { Other generalised idiopathic epilepsies } \\
\text { Epilepsies with seizures precipitated by specific modes of } \\
\quad \text { activation }\end{array}$ \\
\hline $\begin{array}{l}\text { Symptomatic (secondary) } \\
\text { Temporal, frontal, parietal and occipital lobe epilepsies } \\
\text { Chronic progressive epilepsia partialis continua of } \\
\text { childhood } \\
\text { Syndrones characterised by seizures with specilic modes } \\
\text { of precipitation }\end{array}$ & $\begin{array}{l}\text { Cryptogenic or synptomatic } \\
\text { West syndrome (infantile spasins, Blitz-Nick-Salaam } \\
\text { Krämpfe) } \\
\text { Lennox-Gastaut syndrome } \\
\text { Epilepsy with myoclonic-astatic seizures } \\
\text { Epilepsy with myoclonic absences } \\
\text { Symptomatic } \\
\text { Non-specific aetiology } \\
\text { Early myoclonic encephalopathy } \\
\text { Early infintile epileptic encephalopathy } \\
\text { with suppression burst } \\
\text { Other symptomatic generalised } \\
\text { epilepsies } \\
\text { Specific syndromes } \\
\text { Epileptic scizures may complicate many } \\
\text { disease states }\end{array}$ \\
\hline \multicolumn{2}{|l|}{$\begin{array}{l}\text { Defined by seizure type, clinical features, aetiology, } \\
\text { anatomical localisation }\end{array}$} \\
\hline $\begin{array}{l}\text { Undetermined epilepsies } \\
\text { With both generalised and focal scizures } \\
\text { With unequivocal generalised or focal features }\end{array}$ & \\
\hline $\begin{array}{l}\text { Spccial syndromes } \\
\text { Situation related seizures (Gelegenheitsanfälle) } \\
\text { Febrile convulsions } \\
\text { Isolated seizures or isolated status epilepticus }\end{array}$ & \\
\hline $\begin{array}{l}\text { Seizures occurring only when there is an acute or } \\
\text { nonketotic hyperglycaemia }\end{array}$ & xic event due to faclors such as alcohol, drugs, eclampsia, \\
\hline
\end{tabular}




\subsubsection{Problems}

The translation of the definition of epilepsy provided by Gastaut and Kugler (1976) and used by the WHO is:

\section{'A chronic brain disorder of various aetiologies characterised by recurrent seizures dive to excessive discharge of cerebral neurones [...]. Single or occasional epileptic seizures as well as those occurring during an acute illness should not be classified as epilepsy'.}

The problem is that this definition is not operational enough. The definition excludes the use of the term epilepsy for seizures provoked by disorders primarily not involving the brain. The International Classification of the Epilepsies does include these as special syndromes. The term 'chronic' is ambiguous. It does not state the period during which the symptoms must be present before one can decide whether the patient really has epilepsy. This means that patients who suffer from several seizures per year as well as those with one seizure every two years can be included as epileptic patients in epidemiological studies. Whether or not seizures should still be manifest to be regarded as epilepsy not withstanding medication is not clear from this definition. It is also not clear when patients are considered to be cured.

The second part of the definition does exclude seizures directly related to a disease. Examples of such seizures are febrile convulsions and sporadic seizures caused by e.g. alcohol withdrawal. However, others claim that there exists insufficient ground to exclude occasional seizures from the diagnosis (Hopkins, 1987).

The ascertainment of epileptic cases for epidemiological studies may be a problem (Aird et al. 1984). The use of medical records results in an underrepresentation of the milder and nonactive epilepsies as well as of undiagnosed and untreated cases, as some patients may not ask for a medical evaluation of their symptoms (Haerer et al. 1986). The severity of the seizures, the social labelling because of the disease, the educational level or the socio-economic status and consequences may influence the decision to consult a health care professional. Field studies suggest that some 4 to $25 \%$ of the patient with epilepsy were never treated for their seizures (Haerer et al. 1986, Zielinski 1988, Keränen and Riekkinen 1993). The range in percentages may be attributed to different case finding methods and diagnostic criteria.

Patient denial also influences epidemiological data obtained from medical records. In an Italian study denial of the disease was reported in $31 \%$ of a patient population (Maremmani et al. 1991).

Data can be obtained from sources other than the medical record, e.g. death certificates and insurance company data. These alternatives have their own biases. Death certificates are generally available but incomplete in reporting epilepsy. Data obtained from insurance companies mainly present a selected population, which may exclude e.g. unemployed people or people with a low economic status. Additional biases may exist due to patients who do not reveal their disease to an insurance company to prevent exclusion or higher financial contributions.

\subsubsection{Prevalence and incidence}

The number of patients per 1000 persons in a given population at a given time is called the prevalence of the disease. The influence of how epilepsy is defined on prevalence data has been demonstrated in two studies concerning 6000 patients from a general practice (Goodridge and Shorvon 1983a, Cockerell et al. 1995a). 
Table 2.4-I: Prevalence of epilepsy in Europe and United Statcs

\begin{tabular}{|c|c|c|c|c|c|c|}
\hline Country & First author & Definition & Study & Sources" & Age & Prev** \\
\hline \multirow[t]{3}{*}{ Denumark } & Juul- Jensen & epilepsy ${ }^{\prime}$ & retrospective & $\mathrm{MH} / \mathrm{II} / \mathrm{DR}$ & & $12.7^{2}$ \\
\hline & & & 37 ytars & & & \\
\hline & Wagner (1983) & active epilepsy ${ }^{3}$ & retrospective ${ }^{4}$ & $\begin{array}{l}\text { MH/EH/DPP } \\
\text { GP }\end{array}$ & $16-66$ & 4.32 \\
\hline Finland & Keränen (1989) & active epilepsy & $\begin{array}{l}\text { retrospective } \\
19 \text { years }\end{array}$ & $\begin{array}{l}\mathrm{MH} / \mathrm{Ps} / \mathrm{EH} \\
\mathrm{MMR} / \mathrm{HC} / \mathrm{NH} \\
\mathrm{EL} / \mathrm{PR}\end{array}$ & $>15$ & 6.29 \\
\hline \multirow[t]{2}{*}{ Italy } & Granieri (1983) & active epilepsy & $\begin{array}{l}\text { retrospective } \\
18 \text { years }\end{array}$ & $\begin{array}{l}\text { MH/ND/NS } \\
\text { PD/EL/LD/DPP } \\
\mathrm{Ch} / \mathrm{T} / \mathrm{SW} \\
\mathrm{MeH}\end{array}$ & all & $(6.4)^{5}$ \\
\hline & Mareminani (1991) & active epilepsy & retrospective & $\begin{array}{l}\text { H/LD/PR/EHn } \\
\text { SD/SW }\end{array}$ & all & $\begin{array}{l}5.1 \\
(5.2)^{5}\end{array}$ \\
\hline \multirow[t]{2}{*}{ Norway } & De Gruaf (1974) & epilepsy & retrospective & ND/EL/IMR & all & 3.5 \\
\hline & & & 5 years & & & \\
\hline Sweden & Forsgren (1992) & active epilepsy & retrospective & ND/NS/M/Ps & $17^{x}$ & 5.5 \\
\hline & & & 13 years & $\begin{array}{l}\text { GD/EL/SLR } \\
\text { DPP/DN }\end{array}$ & & \\
\hline \multirow{4}{*}{$\begin{array}{l}\text { The Nether- } \\
\text { lands }\end{array}$} & Bongers (1976) & epilepsy & retrospective & GP & all & 2.9 \\
\hline & Rutgers (1984) & $\begin{array}{l}\text { active epilcpsy } \\
\text { last } 2 \text { yeatss }\end{array}$ & prospective & GP & $15-66$ & 4.0 \\
\hline & Crobach (1988) & active epilepsy & retrospective & GP/Ch & all & 3.3 \\
\hline & Metsemakers (1994) & epilepsy ${ }^{7}$ & retrospective & GP & all & 8.5 \\
\hline \multirow[t]{3}{*}{$\begin{array}{l}\text { United King- } \\
\text { dom }\end{array}$} & Crombie (1960) & $\begin{array}{l}\text { active epilepsy } \\
\text { last } 2 \text { years }\end{array}$ & $\begin{array}{l}\text { retrospeclive } \\
3 \text { years }\end{array}$ & GP & all & 4.19 \\
\hline & Goodridge (1983a) & $\begin{array}{l}\text { active epilepsy } \\
\text { last } 2 \text { years }\end{array}$ & $\begin{array}{l}\text { retrospective } \\
35 \text { years }\end{array}$ & GP & all & 5.3 \\
\hline & Cockerell (1995a) & $\begin{array}{l}\text { active epilepsy } \\
\text { last } 2 \text { years }\end{array}$ & $\begin{array}{l}\text { retrospective } \\
10 \text { years }\end{array}$ & GP & all & 4.3 \\
\hline \multirow[t]{3}{*}{ Uniled States } & Hauser $(1975)$ & active epilepsy & $\begin{array}{l}\text { retrospective } \\
25 \text { yuirs }\end{array}$ & HIS/MH & all & 5.7 \\
\hline & Haerer (1986) & active epilepsyn & retrospective & $\begin{array}{l}\text { door-10-door } \\
\text { MI LNH }\end{array}$ & all & 6.78 \\
\hline & Hauser (1991) & uctive epilepsy & $\begin{array}{l}\text { retrospective } \\
40 \text { years }\end{array}$ & $\mathrm{H} \amalg \mathrm{S} / \mathrm{MH}$ & all & 6.8 \\
\hline
\end{tabular}

- $\mathrm{Ch}=$ Chemist, $\mathrm{DR}=$ Death register, EL=EEG-lab, EH=Epilepsy hospilal, H=Hospital, HIS=Hospital information system, $\mathrm{MH}=$ Multiple hospitals, MeH=Mental hospital, $\mathrm{HC}=$ Health centre, $\mathrm{LD}=\mathrm{Local}$ doctors, $\mathrm{ND}=\mathrm{New}$ ology dept, $\mathrm{NS}=$ Neurosurgery dept., $\mathbb{M}=\mathrm{Dept.}$ Internal Medicine, $\mathrm{IR}=$ Insurance register, $S \mathbb{R}=\mathrm{Social}$ insurance register, $\mathrm{SD}=\mathrm{Sch} \infty \mathrm{l}$ doctors, $S W=$ Social workers, $P D=$ Pediatric Dept., $G D=$ Geriatrics Dept., GP=General practitioner, PSD=Psychiatry dept, $\mathrm{DPP}=\mathrm{Doctors}$ with private practice. $\mathrm{DN}=$ District uurses, $\mathrm{DMR}=$ Inst. mentally retarded, $\mathrm{NH}=$ Nursing homes, $\mathrm{PR}=\mathrm{Pharmacy}$ register, $T=$ Teacher,

** per 1000 population

(1) Epilepsy without exclusion of patients seizure free for more than 5 years and not on anti-epileptic drug treatment, (2) Cumulative incidence, (3) Epilepsy $\geq 2$ spontaneous seizures excluding patients with controlled seizures and not receiving treatunent, (4) Registration depending on setting. $1-18$ years, (5) Prevalence corrected toward the Italian population, (6) On AED and having $\geq 1$ seizure in 3 years or not on AED and having $\geq 1$ seizure within last year, (7) active and inactive epilepsy. 
The prevalence of epilepsy excluding febrile seizures but including cases such as single, recurrent, active and inactive epilepsy was 20.3 and 21 in the 1983 and 1993 studies respectively. This number dropped to 17.0 by excluding patients with single seizures. An even lower prevalence - 10.5 - was found by including only those patients receiving anti-epileptic treatment and those having active epilepsy defined as at least one seizure within the last 24 months. Prevalence further decreased to 5.3 in 1983 and to 4.3 in 1993 by including only patients with active epilepsy. In the USA improved case ascertainment strategies resulted in different prevalence rates in the same population studied (Hauser and Kurland 1975, Hauser et al. 1991). The use of broad inclusion criteria - not excluding patients free from seizures for more than 5 years and including patients not on anti-epileptic drug treatment - caused the high prevalence rate of the Danish study (Table 2.4-I) (Juul-Jensen and Foldspang, 1983). The minimal prevalence rate of convulsive disorders was estimated to be $4-6 / 1000$ (Cereghino 1974, Rutgers 1984).

The number of new cases within 1 year per 100000 persons is called the incidence of a disease. It describes the rate with which new cases of the disease occur. The incidence rates suffer the same problems as the prevalence. The classic study of incidence is from Rochester, Minnesota. In this study it was shown that the incidence of recurrent seizures was 44 patients per 100000 persons per year over a 50 year period (Hauser et al. 1993). Similar results with average annual incidence rates for recurrent seizures in between 30 and 50 were reported previously (Hauser 1978, Granieri et al. 1983. Zielinski 1988, Keränen et al. 1989, Forsgren 1990). The incidence increased to 61 per year if cases with single seizures are included (Hauser et al. 1993). Inclusion of febrile seizures leads to an even higher incidence, 115 per year (Hauser, 1978)

Incidence rates vary with age. The incidence rate is highest during the first decade, after which it decreases. An increase in the number of new cases is observed after the sixth decade (Zielinski 1974, Lühdorf et al. 1986, Zielinski 1988, Hauser et al. 1991, Hauser 1992). While the incidence for the population was 44 , the highest incidence, 139, was observed in persons over 75 years of age (Hauser et al. 1993). The increase in the number of tumours and cerebrovascular diseases has been suggested as a possible cause. Another explanation for the increase might be the higher level of case ascertainment for elderly people or more extensive medical care for this group (Schoenberg 1983, Hauser et al. 1993)

The incidence rates for children and elderly persons have been compared over the years. These measurements show that the incidence for children decreases while the incidence rate for the elderly increases (Hauser et al. 1993, Cockerell et al. 1995a). These changes may have been caused by improved perinatal care and increased medical attention for the elderly.

\subsection{Aetiology}

Determining the type of seizure and the type of epilepsy is only half of the diagnostic process. Establishing the aetiology is the other. Aird et al (1984) use both causative and underlying neurochemical and neurophysiological factors to describe the aetiology. These factors may suggest possibilities for the treatment of seizures. The main causative factors for epilepsy are genetic and birth defects, infections, toxic mechanisms, traumata, circulatory causes (e.g. haemorrhage, thrombosis), metabolic and nutritional disorders, neoplasms, heredofamilial and degenerative diseases. The neurochemical description of etiologic factors for epilepsy include metabolic derangements as e.g. hypoxia, hypoglycaemia, electrolyte and acid-base imbalances, vitamin deficiencies, changes in excitatory and inhibitory neurotransmitters, endocrine 
dysfunctions, endogenous toxic states and hyperpyrexia. Most epidemiological studies reporting on aetiology, however, describe only the causative factors. Another approach is to distinguish between cause - a more or less steady-state background factor - and precipitating factors which are short term factors which may cause seizures in susceptible people (Hopkins, 1987).

Table 2.5-1: Ethiological factors in epilepsy

\begin{tabular}{llllllllll}
\hline First author & $\begin{array}{l}\text { De Graal } \\
(1974)\end{array}$ & $\begin{array}{l}\text { Bergamini } \\
(1977)\end{array}$ & $\begin{array}{l}\text { Hauser } \\
(1978)\end{array}$ & $\begin{array}{l}\text { Granieri } \\
(1983)\end{array}$ & $\begin{array}{l}\text { Cowan } \\
(1989)\end{array}$ & $\begin{array}{l}\text { Mcinardi } \\
(1990)\end{array}$ & $\begin{array}{l}\text { Sander } \\
(199)\end{array}$ & $\begin{array}{l}\text { Hauser } \\
(1991)\end{array}$ & $\begin{array}{l}\text { Forsgren } \\
(1992)\end{array}$ \\
\hline Age & $>15 y$ & $1 \mathrm{~m}-21 \mathrm{y}$ & all & all & $<19$ y & all & all & all & $>16$ y \\
Unknown & $68 \%$ & $56 \%$ & $64 \%$ & $60.9 \%$ & $69 \%$ & $28.8 \%$ & $61 \%$ & $76 \%$ & $64.8 \%$ \\
Cer.vase. & $4 \%$ & nav & $5 \%$ & $2.9 \%$ & 3 & $1.9 \%$ & $15 \%$ & $6 \%$ & $11.8 \%$ \\
Cer.tumor & $2 \%$ & nav & $4 \%$ & $1.4 \%$ & 3 & $3.5 \%$ & $6 \%$ & $2 \%$ & $4.7 \%$ \\
Alc./loxic & $3 \%$ & excl. & excl. & nav & $2 \%$ & nav & $6 \%$ & excl. & excl. \\
Post-traum. & $9 \%$ & $12.2 \%$ & $5 \%$ & $6.8 \%$ & $4 \%$ & $10.4 \%$ & $3 \%$ & $5 \%$ & $7.2 \%$ \\
Cong./Gen. & $2 \%$ & $1.9 \%$ & $4 \%$ & $1.1 \%$ & $3 \%$ & $3.2 \%$ & 4 & $5 \%$ & $2.6 \%$ \\
Lufections & $2 \%$ & $11.9 \%$ & $3 \%$ & $3.7 \%$ & $3 \%$ & $5.1 \%$ & $2 \%$ & $4 \%$ & $3.5 \%$ \\
Perinatal & $9 \%$ & $17.8 \%$ & nav & $23.7 \%$ & $7 \%$ & $40.2 \%$ & 4 & nav & $5.4 \%$ \\
Febr.conv. & nav & excl. & nav & nav & 3 & $6.9 \%$ & 4 & nav & nav \\
Other cause & nav & nav & $15 \%$ & nav & 3 & nav & $7 \%$ & $1 \%$ & nav \\
\hline
\end{tabular}

(1) data from tertiary care centre, (2) nav, (3) other and multiple causes $12 \%$, (4) this included congenital perinatal and febrile convuisions, (5)history of febrile convulsions

Studies indicate that in $56-76 \%$ of the patients the aetiology of their epilepsy is unknown (Table 2.5-1). Part of this depends on the assessment of a genetic basis for idiopathic epilepsies. As multiple genes may be involved a family history will underestimate genetic causes. Differences in case finding strategies are also important. For example, a high rate of perinatal causes reported by Granieri et al. (1983) can be explained by the fact that this aetiology was more intensely looked for as there was an urgency in improving the prenatal and perinatal care in the region. The study conducted by Meinardi and Pachlato (1985) shows fewer unknowns and a higher percentage of perinatal factors in a population of a special centre for epilepsy.

The aetiology of epilepsy varies with the age of the patient (Janz 1969, Sander et al. 1990, Hauser 1992). In the neonatal period birth-injuries - haemorrhage and anoxia - may cause epilepsy. Also congenital abnormalities, metabolic disorders and infections can induce epileptic seizures. In early childhood febrile seizures, trauma infection, cerebral degeneration, poisons and metabolic defects may cause epilepsy. In childhood and adolescence idiopathic (primary) epilepsy may manifest itself. In addition to this, trauma, infections and cerebral degeneration can induce epilepsy during this period. It has been reported that in $67.6 \%$ of the patients younger than 15 years the cause of the epilepsy was unknown, while in $20 \%$ a congenital defect, in $4.7 \%$ a trauma and in $4 \%$ an infection was identified as the cause of their disease (Hauser, 1992). In early adult life trauma, tumour or infection may cause epilepsy while juvenile idiopathic epilepsies also occur. Birh injuries and cerebral degeneration can still play a role as a cause of epilepsy in this period 
During late adult life vascular disease, trauma, tumour and cerebral degeneration are the most frequent causes for epilepsy (Marsden and Reynolds, 1988). Also alcohol abuse has been mentioned as a frequent cause of epilepsy. In a study on late-onset epilepsy (starting after the age of 25 years) made up a group of one fourth of all patients, while the cause was unknown in $38 \%$. In $16 \%$ of the patients a brain tumour was the cause, cerebrovascular infarctions were registered in $14 \%$ of the patients. Head trauma accounted for $4 \%$ of the cases and miscellaneous causes such as angioma, brain abscess or leukoencephalopathy accounted for $5 \%$ of the patients (Mouritzen Dam et al. 1985). In a field study in elderly over 65 years the percentage of cases with an unknown cause was $48.9 \%$, while $32.4 \%$ had a vascular cause. A degenerative cause was reported in $11.5 \%$ and neoplasms were reported in $2.7 \%$ of these cases (Hauser, 1992). Others reported from a clinical setting that in patients over 60 years cerebrovascular disease was the cause in $32 \%$, tumours in $14 \%$ while in $25 \%$ the cause was unknown (Lühdorf et al. 1986).

\subsection{Prognosis}

There are four possible courses for epilepsy:

- Seizures remit spontaneously and no treatment is required

- Seizures are controlled with drug treatment and the medication can successfully be withdrawn

- Seizures are controlled but anti-epileptic drug treatment must be continued

- Seizures continue even when on anti-epileptic drug treatment

While problems may occur in all four groups, drug related and/or psychosocial problems can be expected, particularly in the third and the fourth category. As these groups require a lifelong treatment, the etiological classification of the epilepsy may change e.g. due to a gradual manifestation of an oncologic cause. This means that in these patients the correctness of the diagnosis has to be periodically reviewed. A sudden change in effectivenuss of the treatment due to alterations in the metabolism of the drug, concomitant diseases or comedication may occur. Drug toxicity may develop gradually, especially with multiple drugs. Besides this, the compliance can change because of side-effects of the drugs or absence of seizures or due to avoiding the daily confrontation with the disease. Such a change in compliance may lead to recurrence of seizures. Also an irregular life-style may alter the effectiveness of the treatment. Patients in both these categories will need attention from the physician on a regular basis.

\subsubsection{Remission}

The idea that epilepsy is a chronic condition especially if left untreated - seizures beget seizures - has been stated already in the last decades of the $19^{\text {th }}$ century (Gowers, 1885 , reprinted in 1994). This idea has lasted for over 80 years. However, this view is gradually changing. Population studies indicate that $4.1 \%$ to $26 \%$ of the patients with epilepsy do not receive anti-epileptic drug treatment (Zielinski 1974, Goodridge and Shorvon 1983b, Haerer et al. 1986, Keränen and Rjekkinen 1993, Cockerell et al. 1995a). In some of these patients epilepsy remitted spontaneously. It is however difficult to be certain of the diagnosis of epilepsy in these patients who reported seizures in the past but on index day had been free from seizures for several years. The number of patients reported to be seizure free for at least 2 years at 10 and 20 years after onset of the epilepsy were $42 \%$ and $52 \%$ respectively 
(Keränen and Riekkinen, 1993). Some 30\% were seizure free for more than 5 years (Zielinski, 1974). Comparison of incidence and prevalence rates indicates that epilepsy lasts 13 years on average. More recent studies in developing countries also suggest that epilepsy may remit without drug treatment (Sander, 1993).

In the traditional view on epilepsy the prognosis was considered poor with only $20 \%$ going into a long-term remission. In the last two decades studies on remission of seizures show that $35-90 \%$ of patients can achieve a remission of 2 years during some period within 2 to 10 years after starting drug treatment (Shorvon and Reynolds 1982, Elwes et al. 1984, Collaborative Group for Study of Epilepsy 1992). Remission of seizures lasting at least 5 years were reported in $61 \%$ of newly diagnosed patients 10 years after the diagnosis. The chance of having achieved a 5 year remission within 10 and 20 years was $65 \%$ and $76 \%$ respectively (Annegers et al. 1979). A similar result - 68\% seizure free for 5 years or more during nine years of follow-up - was found in a national general practitioners study (Cockerell et al. 1995b).

This means that many patients tend to go into a long-term remission during follow-up. At 20 years after diagnosis $70 \%$ of the patients were in remission (Annegers et al. 1979)

Part of the patients with a remission need medication continuously as the anti-epileptic drugs only suppress the occurrence of seizures instead of curing the disease. While $70 \%$ of the patients were in remission some $50 \%$ were seizure free for 5 years and did not take anticonvulsive medication 20 years after the initial diagnosis, while $20 \%$ of the patients continued to take anti-epileptic drugs although they were free for at least 5 years without seizures (Annegers et al. 1979). Others report that 13\% of the patients which were at least 3 years seizure free after 9 years of follow-up were still taking anti-epileptic drugs (Cockerell et al. 1995b).

Table 2.6-I: Remission rates of scizures in community and hospital based studies.

\begin{tabular}{|c|c|c|c|c|c|c|}
\hline First author & $\begin{array}{l}\text { Annegers } \\
(1979) \\
N=618\end{array}$ & $\begin{array}{l}\text { Elwes } \\
(1984) \\
N=106\end{array}$ & $\begin{array}{l}\text { Zielinski } \\
(1988) \\
N=98\end{array}$ & $\begin{array}{l}\text { CGSE }^{\prime} \\
(1992) \\
N=280\end{array}$ & $\begin{array}{l}\text { Keränen }^{2} \\
(1993) \\
N=33\end{array}$ & $\begin{array}{l}\text { Cockerell } \\
(1995 b) \\
N=564\end{array}$ \\
\hline Study & retrospective & prospective & prospective & prospective & retrospective & prospective \\
\hline Setting & $\begin{array}{l}\text { community } \\
\text { based }\end{array}$ & neur.clin. & $\begin{array}{l}\text { community } \\
\text { based }\end{array}$ & hospital & hospital & $\begin{array}{l}\text { community } \\
\text { based }\end{array}$ \\
\hline $\begin{array}{l}\text { Follow-up } \\
\text { duration }\end{array}$ & $\begin{array}{l}328>10 \text { years } \\
141>20 \text { years }\end{array}$ & $\begin{array}{l}\text { median } 66 \mathrm{mths} \\
\text { (range 6-96) }\end{array}$ & 5 years & $\begin{array}{l}\text { median } 48 \text { mths } \\
\text { (range } 1-87 \text { ) }\end{array}$ & $>2$ years & median 7.1 ys \\
\hline Remission & $\geq 5$ years & $\geq 2$ years & $\geq 2$ years & $\begin{array}{l}\geq 2 \text { and }<3 \\
\text { years }\end{array}$ & $\geq 2$ years & $\begin{array}{l}>3 \text { years } \\
>5 \text { years }\end{array}$ \\
\hline \multirow[t]{2}{*}{$\begin{array}{l}\text { \%opatients } \\
\text { achicving } \\
\text { remission }\end{array}$} & $\begin{array}{l}65 \% \text { at } 10 \text { ys } \\
\text { f.u }{ }^{3} \\
76 \% \text { at } 20 \text { ys } \\
\text { f.u. }{ }^{3}\end{array}$ & $\begin{array}{l}35 \% \text { at } 2 \text { ys } \\
6 u^{3} \\
57 \% \text { at } 3 \text { ys } \\
\text { fu? }\end{array}$ & & $\begin{array}{l}71 \% \text { at } 3 \text { ys } \\
\int .{ }^{3} \\
90 \% \text { at } 5 \text { ys } \\
\text { f.u. }{ }^{3}\end{array}$ & & $\begin{array}{l}68 \% \text { at } 9 \text { ys } \\
\text { f.u. in } 3 \text { year } \\
\text { remission }\end{array}$ \\
\hline & $\begin{array}{l}61 \% \text { at } 10 \text { ys } \\
\text { f.u. } \\
70 \% \text { at } 20 \text { ys } \\
\text { f.u. }\end{array}$ & $\begin{array}{l}79 \% \text { al } 5 \text { ys } \\
\text { f.u }{ }^{3} \\
82 \% \text { at } 8 \text { ys } \\
\text { r.u. } \\
48 \% \text { tree } 2 y^{4} s^{3}\end{array}$ & $\begin{array}{l}6.3 \% \text { at } 5 \text { ys } \\
\text { f.u. }\end{array}$ & & $\begin{array}{l}42 \% \text { at } 10 \text { ys } \\
\text { fu. }{ }^{4} \\
52 \% \text { at } 20 \text { ys } \\
\text { f. }{ }^{4}\end{array}$ & $\begin{array}{l}54 \% \text { at } 9 \text { ys } \\
\text { f.u. in } 5 \text { year } \\
\text { remission }\end{array}$ \\
\hline
\end{tabular}

(1) Collaborative Group for Study of Epilepsy, (2) Untreated epilepsy patients, (3) Cumulative percentage, not considering the continuation of the remission until end of study, (4) Percentage of seizure free patients at moment of follow-up (f.u.). 
It is estimated that in $20-30 \%$ of the patients seizures will continue despite anti-epileptic drug treatment (Annegers et al. 1979, Goodridge and Shorvon 1983b, Shorvon 1991, Cockerell et al. 1995a). Eight percent of the patients does not achieve a seizure free period of one year during 8 years of follow-up. A two year seizure free period was not reached by $18 \%$ of the patients (Elwes et al. 1984). Nevertheless, even those patients who are not in remission 5 years after diagnosis may still have a chance of $33 \%$ of going into a remission during the next 10 years (Annegers et al. 1979).

Differences in remission rates may be attributed to case ascertainment, e.g. hospital based studies contain an overrepresentation of the chronic uncontrolled patients, with newly diagnosed patients and patients in remission are underrepresented. Furthermore, differences in duration of follow-up or discrepancies in the definition of the term remission may account for the differences in the percentages of people who are seizure free (Shorvon, 1984).

Also the definition of epilepsy is important for assessing the remission rates. Several studies show a higher remission rate when patients with possible epilepsy are included in the study (Reynolds 1987, Hart et al. 1990, Collaborative Group for Study of Epilepsy 1992, Cockerell et al. 1995a,b). All studies presented in Table 2.6-I include patients with more than one seizure.

\subsubsection{Problems in establishing prognostic factors}

Although some studies suggest that epilepsy may remit spontaneously most studies appear to support an observation made by Gowers that epilepsy is a process which in case it is not treated early may lead to a chronic state of seizures which are more difficult to treat later on (Elwes et al. 1988, Reynolds 1988, Elwes and Reynolds 1990, Reynolds 1990). In this view factors influencing the outcome of the disease are important.

Studies concerning the prognosis of epilepsy are difficult to assess as their results depend on the medical setting where the data are obtained. In contrast with studies in a clinical setting one may expect higher remission rates of epilepsy in community based studies, given the fact that some of the patients do not visit their physician because the seizures do not worry them (Zielinski 1974, Haerer et al. 1986, Keränen and Riekkinen 1993).

If studies on epilepsy are undertaken with patients who had only one seizure, patients and doctor's delay may introduce a bias towards less severely diseased patients. The longer the delay the higher the probability that the patient suffered another seizure and is not included in the study.

\subsubsection{Prognostic factors}

In the literature various conditions are mentioned that suggest a poor prognosis (Annegers et al. 1979, Shorvon and Reynolds 1982, Elwes et al. 1984, Shorvon 1984, Beghi and Tognoni 1988, van Donselaar 1990, Collaborative Group for Study of Epilepsy (CGSE) 1992):

- A high frequency ( $\geq 2$ per month) of tonic-clonic seizures before treatment

- More than one seizure in the first year of treatment or a relapse early during treatment

- Multiple types of seizures or partial seizures

- Additional neurologic, psychiatric or social handicaps as well as a family history of epilepsy 
Table 2.6-II: Prognostic factors for the course of epilepsy.

\begin{tabular}{|c|c|c|c|c|c|c|}
\hline First author & $\begin{array}{l}\text { Annegers } \\
(1979) \\
N=618\end{array}$ & $\begin{array}{l}\text { Shorvon } \\
(1982) \\
N=94\end{array}$ & $\begin{array}{l}\text { l:lwes } \\
(1984) \\
N=106\end{array}$ & $\begin{array}{l}\text { Hopkins } \\
(1988 \text { ) } \\
N=408\end{array}$ & $\begin{array}{l}\text { CGSE } \\
(1988) \\
N=283\end{array}$ & $\begin{array}{l}\text { v. Donselaar } \\
(1990) \\
N=151\end{array}$ \\
\hline Study & retrospective & prospective & prospective & prospective & prospective & prospective \\
\hline Setting & $\begin{array}{l}\text { community } \\
\text { based }\end{array}$ & neur.clinic & neur clinic & $\begin{array}{l}\text { in/out } \\
\text { private pat }\end{array}$ & multicentre & hospital \\
\hline $\begin{array}{l}\text { Type of } \\
\text { patients }\end{array}$ & new referrals & $\begin{array}{l}\text { new referrals } \\
6-77 \text { years } \\
\mu=23\end{array}$ & $\begin{array}{l}\text { new referrals } \\
6-77 \text { years } \\
\mu=23\end{array}$ & $\begin{array}{l}\text { new referrals } \\
>16 \text { years }\end{array}$ & $\begin{array}{l}\text { new referrals } \\
2-81 \text { years } \\
\mu=19\end{array}$ & $\begin{array}{l}\text { new referrals } \\
15-85 \text { years } \\
\mu=38\end{array}$ \\
\hline $\begin{array}{l}\text { Moment of } \\
\text { inclusion }\end{array}$ & $>2$ seizures & $>2$ seizures & $>2$ seizures & $I^{\text {st }}$ seizure & $\begin{array}{l}\text { start treat- } \\
\text { ment }\end{array}$ & $1^{\text {st }}$ seizure \\
\hline $\begin{array}{l}\text { Therapy } \\
\text { before } 2^{\text {nd }} \\
\text { seizure }\end{array}$ & included & excluded & excluded & included & excluded & excluded \\
\hline Follow-up & $\begin{array}{l}328>10 \text { years } \\
141>20 \text { years }\end{array}$ & 13-33 months & $6-96$ months & 36 months & 2.40 months & 12-24months \\
\hline Age & & not related & & not related & & \\
\hline Sex & not related & not related & & & & \\
\hline $\begin{array}{l}\text { Seizure } \\
\text { type }\end{array}$ & related & & $\begin{array}{l}\text { partial seizu- } \\
\text { res related }\end{array}$ & not related & $\begin{array}{l}\text { multiple sei- } \\
\text { zures related }\end{array}$ & $\begin{array}{l}\text { multiple sei- } \\
\text { zures related }\end{array}$ \\
\hline $\begin{array}{l}\text { Seizures } \\
\text { before/anter } \\
\text { therapy }\end{array}$ & & related & related & & related & \\
\hline $\begin{array}{l}\text { Age at on- } \\
\text { set }\end{array}$ & related & not related & not related & not related & & not related \\
\hline Histories & & not related & $\begin{array}{l}\text { family history } \\
\text { related }\end{array}$ & not related & & not related \\
\hline Time of day & & & not related & related & & related \\
\hline Handicap & related & related & related & & & \\
\hline EEG & & not related & not related & not related & & related \\
\hline
\end{tabular}

Factors not related to the prognosis or factors about which disagreement with respect to their relation to the prognosis of epilepsy exists in the literature (Table 2.6-II) are (Annegers et al. 1979, Shorvon and Reynolds 1982, Elwes et al. 1984, Hopkins et al. 1988, van Donselaar 1990):

- EEG findings after a first idiopathic seizure. Some found a relation between (pretreatment) EEG findings and seizure recurrence. Others, however, did not find such a relation.

- The importance of the time during the day at which the seizure occurs. Some researchers did report a relation between time of the day and the risk of recurrence others deny a prognostic value for this factor. 
- Factors such as gender, age or age at onset. Some report a relation between age at onset and the probability of going into remission. Especially younger patients with idiopathic epilepsy were likely to become seizure free. Such a relation was not observed by others.

\subsection{Some implications of epilepsy}

Epilepsy may have various consequences in daily life. People may experience difficulties in finding and keeping a job, their drivers licence may be withdrawn or they have to find alternative leisure activities.

\subsubsection{Employment}

Difficulties with employment is one of the problems most frequently reported by patients (Thompson and Oxley 1988, Chaplin et al. 1992). The problem seems to be of less relevance during periods of full employment (Crombie et al. 1960). However, during a period of high unemployment it has been shown that $46 \%$ of the potentially economically active patients were unemployed compared with $19 \%$ of a control population (Elwes et al. 1991). The employers as well as colleagues (even within health care environments) have an exaggerated fear of accident-proneness and decrease of productivity (Aspinal 1986, Betts 1986). Contrary to the expectations of employers, sickness absence, injuries and accident frequencies and productivity are about the same for adults with and without epilepsy (Rutgers 1984, Lechtenberg 1985, Dick 1986). Although only some jobs have been affected by statutory barriers, safety concerns were mentioned as the main reason for not hiring people with epilepsy over the years (Craig and Oxley 1986, Hicks and Hicks 1991)

Guidelines have been suggested for employing people with epilepsy. One of the main aspects is an understanding of the basic facts about epilepsy. An applicant with epilepsy should be assessed on an individual basis. The main factors which should be taken into account are the type of epilepsy and its control, associated disorders, characteristics of the individual and suitability for the job (Espir and Floyd, 1986). Job suitability should normally be assumed if a person with epilepsy has the right qualifications and experience (Employment Commission of the International Bureau for Epilepsy, 1989).

\subsubsection{Driving}

Local employment opportunities and public transportation are important factors for the employability of the person with epilepsy as they may experience a restriction of driving privileges (Craig and Oxley, 1988). Several studies have been conducted on the risk of car accidents for epilepsy patients (Parsons 1986, Taylor 1986, Gastaut and Zifkin 1987, Hansotia and Broste 1991). Epilepsy has been mentioned as one of the major causes of collapse behind the steering wheel leading to an accident (Taylor 1986). Statistics show that licensed drivers with epilepsy have only a slightly increased risk of traffic accidents, similar to patients with diabetes (Hansotia and Broste 1991, 1993). Reports on casualties and damage are not consistent (van der Lugt 1972, Gastaut and Zifkin 1987). Whether seizures played a role in accidents may be difficult to establish (Hansotia and Broste 1993). There seems to be a consensus that persons with epilepsy, when licensed to drive, are not at any higher risk of 
having accidents (Commission on Epilepsy, Risk and Insurance, International Bureau for Epilepsy, 1993).

Regulations for holding a drivers licence vary between countries (Aird et al. 1984, Rutgers 1984, Fisher et al. 1994, Krumholz 1994, Shorvon 1995). Some countries require complete freedom from seizures for a period of two years, others require one year. In some countries limited licenses for driving passenger cars are issued for short distance travelling at low density motorways for those people who are dependent on driving for their employment. While restrictions with respect to driving passenger cars may become less strict during the years changes in the limitations for driving heavier and more dangerous vehicles or commercial driving are not expected.

Because of the restrictions, difficulties and costs of obtaining an automobile liability insurance some people with epilepsy do not report their epilepsy to the licensing bureau or drive a car although they are not allowed to do so (van der Lugt 1972, Rutgers 1984). The omission to report having seizures raises the question of who is responsible for reporting the seizures. If physicians were required by law to report the seizure status of their patients to the licensing bureau or when patients have to report their seizure status this would result in illegal driving in $33 \%$ and $53 \%$ of the patients, respectively (Salinsky et al. 1992). In both cases a high rate of non-compliance would be observed, which stresses the importance of having patients educated by their physicians.

\subsubsection{Leisure}

Adverse effects on leisure activities is reported to be a problem by almost one third of the patients ((haplin et al. 1992). Several activities may induce a risk for epilepsy patients when no precautions are taken.

Sports in which head injuries may occur are generally not advisable for people without precautions such as wearing riding caps in horse riding. Of all water sports diving is not advised for persons with recurrent seizures, while the risk of swimming can largely be reduced through a 'buddy system' (Craig and Oxley, 1988). The general approach for people with epilepsy who wish to start some kind of sport activity should be an objective individual assessment whether the activity is feasible given the risk of a seizure.

In about $3 \%$ of new epilepsy patients in the age of 7 to 19 years the first seizures may have been triggered by electronic screen games (Quirk et al. 1995). Television and other visual display units can be considered to be a risk when viewed from a distance less than two meters for a patient in which seizures occur spontaneously and/or are induced by flickering lights or by patterns. Seizures can be prevented easily through covering one eye or the use of polarised glasses and display screens (Harding, 1986)

Although it is generally bulieved that excessive alcohol intake can increase the seizure frequency it is difficult to attribute this increase to alcohol only (Hauser et al. 1988). Continuous alcohol abuse may be associated with poor compliance and disregardance of medication instructions (Chan, 1985). It has been shown that moderate drinking (2-3 glasses/day) did not result in an increase in epileptic seizure frequency. Furthermore, alcohol did not result in a change in plasma drug levels in drugs such as carbamazepine (CBZ), phenobarbiton (PB), phenytoin (PHT) while differences in valproate (VPA) plasma drug levels could not be attributed to alcohol intake (Höppener, 1981). Therefore, a prohibition of alcohol 
for epileptic patients can not be justified, although reservations may exist in those patients who do not comply with the advice provided, have a history of alcohol abuse or have been shown to be sensitive for alcohol.

\subsection{General aspects of anti-epileptic drug treatment}

The occurrence of a seizure will have great impact on the patient as well as on his family. The main objective of drug treatment will be to prevent the occurrence of further fits. The decision to start drug treatment however has consequences for the patient. In order to ensure effective drug treatment these consequences will necessitate an assessment of the pros and cons of drug treatment.

\subsubsection{Reasons for drug treatment}

Justifications for starting pharmacological treatment of epilepsy are its medical and social consequences. As said before, it is commonly assumed that a seizure may increase the chance of the occurrence of additional seizures. This process of 'Bahnung' may eventually lead to recurrent seizures which are difficult to treat (Shorvon and Reynolds 1982, Elwes et al. 1984, Reynolds 1987). Brain damage may develop either due to falling caused by the changes in postural tonus or because of development of neural overactivity inducing a generalised tonicclonic seizure. Status epilepticus is the occurrence of one or multiple seizures for at least 30 minutes during which period the patient is not able to regain normal consciousness (Gastaut and Kugler, 1976). Status epilepticus is accompanied by hypoxia, hypoglycaemia, acidosis and hypotension. Generalised convulsive status epilepticus is a medical emergency, with a mortality rate of about $10 \%$ to $37 \%$ (Aird et al. 1984. Scholtes et al. 1993). Considering these consequences and risks the treatment perspectives become important. As discussed earlier epilepsy has a rather good prognosis with many patients going into remission early during treatment.

\subsubsection{Reasons for refraining from drug treatment}

Being on drug treatment will have consequences of a medical, psychological and/or economical nature for the patient. These consequences may be a reason for not starting drug treatment at all.

From the medical point of view the uncertainty about the fact that the patient has epilepsy or about the specific type of epilepsy can be a reason for refraining from drug treatment. If it is not completely certain that the diagnosis is epilepsy or the type of epilepsy is not well established the best course is to wait and let time decide (Chadwick and Reynolds, 1985). An erroneous diagnosis can cause either refractory epilepsy or the intake of anti-epileptic drugs for nonepileptic paroxysmal attacks of altered awareness. Also the frequency and/or the severity of seizures are important in deciding whether or not to start drug treatment. The impairment caused by the disease should balance or outweigh the impairment caused by the drug treatment. Long-term prophylactic treatment should not be started when seizures appear with years in between, when seizures are mild with negligible psychosocial consequences or when seizures are caused by factors which can be avoided (Jøhannessen et al. 1990).

The attitude of patients towards the drug treatment is important as well. If compliance is not to be expected one should not prescribe drug treatment. One of the reasons is that the 
occurrence of withdrawal seizures due to sudden discontinuation presents considerable risk (Shorvon, 1987). Some state that drug treatment should not be started in alcohol or drug abusers as compliance is expected to be poor in such situations (Jøhannessen et al. 1990).

Side-effects of the drug treatment will probably not outweigh the burden of few, little disabling seizures. The drug treatment may interact with co-medication such as antibiotics, anticoagulants or contraceptives. Besides this, psychological factors can be important. The recurrent intake of medication reminds the patient, the family and colleagues about the fact that the patient is suffering from a chronic disease

Although an early publication of Sonnen (1979) stated that the financial consequences of the treatment of epilepsy are of relevance only in countries such as the United States and India, recent changes in the health care systems cause this factor to be of interest in other countries as well. Perhaps this aspect should be considered to be one of the side-effects of drug treatment (Meinardi, personal communication).

\subsubsection{Goals in anti-epileptic drug treatment}

Anti-epileptic dnug treatment can serve several purposes. The perspective of removing the symptoms and curing the disease is what the physicians aim at, although it is not clear whether the anti-epileptic drugs actually cure the disease. A second goal for anticonvulsive treatment can be the prevention of epilepsy development. This may be achieved through intermittent treatment of fibrile convulsions or temporary treatment after neurosurgical interventions or head trauma. Thirdly, the establishment of a diagnosis ex juvantibus may be the intention of the treatment in those cases in which epilepsy is suspected but can not be diagnosed with certainty

A guideline for approaching the patient is the assumption that the patient wants to get well (Porter, 1984). This sets the goal for the physician: control the seizures and if possible cure the disease. Long-term treatment with anti-epileptic drugs is the normal procedure for the patient who is diagnosed as having epilepsy and experiences seizures which are medically and/or socially disabling. The initiation of the anti-epileptic drug treatment implies that the patient will have to take the drug for several years in a daily regular fashion. Compassionate doctors and responsible patients are necessary for reliable drug intake (Feuerstein et al. 1988).

When the physician faces a patient suffering from a generalised convulsive status epilepticus it is obvious that intervention is necessary. The occurrence of a convulsive status epilepticus is life threatening (Porter 1984, Swartz and Delgado-Escueta 1987. Brodie 1990). Besides identification of the type of status the treatment consists of the correction of causes and precipitating factors.

Initiation of prophylactic anticonvulsive treatment has the goal to prevent the development of epilepsy. One of the diseases which may predispose for developing epilepsy are febrile convulsions. Febrile convulsions are seizures which occur in combination with a febrile disease. They occur mostly in children between the age of 6 months and 5 years. Febrile convulsions may recur: about one third of the patients who had two seizures will have more episodes. About $2 \%$ to $7 \%$ of patients with febrile seizures developed epilepsy (Annegers et al. 1987, Wallace 1987). The prophylactic treatment of febrile seizures with PB or Na-VPA is possible but side-effects such as behavioural disorders, learning disability and hepatotoxicity may occur. Intermittent treatment is another option. Rectal diazepam can be administered by parents to arrest febrile convulsions. 
Prophylactic anti-epileptic drug treatment can be considered also in patients having suffered from head injury or neurosurgery. The number of people in the community suffering from a head injury is large. Each year in the United States some 500.000 people have brain injuries caused by cranial trauma (Hauser and Hesdorffer, 1990). In about 5\% of the head injured patients early seizures occur after the trauma. Phenytoin has been shown to be beneficial for this type of seizures (Temkin et al. 1990). The benefit of other anti-epileptic drugs is not clear. Therefore, also in these patients before initiating anti-epileptic drug treatment one has to weigh the high level of non-compliance and the risk of adverse drug reactions against the uncertain benefit of the treatment of patients with a small risk of actually developing epilepsy (Rimmer and Richens, 1988)

Similar problems arise in the prophylactic treatment of patients who had neurosurgery (Rimmer and Richens 1988, Jennett 1987). Here also a delay in treatment until seizures occur seems more appropriate as little benefit, non-compliance and problems in establishing the moment of withdrawal can be expected when anti-epileptic drug treatment is initiated (Chadwick and Reynolds 1985, Foy et al. 1992)

Finally there is the diagnostic approach. If the diagnosis whether a seizure is epileptic or not can not be established a temporary treatment with an anti-epileptic drug can be used as a diagnostic test. However there is doubt about this procedure. Literature indicates that there is only little room for the diagnosis ex juvantibus (Chadwick and Reynolds 1985, Porter 1984, 1989, Shorvon 1987)

\subsection{Initiation of treatment}

Starting anti-epileptic drug treatment has as a consequence daily intake of anticonvulsive medication for at least two years. As patients perceive this as a cumbersome task, commencing drug treatment also neccesitates to address its withdrawal

\subsubsection{Starting the drug treatment}

The moment drug treatment should be started is not well defined (Rimmer and Richens, 1988). The statement that a patient should have recurrent seizures within a short interval does not specify the duration of this period, which leads to variation in practice. Many physicians would consider a period of one year (Overweg et al. 1981, Rimmer and Richens 1988, Chadwick and Reynolds 1985, Chadwick 1994a). Others would only commence if the recurrence of a seizure takes place within a period of half a year (Sonnen, 1979). Some even start treatment after a first seizure. In a study of 88 patients, with an age of $15-64$ years, it was shown that $31 \%$ of the patients received anti-epileptic drug treatment after their first seizure, while in 2 patients treatment was started to prevent epilepsy (Rutgers, 1984). Van Donselaar (1990) showed that EEG abnormalities after a first idiopathic seizure provided a criterion for selecting patients with a high risk of recurrence, for which drug treatment might be started right away.

The detectability of a seizure may be an important aspect in considerations to commence treatment. Generalised tonic-clonic seizures can hardly be overlooked while others go undetected more easily. This may be the reason why the physician starts treatment earlier when diagnosing tonic-clonic seizures (Porter, 1989). It has been shown that the median number of tonic-clonic seizures before treatment was 3 , while the median number of partial seizures was 20 (Shorvon and Reynolds, 1982). 
Another reason to start the treatment as soon as possible is that early treatment may have been prevented due to patient and/or doctor delay. It has been shown that one third of the patients who experienced a first seizure did not visit a doctor immediately. Within this group $52 \%$ visited their family physician within one year. Half of these patients visited the physician because of a second seizure, while others visited their physician after more than two seizures (Rutgers, 1984). Doctors delay was encountered in $46 \%$ of the patients, mainly in patients who were younger than 20 years at the moment of onset. Some $16 \%$ of the patients had both patient and doctor delay.

It has been shown that in $37 \%$ up to $80 \%$ of the patients with a first seizure a second seizure occurs within one year (Goodridge and Shorvon 1983b, Elwes et al. 1985, Hopkins et al. 1988, Sander et al. 1990). Some suggest early treatment (Elwes et al. 1988, Chandra 1992, Reynolds 1995). However, starting treatment immediately, based on the finding of only one seizure is still questionable. The physician has to establish a correct diagnosis which may be difficult especially if this single event is described incompletely. Furthermore it may be difficult to differentiate between epileptic and pseudo-seizures. Additional studies are needed to answer the question whether treatment after the first seizure prevents chronic epilepsy (First Seizure Trial Group 1993, Chadwick 1995)

Table 2.9-I: Preferred drugs for treatment of specific types of epilepsy. The first and second choice medication is indicated by the number.

\begin{tabular}{|c|c|c|c|c|}
\hline \multirow[b]{2}{*}{$\begin{array}{l}\text { First } \\
\text { authors }\end{array}$} & \multicolumn{4}{|c|}{ Type of epilepsy } \\
\hline & gen.idiopathic & gen.symptoinatic & partial epilepsy & unclassified \\
\hline $\begin{array}{l}\text { Dam } \\
(1982)\end{array}$ & $\begin{array}{l}\text { CBZ,PHT,CIZ, } \\
\text { PB,ETT,PRM, } \\
\text { VPAACZA }\end{array}$ & $\begin{array}{l}\text { SGTC: CBZ,PHT, } \\
\text { CLZ,PB,ETT, } \\
\text { PRM, VPA, } \\
\text { ACZA }\end{array}$ & $\begin{aligned} \text { PEES: } & \text { CBZ,PHT,CLZ, } \\
& \text { PB,ETT,PRM, } \\
& \text { VPA,ACZA } \\
\text { PECS: } & \text { CBZ,PHT, } \\
\text { CLZ,ETT, } & \text { PRM,VPA }\end{aligned}$ & \\
\hline $\begin{array}{l}\text { Meinardi } \\
(1983)\end{array}$ & VPA,PB,ACZA & $\begin{array}{l}\text { VPA,CBZ, PHT,BZP, } \\
\text { ACTH }\end{array}$ & $\begin{array}{l}\text { SP: CBZ,PHT, VPA, } \\
\text { BZP } \\
\text { CP.CBZ,PHT,CLZ, } \\
\text { PRM,VPA }\end{array}$ & \\
\hline $\begin{array}{l}\text { Chadwick } \\
\text { (1990) }\end{array}$ & $\begin{array}{l}\text { SA: I VPA.ESM, } 2 \text { BZP } \\
\text { JME: I VPA } 2 \text { PB } \\
\text { ATC: I VPA } 2 \text { CBZ.PHT }\end{array}$ & $\begin{array}{l}1 \text { VPA,BZP } \\
2 \text { CBZ,PHT,PB }\end{array}$ & $\begin{array}{l}1 \text { CBL.VPA } \\
2 \text { PHT.PB }\end{array}$ & $\begin{array}{l}1 \mathrm{CBZ}, \mathrm{VPA} \\
2 \mathrm{PHT}, \mathrm{PB}\end{array}$ \\
\hline $\begin{array}{l}\text { Meinardi } \\
(1993)\end{array}$ & VPA & VPA & $\begin{array}{l}\text { I CBZ } \\
2 \mathrm{PHT}\end{array}$ & \\
\hline $\begin{array}{l}\text { Chadwick } \\
\text { (1994b) }\end{array}$ & $\begin{aligned} \text { SA: } & 1 \text { VPA.ESM } \\
& 2 \text { BZP.LTG } \\
\text { JME: } & 1 \text { VPA } \\
& 2 \text { PB,LTG } \\
\text { ATC: } & 1 \text { VPA. } \\
& 2 \text { CBZ,PHT,LTG }\end{aligned}$ & $\begin{array}{l}\text { I VPA.BBZI' } \\
2 \text { CI37.I'IT,PB }\end{array}$ & $\begin{array}{l}\text { l CBZ,VPA } \\
2 \text { PHI.PB.VGB }\end{array}$ & $\begin{array}{l}1 \mathrm{VPA} \\
2 \mathrm{CBZ} . \mathrm{PHT}\end{array}$ \\
\hline
\end{tabular}

SA: simple absences, MM: juvenile myoclonic epilepsy, ATC: awakening tonic-clonic seizures, SGTC: secondary generalised tonic clonic. PEES: partial epilepsy elementary symptomatology. PECS: partial epilepsy complex symptomatology. SP: simple partial, CP: complex partial

CBZ; Carbamazepine, PHT: Phenytoin. PB: Phenobarbiton, PRM: Primidon, VPA: Valproate, ESM: Ethosuximide, CLZ: Clonazepun, ETT: Ethotoin. ACZA: Acetizolamide, ACTH: Adrenocorticotropic honnone, BZP: Benzodiazepines, LTG: Lamotrigine VGB: Vigabatrine 


\subsubsection{Drug selection}

The classifications of epilepsies and seizures provide a means for communication. There is no consensus on whether the choice of the applicable drug should be based on the type of the epilepsy or on the type of seizure (Dam et al. 1985, Meinardi 1993). Literature shows that the selection of a drug may be based on the type of epilepsy, the type of seizures or both (Table 2.9-I and Table 2.9-II).

Table 2.9-II: Preferred drugs for treatment of specific seizure types.

\begin{tabular}{|c|c|c|c|c|}
\hline \multirow[b]{2}{*}{ First author } & \multicolumn{4}{|c|}{ Seizure type } \\
\hline & absences & tonic-clonic (GE) & partial & SGTC \\
\hline Jeavons (1977) & $\mathrm{VPA} \rightarrow \mathrm{ESM}$ & $\mathrm{VPA}, \mathrm{CBZ} \rightarrow \mathrm{PHT}$ & $\begin{array}{l}\text { SP: CBZ, VPA } \rightarrow \text { CLP } \\
\text { CP: CBZ } \rightarrow \text { VPA,PHT }\end{array}$ & $\mathrm{VPA}, \mathrm{CBZ} \rightarrow \mathrm{PHT}$ \\
\hline Reynolds (1981) & ESM,CLP,VPA & $\begin{array}{l}\text { PB,PHT,PRM, } \\
\text { CBZ,CLP,VPA }\end{array}$ & $\begin{array}{l}\text { PB,PHT,PRM, } \\
\text { CBZ,CLP,VPA }\end{array}$ & $\begin{array}{l}\text { PB,PHT,PRM, } \\
\text { CBZ,CLP,VPA }\end{array}$ \\
\hline Höppener (1983) & $\begin{array}{l}\text { I VPA,ESM } \\
2 \mathrm{MSM}\end{array}$ & $\begin{array}{l}\text { I CBZ,VPA } \\
2 \text { PB,PRM }\end{array}$ & $\begin{array}{l}\text { I CBZ,PHT } \\
2 \text { VPA,PB,PRM, } \\
\text { CLP,SUL,ACTA, } \\
\text { PCM }\end{array}$ & \\
\hline Shorvon (1985) & ESM,VPA & $\begin{array}{l}\text { CBZ, PHT,PB/PRM, } \\
\text { VPA }\end{array}$ & CBZ,PHT,PB,PRM & CBZ,PHT,PB,PRM \\
\hline Mattson (1989) & ESM,VPA & $\begin{array}{l}\text { I VPA } \\
2 \text { CBZ,PHT,PB,PRM }\end{array}$ & $\begin{array}{l}\text { CBZ,PHT, PB, PRM, } \\
\text { VPA? }\end{array}$ & CBZ,PHT,PB,PRM \\
\hline $\begin{array}{l}\text { Johiunessen } \\
(1990)\end{array}$ & I ESM,VPA & $\begin{array}{l}\text { I CBZ } \\
2 \mathrm{VPA} \\
3 \text { CLP,CLB,PB,PHT }\end{array}$ & $\begin{array}{l}1 \mathrm{CBZ} \\
2 \text { PHT.VPA } \\
3 \text { CL.P }\end{array}$ & $\begin{array}{l}1 \mathrm{CBZ} \\
2 \mathrm{PHT}, \mathrm{VPA} \\
3 \mathrm{CLP}\end{array}$ \\
\hline Scheurer (1990) & $\mathrm{ESM} \rightarrow \mathrm{VPA}$ & $\mathrm{VPA} \rightarrow \mathrm{CBZ}, \mathrm{PHT}, \mathrm{PB}$ & CBZ,PITT,PRM,PB & $\begin{array}{l}\text { CBZ,PHT, PRM,PB, } \\
\text { VPA? }\end{array}$ \\
\hline Mattson (1992) & VPA & CBZ, VPA & $\mathrm{CBZ}$ & $\mathrm{CBZ}$ \\
\hline Wilder (1995) & $\begin{array}{l}\text { ESM,VPA, } \\
\text { LTG,FMT }\end{array}$ & $\begin{array}{l}\text { PHT,CBZ, VPA,GBP, } \\
\text { LTG,FMT }\end{array}$ & $\begin{array}{l}\text { PHT,CHZ,GBP, } \\
\text { LTG,IMT }\end{array}$ & $\begin{array}{l}\text { PHT,CBZ,VPA, } \\
\text { GBP,ITS,FMT }\end{array}$ \\
\hline Brodie (1996) & VPA.ESM & $\begin{array}{l}\text { CBZ,PHT,VPA,PB, } \\
\text { PRM,CLP }\end{array}$ & $\begin{array}{l}\text { CBZ,PHT, VPA,PB, } \\
\text { PRM }\end{array}$ & $\begin{array}{l}\text { CBZ,PHT, VPA,PB, } \\
\text { PRM,CLP }\end{array}$ \\
\hline
\end{tabular}

GE: generalised epilepsy, SGTC: secondary generalised tonic clonic, SP: simple partial, CP: complex partial, a $\rightarrow$ b,c: a followed by $b$ or $\mathrm{c}$

CBZ: Carbamazepine, PHT: Phenytoin, PB: Phenobarbital, PRM: Primidon, VPA: Valproate, ESM: Ethosuximide, MSM Mesuximide, CZP: Clorazepate, CLP: Clonazepan, SUL: Sultiam, ACTA: Acetazolamide, PCM: Phenacemide, CLB: Clobazam, LTG: Lamotrigine, GBP: Gahapentin, FMT: Felbanate

Some use the type of epilepsy for the selection of the drug of first choice. They recommend the use of VPA for idiopathic generalised and generalised symptomatic epilepsy and CBZ for partial epilepsy (Meinardi et al. 1983, Chadwick 1990, Meinardi 1993, Chadwick 1994a,b). Another example is juvenile myoclonic epilepsy which responds only to VPA. Others prefer CBZ for idiopathic generalised and partial epilepsy and differentiate between different types of partial epilepsy (Dam and Kiørboe 1982).

Others prefer the use of the seizure diagnosis to determine the appropriate therapy (Reynolds and Shorvon 1981, Höppener and van der Lugt 1983, Porter 1984, Shorvon 1987, Jøhannessen 1990, Shorvon 1991, Brodie and Dichter 1996). 
Although the seizure type can be used as selection criterion it has been emphasised that also aspects of the type of epilepsy are of importance in making a decision (Dreifuss, 1990). Other investigators state that the seizure type enables to establish a relation between the physiologi$\mathrm{cal}$ and neurochemical factors of seizures and their response to drug treatment. Nevertheless, they advocate a pragmatic approach in the use of classifications for drug selection. Thus, the use of a classification of seizure types or epilepsies should be performed as far as this is relevant for the selection of the appropriate therapy (Aird et al. 1984).

\subsubsection{Monotherapy or polypharmacy}

Until the 1970s the traditional approach to treatment of epilepsy was twofold. In the first approach treatment with one drug was started and gradually the dose was increased until the seizures were controlled or toxic side-effects developed. In the second approach two drugs were used in the hope of a synergistic effect or under the assumption that the toxicity of two drugs in a lower dose would be less than the toxicity of one drug at a higher dose. In both approaches the dosage was changed or other drugs were added if seizures were not controlled. During the seventies a change in the view on prescribing anti-epileptic drugs developed. Studies on the effectiveness of polypharmacy indicated that it is unnecessary in newly diagnosed patients (Reynolds et al. 1976, Shorvon and Reynolds 1977, Shorvon et al. 1978, 1980, Reynolds and Shorvon 1981). The general principle that evolved was that in newly diagnosed patients combination therapy should be tried only when monotherapy with several drugs failed (Dam and Kiørboe 1982, Rowan 1983, Höppener and van der Lugt 1983, Aird et al. 1984, Shorvon 1987. Rimmer and Richens 1988, Porter 1989, Jøhannessen et al. 1990. Scheuer and Pedley 1990)

Reynolds (1980) discusses two studies that suggest that polypharmacy may lead to an increased risk of seizures. Side-effects decreased when fewer drugs were prescribed. However, Lammers et al. (1995) have suggested that this may be due to the decrease of drugload and is not caused by the reduction in number of drugs. As was discussed by Höppener and van der Lugt (1983) the introduction of a second drug may cause changes in antiepileptic drug levels of the newly introduced as well as of the used drug. These changes vary over patients. An additional problem concerning side-effects in polytherapy is the determination of the drug responsible for the adverse reaction. The compliance of the patient may be lower when using polytherapy which may result in incomplete seizure control. Furthermore, the costs of the therapy may be lower if one or more of the prescribed drugs is withdrawn.

However, some patients can benefit from polytherapy. It has been reported that in $11 \%$ of the patients, that were given polytherapy because monotherapy failed, complete seizure control was achieved. A reduction in the seizure frequency was observed in $40 \%$ (Mattson et al. 1985). It has been suggested that a systematic change of drugs is necessary using the most effective drug as a background regimen while the other individual drugs should be added and tested sequentially (Shorvon, 1991)

Insight in drug mechanisms is growing. Anti-epileptic drugs seem to decrease membrane excitability by interacting with neurotransmitter receptors and ion channels (Macdonald and Kelly 1994, Montouris 1995, Brodie and Dichter 1996). More recently new drugs VGB, LTG and GBP have been introduced which can be used as add-on therapy (Wilder, 1995). Combination therapy may become more common as drugs with synergistic effects become available (Chadwick, 1994b). 


\subsubsection{Efficacy of drugs}

All new anti-epileptic drugs are subjected to efficacy trials. Also comparative studies of the relative efficacy of major anti-epileptic drugs have been published. Table 2.9-III lists the studies concerned with CBZ, PHT, VPA, PRM and PB. The assessment of studies on the efficacy of anticonvulsive drugs is difficult because of differences in the studied populations, differences in study design, the small number of patients involved in the studies, differences in outcome measures and short duration of follow-up. The paroxysmal character of the epilepsy makes the duration of follow-up an important aspect. Furthermore, a short follow-up period may result in a bias in favour of drugs for which tolerance can be developed during long-term intake. Because of these problems it is not possible to draw definite conclusions about differences in efficacy of anticonvulsives using results from randomised controlled comparison studies of anti-epileptic drugs (Gram et al. 1982, Chadwick and Turnbull 1985, Beghi et al. 1986).

More recently larger drug trials have been conducted. Mattson et al. (1992) studied the efficacy of CBZ and VPA in a randomised double-blind trial in patients with complex partial seizures and secondary generalised tonic-clonic seizures. They did not find a difference between the two drugs in controlling the tonic-clonic seizures. However, for the complex partial seizures CBZ showed a better control and fewer long term side-effects. They conclude that $\mathrm{CBZ}$ should be used as a drug of first choice for partial epilepsy because about $50 \%$ of the patients with partial complex seizures do also suffer from generalised seizures. The higher number of side-effects for VPA however, may have been caused by an upper range plasma drug level which was higher than is usual in clinical practice (Reynolds et al 1993). Others report that $\mathrm{CBZ}$ and VPA are equally effective while a higher withdrawal rate because of sideeffects was observed for CBZ (Richens et al. 1994, Heller 1995).

\subsubsection{Dose of drugs}

Once a drug has been selected the intention is to control seizures without the development of toxic side-effects. The consensus is that the anti-epileptic drugs can be used more effectively with the assistance of serum level monitoring (Richens and Ahmad 1975. Shorvon et al. 1978, Reynolds 1980, Rimmer and Richens 1988, Commission on Anti-epileptic Drugs of the ILAE, 1993). Considerations about efficacy, toxicity and the plasma drug levels resulted in the concept of therapeutic or optimal range. Within this range seizure control is expected to be optimal while the toxic effects are expected to be minimal

Another important aspect of the therapeutic range is the definition of an upper level above which additional control of seizures is unlikely and toxic side-effects can be expected. Unfortunately, the therapeutic range can not be used to predict the efficacy of the treatment for an individual patient (Shorvon 1987, Lammers 1994). Nevertheless these ranges, which are based on averages for large populations, can also be used for the individual case at onset of therapy. One should be aware that differences in opinion on the appropriate plasma drug levels exist (Table 2.9-IV) 
Table 2.9-IV: Overview of therapeutic ranges in $\mathrm{mg} / \mathrm{l}$ presented in the literature

\begin{tabular}{lllllll}
\hline First author & CBZ & PHT & PB & PRM & VPA & ESM \\
\hline Jeavons (1977) & $4-10$ & $10-25$ & $15-40$ & $n a v^{1}$ & $60-100$ & $40-120$ \\
Schobben (1979) & $4-8$ & $7-12$ & $20-35$ & $7-10$ & $50-80$ & $40-80$ \\
Sonnen (1979) & $5-10$ & $10-20$ & $15-40$ & $4-12$ & $40-80$ \\
Reynolds (1980) & $4-10$ & $10-20$ & $15-40$ & $15-40^{2}$ & $50-100$ & $40-80$ \\
Daun (1982) & $4-10$ & $10-20$ & $15-30$ & nav & nav & $40-90$ \\
Höpjwener (1983) & $4-10$ & $10-20$ & $15-40$ & nav & $?$ & $40-120$ \\
Rowan (1983) & $8-12$ & $15-20$ & $15-40$ & $5-8$ & $?$ & $60-100$ \\
Overweg (1984) & $3-12$ & $10-20$ & $10-40$ & $4-12$ & $30-120$ & $40-120$ \\
Shorvon (1987) & $3.5-11.5$ & $7-20$ & $9-40$ & $9-40^{3}$ & nav. & $40-100$ \\
Rimmer (1988) & $5-10$ & $10-20$ & $15-40$ & $15-40^{1}$ & $50-100$ & $50-100$ \\
Porter (1989) & $4-10$ & $10-20$ & $10-40$ & $5-15$ & $50-100$ & $50-100$ \\
& $7^{3}>8^{4}$ & $18^{3}>20^{4}$ & $35^{3}>40^{4}$ & $10^{3}>12^{4}$ & $80^{3}>100^{4}$ & $80^{3}>100^{4}$ \\
Scheuer (1990) & $8-12$ & $10-20$ & $15-35$ & $5-12$ & $50-120$ & $40-100$ \\
Meijer (1991) & $3-7$ & $10-20$ & $20-40$ & nav & $50-90$ & $50-90$ \\
\hline
\end{tabular}

CBZ: Carbamazepine, PHT: Phenytoin, PB: Phenobarbital, PRM: Primidon, VPA: Valproate, ESM: Ethosuximide (1)nav: not available. (2)Therapeutic range of phenobarbital is used for primidon, (3)Level to be reached in refractory seinures, (4)Toxic level

Some authors do not present the therapeutic range values for Na-VPA because the significance of the drug monitoring for this drug is unknown. As large changes in VPA serum level during the day occur it is not possible to correlate side-effects and serum concentration for this drug (Höppener and van der Lugt, 1983). Differences also exist in the presented optimal serum levels for PRM. Some use the real PRM serum values for establishing the therapeutic range of the drug (Sonnen 1979, Rowan 1983, Overweg 1984, Scheuer and Pedley 1990). Others present the values of PB for PRM because PB is produced during the metabolisation of PRM (Reynolds 1980, Shorvon 1987, Rimmer and Richens 1988, Porter 1989).

Also for carbamazepine the therapeutic ranges vary in the literature. Some found mean blood drug levels of 4.7 to $6.5 \mathrm{mg} / \mathrm{l}$ for carbamazepine in successfully treated patients, while therapy failure was observed at a mean plasma level of $9.8 \mathrm{mg} / \mathrm{l}$ (Ramsay et al 1983). Others use a range of 3-12 mg/l (Overweg 1984, Shorvon 1987), while again others state that the therapeutic range starts at $8 \mathrm{mg} /$ (Rowan 1983, Scheuer and Pedley 1990). It has been mentioned that drug toxicity can be expected starting from $8 \mathrm{mg} / \mathrm{l}$ for this drug (Porter 1989)

A similar variation in therapeutic ranges was observed for PHT, PB and ISM. Lower therapeutic levels are quoted, respectively from $7-15,9-20$ and $40-60 \mathrm{mg} /$, while upper therapeutic levels vary from $12-25,30-40$ and $80-120 \mathrm{mg} /$.

The differences in the patient population or the treatment strategy as well as the possibility that the therapeutic ranges are determined more by the desired anti-epileptic effect than by the appearing side-effects may be the cause for the variation in therapeutic ranges

Besides the interindividual variability in therapeutic values it has been suggested that intra individual variability in therapeutic AED serum levels exist. Schmidt and Janz (1980) showed that the initial therapeutic concentration of the drugs PHT and PB for controlling seizures was 
Table 2.9-IV: Overview of therapeutic ranges in $\mathrm{mg} / \mathrm{l}$ presented in the literature

\begin{tabular}{lllllll}
\hline First author & CBZ & PHT & PB & PRM & VPA & ESM \\
\hline Jeavons (1977) & $4-10$ & $10-25$ & $15-40$ & $n a v^{1}$ & $60-100$ & $40-120$ \\
Schobben (1979) & $4-8$ & $7-12$ & $20-35$ & $7-10$ & $50-80$ & $40-80$ \\
Sonnen (1979) & $5-10$ & $10-20$ & $15-40$ & $4-12$ & $40-80$ \\
Reynolds (1980) & $4-10$ & $10-20$ & $15-40$ & $15-40^{2}$ & $50-100$ & $40-80$ \\
Daun (1982) & $4-10$ & $10-20$ & $15-30$ & nav & nav & $40-90$ \\
Höpjwener (1983) & $4-10$ & $10-20$ & $15-40$ & nav & $?$ & $40-120$ \\
Rowan (1983) & $8-12$ & $15-20$ & $15-40$ & $5-8$ & $?$ & $60-100$ \\
Overweg (1984) & $3-12$ & $10-20$ & $10-40$ & $4-12$ & $30-120$ & $40-120$ \\
Shorvon (1987) & $3.5-11.5$ & $7-20$ & $9-40$ & $9-40^{3}$ & nav. & $40-100$ \\
Rimmer (1988) & $5-10$ & $10-20$ & $15-40$ & $15-40^{1}$ & $50-100$ & $50-100$ \\
Porter (1989) & $4-10$ & $10-20$ & $10-40$ & $5-15$ & $50-100$ & $50-100$ \\
& $7^{3}>8^{4}$ & $18^{3}>20^{4}$ & $35^{3}>40^{4}$ & $10^{3}>12^{4}$ & $80^{3}>100^{4}$ & $80^{3}>100^{4}$ \\
Scheuer (1990) & $8-12$ & $10-20$ & $15-35$ & $5-12$ & $50-120$ & $40-100$ \\
Meijer (1991) & $3-7$ & $10-20$ & $20-40$ & nav & $50-90$ & $50-90$ \\
\hline
\end{tabular}

CBZ: Carbamazepine, PHT: Phenytoin, PB: Phenobarbital, PRM: Primidon, VPA: Valproate, ESM: Ethosuximide (1)nav: not available. (2)Therapeutic range of phenobarbital is used for primidon, (3)Level to be reached in refractory seinures, (4)Toxic level

Some authors do not present the therapeutic range values for Na-VPA because the significance of the drug monitoring for this drug is unknown. As large changes in VPA serum level during the day occur it is not possible to correlate side-effects and serum concentration for this drug (Höppener and van der Lugt, 1983). Differences also exist in the presented optimal serum levels for PRM. Some use the real PRM serum values for establishing the therapeutic range of the drug (Sonnen 1979, Rowan 1983, Overweg 1984, Scheuer and Pedley 1990). Others present the values of PB for PRM because PB is produced during the metabolisation of PRM (Reynolds 1980, Shorvon 1987, Rimmer and Richens 1988, Porter 1989).

Also for carbamazepine the therapeutic ranges vary in the literature. Some found mean blood drug levels of 4.7 to $6.5 \mathrm{mg} / \mathrm{l}$ for carbamazepine in successfully treated patients, while therapy failure was observed at a mean plasma level of $9.8 \mathrm{mg} / \mathrm{l}$ (Ramsay et al 1983). Others use a range of 3-12 mg/l (Overweg 1984, Shorvon 1987), while again others state that the therapeutic range starts at $8 \mathrm{mg} /$ (Rowan 1983, Scheuer and Pedley 1990). It has been mentioned that drug toxicity can be expected starting from $8 \mathrm{mg} / \mathrm{l}$ for this drug (Porter 1989)

A similar variation in therapeutic ranges was observed for PHT, PB and ISM. Lower therapeutic levels are quoted, respectively from $7-15,9-20$ and $40-60 \mathrm{mg} /$, while upper therapeutic levels vary from $12-25,30-40$ and $80-120 \mathrm{mg} /$.

The differences in the patient population or the treatment strategy as well as the possibility that the therapeutic ranges are determined more by the desired anti-epileptic effect than by the appearing side-effects may be the cause for the variation in therapeutic ranges

Besides the interindividual variability in therapeutic values it has been suggested that intra individual variability in therapeutic AED serum levels exist. Schmidt and Janz (1980) showed that the initial therapeutic concentration of the drugs PHT and PB for controlling seizures was 
higher than the AED serum therapeutic concentration measured during the maintenance period of the treatment. In a study on withdrawal of anti-epileptic medication in 1013 patients seizure free for at least 2 years, it was found that $30 \%, 76 \%, 67 \%$ and $51 \%$ of the patients using $\mathrm{CBZ}$, $\mathrm{PB}, \mathrm{PHT}$ or VPA, respectively, had AED serum levels in the subtherapeutic range (Medical Research Council AED Withdrawal Study Group, 1991). These differences in therapeutic levels have been attributed to differences in the activity of the epileptic process.

Also the recommendations on the normal daily doses diverge (Table 2.9-V). For establishing the daily dose some use the body weight of the patient (Dam and Kiørboe 1982, de Krom and van der Lugt 1982, Overweg 1984). Others use a more global approach with a predefined amount of milligrams per day (Sonnen 1979, Aird et al. 1984, Lechtenberg 1985, Shorvon 1987, Rimmer and Richens 1988, Brodie and Dichter 1996).

Table 2.9-V: Normal daily dose of anti-epileptic drugs. In the upper part of the table the daily dose is presented in $\mathrm{mg}$. The lower part of the table presents the daily dose of the $\mathrm{drug}$ in $\mathrm{mg} / \mathrm{kg}$ body weight.

\begin{tabular}{llllllll}
\hline & First author & CBZ & PHT & PB & PRM & VPA & ESM \\
\hline \multirow{4}{*}{ daily } & Sonnen (1979) & $400-1600$ & $250-500$ & $100-200$ & $500-1000$ & $900-2400$ & $500-1500$ \\
& Aird (1984) & $600-1200$ & $100-500$ & $100-300$ & $250-1000$ & $1500-3000$ & $500-1500$ \\
dose & Lechtenberg (1985) & $600-1200$ & $300-400$ & $100-150$ & $750-1500$ & $1000-3000$ & $750-2000$ \\
& Shorvon (1987) & $400-1800$ & $200-500$ & $60-180$ & $250-1000$ & $600-2500$ & $500-1500$ \\
& Rimmer (1988) & $400-1800$ & $150-600$ & $30-240$ & $250-1500$ & $600-3000$ & $500-1500$ \\
& Brodic (1996) & $400-2000$ & $100-700$ & $60-240$ & $250-1500$ & $500-3000$ & $500-2000$ \\
\hline \multirow{4}{*}{$\mathrm{mg} / \mathrm{kg}$} & Daun (1982) & $10-20$ & 5 & $1.5-3.0$ & $12-20$ & $900-1800$ & $20-40$ \\
& de Krom (1982) & $8-12$ & $5-7$ & $2-5$ & nav & $20-30$ & $8-12$ \\
& Ovcrweg (11984) & $15-20$ & 5 & $3-5$ & 10 & 20 & $15-20$ \\
\hline
\end{tabular}

CBZ: Carbanazepine, PHT: Phenyoin, PB: Phenobarbital, PRM: Primidon, VPA: Valproate, ESM: Ethosuximide (1) Maily dose in mg

The relation between dose and seizure control is only statistical. The physician has to decide for the individual patient whether a high dose or not is needed to achieve results immediately (Porter, 1989). Some state that AED serum levels should be in the upper part of the therapeutic range (Rowan 1983). Others found that up to one third of the patients will achieve control of seizures with a level below the therapeutic range (Reynolds et al. 1976, Shorvon et al. 1978, 1980). Control was achieved with 200-300 mg PHT per day or 200-400 mg CBZ per day. Seizure control has been observed even with a low starting dose of e.g. $100 \mathrm{mg}$ PHT or 100-200 mg CBZ (Reynolds 1980. Shorvon et al. 1978). An approach for drug treatment is to start treatment with a low dose and if no effect is observed increase the dose to try to reach the therapeutic range (Höppener and van der Lugt 1983, Shorvon 1985, Chadwick 1990). Another approach is to use the middle of the target range (Mattson et al. 1985, 1992). To assure compliance it is preferable not to increase the dose to a level where toxicity may be expected in spite of recent publications which advocate a return to pre drug level monitoring strategies. 


\subsection{Maintenance}

After drug treatment has been initiated it is necessary to see the patient on a regular basis. During these visits the seizures may remit after a short time of drug treatment, show some response to drugs with a tendency to relapse or turn out to be intractable in which remission is unusual. Furthermore, the correctness of the diagnosis and the development of side-effects have to be assessed. During each of these visits it is advisable to check compliance to support and encourage the patient to comply with the treatment as to improve or maintain seizure control.

\subsubsection{Recurrence of seizures}

After having achieved a remission a relapse may occur. The probability of a relapse during the first 5,10 and 20 years after entering remission is $8 \%, 15 \%$ and $24 \%$, respectively (Annegers et al. 1979). This estimate includes patients on and off anti-epileptic drug treatment.

Recurrence of seizures can be caused by misdiagnosis. Jeavons (1977) stated that 270 of the 1172 patients at his epilepsy clinic did not have epilepsy. Fifty percent of the adults in the nonepilepsy group were misdiagnosed because of syncope. Second to this there were misdiagnoses due to psychiatric disorders. The choice of an ineffective drug may be another reason for occurrence of seizures during treatment. Even when a first choice drug is prescribed it is not necessarily the most effective drug for a specific patient. Suboptimal serum levels can also be a cause of the continuation of seizures

Non-compliance is another reason for continuation of seizures or their reoccurrence after a seizure free period. If complete control of seizures has been achieved the compliance may decrease because of absence of seizures (Feuerstein et al. 1988). Although non-compliance can be the cause of a relapse, the correctness of the diagnosis should be evaluated continuously as a unrecognised concomitant neurological disease may develop.

Finally, there is a group of patients in whom complete seizure control cannot be established although plasma drug levels in the therapeutic range have been reached. Drug resistant epilepsy which is only partly responsive to drug treatment is present in about $20 \%$ of the patients in the UK. Another $20 \%$ of the patients have epilepsy which is completely unresponsive to drug treatment (Shorvon, 1991).

\subsubsection{Drug monitoring}

Plasma drug levels together with clinical findings can be used to determine the effectiveness of the drug treatment and provide a basis for adjustment of the dose of the drug. The practical use of plasma drug levels has been studied amongst others by Beardsley et al. (1983) and Meijer (1991). Beardsley et al. showed in a retrospective study of 164 adult epileptic patients that prescribers did not use the blood levels properly in $17 \%$ of the therapeutic decisions. If they did use the plasma drug levels correctly an improvement in the control of the seizures occurred in $51 \%$ of the patients. Inappropriate decisions caused an improvement in seizure frequency in only $5 \%$ of the patient, while in $32 \%$ of the patients with an inappropriate decision the seizure frequency worsened

Meijer (1991) studied the decisions of 16 physicians. From a retrospective analysis of 1006 therapeutic decisions in 64 patient records he concluded that approximately $66 \%$ of the decisions to adapt the dosage of phenytoin and carbamazepine, and $80 \%$ of the decisions on 
changes in dosage of valproate were done in agreement with the serum concentration and clinical signs. Confronting the physicians with plasma drug levels in the subtherapeutic and toxic range led also to variance in the treatment strategy. Although some differences occurred in cases with subtherapeutic levels, most of the variance in decisions was observed for cases with plasma drug levels in the toxic range.

Anti-epileptic drug monitoring may help in discovering the reason for therapy failure. Changes in seizure control can be attributed to e.g. intra individual variation in pharmacokinetic parameters as a consequence of intermittent disease or to the effects of co-medication on plasma drug levels (Höppener and van der Lugt 1983, Scheuer and Pedley 1990). Drug monitoring can facilitate the diagnosis of symptoms possibly caused by drug toxicity and can help to predict whether a change in the drug dose is likely to result in toxic side-effects (Milano Collaborative Group for Studies on Epilepsy, 1980).

Anti-epileptic drug levels should be used as a guide rather than a rigid goal for the optimal use of the drug. The observed levels depend on the drug half-life and can be affected by the interval between the time of drug intake and the moment of blood sampling. Furthermore, inter-individual differences in optimal levels as well as intra individual variation may occur. It has been shown that patients who are seizure free with levels in the sub therapeutic range do not always benefit from an increase in the dose leading to a therapeutic range level (Woo et al. 1988). Thus, clinical judgement should not be substituted by the therapeutic ranges.

Warnings against the overuse of AED monitoring have been published, as a strict relation between serum levels and efficacy and/or toxicity is not available for an individual patient (Chadwick, 1987). Already in the '70s a set of indications for drug monitoring levels was presented (Kutt and Penry, 1974). More recently, guidelines for therapeutic monitoring were presented which amongst other things state that anti-epileptic drug levels should be obtained after:

- Therapy is initiated

- After a change of dosage and/or drug

- In case of poor seizure control or recurrence of seizures which were well controlled previously

Signs or symptoms of intoxication and a routine control of blood levels once or twice a year are also reasons for monitoring (Commission on Anti-epileptic drugs of the ILAE, 1993).

\subsubsection{Change in dosage or drug}

Reasons for changing drug dosage are incomplete control of seizures and the occurrence of side-effects

The appropriate dose for the individual patient preventing the continuation of the seizures can not be determined a priori (Lammers, 1994). In case of persisting seizures the dose will be increased or the drug will be changed depending on the clinical findings. However, frequent changes in drugs or dosages should be avoided (Rowan, 1983). Although the intention is to achieve complete seizure control it is not clear when the dose should be increased: after a reduction in seizure frequency of less than $50 \%$ or less than $90 \%$ or in case any seizure occurs after a steady state has been reached or only after the second seizure.

One should use sufficient time to evaluate therapeutic efficacy. This means at least that the plasma drug level should have reached a steady state before conclusions about the effectiveness of the drug can be drawn. The time period needed to evaluate the effect of treatment on 
the seizure frequency is rarely mentioned in the literature. It has been suggested that a drug should be tried for a period that would be expected to cover three to five seizures or clusters of seizures, or for at least two months whichever period is the longest (Shorvon, 1991). In this period the drug should be provided in a sufficient dose to reach the upper part of the therapeutic range, confirmed by results from repeated drug monitoring.

\subsubsection{Compliance}

Compliance is the extent to which a patient adheres to a given medical and/or health advice. It concerns the drug intake as well as the keeping of therapeutic appointments (Mattson et al. 1988). In general it is estimated that one third to one half of the patients on chronic medication do not follow the medical advice about the prescription as presented by the physician (Schmidt and Leppik, 1988). Reasons for non-compliance are the loss of individual freedom caused by the need of a regular drug intake, the required changes in life-style or loss of independence because of control by the relatives. Disagreement on the goals for treatment and discordance on the value of the elimination of unhealthy conditions can cause non-compliance as well. Worry about one's health can be caused by a high seizure frequency. Contrary to this, persons with few seizures are more lax about their condition and treatment (Peterson et al. 1982). When a patient disapproves the duration of the treatment the treatment may be rejected. Furthermore, patients and doctors can have a different perception of the risks posed by the disease and the tolerance of the drug dosage. Patients weigh the consequences of compliance against the threat and consequences of having seizures. This process of weighing can result in non-compliance.

A deliberate change of a treatment by the patient herself is called the self-regulation or selfmanagement of the patient. It stresses the fact that it is the patient who has control over the drug intake. Self-regulation also includes the option that patients can take on their own initiative more drugs than prescribed (Thorbecke, 1988).

Contrary to a deliberate change, non-compliance may be due to inadvertency i.e despite the understanding, the acceptance and the desire to adhere to the treatment. This includes noncompliance due to changes in daily pattern during weekends or holidays, or simply forgetting because of absence of a routine for intake of tablets (Höppener and van der Lugt, 1983).

Some suggest that there is no significant difference in seizure control in patients who comply and those who are non-compliant (Schmidt et al. 1988). However, it has been shown that improved seizure control can be expected if compliance is monitored closely (Wannamaker et al. 1980). This may be accomplished by the measurement of anti-epileptic drug levels and patient interview (Leppik, 1988). The reports of a patient may well be a minimum estimate of non-compliance (Trostle, 1988). The additional use of special medication containers and self recording of seizures and drug intake can be easily implemented and may enable a more complete view on compliance. Furthermore, good compliance may be achieved by convenient regimen characteristics such as simplicity, individualisation and low intake frequency (Peterson et al. 1984). Besides this, a good partnership between patient and physician is considered to be of great importance to achieve successful treatment (Green and Simons-Morton 1988, Leppik and Schmidt 1988). According to patients sufficient time, presentation of consistent clinical information and acknowledgement of the role of the patient in controlling the disease will enhance compliance (Feuerstein et al 1988) 


\subsection{Withdrawal}

Many patients will enter a period of prolonged remission. The question of tapering drug treatment will become more relevant to a patient the longer the duration of the seizure-free period gets.

\subsubsection{Reasons for withdrawal}

Among the reasons for a reduction or discontinuation of anti-epileptic drug treatment are factors such as association of the drugs with idiosyncratic and dose related adverse reactions, risks of chronic toxicity and teratogenicity of the drug. Furthermore, the removal of the stigma of epilepsy can be a reason for patients to discuss the option to stop drug treatment (Chadwick, 1988). Successful withdrawal of anti-epileptic treatment can be an advantage when applying for a job and at medical fitness examinations (van Heycop ten Ham, 1980). It is known that many patients stop drug treatment without informing their physician (Zielinski 1974, Goodridge and Shorvon 1983b).

Table 2.11-I: Relapse rates Juring and after withdrawal of anti-epileptic drugs

\begin{tabular}{|c|c|c|c|c|c|c|c|c|}
\hline First author & Study & Sel* & $N$ & $\mathrm{SF}^{* *}$ & Relapses & Taper & Withdrawn & F.U.** \\
\hline $\begin{array}{l}\text { Juul-Jensen } \\
(1964) \\
(19+i 8)\end{array}$ & $\begin{array}{l}\text { prospect } \\
\text { retrospecl }\end{array}$ & $\begin{array}{l}\mathrm{EC} \\
\mathrm{EC}\end{array}$ & $\begin{array}{l}200 \\
196\end{array}$ & $\begin{array}{l}\geq 2 y s \\
\geq 2 y s\end{array}$ & $\begin{array}{l}70(35 \%) \\
79(40 \%)\end{array}$ & $\begin{array}{l}20(28 \%) \\
20(25 \%)\end{array}$ & $\begin{array}{l}50(72 \%) \\
59(75 \%)\end{array}$ & $\begin{array}{l}m=24 \\
m=60\end{array}$ \\
\hline $\begin{array}{l}\text { Oller-Daurella } \\
(1076) \\
(1977) \\
(1987)\end{array}$ & $\begin{array}{l}\text { retrospect } \\
\text { retrospect } \\
\text { retrospect }\end{array}$ & $\begin{array}{l}\text { nav } \\
\text { nav } \\
\text { nav }\end{array}$ & $\begin{array}{l}356 \\
522 \\
608\end{array}$ & $\begin{array}{l}\geq 5 \text { ys } \\
\geq 5 \text { ys } \\
\geq 5 \text { ys }\end{array}$ & & $\begin{array}{l}\text { nav } \\
\text { nav } \\
\text { nav } \\
\text { nav }\end{array}$ & $\begin{array}{l}29 / 138(21 \%) \\
82 / 241(30 \%) \\
119 / 474(24 \%) \\
25 / 134(19 \%)\end{array}$ & $\begin{array}{l}(6-240) \\
m=120 \\
m=180 \\
m=55\end{array}$ \\
\hline $\begin{array}{l}\text { van Hevcop ten } \\
\text { Ham }(1980)\end{array}$ & prospect & $\mathrm{EC}$ & 156 & $\geq 5$ ys & $49(31 \%)$ & $28(57 \%)$ & $21(43 \%)$ & $\geq 24$ \\
\hline $\begin{array}{l}\text { Overweg } \\
\text { (1981) }\end{array}$ & prospect & $\mathrm{EC}$ & 46 & $\geq 3 \mathrm{ys}$ & $29(63 \%)$ & 21 & 8 & $(9-19)$ \\
\hline $\begin{array}{l}\text { Bouma } \\
(1987)\end{array}$ & prospecl & $D C N$ & 116 & $2 y s$ & $26(22 \%)$ & mav & nav & $\begin{array}{l}m=51 \\
(9-120)\end{array}$ \\
\hline $\begin{array}{l}\text { Arts' } \\
(1988)\end{array}$ & prospecl & NDOC & 146 & $\geq 2 y \mathrm{~s}$ & $37(25 \%)$ & $7(19 \%)$ & $30(81 \%)$ & $\begin{array}{l}m=52 \\
(16-113)\end{array}$ \\
\hline $\begin{array}{l}\text { Callaghan } \\
\text { (1988) }\end{array}$ & prospecl & nav & 92 & $2 y s$ & $31(34 \%)$ & $13(45 \%)$ & $18(55 \%)$ & $\begin{array}{l}m=35 \\
(6-62)\end{array}$ \\
\hline $\begin{array}{l}\text { Med.Res.C. } \\
\text { AED.Withdr. } \\
\text { (1991) }\end{array}$ & $\begin{array}{l}\text { rundom. } \\
\text { prospect }\end{array}$ & $\mathrm{MC}$ & 1013 & $\geq 2$ & $\begin{array}{l}221 / 510 \\
(43 \%)\end{array}$ & $\begin{array}{l}107 / 221 \\
(48 \%)\end{array}$ & $\begin{array}{l}|14 / 22| \\
(52 \%)\end{array}$ & $\begin{array}{l}\text { med }=27 \\
(17,42)^{3}\end{array}$ \\
\hline
\end{tabular}

- Selting: EC: Epilepsy Clin., NDOC: Neurology Dept. Outpatient Clin., DCN: Devision of Child Neurology, $\mathrm{MC}$ multiple centres

* Seizure-free period in years

**Follow-up period in months

(1) nav: not available,

(2) Study contains only children,

(3) Definition of epilepsy includes patients with $\geq 2$ afebrile or $\geq 3$ atypical febrile seizures

(4) Median and $25^{\text {th }}$ and $75^{\text {th }}$ percentiles 
Social factors are a main issue for patients deciding not to taper drug truatment. An example of this is the obtaining of the drivers license which may cause a patient to refuse withdrawal of drug treatment (van Heycop ten Ham, 1980). Other patients may refuse withdiawal hecause they fear the consequences of a seizure at work (Pedley, 1988). Because of these perceived risks an analysis of costs and benefits should be made by the physician and the patient before starting an attempt to taper the drug treatment .

The interpretation of studies regarding relapse during and after withdrawal is diflicult. Otten information on the duration of the remission period before commencement of the withdrawal, the seizure types and syndromes, the time taken for the drug withdrawal and diagnostic criteria for recurrent seizures as well as the follow-up period is missing (Chadwick 1988, Pedley 1988). Papers that do mention these data often cannot be compared because of differences in populations studied (Table 2. I 1-1).

Patient characteristics that influence the decision to withdraw anti-epileptic drugs in a positive or negative way have been published (Chadwick and Reynolds 1985, Dean and Penry 1989). One of the factors in favour is the diagnosis of primary generalised epilepsy. It has been shown that absence seizures recur only in $5-20 \%$. Primary generalised tonic-clonic seizures have a recurrence rate of 20-33\% (Janz and Sommer-Burkhardt 1976, Oller-Daurella and Oller 1987, Callaghan et al. 1988). Patients with partial epilepsy show a higher relapse rate; this may be due to the inclusion of symptomatic seizures (Annergers et al. 1979).

Contradictory findings have been reported about the predictive value of the age at onset of epilepsy for the outcome of the withdrawal of the drugs (Janz and Sommer-Burkhardt 1976, Oller-Daurella and Oller 1987, Berg and Shinnar, 1994).

The relapse rate after drug tapering in children has been observed to be somewhat lower than in adult patients, $31 \%$ and $35 \%$ respectively (Callaghan et al. 1988). A literature survey of studies regarding adults and children indicates that the age may be important, especially in conjunction with other factors such as duration, severity and frequency of seizures before treatment (Dean and Penry, 1989). In another survey the opposite is stated, based mainly on studies in children (Hauser and Hesdorffer, 1990). However, more recently it has been shown that children have a lower risk than adults for recurrence of seizures during the first two years after withdrawal (Medical Research Council Anti-epileptic Drug Withdrawal Study Group, 1993)

The number of seizure-free years before withdrawal has been reported as a determinant for the success of withdrawal. Without mentioning the exact data some suggest that there does not seem to be much difference between the relapse rates of patients who are 5 years seizure free and those that are 10 years seizure free (Oller-Daurella et al. 1977). It has been shown that there does not seem to be a difference in recurrence rate after withdrawal for children treated for 2, 3, 4 or 5 years (Arts et al. 1988).

Recommendations on the duration of the period during which the drug is withdrawn vary from four weeks up to several years (Oller-Daurella et al. 1977, van Heycop ten Ham 1980, Overweg et al. 1981, Bouma et al. 1987, Arts et al. 1988, Callaghan et al. 1988). A comparison of seizure recurrence between drug tapering periods of 6 weeks and 9 months did not show a significant difference in risk of recurrent seizures (Tennison et al. 1989, 1994). A duration of at least three month has been advocated, however mainly on basis of data in children. 


\subsubsection{Moment of relapse}

Most of the studies indicate that the number of patients who relapse during the tapering of drug treatment is between $19 \%$ and $57 \%$ (Table 2.11-1). A randomised multicenter trial showed that $52 \%$ of the patients will experience a relapse after withdrawal of drugs (Medical Research Council AED Withdrawal Study Group, 1991). Other studies report that $43 \%$ up to $81 \%$ of the patients had a seizure after the withdrawal was completed. However, three Spanish studies indicate a lower rate of relapses after drug withdrawal. Only 19-30\% of the patients had seizures after discontinuing the treatment. A reason for this difference may be the longer seizure free period ( $\geq 5$ years) before patients were admitted to the tapering. Furthermore, the long period used for drug tapering - for most of the patients up to a year and some even longer - may have had an selective effect on the patients reaching drug withdrawal (OllerDaurella et al 1976, 1977, Oller-Daurela and Oller 1987).

Most of the patients in which the drug is withdrawn relapsed during the first year after the treatment was discontinued (Juul-Jensen 1968, Oller-Daurella et al. 1976, Janz and SommerBurkhardt 1976, Thurston et al. 1972, 1982, Shinnar et al. 1985, Bouma et al. 1987, Callaghan et al. 1988, Medical Research Council Anti-epileptic Drug Withdrawal Study Group, 1991). Most of the relapses occur even earlier after withdrawal, mainly during the first six months.

Recommencing the anti-epileptic treatment after a relapse during drug tapering or after withdrawal results in control of the seizures in many but not all of the patients. In a group of 31 patients who relapsed and where reinstalled on treatment 20 patients $(64.5 \%)$ became seizure free again for 4-50 months, while seven patients (22.5\%) continued to have seizures (Callaghan et al 1988). A study in children showed that one third of the patients whose drug treatment was reintroduced, remained seizure-free after a second withdrawal period, while $50 \%$ were seizure-free for at least six months. In $17 \%$ of the patients seizures were difficult to control (Arts et al. 1988).

\subsection{Summary and conclusion}

Although studies on epilepsy suffer from differences in terminology and case finding strategies they indicate a prevalence rate of $0.4-0.6 \%$. The incidence rate ranges from $0.03-0.05 \%$. The main part of community studies indicate that $60-75 \%$ of the patients have an epilepsy of unknown aetiology.

One of the first principles for the management of epilepsy is to establish the diagnosis as precisely as possible. Because of the paroxysmal character of epilepsy the diagnosis may be difficult to establish. Other diseases such as diabetes, cardiac disease or syncopes can mimic the epileptic seizures. Epilepsy should be treated as early as possible to prevent a chronic state of seizures which may be difficult to treat. The consequences of late treatment can conflict with the time necessary for establishing the correct diagnosis. An incorrect diagnosis can result either in long term anticonvulsive drug intake for non-epileptic causes or in the development of refractory epilepsy. Thus, if the diagnosis is not certain the physician should let time make things clear.

Epilepsy can be treated through regular daily intake of medication. A two year remission of the disease within three years after onset of treatment has been reported in $50-70 \%$ of the patients, while up to $90 \%$ of the patient may show a remission after more than five years. However, patients with additional neurologic, psychiatric or social handicaps seem to have a 
poor prognosis for achieving a remission of seizures. Although insight in drug mechanisms is growing the question whether the disease is cured or seizures are suppressed has not been answered unequivocally

Deciding to start drug treatment implies that the patient will be on anticonvulsants for at least two years. Reasons for the treatment should be sufficiently explained to the patient and his family. Before the treatment is started the physician should educate the patient on the different aspects of the disease, the reason for drug intake, the duration of the treatment, the prognosis and the possibility of side-effects. While a chronic disease such as epilepsy is treated problems of a medical, psychological, social and economical nature may occur. Thus, whether or not to start treatment requires an assessment of the pros and cons of the disease and the cure.

The treatment should be based on a correct diagnosis. There is hardly any room for an ex juvantibus approach to establish the correct diagnosis. There is no consensus whether the selection of the anti-epileptic drug should be based on the seizure type or the type of epilepsy. Monotherapy is preferred above polypharmacy when starting treatment. However, in the future new drugs may make polytherapy a viable option again. The literature shows little consensus regarding the drugs which should be applied for the different types of seizures or epilepsy. Small differences exist in the effectiveness of the drugs for treatment of the different types of epilepsy. Therefore the selection of drugs is based on other aspects such as occurrence of side effects. Also little consensus exists in the literature on the appropriate daily dose and the required anti-epileptic serum levels

The differences in selection of drugs, appropriate doses and required anti-epileptic drug serum levels make the treatment of epilepsy to a large extent based on trial and error. Regular follow-up contacts enable the physician to monitor the effect of the treatment. Although physicians seem to agree on the goal of drug treatment, $20-30 \%$ of the treatment decisions are questionable.

Withdrawal of drug treatment can be started after a seizure free period of two years. There is no consensus on the duration of the tapering period. In the literature the duration varies from several weeks up to many years. In those patients who start drug withdrawal some 20$50 \%$ will suffer a relapse before the drugs are stopped completely. Most of the seizures reoccur early after withdrawal, mainly during the first 6 months. Reintroducing drug treatment will be successful in about $65 \%$ of these patients. The rest of the patients have seizures which are difficult to control.

Patients suffering from seizures will worry about the cause of the attacks. Having been confronted with the diagnosis the patient may experience negative emotions in contacis with other people. Furthermore, patients may loose their driving license and will be afraid about further impacts of the disease on their social life. Commencing anti-epileptic drug treatment will have consequences for the patient (e.g. daily drug intake for at least two to three years). Having been healthy and suddenly being confronted with a disease with paroxysmal symptoms will make the patients tend to deny the illness and keep carrying on their life as they did before the diagnosis. It is generally accepted that at least one third of the patients do not follow the prescription presented by the physician. Part of this non-compliance may be caused unintentionally. However, self-regulation of the treatment is also an important cause. The patient will resent the medical profession if the physician does not seem to really listen to his problems or does not support him. Absence of a good patient-doctor contact will cause 
compliance to decrease. Issues such as accomplishment of the same goals by the patient and his physician and acknowledgement of the role of the patient in the treatment together with close monitoring of the patient during remissions can improve the compliance of the patient.

Drug treatment is only part of the management of epilepsy. Guidance of patients as well as those who make treatment decisions that influence the lives of the patients is also part of the proper management.

\subsection{References}

Aird R.B., Masland R.L., Woodbury D.M., The epilepsies a critical review, Raven Press, New York, 1984.

Annegers J.F., Hauser A., Elveback L.R., Remission of seizures and relapse in patients with epilepsy, Epilepsia, 20, 1979, pp. 729-737.

Annegers J.F., Hauser A., Shirts S.B., Kurland L.T., The risk of epilepsy following febrile convulsions, The New England Journal of Medicine, 316, 1987, pp.493-498.

Arts W.F.M., Visser L.H., Loonen M.C.B., Tjiam A.T., Stroink H., Stuurman P.M., Poortvliet D.C.J., Follow-up of 146 children with epilepsy after withdrawal of anti-epileptic therapy, Epilepsia, 29, 1988, pp. 244-250.

Aspinal A., The real obstacles. In: Epilepsy and employment (Edwards, Espir and Oxley, Eds.), Royal Society of Medicine Scrvices Limited, London, 1986, pp. 19-20.

Beardsley R.S., Freeman J.M., Appel F.A.. Anticonvulsant serum levels are useful only if the physician appropriately uses them: an assessment of the impact of providing serum level data to physicians, Epilepsia, 24, 1983, pp. 330-335.

Beghi E., Di Mascio R., Tognoni G., Drug treatment of epilepsy, outlines, criticism and perspectives, Drugs, 31, 1986, pp. 249-265.

Beghi E., Tognoni G., Prognosis of epilepsy in newly referred patients: A multicenter prospective study, Epilepsia, 29, 3, 1988, pp. 236-243.

Berg A.T., Shinnar S., Relapse following discontinuation of anti-epileptic drugs: a meta-analysis, Neurology, 44, 1994, pp.601-608.

Bergamini L., Bergamasco B., Benna P., Gilli M., Acquired etiological factors in 1.785 epileptic subjects: clinical anamnestic research, Epilcpsia, 18, 1977, pp. 437-444.

Betts T., Employment of people with epilepsy within the National Health Service. In: Epilepsy and employment (Edwards, Espir and Oxley, Eds.), Royal Society of Medicine Services Limited, London, 1986, pp. 59-66.

Bongers E., Coppoolse J., Meinardi H., Posthume E., Zijl C. van. A survey of epilepsy in Zeeland The Netherlands, Instituut voor Epilepsiebestrijding Meer en Bosch - De Cruquiushoeve, Heemstede, 1976.

Bouma P.A.D., Peters A.C.B., Arts R.J.H.M., Stijnen Th, Rossum J. van, Discontinuation of antiepileptic therapy: a prospective study in children, Journal of Neurology. Neurosurgery and Psychiatry, 50, 1987, pp. 1579-1583.

Brodie M J. Status epilepticus in adults. The Lancet, 336, 1990, pp. 551-552

Brodic M.J.. Dichter M.A. Anti-cpileptic drugs, The New England Journal of Medicine, 334, 1996, pp. $168-175$

Callaghan N. Garrett A., Goggin T., Withdrawal of anticonvulsant drugs in patients free of seizures for two years, The New England Journal of Midicimi, 318, 1988, pp. 942-946. 
Cereghino J.J, Epidcmiology of epilepsy, Public Health Reviews. 3, 1974, pp. 91-100.

Cereghino J.J., Brock J.T., Meter J.C. van, Penry J.K., Sinith L.D., White B.G., Carbamazepine for epilepsy, a controlled prospective evaluation, Neurology, 1974, pp. 401-410.

Cereghino J.J., Introduction in anti-epileptic drugs. In: Anti-epileptic drugs, (Levy, Mattson, Meldrum, Penry and Dreifuss, Eds.), $3^{\text {rd }}$ edition, Raven Press, New York, 1989, pp. 23-27.

Chadwick D.W., Overuse of monitoring of blood concentrations of anti-epileptic drugs, British Medical Journal, 294, 1987, pp. 723-724.

Chadwick D., Drug withdrawal and epilepsy, when and how ? Drugs, 35, 1988, pp. 579-583.

Chadwick D., Diagnosis of epilepsy, The Lancet, 336, 1990, pp. 291-296.

Chadwick D., Epilepsy after first scizures: risks and implications, Journal for Neurology, Neurosurgery and Psychiatry, 54, 1991, pp. 385-387.

Chadwick D., Epilepsy, Journal of Neurology, Neurosurgery and Psychiatry, 57, 1994a, pp.264-277.

Chadwick D., Standard approach to anti-epileptic drug tre:atment in the United Kingdom, Epilepsia, 35 (Suppl.4), 1994b, s3-s10

Chadwick D., Case for early treatment is not established, British Midical Journal, 310, 1995, pp.177178.

Chadwick D., Reynolds E.H., When do epileptic patients need treatment? Starting and stopping medication, Brilish Medical Journal, 290, 1985, pp. 1885-1888.

Chadwick D., Tumbull D.M. The comparative efficacy of anti-epileptic drugs for partial and tonicclonic seizures, Journal of Neurology. Neurosurgery and Psychiatry, 48, 1985, pp. 1073-1077.

Chan A.W.K., Alcoholism and epilepsy, Epilepsia, 26, 1985, pp. 323-333.

Chandra B., First seizure in adults: to treat or not to treat, Clinical Neurology and Neurosurgery, 94 (Suppl.), 1992, s61-s63.

Chaplin J.E., Yepez Lasso R., Shorvon S.D., Floyd M., National general practice study of epilepsy: the social and psychological effects of the recent diagnosis of epilepsy, British Medical Journal, 304, 1992, pp. 1416-1418

Cockerell O.C., Eckle 1., Goodridge D.M., Sander J.W., Shorvon S.D., Epilepsy in a population of 6000 re-examined: secular trends in first attendance rates, prevalence, and prognosis, Journal for Neurology, Neurosurgery and Psychiatry, 58, 1995a, pp. 570-576

Cockerell O.C, Johnson A.L., Sander J.W., Hart Y., Shorvon S., Remission of epilcpsy. results from the National Gencral Practice Study of Epilepsy, The Lancet, 346, 1995b, pp. 14(1)-144

Collaborative Group for Study of Epilepsy. Prognosis of epilepsy in newly refurred patients: a multicenter prospective study of the effects of monotherapy on the long-term course of epilepsy. Epilepsia, 33, 1992, pp. 45-51

Commission on Anti-epileptic Drugs of the International League Against Epilepsy, Guidelines for therapeutic monitoring on anti-epileptic drugs, Epilepsia, 34, 4, 1993, pp. 585-587

Commission on Classification and Terminology of the Intemational League Against Epilepsy, Proposal for revised classification of epilepsies and cpileptic syndromes. Fipilepsia, 30, 1989, pp. 389-399.

Commission on Epidemiology and Prognosis of the International Lungue Against Epilepsy, Guidelines for epidemiologic studies on epilepsy, Epilepsia, 34, 4, 1993, pp. 592-596.

Commission on Epilepsy, Risk and Insurance of the Intcmational Bureau for Epilepsy, Workshop on epilepsy, risks and insurance, Epilcpsia, 34, 4, 1993, pp. 590-591.

Cowan L.D., Bodensteiner J.B., Leviton A., Doherty L., Prevalence of the epilepsies in children and adolescents, Epilepsia, 30, 1989, pp. 94-106. 
Craig, A., Oxley J., Statutory and non-statutory barriers to the employment of people with epilepsy. In: Epilepsy and employment (Edwards, Espir and Oxley, Eds.), Royal Society of Medicine Services Limited, London, 1986, pp. 21-31.

Craig A., Oxley J., Social aspects of epilepsy. In: A texlbook of epilepsy (Laidlaw, Richens and Oxley, Eds.), Churchill Livingstone, Edinburgh, 1988, pp. 566-610.

Crobach M.J., Niezink G.M., Leden J. van der, Springer M.P., Epilepsie en huisarts: toeval of hoofdzaak? Nederlands Tijdschrift voor de Geneeskunde, 132, 1988, pp. 1888-1892.

Crombie D.L., Cross K.W., Fry J., Pinsent R.J.F.H., Watts C.A.H., A survey of epilepsy in general practice, British Medical Journal, 1960, pp. 416-422.

Dam M., Gram L., Pedersen B., Orum H., Modern approach to anti-epileptic arug treatment, improving quality of life. The Danish Epilepsy Society, Kobenhavn, 1985.

Dam M., Kiorboe E., Epilepsy, diagnosis and treatment, Scriptor, Copenhagen, 1982.

Dean J.C., Penry J.K., Gencral principles, discontinuation of anti-epileptic drugs. In: Anti-epileptic drugs, (Levy, Mattson, Meldrum. Penry and Drcifuss, Eds.), $3^{\text {rd }}$ edition, Raven Press, New York, 1989, pp. 133-142.

Dichter M.A., Emerging insights into the mechanisms of epilepsy: implications for new anti-epileptic drug development, Epilepsia.35 (Suppl.4), 1994, s5!-s57.

Dick D., Epilepsy in the British steel industry. In: Epilepsy and employment (Edwards, Espir and Oxley, Eds.), Royal Society of Medicine Services Limited, London, 1986, pp. 49-52.

Donselaar C. van, Idiopathic first seizure in adull life, thesis, Rotterdam, 1990.

Drcifuss F.E., The cpilepsies: clinical implications of the international classification, Epilepsia, 31 (Suppl), 1990, s3-s!0

Elwes R.D.C., Chesterman P., Rcynolds E.H., Prognosis after a first untreated tonic-clonic seizure, The Lancet, okt., 1985, pp. 752-753.

Elwes R.D., Johnson C.B., Shorvon S.D., Reynolds E.H., The prognosis for seizure control in newly diagnosed epilepsy, The New England Journal of Medicine, October 1984, pp. 944-947.

Elwes R.D.C., Johnson A.L., Reynolds E.H., The course of untreated epilepsy, British Medical Journal, 297, 1988, pp. 948-297

Elwes R.D.C., Marshall J. Beattic A., Newman P.K., Epilepsy and employment. A community based survey in an area of high uncmployment. Journal of Neurology. Neurosurgery. and Psychiatry, 54, 1991, pp. 200-203

Elwes R.D.C., Reynolds E.H., Should people be treated after a first scizure?, Archives of Neurology, 45, 1988, pp.490-491

Elwes R.D.C., Reynolds E.H., The early prognosis of epilepsy, In: Comprehensive Epileptology (Dam and Gram, Eds.), Ravens Press, New York, 1990, pp. 715-727.

Employment Commission of the Intemational Bureau for Epilepsy, Employing people with epilepsy: principles for good practice, Epilepsia, 30, 1989, pp. 4II-4I2.

Espir M, Floyd M., Epilepsy and recruitment, In: Epilepsy and employment (Edwards, Espir and Oxley, Eds.), Royal Seciety of Medicine Services Limited, London, 1986, pp. 39-46.

Feuerstein M., Licb-Jückstock V., Schnaus H., Springmann E., Weber B., Wunderlich M., Compliance, a joint effort of the patient and his doctor, Epilepsy Research. Suppl. I, 1988, s5 Is56.

First Seizure Trial Group. Randomised clinical trial on the efficacy of anti-epileptic drugs in reducing the risk of relapse after a first unprovoked tonic-clonic seizure, Neurology, 43, 1993, pp 478-483 
Fisher R.S., Parsonage M., Beaussart M., Bladin P., Masland R., Sonnen A.E.H., Rémillard G., Epilepsy and Driving: an intemational perspective, Epilepsia, 35, 3, 1994, pp. 675-684.

Forsgren L., Prospcctive incidence study and clinical characterization of seizures in newly referred adults, Epilepsia, 31, 1990, pp.292-301.

Forsgren L., Prevalence of epilepsy in adults in northern Siveden, Epilepsia, 33, 1992, pp. 450-458.

Foy P.M., Chadwick D.W., Rajgopalan N., Johnson A.L., Shaw M.D.M., Do prophylactic anticonvulsant drugs alter the pattern of seizures after craniotomy, Journal of Neurologv. Neurosurgery and Psychiairy, 55, 1992, pp.753-757.

Gastaut H., Clinical and encephalographic classification of epilcptic seizures, Epilepsia, 11, 1970, pp. 102-113.

Gastaut H., Kugler J., Wörferbuch der Epilepsie, Hippokrates Verlag GmbH, Stuttgart, 1976.

Gastaut H., Zifkin B.G., The risk of automobile accidents with seizures occurring while driving: relation to seizure type, Neurology, 37, 1987, pp.1613-1616.

Goodridge D.M., Shorvon S.D., Epileptic seizures in a population of 6000, I: Demography, diagnosis and classification, and hospital services, Brilish Medical Journal, 287, I983a, pp. 64 I-644.

Goodridge D.M., Shorvon S.D., Epileptic seizures in a population of 6000, II: Treatment and prognosis, British Medical Journal, 287, 1983b, pp. 645-647.

Gowers W.R., Epilepsy and other chronic convulsive diseases; their causes, symptoms and treatment, reprint of the first American edition 1885, Arts en Boeve, Nijmegen, 1994, pp.200.

Graaf A.S. de, Epidemiological aspects of epilcpsy in northern Norway, Epilepsia, 15, 1974, pp. 291299.

Gram L., Draclumann Bentsen K., Pamas J., Flachs H, Controlled trials in epilcpsy: a review, Epilepsia, 23, 1982, pp. 491-519.

Gram L., Wulff K., Rasmussen K.E., Flachs H., Würtz-Jorgensen A., Sommerbeck K.W., Lohren V., Valproate sodium: a controlled clinical trial including monitoring of drug levels, Epilepsia, 18, 1977, pp. 141-148

Granieri E., Rosati G., Tola R., Pavoni M., Paolino E., Pinna L., Monetti V., A descriptive study of epilepsy in the district of Copparo, Italy, 1964-1978, Epilepsia, 24, 1983, pp. 504-514.

Green L.W., Simons-Morton D.G., Denial, delay and disappointment: discovering and overcoming the causes of drug errors and missed appointments. Epilepsy Research, Suppl. 1, 1988, s7-s22.

Haerer A.F., Anderson D.W., Schoenberg B.S., Prevalunce and clinical features of epilepsy in a biracial United States population, Epilepsia, 27, 1986, pp. 66-75

Hansotia P., Broste S.K. The effect of epilepsy and diabetes mellitus on the risk of automobile accidents, The New England Journal of Medicine, 324, 1991, pp.22-26.

Hansotia P., Broste S.K., Epilepsy and traffic safety, Epilepsia, 34, 1993, pp. 852-858.

Harding G., Photosensitive epilepsy and employment. In: Epilepsy and employment (Edwards, Espir and Oxley, Eds.), Royal Society of Medicine Services Limited, London, 1986, pp. 75-87

Hart Y.M., Sander J.W.A.S., Johnson A.L., Shorvon S.D., National General Practice Study of Epilepsy: recurrence after a first seizure. The Lancet, 336, 1990, pp. 127/-1274.

Hauser A.W., Epidemiology of epilepsy. In: Advances in Neurology, vol. 19, (Schoenberg ed.), Raven Press, New York, 1978, pp. 313-339

Hauser A.W., Prevention of post-traumatic epilepsy, The New England Journal of Medicine, 323, 1990, pp. 540-541.

Hauser A.W., Seizure disorders: the changes with age, Epilepsia, 33 (Suppl.4), 1992, s6-s 14. 
Hauser A.W., Annegers J.F., Kurland L.T., Prevalence of epilepsy in Rochester, Minnesota: 19401980, Epilepsia, 32, 1991, pp. 429-445.

Hauser A.W., Annegers J.F., Kurland L.T., Incidence of epilepsy and unprovoked seizures in Rochester, Minnesota: 1935-1984, Epilepsia, 34, 1993, pp.453-468.

Hauser A.W., Hesdorffer D.C., Prognosis. In: Epilepsy, frequency, causes, and consequences, (Hauser and Hessdorffer, Eds.), Epilcpsy foundation of America, Maryland, 1990, pp. 197-243.

Hauser A.W., Kurland L.T., The epidemiology of cpilepsy in Rochester, Minnesota, 1935 through 1967, Epilepsia, 16, 1975, pp. 1-66.

Hauser A.W., Ng S.K.C., Brust J.C.M., Alcohol, seizures and epilepsy, Epilepsia, 29 (Suppl.2), 1988, s66-s78

Heller A.J., Chesterman P., Elwes R.D., Crawford P., Chadwick D., Johnson A.L., Reynolds E.H., Phenobarbitone, phenytoin, carbamazepine, or sodium valproate for newly diagnosed adult epilepsy: a randomised comparative monotherapy trial, Journal of Neurology, Neurosurgery and Psychiatry, 38, 1995, pp.44-50.

Heycop ten Ham M.W. van, Complete recovery from epilepsy ? Discontinuation of antiepileptics after five or more seizure-free years, Huisarts en Wetenschap, 23, 1980, pp. 309-311.

Hicks R.A., Hicks M.J., Attitudes of major employers toward the employment of people with epilepsy: a 30-year study, Epilepsia, 32, 1991, pp. 86-88.

Hopkins A., Definition and epidemiology of epilepsy. In: Epilepsy, (Hopkins, Ed.), Chapman and Hall, London, 1987, pp. 1-18

Hopkins A., Garman A., Clarke C., The first seizure in adult life, the value of clinical features, Electroencephalography, and computerised tomographic scanning in the prediction of seizure recurrence, The Lancet, April 2. 1988, pp. 721-726.

Höppener R.J.E.A., Epilepsy and alcohol, thesis, Maastricht, 1981

Höppener R.J.E.A., Lugt P.J.M. van der, De medicamenteuze behandeling bij epilepsie, Pharmaceulisch Weekblad, 118, 1983, pp. 290-296.

Janz D., Die Epilepsien. Spezielle Pathologie und Therapie, Thieme, Stuttgart; 1969.

Janz D., Sommer-Burkhardt E.M., Discontinuation of anti-epilcptic drugs in patients with epilepsy who have been seizure-free for more than two years. In: Epileptology. Proc of the $7^{\text {th }}$ International Symposium on Epilepsy, (Janz ed.), Thieme-Verlag, Stuttgart, 1976, pp. 228-234.

Jeavons P.M. Choice of drug therapy in epilepsy, The Practitioner, 219, 1977, pp. 542-556.

Jennett B., Epilepsy after head injury and intracranial surgery, In: Epilepsy, (Hopkins, Ed.), Chapman and Hall, London, 1987, pp. 40I-4I2.

Johannessen S.1., Loyning Y., Munthe-Kaas A.W., General Aspects. In: Comprehensive Epileptology (Dam and Gram, Eds.), Ravens Press, New York, 1990, pp. 505-524.

Juul-Jensen $P$., Frequency of recurrence after discontinuance of anticonvulsant therapy in patients with epileptic seizures, Epilepsia, 5, 1964, pp. 352-363

Juul-Jensen $\mathrm{P}$., Frequency of recurrence after discontinuance of anticonvulsant therapy in patients with epileptic seizures, a new follow-up study after 5 years, Epilepsia, 9, 1968, pp. 11-16.

Juul-Jensen P., Foldspang A., Natural history of epileptic seizures, Epilepsia, 24, 1983, pp. 297-312.

Keränen T., Riekkinen P.J., Remission of seizures in untreated epilepsy, British Medical Journal, 307, 1993 , pp. 483.

Keränen T., Riekkinen P.J., Sillanpää M., Incidence and prevalence of epilepsy in adults in eastern Finland, Epilepsia, 30, 1989, pp. 413-421. 
Kosteljanetz M., Christiansen J., Mouritzen Dam A., Stengaard Hansen B., Blatt Lyon B., Pedersen H., Dam M., Carbamazepine vs phenytoin, a controlled clinical trial in focal motor and generalised epilepsy, Archives of Neurology, 36, 1979, pp. 22-24.

Krom M.C.T.F.M. de, Lugt P.J.M. van der, Anti-epileptica 2, Geneesmiddelen Bulletin, 16, 1982, pp. 9-12.

Krumholz A., Driving and epilepsy: a historical perspective and review of current regulations, Epilepsia, 35, 3, 1994, pp. 668-674.

Kutt H., Penry K., Usefulness of blood levels of anti-epileptic drugs, Archives of Neurology, 31, 1974, pp. 283-288.

Laidlaw J., Richens A., Oxley J., Parsonage M., Porter R.J., Classification of epileptic seizures. In: $A$ texibook of epilepsy (Laidlaw, Richens and Oxley, Eds.), Churchill Livingstone, Edinburgh, 1988, pp. $1-20$.

Lammers M.W., Clinimetrics in epileptology, thesis, Nijmegen, 1994

Lammers M.W., Hekster Y.A., Keizer A., Meinardi H., Renier W. O., Monotherapy or polytherapy for epilepsy revisited a quantitative assessment, Epilepsia, 36, 1995, pp.440-446.

Lechtenberg R., The diagnosis and treatment of epilepsy, Macmillan Publishing Company, New York, 1985

Leppik I.E., Compliance during treatment of epilepsy, Epilepsia, 29 (Suppl.), 1988, s79-s84.

Leppik I.E., Schmidt D. Consensus statement on compliance in epilepsy, Epilepsy Research, Suppl.1, 1988, s $179-\mathrm{s} 182$

Lugt P.J.M. van der, Epilepsie en verkeer. De medische geschiktheid van epilepsiepatienten tor deelname aan gemotoriseerd wegverkeer, thesis, Rotterdam, 1972.

Lugt P.J.M. van der, Epilepsie, een ratjetoe van problemen,. In: Man en Paard, Liber Amoricum tgv. het Afscheid van prof. A.Staal. 1990, pp. 125-131

Lühdorf K., Jensen L.K., Plesner A.M., Actiology of seizures in the elderly, Epilepsia, 27, 1986 pp $458-463$

Macdonald R.L., Kelly K.M., Mechanisms of action of currently prescribed and newly developed antiepileptic drugs, Epilepsia, 35 (Suppl 4), 1994, s4l-s50.

Maremmani C., Rossi G., Bonucceli U., Murri L.. Descriptive epidemiologic study of epilepsy syndromes in a district of northwest Tuscany, Italy, Epilepsia, 32, 1991, pp. 294-298.

Marsden C.D., Reynolds E.H. Seizures in adults. In: A lextbook of epilepsy (Laidlaw, Richens and Oxley, Eds.), Churchill Livingstone, Edinburgh, 1988, pp. 144-182

Mattson R.H., General principles, selection of anti-epileptic drug therapy, In: Anti-epileptic drugs, (Levy, Mattson, Meldrum, Penry and Drcifuss, Eds.), $3^{\text {rd }}$ edition, Raven Press, New York, 1989, pp. 103-115

Mattson R.H., Cramer J.A., Collins J.F., VA Epilepsy Co-operative Study Group, Aspects of compliance; taking drugs and keeping clinic appointments. Epilepsy Research, Suppl.1, 1988, sll1-sl17.

Mattson R.H., Cramer J.A., Collins J.F., and the Dept. of Veterans Affairs Epilepsy Co-ciperative Study No. 264 Group, A comparison of valproate with carbamazepine for the treatment of complex partial seizures and secondarily generalised tonic-clonic seizures in adults, The New England Journal of Medicine, 327, 1992, pp. 765-771.

Mattson R.H., Cramer J.A., Collins J.F., Smith D.B., Delgado-Escueta A.V., Comparison of carbamazepine, phenobarbital, phenytoin, and primidone in partial and secondarily generalized tonic-clonic seizures, The New England Journal of Medicine, 313, 1985, pp. 145-151. 
Medical Research Council Anti-epilcptic Drug Withdrawal Study Group, Randomised study of antiepileptic drug withdrawal in patients in remission, The Lancet, 337, 1991, pp. 1175-1180.

Medical Research Council Anti-epileptic Drug Withdrawal Study Group, Prognostic index for recurrence of seizures after remission of epilepsy, British Medical Journal, 306, 1993, pp.13741378.

Meijer J.W A., Knowledge, attitude and practice in anti-epileptic drug monitoring, thesis, Nijmegen, 1991.

Meinardi H., Binnie C.D., Goedhart D., Meijer J.W.A., Developments in pharmacotherapy of epilepsy. In: Epilepsy, an update on research and therapy, Alan R Liss Inc, New York, 1983, pp. 249-265.

Meinardi H., Pachlatko C., Special centres for epilcpsy, In: Comprehensive Epileptology (Dam and Gram, Eds.), Ravens Press, New York, pp. 769-779.

Meinardi H., Epilepsie compendium, Katholieke Universiteit Nijmegen, februari 1993.

Merlis J.K., Proposal for an International Classification of the Epilepsies, Epilepsia, 11, 1970, pp. 114119.

Metsemakers J.F.M. Unlocking patients' records in general practice for research, medical education and quality assurance: the Registration Network Family Practices, thesis, Maastricht, 1994.

Milano Collaborative Group For Studies On Epilepsy, Long-term intensive monitoring in difficult patient. Preliminary results of 16 months of observations-usefulness and limitations. In: Antiepileptic therapy advances in drug monitoring, (Johannessen, Pippenger, Schmidt, Morselli, Richens and Meinardi, Eds.), Ravens Prcss, New York, 1980

Montouris G.D. Practical insights and clinical experience with combinations of the new anti-epileptic drugs, Neurology, 45 (Suppl.2), 1995, s25-s28.

Mouritzen Dam A., Fuglsang-Fredriksen A., Svarre-Olsen U., Dam M., Late-onset epilepsy: aetiologies, types of seizures and value of clinical investigation, EEG, and computerized tomography scan, Epilepsia, 26, 1985, pp. 227-231

Oller-Daurella L., Oller F.V.L., Suppression of anti-epileptic treatment, European Neurology, 27, 1987, pp. 106-113.

Oller-Daurella L., Pamies R., Oller L.F.V., Reduction or discontinuance of anti-epileptic drugs in patients seizure-free for more than 5 years. In: Epileprology. Proc. of the $7^{\text {th }}$ International Symposium on Epilepsy, (Janz ed.). Thieme-Verlag, Stuttgart, 1976, pp. 218-227.

Oller-Daurella L., Oller L.F.V., Pamies R., Clinical, therapeutic and social status of epilepsy patients without seizures for more than five years, in: Epilepsy. The $8^{\text {th }}$ International Symposium, (Penry Ed.), Raven Press, New York, 1977, pp. 69-75

Overweg J., Epilepsie; prognose en bchandeling, Nederlands Tijdschrift voor de Geneeskunde, 128, 1984, pp. 1710-1716.

Overweg J., Withdrawal of anti-epileptic drugs in seizure-free adult patients, prediction of outcome, thesis, Amsterdam 1985, Rodopki.

Overweg J., Rowan A.J., Binnic C.D., Oosting J., Nagelkerke N.J.D., Prediction of seizure recurrence after withdrawal of anti-epileptic drugs, In: Advances in Epileptology: $12^{\text {th }}$ International Symposium, (Dam, Gram and Penry, Eds.), Raven Press, New York, 1981, pp. 503-508.

Parsons M., Fits and other causes of loss of consciousness while driving, Quarterly Journal of Medicine, New Series. 58, 1986, pp.295-303.

Pedley T.A., Discontinuing anti-epileptic drugs, New England Journal of Medicine, 318, 1988, pp. 982-984.

Pellock J.M., Standard approach to anti-epileptic drug treatment in the United States, Epilepsia, 35 (Suppl.4), 1994. s11-s18. 
Peterson G.M., McLean S., Millingen K.S., Determinants of patient compliance with anticonvulsant therapy, Epilepsia, 23, 1982, pp. 607-613

Peterson G.M., McLean S., Millingen K.S., A randomised trial of strategies to improve patient compliance with anticonvulsant therapy, Epilepsia, 25, 1984, pp. 412-417.

Porter R.J., Epilepsy, 100 elementary principles, W.B. Saunders Company Ltd., London, 1984.

Porter R.J. General principles how to usc anti-epileptic drugs. In: Anti-epileptic drugs, (Levy, Mattson, Meldrum, Penry and Dreifuss, Eds.), $3^{\text {rd }}$ edition, Raven Press, New York, 1989, pp. 117-131.

Quirk J.A., Fish D.R., Smith S., Sander J.W., Shorvon S.D., Allen P.J., First seizures associated with playing electronic screen games: a community based study, Annals of Neurology, 37, 1995 , pp.733-737.

Ramsay R.E., Wilder B.J., Berger J.R., Bruni J., A double blind study comparing carbamazepine with phenytoin as initial seizure therapy in adults, Neurology, 1983, pp. 904-910.

Reynolds E.H., Serum levels of anticonvulsant drugs; interpretation and clinical value, Pharmacology and Therapeutics, 8, 1980, pp. 217-235.

Reynolds E.H., Early treatment and prognosis of epilcpsy, Epilepsia, 28, 1987, pp. 97-106.

Reynolds E.H., The prevention of chronic epilepsy, Epilepsia, 29 (Suppl.1), 1988, s25-s28.

Reynolds E.H., Changing view of prognosis of epilepsy, British Medical Journal, 301, 1990, pp. $1112-1114$.

Reynolds E.H, Do anticonvulsants alter the natural course of epilepsy? Treatment should be started as carly as possible, British Medical Journal. 310, 1995, pp. 176.

Reynolds E.H., Chadwick D., Galbraith A.W., One drug (phenytoin) in the treatment of epilepsy, The Lancet, May, 1976, pp. 923-926.

Reynolds E.H., Heller A.J., Chadwick D., Valproate versus carbamazepine for seizures, The New England Journal of Medicine, 328, 1993, pp. 207-208.

Reynolds E.H., Shorvon S.D., Monotherapy or polypharmacy for epilepsy?, Epilepsia, 22, 1981, pp. $1-10$.

Richens A., Ahmad S., Controlled trial of sodium valproate in severe epilepsy, British Medical Journal, 1975, pp. 255-256.

Richens A., Davidson D.L.W., Cartlidge N.E.F., Easter J., EPITEG Collaborative Group, a multicentre comparative trial of sodium valproate and carbamazepine in adult onset epilepsy, Journal of Neurology, Neurosurgery and Psychiatry, 57, 1994, pp.682-687.

Rimmer E.M., Richens A., Clinical pharmacology and medical treatment. In: A textbook of epilepsy (Laidlaw, Richens and Oxley, Eds.). Churchill Livingstone, Edinburgh, 1988, pp. 421-483.

Rodin E.A., Rim C.S., Rennick P.M., The effects of carbamazepine on patients with psychomotor epilepsy: Results of a double blind study, Epilcpsia, 15, 1974, pp. 547-56I.

Rowan A.J., Diagnosis and treatment of epilepsy, Hospital and Community Psychiarry, 34, 1983, pp. 540-547.

Rutgers M.J., Geneeskundige en maatschappelijke aspecten van de zorg voor epilcpsiepatienten in Nederland, thesis, Rotterdam, 1984.

Salinsky M.C., Wegener K., Sinnema F., Epilepsy, driving laws and patient disclosure to physicians, Epilepsia, 33, 1992, pp 469-472.

Sander J.W.A.S., Some aspects of prognosis in the epilepsics: a revicw, Epilepsia, 34. 1993. pp. 10071016 
Sander J.W.A.S., Hart Y.M., Johnson A.L., Shorvon S.D., National General Practice Study of Epilcpsy: newly diagnosed epilcptic seizures in a general population, The Lancet, 336, 1990, pp. 1267-1271.

Scheuer M.L., Pedley T.A., The evaluation and treatment of seizures, The New England Journal of Medicine, 323, 1990, pp. 1468-1474.

Schmidt D. Janz D. Therapeutic plasma concentrations of phenytoin and phenobarbitone. In: Antiepileptic therupy advances in dnig moniloring. (Johannessen, Pippenger, Schmidt, Morselli, Richens and Meinardi, Eds.), Ravens Press, New York, 1980.

Schmidt D., Leppik I.E., Compliance in epilepsy: introduction, Epilepsy Research, Suppl.1, 1988, s3s4.

Schmidt D., Reininghaus R., Winkel R., Relevance of poor compliance for scizure control, Epilepsy Research, Suppl. 1, 1988, s141-s146.

Schobben A.F.A M., Pharmacokinetics and therapeutics in epilepsy, thesis, Nijmegen, 1979, pp.216.

Schoenberg B.S., Epideniological aspects of epilepsy. In: Epilepsy, an update on research and therapy, Alan R.Liss Inc, New York, 1983, pp. 331-343.

Scholtes F.B., Renier W.O., Meinardi H., Generalised convulsive status epilepticus, pathophysiology and treatment, Pharmacyworld and Treatment, 1993, 15, pp. 17-28

Shinnar S., Vining E., Mellits E.D., Discontinuing anti-epileptic medication in children with epilepsy after two years without seizures. The New Lingland Journal of Medicine. 313, 1985, pp.976-980.

Shinnar S., Kang H., Bcrg A.T., Goldensohn E.S., Hauser W.A., Moshé S.L., EEG abnormaties in children with a first unprovoked seizure, Epilepsia, 35, 1994, pp. 471-476.

Shorvon S.D., Reynolds E.H., Unnccussary polypharmacy for epilepsy, British Medical Journal, I, 1977, pp. 1635-1637

Shorvon S.D., Chadwick D., Galbraith A.W., Reynolds E.H., One drug for epilepsy, British Medical Journal, I, 1978. pp. 474-476.

Shorvon S.D., Galbraith A.W., Laundy M., Vydelingum L., Reynolds E.H., Monotherapy for epilepsy. In: Anti-epileptic therapy advances in drug monitoring, (Johannessen, Pippenger, Schmidt, Morselli, Richens and Meinardi, Eds.), Ravens Press, New York, 1980, pp. 213-220.

Shorvon S.D., Reynolds E.H., Early prognosis of epilcpsy, British Medical Journal, 285, 1982, pp. 1699-1701.

Shorvon S.D., The temporal aspects of prognosis in epilepsy, Journal of Neurology. Neurosurgery and Psychiatry, 47, 1984, pp. 1157-1165.

Shorvon S.D. The role of single drug and combination drug therapy in the treatment of epilepsy, in: Madern approach to anti-epileptic drug treatment, (Dam, Gram, and Pedersen, Eds.), The Danish Epilepsy Society, Jorgen Jensens Bogtrykkeri, Kobenhavn, 1985

Shorvon S.D., The treatment of epilepsy by drugs. In: Epilepsy, (Hopkins, Ed.), Chapman and Hall, London, 1987, pp. 229-282

Shorvon S.D., The coursc of untreated epilepsy, British Medical Journal, 297, 1988, pp. 1405.

Shorvon S.D., Epidemiology, classification, natural history and genetics of epilepsy, The Lancet, 336, 1990, pp. 93-96.

Shorvon S.D.. Medical assessment and treatment of chronic epilepsy, all patients should have a long term plan of treatment. British Medical Journal. 302, 1991, pp. 363-366.

Shorvon S.D. Epilepsy and driving. British regulations have recently been eased British Medical Jourmal. 310, Ling, pp.885-886. 
Simonsen N., Zander Olsen P., Kühl V., Lund M. Wendelboe J., A comparative controlled study between carbamazepine and diphenylhydantoin in psychomotor epilepsy, Epilepsia, 17, 1976, pp 169-176.

Sonnen A.E.H., De medicamenteuze behandeling van epilepsic, Nederlands Tijdschrift voor de (ienceskunde, 123, 1979, pp. 485-490.

Swartz B.E., Delgado-Escueta A.V., The management of status epilepticus, In: Epilepsy, (Hopkins, Ed.), Chapman and Hall, London, 1987, pp. 417-442.

Taylor J., Driving as an occupation. In: Epilepsy and employment (Edwards, Espir and Oxley, Eds.), Royal Socicty of Medicine Services Limited, London, 1986, pp. 89-92.

Temkin N.R., Dikmen S.S.,Wilensky A.J., Keihm J., Chabal S., Winn H.R, A randomised doubleblind study of phenytoin for the prevention of post-traumatic seizures, The New England Journal of Medicine, 323, 1990, pp. 497-502.

Tennison M.B., Greenwood R.S., Lewis D.V., Benoit S.E., Rate of taper of anti-epileptic drugs and the risk of seizure recurrence, Annals of Neurology, 26, 1989, pp. 439.

Tennison M., Greenwood R. Lewis D., Thorn M., Discontinuing anti-epileptic drugs in children with epilepsy, The New England Journal of Medicine, 330, 20, 1994, pp. 1407-1410.

Thompson P.J., Oxley J. Socioeconomic accompaniments of severe epilepsy, Epilepsia, 29 (Suppl.1), 1988, pp. s9-s।8.

Thorbecke R., Measurement of compliance through patient interviews, Epilepsy Research, Suppl.1, 1988, pp. 79-83.

Thurston J.H., Thurston D.L.. O'Leary J.. Follow-up study of 148 cases in which therapy had been suspended after prolonged anticonvulsant control. The New England Journal of Medicine, 286, 1972, pp. 169-174.

Thurston J.H., Thurston D.L., Hixon B.B., Keller A.J., Additional follow-up of I48 children 15 to 23 years after withdrawal of anticonvulsant therapy, The New England Journal of Medicine, 306, 1982, pp. 831-836.

Trostle J., Doctors orders and patients' sclf-interest: two views of medication usage?, Epilepsy. Research, Suppl. I, 1988, s57-s69.

Troupin A., Moretti Ojemann L., Halpem L.. Dodrill C., Wilkus R., Friel P., Feigl P., Carbamazcpinea double blind comparison with phenytoin, Neurology, 1977, pp. 511-519.

Tumbull D.M., Rawlins M.D.. Weightman D.. Chadwick D.W., A comparison of phenytoin and valproate in previously untrcatcd adult epileptic patients, Journal of Neurology. Neurosurgery and Psychiatry, 45, 1982, pp. 55-59.

Wagner A.L., A clinical and epidemiological study of adult patients with epilepsy, Acta Neurologica Scandinavia, 94 (Suppl), 1983, s63-s72.

Wallace S.J., Febrile convulsions. In: Epilepsy, (Hopkins, Ed.), Chapman and Hall, London, pp. 443467.

Wannamaker B.B., Morton W.A., Gross A.J., Saunders S., Improvement in the anti-epileptic drug levels following reduction of intervals between clinic visits, lipilepsia, 21, 1980, pp. 155-162.

Wilder B.J., Ramsay R.E., Murphy J.V., Karas B.J., Marquardt K., Hammond E.J., Comparison of valproic acid and phenytoin in newly diagnosed tonic-clonic seizures, Neurology, 33, 1983, pp. 1474-1476.

Wilder B.J., The treatment of epilepsy: an overvicw of clinical practices, Neurology, 45 (Suppl.2), 1995, s7-sll. 
Woo E., Chan Y.M., Yu Y.L., Chan Y.W., Huang C.Y., If a well-stabilised epileptic patient has a subtherapeutic anti-epileptic drug level, should the dose be increased? A randomised prospective study, Epilepsia, 29, 1988, pp. 129-139.

Zielinski J.J., Epileptics not in treatment, Epilepsia, 15, 1974, pp. 203-210.

Zielinski J.J., Epidemiology. In: A textbook of epilepsy (Laidlaw, Richens and Oxley, Eds.), Churchill Livingstone, Edinburgh, 1988, pp. $21-48$. 
CHAPTER 3

\section{System development}

\subsection{Introduction}

Rather than using comparatively domain independent methods such as mathematics or statistics, some decision support systems (DSSs) use a model of the domain based on a symbolic representation of expertise to solve problems. Such DSSs are called knowledge based systems (KBSs). Development of a KBS is a type of task which requires an approach different from conventional system development. In this chapter we will discuss different approaches for KBS development.

KADS - Knowledge Acquisition, Documentation and Structuring - is a representative of structured development methodologies (Breuker and Wielinga 1984a). Besides the provision of socalled elicitation techniques for making knowledge explicit, KADS provides a set of domain independent models which enables analysis of the extracted knowledge. An instantiation of these models is the conceptual model: a description of the problem solving process that may be used for implementation. At the end of this chapter we will present two models which describe different parts of the problem solving process with respect to the drug treatment of epilepsy. Both were developed using the KADS methodology.

\subsection{Methods for KBS development}

A KBS is a model of a problem solving task an expert performs (Jackson 1986, Waterman 1986). This is in contrast with conventional systems which focus on data processing and information exchange tasks as organised around forms (Weitzel and Kerschberg, 1989). During the development of a KBS one has to analyse and model the cognitive processes of e.g. interpreting patient data and applying medical knowledge (Hayes-Roth et al 1983). The cognitive processes are less circumscript than the data processing and information exchange tasks. Building a KBS therefore requires different methods than used in conventional software engineering.

The knowledge applied by neurologists consists of facts and heuristics. This knowledge has to be elicited and encoded, i.e. represented in a symbolic way and it has to be stored in a socalled knowledge base. The process of obtaining the knowledge from the expert and representing it in such a way that it can be formalised in a computer program is called knowledge acquisition. The knowledge acquisition process consists of two separate activities: elicitation and analysis of knowledge. Knowledge acquisition is generally recognised as the main bottleneck in the development of KBSs (Hayes-Roth et al. 1983, Buchanan and Shortliffe, 1984) 
Schreiber and Wielinga (1993) present two views on KBS development:

- The traditional tramsfer view in which the knowledge acquisition process is considered as a process of extracting the knowledge from a human expert and transferring the knowledge into the KBS. An example of this approach is development by prototyping (see below). This approach is often constrained by the representation formalism that is available.

- The modelling view in which the knowledge acquisition process is separated from the implementation of the knowledge. An example of this approach is KADS.

These two views will be described in the following sections.

\subsubsection{Prototyping}

Because it is not always clear what a system should do, users may frequently change previously defined requirements (Wellbank, 1983). Prototyping is used to help the user analyse his ideas on the problem at hand, the functionality of the system and the human-computer interface. In prototyping a system develops in an iterative way. The prototype system is used as a device for interviewing the domain expert on issues relevant for a proper performance of the intended system such as data entry, output of results and response time. Thus, prototyping clarifies aspects of the system's functionality by enabling direct feedback by the user on an implementation of a part of the system (Diaper, 1989). Prototyping can also support the establishment of the necessary co-operative atmosphere between the expert providing the knowledge and the knowledge engineer (Heng, 1987). There are three categories of prototyping: experimental, evolutionary and incremental.

Experimental prototyping is used to show the user different aspects of functionality and to clarify the problem at hand. After a short problem identification phase a model is built. This prototype exhibits limited functional capabilities, low reliability and possibly inefficient performance. The prototype is assessed with the objective to clarify the user requirements and to determine additional requirements. After the assessment the model is discarded. A synonym for this technique is throw-away prototyping (van Dijk et al. 1988).

When the user requirements are available, they may be incomplete or may still be subject to change. Evolutionary protolyping allows a system to evolve according to changing user needs. The process consists of a sequence of prototypes which (hopefully) converge into the final system or parts of it (van Dijk et al. 1988, Tate 1990)

Users may also be able to provide a fairly complete description of the functionality of the system. Clarification of requirements may be necessary on subparts only. In incremental prototyping the complete system design is specified and subtasks are identified. Next these subtasks are implemented, assessed and modified according to the user feedback. In contrast to the evolutionary approach, in incremental prototyping the overall design remains in principle unaltered (Stephens and Bates, 1990)

Experience with prototyping has revealed several disadvantages:

- The task to be performed by the system may change. Major design decisions are made in an early phase of development. In case of incorrect decisions the prototype may not be useful for the next iteration. 
- Maintainability of the evolving system may become a problem (Mayhew and Dearnley, 1990). Although a prototype knowledge base may do well, it may not be possible to scale it up to the full size required.

- Since (part of) the prototype should be realistic the user may be misled and take the prototype for the final system. Strong user reluctance to throw away a prototype that was designed to be discarded has been reported (Stephens and Bates, 1990).

\subsubsection{Structured development}

Prototyping is a kind of code-and-fix oriented approach of system development. In a more rigid approach one uses a predefined framework describing how to analyse the domain and what design issues to take into account. This may facilitate a more comprehensive view on the domain.

\section{Slage based approaches}

Stage based or life-cycle approaches to KBS development divide the development process into separate phases. For each of these phases a set of activities is specified. Different life-cycle models have been presented such as the waterfall model (Royce, 1987), the Hayes-Roth feedback model (Hayes-Roth et al. 1983) and the spiral model (Boehm, 1988). The application of these stage based approaches can be difficult: what the intermediate results of each of the stages should include is only vaguely presented by the authors. Also techniques and tools for supporting the different phases are usually lacking (Fen and Veren, 1991).

A more detailed approach for a stage based development of KBSs is the Pragmatic Knowledge Acquisition Methodology (PKAM) (Grover, 1983). It consists of three phases in which a domain is defined, fundamental knowledge is acquired and the obtained knowledge is improved. This methodology mentions a set of issues to be addressed in each of the phases and contains a number of techniques for knowledge elicitation. Studies on the utility of this approach however are lacking.

\section{KADS}

A more comprehensive methodology is KADS (Knowledge Acquisition, Documentation and Structuring, Breuker and Wielinga 1984a). It describes procedures and documentation, as well as tools and methods for KBS development. KADS emphasises the pre-implementation phases. An extensive analysis of the knowledge is advocated before system design and implementation take place.

The analysis phase in the most recent version of KADS consists of two streams: the external and the internal stream (Breuker and Wielinga 1984ab, Breuker and Wielinga 1987, Breuker et al. 1987, Breuker and Winkels, 1989). In the external stream the analysis of requirements and constraints concerning the system takes place. The internal stream describes the analysis of expertise. The methodology of the external stream is hardly documented. The analysis of the expertise was considered most important (Hickman et al. 1989, Schreiber et al. 1993). Analysis of expertise on different levels of abstraction has to be performed:

- Linguistic analysis, the identification of domain concepts in verbal data

- Conceptual analysis; the integration of individual concepts through formalised description(s), e g. compound concepts, relations and models

- Epistemological analysis; the definition of structural properties of the conceptual knowledge 
- Logical analysis; the definition of the formalisms with which the knowledge and inference making is expressed

- Implementation analysis

The level of abstraction increases from the linguistic to the epistemological level and then decreases again. The most important description of expertise results from the epistemological analysis

In KADS the epistemological description of expertise consists of four layers each describing a different category of knowledge: domain, inference, task and strategic knowledge. The domain layer contains the concepts and rules of the domain without an indication of when and how they can be applied. The inference layer depicts the relation between groups of concepts and inference procedures without an indication of the sequence of their application. This sequence of invocation of the inference procedures is described at the task layer. As the problem solving process may consist of multiple tasks, each of these tasks and the procedure for their invocation is described at the strategic layer

KADS provides a set of tools for knowledge elicitation as well as two facilities that support the analysis of expertise at the epistemological level: a conceptual modelling language and interpretation models. The conceptual modelling language provides a set of primitives for bottom-up development and refinement of the inference layer. These primitives consist of so called metaclasses and knowledge sources. Metaclasses are descriptions of domain concepts in abstract terms, indicating their roles in the reasoning process. The knowledge sources contain the inference procedures. They can be viewed as abstract operations on the metaclasses (Breuker and Wielinga, 1987)

To support the description at the inference layer, interpretation models have been developed. An interpretation model is a domain independent description of a specific type of problem solving task (Wielinga and Breuker, 1987). It provides a structure for analysing the verbalised data. The use of interpretation models resembles the idea of generic tasks: a limited set of general problem solving approaches. These generic tasks describe structures for problem solving which can be instantiated for a specific domain. Brown and Chandrasekaran (1989) define generic tasks as an elementary combination of a problem, a representation and an inference strategy. In contrast, the interpretation models in KADS do not at all address issues related to implementation such as representation aspects. The descriptions of tasks and inference structures in KADS are comparable with data-flow diagrams in conventional software engineering (Schreiber and Wielinga, 1993)

Proponents of structured development advocate an extensive analysis of the domain before commencing the implementation of a DSS. Such an analysis clarifies the view on the domain for which a DSS is developed. Due to the separation of analysis of expertise and its implementation, design decisions can be postponed until a more complete view on the domain and tasks has been obtained. It is assumed that analysis of expertise before implementation assures a more complete description of the expertise and thus a more efficient implementation can be expected (Schreiber et al. 1993) 


\subsubsection{Selection of the development method}

The acceptance of a KBS will depend both on the quality of the advice provided and on the functionality of the system. Although both aspects are important, confidence in the advice is considered a primary issue of concern (O'Keefe et al. 1987, O'Keefe 1989, O'Moore et al. 1990, Wyatt 1991). In prototyping the implementation starts with only a part of the knowledge. This approach makes a less optimal implementation more likely. Incremental improvement of implemented knowledge can cause inconsistencies particularly in complex systems (Nguyen et al. 1985). Given these problems a structured approach for the analysis of expertise in the domain of anti-epileptic drug treatment was considered more appropriate. As KADS provides the most comprehensive approach for structured development of KBSs it was selected as the approach for analysis of expertise in our work.

Prototyping is useful within KADS when it is used as a technique to support analysis and design activities (Nobis and Siedka-Bauer 1988, Tansley and Hayball, 1993). It provides the expert with insight in the procedures which are followed by the system (Hickman et al. 1989). The modelling of expertise in our work was alternated with an implementation of the obtained knowledge to analyse and demonstrate the interaction of the different layers of the model of expertise.

\subsection{Knowledge elicitation}

The process of obtaining knowledge from a human expert is called knowledge elicitation. The extracted knowledge may be modelled, i.e interpreted using some conceptual framework. Although elicitation and modelling are different activities, they are closely related and may direct each other's focus to specific parts of the knowledge.

Indirect techniques such as card sorting, multi-dimensional scaling, cluster analysis, general weighted networks and repertory grids provide a low level approach to knowledge elicitation (Wellbank 1983, Cooke and McDonald 1986, 1987, Burton et al. 1987, Wright and Ayton 1987, Shaw 1988). They enable the exploration of the domain by creating maps displaying the structure of the concepts in the domain. This structure is often derived from a (complete) set of perceived distances between pairs of concepts. The usability of the result depends on the completeness of the set of concepts used for obtaining the distance measures. If the set is not complete bias may result. This will show up in an erroneous description of the structure of domain concepts. How the set of concepts shall (or can) be obtained is not defined. One could question whether the development of a KBS is appropriate when the knowledge of a domain is so little developed that the structure of the domain still has to be identified by these techniques.

Apart from the card sorting technique all of the indirect techniques require the use of a kind of distance matrix in which all possible pairs of concepts are presented. Filling in this matrix is a tedious procedure. The expert may not be convinced of the necessity of such an exercise. Furthermore, the use of indirect techniques will not reveal the actual usage of concepts in the problem solving process (Hoffman, 1987).

Focused interviews, structured interviews, introspection, dialogues, protocol analysis, interruption analysis and questionnaires are direct techniques for knowledge elicitation (Reitman Olsen and Rueter, 1987). With these techniques, the role of concepts and the 
structurs: of the problem solving is derived directly from the verbal descriptions (Breuker and Wielinga 1984b).

A critical issue of direct techniques is that a successful application depends on the observer's skills for interviewing the expert. These techniques are also time consuming. However, most of the burden is on the interviewer, not on the domain expert. The interviewer has to transcribe the recordings of the interviews and to analyse the information obtained. Direct techniques are applied most often (Cullen and Bryman, 1988).

There is no formal methodology for knowledge elicitation (Wellbank 1983, Shadbolt and Burton 1989). The applicability of a knowledge elicitation technique may differ across domains. The suitability of a technique may depend on the personality (introvert/extrovert) and cognitive style (field dependent/independent) of the expert (Burton et al. 1988).

We only used direct techniques for knowledge elicitation. They allow the expert to express his knowledge in a natural way. There was no need to establish a structure of concepts in the domain as this knowledge was available. Our main interest was to obtain insight in how the knowledge is applied when performing tasks in the management of epilepsy patients.

\subsection{Models of anti-epileptic drug treatment}

The interviews and discussions with the expert and the literature review demonstrated that the treatment of epilepsy has different phases

- During the first phase the objective is to obtain enough information to establish a diagnosis. More than one patient-physician contact may be needed.

- The second phase starts when the diagnosis is established. Here the treatment is discussed and selected.

- The third phase consists of the evaluation of the treatment. The drug treatment may be adjusted depending on the symptomatology.

The model of expertise is based on an analysis of this phased approach to the treatment of epilepsy. We focused on the knowledge concerning the initiation and modification of the treatment.

\subsection{Strategic layer}

The management of a chronic disease is composed of a set of procedures for the diagnosis, the initiation of a therapy, the monitoring of the effect of the therapy and if possible the withdrawal of the treatment (Figure 3.5-1). For epilepsy the strategy - followed after the establishment of the diagnosis - can be described as follows. A drug therapy will be initiated with the intention to relief the patient from the seizures. The effectiveness of the drug treatment is checked by monitoring the seizure frequency and the well-being of the patient. The effectiveness of a drug may vary among patients. If the drug treatment proves ineffective the treatment is adjusted (a change in the drug dose or the initiation of another anti-epileptic drug). The effect of the adjustment is monitored. The adjustment of the treatment will end when seizures are controlled i.e. they do not occur anymore. When control of seizures has been established the monitoring of the patient continues for at least two years. After this period tapering of the drug treatment may be started. The results of changes in therapy will again be monitored. If seizures remain controlled the reduction of drug treatment will continue. 


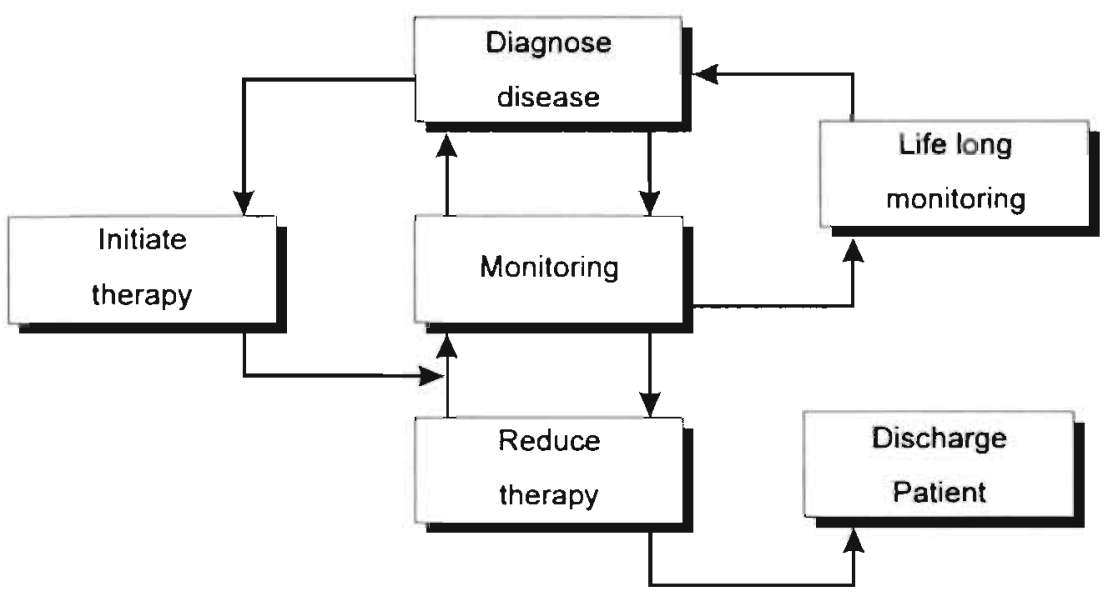

Figure 3.5-I: Strategy for management of epilepsy.

In case drug treatment is ineffective to control seizures completely or when tapering causes reoccurrence of seizures, life-long monitoring may be necessary: an approach in which adjustments of the treatment are made based on balancing the consequences of seizures and sideeffects.

\subsection{Inference structures}

The problem solving structures of both the initiation part and the monitoring part of the treatment were analysed. The analysis of the monitoring part of the treatment was guided by the KADS interpretation models for monitoring and assessment. Due to the lack of appropriate interpretation models for the process of initiating the treatment, we had to construct such a model bottom-up.

\subsubsection{Initiation of drug treatment}

The process for initiation of anti-epileptic drug treatment is decomposed into the subtasks of:

- Selecting the anti-epileptic drug

- Selecting the drug dose and

- Calculating the number of tablets and the intake scheme

For each of these subtasks the relevant domain concepts were extracted from the transcripts of the interviews and dialogues.

The epistemological analysis resulted in an identification of the various roles concepts have in the problem solving process (e.g. the group of main anti-epileptic drugs is used as a set of 
hypotheses from which the most appropriate can be selected; drug side-effects are used as constraints in this selection process). Concepts which have a similar role in the problem solving process are grouped in so-called metaclasses'.

Table 3.6-I: Description of the metaclasses used in the inference model for initiation of anti-epileptic drug treatment.

\begin{tabular}{|c|c|c|}
\hline METACLASS & CONCEPTS & ROL.E \\
\hline SKFLETAL MODEL & prescription data & template of a prescription \\
\hline COMPONENT & $\begin{array}{l}\text { generic drugname } \\
\text { brandname } \\
\text { dose of tablets } \\
\text { number of tablets } \\
\text { intake schemes }\end{array}$ & concepts to be assigned a value \\
\hline RESULT PART & $\begin{array}{l}\text { generic drugname } \\
\text { brundname } \\
\text { dose of tablets } \\
\text { number of tablets } \\
\text { intake schemes }\end{array}$ & instantiated components \\
\hline MODEL & prescription data & suggested drug trealment \\
\hline CASE DESCRIPTION & patient data & portrayal of diseased person \\
\hline HYPOTHESES & anti-epileptic drugs data & $\begin{array}{l}\text { providing options for selection of brand } \\
\text { providing options for selection of daily dose } \\
\text { providing options for selection of dose of tablets }\end{array}$ \\
\hline \multirow[t]{4}{*}{ CONSTRAINTS } & $\begin{array}{l}\text { type of epilepsy } \\
\text { type of seizures } \\
\text { co-medication } \\
\text { drug side-effects } \\
\text { ineflective drugs }\end{array}$ & restrictions for selection of drug \\
\hline & $\begin{array}{l}\text { weight } \\
\text { laboratory results }\end{array}$ & restrictious for selection of daily dose \\
\hline & $\begin{array}{l}\text { generic drugname } \\
\text { brandname } \\
\text { dose of tablets }\end{array}$ & $\begin{array}{l}\text { restrictions for selection of brand } \\
\text { restrictions for selection of dose of tablets } \\
\text { restrictions for assembling of intake scheme }\end{array}$ \\
\hline & $\begin{array}{l}\text { number of tablets } \\
\text { return date }\end{array}$ & $\begin{array}{l}\text { restriclions for assimbling of intake scheme/selection of } \\
\text { total number of tablets }\end{array}$ \\
\hline ALTERNATTVES & $\begin{array}{l}\text { optional generic drug } \\
\text { optional brandname } \\
\text { optional dose of tablets } \\
\text { optional number of lablets } \\
\text { optional intake scheme }\end{array}$ & choices for prescription \\
\hline
\end{tabular}

'The typographic conventions used in the description of inference structures are as follows:

- Tasks are printed in italicised capitals (OBTAIN_DRUGNAME)

- Metaclasses are printed in capitals (c.g. METACLASS)

- Knowledge sources are printed in sinall italicised letters (e.g. knowledge source)

- Individual concepts are printed in small bold capitals (e.g. CONCEPT)

- Values of concepts are printed in sinall capitals (c.g. Valuie)

We use the notation (CONCEPT VALUE) to indicate a concept and its value. 
The metaclasses used in the inference model for initiation of therapy are presented in Table 3.6-I. As concepts may have more than one role in the problem solving process, they can occur in different metaclasses. This also means that a concept (e.g. CARBAMAzEPINE) may occur as a value e.g. in (PRESCRIBED-DRUG CARBAMAZEPINE).

A second part of the elicited knowledge consists of rules, each describing a condition which leads to an assertion of a new fact or an action like the commencement of a task. In our case they are grouped by so called knowledge sources, a set of rules dealing with a specific inference. In an abstract sense a knowledge source is an inference process which instantiates concepts in the output metaclass given instantiated concepts in the input metaclass(es). The inference layer for the initiation of drug treatment is presented in Figure 3.6-I. Metaclasses are represented by rectangles and knowledge sources by ovals. An example of a knowledge source is assign-value which gives a concept in the metaclass RESULT PART a value (e.g. GENERIC DRUGNAME the value CARBAMAZEPINE) based on instantiated concepts in the metaclasses COMPONENT and ALTERNATIVES.

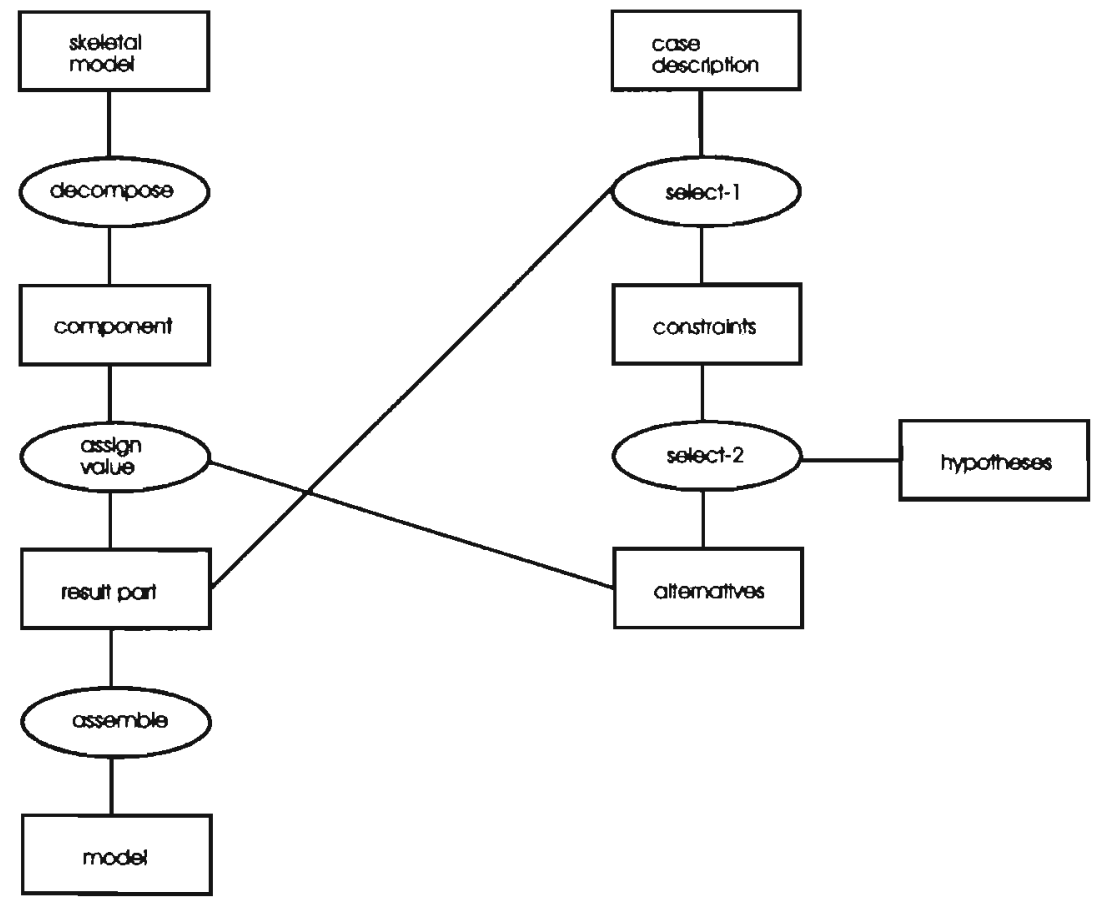

Figure 3.6-I: Inference layer for the initiation of anti-epileptic drug treatment, depicting the metaclasses (rectangles) and knowledge sources (ovals). 
The knowledge sources in Figure 3.6-I are composite ones. By decomposing these knowledge sources into primitive knowledge sources a more detailed view on the inference process can be obtained. Addressing the knowledge sources of the inference layer keeps the knowledge elicitation process focused.

The task structure for the initiation of anti-epileptic drug treatment is presented in Figure 3.6-II. In the task structure, the sequence of application of the knowledge sources is defined as well as the involved metaclasses. In the following the task structure for initiation of a therapy will be discussed and illustrated by the inference model of this task.

Figure 3.6-II: Task layer describing the control structure for the initiation of an anti-epileptic drug treatment.
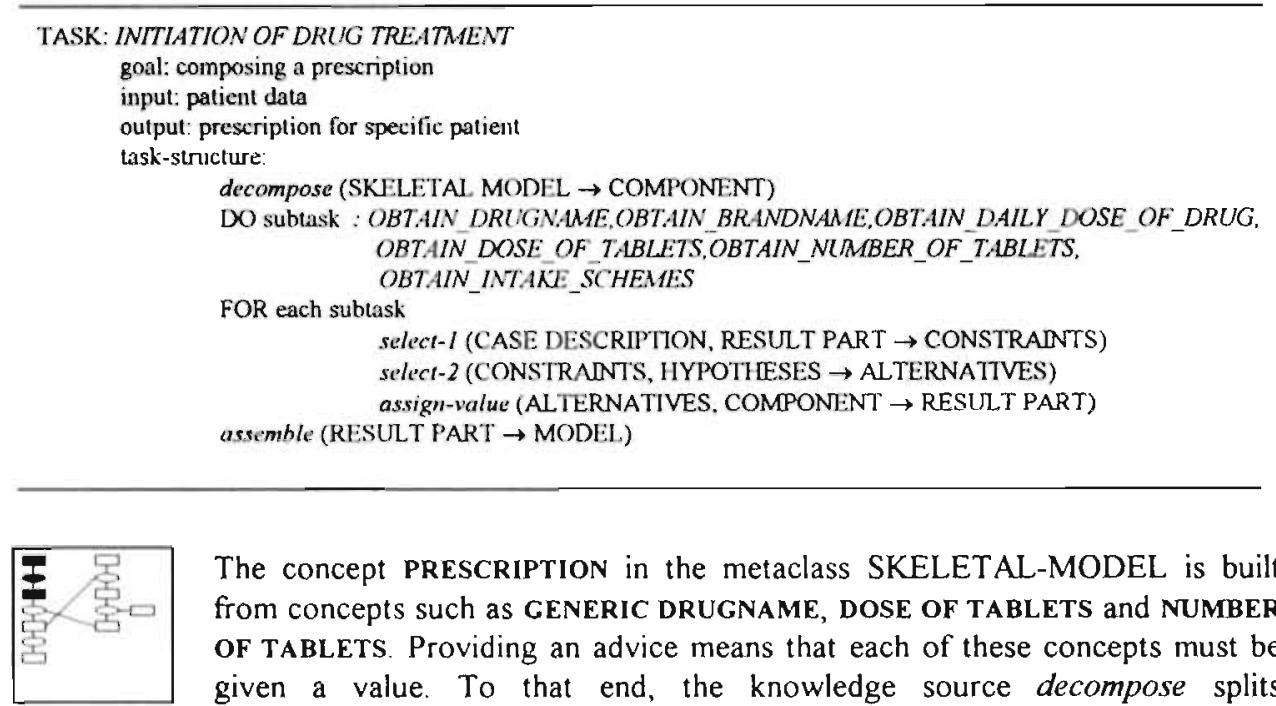

The concept PRESCRIPTION in the metaclass SKELETAL-MODEL is built from concepts such as GENERIC DRUGNAME, DOSE OF TABLETS and NUMBER OF TABLETS. Providing an advice means that each of these concepts must be given a value. To that end, the knowledge source decompose splits PRESCRIPTION into its constituent concepts. These concepts are all contained in the metaclass COMPONENT. ${ }^{2}$

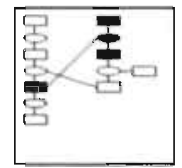

Next several subtasks are to be performed in sequence. As an example we describe the subtask OBTAIN DRUGNAME. This subtask assigns a specific drugname to the concept GENERIC DRUGNAME of the metaclass COMPONENT. To achieve this, information about the patient is extracted from the metaclass CASE DESCRIPTION. For example, the concept TYPE-OFEPILEPSY together with its value PARTIAL EPILEPSY is singled out by the knowledge source select-1. This concept is placed into the metaclass CONSTRAINTS, containing instantiated concepts which narrow down the choice of the drug. Note that the instantiated concepts in RESULT PART can influence the selection

${ }^{2}$ Black rectangles and ovals identify the metaclasses and knowledge sources discussed in the adjacent text. 


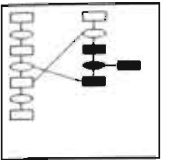

The metaclass HYPOTHESES contains data on the major anti-epileptic drugs. A set of first choice anti-epileptic drugs (CARBAMAZEPINE, PHENYTONN) is derived by select-2 using the information from CONSTRAINTS. This set of drugs becomes the value of the concept OPTIONAL GENERIC DRUGS in the metaclass ALTERNATIVES.

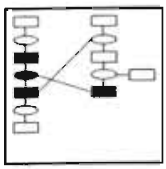

From this set of alternatives a drug (e.g. CARBAMAZEPINE) is selected. The knowledge source assign-value adds this piece of information to the GENERIC DRUGNAME in the metaclass RESULT PART.

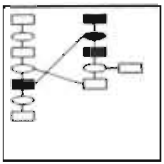

The process is repeated for the other subtasks which each add values to concepts in the metaclass COMPONENT, after obtaining information stored in CASE DESCRIPTION as well as from the intermediate conclusions from metaclass RESULT PART. For example, in the subtask OBTAIN DAILY DO$S E \_O F \_D R U G$, select-I takes the anti-epileptic drug from the GENERIC DRUGNAME in RESULT PART as well as additional restrictive information from the CASE DESCRIPTION such as the LABORATORY-RESULTS and WEIGHT. This information is stored in CONSTRANTS.

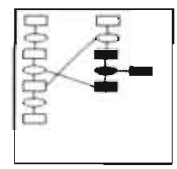

Next, information on DRUG DOSE IN MG/KG, MAXIMUM DOSE and AVAILABLE DOSES of TABLETS is obtained from the drug data in HYPOTHESES. This information is used by the composite knowledge source select-2 to obtain a drug dose for the patient. The dose is adjusted according to the MAXIMUM DOSE, the AVAILABLE DOSE OF TABLETS and the MINIMUM INTAKE FREQUENCY. The calculated dose serves as input for the calculation of values of the concepts OPTIONAL DOSE OF TABLETS and OPTIONAL NUMBER OF TABLETS in ALTERNATIVES.

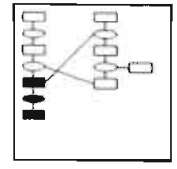

In this way each of the concepts in COMPONENT is assigned a value which is considered most appropriate given the status of the patient and the available medication. When the concepts belonging to the intake scheme are assigned a value, the individual concepts in RESULT PART are restructured by knowledge source assemble into the concept PRESCRIPTION in the metaclass MODEL.

\subsubsection{Monitoring drug treatment}

The KADS library has two interpretation models that are useful for modelling the monitoring of drug treatment: monitoring and assessment. In a monitoring task a comparison of individual data against a norm is made, followed by a classification of the difference, taking into account information from previous patient contacts In the assessment task changes in a group of concepts instead of in a single concept are classified. Table 3.6-III gives an example of the concepts contained by metaclasses in the monitoring process. 
Table 3.6-III: Examples of concepts contained by metaclasses defined in the monitoring process.

\begin{tabular}{|c|c|c|}
\hline METACLASS & CONCEPTS & ROLE \\
\hline CASE DESCRIPTION & patient data & description of cument state of the patient \\
\hline SELECTION CRITERLA & pathophysiological model & description of disease \\
\hline SYSTEM MODEL & physiological model & description of state of a healthy person \\
\hline VARLABLE SET & disease concepts & symptoms related to the patient \\
\hline PARAMETER SET & physiological data of healthy person & symptoms in case not diseased \\
\hline VARIABLE VALUE & $\begin{array}{l}\text { type of epilepsy } \\
\text { type of seizure } \\
\text { frequency of seizures etc. }\end{array}$ & values of symptoms when diseased \\
\hline PARAMETER VALUE & values of physiologic data & values of symptoms when not diseased \\
\hline DIFFERENCE & $\begin{array}{l}\text { presence of epilepsy } \\
\text { presence of seizure } \\
\text { presence of complaints etc. }\end{array}$ & $\begin{array}{l}\text { conclusions on symptoms relevant when } \\
\text { diseased }\end{array}$ \\
\hline HISTORICAL DATA & data from previous contacts & points of reference \\
\hline DISCREPANCY CLASSES & essential data on the patient & course of the disease \\
\hline MODEL SET & prototypical patient descriptions & profiles of standard patients \\
\hline NORM & $\begin{array}{l}\text { standard course of epilepsy } \\
\text { standard course of complaints } \\
\text { standard treaunent procedures etc. }\end{array}$ & $\begin{array}{l}\text { standard values of disease concepts and } \\
\text { treatment strategies }\end{array}$ \\
\hline DECISION CLASS & treatunent procedure & adjustment of treatment \\
\hline
\end{tabular}

Figure 3.6-111: Task structure of the anti-epileptic dng monitoring process.

TASK: MONITORING model-driven

goal: obtain conclusion on progress of disease

input: INITIATION OF DRLIG TREATMENT (MODEL)

output: DISCREPANCY CLASSES

task structure:

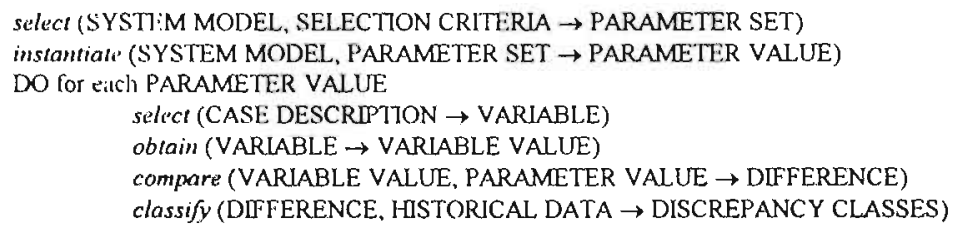

TASK : ASSESSMENT

goal: obtain a treatment procedure input MONITORING (DISCREPANCY CLASSES)

output: DECISION CLASS

task structure

specify (MODEL SET $\rightarrow$ NORMA)

match (DISCREPANCY CLASSES. NORM $\rightarrow$ DECISION CLASS) 


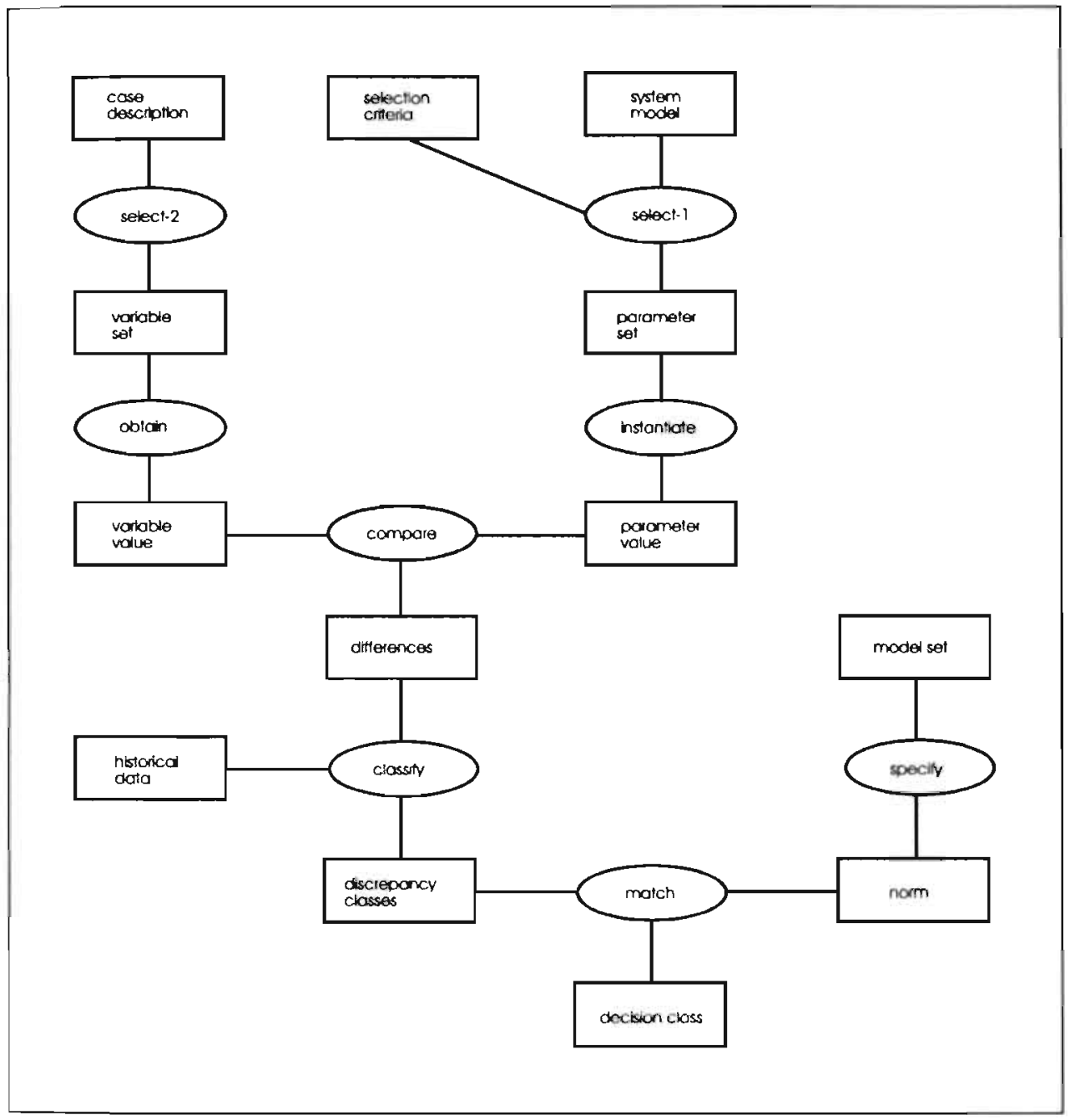

Figure 3.6-rV: Inference model for monitoring drug treatment of epilepsy.

From interviews and on-the-job-recordings it became clear that both a data-driven approach and a model-driven approach to monitoring may be applied. In a data-driven approach it is the patient who takes the initiative by starting to provide information on the effectiveness of the treatment. In the model-driven approach it is the physician who starts asking questions. In both approaches an identical set of issues - but not necessarily in the same sequence - is addressed for analysing the effectiveness of the treatment. In the following discussion the model-driven approach is used since this approach is more structured (see Figure 3.6-III for the task description). In Figure 3.6-IV the inference model of monitoring the drug treatment is presented. 
The interpretation models for the monitoring and assessment tasks are connected through metaclass DISCREPANCY CI.ASSES which contains the concepts that describe the course of the disease in the patient. The data from the concepts in this metaclass are matched with a set of profiles characterising possible courses of the disease. The prototypical profile matching the description of the disease course in the patient under consideration provides information for further treatment.

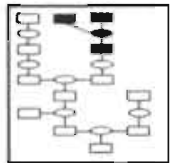

The concepts needed for monitoring the treatment process such as SEIZUREOCCURRENCE, TYPE-OF-SEIZURES, NUMBER-OF-SEIZURES, TYPE-OFEPILEPSY, COMPLAINTS, SIDE-EFFECTS, and COMPLIANCE are contained in the metaclass SELECTION CRITERIA. The SYSTEM MODEL represents the goal of the treatment e.g. the state of a healthy person. The knowledge source select $l$ singles out the relevant concepts from the metaclass SELECTION CRITERIA and obtains the values for these concepts from the SYSTEM MODEL. This piece of information is placed into the metaclass PARAMETER SET. For example, the metaclass PARAMETER SET would contain (SEIZURE-OCCURRENCE NO) for a healthy person.

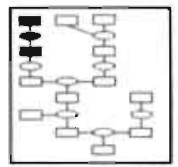

Next the corresponding concepts are singled out by select-2 from the metaclass CASE DESCRIPTION, which contains curTent information on the patient. As an example this could be (SEIZURE-OCCURRENCE YIS). This information is put into the VARIABLE SET

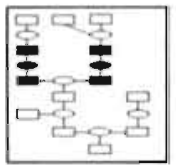

To compare these values, they are retrieved from the metaclasses VARIABLE SET and PARAMETER SET by respectively ubtuin and instantiate. The values are stored into the metaclass VARIABLE VALUE and PARAMETER VALUE respectively.

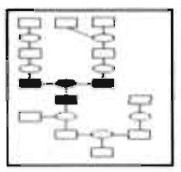

In our example the value of the concept SEIZURE-OCCURRENCE in the patient is different from the value in the physiological model. The conclusion of this comparison is denoted by the concept PRESENCE-OF-SEIZURES with the value PRESENT stored in the metaclass DIFFERENCES

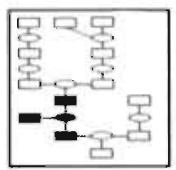

Information on the issue at stake may be compared with the values recorded during previous contacts listed in the metaclass HISTORICAL DATA. The result of the comparisons is contained by the metaclass DISCREPANCY CLASSES. In this case, if the value for PRESENCE-OF-SEIZURES in HISTORICAL DATA is ABSENT, the concept SEIZURE-COURSE in DISCREPANCY CLASSES receives the value INCREASED

By repeating this process for all concepts in metaclass SELECTION CRITERIA, all relevant aspects will be taken from the patient information and compared with historical data.

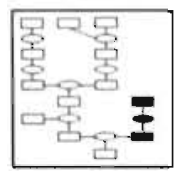

The 'status presence' of disease related items as stored in DISCREPANCY CLASSES is matched with similar items in a set of prototypical cases for which the treatment decision is known (Table 3.6-IV). The MODEL SET contains a description of these prototypical cases. From this metaclass concepts are derived and placed into the metaclass NORM by the knowledge source specify. 
Table 3.6-IV: An example of a description of a case as contained by the metaclass DISCREPANCY CLASSES and a prototypical case as contained by the metaclass NORM.

\begin{tabular}{ll}
\hline Abstract case description (DISCREPANCY CLASSES) & Prototypical case description (NORM) \\
\hline SEIZURE-COURSE INCREASED & SEIZURE-COURSE INCREASED \\
MINOR-SEIZURES ABSENT & MINOR-SEIZURES ABSENT \\
MAJOR-SEIZURES-FREQUENCY INCREASED & MAJOR-SEIZURES-FREQUENCY INCREASED \\
COMPLIANCE AS PRESCRIBED & COMPLIANCE AS PRESCRIBED \\
COMPLAINTS ABSENT & COMPLAINTS ABSENT \\
PLASMA-DRUG-LEVEL LOW THERAPEUTIC & PLASMA-DRUG-LEVEL LOW THERAPEUTIC \\
& TREATMENT-PROCEDURE INCREASEDOSE \\
\hline
\end{tabular}

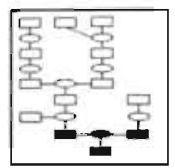

Each of the concepts in DISCREPANCY CLASSES is compared with the corresponding concept in NORM. A set of concepts in DISCREPANCY CLASSES equal to those of the prototypical patient leads to the storing of the concept TREATMENT-PROCEDURE together with its value from the prototypical case in DECISION CLASS. For example, the prototypical case description that matches the description in DISCREPANCY CLASSES contains among other concepts TREATMENT-PROCEDURE with the value INCREASE DOSE. This concept which indicates a further decision with respect to the treatment, is stored by the knowledge source match in the metaclass DECISION CLASS.

Two different types of decision classes were identified. While some decisions can be considered to be relevant only for the present contact, other treatment decisions could lead to treatment changes that have implications for future contacts. For example, switching the type of anti-epileptic drug or the decision to withdraw a drug may require a staged approach. In these procedures, the change in the treatment is stretched out over more than one contact thereby necessitating a monitoring of the consequences of the previous change.

\subsubsection{Additional treatment phases}

At the strategic level two additional phases were mentioned: the life-long monitoring phase and the phase of tapering drug treatment. Both phases are related to the monitoring and assessment tasks. The main difference between monitoring and life-long monitoring is a change in the system model. This is caused by a change in the goal of the drug treatment. Since in monitoring the goal is 'to cure' the patient by controlling the seizures, a physiological model is applied to compare disease concepts with. In life-long monitoring - with the exception of patients with well controlled seizures at a non-toxic level of medication who had recurrence of seizures and for whom therefore withdrawal of medication is no option (or who are known to get reoccurrence of seizures while tapering off the medication) - a balance between 'suppression of seizure and occurrence of side-effects' is required as curing the disease is less likely. Therefore the model is changed into a pathophysiological model of the patient. The withdrawal phase may also require a change in the system model. New seizures which may occur are due to withdrawing the drug instead of due to an ineffective treatment. 


\subsection{Implementation}

The two above described conceptual models were implemented in an experimental system using mul.ISPTM ${ }^{3}$. A layered structure separating the static parts of the knowledge from the procedural parts is used. The static knowledge of the domain layer is contained in frames. These are descriptions of concepts by means of their characteristics, so-called attributes and values. For example, the concept prescription has the attributes drugname, number of tablets and dose of tablets which may have the values carbamazepine, 2 and $200 \mathrm{mg}$, respectively. The domain concepts are arranged into three groups: information about medication, information about epilepsy and information about the patient.

Most of the heuristic knowledge expressed by the expert was captured in IF-THEN rules, which relate a condition to a specific action. Rules which are related to a specific section of the problem solving process are combined in rule sets. In this way the activation of only the relevant part of the knowledge base is possible. In the initiation part 14 of these rule sets with in total 66 rules were used for establishing a drug treatment. The monitoring part contains 965 rules which were combined into 59 rule sets. By using the frame and rule structures the composition of the knowledge as expressed by the expert was preserved in a familiar way. An assessment of the frames could be accomplished easily

Another part of the knowledge concerns the procedural aspects of the problem solving process. This is the task layer of the models described above. This knowledge is separated from the declarative part, but makes use of the information derived from the frames and rules.

A third part of the system is the user interface. The procedure driving the menus is described at the task layer. While in the initiation part we implemented some explanation on why a specific drug is used, in the monitoring part we can only provide a trace of the rules used for obtaining information about the reasoning process of the system.

In the initiation part as well as in the monitoring part a forward-chaining mechanism was used for reasoning. A simple conflict resolution scheme was implemented. The first rule of a set of rules of which the condition part matches the current information is identified. Its conclusion part is executed (an assertion added or a task executed). Next the rule is removed from the set and the process is repeated until the rule set is empty or none of the remaining rules fires.

\subsection{Discussion}

KADS has been presented by its developers as an approach for structured development of KBSs. In the original version of KADS the focus is on the analysis of cognitive processes. The main issue is the analysis of expertise which results in the definition of a model of expertise. This model of expertise is considered a high level specification of the expertise which is required for building the system (Breuker and Wielinga, 1987). This more cognitive oriented approach however widens the gap with implementing a working model. In a later version of KADS less emphasis is put on describing the expertise; a more pragmatic, implementation oriented view has been adopted. The model of expertise is seen as a model which is not a cognitive model of the expert but a specification of the system to be constructed by analysing the expert's problem solving behaviour (Schreiber et al. 1993).

\footnotetext{
3 muLISP is a trademark of Software House, Inc. 3660 Waialae Avenue. Honolulu, Hawaii 96816-3236.
} 
This change in view towards implementation can also be recognised in the changes in the contents of the conceptual model. In the early reports on KADS the conceptual model is a representation of the expertise, contained in a four layer model (Breuker et al. 1987, Schreiber et al. 1988). In the later version, a model of co-operation was added to the conceptual model. This model describes the system-user interaction; tasks to be accomplished by the user and by the system are indicated taking the competence of both agents into account (Breuker and de Greef, 1993). A model of co-operation and particularly a model of communication have been incorporated in the analysis phase (de Greef and Breuker 1992, Tansley and Hayball, 1993).

A third consequence resulting from this shift in attention towards implementation is a change in the project management approach. The early version of KADS is based on the waterfall model for conventional software design. In later reports on KADS the general structure as well as the life-cycle model were modified. A new life-cycle model $\mathrm{CONCH}$ (Client Oriented Normative Control Hierarchy) was introduced, based on the spiral life-cycle model (Boehm 1988, Taylor et al. 1989). A requirements analysis phase is now included. Guidelines and techniques for obtaining these requirements are absent, however (Hickman et al. 1989). More recently, a more detailed description of the processes in the analysis phase has been presented as well as a set of templates for modelling the organisational environment of the KBS (Tansley and Hayball 1993, Schreiber et al. 1994).

Although KADS advocates the use of models for the analysis of expertise, few guidelines with respect to the use and development of models are provided. The use of interpretation models is essential as they are a means for a top-down analysis of the elicited knowledge. Selection and application of these models, however, appears to be difficult. Some of the interpretation models have a very fine granularity. Other models, on the contrary, are less detailed and need extensive refinement. The interpretation models for assessment and monitoring could be used without significant refinement. This seems to be in agreement with the findings of others (Thiemann, 1989).

In case the model library has no interpretation model for the problem at hand, it may be constructed bottom-up. Development of an interpretation model for structuring the verbalised knowledge is supported by the conceptual modelling language. However, the set of primitives presented is only a tentative list of metaclasses and knowledge sources. An extension of the list is necessary, as has been recognised in the literature as well (Linster and Musen 1992, Woodward 1992).

Structured development improves the degree of completeness of the view on the domain but postpones the implementation process. In the knowledge acquisition phase, ideas verbalised by the expert have to be analysed, evaluated and refined. This process requires a spiral life-cycle approach, which enables an incremental evolution of a model of expertise. However, KADS does not offer tools to monitor this incremental process of modelling. Reviewing of intermediate results will require the interaction with a domain expert. In this process the model of expertise is of limited value as it is an abstract representation of his knowledge. Although the inference model will be important during the interaction with the expert, the terminology knowledge sources and metaclasses - as well as the names of the individual knowledge sources and metaclasses have a meaning of their own which is difficult to map on the concepts used by the expert to discuss the domain and his expertise. Also the interaction of the layers of a model will be difficult to comprehend. An extensive translation towards a detailed, less abstract model or an implementation of the model is required. 
As is the case with other approaches for software development KADS provides little support with respect to the evaluation of the quality of models. Work on metrication has taken place in KADS: process related and product related metrics, addressing issues such as duration and milestones, have been developed. A set of primitives which may be used for estimating costs has been defined (Readdie et al. 1989). Also in the new versions of KADS the evaluation of models of expertise needs further consideration. The iterative approach which enables the critiquing of evolving models - as in prototyping - should be included since this leads to a reduction of the risk of building an unsuitable system.

\subsection{References}

Bochm B.W., A spiral model of software development and enhancement, Computer, 3, 1988, pp. 6I72 .

Breuker J., de Greef P., Modelling system-user co-operation in KADS. In: KADS a principled approach to knowledge-based system development, (Schreiber, Wielinga, and Breuker, Eds.) Academic Press, London, 1993, pp. 47-70.

Breuker J., Wielinga B., van Someren M., de Hoog R., Schreiber G., de Greef P., Bredeweg B., Wielemaker J., Billeaut J.P., Davoodi M., Hayward S., Model driven knowledge acquisition, interpretation models, Deliverable task Al, Esprit Project 1098, Dept. Social Science Informatics, University of Amsterdam, 1987.

Breuker J., Wielinga B., Use of models in the interpretation of verbal data. In: Knowledge elicitation for experl systems, a handbook, (Kidd, Ed.), Plenum Press. New York, 1987.

Breuker J, Wiclinga B., Model-driven knowledge acquisition. interpretation models, Deliverable task Al Esprit Project 1098, Dept. Social Science Informatics, University of Amsterdam, 1984a.

Breuker J., Wielinga B., Techniques for knowledge elicitation and analysis, Report 1.5, Dept. Social Science Informatics, University of Amsterdam, 1984b.

Brcuker J., Winkels R., The use of the KADS methodology in designing an intelligent teaching system for diagnosis in physiotherapy. In: Lecture Noles in Medical Informatics 47, Knowledge based systems in medicine, (Talmon and Fox, Eds.), Springer-Verlag. Berlin, 1989, pp. 3-27.

Brown D.C., Chandrasekaran B, Design problem solving, knowledge structures and control strategies, Pitman Publishing, London, 1989.

Buchanan B.G., Shortliffe E.H., Rule-based expert systems, Addison-Wesley, Reading, Massachusetts, 1984

Burton A.M., Shadbolt N.R., Hedgecock A.P., Rugg G., A formal evaluation of knowledge elicitation techniques for expert systems: domain I. In: Research and development in expert systems $\mathrm{W}$, (Moralee, Ed), Cambridge University Press, 1987, pp. 136-145.

Burton A.M., Shadbolt N.R., Rugg G., Hedgecock A.P., Knowledge elicitation techniques in classification domains. In: Proceedings of ICAI-88, (Kodratoff, Ed), Pittman Publishing, London, 1988, pp. 85-90.

Cooke N.M., McDonald J.E., A formal methodology for acquiring and representing expert knowledge, Proceedings of the IEEE, 74, 1986, pp. 1422-1430. 
Cooke N.M., McDonald J.E., The application of psychological scaling techniques to knowledge based s elicitation for knowledge-based systems, International Journal of Man-Machine Studies, 26, 1987. pp.533-550

Cullen J., Bryman A., The knowledge acquisition bottleneck: time for reassessment?, Expert Systems, $5,1988, p p .216-225$

De Greef, P., Breuker J., Analysing system-user cooperation, Knowledge Acquisition, 4, 1992, pp. 89108 .

Diaper D., Designing expert systems, from Dan to Bursheba. In: Knowledge Elicitation, principles, techniques and applications, (Diaper, Ed), Ellis Horwood Limited, Chichester, 1989, pp. 17-46.

Dijk. J.E.M. van, Hilgevoord F.G., Jacques M.T., Otten G.A.M., Sittig A.C., Talmon J.L., Drie methoden voor kennis verwerving, Kennissystemen, 2, 1988, pp. 20-32.

Fen J.A., Veren L.C., Expert system development methodologics in theory and practice. In: Proceedings of the IEEE/ACM Conference on Developing and Managing Expert System Programs, Washington D.C., Sept. 1991, (Fcinstein, Ed), IEEE Computer Society Press, New York, 1991.

Grover M., A pragmatic knowledge acquisition methodology, TRW Defence Systems Group, Redondo Beach, Califomia, 1983.

Hayes-Roth F., Waterman D.A, Lenat D., Buiding expert systems, Addison-Wesley, Reading, Massachusetts. 1983.

Heng M.S.H., Why evolutionary development of expert systems appears to work, Future Generation Computer Systems, 3. 1987, pp. 103-109

Hickman F.R., Killin J.L., Land L., Mulhall T., Poner D. Taylor R.M., Analysis for knowledge-based systems, a practical guide to the KADS methodology, Ellis Horwood Limited, Chichester, 1989.

Hoffman R.R., The problem of extracting knowledge from experts from the perspective of experimental psychology, Artificial Intelligence Magazine. 2, 1987, pp. 53-67.

Jackson P., Introduction to expert systems, International Computer Science Series, Addison-Wesley Publishing Company, Reading, Massachusetts, 1986

Linster M., Musen M.A.. Use of KADS to create a conceptual model of the ONCOCIN task, Knowledge Acquisition, 4, 1992, pp. 55-87.

Mayhew P.J., Dearnley P.A., Organization and management of systems prototyping Information and Sofrware Technology, 32, 1990, pp. 245-252.

Nobis R., Siedka-Bauer H.G.. Prototyping and the LCM, Deliverable G13, NTE-GI3-PR-00I, ESPRIT P1098. NeuTech Entwicklungsgesellschaft mbH \& Co.KG, München, 1988.

Nguyen T., Perkins W.A., Laffey T.J., Checking an cxpert system's knowledge base for consistency and completeness. In: Proceedings of the I.JCAI. 1985, Los Angeles, pp. 375-378

O'Keefe R.M., Balci O., Smith E.P., Validating expert system performance, IEEE Expert, 4, 1987 , pp. $81-90$

O'Keefe R.M., The evaluation of decision-aiding systems: guidelines and methods, Informution and Management, 17, 1989, pp 217-226.

O’Moore R., Clarke K., Smeets R., Brender J., Nykänen P., McNair P., Grimson J., Barbeir B., Items of relevance for the evaluation of knowledge based expert systems and influence from domain characteristics, Deliverable EM-I.I, KAVAS (A I02I). Trinity College, Dublin, 1990.

Readdie M., Streng K.H., Wermser D., KADS metrication, Deliverable G10, SD-G10-R-001, ESPRIT PI098, SD Europe Ltd \& NeuTech Entwicklungsgesellschaft mbH \& Co.KG, München, 1989.

Reitman Olsen J., Rueter H.H., Extracting expertise from experts: methods for knowledge acquisition, Expert Systems, 4, 1987, pp. 152-168. 
Royce W.W., Managing the development of large software systems: concepts and techniques, Proceedings of the $9^{t h}$ International Conference on Sofiware Engineering, Computer Society Press, New York, 1987, pp. 121-125

Schreiber G., Wielinga B., KADS and conventional software engineering. In: KADS a principled approach to KBS development, (Scheiber, Wielinga and Breuker, Eds), Academic Press, London, 1993, pp. 151-165.

Schreiber G., Wielinga B., Breuker J., Introduction and overview on KADS, In: KADS a principled approach to KBS development, (Scheiber, Wielinga and Breuker, Eds), Academic Press, London, 1993, pp. 1-17.

Schreiber G., Wielinga B., Hoog R. de, Akkermans H., Velde W. van de, CommonKADS: a comprehensive methodology for KBS development, IEEE Expert, 12, 1994, pp.28-36.

Schreiber G., Breuker J., Bredeweg B., Wielinga B., Modelling in KBS development, paper presented at the Second Europcan Knowledge Acquisition Workshop '88, Bonn, June 1988.

Shadbolt N., Burton M., Knowledge elicitation. In: Evaluation of human work: practical ergonomics methodology, (Wilson and Corlett. Eds), Taylor and Francis, 1989.

Shaw M.L.G., Knowledge acquisition techniques for knowledge-based systems. In: Proceedings of the ( SC' 'Sl 88, Edmonton, 1988

Stephens M.A., Batıs P.E., Requirements engineering by prototyping: experiences in development of estimating system, Information and Software Technology, 32, 1990, pp. 253-257.

Tate G., Prototyping: helping to build the right software, Information and Software Technology, 32, 1990, pp. 237-244

Tanslcy D.S.W. Hayball C.C., Knowledge-based systems analysis and design: a KADS developer's handhook, Prentic: Hall, New York, 1993.

Taylor R.M., Porter D. Hickman F., Streng K.H., Tansley S., Dorbes G., System evolution, principles and methods (the life-cycle model). ESPRIT project 1098, deliverable Task G9, The KnowledgeBased Systems Centre of Touche Ross Management Consultants, London, 1989

Thiemann J., Wielinga over KADS: weglaten vierde niveau niet verwonderlijk, Kennissystemen, 3, 1989, pp. 3-5

Waterman D.A., A guide to expert systems, Addison-Wesley Publishing Company, Reading, Massachusetts, 1986.

Weitzel J.R., Kerschberg L., Developing knowledge-based systems: reorganizing the system development life cycle, Communications of the $A C M, 32,1989$. pp. 482-488.

Wellbank M., A review of knowledge acquisition techniques for expert systems, British Telecom Research, Martlesham Heath. 1983.

Wielinga B.J., Breuker J.A., Models of expertise. In: Advances in Artificial Intelligence 2, (du Boulay, Hogg, and Stecls. Eds.), Elsevier Science Publishers B.V., Amsterdam, 1987, pp. 497-509.

Woodward J.B., Developing K-ONCOCIN: a case study in the cognitive process of knowledge engineers, Knowledge Acquisition, 4, 1992, pp 237-258.

Wright G. Ayton P., Eliciting and modelling expert knowledge, Decision Support Systems, 3, 1987, pp. $13-26$ 


\section{Reference development}

\subsection{Method for system evaluation}

Evaluation $^{1}$ is generally considered as an essential part in the development of a DSS. Several methodologies have been proposed (Carlson 1974, Spiegelhalter 1983, Green and Keyes 1987, Lundsgaard 1987, Rushby 1988, O’Keefe et al. 1987, O'Keefe 1989, O'Leary and O'Keefe 1989, Rossi-Mori and Ricci 1988, Rossi-Mori et al. 1990, Hollnagel 1989, Preece 1990, Wyatt and Spiegelhalter 1990, O'Moore et al. 1990ab, Rector et al. 1990, Smeets and Talmon 1990, Engelbrecht et al. 1991, Sørgaard 1991, Bankowitz te al. 1992, Clarke et al. 1991, 1994) but none has been accepted as a standard. Usually validation ${ }^{2}$ of a DSS is considered the comerstone of the evaluation process as it addresses among other things the quality of the implemented knowledge. In our case correctness of the output of the system is the issue of interest.

The performance of the system and the neurologist(s) can be expressed by several quality concepts of which correctness and agreement are considered the most important. The correctness metric requires a reference with which the advice of the neurologists and the system can be compared. Correctness then is the percentage of cases which are assigned the reference value for an aspect of the treatment by either the system or a neurologist. Agreement is determined by comparing the treatment proposals of two or more observers without having recourse to a reference. The agreement beyond chance can be expressed by the kappa statistic.

The literature on anti-epileptic drug treatment can be used only as a guideline to assess the correctness of the advice of a system since the recommendations are too general. To assess the correctness of the prescription of an anti-epileptic drug treatment based on data of an individual patient, a reference for each case has to be available. The absence of detailed guidelines for the treatment of an individual patient necessitates consensus about what constitutes good treatment. Human expert(s) must be involved in the development of such a reference. To obtain a generally accepted opinion multiple experts have to be involved. To cope with multiple opinions a method for consensus formation is required.

\footnotetext{
'In general the definitions of ISO/IEC 9126: 1991 (E) are adhered to. The term evaluation incorporates the evaluation preparation - defined as metric selection, rating level definition and assessinent criteria definition - as well as the evaluation procedure i.e. the measurement, rating and assessment part of the evaluation procedure model.

2 Validation is a phase in the evaluation process which is focused on the correctness of the output of the system. It is concemed with the question whether the right system was built. It has to show that the program satisfies the user requirements and is considered to be a 'live' activity in which the software is tested.
} 
such records. In this process the case descriptions were evaluated on their clarity, fidelity and usability to assure that they contained sufficient information to enable diagnosis and treatment decision making. First we will describe the design of our 60 test cases in more detail. Secondly, it is described how from these 60 cases two reference sets for performance evaluation of the system were constructed.

\subsection{Test case design}

Written case descriptions were used in our studies. Although this approach does not mimic the daily patient-doctor contacts, physicians are used to paper cases when discussing and evaluating patients. Hence it is expected that an experimental set-up based on such paper cases will not introduce large biases. Also the fact that during a patient-doctor contact at the outpatient clinic, symptoms caused by an epileptic seizure are normally not observed but communicated verbally makes a written case description sufficiently similar to a patient-doctor contact. Therefore it is safe to assume that relevant information can be presented in writing (as if it were told by an eyewitness), without causing too much deformation of the problem solving process.

\subsubsection{Patient selection}

Patient records formed the basis for our paper cases. Epilepsy patients visiting the outpatient neurology clinic of the University Hospital of Maastricht who were 16 years or older were asked for consent to use anonymously their patient record. In total 70 patients were approached during a period of four months. Patients on anti-epileptic drug medication as well as patients off medication were included. Sixty-six patients gave their permission. Based on the availability of diagnostic information in the patient record 60 cases were finally used for our study.

Information normally gathered during the first few visits to a neurologist such as data concerning the admission of the patient, the patient history and hetero anamnestic information was extracted from the patient record. The family history, the tractus anamneses, psychosocial circumstances, the use of medication, intoxications and data from the physical examination, laboratory and other investigations such as EEG and CT-scans were included as well.

\subsubsection{Validity of paper cases}

The content of the paper cases was first assessed by two neurologists with more than 20 years of practical experience in epilepsy. One of them was professor and head of the Department of Neurology at the University Hospital Maastricht. The other a professor in epileptology and head of a special centre for epilepsy.

The paper cases were presented together with the original diagnosis. The assessors were asked to rate several aspects of the case on a five point scale.

\section{Clarity}

Clarity deals with how the case is presented: can the data be interpreted and is the information sufficient and not contradictory.

In 50 out of our 60 cases $(83.3 \%)$ both neurologists considered the case description to be clear. In five cases both stated that the case description was not clear. 
In the remaining five cases one of the participants did not provide a judgement, due to absence of relevant information or contradictions in the presented information. In three of these cases the other neurologist stated that the description was clear.

\section{Fidelity}

Fidelity deals with whether the case description could come from a real patient.

In fifty-three case descriptions both neurologists considered the case description realistic In four cases at least one neurologist disagreed with the statement that the case had a sufficient fidelity.

\section{Usability}

Usability deals with how well the case description is suited for our purpose. In 47 cases both experts agreed with the statement that the case was usable. In five cases at least one of the participants disagreed with the statement, while in eight cases both participants considered the case not usable.

On the basis of these results 13 case descriptions were refined taking the comments of the experts into account. The remarks mainly concerned unclear symptomatology of seizures and the lack of EEG-findings.

\section{Check on the usability}

After this refinement all 60 cases were assessed on their practical usability through the establishment of a diagnosis and prescription of a therapy by a second neurologist (Neurologist II) from the Neurology Department of the University Hospital in Maastricht.

Neurologist II diagnosed 51 cases as a specific type of epilepsy while in three cases the type of epilepsy was not certain. In six cases Neurologist II felt he needed additional information from a sleep-EEG and refrained from diagnosis.

In 50 of the 51 cases in which a type of epilepsy was diagnosed Neurologist II wrote a prescription. In one case he established a diagnosis but postponed drug treatment to enable registration of the EEG prior to medication.

For the three cases in which the type of epilepsy was uncertain no prescription was provided In the six cases in which Neurologist II requested a sleep-EEG, this information could not be provided and the cases were included unchanged in the set of test cases. The three cases in which the type of epilepsy was uncertain were not modified, as a treatment can be based on such a diagnosis.

\subsubsection{Description of the test cases}

The 60 cases concerned 34 male $(56.6 \%)$ and 26 female patients (43.3\%). The age of the patients ranged from 16 to 72 years. Forty-five percent of the patients was less than 30 years old, $36.6 \%$ was between 30 and 50 years of age while $18.4 \%$ was older than 50 years. Compared with Northern European (adult population) studies of active epilepsy our population is younger (Table 4.2-1). The age distribution resembles closer the adult population of an epilepsy centre (Meinardi 1990), which reflects the selection of problem cases from the Maastricht cohort. 
Table 4.2-I: Age distribution of the cases in our study compared with Northern European adult studies.

\begin{tabular}{llllllll}
\hline $\begin{array}{l}\text { Age } \\
\text { distribution }\end{array}$ & $\begin{array}{l}\text { Our study } \\
\mathrm{n}=60\end{array}$ & $\begin{array}{l}\text { Granf } \\
(1974) \\
\mathrm{n}=489\end{array}$ & $\begin{array}{l}\text { Wagner } \\
(1983) \\
\mathrm{n}=2572\end{array}$ & $\begin{array}{l}\text { Kerainen } \\
(198.9) \\
\mathrm{n}=1233\end{array}$ & $\begin{array}{l}\text { Sander } \\
(1990) \\
\mathrm{n}=425\end{array}$ & $\begin{array}{l}\text { Forsgren } \\
(1992) \\
\mathrm{n}=713\end{array}$ & $\begin{array}{l}\text { Meinardi* } \\
(1990) \\
\mathrm{n}=318\end{array}$ \\
\hline $15-19$ & 18 & 19 & 31.4 & 8.9 & 15.5 & 6.2 & 11.6 \\
$20-29$ & 22 & 30 & & 22.4 & 17.4 & 18.1 & 31.4 \\
$30-39$ & 20 & 13.7 & 34.0 & 18.0 & 14.6 & 21.3 & 17.6 \\
$40-49$ & 22 & 11.9 & & 16.6 & 8.2 & 15.4 & 15.1 \\
$50-59$ & 10 & 11.5 & 34.6 & 16.6 & 12.2 & 15.6 & 8.2 \\
$60-69$ & 8 & 8.2 & & 11.7 & 14.8 & 15.4 & 5.0 \\
$70-79$ & 2 & 5.7 & & 6.3 & 10.1 & 8.0 & \\
$>=80$ & & & & & 7.1 & & \\
\hline
\end{tabular}

Age distribution used: 16-20,21-30 etc

The following types of epilepsy (as validated by our two experts) were present:

- 28 cases $(47 \%)$ had partial epilepsy with secondary generalised partial seizures

- 16 cases (27\%) had primary (idiopathic) generalised epilepsy

- 9 cases $(15 \%)$ had partial epilepsy with complex partial seizures

- 2 cases $(3 \%)$ had partial epilepsy with simple partial seizures

- 5 cases $(8 \%)$ were diagnosed as partial epilepsy with complex partial seizures and partial epilepsy with secondary generalised partial seizures.

Using the classification of the International League Against Epilepsy 44 cases (73.3\%) had partial epilepsy while 16 cases (26.7\%) suffered from (idiopathic) generalised epilepsy.

Table 4.2-11: Distribution (in percentages) of epilepsies in diffenent populations as reported by Overweg (1985). For generalised epilepsy the percentage of cases with idiopathic generalised cpilepsy are presented as well.

\begin{tabular}{|c|c|c|c|c|}
\hline Type of epilepsy & study 1 & study 2 & study 3 & our study \\
\hline Generalised epilepsy & 45.8 & 23.0 & 18.2 & 26.7 \\
\hline Idiopathic & 4.3 .7 & 21.2 & 16.6 & 26.7 \\
\hline Other & 2.1 & 1.8 & 1.6 & 0.0 \\
\hline Partial epilepsy & 52.1 & 73.5 & 63.3 & 73.3 \\
\hline Unclassifiable & 1.7 & 3.4 & 18.4 & 0.0 \\
\hline
\end{tabular}

Overweg (1985) describes three studies which classify epilepsies according to the international classification of epilepsies of Gastaut (Table 4.2-I1). The first study describes the occurrence of epilepsies in a population referred to a single general hospital by primary care physicians. The two other studies describe patient populations in epilepsy centres.

Idiopathic generalised epilepsy occurred in respectively $16.6 \%$ and $21.2 \%$ of the patients of 15 years of age and older coming from epilepsy centres, while in the general hospital this occurred in $43.7 \%$ of the patients. The number of patients with partial epilepsies ranged from $63.3 \%$ up to 
$73.5 \%$. Also with respect to epilepsy syndromes our population resembles the more selected populations of epilepsy centres.

\subsection{Reference development}

Since no objective standard is available for assessing the system, the system was compared with the neurologists. Reference sets were created to obtain sets of cases with which the agreement between neurologists and the system could be assessed. In this section the procedure of constructing reference sets is presented. Two different reference sets were constructed: a diagnostic reference set containing cases for which at least two out of three neurologists agreed about the diagnosis and a treatment reference set for which both a majority agreement with respect to the diagnosis and treatment was obtained.

\subsubsection{Method for consensus formation}

In general two different approaches exist to reduce diversity in opinions among a group of individuals:

- Nominal techniques: a review of anonymous arguments/solutions

- Interactive techniques: a review of onymous arguments/solutions

Social and emotional factors which occur during an interactive group process may influence the way in which an agreement is reached. For example, dominance of some partners in the discussion, the tendency to embark quickly on a solution and the tendency to avoid conflicts, may induce nonoptimal solutions (van der Ven and Delbecq 1971, Delbecq et al. 1975). To avoid these problems, nominal techniques - the Nominal Group Technique (NGT) and the Delphi technique - were developed. In these approaches it remains unknown for the other participants who provided an opinion or solution. This anonymity of views makes it easier to discuss the various options without any prejudice. Nominal techniques have been applied successfully to establish objectives and standards (Loughlin and Moore 1979, Romm and Hulka 1979, Farrel and Scherer 1983, Quaglini et al. 1988, Kors et al. 1989, Kors and van Bemmel 1990), to explore risks and costs and to forecast events (Goodale and Gander 1976, Hill and Goodale 1981, Barret-Connor 1984, Arevalo and Washington 1988). The use of a modified Delphi approach has been shown to be valuable for developing references (Willems et al. 1985).

The NGT and the Delphi technique both consist of three steps: generating and pooling of ideas, feedback and evaluation. In the feedback round of the NGT, participants are expected to explain and discuss different views. In the Delphi procedure statistical information on the generated ideas is provided as feedback to the participants. In the third round participants still present their possibly revised opinions in an anonymous way (Delbecq and van der Ven 1975 , Linstone and Turoff 1975)

In our study we used a Delphi procedure in combination with a consensus meeting to obtain a reference for each case. The Delphi round was used to reduce the variation in prescribing. After the Delphi round the anonymous prescriptions were assessed by a peer review. The annotated prescriptions were discussed in the consensus meeting to reach a reference treatment. 


\subsubsection{Reference development/Acceptance testing}

Complete agreement among neurologists on diagnosis and treatment is expected difficult to achieve given the various opinions in the literature. A majority agreement among the participants was therefore considered to be sufficient for establishing a reference.

Although a neurologist may provide a prescription that is not equal to the reference it may still be considered an acceptable treatment. To assess the acceptability of the (possibly non-reference) prescriptions independent experts are needed to judge the quality of the prescriptions. To avoid to have to rely on the opinion of one single neurologist, each prescription was assessed by two experts

Table 4.3-I: Prescribing (P) and acceptability testing (A) scheme.

\begin{tabular}{cccccc}
\hline Data & Participant & & & & \\
set & A & B & C & D & E \\
\hline I & P & P & P & A & A \\
II & P & P & A & P & A \\
II & P & P & A & A & P \\
IV & P & A & P & P & A \\
V & P & A & P & A & P \\
VI & P & A & A & P & P \\
VII & A & P & P & P & A \\
VIII & A & P & P & A & P \\
IX & A & P & A & P & P \\
X & A & A & P & P & P \\
\hline
\end{tabular}

We designed a scheme for the reference development and acceptability testing procedure with the following properties (Table 4.3-1):

- each participant assessed the acceptability of prescriptions of cases for which he did not prescribe,

- each participant provided prescriptions for the same number of cases,

- each participant assessed the acceptability of the same number of prescriptions,

- each pair of participants was given the same number of cases to provide prescriptions,

- each pair of participants assessed the acceptability of the same number of prescriptions.

The 60 cases were divided into ten sets of six cases in such a way that each neurologist encountered approximately the same case mix (Table 4.3-11).

\subsubsection{Participating neurologists}

Five neurologists were asked to participate in this part of the study. The neurologists were working in neurology departments in five different general hospitals. Their clinical experience in neurology ranged from two to more than 20 years. Two of the participants received their training in neurology from the same supervisor. None of the participants was trained by the 
expert involved in the development of the system. All five neurologists were practising physicians, four as general neurologists, one with more emphasis on epilepsy care. Three participants saw an epilepsy patient every 1-3 days, 2 participants saw at least one epilepsy patient every day. Before the consensus meeting part of our study, one of the neurologists dropped out. In the follow-up part (see chapter 5) he was replaced by a colleague from the same hospital, having an equivalent number of years of clinical experience.

Table 4.3-II: Distribution of diagnoses presented to each participant for prescribing drug treatment.

\begin{tabular}{lrrrrr}
\hline Diagnosis* & N II & N IV & N V & N VI & N VII \\
\hline PEPCS & 6 & 6 & 5 & 5 & 5 \\
PESGPS & 17 & 17 & 17 & 16 & 17 \\
PEPCS+PESGPS & 3 & 4 & 3 & 2 & 3 \\
PESPS & 1 & 1 & 1 & 2 & 1 \\
IGE & 9 & 8 & 10 & 11 & 10 \\
Total & 36 & 36 & 36 & 36 & 36 \\
\hline
\end{tabular}

* PEPCS: partial epilepsy with complex parial seizures. PESGPS: partial epilcpsy with secondary generalised partial seizures, PESPS: partial epilepsy with simple partial seizurcs, IGE: primary (idiopathic) generalised epilepsy.

\subsubsection{Reference development process}

Since the diagnosis may determine the use of specific anti-epileptic drugs a majority agreement on the diagnosis has to be obtained before prescriptions can be compared. The cases for which no majority agreement (two out of three neurologists) on the general diagnosis (partial or generalised epilepsy) was reached were sent back to the neurologists. The various diagnoses established on such a case were given as feedback.

Cases for which the majority of the neurologists agreed on the diagnosis $(3 / 3$ or $2 / 3)$ but for which no majority agreement on the generic drug was obtained were retumed for review as well. Feedback was provided about the different drugs prescribed for the case. Furthermore, information on the frequency of suggested treatments in cases with the same general diagnosis, was given. Because the number of cases for which no agreement on the general diagnosis could be established was rather small, the reviewing of the diagnoses and the review of the generic drug were done simultaneously

After agreement was obtained on the generic drug, the prescribed daily doses were reviewed. For each drug the 5 and 95 percentiles of the daily dose in $\mathrm{mg} / \mathrm{kg}$ body weight were used to keep the number of cases to be reviewed within acceptable limits.

Prescriptions with a drug dose outside this range not caused by rounding off dosages towards an integer number of tablets were classified as outliers and returned for review

\subsection{Statistical analysis}

The $\chi^{2}$ test is used for analysing frequencies of cases in specific categories. It tests whether there is a significant difference between an observed number of cases in the categories and an expected number of cases based on the null hypothesis. The $\chi^{2}$ will be small if the observed and expected frequencies of cases are close to each other (Siegel and Castellan, 1988). 
The McNemar $\chi^{2}$ test is used for the analysis of 'before and after' designs in which the subjects are used as their own controls. The measurements should be made on an nominal or ordinal scale. The number of changes which occur are of importance. The test indicates whether the observed changes are significant (Siegel and Castellan, 1988).

For interpreting the significance of the results from the paired experiments on the acceptabilty scores of the prescriptions for a case the paired Student T-test is used. The differences were expected to be normally distributed. The degrees of freedom is $\mathrm{N}-1$, in which $\mathrm{N}$ is the number of pairs (Hayes, 1980).

The kappa statistic was used for obtaining information on the agreement beyond chance. This statistic was calculated for agreement among pairs of observers as well as for the agreement between one observer and a group (Schouten, 1985).

\subsection{Results}

In this paragraph the results are described of the agreement on the diagnosis and treatment before and after feedback. The agreement of the neurologists with the diagnostic and treatment reference is presented. Furthermore, the agreement between the diagnostic and consensus reference is described.

\subsubsection{Agreement on the diagnosis, generic drug and daily dose}

The degree of agreement among the neurologists on the diagnosis - both at a detailed and a general level - was established. In Table 4.5-I the different diagnostic categories at the general and detailed level are presented. This categorisation is based on the ILAE diagnostic classification (Commission on Classification and Terminology of the ILAE, 1989).

Table 4.5-I: General and detailed diagnoses

\begin{tabular}{ll}
\hline General type of epilepsy & Detailed type of epilepsy \\
\hline Generalised epilepsy & $\begin{array}{l}\text { Primary (idiopathic) generalised epilepsy } \\
\text { Myoclonic epilepsy } \\
\text { Lennox-Gastaut syndrome } \\
\text { West syndrome } \\
\text { Combinations }\end{array}$ \\
Partial epilepsy & $\begin{array}{l}\text { Partial epilepsy with complex partial seizures } \\
\text { Partial epilepsy with simple partial seizures } \\
\text { Partial epilepsy with secondary generalised seizures } \\
\text { Combinations }\end{array}$ \\
Combination & Combination of generalised and partial epilepsy \\
Other & Other suggestions \\
& $\begin{array}{l}\text { Withdrawal seizures } \\
\text { Multifocal epilepsy }\end{array}$ \\
Unknown & Diagnosis unknown or uncertain \\
& Diagnosis unknown or uncertain in combination with any of the above
\end{tabular}


Table 4.5-II presents the degree of agreement on the diagnosis and the prescribed generic drug before feedback. The table is divided into two sections. In the upper part of the table a breakdown of the cases is given according to the agreement with respect to the general and the detailed diagnosis.

Since for each case three neurologists provided a diagnosis and a prescription three $(3 / 3)$ or two neurologists $(2 / 3)$ can give the same diagnosis, or each neurologist may provide a different diagnosis (no). The third row of the upper part of the table presents the number of cases for each level of agreement.

The level of agreement between the three neurologists concerning the medication (stratified with respect to the degree of agreement on the diagnosis) is given in the lower part of the table (Therapy).

Total agreement with respect to the diagnosis (3/3) does not always lead to a total agreement with respect to the medication. Partial agreement with respect to the diagnosis $(2 / 3)$ can still result in total agreement with respect to the treatment, as the third neurologist who disagreed on the diagnosis may still prescribe the same generic drug. Of the 20 cases in which two out of three neurologists agreed on the general diagnosis, all three neurologists agreed about the generic drug in four cases. In 12 of these cases two out of three agreed on the treatment. In ten of these latter cases the pair of neurologists who agreed on the diagnosis also agreed on the generic drug.

Table 4.5-II: Agrecment on the general and detailed diagnosis and the type of generic drug before feedback.

\begin{tabular}{|c|c|c|c|c|c|c|c|c|}
\hline Diagnosis & \multicolumn{4}{|c|}{ general } & \multicolumn{4}{|c|}{ detailed } \\
\hline Agreement on type of epilepsy & $3 / 3$ & \multicolumn{2}{|c|}{$2 / 3$} & no & $3 / 3$ & \multicolumn{2}{|c|}{$2 / 3$} & no \\
\hline Number of cases & 36 & \multicolumn{2}{|c|}{20} & 4 & 20 & \multicolumn{2}{|c|}{26} & 14 \\
\hline Participants & All & Al! & $\begin{array}{l}\text { Diagn } \\
\text { agree }\end{array}$ & All & All & All & $\begin{array}{c}\text { Diagn } \\
\text { agree }\end{array}$ & All \\
\hline Therapy & & & & & & & & \\
\hline Agreement $3 / 3$ & 12 & 4 & 11.a. & 0 & 7 & 6 & ก.a. & 3 \\
\hline Agreement $2 / 3$ & 19 & 12 & 14 & 3 & 10 & 14 & 15 & 10 \\
\hline No ägreement & 5 & 4 & 6 & 1 & 3 & 6 & I! & l \\
\hline
\end{tabular}

Agreement on the diagnosis and the generic drug after feedhack

After the second round the number of cases with a majority agreement on the general diagnosis slightly increased (Table 4.5-III). In 58 out of 60 cases at least two out of three participants agreed on the general diagnosis. Also the agreement with respect to the therapy increased. A majority agreement on both the general diagnosis and the generic drug was reached in 55 out of 60 cases. 
Table 4.5-III: Agreement after foedluack.

\begin{tabular}{|c|c|c|c|c|c|c|c|c|}
\hline Diagnosis & \multicolumn{4}{|c|}{ general } & \multicolumn{4}{|c|}{ detailed } \\
\hline Agreement on type of epilepsy & $3 / 3$ & & & no & $3 / 3$ & & & no \\
\hline Number of cases & 36 & & & 2 & 20 & & & 13 \\
\hline Participants & All & All & $\begin{array}{l}\text { Dingn } \\
\text { agree }\end{array}$ & All & All & All & $\begin{array}{l}\text { Diagn } \\
\text { agree }\end{array}$ & All \\
\hline $\begin{array}{l}\text { Therapy } \\
\text { Agreement } 3 / 3\end{array}$ & 13 & 6 & n.a. & 0 & 8 & 8 & п.а. & 3 \\
\hline Agreement $2 / 3$ & 23 & 14 & 19 & I & 12 & 17 & 19 & 9 \\
\hline No agreement & 0 & 2 & 3 & 1 & 0 & 2 & 8 & 1 \\
\hline
\end{tabular}

For the detailed diagnosis level a majority agreement on both the diagnosis and treatment was reached in $65 \%$ of the cases as compared to $53 \%$ in the first round.

For the general diagnosis level after the first round there were $15(25.0 \%)$ cases with no agreement on either the general diagnosis or generic drug or both. This number decreased to $5(8.3 \%)$ cases after feedback (McNemar $\chi^{2}=8.1 \mathrm{p}<0.01$ ). In three cases disagreement remained with respect to the prescribed generic drug despite the fact that two neurologists agreed on the general type of epilepsy. In two other cases the participants disagreed on the general diagnosis. For the detailed diagnosis in 28 cases $(47 \%$ ) a majority agreement on either the general diagnosis, the generic drug or both was not reached initially. After feedback for 21 cases $(35 \%)$ a majority agreement was not reached.

Agreement on the drug dosage

The upper 95 percentile and the lower 5 percentile $\mathrm{mg} / \mathrm{kg}$ dosage of the drug were calculated for CBZ, VPA and PHT from the cases in which those drugs were prescribed in the second round. These dosages were then used to calculate the maximally and minimally acceptable dose for each case. In 10 cases the prescribed dose was outside these margins. In 7 of these cases the higher or lower dose could be attributed to rounding towards an integer number of tablets. In 3 cases a dose of CBZ was prescribed which was outside the obtained ranges. Review of these cases did not lead to a change in the prescribed dose.

\subsubsection{Description of the reference sets}

Diagnostic reference set

Whether an anti-epileptic drug has to be selected on the basis of a detailed diagnosis or whether a global description of the type of epilepsy is sufficient is not clear from the literature (chapter 2). Therefore, it was decided to use the set of 47 cases where the majority of the neurologists agreed about the detailed diagnosis for the assessment of the system.

The 47 cases in which the majority of the neurologists agreed about the detailed diagnosis is called the diagnosic reference sel. Although each neurologist saw 36 cases the reference cases are not equally divided over the individual neurologists. Table 4.5-IV gives the number of cases which each individual neurologist agreed with the detailed diagnosis for. 
Table 4.5-IV: Number of cases from the diagnostic reference set seen by each neurologist.

\begin{tabular}{|c|c|c|c|c|c|}
\hline Neurologist & III $(\mathrm{n}=36)$ & IV $(n=.36)$ & $V(n=36)$ & $V I(n=36)$ & VU $(n=36)$ \\
\hline Agreement with ref. & 23 & 25 & 21 & 22 & 23 \\
\hline detailed diagnosis & $64 \%$ & $69 \%$ & $58 \%$ & $61 \%$ & $64 \%$ \\
\hline
\end{tabular}

The reference cases were mainly diagnosed as partial epilepsies $(64 \%)$, while $30 \%$ of the cases were diagnosed as primary (idiopathic) generalised epilepsy. Furthermore, twice the neurologists agreed that the type of epilepsy was uncertain or unknown. Once a combination of partial and generalised epilepsy was diagnosed.

One hundred and fourteen prescriptions were generated for the 47 cases by the neurologists that agreed on the detailed diagnosis. In $51.8 \%, 24.6 \%$ and $10.5 \%$ of these prescriptions the selected drug was CBZ, VPA and PHT respectively. Eleven times a neurologist decided not to initiate anti-epileptic drug treatment. For the 47 cases a majority treatment was defined (if it existed) consisting of the opinions of those neurologists which agreed about the detailed diagnosis (Table 4.5-V)

Of the cases diagnosed as a kind of partial epilepsy $63 \%$ received a prescription of $\mathrm{CBZ}$, while in 10\% PHT was the drug prescribed by the majority. In seven cases (23\%) a majority agreement about the treatment was not obtained (No ref) despite the fact that the neurologists agreed about the diagnosis. In one case the reference was not to start drug treatment No drug)

The drug for cases diagnosed as a primary (idiopathic) generalised epilepsy was mainly VPA $(71 \%)$. CBZ was the treatment of the majority in $10 \%$ of these cases. In one case a majority treatment was not obtained.

For the combination of partial and generalised epilepsy $\mathrm{CBZ}$ was used by the majority. For the two cases in which the diagnosis was not certain anti-epileptic drug treatment was not initiated.

Table 4.5-V: Detailed diagnoses and treatment in the diagnostic reference set.

\begin{tabular}{|c|c|c|c|c|c|c|c|c|}
\hline Diagnosis & & Cuses & CBZ & VPA & PHT & No ref & No drug & $\%$ \\
\hline \multirow{4}{*}{$\begin{array}{l}\text { Partial } \\
\text { epilepsy }\end{array}$} & PECPS & 6 & 4 & & & 2 & & 13 \\
\hline & PESGS & 10 & 6 & & 2 & 2 & & 21 \\
\hline & PECPS+PI:SGS & 11 & 8 & & & 2 & 1 & 23 \\
\hline & PESIS & 3 & 1 & & 1 & I & & 6 \\
\hline $\begin{array}{l}\text { Gencralised } \\
\text { epilepsy }\end{array}$ & IGE & 14 & 3 & 10 & & 1 & & 30 \\
\hline Combi & IGIT+PECPS & 1 & 1 & & & & & 2 \\
\hline Unknown & & 2 & & & & & 2 & 4 \\
\hline Total & & 47 & 23 & 10 & 3 & 8 & 3 & 100 \\
\hline
\end{tabular}




\section{Treatment reference set}

In 39 out of the 47 cases a majority agreement on the prescribed treatment was reached. This subset of cases is called the trealmenl reference set.

Cases in which two neurologists agreed about the detailed diagnosis but disagreed about the prescribed drug were excluded, even if the third neurologist who disagreed about the diagnosis agreed on the prescribed drug.

In Table 4.5-VI the number of cases for which the individual neurologists agreed with the reference are presented. Because the neurologists may disagree with the diagnosis or with the prescribed drug the number of cases per neurologist differs.

Table 4.5-VI: Number of cases in which an individual ncurologist agrees with the treatment reference. The number of cases seen by each neurologist and belonging to the diagnostic reference set is indicated by $n$.

\begin{tabular}{llllll}
\hline Neurologist & III $(n=23)$ & IV $(n=25)$ & V $(n=21)$ & V $(n=22)$ & VI $(n=23)$ \\
\hline Agreement with reference drug & 18 & 20 & 14 & 16 & 18 \\
& $78.3 \%$ & $80.0 \%$ & $66.6 \%$ & $72.7 \%$ & $78.3 \%$ \\
\hline
\end{tabular}

Overall, in 18 of the 47 cases (38\%) the majority of the neurologists agreed on the detailed diagnosis as well as on the generic drug and daily dose. In three of these cases no drug treatment was started. In the remaining 15 cases a majority agreement on the generic drug as well as on the daily dose was observed

In 13 of the 14 cases diagnosed as primary (idiopathic) generalised epilepsy, the majority agreed about the drug. In eight of these 13 cases (57\%) at least two neurologists prescribed the same daily dose.

In 22 of the 30 cases diagnosed as a type of partial epilepsy the neurologists agreed about the prescribed drug. In six of these 22 cases $(27 \%)$ the majority agreed about the daily dose. This concerned five cases for which $\mathrm{CBZ}$ and one case for which PHT was prescribed. The neurologists also agreed on the prescribed drug and daily dose for the one case diagnosed as a combination of partial and generalised epilepsy.

\section{Consensus meeting reference}

After the three Delphi rounds the neurologists still varied with respect to the prescribed drug and the daily dose for a specific case. Therefore a consensus meeting was organised. The cases of the diagnostic reference set were discussed. Instead of a reference for an individual case in this consensus meeting guidelines were developed on how to approach a patient with epilepsy in general. A detailed outline of these guidelines is presented in chapter 5 . 
Table 4.5-VII: Comparison of the diagnostic reference set and the consensus.

\begin{tabular}{|c|c|c|c|c|c|c|}
\hline \multirow{8}{*}{$\begin{array}{l}\text { Diagnostic } \\
\text { reference set } \\
(n=47)\end{array}$} & \multirow[b]{2}{*}{ Drug } & \multicolumn{5}{|c|}{ Consensus drug } \\
\hline & & $\mathrm{CBZ}$ & VPA & PHT & No drug & Total \\
\hline & $\mathrm{CBZ}$ & 18 & 2 & 3 & & 23 \\
\hline & VPA & & 10 & & & 10 \\
\hline & $\mathrm{PHT}$ & 3 & & & & 3 \\
\hline & No drug & 1 & & & 2 & 3 \\
\hline & No ret & 4 & 2 & 2 & & 8 \\
\hline & Tota! & 26 & 14 & 5 & 2 & 47 \\
\hline
\end{tabular}

The guidelines defined in the consensus meeting were applied to the diagnostic reference set. Of the 34 cases with partial epilepsy, $26(84 \%)$ should receive CBZ while five cases ( $16 \%$ ) needed PHT according to the consensus (Table 4.5-VII). All 14 cases diagnosed as primary (idiopathic) generalised epilepsy received VPA. For cases in which the type of epilepsy was unknown or uncertain drug treatment was not initiated

In 30 cases (64\%) the majority drug was in accordance with the consensus. Cases in which the reference drug was PHT should have received $\mathrm{CBZ}$ according to the consensus. The case for which treatment was not started although the diagnosis was established should have been treated with CBZ. Using the guidelines a treatment could be defined for those cases for which no reference was obtained by means of the Delphi procedure.

\subsection{References}

Arevalo J.A., Washington E., Cost-effectiveness of the prenatal screening and immunization for hepatitis B virus, Journal of the American Medical Association, 259, 1988, pp 365-369.

Bankowitz R.A., Lave J R., McNcil M.A.. A method for assessing the impact of a computer based decision support system on health care outcomes, Methods of Information in Medicine, 31, 1992, pp.3-11.

Barret-Connor E., Natural history of intestinal parasites in asymptomatic adults, The Journal of Family Practitioners, 19, 1984, pp.635-639.

Carlson E.D., Evaluating the impact of information systems, Management Informatics, 3, 1974, pp.5767.

Clarke K., O'Moore R., Smects R., Talmon J., Brender J., McNair P., Nykänen P., Grimson J., Barber B., A methodology for evaluation of knowledge-based systems. In: Lecrure notes in Medical Informatics 45, Proceedings MIE 91, (Adlassnig, Graber, Bengtsson and Hansen, Eds.), SpringerVerlag, Berlin, 1991, pp. 361-365.

Clarke K., O`Moore R., Smects R., Talmon J., Brender J., McNair P., Nykänen P., Grimson J., Barber B., Methodology for evaluation of knowledge-bascd systems in medicine, Artificial Intelligence in Medicine, 6, 1994, pp.107-121.

Commission on Classification and Terminology of the Intemational League Against Epilepsy, Proposal for revised classification of epilepsies and epileptic syndromes, Epilepsia, 30, 1989, pp. 389-399.

Delbecq A.L., Ven A.H. van der, Gustafson D.H., Group lechniques for program planning: a guide 10 nominal group and Delphi processes, Scott Foresman, Glenvicw IL, 1975. 
Engelbrecht R., Fitter M., Rector A., Requirements for a medical workstation using user-centered design. In: Lecture notes in Medical Informatics 45. Proceedings MIE 91 . (Adlassnig, Graber, Bengtsson and Hansen. Eds.), Springer-Verlag, Berlin, 1991, pp. 140-144.

Farrel P., Scherer K., The Delphi technique as a method for selecting criteria to evaluate nursing care, Nursing Papers, 15, 1983, pp.51-60.

Forsgren L., Prevalence of epilepsy in adults in northem Sweden, Epilepsia, 33, 1992, pp. 450-458

Goodale F., Gander G.W., The future of pathology: a Delphi study by pathology dcpartment chairmen, Journal of Medical Education, 51, 1976, pp.897-903

Graaf A.S de, Epidemiological aspects of epilepsy in northem Norway, Epilepsia, 15, 1974, pp. 291 299.

Green C.J., Keyes M.M., Verification and validation of expert systems. In: Proceedings of the Western Conference on Expert Systems, IEEE Computer Society Press, New York, 1987, pp.38-43.

Hayes W.L., Statistics for the social sciences, $2^{\text {nd }}$ edition, Holt Rineman and Winston, London, 1980.

Hill R.B., Goodale F. The Delphi predictions of pathology chaimen; a six-year retrospective view, Journal of Medical Education, 56, 1981, pp.537-546.

Hollnagel E., Evaluation of expert systems. In: Topics in expert system design, eds. Guida G., Tasso C., Elsevier Science Publishers B.V. (North-Holland), Amsterdam, 1989, pp.377-416

Kors J., Bemmel J.H. van, The Delphi method: a review of its applications in medicine. In: Proceedings MEDINFO'89, (Eds.)Beijing Singapore, 1989, pp. 156-160.

Kors J., Sittig A.C.. Bemmel J.H. van, The Delphi method to validate diagnostic knowledge in computerized HCG interpretation. Methods of Informalion in Medicine. 29. 1990, pp.44-50.

Keränen T., Riekkinen P.J., Sillanpää M., Incidence and prevalence of epilepsy in adults in eastem Finland, Epilepsia, 30, 1989, pp.413-421

Linstone H.A., Turoff M., The Delphi method: techniques and applications, Reading, Mass. AddisonWesley, 1975.

Loughlin K.G., Moore L.F., Using Delphi to achieve congruent objectives and activities in a pediatrics department, Journal of Medical Education, 54, 1979, pp.101-106.

Lundsgaard H.P., Evaluating medical expert systems, Social Science in Medicine. 24, 1987, pp. 805819

Meinardi H., Pachlatko C.. Special centers for epilepsy. In: Comprehensive lipileptology, (Dam and Gram, Eds.), Raven Press, New York, 1990, pp. 769-779

O'Keefe R.M., Balci O., Smith E.P., Validating expert systems performance, IEEE Expert, 4, 1987, pp. 81-89.

O'Keefe R.M., The evaluation of decision-aiding systems: guidelines and methods, Information and Management, 17, 1989, pp.217-226.

O'Leary D., O'Keefe R.M. Verifying and validating expert systems. Tulorial at the IJAI-89, Detroit, 1989.

O'Moore R., Clarke K., Smeets R., Brender J., Nykänen P., McNair P., Grimson J., Barber B., ltems of relevance for the evaluation of knowledge basedlexpert systems and the influence of domain characteristics, KAVAS (A 1021), Report EM-1.1, Trinity College, Dublin, 1990

O'Moore R., Clarke K., Brender J., McNair P., Nykänen P., Smeets R., Talmon J., Grimson J., Barber B., Methodology for evaluation of knowledge based systems, KAVAS (AI021), Report EM-1.2, Trinity College, Dublin, 1990.

Ovenweg J., Withdrawal of anti-epileptic drigs in seizure-free adult patients. prediction of outcome, thesis, Amsterdam, 1985 
Preece A.D., Towards a methodology for evaluating expert systems, Expert Systems, 7, 1990, pp.215223

Quaglini S., Stefanelli M., Barosi G., Benzuini A., A performance evaluation of the expert system ANEMIA, Computers and Biomedical Research, 21, 1988, pp.307-323.

Rector A., Engelbrecht R., Diedrich T., Fieschi M., Fitter M., Moser W., Schneider J., Report on validation and verification requirements and operational criteria, Eurodiabeta (AIM 1019), Deliverable I2, DG-XIII of the Commission of the EC, Brussels, 1990.

Romm F.J., Hulka B.S., Developing criteria for quality of care assessment: effect of the Delphi technique, Health Service Research, 14, 1979, pp.309-312.

Rossi-Mori A., Ricci F.L., On the assessment of medical expert systems. In: Expert systems and decision support in medicine. (Rienhoff, Piccollo and Schneider, Eds.), Lecture notes in medical Informatics 36, Springer Verlag, Berlin, 1988, pp. 292-297.

Russi-Mori A., Pisanelli D.M., Ricci F.L., Evaluation stages and design stups for knowledge-base systems in medicine, Medical Informatics. 15, 1990, pp.191-204.

Rushby J., Quality measures and assurance for AI sofiware, NASA contractor report 4187, SRI Intemational, Menlo Park, 1988.

Sander J.W.A.S., Hart Y.M., Johnson A.L., Shorvon S.D., National General Practice Study of Epilepsy: newly diagnosed epileptic seizures in a general population, The Lancet, 336, 1990, pp. 1267-1271.

Siegel S., Castellan N.J., Nonparametric statistics for the behavioral sciences, $2^{\text {nd }}$ edition, McGrawHill Book Company, New York, 1988.

Schouten H.J.A., Statistical Measurement of interobserver agreement, analysis of agreements and disagreements between observers, thesis, Rotterdam, 1985.

Smeets R., Talmon J., General methodology for the assessment of decision support systems. In: A critique of the evaluation methodology, (Nolan, Ed.), KAVAS (AIM 102.1), Report EM-2.I, Hvidovre Hospital, Hvidovre, 1990.

Sorgaard P.. Evaluating expert system prototypes. Artificial Intelligence and Socicty, 5, 1991, pp.317.

Spiegelhalter D., Evaluation of clinical decision aids. with an application to a system for dyspepsia, Statistics in Medicine, 2, 1983, pp 207-216.

Ven A. van der., Delbecq A.L. Nominal versus group processes for committee decision-making effectiveness, Academy of Management Journal, 6, 1971, pp. 203-212.

Wagner A.L., A clinical and epidemiological study of adult patients with epilepsy, Acta Neurologica Scandinavia, 94 (Suppl.). 1983, pp. 63-72

Willems J.L., Amaud P., Bemmel J.H. van, Bourdillon P.J., Degani R, Dennis B. Harms F.M.A., Macfarlane P.W., Mazzocca G., Meyer J., Ritsema van Eck H.J., Robles de Medina E.O., Zywietz C.. Establishment of a reference library for evaluating computer ECG measurement programs, Computers and Biomedical Research, 18, 1985, pp.439-457.

Wyatt J., Spicgellialter D., Evaluating medical expert systems: what to test and how?. Medical Iniormatics, 15, 1990, pp. 205-217. 



\section{Results on initiation of treatment}

\subsection{Introduction}

In this chapter the results of the analysis of the performance of the system in prescribing antiepileptic drugs in the initiation phase are reported. The advice of the system is compared both with the advice of two neurologists from the same department, one of which provided the knowledge implemented in the system and with that of five neurologists from different neurology departments. In the latter case the final prescriptions of the neurologists as obtained via the Delphi procedure are compared with those of the system.

To assess the acceptability of the advice - independent of whether the advice was according to the majority agreement - the prescriptions were reviewed by two neurologists.

A consensus meeting was held to discuss and possibly reduce the variability among the neurologists that remained after the Delphi procedure. While in the Delphi procedure the objective was to obtain a majority decision for each individual case, a more global approach for treatment was defined in the consensus meeting. These guidelines are used as a reference to compare the system and the neurologists with.

\subsection{Study design}

The goal of the study is to establish the adequacy of the advice of the system by comparing it with the advice of neurologists. A staged approach was adopted for the evaluation.

Five different assessments of the advice of the system were made. For each of the 60 cases of our test set, the expert neurologist (Neurologist I) whose knowledge was implemented in the system, provided a diagnosis and a prescription. The advice of the system, based on the detailed diagnosis of the neurologist and the other relevant patient data, was compared with the advice of this neurologist. This assessment provides some insight in the quality of the knowledge acquisition process.

To assess the impact of local differences a second neurologist (Neurologist II) from the same department as Neurologist $\mathrm{l}$ also provided a detailed diagnosis and a prescription for the 60 cases. A comparison was made with the advice of the system using Neurologist II's diagnosis.

In a third experiment the drug and the daily dose proposed by the system are compared with the treatments suggested by the neurologists after the Delphi method. The diagnostic reference set was used for assessing the system's proposed generic drug, the treatment reference set for assessing the suggested daily dose. This comparison provides a more objective measure of the 
performance of the system as these neurologists did not belong to the department of the expert neurologist.

A prescription different from the majority decision may still be considered a good option for treatment. Therefore, the acceptability of the prescriptions for each case in the diagnostic reference set was assessed by two neurologists who did not provide prescriptions for such a case.

To reduce the interobserver variability to determine the system's correctness, discrepancies in prescriptions were discussed during a consensus meeting. In this meeting general guidelines were proposed by the neurologists. From these guidelines a reference prescription was derived for each case in our diagnostic reference set. The advice of the system and the prescriptions of the individual neurologists were compared with this reference.

In the above design the focus of the assessment gradually shifts from an assessment of the coverage of the implemented knowledge and the correctness of the implementation to an assessment and comparison of the system's and neurologists' performance using a set of cases with reference diagnoses and prescriptions

When discussing the results a difference is made between cases and prescriptions. One has to remember that for each case up to three neurologists prescribe. The sets of cases for which individual neurologists provide a prescription vary. Therefore in most analyses the number of prescriptions is used.

\subsection{Comparing the system with Neurologist I and Neurologist II}

In this paragraph the advice of the system is compared with that of Neurologists I and II, using the 60 test cases. The diagnosis established by the neurologist is entered into the system. Since both neurologists independently diagnose the same case, it is possible that a case receives two different diagnoses. These cases are then entered twice into the system and the treatment advice of the neurologist is compared with that of the system using the diagnosis of that neurologist. In addition to the diagnosis, information about seizures and previous successful treatments, side-effects, use of co-medication, liver and kidney function and weight is entered into the system and used in the reasoning process

\subsubsection{Agreement on the generic drug}

Neurologist 1 provided 59 prescriptions. Although he was able to diagnose the type of epilepsy by using the available information, in one case he did not prescribe a drug because he wanted an additional EEG registration to be made before commencing drug treatment. Monotherapy was used in all cases.

Table 5.3-I: Agreement between Neurologist I, Neurologist II and the system.

\begin{tabular}{|c|c|c|c|c|c|c|}
\hline \multirow{2}{*}{$\begin{array}{l}\text { Gencric drug } \\
\text { System }\end{array}$} & \multicolumn{3}{|c|}{ Neurologist I } & \multicolumn{3}{|c|}{ Neurologist II } \\
\hline & No drug & CBZ. & $\mathrm{Na}-\mathrm{VPA}$ & No drug & $\mathrm{CBZ}$ & Na-VPA \\
\hline No drug & 0 & 0 & 0 & 9 & 0 & 0 \\
\hline CBZ & l & 44 & 0 & 0 & 40 & 0 \\
\hline Na-VPA & 0 & 0 & 15 & 1 & 0 & 10 \\
\hline
\end{tabular}

$\mathrm{CBZ}=$ carbanazepine, $\mathrm{Na}-\mathrm{VPA}=$ sodium valproate 
In 59 out of the 60 cases the system prescribed the same generic drug as Neurologist I (Table 5.3-1). Although the detailed diagnosis is entered, the system reasons with the global diagnosis. Also Neurologist I assigns a particular drug on the basis of the global diagnosis. If the diagnosis was partial epilepsy CBZ was proposed. For the cases diagnosed as primary (idiopathic) generalised epilepsy Na-VPA was selected. In the one case in which the neurologist established a diagnosis but did not provide a prescription the system suggested CBZ since the diagnosis was partial epilepsy.

Neurologist II established a diagnosis in 51 cases while a prescription was provided in 50 cases. Monotherapy was used for all of these cases. In six cases Neurologist Il could not establish the diagnosis and in three cases he was not certain about the diagnosis. In these nine cases Neurologist II decided not to prescribe a drug. In one additional case the neurologist decided not to prescribe a drug although he reached the diagnosis primary (idiopathic) generalised epilepsy. The reason for this was that he wanted to have additional EEG data about the patient. Because anti-epileptic drugs can influence the recordings he decided not to prescribe any drugs until the EEG findings were available

In 50 out of 51 cases (Table 5.3-1) the system and Neurologist II agreed on the drug treatment. Also Neurologist II selects a specific drug on the basis of the global diagnosis. The 39 cases diagnosed by Neurologist II as partial epilepsy received CBZ. In the one case diagnosed as primary (idiopathic) generalised epilepsy and partial epilepsy with secondary generalised partial seizures CBZ was also prescribed. In all ten cases diagnosed as primary (idiopathic) generalised epilepsy Na-VPA was prescribed. The system prescribed the same drugs. For the nine cases in which the neurologist did not provide a diagnosis the system did not suggest a treatment. In the one case in which the neurologist established the diagnosis primary (idiopathic) generalised epilepsy but did not prescribe a drug, the system suggested Na-VPA.

\subsubsection{Agreement on tradename}

When prescribing a drug one can use a generic name, leaving the choice of the brand to the pharmacist, or a tradename. Both neurologists used only tradenames. The system was programmed to do likewise.

The neurologists and the system used only one brand for each of the generic drugs. In case of Na-VPA all three selected Depakine* EC. For CBZ all three used the same brand. Neurologist I used Tegretol ${ }^{\text {w }}$, while Neurologist Il used only the controlled release variant, Tegretol ${ }^{\text {in: }} \mathrm{CR}$. The system uses the same formulation as Neurologist $\mathrm{I}$, hence there is a consistent difference with Neurologist II on the formulation of CBZ.

\subsubsection{Agreement on daily dose}

Both formulations for $\mathrm{CBZ}$ contain the same biologically active substance. The drug is available in the same doses of tablets for both formulations. Therefore, one can compare the prescribed daily doses. In 48 of the 59 cases the system prescribed the same daily dose as Neurologist I. Although the system and the Neurologist II completely agreed in 50 cases about the drug, in only 3 cases the system proposed the same daily dose. Table 5.3-II and Table 5.3III present the results comparing the daily doses of CBZ and Na-VPA prescribed by Neurologist I, Neurologist II and the system. 
Table 5.3-II: Daily dose for CBZ suggested by the system and by Neurologists I and IL.

\begin{tabular}{|c|c|c|c|c|c|c|c|c|}
\hline \multirow[t]{2}{*}{ CBZ } & & \multicolumn{7}{|c|}{ Prescribed daily dose (mg) } \\
\hline & & Neuro & 300 & 400 & 500 & 600 & 700 & 800 \\
\hline \multirow{8}{*}{$\begin{array}{l}\text { System's } \\
\text { daily dose }\end{array}$} & 200 & I & 1 & & & & & \\
\hline & & II & & 2 & & & & \\
\hline & 300 & I & 24 & 3 & & & & \\
\hline & & $\amalg$ & & 1 & 15 & 7 & & \\
\hline & 400 & 1 & 3 & 11 & & 1 & & \\
\hline & & Ш & & & & 6 & 6 & 3 \\
\hline & 500 & I & & 1 & & & & \\
\hline & & II & & & & & & \\
\hline
\end{tabular}

Forty-four times Neurologist I provided a prescription for CBZ. The daily dose of CBZ prescribed by Neurologist 1 ranged from 300 to $600 \mathrm{mg}$ per day. The dosages recommended by the system for $\mathrm{CBZ}$ ranged from 200 to $500 \mathrm{mg}$ per day. In 35 of the 44 prescriptions the daily dose suggested by the system was identical. Five times the neurologist prescribed a higher dose than that proposed by the system. Twice a daily dose two thirds of that prescribed by the neurologist was suggested. Four times the system proposed a higher dose than the neurologist

Table 5.3-III: Daily dose for VPA suggested by the system and by Neurologists I and II.

\begin{tabular}{|c|c|c|c|c|c|c|}
\hline \multirow[t]{2}{*}{ VPA } & & \multicolumn{5}{|c|}{ Prescribed daily dose (mg) } \\
\hline & & Neuro & 900 & 1000 & 1200 & 1500 \\
\hline \multirow{4}{*}{$\begin{array}{l}\text { System's } \\
\text { daily dose } \\
\text { (mg) }\end{array}$} & 900 & 1 & 13 & & 1 & \\
\hline & & [1] & 3 & 4 & 2 & \\
\hline & 1050 & 1 & 1 & & & \\
\hline & & Il & & & & 1 \\
\hline
\end{tabular}

Neurologist II approaches the treatment of partial epilepsy differently. The daily dose for CBZ ranges from 400 to $800 \mathrm{mg}$. In all except one case the system suggested only $50-66 \%$ of the daily dose prescribed by the neurologist.

For Na-VPA Neurologist $\mathrm{l}$ used a daily dose between 900 and $1200 \mathrm{mg}$. The system recommended Na-VPA in a daily dose of 900 or $1050 \mathrm{mg}$ per day. Twice the system suggested a daily dose different from that of the neurologist.

Neurologist II again prescribed higher daily doses for the cases diagnosed as primary (idiopathic) generalised epilepsy. The daily doses ranged from 900 to $1500 \mathrm{mg}$ per day. Also the prescription of Na-VPA differed from that of the system although the differences are smaller as compared to the differences for CBZ. In three of the 10 cases a dose less than $90 \%$ of the neurologist's dose was suggested.

\subsection{Comparing the system with an external reference}

In this paragraph the advice of the system is assessed using the cases from the diagnostic and treatment reference sets (see chapter 4 par. 4.2). 


\subsubsection{Agreement about the drug using the diagnostic reference set}

The agreement of the system with the neurologists on the cases of the diagnostic reference set is presented in Table 5.4-]. The system is compared with only those neurologists who agreed on the detailed diagnosis.

Table 5.4-I: Agreement between the system and the neurologists on the prescribed generic drug using the diagnostic reference set.

\begin{tabular}{|c|c|c|c|c|c|c|c|}
\hline \multirow{3}{*}{\multicolumn{2}{|c|}{$\begin{array}{l}\text { Agreement on: } \\
\text { delailed diagnosis } \\
\text { prescribed drug }\end{array}$}} & \multicolumn{4}{|c|}{ Neurologists } & & \\
\hline & & \multicolumn{2}{|c|}{$3 / 3$} & \multicolumn{2}{|c|}{$2 / 3$} & \multirow{2}{*}{\multicolumn{2}{|c|}{ Total }} \\
\hline & & $3 / 3$ & $2 / 3$ & $2 / 2$ & no & & \\
\hline \multirow[b]{2}{*}{ Agreement } & 3 neuso's & 7 & & & & 7 & \multirow[b]{2}{*}{ Agroement } \\
\hline & 2 neuro's & & 8 & 17 & & 25 & \\
\hline system & I neuro & & 3 & & 8 & 11 & \\
\hline \multirow[t]{2}{*}{ with } & 0 neuro's & I & 1 & 2 & 0 & 4 & Disagreement \\
\hline & Total & 8 & 12 & 19 & 8 & 47 & \\
\hline
\end{tabular}

Full agreement on diagnosis

In 8 cases all three neurologists agreed on the detailed diagnosis and the generic drug (Table 5.4-1). The system agreed in 7 of these cases with the generic drug. The diagnosis of the remaining case was partial epilepsy with secondary generalised seizures. While all three neurologists prescribed PHT the system prescribed CBZ.

There were 12 cases in which two out of three neurologists agreed about the generic drug although all agreed about the detailed diagnosis. The system agreed with the majority in 8 of these cases. In three cases the system recommended the drug prescribed by the third neurologist. In one case the system prescribed a drug which was not prescribed by any of the experts. In this case, diagnosed as partial epilepsy with complex partial seizures and partial epilepsy with secondary generalised seizures, two of the neurologists recommended no drug treatment while the third neurologist decided to prescribe Na-VPA. The system prescribed CBZ.

\section{2/3 agreement on diagnosis}

In 27 cases two out of three neurologists agreed on the detailed diagnosis. In 19 cases these two neurologists also agreed on the generic drug. The system prescribed the same drug in 17 cases (Table 5.4-I). The system prescribed Na-VPA for a case diagnosed as primary (idiopathic) generalised epilepsy while both neurologists prescribed CBZ. In another case, diagnosed as partial epilepsy with simple partial seizures the system suggested CBZ while the neurologists both prescribed PHT for this case.

In 32 of the 47 cases, the generic drug suggested by the system was in agreement with the reference drug. In eleven cases the system proposed a generic drug which was prescribed by only one neurologist. In all eleven cases this neurologist prescribed a drug different from that prescribed by his colleague(s) despite agreement on the detailed diagnosis. In four cases the system suggested a drug not prescribed by any of the neurologists. 


\subsubsection{Variability in neurologist's prescriptions}

In 20 cases of the diagnostic reference set all three neurologists established the same detailed diagnosis and reached a majority agreement on the prescribed drug in all of these cases. In the subset of 27 cases in which 2/3 neurologists agreed on the detailed diagnosis $63 \%$ of the cases had a majority agreement on the prescribed drug. As the selection of the drug is mainly based on the diagnosis, we required total agreement about this issue when comparing the proposed drugs of the system with those of the individual neurologists. Therefore the earlier mentioned subset of 20 cases is used for assessment of the generic drug suggested by the system.

Table 5.4-II presents how often each of the individual neurologists prescribed a generic drug agreed upon by the majority of the neurologists in cases of this subset of 20 cases. All except one of the neurologists prescribed the majority drug in more than $80 \%$ of his cases. One neurologist agreed in only $46 \%$ of the cases.

Table 5.4-1I: Generic drug prescribed in accordance with the majority.

\begin{tabular}{|c|c|c|c|c|c|c|c|c|c|c|c|}
\hline \multicolumn{12}{|c|}{ Agreement of participants and system with drug in $N=20$} \\
\hline \multirow[t]{2}{*}{ Participant } & III & \$ & IV & $S$ & $\mathrm{~V}$ & $\$$ & $\mathrm{~V}$ & $\mathrm{~S}$ & V & $\mathrm{S}$ & system \\
\hline & \multicolumn{2}{|c|}{$n=13$} & \multicolumn{2}{|c|}{$\mathrm{n}=16$} & \multicolumn{2}{|c|}{$n=11$} & \multicolumn{2}{|c|}{$\mathrm{n}=\mathrm{ll}$} & \multicolumn{2}{|c|}{$n=9$} & $n=20$ \\
\hline Drug agreed & 11 & 9 & 15 & 10 & 5 & 9 & 9 & 10 & 8 & 6 & 15 \\
\hline$\%$ & 85 & 75 & 24 & 63 & 46 & 82 & 82 & 91 & 89 & 67 & 75 \\
\hline
\end{tabular}

The system agreed on the prescribed drug with at least two neurologists in 15 of these 20 cases. As a majority was established if a neurologist agreed with at least one of his colleagues it was also examined in what percentage the system agreed with at least one neurologist. In 18 cases $(90 \%)$ the system prescribed the same drug as at least one of the prescribing neurologists

The system's advice was compared with that of an individual neurologist on the basis of the cases seen by both. Three neurologists prescribed more in accordance with the majority than the system. This analysis is in favour of the neurologists as they defined the drug used by the majority, while the system did not

\subsubsection{Agreement about the daily dose}

To obtain insight in the adequacy of the suggested dosage the treatment reference set was used. In 32 out of the 39 cases the system prescribed the same drug. These 32 cases were used to evaluate the dosages prescribed. In Table 5.4-111 a breakdown is presented of how many of these cases are seen by each of the neurologists and by the system.

Table 5.4-III: Agreement with the reference daily Jose.

\begin{tabular}{lcccccc}
\hline Neurologist & $3(\mathrm{n}=14)$ & $4(\mathrm{n}=16)$ & $5(\mathrm{n}=13)$ & $6(\mathrm{n}=14)$ & $7(\mathrm{n}=14)$ & System $(\mathrm{n}=32)$ \\
\hline $\begin{array}{l}\text { Cases for daily dose } \\
\text { comparison }\end{array}$ & 6 & 5 & 6 & 5 & 4 & 11 \\
Agreed daily dose & $43 \%$ & $31 \%$ & $46 \%$ & $36 \%$ & $29 \%$ & 32 \\
\hline
\end{tabular}


A majority dosage (at least two neurologists prescribe the same daily dose) could be obtained in 11 cases (34\%). Within this set of 11 cases the system agreed with the majority of the neurologists in 5 cases (46\%). In $20(63 \%)$ out of the 32 cases the system agreed about the daily dose with at least one neurologist.

Table 5.4-IV:Differences in the daily dose of CBZ (in parentheses the dose prescribed by the system is given as a percentage of the dose prescribed by the neurologists). The number of $100 \mathrm{mg}$ tablets presented in the first row indicates the number of tablets the system preseribed too few or too many.

\begin{tabular}{|c|c|c|c|c|c|c|c|}
\hline $\begin{array}{l}\text { Daily dose } \\
\text { neurologist (mg) }\end{array}$ & -5 tabl. & -4 tabl. & -3 tabl. & -2 lathl & -1 tabl. & 0 tabl. & {$[t .(b)$.} \\
\hline 200 & & & & & & & $2(150 \%)$ \\
\hline 300 & & & & & & $2(100 \%)$ & \\
\hline 400 & & & & & $12(75 \%)$ & $6(100 \%)$ & \\
\hline 600 & & & $9(50 \%)$ & $9(66,7 \%)$ & & & \\
\hline 800 & $1(37.5 \%)$ & $3(50 \%)$ & & & & & \\
\hline 900 & $\mathrm{I}(44 \%)$ & & & & & & \\
\hline
\end{tabular}

In Table 5.4-IV and Table 5.4-V the differences in daily dose between the system and the neurologists are presented for the drugs CBZ and Na-VPA using the 32 cases from the treatment reference set. In this analysis we used the doses of the individual prescriptions of the neurologists.

In 20 of the 32 cases in which the system prescribed the same dug as the majority of the neurologists CBZ was prescribed. In total 45 prescriptions were provided for this drug by the neurologists. The daily dose ranged from 200 to $900 \mathrm{mg}$. A daily dose of more than $600 \mathrm{mg}$ is prescribed 5 times $(12 \%)$. One neurologist prescribed more than $800 \mathrm{mg} \mathrm{CBZ}$.

Table 5.4-V: Differences in prescribed daily dose of CBZ between system and 5 neurologists.

\begin{tabular}{lccccc}
\hline $\begin{array}{l}\text { Difference in CBZ } \\
\text { daily dose (mg) }\end{array}$ & N III & N IV & N V & N VI & N VI \\
\hline-500 & 2 & & & 2 & \\
-400 & 5 & 1 & 3 & 1 & \\
-300 & 1 & & 5 & 3 & \\
-200 & & 6 & & 3 & 3 \\
-100 & & 4 & 1 & 1 & 2 \\
0 & & & & & 2 \\
\hline
\end{tabular}

Table 5.4-IV presents the differences in daily dose for CBZ between the neurologists and the system. In $8(18 \%)$ of these 45 prescriptions the system and a neurologist agreed on the daily dose of CBZ. If a difference of 1 tablet of $100 \mathrm{mg}$ is still acceptable the number of acceptable doses of the system increases to $22(49 \%)$. The system agrees with the neurologists in the lower dosage range. The higher the dose prescribed by the neurologists the larger the 
difference becomes. In 14 prescriptions a neurologist recommended twice or more the daily dose prescribed by the system. Table $5.4-\mathrm{V}$ shows the difference in agreement between the individual neurologists and the system. The system tends to prescribe approximately the same daily doses for CBZ as Neurologist IV and VII.

In 10 cases the system prescribed Na-VPA according to the reference (Table 5.4-VI). Agreement between the neurologists and the system on the prescribed dose of (Na-)VPA occurs in 14 out of 22 prescriptions $(64 \%)$. There are 6 prescriptions where the dose suggested by the system is $90 \%$ of the prescribed dose of the neurologist. This difference in doses is caused by a difference in intake scheme: $3 \times 300 \mathrm{mg}$ versus $2 \times 500 \mathrm{mg}$. Including these cases leads to an agreement in 20 out of 22 prescriptions. In 2 prescriptions ( $9 \%$ ) the system recommended a daily dose which was only $60 \%$ the dose prescribed by one of the neurologists.

Table 5.4-VI: Differences in the daily dose of ( $\mathrm{Na}$-)VPA. The percentages present the dose of the system relative to that of the neurologist. The number of tablets presented in the first row indicates the number of $300 \mathrm{mg}$ tablets the system prescribed too few.

\begin{tabular}{llll}
\hline $\begin{array}{l}\text { Daily dose of } \\
\text { neurologist (mg) }\end{array}$ & -2 tabl. & $-1 / 3$ labl. & 0 tabl. \\
\hline 900 & & & $14(100 \%)$ \\
1000 & & $6(90 \%)$ & \\
1500 & $2(60 \%)$ & & \\
\hline
\end{tabular}

\subsection{Review of the prescriptions}

All prescriptions for each of the 47 cases in the diagnostic reference set were assessed by two neurologists who did not prescribe for such a case. The reviewers judged the overall acceptability of each of the prescriptions on a five point scale (excellent, sufficient, neutral, insufficient, poor). For those prescriptions judged as of possibly poor quality - scored as neutral, insufficient or poor acceptability - the various components of a prescription e.g. the generic drug and the dose of tablets were assessed separately.

\subsubsection{Overview of the results for system and individual neurologists}

In total 114 prescriptions from the neurologists and 47 from the system were reviewed. For all prescriptions of the system both reviewers assessed the overall quality, resulting in 94 reviews. For seven prescriptions of the neurologists a reviewer did not provide an overall judgement. So only 221 rather than 228 review reports for the neurologists' prescriptions are available

In Table 5.5-I more than half of the prescriptions of all but one of the neurologists were considered to be of good quality by the reviewers (range 51 to $63 \%$ ). In less than $20 \%$ (range 13 to $19 \%$ ) their prescriptions were considered inadequate. Compared with these four neurologists a lower percentage of prescriptions of the system was considered to be of good quality but also a smaller percentage of prescriptions were considered inadequate. 
Table 5.5-I: Overall judgements by each of the two reviewers on the prescriptions provided by an individual neurologist. At most two judgements per prescription are available.

\begin{tabular}{|c|c|c|c|c|c|c|c|c|c|c|c|c|}
\hline \multirow{2}{*}{$\frac{\text { Participant }}{\text { Judgement }}$} & \multicolumn{2}{|c|}{ m } & \multicolumn{2}{|c|}{ IV } & \multicolumn{2}{|c|}{$\mathrm{V}$} & \multicolumn{2}{|c|}{$\mathrm{Vl}$} & \multicolumn{2}{|c|}{ VII } & \multicolumn{2}{|c|}{ System } \\
\hline & $\mathrm{n}$ & $\%$ & $n$ & $\%$ & $n$ & $\%$ & $\mathrm{n}$ & $\%$ & $\mathrm{n}$ & $\%$ & $\mathbf{n}$ & $\%$ \\
\hline poor' & 6 & 13 & 8 & 16 & 12 & 29 & 8 & 20 & 8 & 18 & 10 & 11 \\
\hline neutral & 14 & 30 & 15 & $3 !$ & 16 & 39 & 7 & 17 & 14 & 31 & 39 & 42 \\
\hline $\operatorname{good}^{2}$ & 26 & 57 & 26 & 53 & 13 & 32 & 26 & 63 & 22 & 51 & 45 & 48 \\
\hline Total judgements & 46 & 100 & 49 & 100 & 41 & 100 & 41 & 100 & 44 & 100 & 94 & 100 \\
\hline Cases diagn.ref.set & 23 & & 25 & & 21 & & 22 & & 23 & & 47 & \\
\hline Missing judgem. & 0 & & I & & I & & 3 & & 2 & & 0 & \\
\hline
\end{tabular}

(1) poor consists of insufficient and poor, (2) good contains the classes: good and excellent of the five point scale.

In 147 of the 157 review reports indicating a poor or neutral overall acceptability assessments of the prescribed drug were presented. For three of the neurologists more than $50 \%$ of these judgements was positive (range $54 \%$ to $70 \%$ ). Also for the system most (67\%) of the assessments indicated that a proper drug was prescribed (Table 5.5-II). For four of the neurologists $17 \%$ to $36 \%$ of the prescribed drugs were considered not appropriate. The system and one of the neurologists suggested an inappropriate drug in less than $5 \%$ of the prescriptions.

Table 5.5-II: Judgements on the generic drug for prescriptions with an overall poor or neutral quality. For each prescriber the obtained number of judgements indicating that the prescribed drug is appropriate, neutral or inappropriate is presented.

\begin{tabular}{|c|c|c|c|c|c|c|c|c|c|c|c|c|}
\hline \multirow{2}{*}{$\begin{array}{l}\text { Participant } \\
\text { Judgement drug }\end{array}$} & \multicolumn{2}{|c|}{ III } & \multicolumn{2}{|c|}{ IV } & \multicolumn{2}{|c|}{ v } & \multicolumn{2}{|c|}{$\mathrm{VI}$} & \multicolumn{2}{|c|}{ VII } & \multicolumn{2}{|c|}{ System } \\
\hline & $\mathrm{n}$ & $\%$ & $n$ & $\%$ & $n$ & $\%$ & 川 & $\%$ & n & $\%$ & $\mathrm{n}$ & $\%$ \\
\hline inappropriate & 3 & 17 & I & 4 & 5 & 21 & 5 & 36 & 4 & 20 & 1 & 2 \\
\hline neutral & 8 & 44 & 6 & 26 & 6 & 25 & 1 & 7 & 7 & 35 & 15 & 31 \\
\hline appropriate & 7 & 39 & 16 & 70 & 13 & 54 & 8 & 57 & 9 & 45 & 32 & 67 \\
\hline Total judgements & 18 & 100 & 23 & 100 & 24 & 100 & 14 & 100 & 20 & 100 & 48 & 100 \\
\hline Poor/neutral judg. & 20 & & 23 & & 28 & & 15 & & 22 & & 49 & \\
\hline Missing & 2 & & 0 & & 4 & & 1 & & 2 & & 1 & \\
\hline
\end{tabular}

For the prescriptions with a neutral or poor overall quality and an appropriate drug, the quality of the prescribed dose was analysed (Table 5.5-III). For one of these prescriptions a judgement was not provided on this issue. The percentage of judgements suggesting an inappropriate dose ranged from $53 \%$ to $60 \%$ for three of the neurologists, while for two neurologists more than $85 \%$ the daily dose was inappropriate. The system suggested an inappropriate dose in $91 \%$ of these prescriptions. Three neurologists tend to prescribe a (too) high rather than a (too) low dose. Most of the dosages prescribed by the system were judged as being too low.

In 21 of the 157 prescriptions which were considered to be of a (possibly) poor quality the judgement could not be attributed to an inadequate generic drug or daily dose but was caused by an inadequate brand, dose of tablets and/or intake scheme. 
Table 5.5-1II: Judgements provided on the daily dose for prescriptions in which the prescribed drug was considered to be appropriate but the overall judgement indicated (possibly) poor quality. For each neurologist the number of obtained judgements indicating a (too) low, neutral and (too) high daily dose is provided.

\begin{tabular}{|c|c|c|c|c|c|c|c|c|c|c|c|c|}
\hline \multirow{2}{*}{$\frac{\text { Participant }}{\text { Judgm. daily dose }}$} & \multicolumn{2}{|c|}{ III } & \multicolumn{2}{|c|}{ IV } & \multicolumn{2}{|c|}{ V } & \multicolumn{2}{|c|}{$\mathrm{VI}$} & \multicolumn{2}{|c|}{ VII } & \multicolumn{2}{|c|}{ System } \\
\hline & $\mathrm{n}$ & $\%$ & $\mathrm{n}$ & $\%$ & $\mathrm{n}$ & $\%$ & $n$ & $\%$ & $\mathrm{n}$ & $\%$ & $\pi$ & $\%$ \\
\hline$(1 \infty)$ low & 0 & 0 & 7 & 47 & 3 & 23 & 2 & 25 & 6 & 67 & 28 & 88 \\
\hline neutral & 1 & 14 & 6 & 40 & 6 & 46 & 3 & 38 & 1 & 11 & 3 & 9 \\
\hline (too) high & 6 & 86 & 2 & 1.3 & 4 & $3 !$ & 3 & 38 & 2 & 22 & 1 & 3 \\
\hline Tolal judgements & 7 & 100 & 15 & 100 & 13 & 100 & 8 & 100 & 9 & 100 & 32 & 100 \\
\hline Judgen.drug appr. & 7 & & 16 & & 13 & & 8 & & 9 & & 32 & \\
\hline Missing & 0 & & I & & 0 & & 0 & & 0 & & 0 & \\
\hline
\end{tabular}

\subsubsection{Comparing overall scores of system and individual neurologists}

For each case two or three neurologists and the system provided a prescription. The score for a prescription of a neurologist can be compared with the score for the prescription of the system on the same case. The difference between the overall acceptability of the prescription of a neurologist and the system gives an indication which of the two prescriptions is favoured. A positive difference indicat "; that the reviewer favours the prescription of the neurologist, a negative difference is in favour of the system. In Table 5.5-IV the difference scores are presented as a function of

Table 5.5-IV: Difference in overall acceptability scores of the reviewer when comparing the scores of the neurologist with the score of the system.

\begin{tabular}{|c|c|c|c|c|c|c|c|c|c|c|c|c|c|c|c|}
\hline \multirow[t]{2}{*}{ Overall score } & \multicolumn{3}{|c|}{ Reviewer 3} & \multicolumn{3}{|c|}{ Reviewer 4} & \multicolumn{3}{|c|}{ Reviewer 5} & \multicolumn{3}{|c|}{ Reviewer 6} & \multicolumn{3}{|c|}{ Reviewer 7} \\
\hline & - & 0 & + & $=$ & 0 & + & . & 0 & + & - & 0 & + & - & 0 & + \\
\hline n3-syst & & & & 2 & 7 & 2 & l & 4 & 5 & 2 & 8 & 3 & 4 & 6 & 2 \\
\hline n4-syst & 3 & 8 & 0 & & & & 1 & 3 & 9 & 2 & $\bar{y}$ & 0 & 2 & 10 & 2 \\
\hline n5-syst & 4 & 4 & 3 & 4 & 4 & 1 & & & & 4 & 4 & 2 & 4 & 5 & 2 \\
\hline n6-syst & 1 & 9 & । & 3 & 3 & 1 & 3 & 1 & 5 & & & & 4 & 3 & 7 \\
\hline n7-syst & 3 & 6 & 4 & 3 & 4 & 2 & 4 & 3 & 3 & 1 & 7 & 4 & & & \\
\hline Total & 11 & 27 & 8 & 12 & 18 & 6 & 9 & 11 & 22 & 9 & 28 & 9 & 14 & 24 & 13 \\
\hline$\%$ & 24 & 59 & 17 & 33 & 50 & 17 & 21 & 26 & 52 & 20 & 60 & 20 & 28 & 47 & 26 \\
\hline
\end{tabular}

\begin{tabular}{lccccccc}
\hline Overall score & \multicolumn{3}{c}{ Total } & \multicolumn{4}{c}{ Percentage } \\
& \multicolumn{1}{c}{0} & \multicolumn{1}{c}{+} & \multicolumn{1}{c}{0} & + \\
\hline nj-syst & 9 & 25 & 12 & 20 & 54 & 26 \\
n4-syst & 8 & 30 & 11 & 16 & 61 & 23 \\
n3-syst & 16 & 17 & 8 & 39 & 42 & 20 \\
n6-syst & 11 & 16 & 14 & 27 & 39 & 34 \\
n7-syst & 11 & 20 & 13 & 24 & 44 & 29 \\
\hline Total & 55 & 108 & 58 & & & \\
$\%$ & & & & 25 & 49 & 26 \\
\hline
\end{tabular}


the reviewing neurologist. Since in total 221 prescriptions of the neurologists were reviewed, 221 differences could be computed

Twenty prescriber-reviewer combinations exist. In 10 of these combinations the system's prescription was favoured above the neurologists' prescription more often than the reverse. In 7 the neurologists prescription was favoured.

It appears that Neurologist $V$ judges the performance of the system much lower than the other neurologists do. In turn Neurologist $V$ is judged less positively by his colleagues than the system. Overall the system's advice is judged as being of comparable quality as the neurologists.

Exclusion of Neurologist $\mathrm{V}$ as reviewer and as prescriber does not change the conclusion that the system's prescriptions are just as acceptable as the neurologists' prescriptions.

\subsubsection{Variability of the reviewers}

The prescriptions for a case have been independently assessed by two reviewers. This allows the study of the variability in the assessments of the reviewers.

Again we determined for each case the difference between the overall scoring of the recipe of the neurologist and the overall scoring of the corresponding recipe of the system: + indicating that the system's recipe had a better score, 0 indicating equal scores and - indicating that the recipe of the system obtained a lower score than the neurologist's recipe. This comparative score is determined for the assessments of both reviewers

Table 5.5-V gives an overview of how often in the 109 prescriptions of the neurologists for which two assessments were available, two judges give a certain comparative score.

In $14(13 \%)$ prescriptions both reviewers disagreed completely on which prescription is of better quality: one reviewer considered the neurologist's recipe better than the system's, the other reviewer did the reverse. For 42 prescriptions (38\%) both reviewers provided the same assessment for the two recipes.

Table 5.5-V: Agreement between reviewers compa-

ring the overall score for the system

with that for the prescription of a

neu rologist.

\begin{tabular}{crrr}
\hline Judge 1 & Judge 2 & No. cases & $\%$ \\
\hline+ & + & 6 & 5.5 \\
+ & 0 & 32 & 29.4 \\
+ & - & 14 & 12.8 \\
0 & 0 & 2 & 24.8 \\
0 & - & 21 & 19.3 \\
- & - & 9 & 8.3 \\
\hline \multicolumn{2}{l}{ Tolal no or cases } & 109 & 100 \\
\hline
\end{tabular}

The prescription of the system can be considered to be of lower quality when one of the reviewers considered a recipe of the neurologist of better quality and the other reviewer says that the neurologist's recipe is equal or better than the system's prescription. This occurs for 
30 prescriptions of the neurologists (28\%). For 38 pairs of prescriptions $(35 \%)$, the system's prescription is considered better by one reviewer and of equal or better quality than the neurologist's recipe by the other reviewer

\subsection{Results of the consensus meeting}

In this section the results of the consensus meeting are presented. The meeting dealt with general principles and not with individual cases although the diagnostic reference set served as background material for the discussion. Only the issues that are related to the diagnosis, selection of drugs and the initial dose are presented. The discussion resulted in a reference against which the prescriptions of the neurologists and of the system can be compared.

\subsubsection{The use of diagnostic classification schemes}

The diagnostic classification schemes as presented in the literature are considered to be more detailed than necessary for daily medical practice. The reliability of a detailed classification of the epilepsy of a patient may be doubtful. Cases admitted for neurosurgery may require a more detailed classification. Also for research purposes a detailed classification is valuable.

\subsubsection{Initiation of drug treatment}

In the literature a diagnosis ex juvantibus approach as well as initiation of anticonvulsive treatment if the diagnosis is not certain are both considered to be inappropriate (Chadwick and Reynolds 1985, Porter 1984, 1989, Shorvon 1987). In our study drug treatment was initiated twice in the 11 instances that a neurologist could not establish a diagnosis. In the consensus meeting and also in the review process drug treatment was considered unacceptable when no diagnosis is established. It was agreed that one should wait and let time decide what the diagnosis is before drug treatment is started.

Although the neurologists are willing to make a trade-off between the burden of antiepileptic drug treatment and the conseguences and the severeness of the seizures, all agreed that drug treatment should be started as soon as the diagnosis is established and two or more seizures have occurred. Also in the review process refraining from drug treatment was considered unacceptable when the diagnosis was established. However, in daily practice the opinion of the patient should be taken into account. If a patient refuses anticonvulsive treatment, despite knowing the consequences and risks, close monitoring of the patient is required to start the treatment immediately in case he changes his opinion.

\subsubsection{Selection of generic drug}

Based on the literature we concluded that at present there exists no consensus on whether the selection of the drug should be based on the type of epilepsy or on the type of seizures. On the basis of the meeting the neurologists agreed that the type of epilepsy should be the basis for the selection of the anticonvulsive treatment. A differentiation between generalised and partial epilepsy was considered to be sufficient for initial drug selection.

The neurologists agreed that if treatment is started in a newly diagnosed patient monotherapy should be used. The advantages of monotherapy are: a clear relation between drug and 
side-effects, a narrow spectrum of interactions and a better compliance. Polytherapy may be used in those patients where monotherapy proved to be ineffective.

Cases where complete seizure control was achieved in the past with polytherapy while monotherapy was not tried or tried inappropriately, may pose a problem in case of a relapse. Also in these patients the use of monotherapy is preferred even when a relapse occurs

For treatment of primary (idiopathic) generalised epilepsy Na-VPA is preferred. The argument that VPA (Convulex ${ }^{(i)}$ ) does not increase the sodium intake did not outweigh the advantages of Depakine ${ }^{\text {in }}$ EC. Even in case of hypertension the use of VPA is not advised nor has hypertension been mentioned as a contraindication for Na-VPA (Meinardi 1993, Shorvon 1995, Brodie and Dichter 1996).

The neurologists preferred the use of CBZ for treatment of partial epilepsy. Little agreement was observed when to apply PHT. The consensus meeting led to the conclusion that PHT should be the drug of first choice only for patients older than sixty years mainly because of side-effects such as gingival hyperplasia and coarsing of features in younger patients. PHT is a time honoured drug. The therapeutic range can be reached quickly and its use is easy due to the long half-life. Furthermore, the use of PHT for older patients has the advantage that a change in therapy is not necessary in case of surgery as a formulation for parenteral administration is available

\subsubsection{Generic drug or brand, formulation}

All neurologists preferred the use of the brand when prescribing a drug. The advantages mentioned were:

- The quality of a brand is fairly constant. The components as well as the production process have met criteria of quality control

- Variability in drug effectiveness is reduced. The occurrence of seizures can be attributed to a number of known factors.

A special formulation of the drug may prevent the occurrence of side-effects. Fluctuation in the plasma drug level and the occurrence of seizures at a particular time during the day, for example only in the early morning, may be important factors determining the selection of a special formulation of the drug. Furthermore, the compliance of the patient influences the selection of the formulation.

The neurologists agreed that Na-VPA may be prescribed as an uncoated formulation as far as the effectiveness of the drug is concerned. However, they prefer the enteric coated (EC) formulation because gastric distress occurs less often. Besides the $\mathrm{EC}$ formulation the Chrono formulation may be prescribed. This formulation has the advantage of less fluctuation in the plasma drug level while the peak level is reached faster. Nevertheless the EC formulation was preferred, using $300 \mathrm{mg}$ tablets in case the drug is taken three times a day or $500 \mathrm{mg}$ tablets if the drug is taken twice a day

The short half-life of CBZ together with the fluctuations in the plasma drug level require either a frequent drug intake or a controlled release (CR) formulation for this drug. All participants agreed on the use of the CR formulation when $C B Z$ is prescribed. The reference dose of the tablets is considered to be $200 \mathrm{mg}$. Tablets of $400 \mathrm{mg}$ should only be used in case a daily dose of $800 \mathrm{mg}$ or more is prescribed. The use of tablets of $400 \mathrm{mg}$ when a daily dose of less than $800 \mathrm{mg}$ is prescribed is considered to be inappropriate. 


\subsubsection{Daily dose of the drug}

In chapter 2 it has been shown that different opinions exist in the literature about the appropriate daily dose for Na-VPA, CBZ and PHT. Apart from the weight of the patient, which is according to our neurologists the basis for the determination of the daily dose, other factors such as stability of the plasma drug level, the risk of adverse drug reactions, the wish of the patient or the temperament of the neurologist, may influence the prescribed dose.

Although a 'middle of the target range' approach may be applied in selecting the daily dose, all participants agreed that in newly diagnosed patients one should start with a low daily dose and gradually increase the dose based on clinical findings. This is in contrast with the prescriptions they provided in which also a high initial dose occurred.

The ranges of the daily doses for the different drugs used by the neurologists were 900 to $1500 \mathrm{mg}$ for Na-VPA, 200 to $900 \mathrm{mg}$ for CBZ and 150 to $300 \mathrm{mg}$ for PHT. During the discussion the neurologists reached the conclusion that the prescribed daily doses were rather high. They agreed that if:

- Na-VPA or VPA was prescribed one should start with a dose of 900 to $1000 \mathrm{mg}$ per day, depending on the intake frequency

- $\mathrm{CBZ}$ is prescribed and the weight of the patient was less than $80 \mathrm{~kg} 400 \mathrm{mg}$ per day is sufficient. If the weight of the patient is higher than $80 \mathrm{~kg}$ the initial daily dose of the patient should be $600 \mathrm{mg}$

- PHT is selected as the drug for treatment and the weight is less than $80 \mathrm{~kg}$ a daily dose of $200 \mathrm{mg}$ PHT should be prescribed. In case the weight of the patient is higher than $80 \mathrm{~kg}$ they preferred a daily dose of $300 \mathrm{mg}$

One of the reasons for the relatively low daily dose of $\mathrm{CBZ}$ was the risk of adverse drug reactions. Side-effects may have consequences for the compliance. $\mathrm{CBZ}$ strongly induces its own catabolism. Therefore the initial daily dose has to be lower than the daily dose required eventually. The risk of having another seizure was not sufficient for the neurologists to initiate treatment with a higher daily dose. The kindling effect of additional seizures was considered not proven. Nevertheless, they agreed that sufficient prevention against seizures should be guaranteed. In addition to this, the wish of the patient should be taken into account when determining the daily dose. Some of the patients oppose drug treatment and do not like to be reminded several times a day that they are suffering from epilepsy. Others are afraid of having additional seizures and prefer a high dose to suppress further seizures.

\subsubsection{Intake frequency}

To be able to comply. patients need an intake scheme that is easy to handle. Therefore, the neurologists should try to keep the number of intakes per day as low as possible. The drug half-life as well as the formulation are important determinants for establishing the intake frequency. Practical consequences of drug intake have to be considered. While some neurologists combine the intake of the drug with meals, others stated that most patients tend to forget the intake during lunch because of the social consequences. The neurologists agreed that a drug intake of twice a day is most optimal as far as patient compliance is concerned 


\subsubsection{Gradual increase of the dose}

Dose related side-effects can be prevented through a gradual increase of the daily dose. The use of a gradual increase procedure is considered relevant for $\mathrm{CBZ}$. The half-life of $\mathrm{CBZ}$ when starting the intake is about 16 hours while long-term use of the drug decreases the half-life to 8 hours. Side-effects of each dosage can be assessed in a steady state which is reached after five times the half-life after the increase. Theoretically one can increase the dose on the fifth day. However, the neurologists prefer to increase the dose once a week. A more complicated intake scheme may hamper patient compliance. In case of $400 \mathrm{mg}$ the final dose will be reached in the second week, while with $600 \mathrm{mg}$ the final dose will be reached in the third week. The neurologists agreed that reaching an intake of 400 or $600 \mathrm{mg}$ within one week is not appropriate.

\subsection{Comparing the system and the neurologists with the consensus meeting reference}

Based on the guidelines obtained in the consensus meeting, a consensus treatment was established for cases from the diagnostic reference set. The prescriptions of the neurologists as well as the prescriptions of the system were compared with this consensus reference treatment. For the neurologists the number of prescriptions in accordance with the reference was established. The system was compared with the individual neurologists using the same set of cases.

\subsubsection{Agreement on the generic drug}

The group of neurologists provided 114 treatments. Five different drugs were used by the neurologists, whereas in the consensus reference only three drugs were used. The system used only two drugs for treatment (Table 5.7-1).

Anti-epileptic drug therapy was initiated 103 times by the neurologists. Eleven times drug treatment was not started. Seven times this was not according to the consensus meeting reference as this reference stated that drug treatment should be started when the diagnosis is established

Table 5.7-I:Agreement between the group of neurologists and the system with the reference developed during the consensus mecting.

\begin{tabular}{l|rrrrrrr|rrrrr}
\hline $\begin{array}{l}\text { Reference } \\
\text { Treatunent }\end{array}$ & \multicolumn{7}{|c|}{ Neurologists } & \multicolumn{5}{c}{ System } \\
\hline & No & CBZ & PB & PHIT & VF'A & OCI3 & Tolal & No & CBZ & PHT & VPA & Total \\
No & 4 & 0 & 0 & 0 & 0 & 0 & 4 & 2 & 0 & 0 & 0 & 2 \\
CBZ & 6 & 42 & 0 & 9 & 2 & 3 & 62 & 0 & 26 & 0 & 0 & 26 \\
PHT & 0 & 9 & 0 & 2 & 1 & 0 & 12 & 0 & 5 & 0 & 0 & 5 \\
VPA & 1 & 8 & 1 & 1 & 25 & 0 & 36 & 0 & 0 & 0 & 14 & 14 \\
\hline & 11 & 59 & 1 & 12 & 28 & 3 & 114 & 2 & 31 & 0 & 14 & 47 \\
\hline
\end{tabular}

In 73 of the 114 prescriptions (64\%) the selected drug of a neurologist was in accordance with the consensus reference. In two cases two neurologists decided that the type of epilepsy was unknown. In these cases both neurologists did not start drug treatment. 
The system suggested in 42 of the 47 cases (89\%) a drug according to the reference. In 2 cases drug treatment was not started because the type of epilepsy was not certain.

All neurologists prescribed a drug in accordance with the reference in more than half of the cases (range $52 \%$ to $72 \%$ ). The system compared with the individual neurologists suggested a drug according to the consensus consistently more often (range $88 \%$ to $91 \%$ )(Table $5.7-\Pi$ ).

The system suggested CBZ according to the reference consistently more often than the individual neurologist, while the proposal to use VPA was consistently at least as good as that of a neurologist. From the consensus meeting it was concluded that PHT should be used as well. This drug was not recommended by the system. Before the consensus meeting the neurologists disagreed more about the prescription of PHT than about the use of CBZ and VPA. Only twice (17\%) a neurologist used the drug in accordance with the reference, compared to $68 \%$ for $\mathrm{CBZ}$ and $69 \%$ for VPA.

Table 5.7-II: Number of cases for which the system and the individual neurologist recommended the same prescription as the reference.

\begin{tabular}{lllllllllll}
\hline Participant & III & Syst & IV & Syst & $V$ & Syst & V & Syst & VI & Syst \\
\hline Cases & $\mathrm{n}=23$ & $\mathrm{n}=23$ & $\mathrm{n}=25$ & $\mathrm{n}=25$ & $\mathrm{n}=21$ & $\mathrm{n}=21$ & $\mathrm{n}=22$ & $\mathrm{n}=22$ & $\mathrm{n}=23$ & $\mathrm{n}=23$ \\
Correct & 15 & 21 & 18 & 22 & 11 & 19 & 13 & 20 & 16 & 21 \\
Total \% & 65 & 91 & 72 & 88 & 52 & 91 & 59 & 91 & 70 & 91 \\
\hline
\end{tabular}

\subsubsection{Agreement on the daily dose}

For those cases in which the detailed diagnosis established by the neurologist was in agreement with the reference diagnosis and the prescribed drug and the reference drug were the same, the daily doses were compared. In Table 5.7-III prescribed daily doses are expressed as percentages of the reference dose. Since in the case of VPA a range was considered appropriate, doses between $100 \%$ (equivalent to $900 \mathrm{mg}$ ) and $111 \%$ (equivalent to $1000 \mathrm{mg}$ ) are appropriate. For $\mathrm{CBZ}$ the daily dose depends on the weight of the patient.

\section{Total set of prescriptions}

If the neurologists prescribe a drug according to the reference they achieve a better agreement with the reference daily dose than the system (57\% vs $37 \%$ of the prescriptions had the same dosage). When VPA is prescribed little difference between the neurologists and the system was observed $(88 \%$ and $94 \%$ of the prescriptions had the same daily dose as the reference, respectively). The prescribed daily dose of the neurologists ranged from $100 \%$ to $167 \%$ of the reference dose. The system suggested a daily dose ranging from $100 \%$ to $117 \%$ of the reference (Table 5.7-III).

When $\mathrm{CBZ}$ is prescribed - 42 and 62 times by the neurologists and the system respectively the system suggested only twice (3\%) a daily dose equal to that of the consensus, while in $36 \%$ of the prescriptions of the neurologists the dose was according to the reference. In $21 \%$ of the prescriptions the neurologists prescribed a lower daily dose (ranging from $50 \%$ to $75 \%$ of the reference dose), in $43 \%$ the neurologists prescribed a higher daily dose than the reference (ranging from $133 \%$ to $225 \%$ of the reference daily dose). The dosages suggested by the system were lower than the reference in $97 \%$ of the prescriptions (the daily dose ranged from $67 \%$ to $75 \%$ of the reference dose) 
Table 5.7-III: Variations in daily dose for those cases for which the consensus treatment and the detailed diagnosis are agreed upon by the participant.

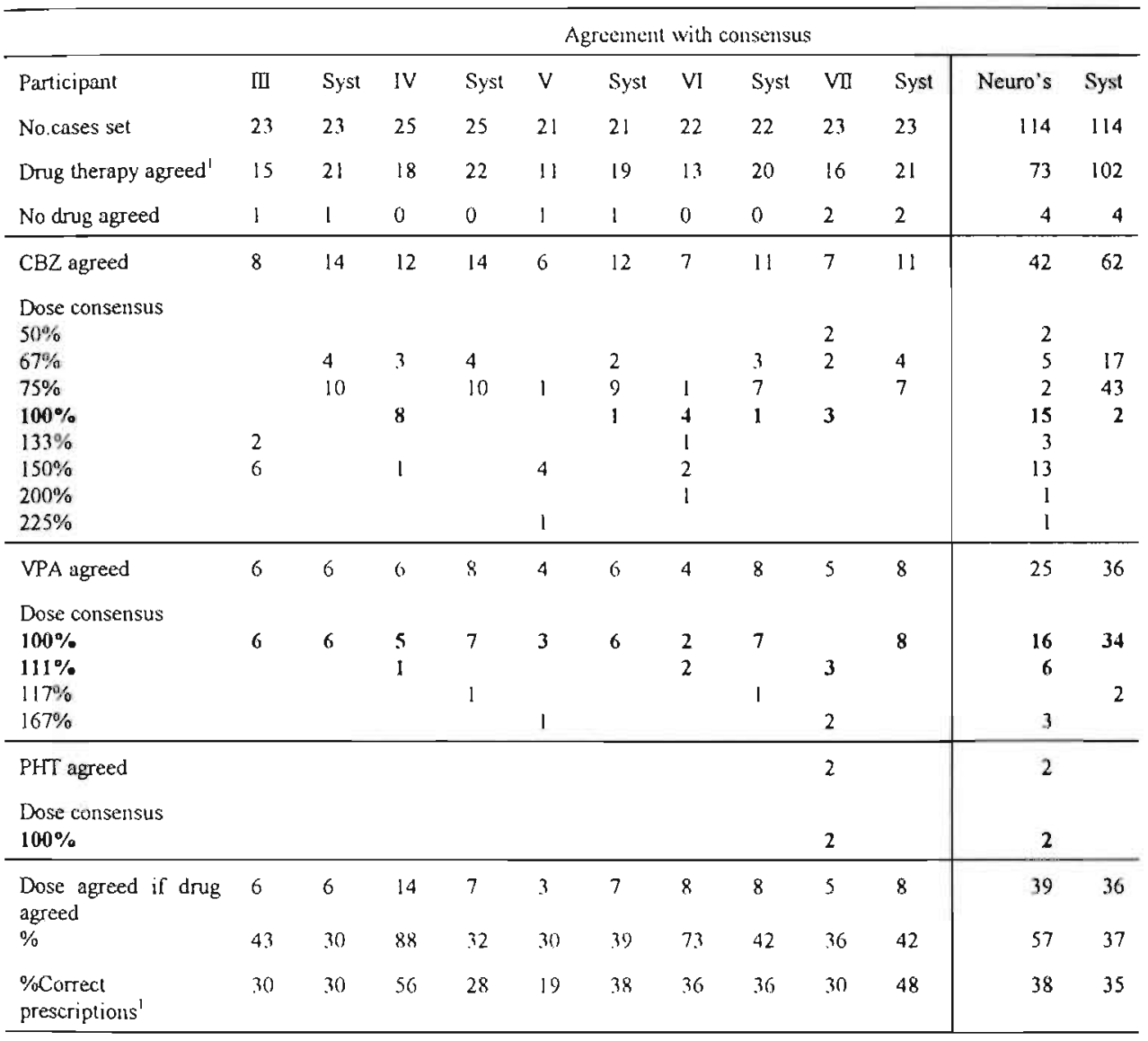

${ }^{1}$ This includes also the cuses for which the reference treatment was to provide no drug treatunent

Agreement in the sets of the individual nenrologist

In those cases in which the individual neurologist prescribes $\mathrm{CBZ}$ in agreement with the reference a daily dose according to the reference was suggested in 0 to $75 \%$ of the prescriptions. The system agreed in $10 \%$ or less for each of the sets seen by an individual neurologist. The system agreed with the reference in less cases than four of the neurologists.

Three neurologists prescribed VPA in a daily dose equal to the reference. Also the system prescribed VPA equal to the reference daily dose in three sets of the individual neurologist.

Agreement on drug and daily dose

All but one of the neurologists provided a prescription (drug and daily dose) according to the reference in less than $40 \%(19 \%$ to $36 \%)$. The other neurologist agreed with the reference in $56 \%$. The system provided a prescription according to the reference in $28 \%$ to $48 \%$ of the cases (for the various sets of cases common with the individual neurologists). Except for one 
of the neurologists the system consistently suggested an equal or higher number of prescriptions in accordance with the reference mainly because of a better agreement on VPA.

\subsection{References}

Chadwick D., Reynolds E.H., When do epileptic putients need treatment? Starting and stopping medication. British Medical Journal, 290. 1985, pp. 1885-1888.

Meinardi H., Epilepsie compendium, Katholicke Universiteit Nijmegen, 1993.

Porter R.J., Epilepsy, 100 elemenlary principles, W.B. Saunders Company Ltd., London, 1984.

Porter R.J. General principles how to use anti-epileptic drugs. In: Anti-epileptic drugs, $3^{\text {rd }}$ edition, (Levy, Mattson, Meldrum, Penry and F.E.Dreifuss, Eds.), Raven Press Ltd., New York, 1989, pp. 117-131.

Shorvon S.D., The trcatment of cpilepsy by drugs. In: Epilepsy. (Hopkins, Ed.), Chapman and Hall, London, 1987, pp. 229-282. 


\section{Results on follow-up of treatment}

\subsection{Introduction}

After a treatment is initiated its effectiveness is assessed during the next patient-doctor contacts; the follow-up. Based on this assessment a new therapy can be prescribed that may be different from the initial treatment. This chapter describes the results of our study concerning this second phase of anti-epileptic drug treatment.

First the treatment proposals provided by the neurologists based on 'paper cases' are compared with the advice provided by our system. The question to be answered is whether the treatments provided by the system are similar to the treatments provided by the neurologists. Several aspects of the treatment suggestions can be studied. We restrict ourselves to the global decisions to adjust the treatment: a continuation of the prescribed treatment ('Equal'), an adjustment of the daily dose of the drug ('Decrease' or 'Increase'), a switch to another antiepileptic drug ('Change') or adding an additional drug to the prescribed treatment ('Extra').

Since no objective standard exists for treatment of epilepsy we use the majority decision of a group of neurologists as the reference. To obtain insight in the variability of treatment decisions and to obtain information about possible alternatives without being dependent on the opinion of a single participant five neurologists were involved in defining a reference treatment for each case. Although consensus development by using a Delphi procedure has been considered, the time investment was deemed to be considerable. Therefore the majority decision among five neurologists was used for obtaining the reference treatment for each case. Comparing the correctness of the neurologists' treatment proposals with the system's output using this reference is disadvantageous for the system. Each of the neurologists has contributed to the reference and needs only to be in agreement with two other neurologists to have a correct proposal. The system needs to be in agreement with three neurologists to be correct. Therefore a second reference is defined by taking the majority of the treatment proposals of the system and the neurologists.

The adequateness of the system is evaluated in the following ways

- Comparison of the agreement between the system and neurologist( $s$ ). This analysis consists of two parts. Firstly the agreement between the system and the individual neurologists as well as among the individual neurologists was determined to establish the position of the system among the neurologists. In this study 265 cases (set 0) are used.

In the second analysis the prescriptions of each neurologist and the system are assessed by comparing them with prescriptions of the other four neurologists. Set 0 is used for this analysis. 
- Evaluation of the system and individual neurologists using the majority decision of the neurologists as the reference. This assessment provides information on how the neurologists and the system agree with a clinical reference. A reference was obtained in 219 of the 265 cases (set I).

- Evaluating the system and the neurologists using the majority decision of the neurologists and the system as the reference. In this study the set of 189 out of 265 cases for which such a reference was established was used (set II)

It is reasonable to assume that case characteristics determine which decision has to be taken. The fact that a majority decision is found in only 219 cases is, however, an indication that individual variation exists. In the second part of this chapter another type of analysis of the follow-ups is presented. Using a non parametric partitioning algorithm, a decision tree has been created based upon the case characteristics. In the ideal situation each terminal or endnode identifies cases of a certain (therapeutic) category. There may be several end nodes which point to the same category. The path from the root to the end-node contains the criteria to reach such an end-node. Four aspects of the derived tree are discussed

- The actual pureness of end-nodes in the decision tree

- The existence of cases with the same combination of patient characteristics that lead to an end-node, for which no reference treatment could be established

- The decision profiles within a impure end-node

- The decisions taken by the system for the cases of an end-node

Based upon this analysis we deduced a set of guidelines to be applied in the assessment of the initiated treatment that lead to specific treatment decisions.

\subsection{Design of test cases}

Like in our study on initiation of the drug treatment, paper cases were used. Information about the first follow-up after treatment initiation was added to the information in the paper cases of our previous study.

From patient records and interviews with neurologists it was determined which information is relevant for evaluating a patient who returns for an assessment of the prescribed treatment. Five characteristics were used to describe the course of the disease: the frequency of the seizures of various types, the compliance, the (kind of) complaints and the plasma drug level of the anti-epileptic drug. As in seizure severity scales the terminology (major and minor seizures) used for the seizure types is related to the severity of the seizures (Duncan and Sander 1991, Cramer 1993).

The following descriptors were used for these characteristics:

- The seizures (both major and minor) may be controlled (absent), decreased (the number of seizures decreased $\geq 50 \%$ ), equal (the number of seizures is unchanged, increased or decreased $<50 \%$ ) or not applicable (for a seizure type which was not present)

- There may be either a good compliance or non-compliance

- There can either be no complaints, dose related or idiosyncratic complaints

- The range of the plasma drug level can be either sub therapeutic, low therapeutic, therapeutic, high therapeutic or toxic 
In total 480 different combinations of these characteristics are possible.

We included - where relevant - other information related to the awareness of the patient, the occurrence of warnings, falling, incontinence, automatisms, convulsion, duration of the seizures and the duration before the patient is able to resume the activity he was pursuing before the seizure. The cause for non-compliance is presented where relevant. Furthermore, in case of complaints a description of the complaint together with information about the start and circumstances was presented. The prescription for the initiation of treatment was presented as well, including the brand of the medication, formulation, dose, number of tablets and number of intakes.

Apart from the detailed descriptors as discussed above a more global classification of the change in seizure frequency is used also (Table 6.2-I). In cases with several seizure types for which only one type of seizures was controlled or decreased in frequency, the reduction was still considered to be significant. Both case types were grouped into the class 'seizures decreased'.

Table 6.2-I: Overview of the seizure frequencies and the used terminology.

\begin{tabular}{|l|l|l|l|}
\hline Major seizures & Minor seizures & Litennediate & Global \\
\hline $\begin{array}{l}\text { equal } \\
\text { equal }\end{array}$ & $\begin{array}{l}\text { n.a. } \\
\text { equal } \\
\text { equal }\end{array}$ & uncontrolled & equal \\
\hline $\begin{array}{l}\text { decreased } \\
\text { n.a. } \\
\text { decreased } \\
\text { decreased } \\
\text { equal } \\
\text { equal } \\
\text { controlled }\end{array}$ & $\begin{array}{l}\text { n.a. } \\
\text { decreased } \\
\text { decreased } \\
\text { equal } \\
\text { decreased } \\
\text { controlled } \\
\text { equal }\end{array}$ & hurdly controlled & decreased \\
\cline { 1 - 2 } $\begin{array}{l}\text { controlled } \\
\text { decreased }\end{array}$ & $\begin{array}{l}\text { decreased } \\
\text { controlled }\end{array}$ & alinost controlled & \\
\hline $\begin{array}{l}\text { controlled } \\
\text { n.a. } \\
\text { controlled }\end{array}$ & $\begin{array}{l}\text { n.a. } \\
\text { controlled } \\
\text { controlled }\end{array}$ & controlled & absent \\
\hline
\end{tabular}

Of the 480 different case descriptions that can be constructed from the case characteristics, 270 follow-up contacts were actually used by:

- Reducing the number of cases with complete seizure control. Only few cases with complete seizure control were included as these cases usually do not entail difficult decisions

- Reducing the number of cases with only one type of seizures. Cases with two different types of seizures were considered of greater interest with regard to careful decision making

- Eliminating less realistic combinations of characteristics

\subsection{Description of the cases}

The 270 cases used in this study were derived from a subset of 43 case descriptions from the diagnostic test set of our study on the initiation of therapy. Each of these cases was the basis 
for one or more cases in the follow-up study. From 22 cases one to five different follow-up contacts were derived; 16 cases were the basis for six to ten different follow-ups. From five cases more than 10 follow-ups were created. The majority of the 43 cases had partial epilepsy. This selection was deliberate as this type of epilepsy is the most difficult to treat and has a larger variety of expressions of seizures (Elwes and Reynolds, 1990).

In 267 of the 270 cases all five neurologists provided a prescription. In the remaining three cases only four neurologists gave a prescription. In two of the 267 cases the system did not present a prescription. So 265 cases remained for comparison.

In this set of 265 cases 238 cases $(89.8 \%$ ) had a diagnosis of partial epilepsy, 12 cases (4.5\%) had idiopathic generalised epilepsy and 15 cases $(5.7 \%)$ had a combination of generalised and partial epilepsy. Three different drugs were prescribed in the 265 cases. CBZ was used in 219 cases $(82.6 \%)$, PHT was used in 36 cases (13.6\%) and Na-VPA was used in 10 cases $(3.8 \%)$.

Table 6.3-I: Distribution of the main characteristics of the paper cases used.

\begin{tabular}{|c|c|c|c|c|c|c|c|c|c|c|}
\hline \multirow{4}{*}{$\begin{array}{l}\text { Chancteristics } \\
\text { Compliance } \\
\text { Complaints } \\
\text { Seizures }\end{array}$} & \multicolumn{10}{|c|}{ Values and frequencies } \\
\hline & \multicolumn{4}{|c|}{ Compliant } & \multicolumn{4}{|c|}{ Non-compliance } & \multirow{2}{*}{\multicolumn{2}{|c|}{ Total }} \\
\hline & \multicolumn{2}{|c|}{ Absent } & \multicolumn{2}{|c|}{ Presient } & \multicolumn{2}{|c|}{ Absent } & \multicolumn{2}{|c|}{ Present } & & \\
\hline & $n$ & $\%$ & n & $\%$ & 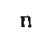 & $\%$ & $n$ & $\%$ & $\mathbf{n}$ & $\%$ \\
\hline controlled & 7 & 2.6 & 17 & 6.4 & 5 & 1.9 & II & 4.2 & 40 & 15.1 \\
\hline almost controlled & 8 & 3.0 & 15 & 5.7 & 6 & 2.3 & 13 & 4.9 & 42 & 15.8 \\
\hline hardly controlled & 20 & 7.6 & 39 & 14.7 & 22 & 8.3 & 34 & 12.8 & 115 & 43.4 \\
\hline uncontrolled & 11 & 4.2 & 18 & 6.8 & 22 & 8.3 & 17 & 6.4 & 68 & 25.7 \\
\hline Total & 46 & 17.4 & 89 & 33.6 & 55 & 20.8 & 75 & 28.3 & 265 & 100 \\
\hline
\end{tabular}

The set of cases is an enriched sample of what one would expect in daily clinical practice. Relatively more cases with (multiple) problems were included (e.g. cases in which seizures were not controlled by the initial treatment and/or cases with side-effects). In our set $95 \%$ of the cases had seizures which were not controlled (Table 6.3-I). This should be contrasted with a clinical setting where about one third of the patients may be controlled by the initial drug treatment (Elwes et al 1984, Beghi and Tognoni 1988,van Donselaar 1990, Collaborative Group for the Study of Epilepsy 1992). Hence it is to be expected that in our study the disagreement between neurologists concerning a specific treatment is larger than that found in daily practice.

\subsubsection{Description of reference set I}

In 219 of the 265 cases the majority of the five neurologists agreed about a specific global treatment decision. In Table 6.3-11 the distribution of the 219 cases with respect to the main characteristics is presented. Seizures were not completely controlled in the majority of these cases $(83.1 \%$ ). In more than half of the cases $(59.8 \%$ ) complaints were present. A combination of incompletely controlled seizures, presence of complaints and non-compliance is observed in $23.3 \%$ of the cases. 
Table 6.3-II: Overview of reference set I.

\begin{tabular}{|c|c|c|c|c|c|c|c|c|c|c|}
\hline \multirow{3}{*}{$\begin{array}{l}\text { Characteristics } \\
\text { Compliance } \\
\text { Complaints }\end{array}$} & \multicolumn{10}{|c|}{ Values and frequencies } \\
\hline & \multicolumn{4}{|c|}{ Compliant } & \multicolumn{4}{|c|}{ Non-compliant } & & \\
\hline & \multicolumn{2}{|c|}{ Absent } & \multicolumn{2}{|c|}{ Present } & \multicolumn{2}{|c|}{ Absent } & \multicolumn{2}{|c|}{ Present } & \multicolumn{2}{|c|}{ Total } \\
\hline Seizures & $\mathrm{n}$ & $\%$ & $\mathrm{n}$ & $\%$ & $\mathrm{n}$ & $\%$ & $\mathrm{n}$ & $\%$ & $\mathrm{n}$ & $\%$ \\
\hline controlled & 7 & 3.2 & 14 & 6.4 & 5 & 2.3 & 11 & 5.0 & 37 & 16.9 \\
\hline a most controlled & 8 & 3.7 & 11 & 5.0 & 5 & 2.3 & 7 & 3.2 & 31 & 14.2 \\
\hline hardly controlled & 16 & 7.3 & 28 & 12.8 & 19 & 8.7 & 27 & 12.3 & 90 & 41,1 \\
\hline uncontrolled & 10 & 4.6 & 16 & 7.3 & 18 & 8.2 & 17 & 7.8 & 61 & 27.9 \\
\hline Total & 41 & 18.7 & 69 & 31.5 & 47 & 21.5 & 62 & 28.3 & 219 & 100 \\
\hline
\end{tabular}

\section{Agreement profiles}

The degree of agreement among the neurologists may vary from case to case. The degree of agreement is indicated by means of agreement profiles. Each entry in the profile indicates the number of neurologists that agree about a treatment decision. For example a 3-2 profile indicates a majority of 3 neurologists while the other two neurologists disagreed with the majority but agreed among themselves.

Table 6.3-MI: Agreement profiles in the group of five neurologists.

\begin{tabular}{lrrrr|rrr}
\hline & \multicolumn{3}{c|}{ Reference set } & \multicolumn{3}{c}{ No reference } \\
\hline Profile & 5 & \multicolumn{1}{c}{$4-1$} & $3-2$ & $3-1-1$ & $2-2-1$ & $2-1-1-1$ & $1-1-1-1-1$ \\
Cases & 58 & 75 & 52 & 34 & 33 & 13 & 0 \\
$\%$ & 21.9 & 28.3 & 19.6 & 12.8 & 12.5 & 4.9 & 0 \\
\hline \multicolumn{3}{c}{ Total } & \multicolumn{3}{c}{$\mathrm{n}=219,82.6 \%$} & & \multicolumn{3}{c}{$\mathrm{n}=46.17 .4 \%$} \\
\hline
\end{tabular}

Table 6.3-IIl shows the distribution of the agreement profiles in the 265 cases. In 219 cases $(82.6 \%)$ a majority decision of the neurologists could be established. In 58 out of 265 cases all five neurologists completely agree on how to treat the patient.

\subsubsection{Description of reference set II}

In 189 of the 265 cases (71.3\%) a majority of at least four out of the six participants - the five neurologists and the system - agreed on the treatment decision. Table 6.3-IV presents the main characteristics of these 189 cases. When this set is compared with the set of 219 cases, minor changes are observed in the frequency of cases with seizures controlled and seizures uncontrolled. The system proposes an approach different from the majority of neurologists mainly in cases with.

- seizures almost controlled

- seizures hardly controlled and complaints

- seizures uncontrolled, complaints absent and non-compliance

- seizures controlled, complaints present and good compliance 
Table 6.3-IV: Overview of reference set II.

\begin{tabular}{|c|c|c|c|c|c|c|c|c|c|c|}
\hline \multirow{4}{*}{$\begin{array}{l}\text { Characteristics } \\
\text { Compliant } \\
\text { Complaints } \\
\text { Seizures }\end{array}$} & \multicolumn{10}{|c|}{ Values and frequencies } \\
\hline & \multicolumn{4}{|c|}{ Compliaut } & \multicolumn{6}{|c|}{ Non-compliant } \\
\hline & \multicolumn{2}{|c|}{ Absent } & \multicolumn{2}{|c|}{ Present } & \multicolumn{2}{|c|}{ Absent } & \multicolumn{2}{|c|}{ Present } & \multicolumn{2}{|c|}{ Total } \\
\hline & $\mathrm{n}$ & $\%$ & $\mathrm{n}$ & $\%$ & n & $\%$ & $\mathrm{n}$ & $\%$ & $\mathrm{n}$ & $\%$ \\
\hline controlled & 7 & 3.8 & 11 & 5.8 & 5 & 2.7 & 11 & 5.8 & 34 & 18.0 \\
\hline almost controlled & 5 & 2.7 & 10 & 5.3 & 5 & 2.7 & 5 & 2.7 & 25 & 13,2 \\
\hline hardly controlled & 15 & 7.9 & 22 & 11.6 & 17 & 9.0 & 21 & 11.1 & 75 & 39.7 \\
\hline uncontrolled & 9 & 4.8 & 16 & 8.5 & 13 & 6.9 & 17 & 9.0 & 55 & 29.1 \\
\hline Total & 36 & 19.1 & 59 & 31.2 & 40 & 21.2 & 54 & 28.6 & 189 & 100 \\
\hline
\end{tabular}

\section{Agreement profiles in reference cases}

The agreement profiles based on all six participants are shown in Table 6.3-V. Inclusion of the system in the judgement procedure yields reference cases in which either at least four neurologists or three neurologists and the system agree about the treatment approach.

Table 6.3-V: Comparison of the agreement in the group of five neurologists with that of the system and the group consisting only of five neurologists.

\begin{tabular}{|c|c|c|c|c|c|c|c|c|c|c|c|c|}
\hline \multirow{2}{*}{\multicolumn{2}{|c|}{$\begin{array}{l}\text { Agrecment among } \\
\text { five neurologists }\end{array}$}} & \multicolumn{11}{|c|}{ five neurologists and the system } \\
\hline & & \multicolumn{4}{|c|}{ Reference $\mathrm{N}=189.713 \%$} & \multicolumn{7}{|c|}{ No reference $\mathrm{N}=76,28.8 \%$} \\
\hline & Profile & 6 & $5-1$ & $4-2$ & $4-1-1$ & $3-3$ & $3-2-1$ & $3-1-1-1$ & $2-2-2$ & $2-2-1-1$ & $2-1-1-1-1$ & To \\
\hline Ref & 5 & 54 & 4 & & & & & & & & & 58 \\
\hline $\mathrm{N}=219$ & 4.1 & & 61 & 9 & 5 & & & & & & & 75 \\
\hline \multirow[t]{2}{*}{$82.6 \%$} & $3-2$ & & & 34 & & 16 & 2 & & & & & 52 \\
\hline & $3-1-1$ & & & & 22 & & 12 & & & & & 34 \\
\hline No ref & $2-2-1$ & & & & & & 26 & & 5 & 2 & & 37 \\
\hline $\begin{array}{l}N=46 \\
17.4 \%\end{array}$ & $2-1-1-1$ & & & & & & & 5 & & 7 & 1 & 13 \\
\hline \multicolumn{2}{|l|}{ Total } & 54 & 65 & 43 & 27 & 16 & 40 & 5 & 5 & 9 & 1 & 268 \\
\hline
\end{tabular}

In 54 of the 265 cases $(20.4 \%)$ the system completely agreed with the five neurologists (profile 6) In $\mathbf{3} 33$ cases the five neurologists had a 5 or 4 -1 agreement profile. When only the neurologists were used as a reference in 86 cases three out of five neurologists agreed about the treatment decision (profiles 3-2 and 3-1-1) Including the system resulted in the definition of a reference in 56 of these cases (profiles 4-2 and 4-1-1). In $16(13.5 \%)$ of the remaining 30 cases the system agreed with the minority of two neurologists. In 12 cases the system proposed a treatment decision equal to that of one of the neurologists.

In 14 of the 265 cases the system suggested a treatment different from any of the treatments suggested by the group of neurologists In nine of these cases there was a reference treatment (5-! and 4-I-! agreement profile), As a 3-3i agreement profile was not sufficient for a case to 
be incorporated as reference case, including the system could not cause cases not in set I to become part of set II. Where a reference had been based on a majority decision of three neurologists the system could either strengthen the agreement or refute it.

\subsubsection{Cases not included in the reference set II}

Reference set II contains 189 cases in which a majority decision was reached. In 76 cases no majority decision could be obtained. Table 6.3-VI gives an overview of the main characteristics of the cases for which a reference treatment could not be obtained. The results can be summarised as:

- In 51 of the 76 cases with no reference a complaint was present, which means that in $31.1 \%$ of the 164 cases in which a complaint was present a reference was not obtained In contrast, in 25 of the 101 cases $(24.8 \%$ ) without a complaint no reference was reached

- In 57 of the cases with no reference, seizures were hardly or almost completely controlled This means that in $36.3 \%$ of the 157 cases in which the anti-epileptic drug treatment seemed to have some but insufficient effect no majority decision could be achieved

- In 17 of the 42 cases $(40.5 \%)$ with seizures which were almost controlled a reference was not obtained.

- In 40 of 115 cases $(34.8 \%$ ) with seizures hardly controlled a reference could not be obtained.

- In 6 of the 40 cases $(15 \%)$ in which seizures were controlled a reference could not be defined. Also for less than one fitth of the cases $(19 \%)$ in which the seizure frequency remained equal to the previous visit a majority agreement could not be obtained

Table 6.3-VI: Description of the cases not included in the reference. In the column ' $\%$ ' the percentage of the total of 76 cases is presented. In the column $\%$ tor the percentage is related to the number of cases in the total test set with the same characteristics.

\begin{tabular}{|c|c|c|c|c|c|c|c|c|c|c|c|c|c|c|c|}
\hline \multirow{3}{*}{$\begin{array}{l}\text { Characteristic } \\
\text { Compliance } \\
\text { Complaints }\end{array}$} & \multicolumn{15}{|c|}{ Values and freyuencies } \\
\hline & \multicolumn{6}{|c|}{ Compliant } & \multicolumn{6}{|c|}{ Non-comp̣tiant } & \multirow{2}{*}{\multicolumn{3}{|c|}{ Total }} \\
\hline & & Ahsent & & & Frese: & & & Abser & & & Presen & & & & \\
\hline Seizures & n & $\%$ & $\% 10 t$ & $n$ & $\%$ & $\because$ tot & n & $\because$ & $\sim \operatorname{tot}$ & n & $\because$ & $*+104$ & $n$ & $\because$ & "stust \\
\hline centrolled & 0 & 0 & a & 6 & 7.9 & 35.1 & 0 & 0 & 0 & 4 & 0 & 0 & 6 & $7 \%$ & 150 \\
\hline almost cont. & 3 & 39 & 37.5 & 5 & 66 & 33.1 & 1 & 1.3 & 167 & $\mathrm{x}$ & 105 & 6.15 & 17 & $2: 4$ & 405 \\
\hline hardly con! & 5 & 6.6 & 25.0 & 17 & 22.4 & 436 & 9 & 66 & 227 & 13 & 171 & $1 \times 2$ & 40 & 826 & $34 x$ \\
\hline uncontr. & 2 & 2.6 & 18.2 & 2 & 2.6 & 111 & 91 & 118 & 409 & 0 & 4 & $\theta$ & 17 & 171 & 191 \\
\hline Totat & 10 & 132 & 217 & 30 & 395 & 337 & 15 & 197 & 273 & $2 !$ & 276 & $2 \times 0$ & 76 & 100 & 100 \\
\hline
\end{tabular}

Agreement profiles in non-reference cases

in $61(80.3 \%)$ of the 76 cases with no reference treatment three out of six participants agree about a specific treatment (profiles $3-3,3-2-1$ and $3-1-1-1$ ) (Table $63-\mathrm{V}$ )

The agreement of each participant with the other participants in these 76 cases is presented in Table 6.3-VII. 'Score' indicates the number of other participants which agree with the participant. 
Table 6.3-VII: Variability in agreement between participants in the 76 cases. The item 'Score' indicates the number of participants which agree with the decision taken by the neurologist or the system.

\begin{tabular}{lrrrrrrrrrrrrr}
\hline & \multicolumn{1}{c}{ NUI } & \multicolumn{3}{c}{ NIV } & \multicolumn{2}{c}{ NV } & \multicolumn{2}{c}{ NVI } & \multicolumn{2}{c}{ NVI } & \multicolumn{2}{c}{ System } \\
\hline Score & n & $\%$ & $\mathrm{n}$ & $\%$ & $\mathrm{n}$ & $\%$ & $\mathrm{n}$ & $\%$ & $\mathrm{n}$ & $\%$ & $\mathrm{n}$ & $\%$ \\
0 & 17 & 22.4 & 12 & 15.8 & 18 & 23.7 & 14 & 18.4 & 11 & 14.5 & 5 & 6.6 \\
1 & 27 & 35.5 & $3 !$ & 40.8 & 15 & 19.7 & 27 & 35.5 & 24 & 31.6 & 24 & 31.6 \\
2 & 32 & 42.1 & 33 & 43.4 & 43 & 56.6 & 35 & 46.1 & 41 & 54.0 & 47 & 61.8 \\
\hline
\end{tabular}

The percentage of cases for which a neurologist prescribed a treatment different from those prescribed by any of the other participants ranges from $14.5 \%$ to $23.7 \%$. The system provided an advice different from those of all neurologist's in only $6.6 \%$ of the cases.

Each of the neurologists agreed with two other participants in $42.1 \%$ to $56.6 \%$ of the cases. The system agreed with two neurologists in $61.8 \%$ of the cases.

\subsubsection{Relevance of characteristics for agreement among neurologists}

Various levels of agreement among the neurologists occur in the cases with a reference treatment (set l). The degree of agreement probably depends on the characteristics of the case. For each of the main features in the case descriptions we determined the level of agreement. The five out of five and four out of five agreement levels are considered to represent high degrees of agreement as changing the opinion of one of the neurologists will not affect the reference treatment.

\section{Seizure froyuency}

In $78 \%$ of the 37 cases in which seizures were completely controlled and in $65.6 \%$ of the 61 cases in which seizures remained equal to the previous visit there is a high degree of agreement on the reference treatment (Table 6.3-VIII). For the cases in which seizures were almost completely controlled a lower $(58.1 \%)$ percentage of cases with a high degree of agreement is observed.

Table 6.3-VII: Distribution of the agreement profiles for the frequencies of the type of seizures.

\begin{tabular}{|c|c|c|c|c|c|c|c|c|c|c|c|c|c|c|}
\hline Characteristic & \multicolumn{9}{|c|}{ Reference treatinent } & \multicolumn{5}{|c|}{ No reference treatmint } \\
\hline Profile & \multicolumn{2}{|c|}{5} & \multicolumn{2}{|c|}{$4-1$} & \multicolumn{2}{|c|}{$3-2$} & \multicolumn{2}{|c|}{$3-1-1$} & Tolal & \multicolumn{2}{|c|}{$2-2-1$} & \multirow{2}{*}{$\begin{array}{l}2-1-1-1 \\
N \%\end{array}$} & \multicolumn{2}{|c|}{ Total } \\
\hline Seizures: & $\mathrm{N}$ & $\%$ & $N$ & $\%$ & $N$ & $\%$ & $N$ & $\%$ & $N \%$ & $\mathrm{~N}$ & $\%$ & & $\mathrm{~N}$ & $\%$ \\
\hline controlled & 17 & 45.6 & 12 & 32.4 & 5 & 13.5 & 3 & 8.1 & 37100 & 3 & 100 & 00 & 3 & 100 \\
\hline almost contr & 8 & 25.8 & 10 & 32.3 & 4 & 12.9 & 9 & 29.0 & 31100 & 5 & 46 & 655 & 11 & 100 \\
\hline hardly contr & 19 & 21.1 & 27 & 30.0 & 32 & 35.6 & 12 & 13.3 & $90 \quad 100$ & 18 & 72 & 728 & 25 & 100 \\
\hline uncontr & 14 & 23.0 & 26 & 42.6 & 11 & 180 & 10 & 16.4 & 61100 & 7 & 100 & 0 & 7 & 100 \\
\hline Total & 58 & 26.5 & 75 & 34.3 & 52 & 23.7 & 34 & 15.5 & 219100 & 33 & 72 & 1328 & 46 & 100 \\
\hline
\end{tabular}


For cases that were hardly controlled the high degree of agreement decreases to $51.1 \%$. This drop in strong agreement can partially be explained by the fact that there seem to be two alternative treatments for certain cases. For example in $35.6 \%$ of the cases in which seizures are hardly controlled the two neurologists who propose a treatment different from the reference agree among themselves (the 3-2 agreement profile). This in contrast with a 3-2 agreement profile in at most $18 \%$ of the other types of seizure frequency.

\section{Compliance}

Compliance appears to have no effect on the degree of agreement among the neurologists (Table 6.3-IX). A high degree of agreement is found in $63.6 \%$ and $57.8 \%$ of the cases with a good compliance and non-compliance, respectively.

Table 6.3-IX: Dist ribution of the agreement profiles for the characteristic 'Compliance'.

\begin{tabular}{|c|c|c|c|c|c|c|c|c|}
\hline \multirow{3}{*}{$\begin{array}{l}\text { Characteristic } \\
\text { Profile } \\
\text { Compliance }\end{array}$} & \multicolumn{5}{|c|}{ Reference trealtnent } & \multicolumn{3}{|c|}{ No reference } \\
\hline & 5 & 4-1 & $3-2$ & $3-1-1$ & Total & $2-2-1$ & $2-1-1-1$ & Total \\
\hline & $N \%$ & $\mathrm{~N} \%$ & $\mathrm{~N} \%$ & $\mathrm{~N} \%$ & $N \quad \%$ & $\mathrm{~N} \%$ & $\mathrm{~N} \%$ & $\mathrm{~N} \%$ \\
\hline $\operatorname{good}$ & $35 \quad 31.8$ & 3531.8 & 2220.0 & $18 \quad 16.4$ & $110 \quad 100$ & 1872 & 728 & 25100 \\
\hline non-compliance & 2321.1 & $40 \quad 36.7$ & 3027.5 & $16 \quad 14.7$ & $109 \quad 100$ & 1571 & 29 & 21100 \\
\hline Total & $58 \quad 26.5$ & $\begin{array}{ll}75 & 34.3\end{array}$ & $52 \quad 23.7$ & $34 \quad 15.5$ & $219 \quad 100$ & 3372 & 1328 & 46100 \\
\hline
\end{tabular}

\section{Complaints}

In $63.7 \%$ of the cases where complaints are absent more than three neurologists agree on the treatment (Table 6.3-X). For cases in which idiosyncratic complaints are present strong agreement occurs in $83.1 \%$ of the cases.

Contrary to this, strong agreement is present in only $38.9 \%$ for cases with dosc related complaints. In $44.4 \%$ of the cases with dose related complaints, there is a $3-2$ agreement pattern, indicating that in these circumstances two alternative treatments may exist.

Table 6.3-X: Distribution of the agreement profiles for the characteristic 'Complaints'.

\begin{tabular}{|c|c|c|c|c|c|c|c|c|c|c|c|c|}
\hline \multirow{3}{*}{$\begin{array}{l}\text { Characteristic } \\
\text { Profile } \\
\text { Complaints }\end{array}$} & \multicolumn{9}{|c|}{ Reference treatınent } & \multicolumn{3}{|c|}{ No reference } \\
\hline & \multicolumn{2}{|r|}{5} & \multicolumn{2}{|c|}{$4-1$} & \multicolumn{2}{|c|}{$3-2$} & \multicolumn{2}{|c|}{$3-1-1$} & Total & $2-2-1$ & $2-1-1-1$ & \multirow{2}{*}{$\begin{array}{l}\text { Total } \\
\text { N } \%\end{array}$} \\
\hline & $N$ & $\%$ & $N$ & $\%$ & $\mathrm{~N}$ & $\%$ & $N$ & $\%$ & $N \%$ & $\mathrm{~N} \%$ & $\mathrm{~N} \%$ & \\
\hline ahsent & 24 & 27.3 & 32 & 36.4 & 17 & 19.3 & 15 & 17.1 & $88 \mathrm{l}(n)$ & 1077 & 323 & 13100 \\
\hline dose related & 6 & 8.3 & 22 & 30.6 & 32 & 44.4 & 12 & 16.6 & 72100 & 2067 & 1033 & 30100 \\
\hline idiosyncratic & 28 & 47.5 & 21 & 35.6 & 3 & 5.1 & 7 & 11.9 & $59 \quad 100$ & 3100 & 00 & 3100 \\
\hline Total & 58 & 26.5 & 75 & 34.3 & 52 & 23.7 & 34 & 15.5 & 219100 & 3372 & 1328 & 46. 100 \\
\hline
\end{tabular}

\section{Plasma drug level}

'Plasma drug level' hardly influences the degree of agreement. Strong agreement is found in more than $55 \%$ of the cases for all plasma drug levels except for levels in the toxic range. Even in the latter strong agreement was found in $50 \%$ of the cases (Table 6.3-XI). 
Table 6.3-XI: Distribution of the agreement profiles for the characteristic 'Plasma drug level'.

\begin{tabular}{|c|c|c|c|c|c|c|c|c|c|c|c|c|}
\hline \multirow{3}{*}{$\begin{array}{l}\text { Characteristic } \\
\text { Profile } \\
\text { Plasma drug level }\end{array}$} & \multicolumn{9}{|c|}{ Reference treatment } & \multicolumn{3}{|c|}{ No reference } \\
\hline & \multicolumn{2}{|c|}{$s$} & \multicolumn{2}{|c|}{$4-1$} & \multicolumn{2}{|c|}{$3-2$} & \multicolumn{2}{|c|}{$3-1-1$} & Total & $2-2-1$ & $2-1-1-1$ & Tola] \\
\hline & $\mathrm{N}$ & $\%$ & $N$ & $\%$ & $\mathrm{~N}$ & $\%$ & $\mathrm{~N}$ & $\%$ & $\mathrm{~N} \%$ & $\mathrm{~N} \%$ & $\mathrm{~N} \%$ & N \% \\
\hline sub ther & 15 & 33.3 & 14 & 31.1 & 12 & 26.7 & 4 & 8.9 & $45 \quad 100$ & 571 & 229 & $7 \quad 100$ \\
\hline low ther & 16 & 36.4 & 14 & 31.8 & 9 & 20.5 & 5 & 11.4 & $44 \quad 100$ & 6100 & 00 & 6100 \\
\hline ther & 11 & 20.8 & 21 & 39.6 & 12 & 22.6 & 9 & 17.0 & $53 \quad 100$ & 770 & 330 & $10 \quad 100$ \\
\hline high ther & 9 & 20.0 & 17 & 37.8 & 9 & 20.0 & 10 & 22.2 & $45 \quad 100$ & 975 & 325 & 12100 \\
\hline toxic & 7 & 21.9 & 9 & 28.1 & 10 & 31.3 & 6 & 18.8 & $32 \quad 100$ & 655 & 546 & 11100 \\
\hline Total & 58 & 26.5 & 75 & 34.3 & 52 & 23.7 & 34 & 15.5 & 219100 & 3372 & 1328 & $46 \quad 100$ \\
\hline
\end{tabular}

\subsubsection{Alternative treatments}

The previous section showed that in more than one third of the cases with hardly controlled seizures as well as in cases with dose related complaints a 3-2 agreement profile was frequently observed. For these two characteristics the alternative treatment is discussed.

\section{Seizure frequency}

In the 32 cases with hardly controlled seizures two neurologists propose an alternative treatment (see Table 6.3-XII). Change of drug is the most common alternative proposal for the reference treatments

Table 6.3-XII: Distribution of the reference treatments and the alternatives for the 32 cases in which a 3-2 agreement pattern was observed.

\begin{tabular}{|c|c|c|c|c|c|c|}
\hline \multirow[t]{2}{*}{ Treatment } & & \multicolumn{5}{|c|}{ Reference treatment } \\
\hline & Decision & Decrease & Equal & Increase & Change & Extra \\
\hline \multirow{4}{*}{$\begin{array}{l}\text { Altenative } \\
\text { tratunent }\end{array}$} & Decrease & & & & d & \\
\hline & Equal & 2 & & & 4 & \\
\hline & Increase & & & & 1 & \\
\hline & Change & 12 & 6 & 1 & & 2 \\
\hline
\end{tabular}

\section{Dose related complaints}

In 32 cases with dose related complaints (Table 6.3-XIII) in most cases (75\%) a change of drug was either the reference treatment or the alternative treatment. With dose related complaints in the history in all cases (9/9) the alternative treatment suggested was a change of drug when the reference decision was to decrease the dose, and also a change of drug in $7 / 12$ cases where the reference was to keep the medication constant. In five cases with dose related complaints where the reference was to continue the treatment, presumably because of seizure related reasons, the alternative was an increase of the dose.

A decision by either the reference or the alternative treatment to increase the dose was rarely observed in combination with an advice to change the drug (3/10) 
Table 6.3-XII: Distribution of the reference treatments and the altematives for the 32 cases with dose related complaints in which a 3-2 agreement pattern was observed.

\begin{tabular}{|c|c|c|c|c|c|c|}
\hline Treatment & & \multicolumn{5}{|c|}{ Reference treatment } \\
\hline \multirow{5}{*}{$\begin{array}{l}\text { Altemative } \\
\text { treatment }\end{array}$} & Decision & Decrease & Equal & Increase & Change & Extra \\
\hline & Decrease & & & & 1 & \\
\hline & Equal & & & 2 & 4 & \\
\hline & Increase & & 5 & & 2 & \\
\hline & Change & 9 & 7 & 1 & & 1 \\
\hline
\end{tabular}

\subsection{Agreement and correctness}

Two of the metrics mainly used when evaluating a DSS are agreement and correctness. The term agreement is used when comparing the advice of the system with that of a neurologist or a group of neurologists. Correctness is used when assessing the advice of the system using a previously defined reference.

\subsubsection{Overview of the decisions in the total set}

Eight different decisions were taken by the neurologists. The decisions to continue the prescribed treatment are grouped into the class 'Equal'. If the daily dose was decreased or increased the decisions are labelled as 'Decrease' and 'Increase'. The class 'Change' contains decisions in which a different anti-epileptic drug was selected for further treatment. This class does not include the addition of a second anti-epileptic drug to the prescribed treatment. The class 'Stop' contains the decision to discontinue the treatment immediately. The class 'Other' contains decisions which differed from adjustment of the treatment, e.g. a referral. Finally, two

Table 6.4-I: Overview of the frequencies of decisions for further treatment of the patient.

\begin{tabular}{|c|c|c|c|c|c|c|c|c|c|c|c|c|c|c|}
\hline \multirow[t]{2}{*}{ Decision } & \multicolumn{2}{|c|}{ Decruase } & \multicolumn{2}{|c|}{ Equal } & \multicolumn{2}{|c|}{ Increase } & \multicolumn{2}{|c|}{ Change } & \multicolumn{2}{|c|}{ Extra } & \multicolumn{2}{|c|}{ Stop } & \multicolumn{2}{|c|}{ Other } \\
\hline & $\mathrm{n}$ & $\%$ & $n$ & $\%$ & $n$ & $\%$ & $\mathrm{n}$ & $\%$ & $n$ & $\%$ & $\mathrm{n}$ & $\%$ & $\mathrm{n}$ & $\%$ \\
\hline III & 50 & 18.9 & 67 & 25.3 & 39 & 14.7 & 96 & 36.2 & 13 & 4.9 & 0 & 0 & 0 & 0 \\
\hline IV & 37 & 14.0 & 113 & 42.6 & 56 & 21.1 & 47 & 17.7 & 9 & 3.4 & 2 & 0.8 & I & 0.4 \\
\hline V & 13 & 4.9 & 30 & 11.3 & 52 & 19.6 & 154 & 58.1 & 14 & 5.3 & 2 & 0.8 & 0 & 0 \\
\hline $\mathrm{VI}$ & 31 & 11.7 & 62 & 23.4 & 65 & 24.5 & 107 & 40.4 & 0 & 0 & 0 & 0 & 0 & 0 \\
\hline VII & 2 & 0.8 & 91 & 34.3 & 34 & 12.8 & 1.35 & 50.9 & 2 & 0.8 & 1 & 0.4 & 0 & 0 \\
\hline System & 11 & 4.2 & 54 & 20.4 & 62 & 2.3 .4 & 136 & 51.3 & 0 & 0 & 2 & 0.8 & 0 & 0 \\
\hline
\end{tabular}

decisions were observed in combination with adding an additional drug to the existing treatment ('Extra'); a continuation of the existing treatment or an alteration of the existing daily dose. Table 6.4-I presents the distribution of the decisions of each of the five neurologists and the system on the 265 cases (in the table the eight types of decisions have been reduced to seven by combining 21 decisions in which an anti-epileptic drug was added to 
the existing treatment and 11 decisions in which both an anti-epileptic drug was added and the original dose was altered into the category 'Extra')

\subsubsection{Differences in treatment approaches}

There are clear differences in the approaches taken by the neurologists. Neurologist IV is the one that most often sticks to the initiated treatment. In $42.6 \%$ of the cases he does not make any adjustment in the dose or the drug. In only $17.7 \%$ he decides to change the treatment. This is in contrast with Neurologist $V$ who predominantly changes the therapy $(58.1 \%$ of the cases) and keeps the drug dose as it was in only a small fraction of the cases.

Also the fraction of cases in which the decision was taken to adjust the dose of the drug shows large variations. While Neurologist III decides to decrease the drug dose in almost one fifth of the cases, Neurologist VII decides to decrease the drug dose in less than $1 \%$.

An increase of the drug dose was decided upon by Neurologist VI in almost a quarter of the cases which is almost twice as often as Neurologist VII.

Adding a second anti-epileptic drug to the existing drug treatment or the immediate stopping of the prescribed drug are not very common decisions. All neurologists decide to switch to polytherapy in less than $6 \%$ of the cases, while the decision to stop the drug is taken by all neurologists in less than $1 \%$ of the cases.

On average, in $25 \%$ of the cases the same treatment was suggested, while in approximately $40 \%$ of the cases the drug was changed. These numbers clearly show that the cases are heavily biased towards cases that are more difficult to treat.

The system proposed to change the drug in more than $50 \%$ of the cases. In less than $5 \%$ of the cases a decrease of the dose of the drug was suggested, while an increase was suggested in almost one fourth of the cases. In $20 \%$ of the cases a continuation of the prescribed treatment was suggested.

Table 6.4-II: $k$-values for the individual neurologists and the system on the total set of 265 cases.

\begin{tabular}{lccccc}
\hline & Neuro II & Neuro IV & Neuro V & Neuro VI & Neuro VI \\
\hline Neuro IV & 0.42 & & & & \\
Neuro V & 0.40 & 0.28 & & & \\
Neuro VI & 0.40 & 0.41 & 0.44 & & \\
Neuro VII & 0.35 & 0.26 & 0.35 & 0.29 & \\
System & 0.42 & 0.35 & 0.56 & 0.46 & 0.40 \\
\hline
\end{tabular}

The agreement beyond what is to be expected by chance alone between two observers can be expressed with the kappa coefficient. $\kappa$-values between 0.21 and 0.40 are considered to indicate fair agreement, those ranging from 0.41 up to 0.60 are considered to denote moderate agreement, while the values between 0.61 and 0.80 and those larger than 0.81 indicate substantial and almost perfect agreement, respectively (Landis and Koch, 1977). 
The $\kappa$-values for the pair wise comparisons of the decisions of neurologists range from 0.26 to 0.44 , while the systen when compared with the individual neurologists has $\mathrm{K}$-values between 0.35 and 0.56 (Table 6.4-II). The agreement of the system with a neurologist is at least as good as the agreement of that neurologist with any of the other neurologists, except for Neurologist IV.

\subsubsection{Agreement between an individual and a group}

The agreement of each individual neurologist with the four remaining neurologists is compared with the agreement of the system with the same group of neurologists. The number of neurologists that took the same decision as the individual neurologist or the system is counted for each case. In Table 6.4-III the distribution of the degree of agreement for each of the neurologists and the system with the other four neurologists is shown for the 265 cases.

The system achieved a significantly higher mean score than all the individual neurologists (paired student t-test, $\mathrm{p}<0.05$ )

Table 6.4-1U: Overview of the scores comparing the decision concerning 265 cases of an individual neurologist with the remaining neurologists. The number of neurologists which agree with the decision for treatment of the individual neurologist is represented by the score.

\begin{tabular}{|c|c|c|c|c|c|c|c|c|c|c|c|c|}
\hline \multirow[b]{2}{*}{ Score } & \multicolumn{2}{|c|}{ N III } & \multicolumn{2}{|c|}{ System } & \multicolumn{2}{|c|}{ NIV } & \multicolumn{2}{|c|}{ System } & \multicolumn{2}{|c|}{$\mathrm{NV}$} & \multicolumn{2}{|c|}{ System } \\
\hline & n & $\%$ & $\mathrm{n}$ & $\%$ & 11 & $\%$ & $\mathrm{n}$ & $\%$ & $n$ & $\%$ & $\mathrm{n}$ & $\%$ \\
\hline 0 & 38 & 14.3 & 18 & 6.8 & 52 & 19.6 & 18 & 6.8 & 43 & 16.2 & 23 & 8.7 \\
\hline 1 & 53 & 20.0 & 42 & 15.8 & 66 & 24.9 & 43 & 16.2 & 42 & 15.8 & 53 & 20.0 \\
\hline 2 & 47 & 17.7 & 62 & 23.4 & 41 & 15.5 & 55 & 20.8 & 60 & 22.6 & 64 & 24.2 \\
\hline 3 & 69 & 26.0 & 84 & 31.7 & 48 & 18.1 & 72 & 27.2 & 62 & 23.4 & 63 & 23.8 \\
\hline 4 & 58 & 21.9 & 59 & 22.3 & 58 & 21.9 & 77 & 29.1 & 58 & 21.9 & 62 & 23.4 \\
\hline \multicolumn{5}{|c|}{$t=3.22 p<0.0005$} & \multicolumn{4}{|c|}{$t=5.96 p<0.0005$} & \multicolumn{4}{|c|}{$t=1.94 p<0.05$} \\
\hline
\end{tabular}

\begin{tabular}{lcccccccc}
\hline & \multicolumn{2}{c}{ Neuro VI } & \multicolumn{2}{c}{ System } & \multicolumn{2}{c}{ Ncuro VII } & \multicolumn{2}{c}{ Systen } \\
\hline Score & $\mathrm{n}$ & $\%$ & $\mathrm{n}$ & $\%$ & $\mathrm{n}$ & $\%$ & $\mathrm{n}$ & $\%$ \\
0 & 37 & 14.0 & 22 & 8.3 & 45 & 17.0 & 22 & 8.3 \\
$\mathrm{I}$ & 47 & 17.7 & 40 & 15.1 & 54 & 20.4 & 48 & 18.1 \\
2 & 55 & 20.8 & 67 & 25.3 & 55 & 20.8 & 61 & 23.0 \\
3 & 68 & 25.7 & 76 & 28.7 & 53 & 20.0 & 61 & 23.0 \\
4 & 58 & 21.9 & 60 & 22.6 & 58 & 21.9 & 73 & 27.5 \\
& & $\mathrm{t}=2.37 \mathrm{p}<0.025$ & & & $1=3.85$ & $0<0.0005$ \\
\hline
\end{tabular}

\section{Complete agreement}

Total agreement among the five neurologists occurs in $21.9 \%$ of the cases. The system takes more decisions which agree with any group of 4 neurologists. In $22.3 \%$ to $29.1 \%$ of the cases, groups of four neurologists agree with the system. The system agrees significantly more with the remaining neurologists than Neurologists IV and VII (McNemar $\chi^{2}=12 p<0.05$, McNemar $\chi^{2}=8.52 \mathrm{p}<0.05$, respectively) 


\section{Majority}

A majority decision was reached by the neurologists in 219 of the 265 cases $(82.6 \%)$. For each neurologist the number of cases in which he agrees with two or more of his colleagues is counted. Also for the system the number of cases in which the system reaches a majority decision with each group of the four remaining neurologists is counted. Each individual neurologist participated in majority agreements in $55.5 \%$ to $68.3 \%$ of the cases, as compared to $71.3 \%$ to $77.4 \%$ when the system replaces the neurologist.

The system achieved a majority agreement (system + two neurologists) significantly more often than four of the individual neurologists (McNemar $\chi^{2}=10.84 p<0.05 ; \chi^{2}=30.45 p<0.05$; $\chi^{2=1.26} \mathrm{NS} ; \chi^{2}=5.96 \mathrm{p}<0.05 ; \chi^{2}=10.74 \mathrm{p}<0.05 ; \mathrm{df}=1$, for Neurologists III to VII, respectively).

\section{Complete disagreement}

A neurologist may take a decision which is not made by any of his colleagues. This occurs in $14.0 \%$ to $19.6 \%$ of the cases for the individual neurologists, whereas for the system this percentage varies between $6.8 \%$ to $8.7 \%$ of the cases. The system had significantly fewer cases with which all neurologists disagreed than each of the neurologists (McNemar $\chi^{2}=8.21$ $p<0.05, \chi^{2}=18.78 p<0.05, \chi^{2}=8.21 p<0.05, \chi^{2}=4.78 p<0.05, \chi^{2}=10.76 p<0.05$ for neurologists III to VII, respectively).

Table 6.4-IV: Agreement of a particular ncurologist and the system with the remaining neurologists, expressed as a kappa value.

\begin{tabular}{lllllllllllll}
\hline \multicolumn{10}{c}{ Assessment of individual neurologist and system by } \\
\hline Group & $4-5-6-7$ & \multicolumn{3}{c}{$3-5-6-7$} & \multicolumn{3}{c}{$3-4-6-7$} & \multicolumn{3}{c}{$3-4-5-7$} & \multicolumn{3}{c}{$3-4-5-6$} \\
Prescriber & N III & Syst & N IV & Syst & N V & Syst & N VI & Syst & N VII & Syst \\
Kappa & 0.39 & 0.44 & 0.34 & 0.46 & 0.36 & 0.43 & 0.39 & 0.46 & 0.35 & 0.47 \\
\hline
\end{tabular}

Agreement beyond chance

The agreement between a particular neurologist and the remaining group of four colleagues can also be expressed by a $\kappa$-value (Schouten, 1985). The $\kappa$-values range from 0.34 to 0.39 (Table 6.3-IV). The agreement of the system with the groups of neurologists ranges from 0.43 to 0.47 and is in all cases higher than the kappa of each individual neurologist.

\subsubsection{Correctness}

In the previous paragraphs the advice of the system was compared with the decisions of individual neurologists. This gave an indication about the capability of the system to make decisions similar to those made by individual neurologists. The correctmess of the decisions of the neurologist and of the system has been assessed using both reference sets.

For set I - the reference based on the neurologists alone - the correctness of the system is somewhat higher than the average correctness of the neurologists $(77.4 \%)$. However, three neurologists score better than the system (Table 6.4-V)

Including the system when establishing the reference increased the correctness for all participants. The system provided a correct advice more often than each individual neurolo- 
gist. This was significantly better for three of the five neurologists $\left(\mathrm{McNemar} \chi^{2}=4.5 \mathrm{p}<0.025\right.$; $\chi^{2}=24.85 \mathrm{p}<0.0005 ; \chi^{2}=0.52 \mathrm{NS} ; \chi^{2}=1.84 \mathrm{NS} ; \chi^{2}=9.13 \mathrm{p}<0.005 ; \mathrm{df}=1$, for neurologists III to VII respectively).

Table 6.4-V: Correctness with the reference of set I (i.e. majority of five neurologists) and set II (i.e. majority of five neurologists and the system).

\begin{tabular}{l|lcccccc}
\hline Set & Participants & N III & N IV & N V & N VI & N VI & System \\
\hline Set I, 3/5 neur. & Cases & 174 & 147 & 180 & 181 & 166 & 171 \\
N=219 & Correciness & 79.5 & 67.1 & 82.2 & 82.6 & 75.8 & 78.1 \\
\hline Set II, 4/6 neur.-syst. & Cases & 155 & 128 & 166 & 161 & 148 & 171 \\
N=189 & Correctness & 82.0 & 67.7 & 87.8 & 85.2 & 78.3 & 90.5 \\
\hline
\end{tabular}

\subsubsection{Variability in agreement in set II}

Since the reference treatment procedure is based on a majority, individual participants may disagree with the reference. In this paragraph the degree of agreement of the individual participants with his colleagues is discussed by.

- Comparing the mean of the scores obtained by each individual participant

- Addressing for each participant the disagreement with the reference treatment

- Studying the complete disagreement of each participant with the rest of the participants Furthermore, for each reference treatment the decisions taken by the group of neurologists and the system are presented.

\section{Overall score}

For the reference cases Table 6.4-VI provides a break-down of the number of participants that agree with a specific neurologist or the system. 'Score' indicates the number of participants a neurologist or the system agrees with. A score of 2 cannot be obtained because the reference contains only the agreement profiles $6,5-1,4-2$ and $4-1-1$. The system achieved a significantly higher score than each individual neurologist does (paired T-test $t=10.68 p<0.05, t=19.62$ $\mathrm{p}<0.05, \mathrm{t}=9.79 \mathrm{p}<0.05, \mathrm{t}=10.11 \mathrm{p}<0.05, \mathrm{t}=15.18 \mathrm{p}<0.05)$.

\section{Disagreement with the reference}

As the reference is defined by at least four participants a score less than 3 indicates disagreement of the participant with the reference. This is observed in 18 cases $(9.5 \%)$ for the system up to 61 cases $(32.3 \%)$ for neurologist IV. The agreement of the system with more than two other participants is better than that of three of the individual neurologists. 
Table 6.4-VI: Variability in the degree of agreement between participants for set II $(n=189)$. The item 'Score' indicates the number of participants which agree with the decision taken by the neurologist or the system.

\begin{tabular}{lrrrrrrrrr}
\hline & \multicolumn{3}{c}{ Neurologist III } & \multicolumn{3}{c}{ Neurologist IV } & \multicolumn{3}{c}{ Neurologist V } \\
\hline Score & $\mathrm{n}$ & $\%$ & cum & $\mathrm{n}$ & $\%$ & cum & $\mathrm{n}$ & $\%$ & cum \\
0 & 17 & 9.0 & 9.0 & 36 & 19.1 & 19.1 & 16 & 8.5 & 8.5 \\
1 & 17 & 9.0 & 18.0 & 25 & 13.2 & 32.3 & 7 & 3.7 & 12.2 \\
3 & 41 & 21.7 & 39.7 & 32 & 16.9 & 49.2 & 55 & 29.1 & 41.3 \\
4 & 60 & 31.7 & 71.4 & 42 & 22.2 & 71.4 & 57 & 30.2 & 71.4 \\
5 & 54 & 28.6 & 100 & 54 & 28.6 & 100 & 54 & 28.6 & 100 \\
Total & 189 & 100 & & 189 & 100 & & 189 & 100 & \\
mean & 3.44 & & & $2.9 \%$ & & & 3.55 & & \\
\hline
\end{tabular}

\begin{tabular}{lrrrrrrrrr}
\hline & \multicolumn{3}{c}{ Neurologist VI } & \multicolumn{4}{c}{ Neurologist VII } & \multicolumn{3}{c}{ System } \\
\hline Score & $\mathrm{n}$ & $\%$ & cum & $\mathrm{n}$ & $\%$ & cum & $\mathrm{n}$ & $\%$ & cum \\
0 & 15 & 7.9 & 7.9 & 26 & 13.8 & 13.8 & 9 & 4.8 & 4.8 \\
$\mathrm{l}$ & 13 & 6.9 & 14.8 & 15 & 7.9 & 21.7 & 9 & 4.8 & 9.5 \\
3 & 48 & 25.4 & 40.2 & 48 & 25.4 & 47.1 & 56 & 29.6 & 39.2 \\
4 & 59 & 31.2 & 71.4 & 46 & 24.3 & 71.4 & 61 & 32.3 & 71.4 \\
5 & 54 & 28.6 & 100 & 54 & 28.6 & 100 & 54 & 28.6 & 100 \\
Total & 189 & 100 & & 189 & 100 & & 189 & 100 & \\
mean & 3.51 & & & 3.24 & & & 3.65 & & \\
\hline
\end{tabular}

\section{('omplete disagreement with the other participants}

The number of cases for which a neurologist disagreed with all other participants (Score 0) ranges from $15(7.9 \%)$ to 36 cases $(19.1 \%)$. In nine cases $(4.8 \%)$ the system proposes a treatment different from any proposal of the neurologists

Table 6.4-VII also gives an overview of the agreement profiles for those cases where the individual participant disagreed completely with the rest of the group. The percentage of cases in which a participant disagreed while all other participants agreed about the treatment decision ranged from $2.7 \%$ to $12.2 \%$ of the cases (three neurologists caused a $5-1$ agreement profile in less than $5 \%$, two neurologists in more than $10 \%$ of the cases). In four cases (2.1\%) the system did not agree with the treatment procedure although all five neurologists did agree completely. Each neurologist as well as the system disagreed completely with the five other participants of which a group of four agreed among themselves (agreement profile 4-1-1) in less than $10 \%$ of the cases 
Table 6.4-VII: Distribution of cases for which the individual participant disagreed completely with the other five participants in cases from set II.

\begin{tabular}{lrrrrrrrrrrrr}
\hline Participant & \multicolumn{2}{c}{$\mathrm{N} \mathrm{WI}$} & \multicolumn{2}{c}{$\mathrm{N} \mathrm{IV}$} & \multicolumn{2}{c}{$\mathrm{N} \mathrm{V}$} & \multicolumn{2}{c}{$\mathrm{N} \mathrm{VI}$} & \multicolumn{2}{c}{$\mathrm{N} \mathrm{VM}$} & \multicolumn{2}{c}{ System } \\
\hline Agreement profile & $\mathrm{n}$ & $\%$ & $\mathrm{n}$ & $\%$ & $\mathrm{n}$ & $\%$ & $\mathrm{n}$ & $\%$ & $\mathrm{n}$ & $\%$ & $\mathrm{n}$ & $\%$ \\
$5-1$ & 5 & 2.7 & 23 & 12.2 & 8 & 4.2 & 6 & 3.2 & 19 & 10.1 & 4 & 2.1 \\
$4-1-1$ & 12 & 6.4 & 13 & 6.9 & 8 & 4.2 & 9 & 4.8 & 7 & 3.7 & 5 & 2.7 \\
Total & 17 & 9.0 & 36 & 19.0 & 16 & 8.5 & 15 & 7.9 & 26 & 13.8 & 9 & 4.8 \\
\hline
\end{tabular}

\section{Difference in treatment decisions}

Four different reference treatments were suggested in the 189 cases. In 10 cases (5.3\%) the dose is decreased, in 38 cases $(20.1 \%)$ the prescribed treatment is continued without any changes, in 41 cases $(21.7 \%)$ the dose is increased and in 100 cases (52.9\%) another drug was selected. The decisions of all neurologists and the system were compared with the reference decision (Table 6.4-VIII). The numbers in the cells on the diagonal (shaded grey) show the agreement of the neurologists and the system with the reference decision. In each cell the following information is provided: the number of decisions, the percentage of these decisions related to the total number of decisions taken ( 945 for the neurologists, 189 for the system) and the percentage of the decisions related to the number of cases in the reference treatment.

Table 6.4-VIn: Overview of all 945 ( $5 \times 189$ ) decisions of the neurologists and the system on the 189 reference cases. Each cell contains information on the number of times a decision is made, as well as the percentage of the total number of decisions and the percentage of the decisions taken (in)correctly. For each cell in the column 'Total' the first row presents the number of times a specific reference decision is made. The second row presents for a specific reference decision the percentage of the total number of decisions (945). The third row presents for the neurologists and the system the percentage of decisions according to the reference.

\begin{tabular}{|c|c|c|c|c|c|c|c|c|c|c|c|c|}
\hline \multirow{3}{*}{$\begin{array}{l}\mathrm{N}=945 \\
\text { Decision } \\
\text { Reference }\end{array}$} & \multicolumn{12}{|c|}{ Participants } \\
\hline & \multicolumn{2}{|c|}{ decrease } & \multicolumn{2}{|c|}{ equal } & \multicolumn{2}{|c|}{ increase } & \multicolumn{2}{|c|}{ change } & \multicolumn{2}{|c|}{ rest } & \multicolumn{2}{|c|}{ Total } \\
\hline & N3-7 & $\mathrm{s}$ & N3-7 & $s$ & N3-7 & $\mathrm{s}$ & N3-7 & $\mathrm{s}$ & N3-7 & $\mathrm{s}$ & N3.7 & $\mathrm{S}$ \\
\hline decrease & 40 & 9 & 5 & 1 & 0 & 0 & 5 & 0 & 0 & 0 & 50 & 10 \\
\hline$\%$ total & 4.2 & 4.8 & 0.5 & 0.5 & 0 & 0 & 0.5 & 0 & 0 & 0 & $s$ & \\
\hline$\%$ (dis)agree & 80.0 & 90.0 & 10.0 & 10.0 & 0 & 0 & 10.0 & 0 & 0 & 0 & 80.0 & 90.0 \\
\hline equal & 4 & 0 & 161 & 31 & 12 & 6 & 11 & 1 & 2 & 0 & 190 & 38 \\
\hline$\%$ total & 0.4 & 0 & 17.0 & 16.4 & 1.3 & 3.2 & 12 & 0.5 & 0.2 & 6 & 20 & \\
\hline$\%$ (dis)agree & 2.1 & 0 & 84.7 & 81.6 & 6.3 & 15.8 & 58 & 2.6 & 1.1 & 0 & 84.7 & 81.6 \\
\hline increase & 0 & 0 & 31 & 0 & 162 & 37 & 8 & 4 & 4 & 0 & 205 & 41 \\
\hline$\%$ total & 0 & 0 & 3.3 & 0 & 17.1 & 19.6 & 0.9 & 2.1 & 0.4 & 0 & 21 & \\
\hline$\%$ (dis)agree & 0 & 0 & 15.1 & 0 & 79.0 & 90.2 & 3.9 & 9.8 & 2.0 & 0 & 79.0 & 90.2 \\
\hline change & 33 & 0 & 44 & 2 & 18 & 2 & 395 & 94 & 10 & 2 & 500 & 100 \\
\hline$\%$ total & 3.5 & 0 & 4.7 & 1.1 & 1.9 & 1.1 & 41.8 & 49.7 & 1.1 & 1.1 & 52 & \\
\hline$\%$ (dis)agree & 6.6 & 0 & 8.8 & 2 & 3.6 & 2.0 & 79.0 & 94.11 & 2.0 & 20 & 79.0 & 94.0 \\
\hline
\end{tabular}


In cases in which the reference treatment was to decrease the drug dose the system made more correct decisions than the neurologists. However, the number of cases in this group is rather small.

The neurologists and the system do not differ much with respect to their correctness for cases in which the reference treatment decision is to continue the existing treatment (equal). For cases with this reference decision almost half $(48.3 \%)$ of the decisions different from the reference were taken by one particular neurologist. In $6(15.8 \%)$ of the 38 cases in which the reference decision was to continue the treatment the system suggested to increase the dose.

The system also made more correct decisions than the neurologists for cases where the reference indicated to increase the drug dose. Of the incorrect decisions proposed by the neurologists $20(46.5 \%)$ were taken by one neurologist. The system decided to change the prescribed drug in $4(9.8 \%)$ of the 41 cases.

The largest difference $(15 \%)$ in correct decisions between the system and the neurologists is found for the reference 'Change'. Again almost half (47.6\%) of the incorrect decisions could be attributed to one particular neurologist.

\subsection{Decision criteria}

It may be expected that characteristics of a case are important for reaching a decision. For each of the main characteristics of a case it was determined whether it could predict the reference treatment. Set I was used for this purpose.

\subsubsection{Treatments indicated by a single attribute}

Only in cases in which idiosyncratic complaints occur there is an almost unanimous decision to change the drug treatment. In only one of the 59 cases the reference treatment was to keep the treatment unchanged. In this case seizures were completely controlled, there existed a good compliance and a high therapeutic plasma drug level was observed (Table 6.5-I)

Table 6.5-I: Breakdown of the reference treatment decisions as a function of the presence of complaints.

\begin{tabular}{lcccccc}
\hline Complaints & \multicolumn{2}{l}{ Absent } & \multicolumn{2}{c}{ Dose relaled } & \multicolumn{2}{l}{ Idiosyncratic } \\
\hline Reftreatment & $\mathrm{N}$ & $\%$ & $\mathrm{~N}$ & $\%$ & $\mathrm{~N}$ & $\%$ \\
decrease & & & 14 & 19.4 & & \\
equal & 34 & 38.6 & 16 & 22.2 & 1 & 1.7 \\
increase & 43 & 48.9 & 1 & 1.4 & & \\
chunge & 10 & 11.4 & 41 & 56.9 & 58 & 98.3 \\
extra & 1 & 1.1 & & & & \\
\hline
\end{tabular}

The system's advice was to change to another drug in 57 of the 59 cases. In the other two cases the system suggested to stop the treatment immediately. In both cases all five neurologists agreed that the drug should be changed and immediate discontinuation of the drug was not deemed necessary. 
As the decision to change the drug when idiosyncratic complaints are present may obscure the possible relation between other case characteristics and treatment decisions, we further analysed the 160 cases without idiosyncratic complaints. In these cases, however, no single characteristic could be identified that leads to a specific treatment decision.

\subsubsection{Treatments ruled out by a single attribute}

Although an attribute may not indicate a specific treatment decision, it may rule out a particular treatment decision. For the 160 reference cases without idiosyncratic complaints, the absence of treatment decisions in the reference is described.

\section{Seizure frequency}

Even when seizures are controlled, one could consider to increase the dose when the plasma drug level is below the therapeutic range. However, when considering cases with completely controlled seizures this decision is not made. Also the decision to change the drug due to e.g. dose related complaints is not made in these cases (Table 6.5-II).

In those cases in which seizures were either almost completely or hardly controlled the dose hardly ever was decreased despite the fact that in more than two thirds of these cases a complaint was present.

The decision to decrease the drug dose is never made when the seizure frequency remains uncontrolled. The presence of complaints in these cases does not lead to a reduction of the dose but mainly to a change of the drug.

The system did not suggest to change or increase the dose in cases in which seizures were completely controlled. For cases in which seizures were still present the system did never decide to decrease the dose of the drug. In the three cases in which the reference treatment was to decrease the drug dose, the system proposed to change to another drug.

Table 6.5-II: Distribution of reference treatments when related to seizure frequency in 160 cases without idiosyncratic complaints.

\begin{tabular}{lcccccccc}
\hline Seizures & \multicolumn{2}{c}{ controlled } & \multicolumn{2}{c}{ almost contr } & \multicolumn{2}{c}{ hardly contr } & \multicolumn{2}{c}{ uncontrolled } \\
\hline Ref treatment & $\mathrm{N}$ & $\%$ & $\mathrm{~N}$ & $\%$ & $\mathrm{~N}$ & $\%$ & $\mathrm{~N}$ & $\%$ \\
decrease & 11 & 36.7 & 2 & 9.5 & 1 & 1.5 & & \\
equal & 19 & 63.3 & 12 & 57.1 & 15 & 22.4 & 4 & 9.5 \\
increase & & 4 & 19.0 & 26 & 38.8 & 14 & 33.3 \\
change & & 3 & 14.3 & 25 & 37.3 & 23 & 54.8 \\
extra & & & & & & 1 & 2.4 \\
\hline
\end{tabular}

\section{Complaints}

When complaints are absent, the decision to decrease the drug dose is never taken by the neurologists or the system.

In only one case with a dose related complaint the neurologists decided to increase the drug dose. The decision profile for this exceptional case was 3-2; two of the neurologists as well as the system decide to change the drug. In none of the cases with a dose related complaint the system proposed to increase the dose. 


\section{Plasma drug level}

For each plasma drug level the four major reference treatments were present. The system applied all but one of the decisions for each of the plasma drug levels. For cases in which the plasma drug level was in the sub therapeutic range the system never decreased the drug dose.

Table 6.5-III: Distribution of the reference treatment decisions when related to the range of the plasma drug level.

\begin{tabular}{lrrrrrrrrrr}
\hline Plasma drug level & \multicolumn{1}{c}{ sub ther. } & \multicolumn{2}{c}{ low ther. } & \multicolumn{2}{c}{ therapeutic } & \multicolumn{2}{c}{ high ther. } & \multicolumn{2}{c}{ toxic } \\
\hline Ref.treatment & $\mathrm{N}$ & $\%$. & $\mathrm{N}$ & $\%$. & $\mathrm{N}$ & $\%$ & $\mathrm{~N}$ & $\%$. & $\mathrm{N}$ & $\%$. \\
decrease & 2 & 5.9 & 1 & 3.2 & 1 & 2.6 & 5 & 16.7 & 5 & 18.5 \\
equal & 10 & 29.4 & 9 & 29.0 & 17 & 44.7 & 8 & 26.7 & 6 & 22.2 \\
increase & 14 & 41.1 & 13 & 41.9 & 13 & 34.2 & 3 & 10.0 & 1 & 3.7 \\
change & 8 & 23.5 & 8 & 25.8 & 7 & 18.4 & 13 & 43.3 & 15 & 55.6 \\
extra & & & & & & & 1 & 3.3 & & \\
\hline
\end{tabular}

\subsection{Treatments indicated by combinations of attributes}

To identify combinations of characteristics which predict the reference treatment an induction algorithm was used to analyse the 219 cases in set 1 . The algorithm iteratively partitions the high-dimensional feature space such that in principle each end-node of the resulting classification tree groups together (almost) only cases with one type of reference treatment. The algorithm has as additional stop criterion that a split has to provide sufficient information. If that is not the case, an impure node is not further splitted (Talmon, 1986).

When we assign to each end-node the treatment decision of the majority of cases in that node the tree has a correctness of $85.4 \%$ on set 1 . In total 14 different end-nodes were created (see figure on next page). In this paragraph first the 'pure' end-nodes, containing cases with the same reference treatment, are discussed. Next the mixed end-nodes, with two different treatments are reviewed.

For the cases in each end-node the treatments suggested by the neurologists and by the system are presented. To assure that there is sufficient support to decide that a combination of case characteristics leads to a specific treatment decision the set of cases for which no majority treatment decision exists was classified according to the criteria described in the decision tree. The assigned treatments were compared with the suggested treatments for these cases. Support for a single treatment decision was considered strong for nodes in which:

- The number of cases from a reference group was relatively large $(\mathrm{N} \geq 5)$

- The number of cases with a treatment other than the main treatment among the cases at an end node is relatively small $(<20 \%)$ and

- The non-reference group that fulfilled the criteria was relatively small when compared with the number of cases from the reference group ( $<20 \%$ of the cases)

Finally the nodes with the largest variation in decisions are discussed briefly. For these nodes little can be concluded about the use of a single treatment given the occurrence of three decisions. 


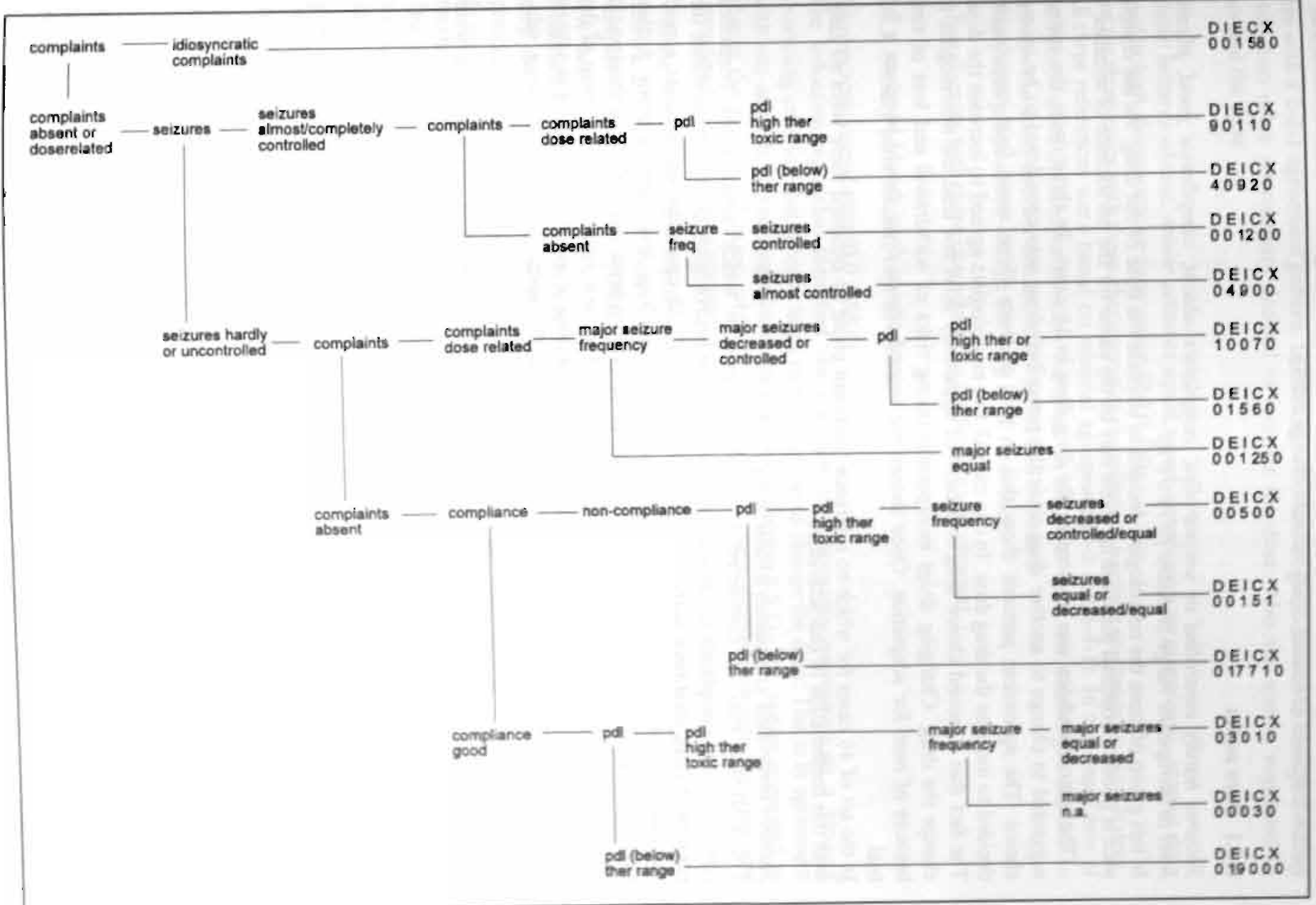


In the following each node is represented by a vector indicating the number of cases with a certain treatment (decrease equal increase change extra).

\subsubsection{Pure nodes}

Seizures hardly controlled or uncontrolled, complaints absent, compliance good, plasma level in therapeutic range or below $(019000)^{1}$

In this group of cases the neurologists decided to increase the dose of the drug. As the compliance is good, suboptimal plasma drug levels may be the cause for the persistence of seizures.

The system's advice was to increase the drug dose in 16 cases. In three cases the system suggested to change to another drug since the prescribed drug was considered to be second choice. The agreement patterns show that in two of these three cases four neurologists decided to increase the drug dose. In one case all five neurologists agreed to increase the dose. The fact that a second choice drug is prescribed apparently does not lead the neurologists to change the drug. Changing drugs incorporates taking risks of withdrawal and loss of time because of need for adaptation. Once chosen the second choice drug should be given a full trial.

In the set of 46 cases for which no reference treatment could be obtained there were no cases with this combination of characteristics.

\section{Seizures controlled, complaints absemt $\left(\begin{array}{lllll}0 & 0 & 12 & 0 & 0\end{array}\right)$}

The treatment may be considered successful in cases in which seizures are controlled completely and complaints are absent. In these cases the neurologists decided to continue the prescribed drug treatment without further changes. In all 12 cases the neurologists agreed completely. The system also decided to continue the drug treatment without further adjustments in all cases. In five of these cases the plasma drug level was below the therapeutic range, while in two cases the plasma drug level was in the toxic range. An adjustment of the drug dose was not suggested. This indicates that plasma drug levels are not applied rigidly to modify the treatment when it is effective in controlling the seizures without inducing sideeffects.

Among the 46 cases without a reference no cases with the same characteristics were observed.

\section{Seizures hardly controlled, non-compliance, plasma drug level high therapeutic or toxic

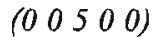

In cases in which seizures were hardly controlled, with non-compliance and a plasma drug level in the high therapeutic or toxic range, the neurologists decided to continue the prescribed treatment. In these five cases the system also decided to continue the prescribed drug treatment.

Non-compliance may explain the persistence of seizures. In these cases it is preferred to improve the compliance as this may lead to improved seizure control.

\footnotetext{
'Decisions taken by the neurologists (Decrease Equal increase Change Extra) are presented in parentheses (D E I C X). In

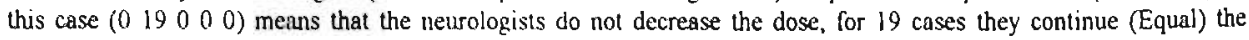
treatment without change, they do not increase, change or add an additional drug.
} 
In the set of cases without a reference treatment the same characteristics were found in one case with a 2-2-1 agreement pattern. In this case two neurologists decided to change the drug while two other neurologists decided to increase the dose. Also the system suggested to increase the dose.

Major seizures absent, minor seizures equal, goad compliance, no complaints, plasma drug level in high therapeutic or toxic range $\left(\begin{array}{lllll}0 & 0 & 0 & 3 & 0\end{array}\right)$

For the three cases with these characteristics the reference suggests to change to another drug. All three references were based on minimal agreement (3-2 and 3-1-1). In two cases two of the neurologists decided to increase the dose. In the other case one neurologist decided to increase the dose, another continued the prescribed treatment.

The system proposed to change the drug in all three cases.

For all cases with these characteristics a reference was obtained

\subsubsection{Nearly pure nodes; two decisions}

\section{Idiosyncratic complaints (0 0 I 580$)$}

The induction algorithm identified the presence of idiosyncratic complaints as a kind of overruling criterion that directly leads to the decision to change the drug. This is in agreement with our analysis of the single case characteristics (section 6.5.1). The system suggested to change the drug in 57 cases, while in two cases the system proposed to stop the treatment immediately as the complaints were very severe.

In the 46 cases of the non reference set, three cases had idiosyncratic complaints. In all three cases a 2-2-1 agreement pattern was present. In two of these cases in which seizures were completely controlled, compliance was good and the plasma drug level was in the sub- and low-therapeutic range two of the neurologists decided to change the drug. An alternative treatment for these cases was proposed by two other neurologists. In one case they decided to stop the prescribed drug without replacement, and in the other case they continued the prescribed drug without adjustments.

In the third case seizures decreased, compliance was good and the plasma drug level was low therapeutic. For this case only one of the neurologists decided to change the prescribed drug. One pair of neurologists decided to increase the drug dose. The other pair continued the treatment without adjustments.

In all three cases the system suggested to change to another drug.

\section{Major seizures equal or minor seizures equal or decreased, and dose related complaints $(001250)$}

In 25 of 26 cases a change of the drug was decided upon. In one case a continuation of the prescribed treatment was the reference. For this case there was a 3-2 agreement profile, in which the two remaining neurologists decided to change the drug

The system used two different approaches for these cases. In 21 cases the system also decided to change the drug, while in 5 cases a continuation of the prescribed treatment was suggested. In all five cases dose related complaints occurred with a plasma drug level below or in the therapeutic range. In two of these cases in which the seizure frequency did not change four of the neurologists decided to change the drug, while the fith chose to continue the 
treatment. In the three remaining cases in which the frequency of minor seizures decreased, a 3-2 decision profile was found, in which continuation of the prescribed treatment was the alternative used by two of the neurologists.

In the set of 46 cases without a reference five cases were found with the same characteristics all of which had a plasma drug level in or above the therapeutic range. In four of the cases (three times 2-2-1 and once a 2-1-1-1 agreement profile) two neurologists agreed to change the drug. In two of these cases two neurologists decided to continue the treatment, while in one case two neurologists decided to decrease the dose. In the remaining case (2-1-1-1) two neurologists decided to continue the prescription.

The system proposed to change the drug in four cases while in one case with a low plasma drug level a continuation of the treatment was suggested, which was also decided upon by two of the neurologists.

While the presence of incompletely or uncontrolled seizures and dose related complaints seems to lead the neurologists towards a change of the drug, the presence of low therapeutic plasma drug levels modifies the decision of the system towards continuation of the treatment.

Major seizures decreased and minor seizures decreased, equal, or absent, or major seizures controlled and minor seizures equal, dose related complaints and plasma level in the high therapeutic or toxic range (1 0070$)$

This node contains eight cases, in seven of which the reference was to change the drug.

A 3-2 decision profile was found in 6 of the cases for which change was the reference. In four of these cases a decrease of the dose was proposed, in one case the dose was decreased and an additional drug was added, in one case a continuation was proposed by the two other neurologists. In one case with seizures hardly controlled, good compliance, dose related complaints and a plasma drug level in the toxic range, a 4-1 agreement profile was observed. In one case with both seizure types decreased, a good compliance and a plasma drug level in the high therapeutic range, three neurologists decided to decrease the dose, while two changed to another drug.

In all 8 cases the system decided to change the drug.

In the set of cases without a reference two cases were found with the same characteristics In both cases two neurologists decided to change the drug while two other neurologists decided to decrease the dose and one neurologist decided to continue the treatment.

In both cases the system decided to change the drug.

Despite some success in controlling the seizures, the presence of dose related complaints and high or toxic range plasma drug levels mainly causes the neurologists to adjust the treatment by changing the drug. As a decrease could lead to further continuation of seizures, the system also suggests to change the drug.

Major seizures equal or major and minor seizures decreased, good compliance, no complaints and plasma drug level in the high therapeutic or toxic range $\left(\begin{array}{lllll}0 & 3 & 0 & 1 & 0\end{array}\right)$

Four cases were found with these characteristics in the set of 219 cases with a reference. In three of these cases the reference was to increase the drug dose (agreement profile 3-1-1 or 41) In one case, with major and minor seizures which remained equal a change of the drug was decided upon by three neurologists, the other two neurologists increased the dose 
In two cases the system decided to increase the dose, while in two cases a change was proposed.

In the 46 cases without a reference, four cases were observed with the same characteristics. In all four cases a 2-2-1 agreement profile was present. In total three different treatment decisions were taken by a pair of neurologists i.e. increase, change or equal.

The system decided to increase the dose in three cases in which seizures were hardly controlled. A change was proposed in a case in which the seizures did not change and the plasma drug level was in the toxic range.

The number of cases in this node is small and an equal number of cases was found in the non reference set. Little more can be concluded than that for these characteristics the neurologists mainly try to achieve complete seizure control by either increasing the dose or changing to another drug.

Seizures almost controlled, complaints absent $(04900)$

A group of 13 cases was identified in which either an increase in the dose or a continuation of the treatment was agreed upon. In 6 cases major seizures were controlled. In five of these the neurologists decided to continue the treatment, although the minor seizures were still incompletely controlled. In one case with a sub therapeutic plasma drug level, the dose was increased. While for cases in which major seizures are controlled the neurologists tend to continue the treatment, for minor seizures the treatment decision is less clear.

The system proposed in all 8 cases with a good compliance to increase the dose. In cases with non-compliance either an increase of the dose -three cases with a (below) therapeutic plasma drug level- or a continuation of the prescribed treatment -two cases with a high therapeutic plasma drug level- was suggested.

In the set of cases without a reference treatment one case was found with the same characteristics. For this case two neurologists agreed about the treatment to continue the treatment. The system also proposed to continue the treatment without adjustments as noncompliance and a toxic range plasma drug level were present.

The system increases the dose if seizures are not completely controlled unless noncompliance and high therapeutic or toxic plasma drug levels are present. The neurologists mainly tend to continue the prescription although seizures are not completely controlled and compliance is good.

\subsubsection{Mixed nodes; three decisions}

In five nodes covering 70 cases three different reference treatment decisions were observed. The system suggested the same treatment as the reference in 45 cases (64\%). In nine cases the system agreed with two neurologists who did not agree with the reference but agreed among themselves on a different treatment (Table 6.6-I). 
Table 6.6-I: Overview of the reference decisions and the advice of the system concerning the 70 cases in the 5 nodes in which three different decisions were observed.

\begin{tabular}{l|lccccc}
\hline Treatment & \multicolumn{6}{|c}{ Reference } \\
\hline \multirow{3}{*}{ System } & Decisions & Decreasc & Equal & Increase & Change & eXtra \\
& Decrease & 9 & 2 & & & \\
& Equal & 2 & 12 & 1 & 4 & \\
& Increase & & 6 & 16 & 3 & 1 \\
& Change & 2 & 3 & 1 & 8 & \\
\hline
\end{tabular}

In two of the five nodes the correctness of the system is more than $70 \%$, in one node more than $60 \%$ was reached, while twice the system achieved a correctness of less than $60 \%$. In all nodes except node $\left(\begin{array}{lllll}9 & 0 & l & 1 & 0\end{array}\right)$ more than $50 \%$ of the reference treatments is based on a minimal agreement pattern (3-2 or 3-1-1). In (901llo) this was $27 \%$.

Only for node $\left(\begin{array}{lllll}9 & 0 & 1 & 1 & 0\end{array}\right)$ one can speak of an indication for a reference treatment (Table 6.6-II). In this node with seizures almost or completely controlled, dose related complaints and a plasma drug level in the high therapeutic or toxic range, 3 cases have a minimal agreement pattern (3-1-1 or 3-2). For 7 of the 9 cases in which both seizure types are completely controlled and compliance is good the system as well as the neurologists decided to decrease the drug dose. No cases with these characteristics were observed in the non-reference group. In the case in which both the neurologists and the system decided to change to another drug, the seizures were almost controlled and a high therapeutic plasma drug level was observed. Four neurologists agreed on this treatment.

Table 6.6-II: Distribution of the reference decision and the treatment decision suggested by the system for node $\left(\begin{array}{lllll}9 & 0 & 1 & 1 & 0\end{array}\right)$

\begin{tabular}{l|lccc}
\hline Treatment & \multicolumn{4}{|c}{ Reference } \\
\hline \multirow{3}{*}{ System } & Decisions & Decrease & Equal & Change \\
& Decrease & 7 & 1 & \\
& Equal & 1 & & \\
& Change & 1 & & 1 \\
\hline
\end{tabular}

The non-reference group of this node contained five cases ( $31 \%$ of the total set) with the same characteristics. All of these cases had one type of seizures controlled while the other decreased

In node ( $\left.\begin{array}{lllll}4 & 0 & 9 & 2 & 0\end{array}\right)$ with cases with seizures almost or completely controlled, dose related complaints and a plasma drug level in or below the therapeutic range, the neurologists as well as the system mainly decide to continue the treatment. The dose was decreased in cases in which compliance was good and complaints were severe. In case of severe complaints and non-compliance a change of the drug was suggested. One single approach for treatment for 
these cases is not indicated. The system and the neurologists agree in $73 \%$ of the cases covering three different decisions.

Table 6.6-III: Decisions of the neurologists and the system for node $(40920)$.

\begin{tabular}{l|lccc}
\hline Treatment & \multicolumn{4}{|c}{ Reference } \\
\hline \multirow{3}{*}{ System } & Decisions & Decrease & Equal & Change \\
& Decrease & 2 & 1 & \\
& Equal & 1 & 8 & 1 \\
& Change & 1 & & 1 \\
\hline
\end{tabular}

Differences in treatment between the system and the neurologists were observed for three nodes $\left(\begin{array}{llll}0 & 17 & 7 & 1\end{array}\right),\left(\begin{array}{lllll}0 & 0 & 5 & 1\end{array}\right)$ and $(01560)$

In node $\left(\begin{array}{llll}0 & 17 & 7 & 1\end{array}\right)$, the neurologists used one treatment decision in $68 \%$ of the cases in which seizures are hardly or uncontrolled, complaints are absent, non-compliance and a plasma drug level in or below the therapeutic range (Table 6.6-IV). The neurologists decided mainly to increase the dose, while in 7 cases the treatment is continued without further adjustments. In $68 \%$ of the cases agreement between the system and the reference was observed. The system adheres to a larger extent to the decision to increase the dose for these cases ( $92 \%$ ).

Table 6.6-IV: Decisions of the neurologists and the system for node $\left(\begin{array}{llllll}0 & 17 & 7 & 1 & 0\end{array}\right)$.

\begin{tabular}{l|lccc}
\hline Treatment & \multicolumn{4}{|c}{ Reference } \\
& Decisions & Equal & Increase & Change \\
System & Equal & 1 & 1 & \\
& Increase & 6 & 16 & 1 \\
\hline
\end{tabular}

In node $\left(\begin{array}{lllll}0 & 0 & 1 & 5 & 1\end{array}\right)$ in which seizures are hardly controlled, non-compliance is present and a plasma drug level in the high therapeutic or toxic range is observed the main reference treatment is a change of the drug. In one case the dose was kept equal, while in another case a second anti-epileptic drug was added. The system did not suggest a specific treatment for the cases at this node. In three cases the system decided to continue the prescription, in three other cases an increase of the dose was proposed. In one case a change of the drug was suggested. Two of these decisions coincide with the reference. 
Table 6.6-V: Overview of the decisions taken by the neurologists and the system on node ( 01560 )

\begin{tabular}{l|lccc}
\hline \multirow{2}{*}{ Treatment } & \multicolumn{4}{c}{ Reference } \\
\hline \multirow{3}{*}{ System } & Decisions & Equal & Increase & Change \\
& Equal & 2 & 1 & 1 \\
& Change & 3 & 5 \\
\hline
\end{tabular}

In node ( $\left.\begin{array}{lllll}0 & 1 & 5 & 6 & 0\end{array}\right)$ in which seizures are hardly controlled, dose related complaints are present and the plasma drug level was in or below the therapeutic range all reference treatments were based on minimal agreement (3-2 and 3-1-1).

The single decision to increase the dose was taken by three of the neurologists, while the other two proposed to change the drug. The system suggests only two different treatments of which nine $(75 \%)$ are a change of the drug. The neurologists however use a continuation of the treatment almost as often as a change of the treatment (Table 6.6-V).

\subsection{References}

Beghi E., Tognoni G., Prognosis of epilepsy in newly referred patients: A multicentre prospective study, Epilepsia, 29, 1988, pp. 236-243.

Collaborative Group for Study of Epilepsy, Prognosis of epilepsy in newly referred patients: a multicintre prospective study of the effects of monotherapy on the long-term course of epilepsy, Epilepvia, 33, 1992, pp. 45-51.

Cramer J.A., A clinimetric approach to assessing quality of life in epilepsy, Epilepsia, 34, 1993, (Suppl.), 4, s8-s13.

Donselaar C. van, Idioparhic first seizure in adult life, thesis, Rotterdam, 1990.

Duncan J.S., Sander J.W.A.S., The Chalfont seizure severity scale. Journal of Neurology, Neurosurgery and Psychiatry, 54, 1991, pp. 873-876.

Elwes R.D., Johnson C.B., Shorvon S.D., Reynolds E.H., The prognosis for seizure control in newly diagnosed epilepsy, The New England Journal of Medicine, 40, 1984, pp. 944-947.

Elwes R.D.C., Reynolds E.H., The early prognosis of epilepsy. In: Comprehensive Epileptology, (Dam and Gram, Eds.), Ravens Press, New York, 1990, pp. 715-727.

Landis J.R., Koch G.G., The measurement of ubserver agreement for categorical data, Biometrics, 1977,33 , pp. 159-174

Schouten H.J.A., Statistical measurement of inlerobserver agreement, analysis of agreements and disagreements between observers, thesis, Rotterdam, 1985.

Talmon J.L., A multiclass nonparametric partitioning algorithm, Pattern Recognition Letters, 1986, 4, pp. 31-38. 


\section{Conclusions and discussion}

\subsection{Introduction}

In this thesis the development and assessment of a computerised model of expert knowledge for prescribing anti-epileptic drugs was described.

The research question investigated was:

- Is it possible to develop a decision support system that provides adequate advice concerning anti-epileptic drug therapy?

Both the initiation of anti-epileptic drug treatment and the adjustment of the treatment during a first follow-up contact were studied.

\subsection{System development}

A structured approach for system development, the KADS methodology, was used At the time we used KADS, its orientation was mainly towards the analysis of the cognitive process. In the more recent literature on KADS, this orientation shifted towards a more pragmatic approach regarding the implementation of models (Schreiber et al. 1993, Tansley and Hayball 1993).

In the early versions of KADS a waterfall-based model for system development was used. Limited attention was paid to project management in these versions. Later, a spiral life-cycle model was introduced for project management (Hickman et al. 1989)

KADS provides little support with respect to the evaluation of the quality of the developed models. The little work on metrication that has taken place was mainly directed towards project management (Readdie et al. 1989). It is the philosophy of KADS that because of the extensive analysis of knowledge there is a better chance that the right implementation is made. However, we recognised that also implementation of the (partial) model of expertise could be of value to support elicitation and analysis. In this context it would be appropriate if KADS would include a reference to a recommended evaluation methodology.

Several problems were encountered with the application of KADS. The use of interpretation models appeared to be difficult. This could party be attributed to differences in granularity 
between models. Furthermore, the model library was incomplete, requiring the design of additional interpretation models. Although development of interpretation models is supported by a conceptual modelling language, this language only provides a tentative list of metaclasses and knowledge sources. Moreover, due to the high level of abstraction of the models they need to be translated before they can be presented to a domain expert.

The goal of our study was the development of a computerised model for prescribing antiepileptic drug treatment. The expertise for such a knowledge-based system may be obtained from a single expert or multiple experts. In our case we decided to use only one expert because of the availability of the extensive literature. It was expected that one neurologist was enough to disambiguate any problems found in the literature and to offer additional information. The plan was to assess and - if necessary to adapt - the developed models using experts that are successively less involved in the development of the system and less aware of the working procedures of the expert who provided the knowledge. In retrospect, we expect that involvement of multiple experts during the development could have reduced some of the evaluation efforts and would have made us aware of some of the observed problems earlier in the development process

The developed models for the initiation and monitoring of anti-epileptic drug treatment were implemented in an experimental system using muLISP ${ }^{\mathrm{rm}}$. The system has a layered structure separating the static parts of the knowledge from the procedural parts. A frame and rule based structure was used. Rules related to a specific section of the problem solving process were combined in rule sets which can be used when a knowledge source is activated. In the initiation part as well as in the monitoring part a forward-chaining mechanism was used for reasoning. Most of the rule sets belong to the assessment task of the monitoring part of drug treatment. The use of the frame and rule structures preserved the structure of the knowledge in a way recognisable to the expert. Therefore, a static assessment was possible.

\subsection{Reference development procedure}

Detailed guidelines for anti-epileptic drug treatment of individual patients are not available. Therefore a reference had to be developed to assess the system's advice. This reference was also used to obtain insight in the variability of the treatment decisions of neurologists. Paper cases based on real patient data were designed and used for the determination of the reference. The ultimate goal was to have a set of cases with for each case an agreed upon diagnosis and an agreed upon prescription. The majority decision among three neurologists - if it existed was taken as reference.

A Delphi-like approach was used to reduce the variability and remove erroneous treatment suggestions of the neurologists. The use of statistical feedback significantly increased the number of cases in which a majority decision on the drug was reached.

Due to the variation in the prescribed daily dose among the neurologists we applied a range of acceptable daily doses to reduce the outliers. Feedback was less successful in this situation because part of the outliers could be attributed to rounding off dosages toward an integer number of tablets and part of the outliers concerned mainly one participant who did not change his prescriptions 
In our initial set of 60 cases the neurologists achieved a majority on the detailed diagnosis in 47 cases (the diagnostic reference set). In 39 of these 47 cases they achieved also a majority decision on the selected drug (the treatment reference set)

A prescription not equal to that of the majority may still be considered an acceptable treatment. Therefore, after the Delphi round the prescriptions were assessed on their acceptability by two independent neurologists.

A consensus meeting was organised to establish objective decision criteria. The annotated prescriptions containing information on the acceptability were discussed in a consensus meeting. For each of the 47 cases in the diagnostic reference set a reference treatment could be obtained on the basis of guidelines. The discussion led to a better agreement among the neurologists with respect to the use of phenytoin.

The Delphi-approach could also have been used to establish a reference for the assessment of the treatment decisions. However, the number of cases needed to investigate the decision process would require too much time from the neurologists to achieve a reference. Instead of this, the decisions of five neurologists on 265 cases are used to obtain two reference subsets: a clinical reference consisting of the majority decision of the five neurologists and a reference consisting of the majority decision of both the neurologists and the system. Whereas the clinical reference indicates the minimal level of agreement of the system with the neurologists as the system was not involved in defining the reference, the second reference enables a more balanced assessment of the neurologists' and the system's performance.

\subsection{Initiating anti-epileptic drug treatment}

For the evaluation of the initiation of drug treatment a staged approach was used. The focus gradually shifts from an assessment of the coverage and correctness of the implemented knowledge to an evaluation of the system's and neurologists' performance using reference diagnoses and prescriptions.

\section{Quality of the knowledge acquisition}

The prescriptions of the neurologist who provided the implemented knowledge and the advice of the system were compared. The 60 paper cases were used. In all but one case the neurologist and the system agreed on the generic drug as well as on the selected brand and formulation.

In $80 \%$ of the prescriptions for carbamazepine the daily dose was identical to that of the neurologist. The system suggested a lower dose for carbamazepine $(66 \%-75 \%$ of the daily dose) in $11 \%$ of the cases and a higher dose (125-133\% of the daily dose) in $9 \%$ of the cases.

When prescribing sodium-valproate the neurologist and the system differed in two of the fifteen cases (13\%). Once a higher dose (116\% of the daily dose) and once a lower dose (75\% of the daily dose) was prescribed.

In one case the neurologist did not provide a prescription (because although a diagnosis was established he wanted additional information from the EEG). This showed that despite the fact that a diagnosis is established, drug treatment need not be started. The system was programmed to initiate a drug treatment when a diagnosis is established.

From this experiment it was concluded that the knowledge acquisition was appropriate. 


\section{Local differences}

The same 60 cases were presented to another neurologist (Neurologist II) from the same department as the expert neurologist.

Neurologist II and the system use the same drugs for treatment of epilepsy. The neurologist did not initiate drug treatment when the diagnosis was unknown. Again in one case the system suggested to start anti-convulsive treatment as a diagnosis was established. The neurologist, however, refrained from drug treatment as he also felt he needed additional information about the EEG

A difference in the formulation as well as in the daily dose was observed for carbamazepine. Neurologist II and the system do not seem to agree as far as the daily dose is concerned. The system suggested $50 \%$ to $75 \%$ of the daily doses for carbamazepine prescribed by the neurologist. With respect to the formulation the neurologist preferred the controlled release variant

The daily dose for sodium-valproate differed less. In three out of 10 cases an identical dose was prescribed, while in 4 cases $90 \%$ of the daily dose of the neurologist was prescribed. This difference in the daily dose of sodium-valproate could be attributed to the use of a different intake scheme. The neurologist preferred a tablet intake twice a day, thereby being able to prescribe tablets of $500 \mathrm{mg}$ instead of $300 \mathrm{mg}$. Still there were three cases in which Neurologist II preferred to start with a higher dose. In these cases, 70-75\% of the dose of the neurologist was suggested by the system.

The experiment showed that even within the same department different approaches exist. Neurologist 1 starts with a relatively low dose which may be increased when treatment proves not to be effective. Neurologist II on the other hand seems to adhere more to a 'middle of the target range' approach, reducing the dose when side-effects occur.

Some investigators suggest that anti-epileptic drugs have a certain specificity for certain types of epilepsy (Porter 1984, Mattson et al. 1988, 1992). However, conclusions about differences in efficacy of anti-epileptic drugs in controlling particular seizure types or epilepsies are difficult to draw, due to differences in patient populations and study designs (Gram et al. 1982, Chadwick and Tumbull 1985, Chadwick 1994ab, Brodie and Dichter, 1996). Both our neurologists consistently used different drugs for the different types of epilepsy: carbamazepine for treatment of partial epilepsy and sodium-valproate for treatment of idiopathic generalised epilepsy

Little difference was expected with respect to the daily dose; both neurologists worked in the same department and agreed when selecting the anti-epileptic drug for treatment. However, the different approaches - start with a low dose and increase when necessary versus start with a high dose and decrease in case of side-effects - suggests differences in the assessment of the risk of occurrence and consequences of seizures and complaints. Differences in treatment policy were also observed in recent literature (Mattson et al. 1992, Reynolds et al. 1993, Heller et al. 1995).

To be able to cope with such differences - given that consensus on this issue not yet exists systems that provide therapeutic advice should be tuneable to different treatment styles. Whether the dosage schemes for a given treatment style are 'hand-coded' or user-adjustable remains an issue for discussion 


\section{Impact of local preferences}

The cases in the diagnostic reference set were used to assess the prescribed drug.

The system prescribed a drug in accordance to this reference in $75 \%$ of the cases (range neurologists $46 \%$ to $94 \%$ ). Since the system was not involved in the establishment of the reference a bias in favour of the neurologists is present as each neurologist

- was allowed to change his prescription in the Delphi procedure

- only had to agree with one of his two colleagues to define a reference.

In $90 \%$ of the cases there was at least one neurologist that prescribed the same generic drug as the system did.

In 32 of the 39 cases of the treatment reference set the system prescribed the same generic drug as the treatment reference. A reference for the daily dose (defined as a dose prescribed by two neurologists who agreed on the prescribed drug) could be established in a subset of 11 cases. The system agreed in 5 of these cases (46\%). In $62.5 \%$ of these cases the system suggested a daily dose identical to that of at least one of the neurologists. The individual neurologists agreed with the reference daily dose in $75 \%$ to $100 \%$ of the cases.

Differences in doses mainly occurred in cases in which carbamazepine was the reference drug. The neurologists seem to adhere more to a 'middle of the target range' approach, whereas the system prescribed according to a 'minimal therapy' principle. The fact that carbamazepine induces its own catabolism, which may necessitate a dose higher than the initial dose for controlling the seizures, may have been a reason for the higher daily dose prescribed by the neurologists. Another reason for the difference may be due to the risks of dose related complaints after an initial dose of carbamazepine. The balancing of the risks of having another seizure and the occurrence of side-effects may cause variations in the prescribed daily dose. The system does not balance these risks explicitly

\section{Judgemen of quality of prescriptions}

The overall acceptability (as judged by two independent reviewers) of the system's advice was compared with the acceptability of the advice of each of the neurologists. Little difference was observed between the overall acceptability of the prescriptions of the system and those of the prescribing neurologists. The system obtained a lower number of judgements indicating a poor acceptability $(11 \%)$ than the neurologists (range 13\% to $29 \%$ ). On the other hand also the number of prescriptions with a good acceptability (48\%) were fewer than those of most of the neurologists (range $32 \%$ to $63 \%$ ).

\section{Judgement on selected drug and daily dose}

It is apparent from the literature that there exists disagreement about the choice of antiepileptic drugs. Also our study showed disagreement about the appropriateness of the prescribed drug. A 'neutral' or 'poor' overall acceptability judgement was given 157 (49.8\%) times. For these prescriptions judgements were also given with respect to the prescribed drug and the daily dose. In 62 (39.5\%) of these reviews the drug selection was judged 'neutral' or 'inappropriate'. For three of the neurologists more than $50 \%$ of the judgements indicated that an appropriate drug was prescribed (range $54 \%$ to $70 \%$ ). Also the system mainly $(67 \%)$ obtained a positive judgement for the suggested drug. Except for one of the neurologists the drug was considered inappropriate in $17 \%$ to $36 \%$. For the remaining neurologist as well as for the system less than $5 \%$ of the judgements indicated that an inappropriate drug was prescribed. 
The system's choice of a specific drug is therefore less often the cause for a low acceptability of the prescriptions than for four of the neurologists.

The doses to control seizures recommended in the literature vary. The initial doses for carbamazepine ranges from 200 to $800 \mathrm{mg}$ whereas the dose for sodium-valproate ranged from $600 \mathrm{mg}$ to $3000 \mathrm{mg}$ (Sonnen 1979, Shorvon et al. 1980, Aird et al. 1984, Lechtenberg 1985, Shorvon 1987, Rimmer and Richens 1988, Brodie and Dichter 1996). Also in our study differences in the daily doses of the prescriptions of the neurologists were observed. For the individual neurologists the percentage of judgements indicating an inappropriate dose ranged from $53 \%$ to $60 \%$ for three of the neurologists, while for two neurologists more than $85 \%$ of the judgements indicated that the daily dose was inappropriate. For the system in $91 \%$ of these judgements the dose was considered inappropriate. All but one of the judgements indicated that the daily dose was (too) low. Overall there is little difference between the acceptability of the advice of the system and that of the neurologists.

It has been recently suggested that the main anti-epileptic drugs differ little with respect to their effectiveness of controlling seizures (Richens et al. 1994, Heller et al. 1995). Nevertheless, the approach in which carbamazepine and phenytoin are the first choice drugs for partial epilepsy and valproate for generalised epilepsy is favoured in daily practice (Chadwick 1994ab, Shorvon 1995, Brodie and Dichter 1996). This was also observed in our study. As far as the proposed drug is concerned the advice of the system appears to be sound

Although doses of anti-epileptic drugs are not predictive for efficacy (Shorvon 1987, Commission on Anti-epileptic Drugs 1993, Lammers 1994) disagreement about the prescribed daily dose in our study turned out to be the main reason for a low acceptance of prescriptions of the system.

\section{Consensus meeting}

To establish an objective reference, differences among the neurologists were discussed during a consensus meeting. It was concluded that the initial daily dose for carbamazepine and sodiumvalproate as prescribed by the neurologists was rather high. Consensus was reached about the fact that the initial daily dose of carbamazepine for patients with a body weight up to $80 \mathrm{~kg}$ should be $400 \mathrm{mg}$, while patients with a higher body weight should receive $600 \mathrm{mg}$ per day. An appropriate initial dose of sodium-valproate was considered to be 900 to $1000 \mathrm{mg}$ depending on whether two or three intakes per day are used. For the system this means an adjustment of its 'minimal effective dose' with respect to carbamazepine, while the system's sodiumvalproate minimal effective dose agreed with the one proposed by the neurologists.

A relation between the drug dose and the efficacy of the drug has not been found so far (I.ammers, 1994). It has been suggested that the policy to achieve therapeutic plasma levels soon after the onset of treatment may induce side-effects (Reynolds et al. 1993, Heller et al. 1995). The adverse drug effects may contribute significantly to decreased patient compliance (Allen 1995). Increasing attention is paid to quality-of-life issues such as behavioural and cognitive effects and the ability to sustain as normal a life as possible while receiving medication (Pellock 1994). These arguments together with reports from the literature which show that the view with respect to the prognosis of epilepsy is changing from a chronic outcome towards a more benign course with a good chance of achieving a remission, lead to the conclusion that there is little room left for a 'middle of the target' policy in drug treatment of epilepsy. The consensus on the daily dose as achieved in our study is near the lower range of 
the daily doses found in the literature, which suggests that also the neurologists in our study agree most with the treatment policy of 'minimal therapy'.

\section{Comparing the proposed drug with the consensus}

The guidelines from the consensus meeting were used for establishing a consensus meeting reference prescription for each case. Comparing prescriptions of the neurologists and the advice of the system with this consensus meeting reference showed that all neurologists prescribed a drug in accordance with the reference in more than half of the cases (range $52 \%$ to $72 \%$ ). The system reached a consistently better agreement (range $88 \%$ to $91 \%$ ) with the reference drug on the subsets of cases seen by the individual neurologists.

The consensus meeting advised that phenytoin should be used as well. This drug was not recommended by the system as a first choice. The neurologists disagreed more with the consensus meeting reference about the prescription of phenytoin than about the use of carbamazepine and valproate. Only twice (17\%) a neurologist used the drug in accordance with the consensus meeting reference, compared to $68 \%$ for carbamazepine and $69 \%$ for valproate.

\section{Comparing the proposed daily dose with the consensus}

If the group of neurologists prescribed a drug according to the consensus meeting reference, they achieved a better agreement with the reference daily dose than when the system agreed with the drug reference. When valproate is prescribed according to the consensus, little difference between the neurologists and the system was observed ( $88 \%$ vs. $94 \%$ ). For carbamazepine the system suggested only twice (3\%) a daily dose equal to that of the reference, while in $36 \%$ of the prescriptions of the neurologists the dose was in accordance with the consensus meeting reference.

\section{Correctness of the prescribed drug and the daily dose}

All but one of the neurologists provided a prescription - taking also the decision "no drug treatment' into account - according to the consensus (drug and daily dose) in less than $40 \%$ (19\% to $36 \%$ ). The other neurologist agreed with the reference in $56 \%$. The system prescribed according to the consensus reference in $28 \%$ to $48 \%$ of the cases (for the various sets of cases common with the individual neurologists), which was equal to or better than most of the neurologists did

Since the dosage prescribed by the system can be easily corrected to agree with the consensus, it can be concluded that the system is suitable for providing advice regarding the initiation of anti-epileptic drug treatment.

\subsection{Follow-up of treatment}

The second evaluation of the system concerned the adjustment of the treatment based on information obtained in the first follow-up contact. The global decisions of the system (continuation, increase or decrease of the daily dose or change of the prescribed drug) were compared with the decisions taken by five neurologists. Three different analyses were performed. 
First, the agreement between the system and the neurologists was studied on a set of 265 cases. The system had a moderate agreement (as measured with the kappa-statistic) with three individual neurologists on the treatment decision. Only two pairs of neurologists showed such a moderate agreement, all other combinations of neurologists had a lower level of agreement.

The system reached a fair agreement beyond chance with each of the subgroups of four neurologists, whereas the individual neurologists only reach a moderate agreement with their group of peers. Also the system takes less decisions with which the neurologists completely disagree.

In a second analysis a clinical reference was developed, being the majority decision of the five neurologists. A reference treatment decision was obtained in 219 cases (set I). Although this reference was positively biased towards the neurologists as they contributed to the reference, the system reached a correctness of $78.1 \%$ for these cases which was comparable with the average correctness of the neurologists $77.4 \%$ (range $67.1 \%$ to $82.6 \%$ )

To correct for the bias a second reference of 189 cases was developed based on the treatment decisions of the neurologists as well as of the system (set II). In this set the system reached a correctness of $90.5 \%$, whereas the average correctness within the group of neurologists was $81.9 \%$ (range $67.7 \%$ to $87.8 \%$ ). The system achieved a significantly higher correctness than three of the neurologists.

Also for the set of 76 cases for which no reference treatment decision could be obtained, the system proposed fewer treatment decisions (5\%) which were different from the decisions taken by any of the neurologists than any of the individual neurologists did (range $8 \%$ to 19\%).

\section{Criteria for adjustment of the therapy}

Set I was used to investigate whether criteria could be derived that are used by the neurologists for certain treatment decisions. With the help of machine learning techniques a decision tree was constructed. The algorithm tries to build a tree such that the end nodes contain sets of cases with a single therapy. Stop criteria may prevent splits that have insufficient predictive value. Hence, impure end nodes may occur. The internal nodes contain criteria indicating which way to proceed in the tree. The tree had 14 end nodes. Support for a treatment decision was considered to be strong for six nodes. From the criteria in the tree that lead to these nodes the following guidelines for adjustment of the treatment were derived:

- In cases in which seizures are completely controlled and complaints are absent the prescribed treatment is continued. In the literature it is mentioned that an increase of the drug dose was a common decision if drug plasma levels were low (Meijer, 1991). In our study, however, as also has been recommended in the literature (Shorvon, 1995), neurologists did not apply serum levels as rigidly in these cases. Also the system decided to continue the treatment without adjustments for these cases.

- In cases with idiosyncratic complaints a change of the drug is appropriate. For the neurologists as well as for the system the presence of idiosyncratic complaints leads to the use of another drug.

- The drug dose is increased when seizures are hardly controlled (the seizure frequency decreased, or one type of seizures remained the same, while the other type decreased or was controlled) or uncontrolled (the frequency of the seizure type(s) did not change), in combination with absence of complaints, a good compliance and a plasma drug level below or in the therapeutic range. It is stated in the literature that often the dose is 
increased without checking the plasma drug level (Meijer 1991). In our study however, neurologists appear to take the plasma drug level as well as information about complaints and compliance into account to decide whether or not to increase the dose. The system reached the same treatment decision in all except three cases in which the prescribed drug was considered to be second choice. Receni studies indicate that as far as controlling the seizures is concerned little difference is observed in effectiveness of the main anti-epileptic drugs (Richens et al 1994, Heller et al. 1995). Given the risks of drug withdrawal and time loss, once a second choice drug has been initiated it should be given a full trial.

- In cases with uncontrolled major seizures and dose related complaints the prescribed drug is changed. In 21 of the 26 cases the system also decides to change the drug. In all five cases dose related complaints occurred at a (below) therapeutic plasma drug level immediately after initiation of the treatment. These complaints may be transient in contrast with those occurring in combination with a high or toxic range plasma dnug level. A continuation of the treatment was proposed by the system and agreed at by at least one of the neurologists.

- The prescribed drug is changed in cases in which some success is achieved in controlling the seizures (either major seizures decreased while minor seizures were not controlled or major seizures controlled and minor seizures remained equal), dose related complaints are present and the plasma drug level is in the high therapeutic or toxic range. These findings are in accordance with the findings of others who considered an increase of the dose in case of a (near) toxic serum concentration to be a dubious decision (Meijer, 1991). The plasma drug level is used as indication whether toxicity develops and additional anticonvulsive action is small (Shorvon, 1995). The system decided to change the drug in all eight cases.

- The drug treatment is continued in cases in which the seizure type(s) decreased or in which one type of seizures was controlled while the other remained equal, with noncompliance and a plasma drug level in the high therapeutic or toxic range

For $39.3 \%$ of the cases in set I criteria leading to a single therapy could not be obtained. The reason could be the way in which the cases were described and analysed, or the existence of alternative treatments. The first reason can largely be rejected as a detailed analysis did not show any discriminative factors not taken into account in the analysis. There is some evidenci that alternative treatments may exist for some subgroups. For some groups of cases a single reference treatment does not seem to exist, although in individual cases a reference treatment was defined by the neurologists

\subsection{General conclusion and future prospects}

The question whether it is possible to provide adequate advice for guiding anti-epileptic drug treatment by means of a computerised model can be answered in the affirmative

1. For the initiation of the therapy and the monitoring of the treatment, the agreement of the system with individual neurologists was in general at least as good as the agreement among the neurologists

2. For the monitoring of the treatment the correctness of the system on a clinical reference was as good as the correctness of the neurologists even though the system was not involved in establishing the reference while the neurologists were.

It was possible to elicit the necessary knowledge from an expert neurologist. 
With respect to the question whether the system suggested an adequate anti-epileptic drug it was concluded that the system proposed an adequate anti-epileptic drug when initiating drug treatment. When comparing the overall acceptability (as judged by two independent reviewers) of the prescriptions there was little difference between the neurologists and the system. The neutral or poor quality overall judgement was in the case of the system due to a (too) low prescribed dose.

It was apparent that even after a two-staged Delphi procedure there existed a relatively large inter-individual variability between the neurologists. A similar variability was observed among the reviewers. Because of this variability in opinions with respect to the way how to initiate the therapy a consensus meeting was organised. When assessing the correctness of the prescriptions (in relation to the reference obtained in the consensus meeting) the system's proposals for initiating anti-epileptic drug treatment were better than those of most of the individual neurologists.

With respect to the follow-up the system appears to be as good as the neurologists in taking treatment decisions. There is a moderate agreement between the treatment proposals of individual neurologists and the system. The system had a consistently better agreement with a group of four neurologists than each other neurologist individually. Comparison of the system's prescriptions with a reference defined by the group of neurologists (clinical reference) showed that the system reached a correctness comparable to that of the individual neurologists Compared with the reference based on the decisions of the neurologist as well as the system, the system reached a better correctness than three of the neurologists.

As far as the criteria for adjustment of the treatment are concerned, six guidelines for adjustment were identified. For each of these guidelines the system seems to apply the same treatment decisions.

The described system is an implementation of the elicited knowledge on anti-epileptic drug treatment rather than a prototype of a system that can be used in clinical practice. It lacks the interface with a clinical information system as well as a user intertace that will meet the requirements of the clinician. Also relevant functionalities for clinical use are lacking. One of the aspects which will require further development is the explanation facility. Although a trace of the rules used for obtaining an advice may be provided, they have to be reworded to enable interpretation of the reasoning process of the system by a neurologist.

Despite these shortcomings our studies indicate that the developed models are fit for integration with other software such as electronic medical records or clinical information systems. The model also would be eminently suited for incorporation into an interactive teaching system for neurological residents. The cases used in the consensus meeting could also be used for this purpose.

Because of the observed inter-individual variability it is advisable to further develop the system such that it can be installed in a neurologist's office initially for testing purposes. In these circumstances the reasons for deviating from the system's advice should be analysed. In this way information can be obtained that will assist in the further development of guidelines for treatment of epilepsy. At the same time such guidelines become available in computerised form to ease their application for individual patients. Affer the system is released for use and knowledge with respect to the treatment is maintained the system can be used by the neurologists for a second opinion. 
Also the system could be extended to incorporate the diagnostic process. It can be expected that future changes in the diagnostic criteria will be less than in the past. The current criteria have been reviewed and established over the years and are generally accepted.

An organisation should volunteer to organise regular consensus meetings in order to keep diagnosis and treatment protocols up to date. This knowledge could be stored in a knowledge base and be distributed among the neurologists.

\subsection{References}

Aird R.B., Masland R.L., Woodbury D.M., The epilepsies a critical review, Raven Press, New York, 1984.

Allen F.H., Entering a new drug era: practical issues in the treatment of epilepsy, Neurology, 45, 1995, (Suppl 2), s5-s6.

Brodie M.J., Dichter M.A., Antiepileptic drugs. The New England Journal of Medicine, 334, 1996, pp.168-175.

Chadwick D., Epilepsy, Journal of Neurology, Neurosurgery and Psychiatry, 57, 1994a, pp.264-277.

Chadwick D., Standard approach to anticpileptic drug treatment in the United Kingdom, Epilepsia, 35 (Suppl.4), 1994b, s3-s 10.

Chadwick D., Tumbull D.M., The comparative efficacy of antiepileptic drugs for partial and tonicclonic seizures, Journal of Neurology, Neurosurgery and Psychiairy, 48, 1985, pp. 1073-1077.

Commission on Antiepileptic Drugs of the International League Against Epilepsy, Guidelines for therapeutic monitoring on antiepileptic drugs, Epilepsia, 34, 4, 1993, pp 585-587.

Dreifuss F.E., Risk benefit analysis in the management of the child with cpilepsy. In: Modern approach 10 antiepileptic drug treatment, improving quality of life, (Dam, Gram, Pcdersen and orum, Eds.), The Danish Epilepsy Society, Kobenhavn, 1985.

Dreifuss F.E, The epilepsies: clinical implications of the international classification, Epilepsia, 31 , (Suppl. 3), 1990, s3-s10.

Gram L., Drachmann Bentsen K., Parnas J., Flachs H., Controlled trials in epilepsy: a review, Epilepsia, 23. 1982, pp. 49I-519.

Heller A.J., Chesteman P., Elwes R.D., Crawford P., Chadwick D., Johnson A.L., Reynolds E.H., Phenobarbitone, phenytoin, carbamazepine, or sodium valproatc for newly diagnosed adult epilcpsy: a randomised comparative monotherapy trial, Journal of Neurology. Neurosurgery and Psychiary, 38,1995 , pp. 44-50.

Hicknan F.R., Killin J.L, Land L., Mulhall T., Porter D., Taylor R.M., Analysis for knowledge-based systems, a practical guide to the KADS methodology, Ellis Horwood Limited, Chichester, 1989.

Lammers M.W., Clinimetrics in epileprology, thesis, Nijmegen, 1994.

Lechtenberg R., The diagnosis and ireatment of epilepsy, Macmillan Publishing Company, New York, 1985.

Mattson R.H., Cramer J.A., Collins J.F., VA Epilepsy Cooperative Study Group, Aspects of compliance; taking drugs and keeping clinic appointments. In: Compliance in Epilepsy (Schmidt and Leppik, Eds.), Elsevier Science Publishers B.V., Amsterdam, 1988, pp. III-117.

Mattson R.H., Cramer J A. Collins J.F., and the Dept. of Veterans Affairs Epilepsy Cooperative Study No 264 Group, A comparison of valproate with carbamazepine for the treatment of complex partial seizures and secondarily generalised tonic-clonic seizures in adults, The New England Journal of Medicine, 327, 1992, pp. 765-771 
Meijer J.W.A., Knowledge, attitude and practice in antiepileptic drug monitoring, thesis, Nijmegen, 1991

Pellock J.M., Standard approach to antiepileptic drug treatment in the United States, Epilepsia, 35(Suppl.4), 1994, s11-s 18.

Porter R.J., Epilepsy, 100 elementary principles, W.B. Saunders Company Ltd., London, 1984.

Principe J.C., Smith J.R., Microcomputer-based system for the detection and quantification of petit mal epilepsy, Computers in Biology and Medicine, 1982, 12, pp. 87-95

Readdie M., Streng K.H., Wermser D, KADS metrication, Deliverable G10. SD-G10-R-001, ESPRT P1098, SD Europe Ltd \& NeuTech Entwicklungsgesellschaft mbH \& Co.KG, 1989.

Reynolds E.H., Heller A.J., Chadwick D., Valproate versus carbamazepine for seizures, The New England Journal of Medicine, 328, 1993, pp. 207-208.

Richens A., Davidson D.L.W., Cartlidge N.E.F., Easter D.J., A multicentre comparitive trial of sodium valproate and carbamazepine in adult onset epilepsy. Journal of Neurology. Neurosurgery and Psychiatry, 1994, 57, pp. 682-687.

Rimmer E.M., Richens A., Clinical phamacology and medical treatment. In: A textbook of epilepsy, (Laidlaw, Richens and Oxley, Eds.), $3^{\text {rd }}$ edition, Churchill Livingstone, Edinburgh, 1988, pp. 421483.

Scheiber G., Wielinga B., Breuker J., KADS a principled approach to KBSS development, Academic Press, London, 1993.

Shorvon S.D., Galbraith A.W.. Laundy M., Vydelingum L., Reynolds E.H., Monotherapy for epilepsy. In: Antiepileptic therapy: advances in drug monitoring. (Johannessen, Pippenger, Schmidt, Morselli, Richens and Meinardi, Eds.). Raven Press, New York, 1980, pp. 213-220.

Shorvon S.D., The treatment of epilepsy by drugs. In: Epilepsy (Hopkins, Ed.), Chapman and Hall, London, 1987, pp. 229-282.

Shorvon S., The drug treatment of epilepsy. In: Epilepsy, (Hopkins, Shorvon and Cascino, Eds.), $2^{\text {nd }}$ edition, Chapman and Hall Medical, London, 1995.

Sonnen A.E.H., De medicamenteuze behandeling van epilepsie, Nedcrlands Tijdschrift voor de Geneeskunde, 123, 1979, pp. 485-490.

Tansley D.S.W., Hayball C.C., Knowledge-based systems analysis and design, a KADS developer's handbook, Prentice Hall, New York, 1993.

Ward C.D., Decision making in refractory epilepsy: an analysis based on computer simulation, Journal of Neurology: Neurosurgery and Psychiatry, 1989, 49, pp. 781-784. 


\section{Summary}

Given the ageing of the population and the expected growth in the number of patients with multiple problems, an increase can be expected in the amount of information that has to be processed when dealing with an individual patient. Simultaneously there is a pressure to practice medicine in a cost-effective way. The clinician who wants to do the best for his patient must try to maximise the expected utility of his actions. The expectations of patients with respect to the capabilities of medicine complicate this process as conflicts may arise between the requests of individual patients and societal needs. Physicians need tools to assist them in mastering the growing amount of increasingly more complex medical knowledge to assure optimal care. Information technology may assist the physician with clinical decision making in daily practice to achieve best medical practice. Also in the domain of epilepsy - a disorder of the brain which manifests itself through brief usually unprovoked stereotypical disturbances of behaviour, emotion, motor function or sensation - supporting the doctor by a 'computer fed by a team of specialists' was considered as an option to improve patient management.

In this thesis the development of a computerised model of the expert knowledge used for prescribing anti-epileptic drugs is described. It was studied whether such a model can provide advice of a good quality. The advice concerns:

1. the initiation of anti-epileptic drug treatment after the diagnosis has been established and

2. the adjustment of the drug treatment after assessment of its effectiveness.

\subsection{The domain of epilepsy}

In chapter 2 a review of the literature with respect to diagnosis and treatment of epilepsy is presented. Problems discussed are related to

- the definition of epilepsy which can be considered as a framework rather than a fixed set of criteria for diagnostic interpretation of symptoms.

- the presence of different approaches for deciding on drug treatment.

- the disagreement on therapeutic ranges of drugs and the usage of plasma drug levels

- the psychological and social consequences of the long-term character of the treatment procedures. 
Although guidelines with respect to diagnosis and treatment of epilepsy are available the actual context for their application is usually missing. Guidelines to enhance the decision making process of the physician in managing a specific patient could therefore be of value.

\subsection{Building a model of expertise}

The KADS structured approach for knowledge based systems development was used. Two inference models describing the initiation and the monitoring of the treatment in patients with epilepsy were developed (Chapter 3 )

At the time we used KADS two main problems were encountered:

- KADS orientation towards the analysis of the cognitive process instead of the implementation of a model of expertise.

- the lack of support for the evaluation of the quality of the models.

Over the years the orientation of KADS has shifted from the analysis of cognitive processes towards a more pragmatic approach for implementation of models. Some work on metrication has also taken place. This work, however, is mainly directed towards project management. Little has been done with respect to the evaluation of models. Integrating KADS with an evaluation methodology could enhance user acceptance of a decision support system.

\subsection{Reference development}

The evaluation of computerised models is a difficult but essential part of the development of a decision support system. Several methodologies have been proposed in the literature. Most methodologies consider validation of a decision support system as the most important part of the evaluation process. The assessment of the correctness of the output of the system is the main issue in this phase. An important problem in the evaluation of decision support systems is the development of a reference for assessment of the system's advice. As described in chapter 2 an objectively correct treatment for an individual patient is not available. This means that a reference for evaluating the advice of the system had to be developed by a group of neurologists.

\section{Reference for the initiation of treatment}

For the initiation of the treatment sixty paper cases were constructed describing the clinical presentation of a patient's first two or three visits (Chapter 4). For each case three neurologists were asked to provide a prescription. By means of a two step Delphi-like approach addressing the diagnosis and the prescribed drug, the variability in prescribing could be reduced as far as the drug was concerned. The feedback round on the daily dose was less successful in reducing the variance in dosage.

Two reference sets were developed, a diagnostic reference set for the assessment of the prescribed drug and a treatment reference set for the assessment of the daily dose.

Because a treatment not equal to that provided by the majority may still be considered an acceptable treatment, the acceptability of the prescriptions was assessed by two independent neurologists after the Delphi round.

The variability among the neurologists made it difficult to obtain a set of cases for which the neurologists agreed about the diagnosis as well as about the treatment. Therefore a consensus 
meeting was organised to obtain more objective decision criteria. For the cases of the diagnostic reference set a consensus reference treatment was obtained on the basis of the guidelines defined in the meeting. The consensus meeting led to a better agreement among the neurologists with respect to the use of phenytoin.

\section{Reference for the follow-up of treatment}

The assessment of the advice in a first follow-up contact also required the use of a majority decision of a group of neurologists as no objective standard existed. Two reference sets were obtained (Chapter 6). A clinical reference consisted of cases about which the majority of the neurologists agreed. A second reference consisted of cases about which a majority decision of both the neurologists and the system could be obtained.

In addition to this more global approach we identified case characteristics, using machine learning techniques, which the neurologists may have used when deciding about the adjustment of the treatment.

\subsection{Evaluation of the initiation of the treatment}

Comparing the system's advice for cases in the diagnostic reference set with the prescriptions of the neurologist showed that the system prescribed an anti-epileptic drug in accordance with the reference as least as often as an individual neurologist (Chapter 5).

The system prescribed a daily dose in accordance to the reference for sodium-valproate. For carbamazepine the system predominantly prescribed a dose lower than the neurologists did. A difference in treatment approach apparently existed. While the neurologists adhered more to a 'middle of the target' approach the system prescribed according to the 'minimal therapy' principle which means that initially a low dose is used which is increased if seizures do not remit.

The acceptability analysis showed little difference in overall acceptability between the prescriptions of the system and the neurologists. The system received a lower number of judgements indicating a poor acceptability than the individual neurologists. On the other hand the number of prescriptions for which a good acceptability was observed was lower than those of most of the neurologists. The system suggested more often an acceptable drug than most of the neurologists. If the acceptance of the suggested prescription of the system was judged low this was mainly due to a too low daily dose.

This evaluation of the system's advice for the reference cases and the assessment of the acceptability provided an indication of the performance of the system but the variability in prescribing among the neurologists did not enable us to make a firm statement about the system's correctness. To reduce the variability a consensus meeting was organised. It appeared that the daily dose initially prescribed by the neurologists was rather high

The system as well as the group of neurologists for sodium-valproate mainly prescribed a daily dose in accordance with the consensus guidelines. For carbamazepine the system prescribed less often a daily dose in accordance with the consensus guidelines than the group of neurologists.

Except for one of the neurologists who prescribed according to the consensus in more than half of the cases, the system suggested an equal or higher number of prescriptions (drug and daily dose) according to the consensus than the other neurologists did. 


\subsection{Evaluation of the follow-up of the treatment}

To assess whether the system takes adequate decisions during monitoring the drug treatment, the global treatment decisions of the system were compared with the decisions of the five neurologists.

The system reached a moderate agreement on the treatment decision - as expressed with the $\kappa$-statistic - with three of the individual neurologists on the treatment decision. Only twice a pair of neurologists showed a moderate agreement, while the other combinations of two neurologists had a lower level of agreement. This shows that the system agreed better with each individual neurologist than any of the neurologist with their colleagues.

Comparing the agreement between the system and a group of four neurologists with that of an individual neurologist with the same four neurologists showed that the system reached a fair agreement, while each of the individual neurologists moderately agreed with their four colleagues.

A clinical reference was developed, based on the treatment decisions of the majority of the five neurologists. For 219 out of 265 cases such a majority decision could be obtained. The system reached a correctness comparable to that of the individual neurologist on this set of cases. This reference was positively biased towards the neurologists, as they contributed to the definition of the reference treatment decision while the system did not. Including the system's treatment decision in the reference creation process led to a subset of 189 cases in which a majority decision could be obtained. Within this subset the system reached a higher correctness than the neurologists. The analysis of the set of 76 cases for which no reference treatment could be obtained showed that the system suggested fewer treatment decisions different from the decisions taken by any of the neurologists, than each of the individual neurologists did.

Case characteristics may be expected to be important for reaching specific treatment decisions. To obtain insight in the decision making process of the group of neurologists a decision tree was constructed with the help of machine learning techniques. The tree consists of branches which describe a path in theory leading to one specific treatment. Each path may use different case characteristics. An end-node presents the number of cases for each different treatment that fulfil the criteria belonging to the specific branch.

An assessment of the criteria for adjustment of the therapy showed that for six branches the end-nodes contained cases of predominantly one treatment. These criteria were taken as guidelines for follow-up of the treatment. The system uses the same treatment decisions for cases described by each of these guidelines

\subsection{General conclusion}

The question whether a computerised model based on expert knowledge can provide quality advice on anti-epileptic drug treatment can be answered in the affirmative. Comparing the system with the diagnostic reference and the consensus reference showed that it provides a correct advice for initiating anti-epileptic drug treatment. Poor acceptability of an advice could be attributed to a low dose. This part of the knowledge base can easily be corrected.

Also for the adjustment of the initiated treatment the system proposed treatment decisions which were in good agreement with those of the individual neurologists as well as with those 
of a group of neurologists. Because of the detected inter-individual differences it is advisable to further develop the system (including a user-friendly user interface) and install it for testing in a neurologist's office to analyse reasons for deviations from the system's advice. When the knowledge base is maintained over time the system's advice can be used as a second opinion or for educational purposes 


\section{Samenvatting}

Door de stijgende leeftijd van de bevolking en de verwachte groei van het aantal patienten met meer dan één gezondheidsprobleem, kan men een toename verwachten van de hoeveelheid informatie die verwerkt moet worden bij het behandelen van een individuele patient. Gelijktijdig wordt er druk uitgeoefend om zo kosten-effectief mogelijk te werken in de gezondheidszorg. De clinicus die het beste voor zijn patient wil bereiken moet trachten de verwachte opbrengst van zijn activiteiten te maximaliseren. De verwachtingen van patienten ten aanzien van de mogelijkheden van de geneeskunde bemoeilijken dit proces doordat er conflicten kunnen optreden tussen wensen van de individuele patient en het maatschappelijke belang. Artsen hebben behoefte aan methoden die helpen bij het verwerken van de groeiende hoeveelheid van steeds complexere medische kennis om optimale zorg te waarborgen. Informatietechnologie kan de arts bij zijn dagelijkse klinische besluitvorming helpen optimale zorg te verlenen. Ook op het gebied van epilepsie - een aandoening van de hersenen welke zich manifesteert door korte, meestal niet geprovoceerde stereotypische verstoringen van gedrag, emotie, motoriek of gevoel - werd ondersteuning van de arts door 'een computer gevoed door een team van specialisten' gezien als mogelijkheid om het beleid met betrekking tot de patient te verbeteren.

In dit proefschrift wordt de ontwikkeling van een geautomatiseerd model van de kennis van een expert met betrekking tot het voorschrijven van anti-epileptische medicatie beschreven. $\mathrm{Er}$ werd onderzocht of een dergelijk model een kwalitatief goed advies kan geven. Dit advies betreft:

1. het starten van de behandeling met anti-epileptica nadat de diagnose gesteld is en

2. de aanpassing van de medicamenteuze behandeling na beoordeling van de effectiviteit.

\section{Epilepsie}

In hoofdstuk 2 wordt een overzicht van de literatuur met betrekking tot diagnostick en behandeling van epilepsie gepresenteerd. Verschillende problemen worden besproken:

- de definitie van epilepsie, die eerder beschouwd kan worden als een raamwerk in plaats van een vastgestelde set van criteria voor diagnostische interpretatie van symptomen

- de verschillende benaderingen met betrekking tot beslissingen over de medicamenteuze behandeling

- het verschil in mening betreffende de therapeutische range van de medicamenten en het gebruik van plasmaspiegels bij de instelling en bewaking van de therapie

- de psychische en sociale consequenties van het lange termijn karakter van de behandelingsprocedure

Ofschoon richtlijnen voor diagnostiek en behandeling van epilepsie beschikbaar zijn, ontbreekt hierin meestal de context voor de toepassing ervan. Ondersteuning van het besluitvormingsproces van de arts middels uitgebreidere richtlijnen betreffende de behandeling van de specifieke patient kunnen van waarde zijn. 


\section{Het bouwen van een kennismodel}

KADS, een gestructureerde benadering voor de ontwikkeling van kennissystemen, werd gebruikt om de kennis over de behandeling van epilepsie te modelleren. Twee inferentie modellen - een voor de initiatie en een voor het bewaken van de medicamenteuze behandeling van patienten met epilepsie - werden ontwikkeld.

Bij het gebruik van KADS werden twee belangrijke problemen geconstateerd:

- de gerichtheid van KADS op het analyseren van het cognitieve proces in plaats van op de implementatie van een kennismodel.

- het ontbreken van ondersteuning voor het evalueren van de kwaliteit van de modellen.

Door de jaren heen is de nadruk van KADS op de analyse van cognitieve processen steeds meer verschoven in de richting van een meer pragmatische benadering voor implementatie van modellen. Verschillende metrieken zijn ontwikkeld. Dit werk was voornamelijk gericht op projectmanagement. Met betrekking tot de evaluatie van modellen heeft weinig onderzoek plaatsgevonden. Integratie van KADS met een evaluatiemethode kan de acceptatie van een besluitvormingondersteunend systeem door de gebruiker verbeteren

\section{Ontwikkeling van referentie sets}

De evaluatie van een implementatie van een kennismodel is een moeilijk maar essentieel onderdeel van de ontwikkeling van een besluitvormingondersteunend systeem. Verschillende methoden zijn beschreven in de literatuur. De meeste methoden beschouwen validatie van het besluitvormingondersteunend systeem als het belangrijkste onderdeel van het evaluatieproces. De beoordeling van de correctheid van de output van het systeem is hierbij het belangrijkste onderwerp. Voor de evaluatie van een besluitvormingondersteunend systeem is de ontwikkeling van een referentie, waarmee het advies van het systeem kan worden beoordeeld, belangrijk. Zoals beschreven in hoofdstuk 2 zijn er geen objectieve criteria voor correcte behandeling van een individuele patient beschikbaar. Dit betekent dat een referentie voor de evaluatie van het systeem ontwikkeld moet worden door een groep van op dit gebied deskundige neurologen

\section{Referentie voor het initieren van de behandeling}

Voor het initierren van de behandeling werden zestig papieren casus ontwikkeld, die elk een klinische presentatie van de eerste twee of drie poliklinische bezoeken van de patient beschrijven (hoofdstuk 4). Voor elk van deze casus werden drie neurologen verzocht een recept te schrijven. Met behulp van een twee stappen Delphi-achtige benadering, die zowel de diagnose als het voorgeschreven medicament betrof, kon de variabiliteit in voorschrijven van het soort medicament worden beperkt. De feedbackronde voor de dagelijkse dosering was minder succesvol in het reduceren van de variabiliteit

Twee referentie sets werden ontwikkeld, een diagnostiek referentie set voor de beoordeling van het voorgeschreven medicament en een therapeutische referentie set die gebruikt werd voor de beoordeling van de dagelijkse dosering.

Omdat een behandeling die niet in overeenstemming is met die voorgeschreven door de meerderheid nog steeds acceptabel kan zijn, werd na de Delphi ronde de aanvaardbaarheid van de recepten beoordeeld door twee onafhankelijke neurologen 
De variabiliteit onder de neurologen maakte het moeilijk een set van casus te verkrijgen met volledige overeenstemming op zowel de diagnose als de behandeling. Daarom werd een consensus bijeenkomst georganiseerd om een objectief criterium te verkrijgen. Voor de casus uit de diagnostiek referentie set werd een consensus referentie behandeling opgesteld op basis van de richtlijnen van de consensus bijeenkomst. De consensus bijeenkomst leidde tot een betere overeenstemming onder de neurologen met betrekking tot het gebruik van met name phenytoine.

\section{Referentie voor de follow-up van de behandeling}

Voor de beoordeling van het advies in een eerste follow-up contact moest een meerderheidsbeslissing van een groep van neurologen gebruikt worden aangezien hiervoor geen objectieve standaard bestaat. Twee referentie sets werden verkregen (hoofdstuk 6). Een klinische referentie set bestaande uit casus waarbij de meerderheid van de neurologen het eens was en een tweede referentie set bestaand uit casus waarbij een meerderheidsbeslissing verkregen werd op basis van zowel de uitspraken van de neurologen en de adviezen van het systeem Verder identificeerden we karakteristieken van de casus, middels machinaal leren, die de neurologen gebruikt kunnen hebben bij het beslissen over het aanpassen van de behandeling.

\section{Evaluatie van de initiatie van de behandeling}

Een vergelijking van de adviezen van het systeem, voor de casus uit de diagnostiek referentie set, met de recepten van de neurologen toonde aan dat het systeem ten minste net zo vaak als een individuele neuroloog anti-epileptica voorschrijft in overeenstemming met de referentie (hoofdstuk 5).

Het systeem adviseert voor natrium-valproaat een dagelijkse dosering in overeenstemming met de referentie. Voor carbamazepine suggereert het systeem vournamelijk een lagere dosering dan de neurologen. Klaarblijkelijk bestond er een verschil in benadering bij de behandeling. Terwijl de neurologen meer aansluiten bij een 'middle of the target' aanpak, adviseerde het systeem meer volgens het 'minimale therapie' principe, hetgeen betekent dat bij aanvang een lage dosering van het medicament wordt geadviseerd die verhoogd wordt indien de toevallen niet afnemen.

De analyse van de aanvaardbaarheid van de medicamenteuze adviezen toonde op globaal niveau weinig verschil in acceptatie tussen de recepten van het systeem en die van de neurologen. Het systeem had een kleiner aantal oordelen met een lage graad van acceptatie dan de individuele neurologen. Echter het aantal recepten met een hoge acceptatie was lager dan dat van de meeste neurologen. Het systeem adviseerde vaker een acceptabel medicament dan de meeste neurologen. Indien de acceptatiegraad van het door het systeem geadviseerde recept laag was werd dit meestal veroorzaakt door een te lage dagelijkse dosering

De evaluatie van de adviezen van het systeem voor de referentie casus en de beoordeling van de aanvaardbaarheid gaven een indruk van de prestatie van het systeem. De variabiliteit onder de neurologen maakte het echter moeilijk een harde uitspraak te doen over de correctheid van het systeem. Om de variabiliteit te verminderen werd een consensus bijeenkomst gehouden. Hierbij kwam naar voren dat de dagelijkse dosering die initieel door de neurologen werd voorgeschreven, vrij hoog was. 
Zowel het systeem als de groep van neurologen schreven voor natrium-valproaat meestal een dagelijkse dosering voor in overeenstemming met de consensus richtlijn. Voor carbamazepine adviseerde het systeem minder vaak een dagelijkse dosering in overeenstemming met de consensus richtlijnen dan de groep neurologen

Het systeem suggereerde een gelijk of hoger aantal recepten (medicament en dagelijkse dosering) in overeenstemming met de consensus dan vrijwel alle neurologen. Slechts een neuroloog schreef in meer dan de helft van de casus een recept in overeenstemming met de consensus voor en was hierbij beter dan het systeem.

\section{Evaluatie van de follow-up van de behandeling}

Om te beoordelen of het systeem adequate beslissingen neemt tijdens het bewaken van de medicamenteuze behandeling, werden de globale beslissingen betreffende de behandeling zoals voorgeschreven door het systeem vergeleken met de beslissingen van de vijf neurologen.

Het systeem bereikte een matige overeenstemming met betrekking tot het behandelingsbesluit - uitgedrukt in de $\mathrm{k}$-waarde - met drie van de individuele neurologen. Slechts twee paren van neurologen hadden een matige overeenstemming; de overige combinaties van twee neurologen hadden een lagere graad van overeenstemming. Het systeem stemde dus betere overeen met elk van de individuele neurologen dan elk van de neurologen met zijn collega's

De vergelijking van de overeenstemming tussen het systeem en een groep van vier neurologen met de overeenstemming tussen een individuele neuroloog en dezelfde groep van vier neurologen, wees uit dat het systeem een vrij goede overeenstemming bereikte, terwijl elk van de individuele neurologen matig overeenstemde met zijn vier collega's

Een klinische referentie werd ontwikkeld, gebaseerd op de behandelingsbesluiten van de meerderheid van de vijf neurologen. Voor 219 van de 265 casus kon een dergelijke meerderheidsbeslissing verkregen worden. Op deze set van casus bereikte het systeem een correctheid vergelijkbaar met die van de individuele neuroloog. Deze referentie heeft echter een bias ten gunste van de neurologen doordat deze laatsten bijdragen aan de definitie van de referentie voor de behandeling, terwijl het systeem dit niet deed. Door het advies van het systeem te betrekken bij de definitie van de referentie behandeling werd voor een subset van 189 casus een meerderheidsoordeel verkregen. Binnen deze subset van casus bereikte het systeem een betere correctheid dan de neurologen. De analyse van de 76 casus waarbij geen referentie verkregen kon worden, toonde aan dat het systeem minder vaak dan elk van de individuele neurologen een behandelingsbesluit adviseerde dat door geen van de neurologen was voorgesteld.

Karakteristieken van een casus kunnen belangrijk zijn voor het bereiken van specifieke behandelingsbesluiten. Om meer inzicht te krijgen in het besluitvormingsproces van de groep van neurologen werd een beslisboom geconstrueerd met behulp van machinaal leren. De boom bestaat uit paden die elk leiden tot een eindknoop waaraan in principe één specifieke behandeling toegewezen kan worden. Elk pad gebruikt verschillende karakteristieken van een casus. Een eindknoop geeft het aantal casus weer voor elk van de verschillende behandelingsbesluiten, dat aan de criteria voldoet behorende bij het specifieke pad, dat naar deze eindknoop leidt. 
Een analyse van de criteria voor aanpassing van de therapie toonde aan dat bij zes paden de eindknopen casus bevatten met voornamelijk één behandelingsbesluit. Deze criteria werden gebruikt als richtlijnen voor de follow-up van de behandeling. Voor elk van deze richtlijnen adviseer het systeem dezelfde behandelingsbesluiten als de groep neurologen

\section{Algemene conclusies}

De vraag of een geautomatiseerd model gebaseerd op kennis van een expert een kwalitatief goed advies voor de medicamenteuze behandeling van epilepsie kan geven kan bevestigend beantwoord worden. Een vergelijking van de adviezen met de diagnostiek referentie en de consensus referentie toonde aan dat het systeem een correct advies geeft voor het initiëren van de behandeling met anti-epileptica. Een lage graad van aanvaardbaarheid van een advies kon worden toegeschreven aan een (te) lage dagelijkse dosering. Dit deel van het kennisbestand kan gemakkelijk aangepast worden.

Ook voor de aanpassing van de geïnitieerde behandeling stelde het systeem behandelingsbesluiten voor die een goede overeenstemming hadden met zowel de individuele neurologen als groepen van neurologen.

Vanwege de waargenomen inter-individuele verschillen is het raadzaam het systeem verder te ontwikkelen (inclusief een gebruikersvriendelijke interface) en het voor verdere tests te installeren in een neurologische polikliniek, om zo de redenen voor het niet volgen van het advies van het systeem te kunnen analyseren. Als het kennisbestand van het systeem onderhouden wordt, kan het gebruikt worden voor een second opinion, of voor onderwijsdoeleinden. 


\section{Tot slot}

Het schrijven van een proefschrift is niet mogelijk zonder de hulp en de steun van anderen. Dit is reeds vele malen geschreven in het dankwoord van een proefschrift. Ook bij het schrijven van dit proefschrift werd het wederom proefondervindelijk vastgesteld. Daarom wil ik hier enkele mensen speciaal bedanken die voor mij van groot belang zijn geweest in de afgelopen jaren.

Wijlen Prof.dr.van der Lugt voor zijn bereidwilligheid tot een reflecterende kijk op het medisch denken en handelen en het stimuleren van een idee van een tweede jaars student geneeskunde.

Prof.dr.ir.Hasman: Beste Arie, het idee medische kennis te standaardiseren vond bij jou gehoor. Bedankt voor je interesse en de deskundige begeleiding met name in de laatste fase van het proefschrift.

Prof.dr.Meinardi: Beste Harry, je interesse, je praktische kijk, je gevoel voor humor en je inzet hebben me vaak verwonderd. Bedankt voor je stimulerende invloed.

Prof dr.Troost: Beste Jaap, bedankt voor je positieve commentaar en de duwtjes in de goede richting

Dr.ir. Talmon: Beste Jan, een speciaal woord van dank geldt jou. Je enorme energie en je flexibiliteit zijn essentieel voor een promovendus.

Drs. Groothuis en drs. Tange: Beste Siebren en Huibert, gelukkig was er ook nog iets anders dan promoveren. Ik wens jullie alle twee veel succes met jullie promotie.

Drs.Kubben, internist. Beste Frank, je belangstelling en vertrouwen is voor menig mens een verademing. Ik wens je veel succes met je promotie.

Ir.Stallenberg: Beste Gerard, je nuchtere kijk op zaken en je gevoel voor humor worden zeer op prijs gesteld. Onze wandelingen waren een welkome afwisseling. Arigato.

Verder wil ik de neurologen die aan het onderzoek hebben meegewerkt bedanken voor het beschikbaar stellen van hun tijd en hun bereidwilligheid om op een andere manier naar medisch handelen te kijken. Dr.H.Schouten en dr. A.van Kesteren wil ik bedanken voor hun statistische adviezen

Dat de laatste loodjes het zwaarst wegen heefl naast enkele goede vrienden met name de fam. moeten ervaren. Mijn dank gaat dan ook uit naar hen wier steun ik heb ontvangen, in het bijzonder naar mijn moeder, voor al haar goede zorgen, mijn vader, voor al zijn inspanningen, en Marjo, Desirée en Vincent voor hun warme belangstelling.

Tot slot wil ik Eline, mijn vrouw, bedanken voor haar steun en bereidheid mijn werk te laten prevaleren boven andere belangrijke zaken. Dankzij jouw laatste zetjes is het gelukt. Het is af. 

Decisions

and Strategies

in Drug Treatment

of Epilepsy

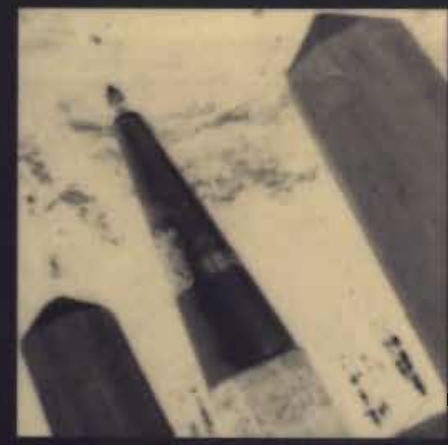

Raymond Smeets 
Decisions and strategies in drug treatment of epilepsy 


\title{
Decisions and strategies in drug treatment of epilepsy
}

\author{
PROEFSCHRIFT
}

ter verkrijging van de graad van doctor aan de Universiteit Maastricht, op gezag van de Rector Magnificus, Prof.mr.M.J.Cohen, volgens het besluit van het College van Dekanen, in het openbaar te verdedigen op

vrijdag 29 november 1996 om 12.00 uur

door

Raymond Pierre Angèle Marie Smeets

geboren te Rothem-Meerssen in 1961 
Promotores:

Co-promotor:

Beoordelingscommissie:
Prof.dr.ir. A. Hasman

Prof.dr. H. Meinardi

Prof.dr. J. Troost

Dr.ir. J.L. Talmon

\author{
Prof.dr. J.A. Knottnerus (voorzitter) \\ Dr. J. van der Lei (Erasmus Universiteit Rotterdam) \\ Prof.dr. P. Pop \\ Dr. W.O. Renier (Katholieke Universiteit Nijmegen) \\ Prof.dr. R.W. Stockbrügger
}

ISBN: $90-9010008-3$

(c) R.P.A.M. Smeets, Maastricht, 1996.

Produktie: Datawyse, Maastricht

Omslag: Consultatie: John van de Poel, Ruud Groothedde.

Foto's: Borders-No Borders, Herbert Rikers, Bunde.

Lay-out: Datawyse, Joop Caris.

Dit proefschrift kwam tot stand in het kader van het instituut ExTra, deeluitmakendvan de landelijke onderzoekschool CaRe (Netherlands School of Primary Care Research), in 1995 erkend door de KNAW.

Het in dit proefschrif gepresenteerde onderzoek werd gesteund door subsidies van het Nationaal Epilepsie Fonds - De Macht van het Kleine en de SWOL.

Met dank aan het Nationaal Epilepsie Fonds - De Macht van het Kleine, Ciba Geigy B.V.. Dumex B.V., Janssen-Cilag B.V., Karwijk Fama B.V., Novo Nordisk Farma B.V., Schering-Plough B.V., Yamanouchi Farma B.V., inzake totstandkoming van dit proefschrift. 


\section{Voor Eline.}





\section{CONTENTS}

\section{CHAPTER 1 INTRODUCTION}

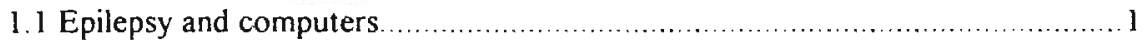

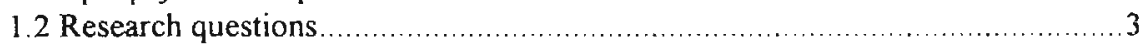

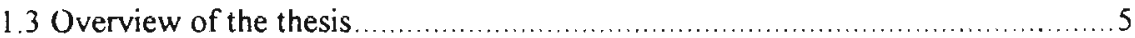

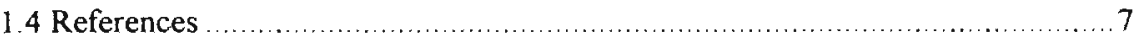

\section{CHAPTER 2 EPILEPSY}

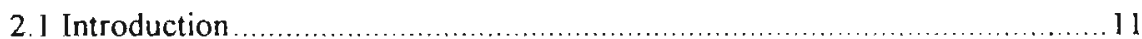

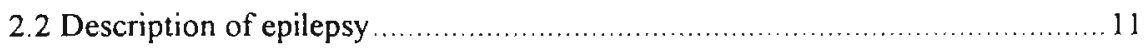

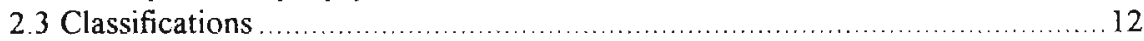

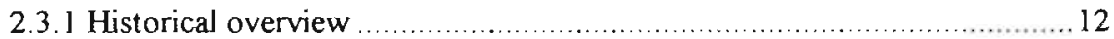

2.3.2 Importance of different classifications ............................................ 13

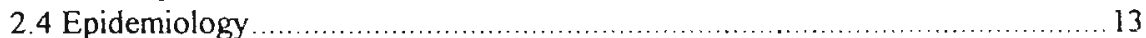

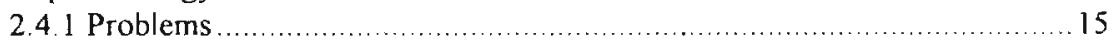

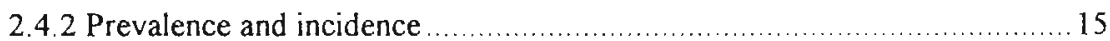

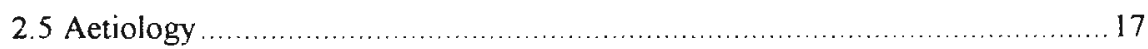

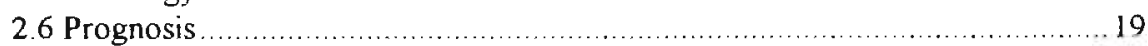

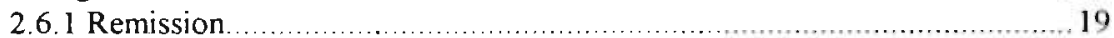

2.6.2 Problems in establishing prognostic factors ................................. 21

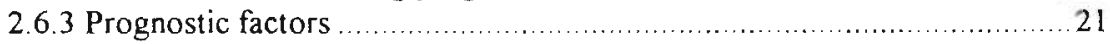

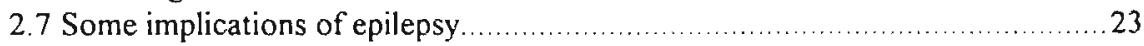

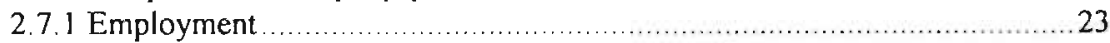

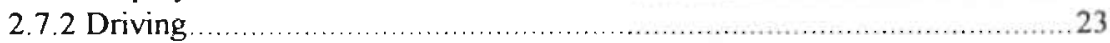

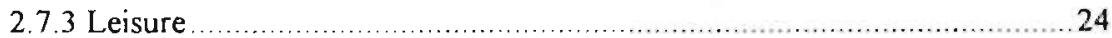

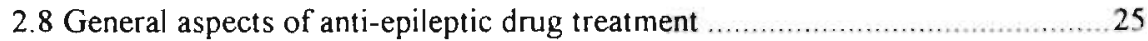

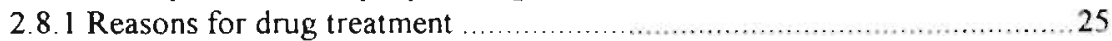

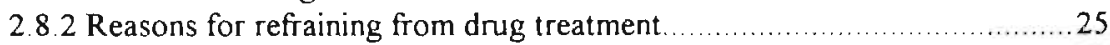

2.8.3 Goals in anti-epileptic drug treatment .....................................26

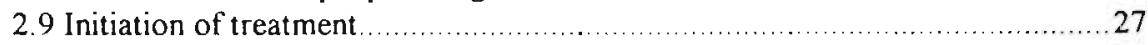

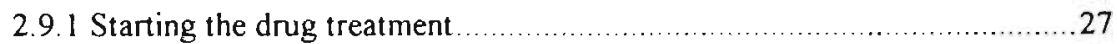

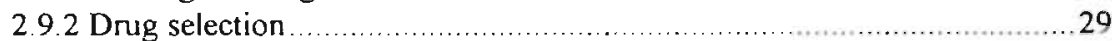

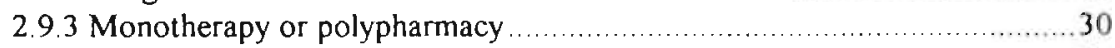

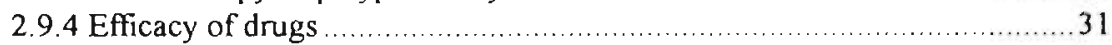

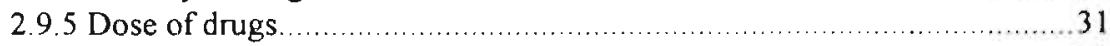

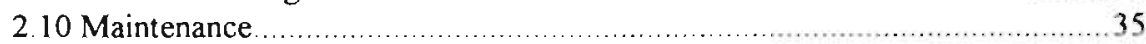

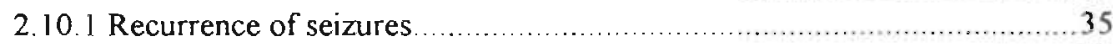

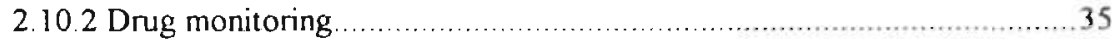

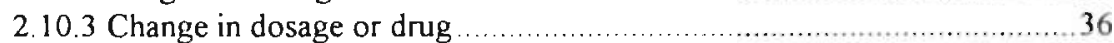

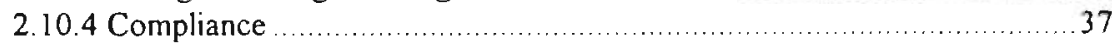

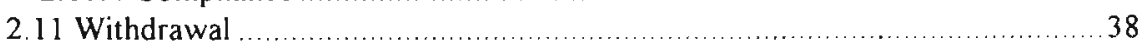

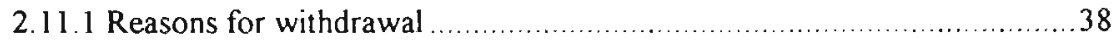

2.11.2 Moment of relapse......................................................... 40

2.12 Summary and conclusion......................................................... 40

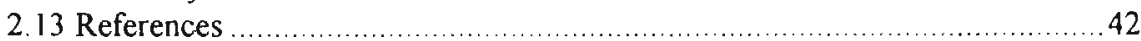




\section{CHAPTER 3 SYSTEM DEVELOPMENT}

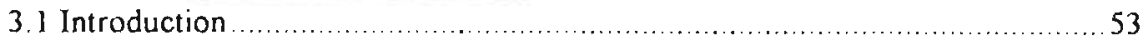

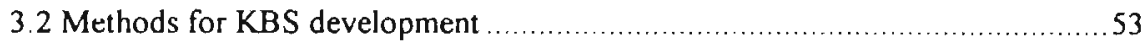

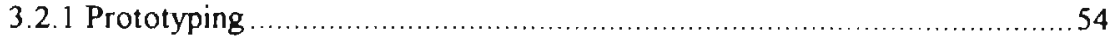

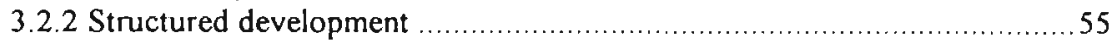

3.2.3 Selection of the development method ........................................ 57

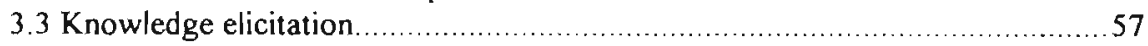

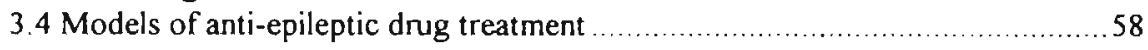

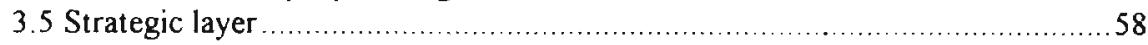

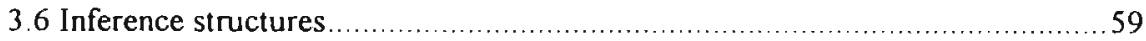

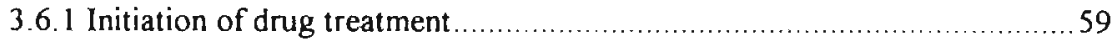

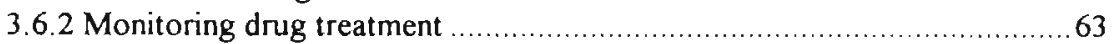

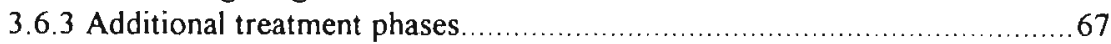

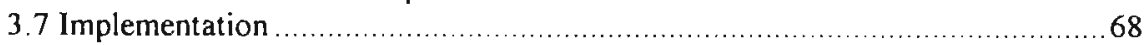

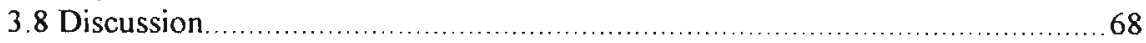

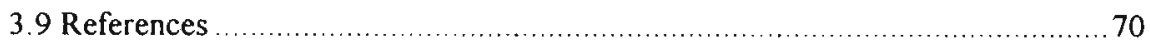

CHAPTER 4 REFERENCE DEVELOPMENT

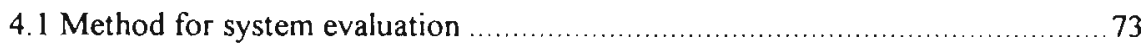

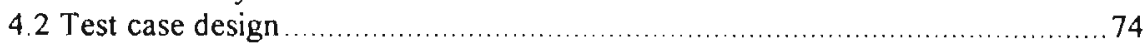

4.2.1 Patient selection .................................................................... 74

4.2.2 Validity of paper cases ............................................................. 74

4,2.3 Description of the test cases ................................................. 75

4.3 Reference development ........................................................... 77

4.3.1 Method for consensus formation .............................................. 77

4.3.2 Reference development/Acceptance testing ...................................78

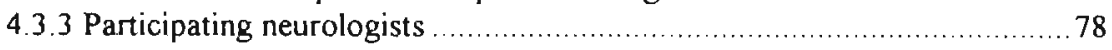

4.3.4 Reference development process ................................................ 79

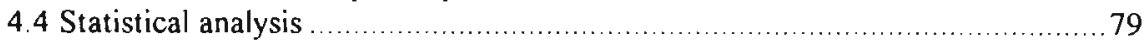

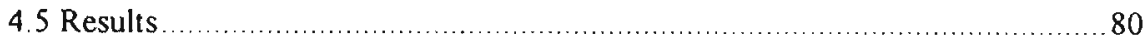

4.5.1 Agreement on the diagnosis, generic drug and daily dose ................... 80

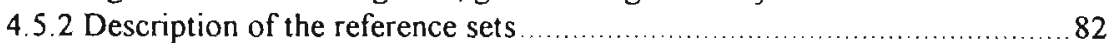

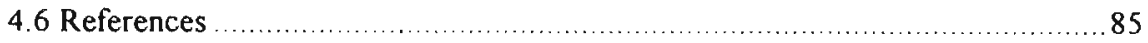

CHAPTER 5 RESULTS ON INITIATION OF TREATMENT

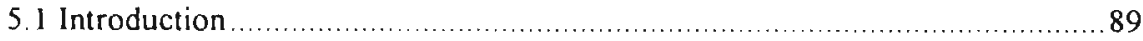

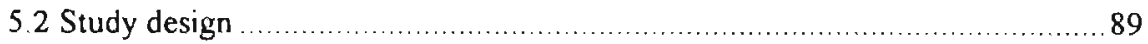

5.3 Comparing the system with Neurologist I and Neurologist II _................... 90

5.3.1 Agreement on the generic drug ................................................. 90

5.3.2 Agreement on tradename ....................................................... 91

5.3 .3 Agreement on daily dose ....................................................... 91

5.4 Comparing the system with an external reference .................................. 92

5.4.1 Agreement about the drug using the diagnostic reference set ................93

5.4 .2 Variability in neurologist's prescriptions ....................................... 94

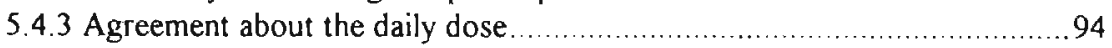

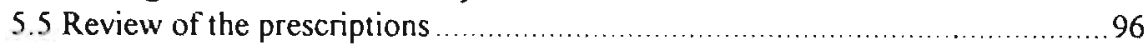


5.5.1 Overview of the results for system and individual neurologists...............96

5.5.2 Comparing overall scores of system and individual neurologists..............98

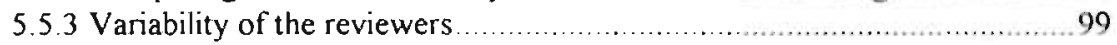

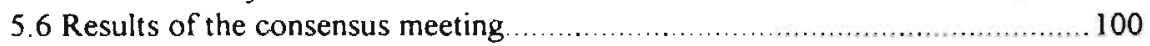

5.6.1 The use of diagnostic classification schemes ............................ 100

5.6 .2 Initiation of drug treatment ..................................................... 100

5.6 .3 Selection of generic drug .................................................... 100

5.6 .4 Generic drug or brand, formulation .......................................... 101

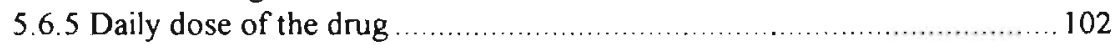

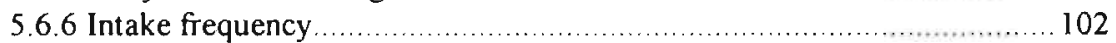

5.6 .7 Gradual increase of the dose ............................................. 103

5.7 Comparing the system and the neurologists with the consensus meeting

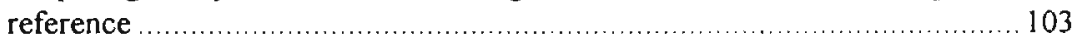

5.7.1 Agreement on the generic drug .............................................. 103

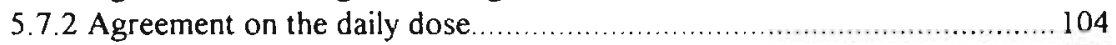

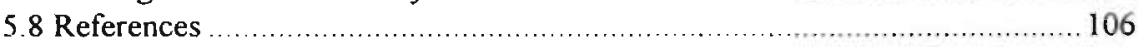

CHAPTER 6 RESULTS ON FOLLOW-UP OF TREATMENT

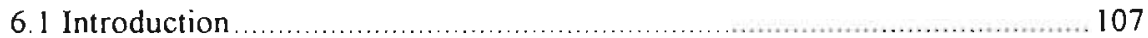

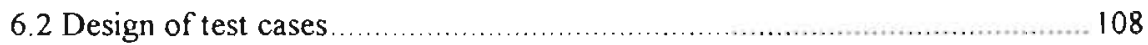

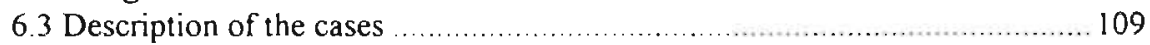

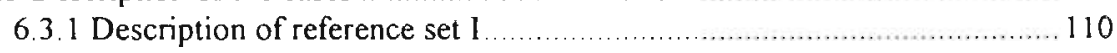

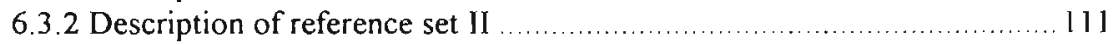

6.3.3 Cases not included in the reference set II .................................. 113

6.3.4 Relevance of characteristics for agreement among neurologists ............. 114

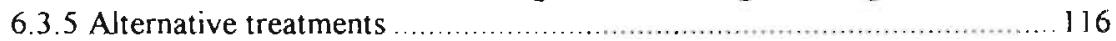

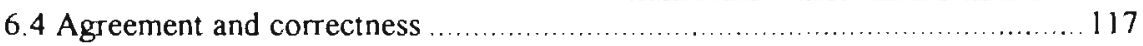

6.4.1 Overview of the decisions in the total set ................................. 117

6.4.2 Differences in treatment approaches ........................................... 118

6.4.3 Agreement between an individual and a group .............................. 119

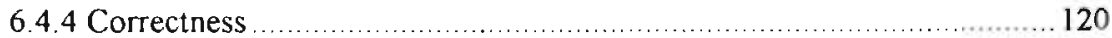

6.4.5 Variability in agreement in set II .......................................... 121

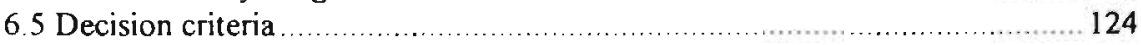

6.5.1 Treatments indicated by a single attribute ................................ 124

6.5.2 Treatments ruled out by a single attribute ................................. 125

6.6 Treatments indicated by combinations of attributes .............................. 126

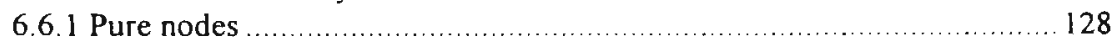

6.6.2 Nearly pure nodes; two decisions ............................................. 129

6.6.3 Mixed nodes; three decisions ................................................ 13 ]

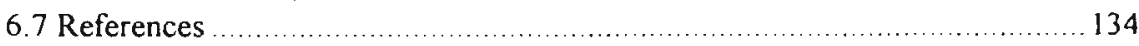


CHAPTER 7 CONCLUSIONS AND DISCUSSION

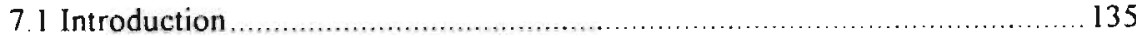

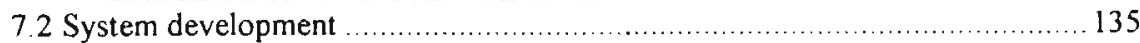

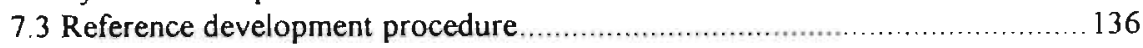

7.4 Initiating anti-epileptic drug treatment ............................................ 137

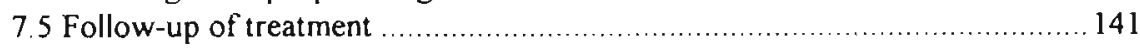

7.6 General conclusion and future prospects ...................................... 143

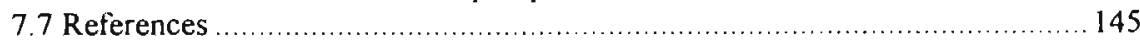

CHAPTER 8 SUMMARY

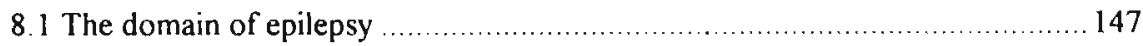

8.2 Building a model of expertise .................................................. 148

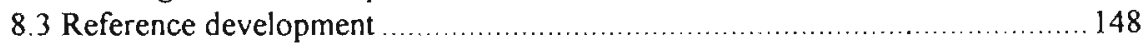

8.4 Evaluation of the initiation of the treatment ...................................... 149

8.5 Evaluation of the follow-up of the treatment ..................................... 150

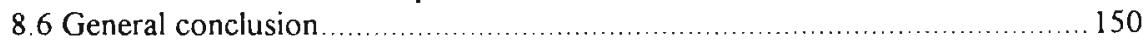

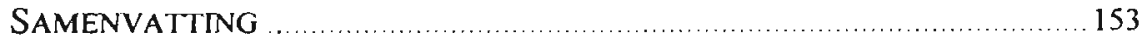

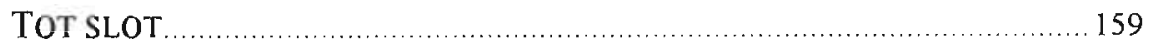

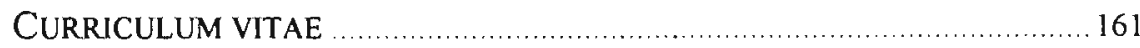




\section{Introduction}

\subsection{Epilepsy and computers}

Epilepsy is a disorder which manifests itself through brief, usually unprovoked stereotypical disturbances of behaviour, emotion, motor function or sensation. The occurrence of these symptoms will have a great impact on the daily life of the patient and his family. Such seizures are normally treated with anti-epileptic drugs. Although doctors may have the same education, it seems that some are more successful than others in the treatment of patients. Part of this difference may be attributed to experience. The use of a 'computer fed by a team of specialists' to support the doctor has been considered an option to improve patient management in the domain of epilepsy (Meinardi, 1985).

Computer programs, either embedded or stand-alone, which are designed to help health professionals in patient management by suggesting or providing patient specific advice or treatment are called decision support systems (DSSs). Evidence, especially the more recent, suggests that this type of computerised decision support can improve physician performance (Sutton 1989, Paterson-Brown and Vipond 1990, Johnston et al. 1994, Sullivan and Mitchell 1995). DSSs analyse and reason about patient data and support the diagnosis, treatment and disease prevention tasks by producing relevant conclusions or by (suggesting) actions for a given problem of a specific patient. This contrasts with clinical information systems and literature reference systems. These latter systems provide information but do not specify how to apply it in the decision making process for the specific problem of an individual patient (Perreault and Wiederhold, 1990).

Taking into account under which circumstances the advice is generated three types of DSSs can be discerned

- Solicited advice systems i.e. systems providing advice upon request of the user

- Unsolicited advice systems i.e systems providing suggestions without a specific request of the user

- Autonomous systems or closed-loop systems i.e. systems undertaking medical actions without having a physician in the loop

Each of these types of systems requires a different involvement from the user. In case of a system providing solicited advice, the physician has to make a request for information and to take a decision on the applicability of the information provided. 
Systems providing unsolicited advice do so on the basis of existing patient data. The user decides whether or not to act on the advice. Autonomous systems both analyse the information and apply the advice for a patient specific problem. The user has to decide whether to apply the system for a specific task.

Studies of the impact of DSSs in various domains have shown that such systems can enhance patient care through (see e.g. Johnston et al. 1994 for a review):

- Improvement of the diagnostic accuracy

- More effective control of the disease by:

a. Having serum levels of therapeutics in accepted ranges more often

b. Earlier achievement of therapeutic doses

c. A reduction of adverse drug reactions

$\mathrm{d}$. Earlier change of ineffective treatments

e. Improvement of uniformity in drug treatment

- Increase in the use of preventive procedures

- Improvement of patient follow-up

In the management of patients with epilepsy three sub-domains can be considered as problem areas for which it may be worthwhile to develop a DSS:

- The diagnostic process

- The decision whether or not to start drug treatment

- Drug treatment

Establishing the correct diagnosis is an essential part in the decision making process. Uncertainty about the fact whether a patient has epilepsy may be a reason for refraining from drug treatment (Chadwick and Reynolds, 1985). Decision support in this domain has been considered. The definitions of disease concepts are not stable or specific enough to enable optimal diagnostic support. For example, recently a new set of definitions has been proposed (Commission on Epidemiology and Prognosis LAE, 1993). Others who developed procedures for diagnostic support encountered problems related to definitions (Principe and Smith 1982, Korpinen et al 1994). Hence a diagnostic decision support system seems less promising.

The decision to start anti-epileptic drug treatment has as a consequence the intake of a drug for at least two years in a regular, daily fashion. The risks of recurrence of seizures has to balance or outweigh the disadvantages of the treatment. In this process of decision making issues such as consequences of the seizures, side-effects, compliance and psycho-social consequences of the drug intake have to be addressed (Dreifuss, 1985). Little guidance was found in the literature on how to incorporate issues such as compliance and psycho-social aspects in the decision making (Chadwick and Reynolds 1985, Jøhannessen et al. 1990). Building decision support for this domain would rely to a large extent on common sense and subtleties that are difficult to formalise. The literature still is not conclusive about the perceived risks of seizures and the moment of commencing treatment (Chadwick 1995, Reynolds 1995).

Once the decision to start treatment has been taken, problems such as the selection of the drug, the determination of an adequate drug dose regime and the adjustment of initiated treatment will have to be addressed. Multiple reasons for adjustment of the treatment can occur (Beardsley et al. 1983, Ward 1989, Meyer 1991, Dichter 1993). 
Insufficient effectiveness of the drug is a common problem. Also side-effects may warrant modification of the treatment. Decision making may be complicated by noncompliance of the patient. More or less formalised but still not uniform procedures for drug treatment are discussed in the literature. Guidance in dosage determination has been provided by models which mainly address the pharmacodynamics of drug treatment (van der Kleijn 1983, Peterson 1983). The domain is relatively stable, as only a few new drugs enter the market. It has been assumed that models which support the physician in treatment may improve patient care (Meinardi 1985, Rimmer and Richens 1988).

It was concluded on the basis of the perceived problems in the three sub-domains that drug treatment was the most suitable domain for decision support. Our study therefore focuses on the elicitation, implementation and assessment of knowledge of treatment procedures for anti-epileptic drugs.

Automated decision support addresses the system of medical practice as a whole including health care professionals and patients (Musen, 1993). However, before one starts to study how a DSS has to be integrated in the system of medical practice it is necessary to establish the efficacy of a DSS. If a DSS has a low efficacy in a domain then one should not put it into routine use. Despite considerable development and evaluation efforts, few DSSs are used in daily practice (Nykänen 1990, O'Moore et al. 1990, Heathfield and Wyatt 1993, de Dombal 1993). Factors hampering the acceptance of DSSs for routine use in medical care may have a technical, economic, organisational, psychological and/or medical origin. It has been suggested that by taking these factors into account the introduction of DSSs in the medical domain may be stimulated (van der Lei, 1993).

A generally accepted evaluation methodology which addresses the above mentioned problem areas is needed. Most existing evaluation methodologies have as a common denominator at least the validation of a system, of which the assessment of the implemented knowledge is considered to be the cornerstone (Spiegelhalter 1983, Green and Keyes 1987, O'Keefe et al. 1987, O'Keefe 1989. O'Leary and O'Keefe 1989, RossiMori et al. 1990, Wyatt and Spiegelhalter 1990, Engelbrecht et al. 1991, Grant et al. 1991, Clarke et al. 1994). We restricted ourselves to this type of assessment.

\subsection{Research questions}

In this thesis the main research question is

"Is it possible to develop decision support that provides adequate advice concerning anti-epileptic drug treatment?"

To answer this question we elicited and analysed the knowledge of an exper neurologist. This knowledge was implemented in a prototype system. The developed system can give advice in two different situations:

- the initiation of the anticonvulsive treatment after the diagnosis has been established and

- the adjustment of the drug treatment after an assessment of its effectiveness. 
The adequacy of the system's advice for initiation of anti-epileptic drug treatment is evaluated by addressing the following questions:

1. Does the system suggest an adequate anti-epileptic drug when initiating drug treatment?

2. Does the system provide an adequate daily dose when initiating drug treatment?

When an objectively correct treatment would be available for each case, such a treatment could serve as reference for answering the above listed questions. In the management of epilepsy there is no such objectively correct treatment (see chapter 2). Therefore we compare the system's advice with the suggestions of a group of neurologists.

For the initiation of drug treatment a Delphi procedure was followed to reduce variability and remove erroneous treatment suggestions of the neurologists. From the set of 60 cases we obtained a diagnostic reference set where the majority of three neurologists agreed on the detailed diagnosis. This diagnostic reference set was reduced in size to a treatment reference set where the majority of the neurologists agreed on the generic drug.

The diagnostic reference set is used for assessment of the proposed generic drug; the treatment reference set for assessment of the proposed daily dose of the drug. For both sets of cases the agreement between the system and individual neurologists was determined. It was decided that the system's advice would be considered adequate when it conforms to the reference as good as or better than the individual neurologists. If the system does not agree with the majority but gave an advice equal to that of one neurologist such an advice can be considered an alternative treatment.

As more than one treatment may be appropriate for a case the acceptability of the prescriptions for cases in the diagnostic reference set was assessed by two independent (non prescribing) neurologists. The question addressed is:

3. What is the overall acceptability of the advice of the system compared with that of the neurologists?

If the prescription was considered of poor quality, the judgement on the appropriateness of the generic drug and the prescribed daily dose was analysed to determine which of these aspects was the reason for the poor quality.

The comparison of the neurologist's prescriptions with the system's advice for the cases in the reference sets and the assessment of the acceptability provided an indication about the performance of the system. However, it did not enable us to make a statement about the correctness of the detailed prescriptions due to the variance in prescribing among the neurologists. To reduce the variability in prescribing among the neurologists we decided to have a consensus meeting in which the prescriptions for each of the cases were discussed. During this discussion global guidelines were agreed upon. These guidelines could be used to determine a reference treatment for each of the cases. The advice of the system as well as the prescriptions of the neurologists are compared with this reference. The question addressed was:

4. What is the correctness of the system's prescriptions compared with the correctness of the neurologists' prescriptions? 
The adjustment of drug treatment is another part of the process for which an advice may be provided by the system. This is evaluated by addressing the question:

5. Does the system take adequate treatment decisions while monitoring drug treatment?

The global decisions to adjust the treatment - i.e. continue, decrease or increase the daily dose or change of drug - were used for comparison.

On the basis of a set of 265 descriptions of follow-up contacts the agreement of the system with each individual neurologist is determined. A comparison between the agreement of an individual neurologist and the remaining group of four neurologists and between the system and this group is made to obtain information about the adequacy of the system.

Based on the 265 follow-up descriptions two reference treatment sets were established to determine the performance of the system. A clinical reference set contained cases for which a majority decision of the five neurologists could be established. The second reference used the majority decision obtained from both the five neurologists and the system. To obtain a more tangible measure, the agreement between the system and the neurologists was expressed as a score, the level of agreement, indicating the number of neurologists which agree or disagree with the system.

To compare the decision making process of the group of neurologists with that of the system a classification tree is constructed based on case characteristics and the treatment decisions. The end-nodes of the tree present sets of cases which share particular case characteristics. The path to an end-node which contains mainly cases with one treatment decision indicates a guideline which may have been applied by the group of neurologists. End-nodes with several treatment decisions indicate categories of cases for which guidelines are difficult to obtain. For each of the major end-nodes the decision making process of the neurologists and the system are compared. The question addressed here is:

6. How do the treatment decisions of the system compare with those of the group of neurologists for the cases that meet the guidelines derived from the neurologists' decisions?

\subsection{Overview of the thesis}

In chapter 2 a review of the literature on epilepsy and its treatment is presented. The chapter is divided into two parts. In the first part a description of epilepsy and an overview of the diagnostic classifications are presented. Studies on prevalence and incidence in the United States and European countries indicate how common epilepsy is. In the second part treatment of epilepsy is discussed including aspects of drug treatment such as initiation, maintenance and withdrawal of drug treatment. This information was used during the design of the system.

Since the literature does not provide enough information about the criteria to be used in the decision making process of drug treatment it was decided also to elicit this knowledge from an expert. The amount of data required for commencing and monitoring the drug treatment makes the use of flow charts unfeasible and calls for 
computer support. Several approaches for knowledge acquisition are reviewed in chapter 3. Models describing the initiation and monitoring part of the treatment process are presented. Problems in analysing expertise using KADS are discussed.

The evaluation of the system focused on the implemented knowledge. As a standard for anti-epileptic dnug treatment was not available, a reference treatment had to be developed for a set of test cases. A Delphi-like approach in combination with a consensus meeting was selected for establishing a reference with which to compare the system. In chapter 4 the construction of the test cases and development of the reference are presented.

Chapter 5 addresses the assessment of the system's advice for initiating anti-epileptic drug treatment. Three analyses are presented in which the advice of the system is compared with the prescriptions of:

- The neurologist who provided the expertise to investigate whether the domain was sufficiently covered during the knowledge elicitation process

- A neurologist from the same department not involved in the development of the system to obtain some information on local differences in treatment

- A group of five neurologists from five different general hospitals in The Netherlands to assess the impact of individual preferences

In each of the experiments the prescribed drug and the daily dose are assessed. A comparison of the performance of individual neurologists and of the system is presented

To be able to determine the correctness of prescriptions a consensus meeting was held to define a treatment reference. The reference determined the required generic drug as well as the daily dose. The advice of the system as well as the prescriptions of the neurologists are compared with this reference.

In chapter 6 the advice of the system is assessed using case descriptions, in which drug treatment has been started, who return for a follow-up. In this chapter the treatment decisions are assessed at a global level: increase, decrease or keep the dose as it is or change the type of drug. The chapter consists of two parts. In the first part the advice of the system is addressed. Three different analyses are presented:

- The degree of agreement between the system and the neurologist(s) is determined to establish how good the system is in relation to the individual neurologist

- A clinical reference based on the majority decision of the group of neurologists was used to assess the correctness of the system and the neurologists

- A comparison using a reference based on the majority decision of both the neurologists and the system provided information on how the system performed when the system is considered as a peer

One may expect that case characteristics determine which treatment decision is taken. The second part of chapter 6 describes the results of an analysis leading to criteria that may have been used by the neurologists in arriving at a treatment proposal. The treatment decisions of the system are compared with them. 
In chapter 7 the final conclusions are presented and discussed. Directions for future research are indicated as well.

\subsection{References}

Beardsley R.S., Freeman J.M., Appel F.A., Anti-convulsant serum levels are useful only if the physician appropriately uses them: an assessment of the impact of providing serum level data to physicians, Epilepsia, 24, 1983, pp. 330-335.

Chadwick D., Reynolds E.H., When do epileptic patients need treatment? Starting and stopping medication, British Medical Journal, 290, 1985, pp. 1885-1888.

Chadwick D., Case for early treatment is not established, British Medical Journal, 1995, 310 , pp.177-178.

Clarke K., O'Moore R., Smeets R., Talmon J., Brender J., McNair P., Nykänen P., Grimson J., Barber B.A., A methodology for evaluation of knowledge-based systems in medicine, Artificial Intelligence in Medicine, 1994, 6, pp. 107-121.

Commission on Epidemiology and Prognosis of the International League Against Epilepsy, Guidelines for epidemiologic studies on epilepsy, Epilepsia, 34, 1993, pp.592-596.

Dichter M.A., The premise, the promise, and the problems with basic research in epilepsy, Epilepsia, 34, 1993, pp.791-799.

Dombal F.T de, Background and current state-of-the-art Technology and Healih Care. European Society for Engineering and Medicine. Vol. I, April 1993, pp. 77-83.

Dreifuss F.E., Risk benefit analysis in the management of the child with epilepsy. In: Modern approach to anti-epileptic drug treatment, improving quality of life, (Dam, Gram, Pedcrsen and Ørum, Eds.), The Danish Epilepsy Society, Kobenhavn, 1985.

Engelbrecht R., Fitter M., Rector A., Requirements for a medical workstation using usercentred design. In: Proceedings MIE '9l (Adlassnig, Graber, Bengtsson and Hansen, Eds.), Lecture Notes in Medical Informatics, vol 45. Springer-Verlag, Berlin, 1991, pp. 140-144.

Grant A., Parker-Jones C., White R., Cramp D, Barreiron A., Mira P., Artal A., Monteror J., Evaluation of knowledge based systems from the user perspective. In: Knowledge-hased systems in Medicine: Methods Applications and Evaluation, (Talmon and Fox, Eds.), Lecture Notes in Mcdical Informatics, vol 47, Springer Verlag, Berlin, 1991, pp. 312-324

Green C.J., Keyes M.M., Verification and validation of expert systems. In: Proceedings Western Conference on expert systems Anaheim, IEEE Computer Socicty Press, New York, 1987, pp. 38-43.

Heathfield H.A., Wyatt J., Philosophies for the design and development of clinical dicision support systems. Methods of Information in Medicine, 32, J993, pp. 1-8

Johannessen S.I., Loyning Y., Munthe-Kaas A.W. General Aspects. In: Comprehensive Epileptology (Dam and Gram, Eds.), Raven Press, New York, 1990, pp 505-524.

Johnston M.E., Langton K.B., Hayncs R.B., Mathicu A., Effects of computer-based clinical decision support systems on clinician performance and patient outcome, a critical appraisal of research, Annals of Internal Medicine, 120, 1994, pp. $135-142$.

Kleyn E. van der, Continuous monitoring of plasma anti-epileptic drug levels, in: Current therapy in epilepsy, Acta Ncurologica Scandinavia, 97 (Suppl.), 1983, pp.91-114.

Korpinen L., Pietilä T., Peltola J., Nissilä M., Keränen T., Tuovinen T., Falck B., Petránek E.S., Frey H., Evaluation of epilepsy expert, a decision support system, Computer Methods and Programs in Biomedicine. 45. 1994, pp.223-231. 
Lei, J. van der, Experience from computer based records for computer assisted decision making. Methods of Information in Medicine, 32, 1993, pp. 14-15

Meinardi H., Epileptology, military art of healing or science. Inaugural address, Catholic University of Nijmegen, 22 Februari 1985.

Meyer J.W.A. Knowledge, attitude and practice in anti-epileptic drug monitoring, thesis, Nijmegen, 1991.

Musen M., Architectures for architects, Methods of Information in Medicine, 32, 1993, pp. 1213.

Nykänen P. Issues on the evaluation of computer-based support to clinical decision making, Report of the SYDPOL-working group, Institute of informatics, Oslo University, Research report 127, 1990.

O'Keefe R.M., Balci O., Smith E.P., Validating expert systems performance. IEEE Expert, Winter, 1987, pp. $81-89$.

O'Keefe R.M. The evaluation of decision-aiding systems, guidelines and methods, Information and Management, 17, 1989, pp.217-226.

O'Leary D., O'Keefe R. Verifying and validating expert systems, Tutorial at the 11 th IJAI-89, Detroit USA, 1989.

O'Moore R., Clarke K, Smeets R., Brender J., Nykänen P., McNair P., Grimson J., Barber B.: Items of Relevance for the Evaluation of Knowledge Based / Expert Systems and Infuence from Domain Characteristics. KAVAS (AJ021) Report EM 1.l., Trinity College, Dublin, 1990.

Paterson-Brown S., Vipond M.N., Madern aids to clinical decision making in the acute abdomen, British Journal for Surgery, 77, 1990, pp. 13-18.

Perreault L., Wiederhold G., System design and evaluation. In: Medical Informatics. Computer applications in healih care (Shortliffe, Perreault, Wiederhold and Fagan, Eds.), Addison-Wesley Publishing Company, Reading, Mass. 1990.

Peterson G.M., Computer-assisted drug therapy for epilepsy, Drig Intellingence and Clinical Pharmacy, 1983, 17, pp.123-125.

Principe J.C. Smith J.R., Microcomputer-based system for the detection and quantification of petit mal epilepsy, Computers in Biology and Medicine, 12, 1982, pp.87-95.

Reynolds E.H., Do anticonvulsants alter the natural course of epilepsy? Treatment should be started as early as possible, British Medical Journal, 1995, 310. pp. 176.

Rimmer E.M., Richons A., Clinical pharmacology and medical treatment. In: A textbook of upilepsy, (Laidlaw, Richens and Oxley, Eds.), 3rd. edition, Churchill Livingstone, Edinburgh, 1988, pp. 421-483.

Rossi-Mori, A., Pisanelli, D.M., Ricci, F.L. Evaluation stages and design steps for knowledgebase systems in medicine. Medical Informatics, vol. 15, no.3, p.191-204, 1990.

Spiegelhalter D.J. Evaluation of clinical decision-aids with an application to a system for dyspepsia, Statistics in Medicine. 2, 1983, pp.207-216.

Sullivan F., Mitchell E., Has general practitioner computing made a difference to patient care? A systematic review of published reports, British Medical Joumal, 311, 1995, pp.848-852.

Sutton, G.C., Computer-aided diagnosis a review, British Journal for Surgery, 76, 1989, p.8285 .

Ward C.D.. Decision making in refractory epilepsy. an analysis based on computer simulation, Journal ut Neurology. Neurosurgery and Psychiatry, 1989, 49, pp: 781-784. 
Wyatt J., Spiegelhalter, D. Evaluating medical expert systems: what to test and how? Medical Informatics, 15, 1990, pp.205-217. 


\section{Epilepsy}

\subsection{Introduction}

In this chapter both a scientific and a practical view on epilepsy are presented. The scientific part starts with defining the disease and provides a historical overview of various classifications. It shows the impact of technological and pharmacological research on the development of definitions and classifications. Furthermore, epidemiological data are presented. The prognosis and the implications of epilepsy for daily life of the patient will be addressed. Next the view is shifted towards daily practice. The problems the physician encounters when trying to relieve the patient from his seizures by using anti-epileptic drugs (AEDs) are discussed. After the diagnosis is established, a trade-off between the pros and the cons of drug treatment has to be made, given the specific situation of the patient. lssues such as when to start the drug treatment, which drug to select and what initial dose to prescribe will be discussed. As seizures may recur the start of drug treatment for epilepsy implies a longterm commitment to taking medication. Reasons for recurrence of seizures will be addressed. Furthermore the role of anti-epileptic drug monitoring in the treatment process will be discussed. Like in other diseases requiring long-term prophylactic drug treatment such as diabetes or hypertension non-compliance may occur. Causes of non-compliance will be mentioned. Many patients will show an improvement of their seizures. In this case drug withdrawal will be suggested by the patient or the doctor. Aspects of drug withdrawal and the risks of relapse will be described.

\subsection{Description of epilepsy}

Epilepsy is a paroxysmal disturbance of the functioning of the central nervous system. This disturbance is recurrent, stereotype in character and associated with spontaneous, excessive, synchronous and self-limiting neuronal discharge (Aird et al. 1984). It manifests itself in clinically detectable events, i.e. seizures which are perceptible for the subject and/or an observer. Thus, an epileptiform discharge in the EEG registration without a clinical manifestation is not called a seizure. The clinical detectability of an event may change and depends on the tests applied for its registration. For example, a 'bed-side' registration may not detect repeated cognitive impairment during a day while an advanced psychological test can register it 


\subsection{Classifications}

The classification of seizures and epileptic syndromes has changed over the years. This was caused partially by technical and pharmaceutical developments as a historical overview indicates.

\subsubsection{Historical overview}

The early classification of epilepsies was based on the aetiology, symptomatology or anatomy. The aetiology divided the epilepsies into two groups: those which could be attributed to gross cerebral lesions (symptomatic) and those without any identifiable cause (idiopathic) (Laidlaw et al. 1988)

The clinical findings regarding seizures were the basis for differentiation in classifications based on the symptomatology. It was not until 1946 that one could prescribe a drug treatment on the basis of the type of seizure present. At that time trimethadion was discovered. The drug turned out to be most effective against absence seizures (2-15 seconds disturbance or loss of higher psychic capabilities). This finding led to the conclusion that the drug treatment could depend on the type of seizure patterns and urged the need for a better classification of seizures (Cereghino, 1989).

The development of EEG-registration techniques in the late forties and the recognition of typical EEG patterns introduced another factor that could be used in the classification of epilepsies. Classifications of epilepsy based on aetiology (i.e. idiopathic or symptomatic) alone were considered to be inadequate for clinical applications at that time. The use of an EEG registration was required. This technological development resulted in 1954 in a new classification of the epilepsies into centre-encephalic and focal or cortical epilepsies (Dam and Kiørboe, 1982).

Table 2.3-I: International classification of epileptic seizures

\begin{tabular}{|c|c|c|}
\hline Partial scizures & Generalised seizures & Unclassified epileptic seizures \\
\hline A. Simple partial seizures & A. I. Absence seizures & (inadequate or incomplete \\
\hline 1. with molor syinploms & 2. Atypical absence seizures & data) \\
\hline 2. with somatosensory or & B. Myoclonic seizures & \\
\hline special sensory symptorns & C. Clonic seizures & \\
\hline 3. with autonomic symptoms & D. Tonic seizures & \\
\hline 4. with psychic symptoms & E. Tonic-clonic seizures & \\
\hline & F. Atonic seizures & \\
\hline
\end{tabular}

B. Complex partial scizures

Beginning as simple partial seizures and progressing to impainnent of consciousness

with no other features

with features as in A. $1-4$

with automatisnis

With impainment of consciousness at onset

with no other features

with features as in A. 14

with automatisms 
In 1969 a consensus classification for the epileptic seizures was accepted by the International League Against Epilepsy (ILAE) (Gastaut, 1970). A distinction was made between seizures starting locally and seizures being generalised from the onset. The epileptic seizures were categorised based on factors such as clinical features, EEG findings, age at onset, anatomic basis and the aetiology.

Further modification of the classification of epileptic seizures took place in 1981 when the attributes for classification were reduced to clinical manifestations (preferably recorded on videotape) and related EEG findings. This classification was accepted by the ILAE (Table 2.31). The eliminated factors such as aetiology, age at onset and anatomy were taken into account in the classification of epilepsies.

Besides the classification of seizures also a classification of the epilepsies was proposed (Merlis, 1970). The classification of epilepsies distinguished between generalised and partial epilepsies. Clinical characteristics such as aetiology, seizures, neurological status and age of onset were also used for classifying epilepsies. Still much overlap was present in the attributes used for classifying the seizures and epilepsies. In 1985 a new classification for epilepsies was proposed but it was not accepted until 1989 (Table 2.4-II). This classification retains the distinction of epilepsies in generalised and partial (localisation related) categories, based on the localisation of the onset of the seizures, on the cause (both idiopathic or symptomatic) and the age at onset. Furthermore two new categories were added:

- Epilepsies and syndromes of which it is not clear whether they are focal or generalised

- A category of special syndromes which is used for seizures occurring in special circumstances such as fever, intoxication or use of drugs (Commission on Classification and Terminology of the ILAE, 1989).

\subsubsection{Importance of different classifications}

Selection of the drug treatment can be based on the diagnosed seizure type and/or the type of epilepsy. The role of these detailed classification schemes in patient management is, however, rather limited. The seizure classification is mainly based on clinical findings and to a lesser extent connected with the neurophysiological and chemical basis of the convulsions. In fact the classification scheme is more detailed than the knowledge about basic mechanisms of available anti-epileptic drugs (Dichter 1994, Macdonald and Kelly 1994, Montouris 1995, Brodie and Dichter 1996).

The classification of the epilepsies includes factors such as aetiology and natural history which allows for a more complete description of the patient enabling an assessment of the prognosis. Although there are special types of epilepsy which require a specific drug treatment the classification of epilepsies is more detailed then necessary for drug treatment

More recently, new definitions of epilepsy and seizures and seizure type classification have been proposed to bridge the gap between research and patient management (Commission on Epidemiology and Prognosis of the ILAE, 1993)

\subsection{Epidemiology}

There are two main problems in obtaining statistics on epilepsy. The first problem is related to the definition of the disease. The second problem is caused by the sources from which data are gathered 
Table 2.4-II: International classification of epilepsics, epilcptic syndromes and related seizure disorders (ICE)

\begin{tabular}{|c|c|}
\hline Localisation related (focal, local, partial) & Generalised \\
\hline $\begin{array}{l}\text { Idiopathic (Primary) } \\
\text { Benign childhood epilepsy with centre-temporal spike } \\
\text { Childhood epilepsy with occipital paroxysins } \\
\text { Primary reading epilepsy }\end{array}$ & $\begin{array}{l}\text { Idiopathic (primary) } \\
\text { Benign neonatal farnilial convulsions } \\
\text { Benign neonatal convulsions } \\
\text { Benign myoclonic epilepsy in infancy } \\
\text { Childhood absence epilepsy (pyknolepsy) } \\
\text { Juvenile absence epilepsy } \\
\text { Juvenile myoclonic epilepsy (impulsive petit mal) } \\
\text { Epilepsies with grand mal seizures (GTCS) on awakening } \\
\text { Other generalised idiopathic epilepsies } \\
\text { Epilepsies with seizures precipitated by specific modes of } \\
\quad \text { activation }\end{array}$ \\
\hline $\begin{array}{l}\text { Symptomatic (secondary) } \\
\text { Temporal, frontal, parietal and occipital lobe epilepsies } \\
\text { Chronic progressive epilepsia partialis continua of } \\
\text { childhood } \\
\text { Syndrones characterised by seizures with specilic modes } \\
\text { of precipitation }\end{array}$ & $\begin{array}{l}\text { Cryptogenic or synptomatic } \\
\text { West syndrome (infantile spasins, Blitz-Nick-Salaam } \\
\text { Krämpfe) } \\
\text { Lennox-Gastaut syndrome } \\
\text { Epilepsy with myoclonic-astatic seizures } \\
\text { Epilepsy with myoclonic absences } \\
\text { Symptomatic } \\
\text { Non-specific aetiology } \\
\text { Early myoclonic encephalopathy } \\
\text { Early infintile epileptic encephalopathy } \\
\text { with suppression burst } \\
\text { Other symptomatic generalised } \\
\text { epilepsies } \\
\text { Specific syndromes } \\
\text { Epileptic scizures may complicate many } \\
\text { disease states }\end{array}$ \\
\hline \multicolumn{2}{|l|}{$\begin{array}{l}\text { Defined by seizure type, clinical features, aetiology, } \\
\text { anatomical localisation }\end{array}$} \\
\hline $\begin{array}{l}\text { Undetermined epilepsies } \\
\text { With both generalised and focal scizures } \\
\text { With unequivocal generalised or focal features }\end{array}$ & \\
\hline $\begin{array}{l}\text { Spccial syndromes } \\
\text { Situation related seizures (Gelegenheitsanfälle) } \\
\text { Febrile convulsions } \\
\text { Isolated seizures or isolated status epilepticus }\end{array}$ & \\
\hline $\begin{array}{l}\text { Seizures occurring only when there is an acute or } \\
\text { nonketotic hyperglycaemia }\end{array}$ & xic event due to faclors such as alcohol, drugs, eclampsia, \\
\hline
\end{tabular}




\subsubsection{Problems}

The translation of the definition of epilepsy provided by Gastaut and Kugler (1976) and used by the WHO is:

\section{'A chronic brain disorder of various aetiologies characterised by recurrent seizures dive to excessive discharge of cerebral neurones [...]. Single or occasional epileptic seizures as well as those occurring during an acute illness should not be classified as epilepsy'.}

The problem is that this definition is not operational enough. The definition excludes the use of the term epilepsy for seizures provoked by disorders primarily not involving the brain. The International Classification of the Epilepsies does include these as special syndromes. The term 'chronic' is ambiguous. It does not state the period during which the symptoms must be present before one can decide whether the patient really has epilepsy. This means that patients who suffer from several seizures per year as well as those with one seizure every two years can be included as epileptic patients in epidemiological studies. Whether or not seizures should still be manifest to be regarded as epilepsy not withstanding medication is not clear from this definition. It is also not clear when patients are considered to be cured.

The second part of the definition does exclude seizures directly related to a disease. Examples of such seizures are febrile convulsions and sporadic seizures caused by e.g. alcohol withdrawal. However, others claim that there exists insufficient ground to exclude occasional seizures from the diagnosis (Hopkins, 1987).

The ascertainment of epileptic cases for epidemiological studies may be a problem (Aird et al. 1984). The use of medical records results in an underrepresentation of the milder and nonactive epilepsies as well as of undiagnosed and untreated cases, as some patients may not ask for a medical evaluation of their symptoms (Haerer et al. 1986). The severity of the seizures, the social labelling because of the disease, the educational level or the socio-economic status and consequences may influence the decision to consult a health care professional. Field studies suggest that some 4 to $25 \%$ of the patient with epilepsy were never treated for their seizures (Haerer et al. 1986, Zielinski 1988, Keränen and Riekkinen 1993). The range in percentages may be attributed to different case finding methods and diagnostic criteria.

Patient denial also influences epidemiological data obtained from medical records. In an Italian study denial of the disease was reported in $31 \%$ of a patient population (Maremmani et al. 1991).

Data can be obtained from sources other than the medical record, e.g. death certificates and insurance company data. These alternatives have their own biases. Death certificates are generally available but incomplete in reporting epilepsy. Data obtained from insurance companies mainly present a selected population, which may exclude e.g. unemployed people or people with a low economic status. Additional biases may exist due to patients who do not reveal their disease to an insurance company to prevent exclusion or higher financial contributions.

\subsubsection{Prevalence and incidence}

The number of patients per 1000 persons in a given population at a given time is called the prevalence of the disease. The influence of how epilepsy is defined on prevalence data has been demonstrated in two studies concerning 6000 patients from a general practice (Goodridge and Shorvon 1983a, Cockerell et al. 1995a). 
Table 2.4-I: Prevalence of epilepsy in Europe and United Statcs

\begin{tabular}{|c|c|c|c|c|c|c|}
\hline Country & First author & Definition & Study & Sources" & Age & Prev** \\
\hline \multirow[t]{3}{*}{ Denumark } & Juul- Jensen & epilepsy ${ }^{\prime}$ & retrospective & $\mathrm{MH} / \mathrm{II} / \mathrm{DR}$ & & $12.7^{2}$ \\
\hline & & & 37 ytars & & & \\
\hline & Wagner (1983) & active epilepsy ${ }^{3}$ & retrospective ${ }^{4}$ & $\begin{array}{l}\text { MH/EH/DPP } \\
\text { GP }\end{array}$ & $16-66$ & 4.32 \\
\hline Finland & Keränen (1989) & active epilepsy & $\begin{array}{l}\text { retrospective } \\
19 \text { years }\end{array}$ & $\begin{array}{l}\mathrm{MH} / \mathrm{Ps} / \mathrm{EH} \\
\mathrm{MMR} / \mathrm{HC} / \mathrm{NH} \\
\mathrm{EL} / \mathrm{PR}\end{array}$ & $>15$ & 6.29 \\
\hline \multirow[t]{2}{*}{ Italy } & Granieri (1983) & active epilepsy & $\begin{array}{l}\text { retrospective } \\
18 \text { years }\end{array}$ & $\begin{array}{l}\text { MH/ND/NS } \\
\text { PD/EL/LD/DPP } \\
\mathrm{Ch} / \mathrm{T} / \mathrm{SW} \\
\mathrm{MeH}\end{array}$ & all & $(6.4)^{5}$ \\
\hline & Mareminani (1991) & active epilepsy & retrospective & $\begin{array}{l}\text { H/LD/PR/EHn } \\
\text { SD/SW }\end{array}$ & all & $\begin{array}{l}5.1 \\
(5.2)^{5}\end{array}$ \\
\hline \multirow[t]{2}{*}{ Norway } & De Gruaf (1974) & epilepsy & retrospective & ND/EL/IMR & all & 3.5 \\
\hline & & & 5 years & & & \\
\hline Sweden & Forsgren (1992) & active epilepsy & retrospective & ND/NS/M/Ps & $17^{x}$ & 5.5 \\
\hline & & & 13 years & $\begin{array}{l}\text { GD/EL/SLR } \\
\text { DPP/DN }\end{array}$ & & \\
\hline \multirow{4}{*}{$\begin{array}{l}\text { The Nether- } \\
\text { lands }\end{array}$} & Bongers (1976) & epilepsy & retrospective & GP & all & 2.9 \\
\hline & Rutgers (1984) & $\begin{array}{l}\text { active epilcpsy } \\
\text { last } 2 \text { yeatss }\end{array}$ & prospective & GP & $15-66$ & 4.0 \\
\hline & Crobach (1988) & active epilepsy & retrospective & GP/Ch & all & 3.3 \\
\hline & Metsemakers (1994) & epilepsy ${ }^{7}$ & retrospective & GP & all & 8.5 \\
\hline \multirow[t]{3}{*}{$\begin{array}{l}\text { United King- } \\
\text { dom }\end{array}$} & Crombie (1960) & $\begin{array}{l}\text { active epilepsy } \\
\text { last } 2 \text { years }\end{array}$ & $\begin{array}{l}\text { retrospeclive } \\
3 \text { years }\end{array}$ & GP & all & 4.19 \\
\hline & Goodridge (1983a) & $\begin{array}{l}\text { active epilepsy } \\
\text { last } 2 \text { years }\end{array}$ & $\begin{array}{l}\text { retrospective } \\
35 \text { years }\end{array}$ & GP & all & 5.3 \\
\hline & Cockerell (1995a) & $\begin{array}{l}\text { active epilepsy } \\
\text { last } 2 \text { years }\end{array}$ & $\begin{array}{l}\text { retrospective } \\
10 \text { years }\end{array}$ & GP & all & 4.3 \\
\hline \multirow[t]{3}{*}{ Uniled States } & Hauser $(1975)$ & active epilepsy & $\begin{array}{l}\text { retrospective } \\
25 \text { yuirs }\end{array}$ & HIS/MH & all & 5.7 \\
\hline & Haerer (1986) & active epilepsyn & retrospective & $\begin{array}{l}\text { door-10-door } \\
\text { MI LNH }\end{array}$ & all & 6.78 \\
\hline & Hauser (1991) & uctive epilepsy & $\begin{array}{l}\text { retrospective } \\
40 \text { years }\end{array}$ & $\mathrm{H} \amalg \mathrm{S} / \mathrm{MH}$ & all & 6.8 \\
\hline
\end{tabular}

- $\mathrm{Ch}=$ Chemist, $\mathrm{DR}=$ Death register, EL=EEG-lab, EH=Epilepsy hospilal, H=Hospital, HIS=Hospital information system, $\mathrm{MH}=$ Multiple hospitals, MeH=Mental hospital, $\mathrm{HC}=$ Health centre, $\mathrm{LD}=\mathrm{Local}$ doctors, $\mathrm{ND}=\mathrm{New}$ ology dept, $\mathrm{NS}=$ Neurosurgery dept., $\mathbb{M}=\mathrm{Dept.}$ Internal Medicine, $\mathrm{IR}=$ Insurance register, $S \mathbb{R}=\mathrm{Social}$ insurance register, $\mathrm{SD}=\mathrm{Sch} \infty \mathrm{l}$ doctors, $S W=$ Social workers, $P D=$ Pediatric Dept., $G D=$ Geriatrics Dept., GP=General practitioner, PSD=Psychiatry dept, $\mathrm{DPP}=\mathrm{Doctors}$ with private practice. $\mathrm{DN}=$ District uurses, $\mathrm{DMR}=$ Inst. mentally retarded, $\mathrm{NH}=$ Nursing homes, $\mathrm{PR}=\mathrm{Pharmacy}$ register, $T=$ Teacher,

** per 1000 population

(1) Epilepsy without exclusion of patients seizure free for more than 5 years and not on anti-epileptic drug treatment, (2) Cumulative incidence, (3) Epilepsy $\geq 2$ spontaneous seizures excluding patients with controlled seizures and not receiving treatunent, (4) Registration depending on setting. $1-18$ years, (5) Prevalence corrected toward the Italian population, (6) On AED and having $\geq 1$ seizure in 3 years or not on AED and having $\geq 1$ seizure within last year, (7) active and inactive epilepsy. 
The prevalence of epilepsy excluding febrile seizures but including cases such as single, recurrent, active and inactive epilepsy was 20.3 and 21 in the 1983 and 1993 studies respectively. This number dropped to 17.0 by excluding patients with single seizures. An even lower prevalence - 10.5 - was found by including only those patients receiving anti-epileptic treatment and those having active epilepsy defined as at least one seizure within the last 24 months. Prevalence further decreased to 5.3 in 1983 and to 4.3 in 1993 by including only patients with active epilepsy. In the USA improved case ascertainment strategies resulted in different prevalence rates in the same population studied (Hauser and Kurland 1975, Hauser et al. 1991). The use of broad inclusion criteria - not excluding patients free from seizures for more than 5 years and including patients not on anti-epileptic drug treatment - caused the high prevalence rate of the Danish study (Table 2.4-I) (Juul-Jensen and Foldspang, 1983). The minimal prevalence rate of convulsive disorders was estimated to be $4-6 / 1000$ (Cereghino 1974, Rutgers 1984).

The number of new cases within 1 year per 100000 persons is called the incidence of a disease. It describes the rate with which new cases of the disease occur. The incidence rates suffer the same problems as the prevalence. The classic study of incidence is from Rochester, Minnesota. In this study it was shown that the incidence of recurrent seizures was 44 patients per 100000 persons per year over a 50 year period (Hauser et al. 1993). Similar results with average annual incidence rates for recurrent seizures in between 30 and 50 were reported previously (Hauser 1978, Granieri et al. 1983. Zielinski 1988, Keränen et al. 1989, Forsgren 1990). The incidence increased to 61 per year if cases with single seizures are included (Hauser et al. 1993). Inclusion of febrile seizures leads to an even higher incidence, 115 per year (Hauser, 1978)

Incidence rates vary with age. The incidence rate is highest during the first decade, after which it decreases. An increase in the number of new cases is observed after the sixth decade (Zielinski 1974, Lühdorf et al. 1986, Zielinski 1988, Hauser et al. 1991, Hauser 1992). While the incidence for the population was 44 , the highest incidence, 139, was observed in persons over 75 years of age (Hauser et al. 1993). The increase in the number of tumours and cerebrovascular diseases has been suggested as a possible cause. Another explanation for the increase might be the higher level of case ascertainment for elderly people or more extensive medical care for this group (Schoenberg 1983, Hauser et al. 1993)

The incidence rates for children and elderly persons have been compared over the years. These measurements show that the incidence for children decreases while the incidence rate for the elderly increases (Hauser et al. 1993, Cockerell et al. 1995a). These changes may have been caused by improved perinatal care and increased medical attention for the elderly.

\subsection{Aetiology}

Determining the type of seizure and the type of epilepsy is only half of the diagnostic process. Establishing the aetiology is the other. Aird et al (1984) use both causative and underlying neurochemical and neurophysiological factors to describe the aetiology. These factors may suggest possibilities for the treatment of seizures. The main causative factors for epilepsy are genetic and birth defects, infections, toxic mechanisms, traumata, circulatory causes (e.g. haemorrhage, thrombosis), metabolic and nutritional disorders, neoplasms, heredofamilial and degenerative diseases. The neurochemical description of etiologic factors for epilepsy include metabolic derangements as e.g. hypoxia, hypoglycaemia, electrolyte and acid-base imbalances, vitamin deficiencies, changes in excitatory and inhibitory neurotransmitters, endocrine 
dysfunctions, endogenous toxic states and hyperpyrexia. Most epidemiological studies reporting on aetiology, however, describe only the causative factors. Another approach is to distinguish between cause - a more or less steady-state background factor - and precipitating factors which are short term factors which may cause seizures in susceptible people (Hopkins, 1987).

Table 2.5-1: Ethiological factors in epilepsy

\begin{tabular}{llllllllll}
\hline First author & $\begin{array}{l}\text { De Graal } \\
(1974)\end{array}$ & $\begin{array}{l}\text { Bergamini } \\
(1977)\end{array}$ & $\begin{array}{l}\text { Hauser } \\
(1978)\end{array}$ & $\begin{array}{l}\text { Granieri } \\
(1983)\end{array}$ & $\begin{array}{l}\text { Cowan } \\
(1989)\end{array}$ & $\begin{array}{l}\text { Mcinardi } \\
(1990)\end{array}$ & $\begin{array}{l}\text { Sander } \\
(199)\end{array}$ & $\begin{array}{l}\text { Hauser } \\
(1991)\end{array}$ & $\begin{array}{l}\text { Forsgren } \\
(1992)\end{array}$ \\
\hline Age & $>15 y$ & $1 \mathrm{~m}-21 \mathrm{y}$ & all & all & $<19$ y & all & all & all & $>16$ y \\
Unknown & $68 \%$ & $56 \%$ & $64 \%$ & $60.9 \%$ & $69 \%$ & $28.8 \%$ & $61 \%$ & $76 \%$ & $64.8 \%$ \\
Cer.vase. & $4 \%$ & nav & $5 \%$ & $2.9 \%$ & 3 & $1.9 \%$ & $15 \%$ & $6 \%$ & $11.8 \%$ \\
Cer.tumor & $2 \%$ & nav & $4 \%$ & $1.4 \%$ & 3 & $3.5 \%$ & $6 \%$ & $2 \%$ & $4.7 \%$ \\
Alc./loxic & $3 \%$ & excl. & excl. & nav & $2 \%$ & nav & $6 \%$ & excl. & excl. \\
Post-traum. & $9 \%$ & $12.2 \%$ & $5 \%$ & $6.8 \%$ & $4 \%$ & $10.4 \%$ & $3 \%$ & $5 \%$ & $7.2 \%$ \\
Cong./Gen. & $2 \%$ & $1.9 \%$ & $4 \%$ & $1.1 \%$ & $3 \%$ & $3.2 \%$ & 4 & $5 \%$ & $2.6 \%$ \\
Lufections & $2 \%$ & $11.9 \%$ & $3 \%$ & $3.7 \%$ & $3 \%$ & $5.1 \%$ & $2 \%$ & $4 \%$ & $3.5 \%$ \\
Perinatal & $9 \%$ & $17.8 \%$ & nav & $23.7 \%$ & $7 \%$ & $40.2 \%$ & 4 & nav & $5.4 \%$ \\
Febr.conv. & nav & excl. & nav & nav & 3 & $6.9 \%$ & 4 & nav & nav \\
Other cause & nav & nav & $15 \%$ & nav & 3 & nav & $7 \%$ & $1 \%$ & nav \\
\hline
\end{tabular}

(1) data from tertiary care centre, (2) nav, (3) other and multiple causes $12 \%$, (4) this included congenital perinatal and febrile convuisions, (5)history of febrile convulsions

Studies indicate that in $56-76 \%$ of the patients the aetiology of their epilepsy is unknown (Table 2.5-1). Part of this depends on the assessment of a genetic basis for idiopathic epilepsies. As multiple genes may be involved a family history will underestimate genetic causes. Differences in case finding strategies are also important. For example, a high rate of perinatal causes reported by Granieri et al. (1983) can be explained by the fact that this aetiology was more intensely looked for as there was an urgency in improving the prenatal and perinatal care in the region. The study conducted by Meinardi and Pachlato (1985) shows fewer unknowns and a higher percentage of perinatal factors in a population of a special centre for epilepsy.

The aetiology of epilepsy varies with the age of the patient (Janz 1969, Sander et al. 1990, Hauser 1992). In the neonatal period birth-injuries - haemorrhage and anoxia - may cause epilepsy. Also congenital abnormalities, metabolic disorders and infections can induce epileptic seizures. In early childhood febrile seizures, trauma infection, cerebral degeneration, poisons and metabolic defects may cause epilepsy. In childhood and adolescence idiopathic (primary) epilepsy may manifest itself. In addition to this, trauma, infections and cerebral degeneration can induce epilepsy during this period. It has been reported that in $67.6 \%$ of the patients younger than 15 years the cause of the epilepsy was unknown, while in $20 \%$ a congenital defect, in $4.7 \%$ a trauma and in $4 \%$ an infection was identified as the cause of their disease (Hauser, 1992). In early adult life trauma, tumour or infection may cause epilepsy while juvenile idiopathic epilepsies also occur. Birh injuries and cerebral degeneration can still play a role as a cause of epilepsy in this period 
During late adult life vascular disease, trauma, tumour and cerebral degeneration are the most frequent causes for epilepsy (Marsden and Reynolds, 1988). Also alcohol abuse has been mentioned as a frequent cause of epilepsy. In a study on late-onset epilepsy (starting after the age of 25 years) made up a group of one fourth of all patients, while the cause was unknown in $38 \%$. In $16 \%$ of the patients a brain tumour was the cause, cerebrovascular infarctions were registered in $14 \%$ of the patients. Head trauma accounted for $4 \%$ of the cases and miscellaneous causes such as angioma, brain abscess or leukoencephalopathy accounted for $5 \%$ of the patients (Mouritzen Dam et al. 1985). In a field study in elderly over 65 years the percentage of cases with an unknown cause was $48.9 \%$, while $32.4 \%$ had a vascular cause. A degenerative cause was reported in $11.5 \%$ and neoplasms were reported in $2.7 \%$ of these cases (Hauser, 1992). Others reported from a clinical setting that in patients over 60 years cerebrovascular disease was the cause in $32 \%$, tumours in $14 \%$ while in $25 \%$ the cause was unknown (Lühdorf et al. 1986).

\subsection{Prognosis}

There are four possible courses for epilepsy:

- Seizures remit spontaneously and no treatment is required

- Seizures are controlled with drug treatment and the medication can successfully be withdrawn

- Seizures are controlled but anti-epileptic drug treatment must be continued

- Seizures continue even when on anti-epileptic drug treatment

While problems may occur in all four groups, drug related and/or psychosocial problems can be expected, particularly in the third and the fourth category. As these groups require a lifelong treatment, the etiological classification of the epilepsy may change e.g. due to a gradual manifestation of an oncologic cause. This means that in these patients the correctness of the diagnosis has to be periodically reviewed. A sudden change in effectivenuss of the treatment due to alterations in the metabolism of the drug, concomitant diseases or comedication may occur. Drug toxicity may develop gradually, especially with multiple drugs. Besides this, the compliance can change because of side-effects of the drugs or absence of seizures or due to avoiding the daily confrontation with the disease. Such a change in compliance may lead to recurrence of seizures. Also an irregular life-style may alter the effectiveness of the treatment. Patients in both these categories will need attention from the physician on a regular basis.

\subsubsection{Remission}

The idea that epilepsy is a chronic condition especially if left untreated - seizures beget seizures - has been stated already in the last decades of the $19^{\text {th }}$ century (Gowers, 1885 , reprinted in 1994). This idea has lasted for over 80 years. However, this view is gradually changing. Population studies indicate that $4.1 \%$ to $26 \%$ of the patients with epilepsy do not receive anti-epileptic drug treatment (Zielinski 1974, Goodridge and Shorvon 1983b, Haerer et al. 1986, Keränen and Rjekkinen 1993, Cockerell et al. 1995a). In some of these patients epilepsy remitted spontaneously. It is however difficult to be certain of the diagnosis of epilepsy in these patients who reported seizures in the past but on index day had been free from seizures for several years. The number of patients reported to be seizure free for at least 2 years at 10 and 20 years after onset of the epilepsy were $42 \%$ and $52 \%$ respectively 
(Keränen and Riekkinen, 1993). Some 30\% were seizure free for more than 5 years (Zielinski, 1974). Comparison of incidence and prevalence rates indicates that epilepsy lasts 13 years on average. More recent studies in developing countries also suggest that epilepsy may remit without drug treatment (Sander, 1993).

In the traditional view on epilepsy the prognosis was considered poor with only $20 \%$ going into a long-term remission. In the last two decades studies on remission of seizures show that $35-90 \%$ of patients can achieve a remission of 2 years during some period within 2 to 10 years after starting drug treatment (Shorvon and Reynolds 1982, Elwes et al. 1984, Collaborative Group for Study of Epilepsy 1992). Remission of seizures lasting at least 5 years were reported in $61 \%$ of newly diagnosed patients 10 years after the diagnosis. The chance of having achieved a 5 year remission within 10 and 20 years was $65 \%$ and $76 \%$ respectively (Annegers et al. 1979). A similar result - 68\% seizure free for 5 years or more during nine years of follow-up - was found in a national general practitioners study (Cockerell et al. 1995b).

This means that many patients tend to go into a long-term remission during follow-up. At 20 years after diagnosis $70 \%$ of the patients were in remission (Annegers et al. 1979)

Part of the patients with a remission need medication continuously as the anti-epileptic drugs only suppress the occurrence of seizures instead of curing the disease. While $70 \%$ of the patients were in remission some $50 \%$ were seizure free for 5 years and did not take anticonvulsive medication 20 years after the initial diagnosis, while $20 \%$ of the patients continued to take anti-epileptic drugs although they were free for at least 5 years without seizures (Annegers et al. 1979). Others report that 13\% of the patients which were at least 3 years seizure free after 9 years of follow-up were still taking anti-epileptic drugs (Cockerell et al. 1995b).

Table 2.6-I: Remission rates of scizures in community and hospital based studies.

\begin{tabular}{|c|c|c|c|c|c|c|}
\hline First author & $\begin{array}{l}\text { Annegers } \\
(1979) \\
N=618\end{array}$ & $\begin{array}{l}\text { Elwes } \\
(1984) \\
N=106\end{array}$ & $\begin{array}{l}\text { Zielinski } \\
(1988) \\
N=98\end{array}$ & $\begin{array}{l}\text { CGSE }^{\prime} \\
(1992) \\
N=280\end{array}$ & $\begin{array}{l}\text { Keränen }^{2} \\
(1993) \\
N=33\end{array}$ & $\begin{array}{l}\text { Cockerell } \\
(1995 b) \\
N=564\end{array}$ \\
\hline Study & retrospective & prospective & prospective & prospective & retrospective & prospective \\
\hline Setting & $\begin{array}{l}\text { community } \\
\text { based }\end{array}$ & neur.clin. & $\begin{array}{l}\text { community } \\
\text { based }\end{array}$ & hospital & hospital & $\begin{array}{l}\text { community } \\
\text { based }\end{array}$ \\
\hline $\begin{array}{l}\text { Follow-up } \\
\text { duration }\end{array}$ & $\begin{array}{l}328>10 \text { years } \\
141>20 \text { years }\end{array}$ & $\begin{array}{l}\text { median } 66 \mathrm{mths} \\
\text { (range 6-96) }\end{array}$ & 5 years & $\begin{array}{l}\text { median } 48 \text { mths } \\
\text { (range } 1-87 \text { ) }\end{array}$ & $>2$ years & median 7.1 ys \\
\hline Remission & $\geq 5$ years & $\geq 2$ years & $\geq 2$ years & $\begin{array}{l}\geq 2 \text { and }<3 \\
\text { years }\end{array}$ & $\geq 2$ years & $\begin{array}{l}>3 \text { years } \\
>5 \text { years }\end{array}$ \\
\hline \multirow[t]{2}{*}{$\begin{array}{l}\text { \%opatients } \\
\text { achicving } \\
\text { remission }\end{array}$} & $\begin{array}{l}65 \% \text { at } 10 \text { ys } \\
\text { f.u }{ }^{3} \\
76 \% \text { at } 20 \text { ys } \\
\text { f.u. }{ }^{3}\end{array}$ & $\begin{array}{l}35 \% \text { at } 2 \text { ys } \\
6 u^{3} \\
57 \% \text { at } 3 \text { ys } \\
\text { fu? }\end{array}$ & & $\begin{array}{l}71 \% \text { at } 3 \text { ys } \\
\int .{ }^{3} \\
90 \% \text { at } 5 \text { ys } \\
\text { f.u. }{ }^{3}\end{array}$ & & $\begin{array}{l}68 \% \text { at } 9 \text { ys } \\
\text { f.u. in } 3 \text { year } \\
\text { remission }\end{array}$ \\
\hline & $\begin{array}{l}61 \% \text { at } 10 \text { ys } \\
\text { f.u. } \\
70 \% \text { at } 20 \text { ys } \\
\text { f.u. }\end{array}$ & $\begin{array}{l}79 \% \text { al } 5 \text { ys } \\
\text { f.u }{ }^{3} \\
82 \% \text { at } 8 \text { ys } \\
\text { r.u. } \\
48 \% \text { tree } 2 y^{4} s^{3}\end{array}$ & $\begin{array}{l}6.3 \% \text { at } 5 \text { ys } \\
\text { f.u. }\end{array}$ & & $\begin{array}{l}42 \% \text { at } 10 \text { ys } \\
\text { fu. }{ }^{4} \\
52 \% \text { at } 20 \text { ys } \\
\text { f. }{ }^{4}\end{array}$ & $\begin{array}{l}54 \% \text { at } 9 \text { ys } \\
\text { f.u. in } 5 \text { year } \\
\text { remission }\end{array}$ \\
\hline
\end{tabular}

(1) Collaborative Group for Study of Epilepsy, (2) Untreated epilepsy patients, (3) Cumulative percentage, not considering the continuation of the remission until end of study, (4) Percentage of seizure free patients at moment of follow-up (f.u.). 
It is estimated that in $20-30 \%$ of the patients seizures will continue despite anti-epileptic drug treatment (Annegers et al. 1979, Goodridge and Shorvon 1983b, Shorvon 1991, Cockerell et al. 1995a). Eight percent of the patients does not achieve a seizure free period of one year during 8 years of follow-up. A two year seizure free period was not reached by $18 \%$ of the patients (Elwes et al. 1984). Nevertheless, even those patients who are not in remission 5 years after diagnosis may still have a chance of $33 \%$ of going into a remission during the next 10 years (Annegers et al. 1979).

Differences in remission rates may be attributed to case ascertainment, e.g. hospital based studies contain an overrepresentation of the chronic uncontrolled patients, with newly diagnosed patients and patients in remission are underrepresented. Furthermore, differences in duration of follow-up or discrepancies in the definition of the term remission may account for the differences in the percentages of people who are seizure free (Shorvon, 1984).

Also the definition of epilepsy is important for assessing the remission rates. Several studies show a higher remission rate when patients with possible epilepsy are included in the study (Reynolds 1987, Hart et al. 1990, Collaborative Group for Study of Epilepsy 1992, Cockerell et al. 1995a,b). All studies presented in Table 2.6-I include patients with more than one seizure.

\subsubsection{Problems in establishing prognostic factors}

Although some studies suggest that epilepsy may remit spontaneously most studies appear to support an observation made by Gowers that epilepsy is a process which in case it is not treated early may lead to a chronic state of seizures which are more difficult to treat later on (Elwes et al. 1988, Reynolds 1988, Elwes and Reynolds 1990, Reynolds 1990). In this view factors influencing the outcome of the disease are important.

Studies concerning the prognosis of epilepsy are difficult to assess as their results depend on the medical setting where the data are obtained. In contrast with studies in a clinical setting one may expect higher remission rates of epilepsy in community based studies, given the fact that some of the patients do not visit their physician because the seizures do not worry them (Zielinski 1974, Haerer et al. 1986, Keränen and Riekkinen 1993).

If studies on epilepsy are undertaken with patients who had only one seizure, patients and doctor's delay may introduce a bias towards less severely diseased patients. The longer the delay the higher the probability that the patient suffered another seizure and is not included in the study.

\subsubsection{Prognostic factors}

In the literature various conditions are mentioned that suggest a poor prognosis (Annegers et al. 1979, Shorvon and Reynolds 1982, Elwes et al. 1984, Shorvon 1984, Beghi and Tognoni 1988, van Donselaar 1990, Collaborative Group for Study of Epilepsy (CGSE) 1992):

- A high frequency ( $\geq 2$ per month) of tonic-clonic seizures before treatment

- More than one seizure in the first year of treatment or a relapse early during treatment

- Multiple types of seizures or partial seizures

- Additional neurologic, psychiatric or social handicaps as well as a family history of epilepsy 
Table 2.6-II: Prognostic factors for the course of epilepsy.

\begin{tabular}{|c|c|c|c|c|c|c|}
\hline First author & $\begin{array}{l}\text { Annegers } \\
(1979) \\
N=618\end{array}$ & $\begin{array}{l}\text { Shorvon } \\
(1982) \\
N=94\end{array}$ & $\begin{array}{l}\text { l:lwes } \\
(1984) \\
N=106\end{array}$ & $\begin{array}{l}\text { Hopkins } \\
(1988 \text { ) } \\
N=408\end{array}$ & $\begin{array}{l}\text { CGSE } \\
(1988) \\
N=283\end{array}$ & $\begin{array}{l}\text { v. Donselaar } \\
(1990) \\
N=151\end{array}$ \\
\hline Study & retrospective & prospective & prospective & prospective & prospective & prospective \\
\hline Setting & $\begin{array}{l}\text { community } \\
\text { based }\end{array}$ & neur.clinic & neur clinic & $\begin{array}{l}\text { in/out } \\
\text { private pat }\end{array}$ & multicentre & hospital \\
\hline $\begin{array}{l}\text { Type of } \\
\text { patients }\end{array}$ & new referrals & $\begin{array}{l}\text { new referrals } \\
6-77 \text { years } \\
\mu=23\end{array}$ & $\begin{array}{l}\text { new referrals } \\
6-77 \text { years } \\
\mu=23\end{array}$ & $\begin{array}{l}\text { new referrals } \\
>16 \text { years }\end{array}$ & $\begin{array}{l}\text { new referrals } \\
2-81 \text { years } \\
\mu=19\end{array}$ & $\begin{array}{l}\text { new referrals } \\
15-85 \text { years } \\
\mu=38\end{array}$ \\
\hline $\begin{array}{l}\text { Moment of } \\
\text { inclusion }\end{array}$ & $>2$ seizures & $>2$ seizures & $>2$ seizures & $I^{\text {st }}$ seizure & $\begin{array}{l}\text { start treat- } \\
\text { ment }\end{array}$ & $1^{\text {st }}$ seizure \\
\hline $\begin{array}{l}\text { Therapy } \\
\text { before } 2^{\text {nd }} \\
\text { seizure }\end{array}$ & included & excluded & excluded & included & excluded & excluded \\
\hline Follow-up & $\begin{array}{l}328>10 \text { years } \\
141>20 \text { years }\end{array}$ & 13-33 months & $6-96$ months & 36 months & 2.40 months & 12-24months \\
\hline Age & & not related & & not related & & \\
\hline Sex & not related & not related & & & & \\
\hline $\begin{array}{l}\text { Seizure } \\
\text { type }\end{array}$ & related & & $\begin{array}{l}\text { partial seizu- } \\
\text { res related }\end{array}$ & not related & $\begin{array}{l}\text { multiple sei- } \\
\text { zures related }\end{array}$ & $\begin{array}{l}\text { multiple sei- } \\
\text { zures related }\end{array}$ \\
\hline $\begin{array}{l}\text { Seizures } \\
\text { before/anter } \\
\text { therapy }\end{array}$ & & related & related & & related & \\
\hline $\begin{array}{l}\text { Age at on- } \\
\text { set }\end{array}$ & related & not related & not related & not related & & not related \\
\hline Histories & & not related & $\begin{array}{l}\text { family history } \\
\text { related }\end{array}$ & not related & & not related \\
\hline Time of day & & & not related & related & & related \\
\hline Handicap & related & related & related & & & \\
\hline EEG & & not related & not related & not related & & related \\
\hline
\end{tabular}

Factors not related to the prognosis or factors about which disagreement with respect to their relation to the prognosis of epilepsy exists in the literature (Table 2.6-II) are (Annegers et al. 1979, Shorvon and Reynolds 1982, Elwes et al. 1984, Hopkins et al. 1988, van Donselaar 1990):

- EEG findings after a first idiopathic seizure. Some found a relation between (pretreatment) EEG findings and seizure recurrence. Others, however, did not find such a relation.

- The importance of the time during the day at which the seizure occurs. Some researchers did report a relation between time of the day and the risk of recurrence others deny a prognostic value for this factor. 
- Factors such as gender, age or age at onset. Some report a relation between age at onset and the probability of going into remission. Especially younger patients with idiopathic epilepsy were likely to become seizure free. Such a relation was not observed by others.

\subsection{Some implications of epilepsy}

Epilepsy may have various consequences in daily life. People may experience difficulties in finding and keeping a job, their drivers licence may be withdrawn or they have to find alternative leisure activities.

\subsubsection{Employment}

Difficulties with employment is one of the problems most frequently reported by patients (Thompson and Oxley 1988, Chaplin et al. 1992). The problem seems to be of less relevance during periods of full employment (Crombie et al. 1960). However, during a period of high unemployment it has been shown that $46 \%$ of the potentially economically active patients were unemployed compared with $19 \%$ of a control population (Elwes et al. 1991). The employers as well as colleagues (even within health care environments) have an exaggerated fear of accident-proneness and decrease of productivity (Aspinal 1986, Betts 1986). Contrary to the expectations of employers, sickness absence, injuries and accident frequencies and productivity are about the same for adults with and without epilepsy (Rutgers 1984, Lechtenberg 1985, Dick 1986). Although only some jobs have been affected by statutory barriers, safety concerns were mentioned as the main reason for not hiring people with epilepsy over the years (Craig and Oxley 1986, Hicks and Hicks 1991)

Guidelines have been suggested for employing people with epilepsy. One of the main aspects is an understanding of the basic facts about epilepsy. An applicant with epilepsy should be assessed on an individual basis. The main factors which should be taken into account are the type of epilepsy and its control, associated disorders, characteristics of the individual and suitability for the job (Espir and Floyd, 1986). Job suitability should normally be assumed if a person with epilepsy has the right qualifications and experience (Employment Commission of the International Bureau for Epilepsy, 1989).

\subsubsection{Driving}

Local employment opportunities and public transportation are important factors for the employability of the person with epilepsy as they may experience a restriction of driving privileges (Craig and Oxley, 1988). Several studies have been conducted on the risk of car accidents for epilepsy patients (Parsons 1986, Taylor 1986, Gastaut and Zifkin 1987, Hansotia and Broste 1991). Epilepsy has been mentioned as one of the major causes of collapse behind the steering wheel leading to an accident (Taylor 1986). Statistics show that licensed drivers with epilepsy have only a slightly increased risk of traffic accidents, similar to patients with diabetes (Hansotia and Broste 1991, 1993). Reports on casualties and damage are not consistent (van der Lugt 1972, Gastaut and Zifkin 1987). Whether seizures played a role in accidents may be difficult to establish (Hansotia and Broste 1993). There seems to be a consensus that persons with epilepsy, when licensed to drive, are not at any higher risk of 
having accidents (Commission on Epilepsy, Risk and Insurance, International Bureau for Epilepsy, 1993).

Regulations for holding a drivers licence vary between countries (Aird et al. 1984, Rutgers 1984, Fisher et al. 1994, Krumholz 1994, Shorvon 1995). Some countries require complete freedom from seizures for a period of two years, others require one year. In some countries limited licenses for driving passenger cars are issued for short distance travelling at low density motorways for those people who are dependent on driving for their employment. While restrictions with respect to driving passenger cars may become less strict during the years changes in the limitations for driving heavier and more dangerous vehicles or commercial driving are not expected.

Because of the restrictions, difficulties and costs of obtaining an automobile liability insurance some people with epilepsy do not report their epilepsy to the licensing bureau or drive a car although they are not allowed to do so (van der Lugt 1972, Rutgers 1984). The omission to report having seizures raises the question of who is responsible for reporting the seizures. If physicians were required by law to report the seizure status of their patients to the licensing bureau or when patients have to report their seizure status this would result in illegal driving in $33 \%$ and $53 \%$ of the patients, respectively (Salinsky et al. 1992). In both cases a high rate of non-compliance would be observed, which stresses the importance of having patients educated by their physicians.

\subsubsection{Leisure}

Adverse effects on leisure activities is reported to be a problem by almost one third of the patients ((haplin et al. 1992). Several activities may induce a risk for epilepsy patients when no precautions are taken.

Sports in which head injuries may occur are generally not advisable for people without precautions such as wearing riding caps in horse riding. Of all water sports diving is not advised for persons with recurrent seizures, while the risk of swimming can largely be reduced through a 'buddy system' (Craig and Oxley, 1988). The general approach for people with epilepsy who wish to start some kind of sport activity should be an objective individual assessment whether the activity is feasible given the risk of a seizure.

In about $3 \%$ of new epilepsy patients in the age of 7 to 19 years the first seizures may have been triggered by electronic screen games (Quirk et al. 1995). Television and other visual display units can be considered to be a risk when viewed from a distance less than two meters for a patient in which seizures occur spontaneously and/or are induced by flickering lights or by patterns. Seizures can be prevented easily through covering one eye or the use of polarised glasses and display screens (Harding, 1986)

Although it is generally bulieved that excessive alcohol intake can increase the seizure frequency it is difficult to attribute this increase to alcohol only (Hauser et al. 1988). Continuous alcohol abuse may be associated with poor compliance and disregardance of medication instructions (Chan, 1985). It has been shown that moderate drinking (2-3 glasses/day) did not result in an increase in epileptic seizure frequency. Furthermore, alcohol did not result in a change in plasma drug levels in drugs such as carbamazepine (CBZ), phenobarbiton (PB), phenytoin (PHT) while differences in valproate (VPA) plasma drug levels could not be attributed to alcohol intake (Höppener, 1981). Therefore, a prohibition of alcohol 
for epileptic patients can not be justified, although reservations may exist in those patients who do not comply with the advice provided, have a history of alcohol abuse or have been shown to be sensitive for alcohol.

\subsection{General aspects of anti-epileptic drug treatment}

The occurrence of a seizure will have great impact on the patient as well as on his family. The main objective of drug treatment will be to prevent the occurrence of further fits. The decision to start drug treatment however has consequences for the patient. In order to ensure effective drug treatment these consequences will necessitate an assessment of the pros and cons of drug treatment.

\subsubsection{Reasons for drug treatment}

Justifications for starting pharmacological treatment of epilepsy are its medical and social consequences. As said before, it is commonly assumed that a seizure may increase the chance of the occurrence of additional seizures. This process of 'Bahnung' may eventually lead to recurrent seizures which are difficult to treat (Shorvon and Reynolds 1982, Elwes et al. 1984, Reynolds 1987). Brain damage may develop either due to falling caused by the changes in postural tonus or because of development of neural overactivity inducing a generalised tonicclonic seizure. Status epilepticus is the occurrence of one or multiple seizures for at least 30 minutes during which period the patient is not able to regain normal consciousness (Gastaut and Kugler, 1976). Status epilepticus is accompanied by hypoxia, hypoglycaemia, acidosis and hypotension. Generalised convulsive status epilepticus is a medical emergency, with a mortality rate of about $10 \%$ to $37 \%$ (Aird et al. 1984. Scholtes et al. 1993). Considering these consequences and risks the treatment perspectives become important. As discussed earlier epilepsy has a rather good prognosis with many patients going into remission early during treatment.

\subsubsection{Reasons for refraining from drug treatment}

Being on drug treatment will have consequences of a medical, psychological and/or economical nature for the patient. These consequences may be a reason for not starting drug treatment at all.

From the medical point of view the uncertainty about the fact that the patient has epilepsy or about the specific type of epilepsy can be a reason for refraining from drug treatment. If it is not completely certain that the diagnosis is epilepsy or the type of epilepsy is not well established the best course is to wait and let time decide (Chadwick and Reynolds, 1985). An erroneous diagnosis can cause either refractory epilepsy or the intake of anti-epileptic drugs for nonepileptic paroxysmal attacks of altered awareness. Also the frequency and/or the severity of seizures are important in deciding whether or not to start drug treatment. The impairment caused by the disease should balance or outweigh the impairment caused by the drug treatment. Long-term prophylactic treatment should not be started when seizures appear with years in between, when seizures are mild with negligible psychosocial consequences or when seizures are caused by factors which can be avoided (Jøhannessen et al. 1990).

The attitude of patients towards the drug treatment is important as well. If compliance is not to be expected one should not prescribe drug treatment. One of the reasons is that the 
occurrence of withdrawal seizures due to sudden discontinuation presents considerable risk (Shorvon, 1987). Some state that drug treatment should not be started in alcohol or drug abusers as compliance is expected to be poor in such situations (Jøhannessen et al. 1990).

Side-effects of the drug treatment will probably not outweigh the burden of few, little disabling seizures. The drug treatment may interact with co-medication such as antibiotics, anticoagulants or contraceptives. Besides this, psychological factors can be important. The recurrent intake of medication reminds the patient, the family and colleagues about the fact that the patient is suffering from a chronic disease

Although an early publication of Sonnen (1979) stated that the financial consequences of the treatment of epilepsy are of relevance only in countries such as the United States and India, recent changes in the health care systems cause this factor to be of interest in other countries as well. Perhaps this aspect should be considered to be one of the side-effects of drug treatment (Meinardi, personal communication).

\subsubsection{Goals in anti-epileptic drug treatment}

Anti-epileptic dnug treatment can serve several purposes. The perspective of removing the symptoms and curing the disease is what the physicians aim at, although it is not clear whether the anti-epileptic drugs actually cure the disease. A second goal for anticonvulsive treatment can be the prevention of epilepsy development. This may be achieved through intermittent treatment of fibrile convulsions or temporary treatment after neurosurgical interventions or head trauma. Thirdly, the establishment of a diagnosis ex juvantibus may be the intention of the treatment in those cases in which epilepsy is suspected but can not be diagnosed with certainty

A guideline for approaching the patient is the assumption that the patient wants to get well (Porter, 1984). This sets the goal for the physician: control the seizures and if possible cure the disease. Long-term treatment with anti-epileptic drugs is the normal procedure for the patient who is diagnosed as having epilepsy and experiences seizures which are medically and/or socially disabling. The initiation of the anti-epileptic drug treatment implies that the patient will have to take the drug for several years in a daily regular fashion. Compassionate doctors and responsible patients are necessary for reliable drug intake (Feuerstein et al. 1988).

When the physician faces a patient suffering from a generalised convulsive status epilepticus it is obvious that intervention is necessary. The occurrence of a convulsive status epilepticus is life threatening (Porter 1984, Swartz and Delgado-Escueta 1987. Brodie 1990). Besides identification of the type of status the treatment consists of the correction of causes and precipitating factors.

Initiation of prophylactic anticonvulsive treatment has the goal to prevent the development of epilepsy. One of the diseases which may predispose for developing epilepsy are febrile convulsions. Febrile convulsions are seizures which occur in combination with a febrile disease. They occur mostly in children between the age of 6 months and 5 years. Febrile convulsions may recur: about one third of the patients who had two seizures will have more episodes. About $2 \%$ to $7 \%$ of patients with febrile seizures developed epilepsy (Annegers et al. 1987, Wallace 1987). The prophylactic treatment of febrile seizures with PB or Na-VPA is possible but side-effects such as behavioural disorders, learning disability and hepatotoxicity may occur. Intermittent treatment is another option. Rectal diazepam can be administered by parents to arrest febrile convulsions. 
Prophylactic anti-epileptic drug treatment can be considered also in patients having suffered from head injury or neurosurgery. The number of people in the community suffering from a head injury is large. Each year in the United States some 500.000 people have brain injuries caused by cranial trauma (Hauser and Hesdorffer, 1990). In about 5\% of the head injured patients early seizures occur after the trauma. Phenytoin has been shown to be beneficial for this type of seizures (Temkin et al. 1990). The benefit of other anti-epileptic drugs is not clear. Therefore, also in these patients before initiating anti-epileptic drug treatment one has to weigh the high level of non-compliance and the risk of adverse drug reactions against the uncertain benefit of the treatment of patients with a small risk of actually developing epilepsy (Rimmer and Richens, 1988)

Similar problems arise in the prophylactic treatment of patients who had neurosurgery (Rimmer and Richens 1988, Jennett 1987). Here also a delay in treatment until seizures occur seems more appropriate as little benefit, non-compliance and problems in establishing the moment of withdrawal can be expected when anti-epileptic drug treatment is initiated (Chadwick and Reynolds 1985, Foy et al. 1992)

Finally there is the diagnostic approach. If the diagnosis whether a seizure is epileptic or not can not be established a temporary treatment with an anti-epileptic drug can be used as a diagnostic test. However there is doubt about this procedure. Literature indicates that there is only little room for the diagnosis ex juvantibus (Chadwick and Reynolds 1985, Porter 1984, 1989, Shorvon 1987)

\subsection{Initiation of treatment}

Starting anti-epileptic drug treatment has as a consequence daily intake of anticonvulsive medication for at least two years. As patients perceive this as a cumbersome task, commencing drug treatment also neccesitates to address its withdrawal

\subsubsection{Starting the drug treatment}

The moment drug treatment should be started is not well defined (Rimmer and Richens, 1988). The statement that a patient should have recurrent seizures within a short interval does not specify the duration of this period, which leads to variation in practice. Many physicians would consider a period of one year (Overweg et al. 1981, Rimmer and Richens 1988, Chadwick and Reynolds 1985, Chadwick 1994a). Others would only commence if the recurrence of a seizure takes place within a period of half a year (Sonnen, 1979). Some even start treatment after a first seizure. In a study of 88 patients, with an age of $15-64$ years, it was shown that $31 \%$ of the patients received anti-epileptic drug treatment after their first seizure, while in 2 patients treatment was started to prevent epilepsy (Rutgers, 1984). Van Donselaar (1990) showed that EEG abnormalities after a first idiopathic seizure provided a criterion for selecting patients with a high risk of recurrence, for which drug treatment might be started right away.

The detectability of a seizure may be an important aspect in considerations to commence treatment. Generalised tonic-clonic seizures can hardly be overlooked while others go undetected more easily. This may be the reason why the physician starts treatment earlier when diagnosing tonic-clonic seizures (Porter, 1989). It has been shown that the median number of tonic-clonic seizures before treatment was 3 , while the median number of partial seizures was 20 (Shorvon and Reynolds, 1982). 
Another reason to start the treatment as soon as possible is that early treatment may have been prevented due to patient and/or doctor delay. It has been shown that one third of the patients who experienced a first seizure did not visit a doctor immediately. Within this group $52 \%$ visited their family physician within one year. Half of these patients visited the physician because of a second seizure, while others visited their physician after more than two seizures (Rutgers, 1984). Doctors delay was encountered in $46 \%$ of the patients, mainly in patients who were younger than 20 years at the moment of onset. Some $16 \%$ of the patients had both patient and doctor delay.

It has been shown that in $37 \%$ up to $80 \%$ of the patients with a first seizure a second seizure occurs within one year (Goodridge and Shorvon 1983b, Elwes et al. 1985, Hopkins et al. 1988, Sander et al. 1990). Some suggest early treatment (Elwes et al. 1988, Chandra 1992, Reynolds 1995). However, starting treatment immediately, based on the finding of only one seizure is still questionable. The physician has to establish a correct diagnosis which may be difficult especially if this single event is described incompletely. Furthermore it may be difficult to differentiate between epileptic and pseudo-seizures. Additional studies are needed to answer the question whether treatment after the first seizure prevents chronic epilepsy (First Seizure Trial Group 1993, Chadwick 1995)

Table 2.9-I: Preferred drugs for treatment of specific types of epilepsy. The first and second choice medication is indicated by the number.

\begin{tabular}{|c|c|c|c|c|}
\hline \multirow[b]{2}{*}{$\begin{array}{l}\text { First } \\
\text { authors }\end{array}$} & \multicolumn{4}{|c|}{ Type of epilepsy } \\
\hline & gen.idiopathic & gen.symptoinatic & partial epilepsy & unclassified \\
\hline $\begin{array}{l}\text { Dam } \\
(1982)\end{array}$ & $\begin{array}{l}\text { CBZ,PHT,CIZ, } \\
\text { PB,ETT,PRM, } \\
\text { VPAACZA }\end{array}$ & $\begin{array}{l}\text { SGTC: CBZ,PHT, } \\
\text { CLZ,PB,ETT, } \\
\text { PRM, VPA, } \\
\text { ACZA }\end{array}$ & $\begin{aligned} \text { PEES: } & \text { CBZ,PHT,CLZ, } \\
& \text { PB,ETT,PRM, } \\
& \text { VPA,ACZA } \\
\text { PECS: } & \text { CBZ,PHT, } \\
\text { CLZ,ETT, } & \text { PRM,VPA }\end{aligned}$ & \\
\hline $\begin{array}{l}\text { Meinardi } \\
(1983)\end{array}$ & VPA,PB,ACZA & $\begin{array}{l}\text { VPA,CBZ, PHT,BZP, } \\
\text { ACTH }\end{array}$ & $\begin{array}{l}\text { SP: CBZ,PHT, VPA, } \\
\text { BZP } \\
\text { CP.CBZ,PHT,CLZ, } \\
\text { PRM,VPA }\end{array}$ & \\
\hline $\begin{array}{l}\text { Chadwick } \\
\text { (1990) }\end{array}$ & $\begin{array}{l}\text { SA: I VPA.ESM, } 2 \text { BZP } \\
\text { JME: I VPA } 2 \text { PB } \\
\text { ATC: I VPA } 2 \text { CBZ.PHT }\end{array}$ & $\begin{array}{l}1 \text { VPA,BZP } \\
2 \text { CBZ,PHT,PB }\end{array}$ & $\begin{array}{l}1 \text { CBL.VPA } \\
2 \text { PHT.PB }\end{array}$ & $\begin{array}{l}1 \mathrm{CBZ}, \mathrm{VPA} \\
2 \mathrm{PHT}, \mathrm{PB}\end{array}$ \\
\hline $\begin{array}{l}\text { Meinardi } \\
(1993)\end{array}$ & VPA & VPA & $\begin{array}{l}\text { I CBZ } \\
2 \mathrm{PHT}\end{array}$ & \\
\hline $\begin{array}{l}\text { Chadwick } \\
\text { (1994b) }\end{array}$ & $\begin{aligned} \text { SA: } & 1 \text { VPA.ESM } \\
& 2 \text { BZP.LTG } \\
\text { JME: } & 1 \text { VPA } \\
& 2 \text { PB,LTG } \\
\text { ATC: } & 1 \text { VPA. } \\
& 2 \text { CBZ,PHT,LTG }\end{aligned}$ & $\begin{array}{l}\text { I VPA.BBZI' } \\
2 \text { CI37.I'IT,PB }\end{array}$ & $\begin{array}{l}\text { l CBZ,VPA } \\
2 \text { PHI.PB.VGB }\end{array}$ & $\begin{array}{l}1 \mathrm{VPA} \\
2 \mathrm{CBZ} . \mathrm{PHT}\end{array}$ \\
\hline
\end{tabular}

SA: simple absences, MM: juvenile myoclonic epilepsy, ATC: awakening tonic-clonic seizures, SGTC: secondary generalised tonic clonic. PEES: partial epilepsy elementary symptomatology. PECS: partial epilepsy complex symptomatology. SP: simple partial, CP: complex partial

CBZ; Carbamazepine, PHT: Phenytoin. PB: Phenobarbiton, PRM: Primidon, VPA: Valproate, ESM: Ethosuximide, CLZ: Clonazepun, ETT: Ethotoin. ACZA: Acetizolamide, ACTH: Adrenocorticotropic honnone, BZP: Benzodiazepines, LTG: Lamotrigine VGB: Vigabatrine 


\subsubsection{Drug selection}

The classifications of epilepsies and seizures provide a means for communication. There is no consensus on whether the choice of the applicable drug should be based on the type of the epilepsy or on the type of seizure (Dam et al. 1985, Meinardi 1993). Literature shows that the selection of a drug may be based on the type of epilepsy, the type of seizures or both (Table 2.9-I and Table 2.9-II).

Table 2.9-II: Preferred drugs for treatment of specific seizure types.

\begin{tabular}{|c|c|c|c|c|}
\hline \multirow[b]{2}{*}{ First author } & \multicolumn{4}{|c|}{ Seizure type } \\
\hline & absences & tonic-clonic (GE) & partial & SGTC \\
\hline Jeavons (1977) & $\mathrm{VPA} \rightarrow \mathrm{ESM}$ & $\mathrm{VPA}, \mathrm{CBZ} \rightarrow \mathrm{PHT}$ & $\begin{array}{l}\text { SP: CBZ, VPA } \rightarrow \text { CLP } \\
\text { CP: CBZ } \rightarrow \text { VPA,PHT }\end{array}$ & $\mathrm{VPA}, \mathrm{CBZ} \rightarrow \mathrm{PHT}$ \\
\hline Reynolds (1981) & ESM,CLP,VPA & $\begin{array}{l}\text { PB,PHT,PRM, } \\
\text { CBZ,CLP,VPA }\end{array}$ & $\begin{array}{l}\text { PB,PHT,PRM, } \\
\text { CBZ,CLP,VPA }\end{array}$ & $\begin{array}{l}\text { PB,PHT,PRM, } \\
\text { CBZ,CLP,VPA }\end{array}$ \\
\hline Höppener (1983) & $\begin{array}{l}\text { I VPA,ESM } \\
2 \mathrm{MSM}\end{array}$ & $\begin{array}{l}\text { I CBZ,VPA } \\
2 \text { PB,PRM }\end{array}$ & $\begin{array}{l}\text { I CBZ,PHT } \\
2 \text { VPA,PB,PRM, } \\
\text { CLP,SUL,ACTA, } \\
\text { PCM }\end{array}$ & \\
\hline Shorvon (1985) & ESM,VPA & $\begin{array}{l}\text { CBZ, PHT,PB/PRM, } \\
\text { VPA }\end{array}$ & CBZ,PHT,PB,PRM & CBZ,PHT,PB,PRM \\
\hline Mattson (1989) & ESM,VPA & $\begin{array}{l}\text { I VPA } \\
2 \text { CBZ,PHT,PB,PRM }\end{array}$ & $\begin{array}{l}\text { CBZ,PHT, PB, PRM, } \\
\text { VPA? }\end{array}$ & CBZ,PHT,PB,PRM \\
\hline $\begin{array}{l}\text { Johiunessen } \\
(1990)\end{array}$ & I ESM,VPA & $\begin{array}{l}\text { I CBZ } \\
2 \mathrm{VPA} \\
3 \text { CLP,CLB,PB,PHT }\end{array}$ & $\begin{array}{l}1 \mathrm{CBZ} \\
2 \text { PHT.VPA } \\
3 \text { CL.P }\end{array}$ & $\begin{array}{l}1 \mathrm{CBZ} \\
2 \mathrm{PHT}, \mathrm{VPA} \\
3 \mathrm{CLP}\end{array}$ \\
\hline Scheurer (1990) & $\mathrm{ESM} \rightarrow \mathrm{VPA}$ & $\mathrm{VPA} \rightarrow \mathrm{CBZ}, \mathrm{PHT}, \mathrm{PB}$ & CBZ,PITT,PRM,PB & $\begin{array}{l}\text { CBZ,PHT, PRM,PB, } \\
\text { VPA? }\end{array}$ \\
\hline Mattson (1992) & VPA & CBZ, VPA & $\mathrm{CBZ}$ & $\mathrm{CBZ}$ \\
\hline Wilder (1995) & $\begin{array}{l}\text { ESM,VPA, } \\
\text { LTG,FMT }\end{array}$ & $\begin{array}{l}\text { PHT,CBZ, VPA,GBP, } \\
\text { LTG,FMT }\end{array}$ & $\begin{array}{l}\text { PHT,CHZ,GBP, } \\
\text { LTG,IMT }\end{array}$ & $\begin{array}{l}\text { PHT,CBZ,VPA, } \\
\text { GBP,ITS,FMT }\end{array}$ \\
\hline Brodie (1996) & VPA.ESM & $\begin{array}{l}\text { CBZ,PHT,VPA,PB, } \\
\text { PRM,CLP }\end{array}$ & $\begin{array}{l}\text { CBZ,PHT, VPA,PB, } \\
\text { PRM }\end{array}$ & $\begin{array}{l}\text { CBZ,PHT, VPA,PB, } \\
\text { PRM,CLP }\end{array}$ \\
\hline
\end{tabular}

GE: generalised epilepsy, SGTC: secondary generalised tonic clonic, SP: simple partial, CP: complex partial, a $\rightarrow$ b,c: a followed by $b$ or $\mathrm{c}$

CBZ: Carbamazepine, PHT: Phenytoin, PB: Phenobarbital, PRM: Primidon, VPA: Valproate, ESM: Ethosuximide, MSM Mesuximide, CZP: Clorazepate, CLP: Clonazepan, SUL: Sultiam, ACTA: Acetazolamide, PCM: Phenacemide, CLB: Clobazam, LTG: Lamotrigine, GBP: Gahapentin, FMT: Felbanate

Some use the type of epilepsy for the selection of the drug of first choice. They recommend the use of VPA for idiopathic generalised and generalised symptomatic epilepsy and CBZ for partial epilepsy (Meinardi et al. 1983, Chadwick 1990, Meinardi 1993, Chadwick 1994a,b). Another example is juvenile myoclonic epilepsy which responds only to VPA. Others prefer CBZ for idiopathic generalised and partial epilepsy and differentiate between different types of partial epilepsy (Dam and Kiørboe 1982).

Others prefer the use of the seizure diagnosis to determine the appropriate therapy (Reynolds and Shorvon 1981, Höppener and van der Lugt 1983, Porter 1984, Shorvon 1987, Jøhannessen 1990, Shorvon 1991, Brodie and Dichter 1996). 
Although the seizure type can be used as selection criterion it has been emphasised that also aspects of the type of epilepsy are of importance in making a decision (Dreifuss, 1990). Other investigators state that the seizure type enables to establish a relation between the physiologi$\mathrm{cal}$ and neurochemical factors of seizures and their response to drug treatment. Nevertheless, they advocate a pragmatic approach in the use of classifications for drug selection. Thus, the use of a classification of seizure types or epilepsies should be performed as far as this is relevant for the selection of the appropriate therapy (Aird et al. 1984).

\subsubsection{Monotherapy or polypharmacy}

Until the 1970s the traditional approach to treatment of epilepsy was twofold. In the first approach treatment with one drug was started and gradually the dose was increased until the seizures were controlled or toxic side-effects developed. In the second approach two drugs were used in the hope of a synergistic effect or under the assumption that the toxicity of two drugs in a lower dose would be less than the toxicity of one drug at a higher dose. In both approaches the dosage was changed or other drugs were added if seizures were not controlled. During the seventies a change in the view on prescribing anti-epileptic drugs developed. Studies on the effectiveness of polypharmacy indicated that it is unnecessary in newly diagnosed patients (Reynolds et al. 1976, Shorvon and Reynolds 1977, Shorvon et al. 1978, 1980, Reynolds and Shorvon 1981). The general principle that evolved was that in newly diagnosed patients combination therapy should be tried only when monotherapy with several drugs failed (Dam and Kiørboe 1982, Rowan 1983, Höppener and van der Lugt 1983, Aird et al. 1984, Shorvon 1987. Rimmer and Richens 1988, Porter 1989, Jøhannessen et al. 1990. Scheuer and Pedley 1990)

Reynolds (1980) discusses two studies that suggest that polypharmacy may lead to an increased risk of seizures. Side-effects decreased when fewer drugs were prescribed. However, Lammers et al. (1995) have suggested that this may be due to the decrease of drugload and is not caused by the reduction in number of drugs. As was discussed by Höppener and van der Lugt (1983) the introduction of a second drug may cause changes in antiepileptic drug levels of the newly introduced as well as of the used drug. These changes vary over patients. An additional problem concerning side-effects in polytherapy is the determination of the drug responsible for the adverse reaction. The compliance of the patient may be lower when using polytherapy which may result in incomplete seizure control. Furthermore, the costs of the therapy may be lower if one or more of the prescribed drugs is withdrawn.

However, some patients can benefit from polytherapy. It has been reported that in $11 \%$ of the patients, that were given polytherapy because monotherapy failed, complete seizure control was achieved. A reduction in the seizure frequency was observed in $40 \%$ (Mattson et al. 1985). It has been suggested that a systematic change of drugs is necessary using the most effective drug as a background regimen while the other individual drugs should be added and tested sequentially (Shorvon, 1991)

Insight in drug mechanisms is growing. Anti-epileptic drugs seem to decrease membrane excitability by interacting with neurotransmitter receptors and ion channels (Macdonald and Kelly 1994, Montouris 1995, Brodie and Dichter 1996). More recently new drugs VGB, LTG and GBP have been introduced which can be used as add-on therapy (Wilder, 1995). Combination therapy may become more common as drugs with synergistic effects become available (Chadwick, 1994b). 


\subsubsection{Efficacy of drugs}

All new anti-epileptic drugs are subjected to efficacy trials. Also comparative studies of the relative efficacy of major anti-epileptic drugs have been published. Table 2.9-III lists the studies concerned with CBZ, PHT, VPA, PRM and PB. The assessment of studies on the efficacy of anticonvulsive drugs is difficult because of differences in the studied populations, differences in study design, the small number of patients involved in the studies, differences in outcome measures and short duration of follow-up. The paroxysmal character of the epilepsy makes the duration of follow-up an important aspect. Furthermore, a short follow-up period may result in a bias in favour of drugs for which tolerance can be developed during long-term intake. Because of these problems it is not possible to draw definite conclusions about differences in efficacy of anticonvulsives using results from randomised controlled comparison studies of anti-epileptic drugs (Gram et al. 1982, Chadwick and Turnbull 1985, Beghi et al. 1986).

More recently larger drug trials have been conducted. Mattson et al. (1992) studied the efficacy of CBZ and VPA in a randomised double-blind trial in patients with complex partial seizures and secondary generalised tonic-clonic seizures. They did not find a difference between the two drugs in controlling the tonic-clonic seizures. However, for the complex partial seizures CBZ showed a better control and fewer long term side-effects. They conclude that $\mathrm{CBZ}$ should be used as a drug of first choice for partial epilepsy because about $50 \%$ of the patients with partial complex seizures do also suffer from generalised seizures. The higher number of side-effects for VPA however, may have been caused by an upper range plasma drug level which was higher than is usual in clinical practice (Reynolds et al 1993). Others report that $\mathrm{CBZ}$ and VPA are equally effective while a higher withdrawal rate because of sideeffects was observed for CBZ (Richens et al. 1994, Heller 1995).

\subsubsection{Dose of drugs}

Once a drug has been selected the intention is to control seizures without the development of toxic side-effects. The consensus is that the anti-epileptic drugs can be used more effectively with the assistance of serum level monitoring (Richens and Ahmad 1975. Shorvon et al. 1978, Reynolds 1980, Rimmer and Richens 1988, Commission on Anti-epileptic Drugs of the ILAE, 1993). Considerations about efficacy, toxicity and the plasma drug levels resulted in the concept of therapeutic or optimal range. Within this range seizure control is expected to be optimal while the toxic effects are expected to be minimal

Another important aspect of the therapeutic range is the definition of an upper level above which additional control of seizures is unlikely and toxic side-effects can be expected. Unfortunately, the therapeutic range can not be used to predict the efficacy of the treatment for an individual patient (Shorvon 1987, Lammers 1994). Nevertheless these ranges, which are based on averages for large populations, can also be used for the individual case at onset of therapy. One should be aware that differences in opinion on the appropriate plasma drug levels exist (Table 2.9-IV) 
Table 2.9-IV: Overview of therapeutic ranges in $\mathrm{mg} / \mathrm{l}$ presented in the literature

\begin{tabular}{lllllll}
\hline First author & CBZ & PHT & PB & PRM & VPA & ESM \\
\hline Jeavons (1977) & $4-10$ & $10-25$ & $15-40$ & $n a v^{1}$ & $60-100$ & $40-120$ \\
Schobben (1979) & $4-8$ & $7-12$ & $20-35$ & $7-10$ & $50-80$ & $40-80$ \\
Sonnen (1979) & $5-10$ & $10-20$ & $15-40$ & $4-12$ & $40-80$ \\
Reynolds (1980) & $4-10$ & $10-20$ & $15-40$ & $15-40^{2}$ & $50-100$ & $40-80$ \\
Daun (1982) & $4-10$ & $10-20$ & $15-30$ & nav & nav & $40-90$ \\
Höpjwener (1983) & $4-10$ & $10-20$ & $15-40$ & nav & $?$ & $40-120$ \\
Rowan (1983) & $8-12$ & $15-20$ & $15-40$ & $5-8$ & $?$ & $60-100$ \\
Overweg (1984) & $3-12$ & $10-20$ & $10-40$ & $4-12$ & $30-120$ & $40-120$ \\
Shorvon (1987) & $3.5-11.5$ & $7-20$ & $9-40$ & $9-40^{3}$ & nav. & $40-100$ \\
Rimmer (1988) & $5-10$ & $10-20$ & $15-40$ & $15-40^{1}$ & $50-100$ & $50-100$ \\
Porter (1989) & $4-10$ & $10-20$ & $10-40$ & $5-15$ & $50-100$ & $50-100$ \\
& $7^{3}>8^{4}$ & $18^{3}>20^{4}$ & $35^{3}>40^{4}$ & $10^{3}>12^{4}$ & $80^{3}>100^{4}$ & $80^{3}>100^{4}$ \\
Scheuer (1990) & $8-12$ & $10-20$ & $15-35$ & $5-12$ & $50-120$ & $40-100$ \\
Meijer (1991) & $3-7$ & $10-20$ & $20-40$ & nav & $50-90$ & $50-90$ \\
\hline
\end{tabular}

CBZ: Carbamazepine, PHT: Phenytoin, PB: Phenobarbital, PRM: Primidon, VPA: Valproate, ESM: Ethosuximide (1)nav: not available. (2)Therapeutic range of phenobarbital is used for primidon, (3)Level to be reached in refractory seinures, (4)Toxic level

Some authors do not present the therapeutic range values for Na-VPA because the significance of the drug monitoring for this drug is unknown. As large changes in VPA serum level during the day occur it is not possible to correlate side-effects and serum concentration for this drug (Höppener and van der Lugt, 1983). Differences also exist in the presented optimal serum levels for PRM. Some use the real PRM serum values for establishing the therapeutic range of the drug (Sonnen 1979, Rowan 1983, Overweg 1984, Scheuer and Pedley 1990). Others present the values of PB for PRM because PB is produced during the metabolisation of PRM (Reynolds 1980, Shorvon 1987, Rimmer and Richens 1988, Porter 1989).

Also for carbamazepine the therapeutic ranges vary in the literature. Some found mean blood drug levels of 4.7 to $6.5 \mathrm{mg} / \mathrm{l}$ for carbamazepine in successfully treated patients, while therapy failure was observed at a mean plasma level of $9.8 \mathrm{mg} / \mathrm{l}$ (Ramsay et al 1983). Others use a range of 3-12 mg/l (Overweg 1984, Shorvon 1987), while again others state that the therapeutic range starts at $8 \mathrm{mg} /$ (Rowan 1983, Scheuer and Pedley 1990). It has been mentioned that drug toxicity can be expected starting from $8 \mathrm{mg} / \mathrm{l}$ for this drug (Porter 1989)

A similar variation in therapeutic ranges was observed for PHT, PB and ISM. Lower therapeutic levels are quoted, respectively from $7-15,9-20$ and $40-60 \mathrm{mg} /$, while upper therapeutic levels vary from $12-25,30-40$ and $80-120 \mathrm{mg} /$.

The differences in the patient population or the treatment strategy as well as the possibility that the therapeutic ranges are determined more by the desired anti-epileptic effect than by the appearing side-effects may be the cause for the variation in therapeutic ranges

Besides the interindividual variability in therapeutic values it has been suggested that intra individual variability in therapeutic AED serum levels exist. Schmidt and Janz (1980) showed that the initial therapeutic concentration of the drugs PHT and PB for controlling seizures was 
Table 2.9-IV: Overview of therapeutic ranges in $\mathrm{mg} / \mathrm{l}$ presented in the literature

\begin{tabular}{lllllll}
\hline First author & CBZ & PHT & PB & PRM & VPA & ESM \\
\hline Jeavons (1977) & $4-10$ & $10-25$ & $15-40$ & $n a v^{1}$ & $60-100$ & $40-120$ \\
Schobben (1979) & $4-8$ & $7-12$ & $20-35$ & $7-10$ & $50-80$ & $40-80$ \\
Sonnen (1979) & $5-10$ & $10-20$ & $15-40$ & $4-12$ & $40-80$ \\
Reynolds (1980) & $4-10$ & $10-20$ & $15-40$ & $15-40^{2}$ & $50-100$ & $40-80$ \\
Daun (1982) & $4-10$ & $10-20$ & $15-30$ & nav & nav & $40-90$ \\
Höpjwener (1983) & $4-10$ & $10-20$ & $15-40$ & nav & $?$ & $40-120$ \\
Rowan (1983) & $8-12$ & $15-20$ & $15-40$ & $5-8$ & $?$ & $60-100$ \\
Overweg (1984) & $3-12$ & $10-20$ & $10-40$ & $4-12$ & $30-120$ & $40-120$ \\
Shorvon (1987) & $3.5-11.5$ & $7-20$ & $9-40$ & $9-40^{3}$ & nav. & $40-100$ \\
Rimmer (1988) & $5-10$ & $10-20$ & $15-40$ & $15-40^{1}$ & $50-100$ & $50-100$ \\
Porter (1989) & $4-10$ & $10-20$ & $10-40$ & $5-15$ & $50-100$ & $50-100$ \\
& $7^{3}>8^{4}$ & $18^{3}>20^{4}$ & $35^{3}>40^{4}$ & $10^{3}>12^{4}$ & $80^{3}>100^{4}$ & $80^{3}>100^{4}$ \\
Scheuer (1990) & $8-12$ & $10-20$ & $15-35$ & $5-12$ & $50-120$ & $40-100$ \\
Meijer (1991) & $3-7$ & $10-20$ & $20-40$ & nav & $50-90$ & $50-90$ \\
\hline
\end{tabular}

CBZ: Carbamazepine, PHT: Phenytoin, PB: Phenobarbital, PRM: Primidon, VPA: Valproate, ESM: Ethosuximide (1)nav: not available. (2)Therapeutic range of phenobarbital is used for primidon, (3)Level to be reached in refractory seinures, (4)Toxic level

Some authors do not present the therapeutic range values for Na-VPA because the significance of the drug monitoring for this drug is unknown. As large changes in VPA serum level during the day occur it is not possible to correlate side-effects and serum concentration for this drug (Höppener and van der Lugt, 1983). Differences also exist in the presented optimal serum levels for PRM. Some use the real PRM serum values for establishing the therapeutic range of the drug (Sonnen 1979, Rowan 1983, Overweg 1984, Scheuer and Pedley 1990). Others present the values of PB for PRM because PB is produced during the metabolisation of PRM (Reynolds 1980, Shorvon 1987, Rimmer and Richens 1988, Porter 1989).

Also for carbamazepine the therapeutic ranges vary in the literature. Some found mean blood drug levels of 4.7 to $6.5 \mathrm{mg} / \mathrm{l}$ for carbamazepine in successfully treated patients, while therapy failure was observed at a mean plasma level of $9.8 \mathrm{mg} / \mathrm{l}$ (Ramsay et al 1983). Others use a range of 3-12 mg/l (Overweg 1984, Shorvon 1987), while again others state that the therapeutic range starts at $8 \mathrm{mg} /$ (Rowan 1983, Scheuer and Pedley 1990). It has been mentioned that drug toxicity can be expected starting from $8 \mathrm{mg} / \mathrm{l}$ for this drug (Porter 1989)

A similar variation in therapeutic ranges was observed for PHT, PB and ISM. Lower therapeutic levels are quoted, respectively from $7-15,9-20$ and $40-60 \mathrm{mg} /$, while upper therapeutic levels vary from $12-25,30-40$ and $80-120 \mathrm{mg} /$.

The differences in the patient population or the treatment strategy as well as the possibility that the therapeutic ranges are determined more by the desired anti-epileptic effect than by the appearing side-effects may be the cause for the variation in therapeutic ranges

Besides the interindividual variability in therapeutic values it has been suggested that intra individual variability in therapeutic AED serum levels exist. Schmidt and Janz (1980) showed that the initial therapeutic concentration of the drugs PHT and PB for controlling seizures was 
higher than the AED serum therapeutic concentration measured during the maintenance period of the treatment. In a study on withdrawal of anti-epileptic medication in 1013 patients seizure free for at least 2 years, it was found that $30 \%, 76 \%, 67 \%$ and $51 \%$ of the patients using $\mathrm{CBZ}$, $\mathrm{PB}, \mathrm{PHT}$ or VPA, respectively, had AED serum levels in the subtherapeutic range (Medical Research Council AED Withdrawal Study Group, 1991). These differences in therapeutic levels have been attributed to differences in the activity of the epileptic process.

Also the recommendations on the normal daily doses diverge (Table 2.9-V). For establishing the daily dose some use the body weight of the patient (Dam and Kiørboe 1982, de Krom and van der Lugt 1982, Overweg 1984). Others use a more global approach with a predefined amount of milligrams per day (Sonnen 1979, Aird et al. 1984, Lechtenberg 1985, Shorvon 1987, Rimmer and Richens 1988, Brodie and Dichter 1996).

Table 2.9-V: Normal daily dose of anti-epileptic drugs. In the upper part of the table the daily dose is presented in $\mathrm{mg}$. The lower part of the table presents the daily dose of the $\mathrm{drug}$ in $\mathrm{mg} / \mathrm{kg}$ body weight.

\begin{tabular}{llllllll}
\hline & First author & CBZ & PHT & PB & PRM & VPA & ESM \\
\hline \multirow{4}{*}{ daily } & Sonnen (1979) & $400-1600$ & $250-500$ & $100-200$ & $500-1000$ & $900-2400$ & $500-1500$ \\
& Aird (1984) & $600-1200$ & $100-500$ & $100-300$ & $250-1000$ & $1500-3000$ & $500-1500$ \\
dose & Lechtenberg (1985) & $600-1200$ & $300-400$ & $100-150$ & $750-1500$ & $1000-3000$ & $750-2000$ \\
& Shorvon (1987) & $400-1800$ & $200-500$ & $60-180$ & $250-1000$ & $600-2500$ & $500-1500$ \\
& Rimmer (1988) & $400-1800$ & $150-600$ & $30-240$ & $250-1500$ & $600-3000$ & $500-1500$ \\
& Brodic (1996) & $400-2000$ & $100-700$ & $60-240$ & $250-1500$ & $500-3000$ & $500-2000$ \\
\hline \multirow{4}{*}{$\mathrm{mg} / \mathrm{kg}$} & Daun (1982) & $10-20$ & 5 & $1.5-3.0$ & $12-20$ & $900-1800$ & $20-40$ \\
& de Krom (1982) & $8-12$ & $5-7$ & $2-5$ & nav & $20-30$ & $8-12$ \\
& Ovcrweg (11984) & $15-20$ & 5 & $3-5$ & 10 & 20 & $15-20$ \\
\hline
\end{tabular}

CBZ: Carbanazepine, PHT: Phenyoin, PB: Phenobarbital, PRM: Primidon, VPA: Valproate, ESM: Ethosuximide (1) Maily dose in mg

The relation between dose and seizure control is only statistical. The physician has to decide for the individual patient whether a high dose or not is needed to achieve results immediately (Porter, 1989). Some state that AED serum levels should be in the upper part of the therapeutic range (Rowan 1983). Others found that up to one third of the patients will achieve control of seizures with a level below the therapeutic range (Reynolds et al. 1976, Shorvon et al. 1978, 1980). Control was achieved with 200-300 mg PHT per day or 200-400 mg CBZ per day. Seizure control has been observed even with a low starting dose of e.g. $100 \mathrm{mg}$ PHT or 100-200 mg CBZ (Reynolds 1980. Shorvon et al. 1978). An approach for drug treatment is to start treatment with a low dose and if no effect is observed increase the dose to try to reach the therapeutic range (Höppener and van der Lugt 1983, Shorvon 1985, Chadwick 1990). Another approach is to use the middle of the target range (Mattson et al. 1985, 1992). To assure compliance it is preferable not to increase the dose to a level where toxicity may be expected in spite of recent publications which advocate a return to pre drug level monitoring strategies. 


\subsection{Maintenance}

After drug treatment has been initiated it is necessary to see the patient on a regular basis. During these visits the seizures may remit after a short time of drug treatment, show some response to drugs with a tendency to relapse or turn out to be intractable in which remission is unusual. Furthermore, the correctness of the diagnosis and the development of side-effects have to be assessed. During each of these visits it is advisable to check compliance to support and encourage the patient to comply with the treatment as to improve or maintain seizure control.

\subsubsection{Recurrence of seizures}

After having achieved a remission a relapse may occur. The probability of a relapse during the first 5,10 and 20 years after entering remission is $8 \%, 15 \%$ and $24 \%$, respectively (Annegers et al. 1979). This estimate includes patients on and off anti-epileptic drug treatment.

Recurrence of seizures can be caused by misdiagnosis. Jeavons (1977) stated that 270 of the 1172 patients at his epilepsy clinic did not have epilepsy. Fifty percent of the adults in the nonepilepsy group were misdiagnosed because of syncope. Second to this there were misdiagnoses due to psychiatric disorders. The choice of an ineffective drug may be another reason for occurrence of seizures during treatment. Even when a first choice drug is prescribed it is not necessarily the most effective drug for a specific patient. Suboptimal serum levels can also be a cause of the continuation of seizures

Non-compliance is another reason for continuation of seizures or their reoccurrence after a seizure free period. If complete control of seizures has been achieved the compliance may decrease because of absence of seizures (Feuerstein et al. 1988). Although non-compliance can be the cause of a relapse, the correctness of the diagnosis should be evaluated continuously as a unrecognised concomitant neurological disease may develop.

Finally, there is a group of patients in whom complete seizure control cannot be established although plasma drug levels in the therapeutic range have been reached. Drug resistant epilepsy which is only partly responsive to drug treatment is present in about $20 \%$ of the patients in the UK. Another $20 \%$ of the patients have epilepsy which is completely unresponsive to drug treatment (Shorvon, 1991).

\subsubsection{Drug monitoring}

Plasma drug levels together with clinical findings can be used to determine the effectiveness of the drug treatment and provide a basis for adjustment of the dose of the drug. The practical use of plasma drug levels has been studied amongst others by Beardsley et al. (1983) and Meijer (1991). Beardsley et al. showed in a retrospective study of 164 adult epileptic patients that prescribers did not use the blood levels properly in $17 \%$ of the therapeutic decisions. If they did use the plasma drug levels correctly an improvement in the control of the seizures occurred in $51 \%$ of the patients. Inappropriate decisions caused an improvement in seizure frequency in only $5 \%$ of the patient, while in $32 \%$ of the patients with an inappropriate decision the seizure frequency worsened

Meijer (1991) studied the decisions of 16 physicians. From a retrospective analysis of 1006 therapeutic decisions in 64 patient records he concluded that approximately $66 \%$ of the decisions to adapt the dosage of phenytoin and carbamazepine, and $80 \%$ of the decisions on 
changes in dosage of valproate were done in agreement with the serum concentration and clinical signs. Confronting the physicians with plasma drug levels in the subtherapeutic and toxic range led also to variance in the treatment strategy. Although some differences occurred in cases with subtherapeutic levels, most of the variance in decisions was observed for cases with plasma drug levels in the toxic range.

Anti-epileptic drug monitoring may help in discovering the reason for therapy failure. Changes in seizure control can be attributed to e.g. intra individual variation in pharmacokinetic parameters as a consequence of intermittent disease or to the effects of co-medication on plasma drug levels (Höppener and van der Lugt 1983, Scheuer and Pedley 1990). Drug monitoring can facilitate the diagnosis of symptoms possibly caused by drug toxicity and can help to predict whether a change in the drug dose is likely to result in toxic side-effects (Milano Collaborative Group for Studies on Epilepsy, 1980).

Anti-epileptic drug levels should be used as a guide rather than a rigid goal for the optimal use of the drug. The observed levels depend on the drug half-life and can be affected by the interval between the time of drug intake and the moment of blood sampling. Furthermore, inter-individual differences in optimal levels as well as intra individual variation may occur. It has been shown that patients who are seizure free with levels in the sub therapeutic range do not always benefit from an increase in the dose leading to a therapeutic range level (Woo et al. 1988). Thus, clinical judgement should not be substituted by the therapeutic ranges.

Warnings against the overuse of AED monitoring have been published, as a strict relation between serum levels and efficacy and/or toxicity is not available for an individual patient (Chadwick, 1987). Already in the '70s a set of indications for drug monitoring levels was presented (Kutt and Penry, 1974). More recently, guidelines for therapeutic monitoring were presented which amongst other things state that anti-epileptic drug levels should be obtained after:

- Therapy is initiated

- After a change of dosage and/or drug

- In case of poor seizure control or recurrence of seizures which were well controlled previously

Signs or symptoms of intoxication and a routine control of blood levels once or twice a year are also reasons for monitoring (Commission on Anti-epileptic drugs of the ILAE, 1993).

\subsubsection{Change in dosage or drug}

Reasons for changing drug dosage are incomplete control of seizures and the occurrence of side-effects

The appropriate dose for the individual patient preventing the continuation of the seizures can not be determined a priori (Lammers, 1994). In case of persisting seizures the dose will be increased or the drug will be changed depending on the clinical findings. However, frequent changes in drugs or dosages should be avoided (Rowan, 1983). Although the intention is to achieve complete seizure control it is not clear when the dose should be increased: after a reduction in seizure frequency of less than $50 \%$ or less than $90 \%$ or in case any seizure occurs after a steady state has been reached or only after the second seizure.

One should use sufficient time to evaluate therapeutic efficacy. This means at least that the plasma drug level should have reached a steady state before conclusions about the effectiveness of the drug can be drawn. The time period needed to evaluate the effect of treatment on 
the seizure frequency is rarely mentioned in the literature. It has been suggested that a drug should be tried for a period that would be expected to cover three to five seizures or clusters of seizures, or for at least two months whichever period is the longest (Shorvon, 1991). In this period the drug should be provided in a sufficient dose to reach the upper part of the therapeutic range, confirmed by results from repeated drug monitoring.

\subsubsection{Compliance}

Compliance is the extent to which a patient adheres to a given medical and/or health advice. It concerns the drug intake as well as the keeping of therapeutic appointments (Mattson et al. 1988). In general it is estimated that one third to one half of the patients on chronic medication do not follow the medical advice about the prescription as presented by the physician (Schmidt and Leppik, 1988). Reasons for non-compliance are the loss of individual freedom caused by the need of a regular drug intake, the required changes in life-style or loss of independence because of control by the relatives. Disagreement on the goals for treatment and discordance on the value of the elimination of unhealthy conditions can cause non-compliance as well. Worry about one's health can be caused by a high seizure frequency. Contrary to this, persons with few seizures are more lax about their condition and treatment (Peterson et al. 1982). When a patient disapproves the duration of the treatment the treatment may be rejected. Furthermore, patients and doctors can have a different perception of the risks posed by the disease and the tolerance of the drug dosage. Patients weigh the consequences of compliance against the threat and consequences of having seizures. This process of weighing can result in non-compliance.

A deliberate change of a treatment by the patient herself is called the self-regulation or selfmanagement of the patient. It stresses the fact that it is the patient who has control over the drug intake. Self-regulation also includes the option that patients can take on their own initiative more drugs than prescribed (Thorbecke, 1988).

Contrary to a deliberate change, non-compliance may be due to inadvertency i.e despite the understanding, the acceptance and the desire to adhere to the treatment. This includes noncompliance due to changes in daily pattern during weekends or holidays, or simply forgetting because of absence of a routine for intake of tablets (Höppener and van der Lugt, 1983).

Some suggest that there is no significant difference in seizure control in patients who comply and those who are non-compliant (Schmidt et al. 1988). However, it has been shown that improved seizure control can be expected if compliance is monitored closely (Wannamaker et al. 1980). This may be accomplished by the measurement of anti-epileptic drug levels and patient interview (Leppik, 1988). The reports of a patient may well be a minimum estimate of non-compliance (Trostle, 1988). The additional use of special medication containers and self recording of seizures and drug intake can be easily implemented and may enable a more complete view on compliance. Furthermore, good compliance may be achieved by convenient regimen characteristics such as simplicity, individualisation and low intake frequency (Peterson et al. 1984). Besides this, a good partnership between patient and physician is considered to be of great importance to achieve successful treatment (Green and Simons-Morton 1988, Leppik and Schmidt 1988). According to patients sufficient time, presentation of consistent clinical information and acknowledgement of the role of the patient in controlling the disease will enhance compliance (Feuerstein et al 1988) 


\subsection{Withdrawal}

Many patients will enter a period of prolonged remission. The question of tapering drug treatment will become more relevant to a patient the longer the duration of the seizure-free period gets.

\subsubsection{Reasons for withdrawal}

Among the reasons for a reduction or discontinuation of anti-epileptic drug treatment are factors such as association of the drugs with idiosyncratic and dose related adverse reactions, risks of chronic toxicity and teratogenicity of the drug. Furthermore, the removal of the stigma of epilepsy can be a reason for patients to discuss the option to stop drug treatment (Chadwick, 1988). Successful withdrawal of anti-epileptic treatment can be an advantage when applying for a job and at medical fitness examinations (van Heycop ten Ham, 1980). It is known that many patients stop drug treatment without informing their physician (Zielinski 1974, Goodridge and Shorvon 1983b).

Table 2.11-I: Relapse rates Juring and after withdrawal of anti-epileptic drugs

\begin{tabular}{|c|c|c|c|c|c|c|c|c|}
\hline First author & Study & Sel* & $N$ & $\mathrm{SF}^{* *}$ & Relapses & Taper & Withdrawn & F.U.** \\
\hline $\begin{array}{l}\text { Juul-Jensen } \\
(1964) \\
(19+i 8)\end{array}$ & $\begin{array}{l}\text { prospect } \\
\text { retrospecl }\end{array}$ & $\begin{array}{l}\mathrm{EC} \\
\mathrm{EC}\end{array}$ & $\begin{array}{l}200 \\
196\end{array}$ & $\begin{array}{l}\geq 2 y s \\
\geq 2 y s\end{array}$ & $\begin{array}{l}70(35 \%) \\
79(40 \%)\end{array}$ & $\begin{array}{l}20(28 \%) \\
20(25 \%)\end{array}$ & $\begin{array}{l}50(72 \%) \\
59(75 \%)\end{array}$ & $\begin{array}{l}m=24 \\
m=60\end{array}$ \\
\hline $\begin{array}{l}\text { Oller-Daurella } \\
(1076) \\
(1977) \\
(1987)\end{array}$ & $\begin{array}{l}\text { retrospect } \\
\text { retrospect } \\
\text { retrospect }\end{array}$ & $\begin{array}{l}\text { nav } \\
\text { nav } \\
\text { nav }\end{array}$ & $\begin{array}{l}356 \\
522 \\
608\end{array}$ & $\begin{array}{l}\geq 5 \text { ys } \\
\geq 5 \text { ys } \\
\geq 5 \text { ys }\end{array}$ & & $\begin{array}{l}\text { nav } \\
\text { nav } \\
\text { nav } \\
\text { nav }\end{array}$ & $\begin{array}{l}29 / 138(21 \%) \\
82 / 241(30 \%) \\
119 / 474(24 \%) \\
25 / 134(19 \%)\end{array}$ & $\begin{array}{l}(6-240) \\
m=120 \\
m=180 \\
m=55\end{array}$ \\
\hline $\begin{array}{l}\text { van Hevcop ten } \\
\text { Ham }(1980)\end{array}$ & prospect & $\mathrm{EC}$ & 156 & $\geq 5$ ys & $49(31 \%)$ & $28(57 \%)$ & $21(43 \%)$ & $\geq 24$ \\
\hline $\begin{array}{l}\text { Overweg } \\
\text { (1981) }\end{array}$ & prospect & $\mathrm{EC}$ & 46 & $\geq 3 \mathrm{ys}$ & $29(63 \%)$ & 21 & 8 & $(9-19)$ \\
\hline $\begin{array}{l}\text { Bouma } \\
(1987)\end{array}$ & prospecl & $D C N$ & 116 & $2 y s$ & $26(22 \%)$ & mav & nav & $\begin{array}{l}m=51 \\
(9-120)\end{array}$ \\
\hline $\begin{array}{l}\text { Arts' } \\
(1988)\end{array}$ & prospecl & NDOC & 146 & $\geq 2 y \mathrm{~s}$ & $37(25 \%)$ & $7(19 \%)$ & $30(81 \%)$ & $\begin{array}{l}m=52 \\
(16-113)\end{array}$ \\
\hline $\begin{array}{l}\text { Callaghan } \\
\text { (1988) }\end{array}$ & prospecl & nav & 92 & $2 y s$ & $31(34 \%)$ & $13(45 \%)$ & $18(55 \%)$ & $\begin{array}{l}m=35 \\
(6-62)\end{array}$ \\
\hline $\begin{array}{l}\text { Med.Res.C. } \\
\text { AED.Withdr. } \\
\text { (1991) }\end{array}$ & $\begin{array}{l}\text { rundom. } \\
\text { prospect }\end{array}$ & $\mathrm{MC}$ & 1013 & $\geq 2$ & $\begin{array}{l}221 / 510 \\
(43 \%)\end{array}$ & $\begin{array}{l}107 / 221 \\
(48 \%)\end{array}$ & $\begin{array}{l}|14 / 22| \\
(52 \%)\end{array}$ & $\begin{array}{l}\text { med }=27 \\
(17,42)^{3}\end{array}$ \\
\hline
\end{tabular}

- Selting: EC: Epilepsy Clin., NDOC: Neurology Dept. Outpatient Clin., DCN: Devision of Child Neurology, $\mathrm{MC}$ multiple centres

* Seizure-free period in years

**Follow-up period in months

(1) nav: not available,

(2) Study contains only children,

(3) Definition of epilepsy includes patients with $\geq 2$ afebrile or $\geq 3$ atypical febrile seizures

(4) Median and $25^{\text {th }}$ and $75^{\text {th }}$ percentiles 
Social factors are a main issue for patients deciding not to taper drug truatment. An example of this is the obtaining of the drivers license which may cause a patient to refuse withdrawal of drug treatment (van Heycop ten Ham, 1980). Other patients may refuse withdiawal hecause they fear the consequences of a seizure at work (Pedley, 1988). Because of these perceived risks an analysis of costs and benefits should be made by the physician and the patient before starting an attempt to taper the drug treatment .

The interpretation of studies regarding relapse during and after withdrawal is diflicult. Otten information on the duration of the remission period before commencement of the withdrawal, the seizure types and syndromes, the time taken for the drug withdrawal and diagnostic criteria for recurrent seizures as well as the follow-up period is missing (Chadwick 1988, Pedley 1988). Papers that do mention these data often cannot be compared because of differences in populations studied (Table 2. I 1-1).

Patient characteristics that influence the decision to withdraw anti-epileptic drugs in a positive or negative way have been published (Chadwick and Reynolds 1985, Dean and Penry 1989). One of the factors in favour is the diagnosis of primary generalised epilepsy. It has been shown that absence seizures recur only in $5-20 \%$. Primary generalised tonic-clonic seizures have a recurrence rate of 20-33\% (Janz and Sommer-Burkhardt 1976, Oller-Daurella and Oller 1987, Callaghan et al. 1988). Patients with partial epilepsy show a higher relapse rate; this may be due to the inclusion of symptomatic seizures (Annergers et al. 1979).

Contradictory findings have been reported about the predictive value of the age at onset of epilepsy for the outcome of the withdrawal of the drugs (Janz and Sommer-Burkhardt 1976, Oller-Daurella and Oller 1987, Berg and Shinnar, 1994).

The relapse rate after drug tapering in children has been observed to be somewhat lower than in adult patients, $31 \%$ and $35 \%$ respectively (Callaghan et al. 1988). A literature survey of studies regarding adults and children indicates that the age may be important, especially in conjunction with other factors such as duration, severity and frequency of seizures before treatment (Dean and Penry, 1989). In another survey the opposite is stated, based mainly on studies in children (Hauser and Hesdorffer, 1990). However, more recently it has been shown that children have a lower risk than adults for recurrence of seizures during the first two years after withdrawal (Medical Research Council Anti-epileptic Drug Withdrawal Study Group, 1993)

The number of seizure-free years before withdrawal has been reported as a determinant for the success of withdrawal. Without mentioning the exact data some suggest that there does not seem to be much difference between the relapse rates of patients who are 5 years seizure free and those that are 10 years seizure free (Oller-Daurella et al. 1977). It has been shown that there does not seem to be a difference in recurrence rate after withdrawal for children treated for 2, 3, 4 or 5 years (Arts et al. 1988).

Recommendations on the duration of the period during which the drug is withdrawn vary from four weeks up to several years (Oller-Daurella et al. 1977, van Heycop ten Ham 1980, Overweg et al. 1981, Bouma et al. 1987, Arts et al. 1988, Callaghan et al. 1988). A comparison of seizure recurrence between drug tapering periods of 6 weeks and 9 months did not show a significant difference in risk of recurrent seizures (Tennison et al. 1989, 1994). A duration of at least three month has been advocated, however mainly on basis of data in children. 


\subsubsection{Moment of relapse}

Most of the studies indicate that the number of patients who relapse during the tapering of drug treatment is between $19 \%$ and $57 \%$ (Table 2.11-1). A randomised multicenter trial showed that $52 \%$ of the patients will experience a relapse after withdrawal of drugs (Medical Research Council AED Withdrawal Study Group, 1991). Other studies report that $43 \%$ up to $81 \%$ of the patients had a seizure after the withdrawal was completed. However, three Spanish studies indicate a lower rate of relapses after drug withdrawal. Only 19-30\% of the patients had seizures after discontinuing the treatment. A reason for this difference may be the longer seizure free period ( $\geq 5$ years) before patients were admitted to the tapering. Furthermore, the long period used for drug tapering - for most of the patients up to a year and some even longer - may have had an selective effect on the patients reaching drug withdrawal (OllerDaurella et al 1976, 1977, Oller-Daurela and Oller 1987).

Most of the patients in which the drug is withdrawn relapsed during the first year after the treatment was discontinued (Juul-Jensen 1968, Oller-Daurella et al. 1976, Janz and SommerBurkhardt 1976, Thurston et al. 1972, 1982, Shinnar et al. 1985, Bouma et al. 1987, Callaghan et al. 1988, Medical Research Council Anti-epileptic Drug Withdrawal Study Group, 1991). Most of the relapses occur even earlier after withdrawal, mainly during the first six months.

Recommencing the anti-epileptic treatment after a relapse during drug tapering or after withdrawal results in control of the seizures in many but not all of the patients. In a group of 31 patients who relapsed and where reinstalled on treatment 20 patients $(64.5 \%)$ became seizure free again for 4-50 months, while seven patients (22.5\%) continued to have seizures (Callaghan et al 1988). A study in children showed that one third of the patients whose drug treatment was reintroduced, remained seizure-free after a second withdrawal period, while $50 \%$ were seizure-free for at least six months. In $17 \%$ of the patients seizures were difficult to control (Arts et al. 1988).

\subsection{Summary and conclusion}

Although studies on epilepsy suffer from differences in terminology and case finding strategies they indicate a prevalence rate of $0.4-0.6 \%$. The incidence rate ranges from $0.03-0.05 \%$. The main part of community studies indicate that $60-75 \%$ of the patients have an epilepsy of unknown aetiology.

One of the first principles for the management of epilepsy is to establish the diagnosis as precisely as possible. Because of the paroxysmal character of epilepsy the diagnosis may be difficult to establish. Other diseases such as diabetes, cardiac disease or syncopes can mimic the epileptic seizures. Epilepsy should be treated as early as possible to prevent a chronic state of seizures which may be difficult to treat. The consequences of late treatment can conflict with the time necessary for establishing the correct diagnosis. An incorrect diagnosis can result either in long term anticonvulsive drug intake for non-epileptic causes or in the development of refractory epilepsy. Thus, if the diagnosis is not certain the physician should let time make things clear.

Epilepsy can be treated through regular daily intake of medication. A two year remission of the disease within three years after onset of treatment has been reported in $50-70 \%$ of the patients, while up to $90 \%$ of the patient may show a remission after more than five years. However, patients with additional neurologic, psychiatric or social handicaps seem to have a 
poor prognosis for achieving a remission of seizures. Although insight in drug mechanisms is growing the question whether the disease is cured or seizures are suppressed has not been answered unequivocally

Deciding to start drug treatment implies that the patient will be on anticonvulsants for at least two years. Reasons for the treatment should be sufficiently explained to the patient and his family. Before the treatment is started the physician should educate the patient on the different aspects of the disease, the reason for drug intake, the duration of the treatment, the prognosis and the possibility of side-effects. While a chronic disease such as epilepsy is treated problems of a medical, psychological, social and economical nature may occur. Thus, whether or not to start treatment requires an assessment of the pros and cons of the disease and the cure.

The treatment should be based on a correct diagnosis. There is hardly any room for an ex juvantibus approach to establish the correct diagnosis. There is no consensus whether the selection of the anti-epileptic drug should be based on the seizure type or the type of epilepsy. Monotherapy is preferred above polypharmacy when starting treatment. However, in the future new drugs may make polytherapy a viable option again. The literature shows little consensus regarding the drugs which should be applied for the different types of seizures or epilepsy. Small differences exist in the effectiveness of the drugs for treatment of the different types of epilepsy. Therefore the selection of drugs is based on other aspects such as occurrence of side effects. Also little consensus exists in the literature on the appropriate daily dose and the required anti-epileptic serum levels

The differences in selection of drugs, appropriate doses and required anti-epileptic drug serum levels make the treatment of epilepsy to a large extent based on trial and error. Regular follow-up contacts enable the physician to monitor the effect of the treatment. Although physicians seem to agree on the goal of drug treatment, $20-30 \%$ of the treatment decisions are questionable.

Withdrawal of drug treatment can be started after a seizure free period of two years. There is no consensus on the duration of the tapering period. In the literature the duration varies from several weeks up to many years. In those patients who start drug withdrawal some 20$50 \%$ will suffer a relapse before the drugs are stopped completely. Most of the seizures reoccur early after withdrawal, mainly during the first 6 months. Reintroducing drug treatment will be successful in about $65 \%$ of these patients. The rest of the patients have seizures which are difficult to control.

Patients suffering from seizures will worry about the cause of the attacks. Having been confronted with the diagnosis the patient may experience negative emotions in contacis with other people. Furthermore, patients may loose their driving license and will be afraid about further impacts of the disease on their social life. Commencing anti-epileptic drug treatment will have consequences for the patient (e.g. daily drug intake for at least two to three years). Having been healthy and suddenly being confronted with a disease with paroxysmal symptoms will make the patients tend to deny the illness and keep carrying on their life as they did before the diagnosis. It is generally accepted that at least one third of the patients do not follow the prescription presented by the physician. Part of this non-compliance may be caused unintentionally. However, self-regulation of the treatment is also an important cause. The patient will resent the medical profession if the physician does not seem to really listen to his problems or does not support him. Absence of a good patient-doctor contact will cause 
compliance to decrease. Issues such as accomplishment of the same goals by the patient and his physician and acknowledgement of the role of the patient in the treatment together with close monitoring of the patient during remissions can improve the compliance of the patient.

Drug treatment is only part of the management of epilepsy. Guidance of patients as well as those who make treatment decisions that influence the lives of the patients is also part of the proper management.

\subsection{References}

Aird R.B., Masland R.L., Woodbury D.M., The epilepsies a critical review, Raven Press, New York, 1984.

Annegers J.F., Hauser A., Elveback L.R., Remission of seizures and relapse in patients with epilepsy, Epilepsia, 20, 1979, pp. 729-737.

Annegers J.F., Hauser A., Shirts S.B., Kurland L.T., The risk of epilepsy following febrile convulsions, The New England Journal of Medicine, 316, 1987, pp.493-498.

Arts W.F.M., Visser L.H., Loonen M.C.B., Tjiam A.T., Stroink H., Stuurman P.M., Poortvliet D.C.J., Follow-up of 146 children with epilepsy after withdrawal of anti-epileptic therapy, Epilepsia, 29, 1988, pp. 244-250.

Aspinal A., The real obstacles. In: Epilepsy and employment (Edwards, Espir and Oxley, Eds.), Royal Society of Medicine Scrvices Limited, London, 1986, pp. 19-20.

Beardsley R.S., Freeman J.M., Appel F.A.. Anticonvulsant serum levels are useful only if the physician appropriately uses them: an assessment of the impact of providing serum level data to physicians, Epilepsia, 24, 1983, pp. 330-335.

Beghi E., Di Mascio R., Tognoni G., Drug treatment of epilepsy, outlines, criticism and perspectives, Drugs, 31, 1986, pp. 249-265.

Beghi E., Tognoni G., Prognosis of epilepsy in newly referred patients: A multicenter prospective study, Epilepsia, 29, 3, 1988, pp. 236-243.

Berg A.T., Shinnar S., Relapse following discontinuation of anti-epileptic drugs: a meta-analysis, Neurology, 44, 1994, pp.601-608.

Bergamini L., Bergamasco B., Benna P., Gilli M., Acquired etiological factors in 1.785 epileptic subjects: clinical anamnestic research, Epilcpsia, 18, 1977, pp. 437-444.

Betts T., Employment of people with epilepsy within the National Health Service. In: Epilepsy and employment (Edwards, Espir and Oxley, Eds.), Royal Society of Medicine Services Limited, London, 1986, pp. 59-66.

Bongers E., Coppoolse J., Meinardi H., Posthume E., Zijl C. van. A survey of epilepsy in Zeeland The Netherlands, Instituut voor Epilepsiebestrijding Meer en Bosch - De Cruquiushoeve, Heemstede, 1976.

Bouma P.A.D., Peters A.C.B., Arts R.J.H.M., Stijnen Th, Rossum J. van, Discontinuation of antiepileptic therapy: a prospective study in children, Journal of Neurology. Neurosurgery and Psychiatry, 50, 1987, pp. 1579-1583.

Brodie M J. Status epilepticus in adults. The Lancet, 336, 1990, pp. 551-552

Brodic M.J.. Dichter M.A. Anti-cpileptic drugs, The New England Journal of Medicine, 334, 1996, pp. $168-175$

Callaghan N. Garrett A., Goggin T., Withdrawal of anticonvulsant drugs in patients free of seizures for two years, The New England Journal of Midicimi, 318, 1988, pp. 942-946. 
Cereghino J.J, Epidcmiology of epilepsy, Public Health Reviews. 3, 1974, pp. 91-100.

Cereghino J.J., Brock J.T., Meter J.C. van, Penry J.K., Sinith L.D., White B.G., Carbamazepine for epilepsy, a controlled prospective evaluation, Neurology, 1974, pp. 401-410.

Cereghino J.J., Introduction in anti-epileptic drugs. In: Anti-epileptic drugs, (Levy, Mattson, Meldrum, Penry and Dreifuss, Eds.), $3^{\text {rd }}$ edition, Raven Press, New York, 1989, pp. 23-27.

Chadwick D.W., Overuse of monitoring of blood concentrations of anti-epileptic drugs, British Medical Journal, 294, 1987, pp. 723-724.

Chadwick D., Drug withdrawal and epilepsy, when and how ? Drugs, 35, 1988, pp. 579-583.

Chadwick D., Diagnosis of epilepsy, The Lancet, 336, 1990, pp. 291-296.

Chadwick D., Epilepsy after first scizures: risks and implications, Journal for Neurology, Neurosurgery and Psychiatry, 54, 1991, pp. 385-387.

Chadwick D., Epilepsy, Journal of Neurology, Neurosurgery and Psychiatry, 57, 1994a, pp.264-277.

Chadwick D., Standard approach to anti-epileptic drug tre:atment in the United Kingdom, Epilepsia, 35 (Suppl.4), 1994b, s3-s10

Chadwick D., Case for early treatment is not established, British Midical Journal, 310, 1995, pp.177178.

Chadwick D., Reynolds E.H., When do epileptic patients need treatment? Starting and stopping medication, Brilish Medical Journal, 290, 1985, pp. 1885-1888.

Chadwick D., Tumbull D.M. The comparative efficacy of anti-epileptic drugs for partial and tonicclonic seizures, Journal of Neurology. Neurosurgery and Psychiatry, 48, 1985, pp. 1073-1077.

Chan A.W.K., Alcoholism and epilepsy, Epilepsia, 26, 1985, pp. 323-333.

Chandra B., First seizure in adults: to treat or not to treat, Clinical Neurology and Neurosurgery, 94 (Suppl.), 1992, s61-s63.

Chaplin J.E., Yepez Lasso R., Shorvon S.D., Floyd M., National general practice study of epilepsy: the social and psychological effects of the recent diagnosis of epilepsy, British Medical Journal, 304, 1992, pp. 1416-1418

Cockerell O.C., Eckle 1., Goodridge D.M., Sander J.W., Shorvon S.D., Epilepsy in a population of 6000 re-examined: secular trends in first attendance rates, prevalence, and prognosis, Journal for Neurology, Neurosurgery and Psychiatry, 58, 1995a, pp. 570-576

Cockerell O.C, Johnson A.L., Sander J.W., Hart Y., Shorvon S., Remission of epilcpsy. results from the National Gencral Practice Study of Epilepsy, The Lancet, 346, 1995b, pp. 14(1)-144

Collaborative Group for Study of Epilepsy. Prognosis of epilepsy in newly refurred patients: a multicenter prospective study of the effects of monotherapy on the long-term course of epilepsy. Epilepsia, 33, 1992, pp. 45-51

Commission on Anti-epileptic Drugs of the International League Against Epilepsy, Guidelines for therapeutic monitoring on anti-epileptic drugs, Epilepsia, 34, 4, 1993, pp. 585-587

Commission on Classification and Terminology of the Intemational League Against Epilepsy, Proposal for revised classification of epilepsies and cpileptic syndromes. Fipilepsia, 30, 1989, pp. 389-399.

Commission on Epidemiology and Prognosis of the International Lungue Against Epilepsy, Guidelines for epidemiologic studies on epilepsy, Epilepsia, 34, 4, 1993, pp. 592-596.

Commission on Epilepsy, Risk and Insurance of the Intcmational Bureau for Epilepsy, Workshop on epilepsy, risks and insurance, Epilcpsia, 34, 4, 1993, pp. 590-591.

Cowan L.D., Bodensteiner J.B., Leviton A., Doherty L., Prevalence of the epilepsies in children and adolescents, Epilepsia, 30, 1989, pp. 94-106. 
Craig, A., Oxley J., Statutory and non-statutory barriers to the employment of people with epilepsy. In: Epilepsy and employment (Edwards, Espir and Oxley, Eds.), Royal Society of Medicine Services Limited, London, 1986, pp. 21-31.

Craig A., Oxley J., Social aspects of epilepsy. In: A texlbook of epilepsy (Laidlaw, Richens and Oxley, Eds.), Churchill Livingstone, Edinburgh, 1988, pp. 566-610.

Crobach M.J., Niezink G.M., Leden J. van der, Springer M.P., Epilepsie en huisarts: toeval of hoofdzaak? Nederlands Tijdschrift voor de Geneeskunde, 132, 1988, pp. 1888-1892.

Crombie D.L., Cross K.W., Fry J., Pinsent R.J.F.H., Watts C.A.H., A survey of epilepsy in general practice, British Medical Journal, 1960, pp. 416-422.

Dam M., Gram L., Pedersen B., Orum H., Modern approach to anti-epileptic arug treatment, improving quality of life. The Danish Epilepsy Society, Kobenhavn, 1985.

Dam M., Kiorboe E., Epilepsy, diagnosis and treatment, Scriptor, Copenhagen, 1982.

Dean J.C., Penry J.K., Gencral principles, discontinuation of anti-epileptic drugs. In: Anti-epileptic drugs, (Levy, Mattson, Meldrum. Penry and Drcifuss, Eds.), $3^{\text {rd }}$ edition, Raven Press, New York, 1989, pp. 133-142.

Dichter M.A., Emerging insights into the mechanisms of epilepsy: implications for new anti-epileptic drug development, Epilepsia.35 (Suppl.4), 1994, s5!-s57.

Dick D., Epilepsy in the British steel industry. In: Epilepsy and employment (Edwards, Espir and Oxley, Eds.), Royal Society of Medicine Services Limited, London, 1986, pp. 49-52.

Donselaar C. van, Idiopathic first seizure in adull life, thesis, Rotterdam, 1990.

Drcifuss F.E., The cpilepsies: clinical implications of the international classification, Epilepsia, 31 (Suppl), 1990, s3-s!0

Elwes R.D.C., Chesterman P., Rcynolds E.H., Prognosis after a first untreated tonic-clonic seizure, The Lancet, okt., 1985, pp. 752-753.

Elwes R.D., Johnson C.B., Shorvon S.D., Reynolds E.H., The prognosis for seizure control in newly diagnosed epilepsy, The New England Journal of Medicine, October 1984, pp. 944-947.

Elwes R.D.C., Johnson A.L., Reynolds E.H., The course of untreated epilepsy, British Medical Journal, 297, 1988, pp. 948-297

Elwes R.D.C., Marshall J. Beattic A., Newman P.K., Epilepsy and employment. A community based survey in an area of high uncmployment. Journal of Neurology. Neurosurgery. and Psychiatry, 54, 1991, pp. 200-203

Elwes R.D.C., Reynolds E.H., Should people be treated after a first scizure?, Archives of Neurology, 45, 1988, pp.490-491

Elwes R.D.C., Reynolds E.H., The early prognosis of epilepsy, In: Comprehensive Epileptology (Dam and Gram, Eds.), Ravens Press, New York, 1990, pp. 715-727.

Employment Commission of the Intemational Bureau for Epilepsy, Employing people with epilepsy: principles for good practice, Epilepsia, 30, 1989, pp. 4II-4I2.

Espir M, Floyd M., Epilepsy and recruitment, In: Epilepsy and employment (Edwards, Espir and Oxley, Eds.), Royal Seciety of Medicine Services Limited, London, 1986, pp. 39-46.

Feuerstein M., Licb-Jückstock V., Schnaus H., Springmann E., Weber B., Wunderlich M., Compliance, a joint effort of the patient and his doctor, Epilepsy Research. Suppl. I, 1988, s5 Is56.

First Seizure Trial Group. Randomised clinical trial on the efficacy of anti-epileptic drugs in reducing the risk of relapse after a first unprovoked tonic-clonic seizure, Neurology, 43, 1993, pp 478-483 
Fisher R.S., Parsonage M., Beaussart M., Bladin P., Masland R., Sonnen A.E.H., Rémillard G., Epilepsy and Driving: an intemational perspective, Epilepsia, 35, 3, 1994, pp. 675-684.

Forsgren L., Prospcctive incidence study and clinical characterization of seizures in newly referred adults, Epilepsia, 31, 1990, pp.292-301.

Forsgren L., Prevalence of epilepsy in adults in northern Siveden, Epilepsia, 33, 1992, pp. 450-458.

Foy P.M., Chadwick D.W., Rajgopalan N., Johnson A.L., Shaw M.D.M., Do prophylactic anticonvulsant drugs alter the pattern of seizures after craniotomy, Journal of Neurologv. Neurosurgery and Psychiairy, 55, 1992, pp.753-757.

Gastaut H., Clinical and encephalographic classification of epilcptic seizures, Epilepsia, 11, 1970, pp. 102-113.

Gastaut H., Kugler J., Wörferbuch der Epilepsie, Hippokrates Verlag GmbH, Stuttgart, 1976.

Gastaut H., Zifkin B.G., The risk of automobile accidents with seizures occurring while driving: relation to seizure type, Neurology, 37, 1987, pp.1613-1616.

Goodridge D.M., Shorvon S.D., Epileptic seizures in a population of 6000, I: Demography, diagnosis and classification, and hospital services, Brilish Medical Journal, 287, I983a, pp. 64 I-644.

Goodridge D.M., Shorvon S.D., Epileptic seizures in a population of 6000, II: Treatment and prognosis, British Medical Journal, 287, 1983b, pp. 645-647.

Gowers W.R., Epilepsy and other chronic convulsive diseases; their causes, symptoms and treatment, reprint of the first American edition 1885, Arts en Boeve, Nijmegen, 1994, pp.200.

Graaf A.S. de, Epidemiological aspects of epilcpsy in northern Norway, Epilepsia, 15, 1974, pp. 291299.

Gram L., Draclumann Bentsen K., Pamas J., Flachs H, Controlled trials in epilcpsy: a review, Epilepsia, 23, 1982, pp. 491-519.

Gram L., Wulff K., Rasmussen K.E., Flachs H., Würtz-Jorgensen A., Sommerbeck K.W., Lohren V., Valproate sodium: a controlled clinical trial including monitoring of drug levels, Epilepsia, 18, 1977, pp. 141-148

Granieri E., Rosati G., Tola R., Pavoni M., Paolino E., Pinna L., Monetti V., A descriptive study of epilepsy in the district of Copparo, Italy, 1964-1978, Epilepsia, 24, 1983, pp. 504-514.

Green L.W., Simons-Morton D.G., Denial, delay and disappointment: discovering and overcoming the causes of drug errors and missed appointments. Epilepsy Research, Suppl. 1, 1988, s7-s22.

Haerer A.F., Anderson D.W., Schoenberg B.S., Prevalunce and clinical features of epilepsy in a biracial United States population, Epilepsia, 27, 1986, pp. 66-75

Hansotia P., Broste S.K. The effect of epilepsy and diabetes mellitus on the risk of automobile accidents, The New England Journal of Medicine, 324, 1991, pp.22-26.

Hansotia P., Broste S.K., Epilepsy and traffic safety, Epilepsia, 34, 1993, pp. 852-858.

Harding G., Photosensitive epilepsy and employment. In: Epilepsy and employment (Edwards, Espir and Oxley, Eds.), Royal Society of Medicine Services Limited, London, 1986, pp. 75-87

Hart Y.M., Sander J.W.A.S., Johnson A.L., Shorvon S.D., National General Practice Study of Epilepsy: recurrence after a first seizure. The Lancet, 336, 1990, pp. 127/-1274.

Hauser A.W., Epidemiology of epilepsy. In: Advances in Neurology, vol. 19, (Schoenberg ed.), Raven Press, New York, 1978, pp. 313-339

Hauser A.W., Prevention of post-traumatic epilepsy, The New England Journal of Medicine, 323, 1990, pp. 540-541.

Hauser A.W., Seizure disorders: the changes with age, Epilepsia, 33 (Suppl.4), 1992, s6-s 14. 
Hauser A.W., Annegers J.F., Kurland L.T., Prevalence of epilepsy in Rochester, Minnesota: 19401980, Epilepsia, 32, 1991, pp. 429-445.

Hauser A.W., Annegers J.F., Kurland L.T., Incidence of epilepsy and unprovoked seizures in Rochester, Minnesota: 1935-1984, Epilepsia, 34, 1993, pp.453-468.

Hauser A.W., Hesdorffer D.C., Prognosis. In: Epilepsy, frequency, causes, and consequences, (Hauser and Hessdorffer, Eds.), Epilcpsy foundation of America, Maryland, 1990, pp. 197-243.

Hauser A.W., Kurland L.T., The epidemiology of cpilepsy in Rochester, Minnesota, 1935 through 1967, Epilepsia, 16, 1975, pp. 1-66.

Hauser A.W., Ng S.K.C., Brust J.C.M., Alcohol, seizures and epilepsy, Epilepsia, 29 (Suppl.2), 1988, s66-s78

Heller A.J., Chesterman P., Elwes R.D., Crawford P., Chadwick D., Johnson A.L., Reynolds E.H., Phenobarbitone, phenytoin, carbamazepine, or sodium valproate for newly diagnosed adult epilepsy: a randomised comparative monotherapy trial, Journal of Neurology, Neurosurgery and Psychiatry, 38, 1995, pp.44-50.

Heycop ten Ham M.W. van, Complete recovery from epilepsy ? Discontinuation of antiepileptics after five or more seizure-free years, Huisarts en Wetenschap, 23, 1980, pp. 309-311.

Hicks R.A., Hicks M.J., Attitudes of major employers toward the employment of people with epilepsy: a 30-year study, Epilepsia, 32, 1991, pp. 86-88.

Hopkins A., Definition and epidemiology of epilepsy. In: Epilepsy, (Hopkins, Ed.), Chapman and Hall, London, 1987, pp. 1-18

Hopkins A., Garman A., Clarke C., The first seizure in adult life, the value of clinical features, Electroencephalography, and computerised tomographic scanning in the prediction of seizure recurrence, The Lancet, April 2. 1988, pp. 721-726.

Höppener R.J.E.A., Epilepsy and alcohol, thesis, Maastricht, 1981

Höppener R.J.E.A., Lugt P.J.M. van der, De medicamenteuze behandeling bij epilepsie, Pharmaceulisch Weekblad, 118, 1983, pp. 290-296.

Janz D., Die Epilepsien. Spezielle Pathologie und Therapie, Thieme, Stuttgart; 1969.

Janz D., Sommer-Burkhardt E.M., Discontinuation of anti-epilcptic drugs in patients with epilepsy who have been seizure-free for more than two years. In: Epileptology. Proc of the $7^{\text {th }}$ International Symposium on Epilepsy, (Janz ed.), Thieme-Verlag, Stuttgart, 1976, pp. 228-234.

Jeavons P.M. Choice of drug therapy in epilepsy, The Practitioner, 219, 1977, pp. 542-556.

Jennett B., Epilepsy after head injury and intracranial surgery, In: Epilepsy, (Hopkins, Ed.), Chapman and Hall, London, 1987, pp. 40I-4I2.

Johannessen S.1., Loyning Y., Munthe-Kaas A.W., General Aspects. In: Comprehensive Epileptology (Dam and Gram, Eds.), Ravens Press, New York, 1990, pp. 505-524.

Juul-Jensen $P$., Frequency of recurrence after discontinuance of anticonvulsant therapy in patients with epileptic seizures, Epilepsia, 5, 1964, pp. 352-363

Juul-Jensen $\mathrm{P}$., Frequency of recurrence after discontinuance of anticonvulsant therapy in patients with epileptic seizures, a new follow-up study after 5 years, Epilepsia, 9, 1968, pp. 11-16.

Juul-Jensen P., Foldspang A., Natural history of epileptic seizures, Epilepsia, 24, 1983, pp. 297-312.

Keränen T., Riekkinen P.J., Remission of seizures in untreated epilepsy, British Medical Journal, 307, 1993 , pp. 483.

Keränen T., Riekkinen P.J., Sillanpää M., Incidence and prevalence of epilepsy in adults in eastern Finland, Epilepsia, 30, 1989, pp. 413-421. 
Kosteljanetz M., Christiansen J., Mouritzen Dam A., Stengaard Hansen B., Blatt Lyon B., Pedersen H., Dam M., Carbamazepine vs phenytoin, a controlled clinical trial in focal motor and generalised epilepsy, Archives of Neurology, 36, 1979, pp. 22-24.

Krom M.C.T.F.M. de, Lugt P.J.M. van der, Anti-epileptica 2, Geneesmiddelen Bulletin, 16, 1982, pp. 9-12.

Krumholz A., Driving and epilepsy: a historical perspective and review of current regulations, Epilepsia, 35, 3, 1994, pp. 668-674.

Kutt H., Penry K., Usefulness of blood levels of anti-epileptic drugs, Archives of Neurology, 31, 1974, pp. 283-288.

Laidlaw J., Richens A., Oxley J., Parsonage M., Porter R.J., Classification of epileptic seizures. In: $A$ texibook of epilepsy (Laidlaw, Richens and Oxley, Eds.), Churchill Livingstone, Edinburgh, 1988, pp. $1-20$.

Lammers M.W., Clinimetrics in epileptology, thesis, Nijmegen, 1994

Lammers M.W., Hekster Y.A., Keizer A., Meinardi H., Renier W. O., Monotherapy or polytherapy for epilepsy revisited a quantitative assessment, Epilepsia, 36, 1995, pp.440-446.

Lechtenberg R., The diagnosis and treatment of epilepsy, Macmillan Publishing Company, New York, 1985

Leppik I.E., Compliance during treatment of epilepsy, Epilepsia, 29 (Suppl.), 1988, s79-s84.

Leppik I.E., Schmidt D. Consensus statement on compliance in epilepsy, Epilepsy Research, Suppl.1, 1988, s $179-\mathrm{s} 182$

Lugt P.J.M. van der, Epilepsie en verkeer. De medische geschiktheid van epilepsiepatienten tor deelname aan gemotoriseerd wegverkeer, thesis, Rotterdam, 1972.

Lugt P.J.M. van der, Epilepsie, een ratjetoe van problemen,. In: Man en Paard, Liber Amoricum tgv. het Afscheid van prof. A.Staal. 1990, pp. 125-131

Lühdorf K., Jensen L.K., Plesner A.M., Actiology of seizures in the elderly, Epilepsia, 27, 1986 pp $458-463$

Macdonald R.L., Kelly K.M., Mechanisms of action of currently prescribed and newly developed antiepileptic drugs, Epilepsia, 35 (Suppl 4), 1994, s4l-s50.

Maremmani C., Rossi G., Bonucceli U., Murri L.. Descriptive epidemiologic study of epilepsy syndromes in a district of northwest Tuscany, Italy, Epilepsia, 32, 1991, pp. 294-298.

Marsden C.D., Reynolds E.H. Seizures in adults. In: A lextbook of epilepsy (Laidlaw, Richens and Oxley, Eds.), Churchill Livingstone, Edinburgh, 1988, pp. 144-182

Mattson R.H., General principles, selection of anti-epileptic drug therapy, In: Anti-epileptic drugs, (Levy, Mattson, Meldrum, Penry and Drcifuss, Eds.), $3^{\text {rd }}$ edition, Raven Press, New York, 1989, pp. 103-115

Mattson R.H., Cramer J.A., Collins J.F., VA Epilepsy Co-operative Study Group, Aspects of compliance; taking drugs and keeping clinic appointments. Epilepsy Research, Suppl.1, 1988, sll1-sl17.

Mattson R.H., Cramer J.A., Collins J.F., and the Dept. of Veterans Affairs Epilepsy Co-ciperative Study No. 264 Group, A comparison of valproate with carbamazepine for the treatment of complex partial seizures and secondarily generalised tonic-clonic seizures in adults, The New England Journal of Medicine, 327, 1992, pp. 765-771.

Mattson R.H., Cramer J.A., Collins J.F., Smith D.B., Delgado-Escueta A.V., Comparison of carbamazepine, phenobarbital, phenytoin, and primidone in partial and secondarily generalized tonic-clonic seizures, The New England Journal of Medicine, 313, 1985, pp. 145-151. 
Medical Research Council Anti-epilcptic Drug Withdrawal Study Group, Randomised study of antiepileptic drug withdrawal in patients in remission, The Lancet, 337, 1991, pp. 1175-1180.

Medical Research Council Anti-epileptic Drug Withdrawal Study Group, Prognostic index for recurrence of seizures after remission of epilepsy, British Medical Journal, 306, 1993, pp.13741378.

Meijer J.W A., Knowledge, attitude and practice in anti-epileptic drug monitoring, thesis, Nijmegen, 1991.

Meinardi H., Binnie C.D., Goedhart D., Meijer J.W.A., Developments in pharmacotherapy of epilepsy. In: Epilepsy, an update on research and therapy, Alan R Liss Inc, New York, 1983, pp. 249-265.

Meinardi H., Pachlatko C., Special centres for epilcpsy, In: Comprehensive Epileptology (Dam and Gram, Eds.), Ravens Press, New York, pp. 769-779.

Meinardi H., Epilepsie compendium, Katholieke Universiteit Nijmegen, februari 1993.

Merlis J.K., Proposal for an International Classification of the Epilepsies, Epilepsia, 11, 1970, pp. 114119.

Metsemakers J.F.M. Unlocking patients' records in general practice for research, medical education and quality assurance: the Registration Network Family Practices, thesis, Maastricht, 1994.

Milano Collaborative Group For Studies On Epilepsy, Long-term intensive monitoring in difficult patient. Preliminary results of 16 months of observations-usefulness and limitations. In: Antiepileptic therapy advances in drug monitoring, (Johannessen, Pippenger, Schmidt, Morselli, Richens and Meinardi, Eds.), Ravens Prcss, New York, 1980

Montouris G.D. Practical insights and clinical experience with combinations of the new anti-epileptic drugs, Neurology, 45 (Suppl.2), 1995, s25-s28.

Mouritzen Dam A., Fuglsang-Fredriksen A., Svarre-Olsen U., Dam M., Late-onset epilepsy: aetiologies, types of seizures and value of clinical investigation, EEG, and computerized tomography scan, Epilepsia, 26, 1985, pp. 227-231

Oller-Daurella L., Oller F.V.L., Suppression of anti-epileptic treatment, European Neurology, 27, 1987, pp. 106-113.

Oller-Daurella L., Pamies R., Oller L.F.V., Reduction or discontinuance of anti-epileptic drugs in patients seizure-free for more than 5 years. In: Epileprology. Proc. of the $7^{\text {th }}$ International Symposium on Epilepsy, (Janz ed.). Thieme-Verlag, Stuttgart, 1976, pp. 218-227.

Oller-Daurella L., Oller L.F.V., Pamies R., Clinical, therapeutic and social status of epilepsy patients without seizures for more than five years, in: Epilepsy. The $8^{\text {th }}$ International Symposium, (Penry Ed.), Raven Press, New York, 1977, pp. 69-75

Overweg J., Epilepsie; prognose en bchandeling, Nederlands Tijdschrift voor de Geneeskunde, 128, 1984, pp. 1710-1716.

Overweg J., Withdrawal of anti-epileptic drugs in seizure-free adult patients, prediction of outcome, thesis, Amsterdam 1985, Rodopki.

Overweg J., Rowan A.J., Binnic C.D., Oosting J., Nagelkerke N.J.D., Prediction of seizure recurrence after withdrawal of anti-epileptic drugs, In: Advances in Epileptology: $12^{\text {th }}$ International Symposium, (Dam, Gram and Penry, Eds.), Raven Press, New York, 1981, pp. 503-508.

Parsons M., Fits and other causes of loss of consciousness while driving, Quarterly Journal of Medicine, New Series. 58, 1986, pp.295-303.

Pedley T.A., Discontinuing anti-epileptic drugs, New England Journal of Medicine, 318, 1988, pp. 982-984.

Pellock J.M., Standard approach to anti-epileptic drug treatment in the United States, Epilepsia, 35 (Suppl.4), 1994. s11-s18. 
Peterson G.M., McLean S., Millingen K.S., Determinants of patient compliance with anticonvulsant therapy, Epilepsia, 23, 1982, pp. 607-613

Peterson G.M., McLean S., Millingen K.S., A randomised trial of strategies to improve patient compliance with anticonvulsant therapy, Epilepsia, 25, 1984, pp. 412-417.

Porter R.J., Epilepsy, 100 elementary principles, W.B. Saunders Company Ltd., London, 1984.

Porter R.J. General principles how to usc anti-epileptic drugs. In: Anti-epileptic drugs, (Levy, Mattson, Meldrum, Penry and Dreifuss, Eds.), $3^{\text {rd }}$ edition, Raven Press, New York, 1989, pp. 117-131.

Quirk J.A., Fish D.R., Smith S., Sander J.W., Shorvon S.D., Allen P.J., First seizures associated with playing electronic screen games: a community based study, Annals of Neurology, 37, 1995 , pp.733-737.

Ramsay R.E., Wilder B.J., Berger J.R., Bruni J., A double blind study comparing carbamazepine with phenytoin as initial seizure therapy in adults, Neurology, 1983, pp. 904-910.

Reynolds E.H., Serum levels of anticonvulsant drugs; interpretation and clinical value, Pharmacology and Therapeutics, 8, 1980, pp. 217-235.

Reynolds E.H., Early treatment and prognosis of epilcpsy, Epilepsia, 28, 1987, pp. 97-106.

Reynolds E.H., The prevention of chronic epilepsy, Epilepsia, 29 (Suppl.1), 1988, s25-s28.

Reynolds E.H., Changing view of prognosis of epilepsy, British Medical Journal, 301, 1990, pp. $1112-1114$.

Reynolds E.H, Do anticonvulsants alter the natural course of epilepsy? Treatment should be started as carly as possible, British Medical Journal. 310, 1995, pp. 176.

Reynolds E.H., Chadwick D., Galbraith A.W., One drug (phenytoin) in the treatment of epilepsy, The Lancet, May, 1976, pp. 923-926.

Reynolds E.H., Heller A.J., Chadwick D., Valproate versus carbamazepine for seizures, The New England Journal of Medicine, 328, 1993, pp. 207-208.

Reynolds E.H., Shorvon S.D., Monotherapy or polypharmacy for epilepsy?, Epilepsia, 22, 1981, pp. $1-10$.

Richens A., Ahmad S., Controlled trial of sodium valproate in severe epilepsy, British Medical Journal, 1975, pp. 255-256.

Richens A., Davidson D.L.W., Cartlidge N.E.F., Easter J., EPITEG Collaborative Group, a multicentre comparative trial of sodium valproate and carbamazepine in adult onset epilepsy, Journal of Neurology, Neurosurgery and Psychiatry, 57, 1994, pp.682-687.

Rimmer E.M., Richens A., Clinical pharmacology and medical treatment. In: A textbook of epilepsy (Laidlaw, Richens and Oxley, Eds.). Churchill Livingstone, Edinburgh, 1988, pp. 421-483.

Rodin E.A., Rim C.S., Rennick P.M., The effects of carbamazepine on patients with psychomotor epilepsy: Results of a double blind study, Epilcpsia, 15, 1974, pp. 547-56I.

Rowan A.J., Diagnosis and treatment of epilepsy, Hospital and Community Psychiarry, 34, 1983, pp. 540-547.

Rutgers M.J., Geneeskundige en maatschappelijke aspecten van de zorg voor epilcpsiepatienten in Nederland, thesis, Rotterdam, 1984.

Salinsky M.C., Wegener K., Sinnema F., Epilepsy, driving laws and patient disclosure to physicians, Epilepsia, 33, 1992, pp 469-472.

Sander J.W.A.S., Some aspects of prognosis in the epilepsics: a revicw, Epilepsia, 34. 1993. pp. 10071016 
Sander J.W.A.S., Hart Y.M., Johnson A.L., Shorvon S.D., National General Practice Study of Epilcpsy: newly diagnosed epilcptic seizures in a general population, The Lancet, 336, 1990, pp. 1267-1271.

Scheuer M.L., Pedley T.A., The evaluation and treatment of seizures, The New England Journal of Medicine, 323, 1990, pp. 1468-1474.

Schmidt D. Janz D. Therapeutic plasma concentrations of phenytoin and phenobarbitone. In: Antiepileptic therupy advances in dnig moniloring. (Johannessen, Pippenger, Schmidt, Morselli, Richens and Meinardi, Eds.), Ravens Press, New York, 1980.

Schmidt D., Leppik I.E., Compliance in epilepsy: introduction, Epilepsy Research, Suppl.1, 1988, s3s4.

Schmidt D., Reininghaus R., Winkel R., Relevance of poor compliance for scizure control, Epilepsy Research, Suppl. 1, 1988, s141-s146.

Schobben A.F.A M., Pharmacokinetics and therapeutics in epilepsy, thesis, Nijmegen, 1979, pp.216.

Schoenberg B.S., Epideniological aspects of epilepsy. In: Epilepsy, an update on research and therapy, Alan R.Liss Inc, New York, 1983, pp. 331-343.

Scholtes F.B., Renier W.O., Meinardi H., Generalised convulsive status epilepticus, pathophysiology and treatment, Pharmacyworld and Treatment, 1993, 15, pp. 17-28

Shinnar S., Vining E., Mellits E.D., Discontinuing anti-epileptic medication in children with epilepsy after two years without seizures. The New Lingland Journal of Medicine. 313, 1985, pp.976-980.

Shinnar S., Kang H., Bcrg A.T., Goldensohn E.S., Hauser W.A., Moshé S.L., EEG abnormaties in children with a first unprovoked seizure, Epilepsia, 35, 1994, pp. 471-476.

Shorvon S.D., Reynolds E.H., Unnccussary polypharmacy for epilepsy, British Medical Journal, I, 1977, pp. 1635-1637

Shorvon S.D., Chadwick D., Galbraith A.W., Reynolds E.H., One drug for epilepsy, British Medical Journal, I, 1978. pp. 474-476.

Shorvon S.D., Galbraith A.W., Laundy M., Vydelingum L., Reynolds E.H., Monotherapy for epilepsy. In: Anti-epileptic therapy advances in drug monitoring, (Johannessen, Pippenger, Schmidt, Morselli, Richens and Meinardi, Eds.), Ravens Press, New York, 1980, pp. 213-220.

Shorvon S.D., Reynolds E.H., Early prognosis of epilcpsy, British Medical Journal, 285, 1982, pp. 1699-1701.

Shorvon S.D., The temporal aspects of prognosis in epilepsy, Journal of Neurology. Neurosurgery and Psychiatry, 47, 1984, pp. 1157-1165.

Shorvon S.D. The role of single drug and combination drug therapy in the treatment of epilepsy, in: Madern approach to anti-epileptic drug treatment, (Dam, Gram, and Pedersen, Eds.), The Danish Epilepsy Society, Jorgen Jensens Bogtrykkeri, Kobenhavn, 1985

Shorvon S.D., The treatment of epilepsy by drugs. In: Epilepsy, (Hopkins, Ed.), Chapman and Hall, London, 1987, pp. 229-282

Shorvon S.D., The coursc of untreated epilepsy, British Medical Journal, 297, 1988, pp. 1405.

Shorvon S.D., Epidemiology, classification, natural history and genetics of epilepsy, The Lancet, 336, 1990, pp. 93-96.

Shorvon S.D.. Medical assessment and treatment of chronic epilepsy, all patients should have a long term plan of treatment. British Medical Journal. 302, 1991, pp. 363-366.

Shorvon S.D. Epilepsy and driving. British regulations have recently been eased British Medical Jourmal. 310, Ling, pp.885-886. 
Simonsen N., Zander Olsen P., Kühl V., Lund M. Wendelboe J., A comparative controlled study between carbamazepine and diphenylhydantoin in psychomotor epilepsy, Epilepsia, 17, 1976, pp 169-176.

Sonnen A.E.H., De medicamenteuze behandeling van epilepsic, Nederlands Tijdschrift voor de (ienceskunde, 123, 1979, pp. 485-490.

Swartz B.E., Delgado-Escueta A.V., The management of status epilepticus, In: Epilepsy, (Hopkins, Ed.), Chapman and Hall, London, 1987, pp. 417-442.

Taylor J., Driving as an occupation. In: Epilepsy and employment (Edwards, Espir and Oxley, Eds.), Royal Socicty of Medicine Services Limited, London, 1986, pp. 89-92.

Temkin N.R., Dikmen S.S.,Wilensky A.J., Keihm J., Chabal S., Winn H.R, A randomised doubleblind study of phenytoin for the prevention of post-traumatic seizures, The New England Journal of Medicine, 323, 1990, pp. 497-502.

Tennison M.B., Greenwood R.S., Lewis D.V., Benoit S.E., Rate of taper of anti-epileptic drugs and the risk of seizure recurrence, Annals of Neurology, 26, 1989, pp. 439.

Tennison M., Greenwood R. Lewis D., Thorn M., Discontinuing anti-epileptic drugs in children with epilepsy, The New England Journal of Medicine, 330, 20, 1994, pp. 1407-1410.

Thompson P.J., Oxley J. Socioeconomic accompaniments of severe epilepsy, Epilepsia, 29 (Suppl.1), 1988, pp. s9-s।8.

Thorbecke R., Measurement of compliance through patient interviews, Epilepsy Research, Suppl.1, 1988, pp. 79-83.

Thurston J.H., Thurston D.L.. O'Leary J.. Follow-up study of 148 cases in which therapy had been suspended after prolonged anticonvulsant control. The New England Journal of Medicine, 286, 1972, pp. 169-174.

Thurston J.H., Thurston D.L., Hixon B.B., Keller A.J., Additional follow-up of I48 children 15 to 23 years after withdrawal of anticonvulsant therapy, The New England Journal of Medicine, 306, 1982, pp. 831-836.

Trostle J., Doctors orders and patients' sclf-interest: two views of medication usage?, Epilepsy. Research, Suppl. I, 1988, s57-s69.

Troupin A., Moretti Ojemann L., Halpem L.. Dodrill C., Wilkus R., Friel P., Feigl P., Carbamazcpinea double blind comparison with phenytoin, Neurology, 1977, pp. 511-519.

Tumbull D.M., Rawlins M.D.. Weightman D.. Chadwick D.W., A comparison of phenytoin and valproate in previously untrcatcd adult epileptic patients, Journal of Neurology. Neurosurgery and Psychiatry, 45, 1982, pp. 55-59.

Wagner A.L., A clinical and epidemiological study of adult patients with epilepsy, Acta Neurologica Scandinavia, 94 (Suppl), 1983, s63-s72.

Wallace S.J., Febrile convulsions. In: Epilepsy, (Hopkins, Ed.), Chapman and Hall, London, pp. 443467.

Wannamaker B.B., Morton W.A., Gross A.J., Saunders S., Improvement in the anti-epileptic drug levels following reduction of intervals between clinic visits, lipilepsia, 21, 1980, pp. 155-162.

Wilder B.J., Ramsay R.E., Murphy J.V., Karas B.J., Marquardt K., Hammond E.J., Comparison of valproic acid and phenytoin in newly diagnosed tonic-clonic seizures, Neurology, 33, 1983, pp. 1474-1476.

Wilder B.J., The treatment of epilepsy: an overvicw of clinical practices, Neurology, 45 (Suppl.2), 1995, s7-sll. 
Woo E., Chan Y.M., Yu Y.L., Chan Y.W., Huang C.Y., If a well-stabilised epileptic patient has a subtherapeutic anti-epileptic drug level, should the dose be increased? A randomised prospective study, Epilepsia, 29, 1988, pp. 129-139.

Zielinski J.J., Epileptics not in treatment, Epilepsia, 15, 1974, pp. 203-210.

Zielinski J.J., Epidemiology. In: A textbook of epilepsy (Laidlaw, Richens and Oxley, Eds.), Churchill Livingstone, Edinburgh, 1988, pp. $21-48$. 
CHAPTER 3

\section{System development}

\subsection{Introduction}

Rather than using comparatively domain independent methods such as mathematics or statistics, some decision support systems (DSSs) use a model of the domain based on a symbolic representation of expertise to solve problems. Such DSSs are called knowledge based systems (KBSs). Development of a KBS is a type of task which requires an approach different from conventional system development. In this chapter we will discuss different approaches for KBS development.

KADS - Knowledge Acquisition, Documentation and Structuring - is a representative of structured development methodologies (Breuker and Wielinga 1984a). Besides the provision of socalled elicitation techniques for making knowledge explicit, KADS provides a set of domain independent models which enables analysis of the extracted knowledge. An instantiation of these models is the conceptual model: a description of the problem solving process that may be used for implementation. At the end of this chapter we will present two models which describe different parts of the problem solving process with respect to the drug treatment of epilepsy. Both were developed using the KADS methodology.

\subsection{Methods for KBS development}

A KBS is a model of a problem solving task an expert performs (Jackson 1986, Waterman 1986). This is in contrast with conventional systems which focus on data processing and information exchange tasks as organised around forms (Weitzel and Kerschberg, 1989). During the development of a KBS one has to analyse and model the cognitive processes of e.g. interpreting patient data and applying medical knowledge (Hayes-Roth et al 1983). The cognitive processes are less circumscript than the data processing and information exchange tasks. Building a KBS therefore requires different methods than used in conventional software engineering.

The knowledge applied by neurologists consists of facts and heuristics. This knowledge has to be elicited and encoded, i.e. represented in a symbolic way and it has to be stored in a socalled knowledge base. The process of obtaining the knowledge from the expert and representing it in such a way that it can be formalised in a computer program is called knowledge acquisition. The knowledge acquisition process consists of two separate activities: elicitation and analysis of knowledge. Knowledge acquisition is generally recognised as the main bottleneck in the development of KBSs (Hayes-Roth et al. 1983, Buchanan and Shortliffe, 1984) 
Schreiber and Wielinga (1993) present two views on KBS development:

- The traditional tramsfer view in which the knowledge acquisition process is considered as a process of extracting the knowledge from a human expert and transferring the knowledge into the KBS. An example of this approach is development by prototyping (see below). This approach is often constrained by the representation formalism that is available.

- The modelling view in which the knowledge acquisition process is separated from the implementation of the knowledge. An example of this approach is KADS.

These two views will be described in the following sections.

\subsubsection{Prototyping}

Because it is not always clear what a system should do, users may frequently change previously defined requirements (Wellbank, 1983). Prototyping is used to help the user analyse his ideas on the problem at hand, the functionality of the system and the human-computer interface. In prototyping a system develops in an iterative way. The prototype system is used as a device for interviewing the domain expert on issues relevant for a proper performance of the intended system such as data entry, output of results and response time. Thus, prototyping clarifies aspects of the system's functionality by enabling direct feedback by the user on an implementation of a part of the system (Diaper, 1989). Prototyping can also support the establishment of the necessary co-operative atmosphere between the expert providing the knowledge and the knowledge engineer (Heng, 1987). There are three categories of prototyping: experimental, evolutionary and incremental.

Experimental prototyping is used to show the user different aspects of functionality and to clarify the problem at hand. After a short problem identification phase a model is built. This prototype exhibits limited functional capabilities, low reliability and possibly inefficient performance. The prototype is assessed with the objective to clarify the user requirements and to determine additional requirements. After the assessment the model is discarded. A synonym for this technique is throw-away prototyping (van Dijk et al. 1988).

When the user requirements are available, they may be incomplete or may still be subject to change. Evolutionary protolyping allows a system to evolve according to changing user needs. The process consists of a sequence of prototypes which (hopefully) converge into the final system or parts of it (van Dijk et al. 1988, Tate 1990)

Users may also be able to provide a fairly complete description of the functionality of the system. Clarification of requirements may be necessary on subparts only. In incremental prototyping the complete system design is specified and subtasks are identified. Next these subtasks are implemented, assessed and modified according to the user feedback. In contrast to the evolutionary approach, in incremental prototyping the overall design remains in principle unaltered (Stephens and Bates, 1990)

Experience with prototyping has revealed several disadvantages:

- The task to be performed by the system may change. Major design decisions are made in an early phase of development. In case of incorrect decisions the prototype may not be useful for the next iteration. 
- Maintainability of the evolving system may become a problem (Mayhew and Dearnley, 1990). Although a prototype knowledge base may do well, it may not be possible to scale it up to the full size required.

- Since (part of) the prototype should be realistic the user may be misled and take the prototype for the final system. Strong user reluctance to throw away a prototype that was designed to be discarded has been reported (Stephens and Bates, 1990).

\subsubsection{Structured development}

Prototyping is a kind of code-and-fix oriented approach of system development. In a more rigid approach one uses a predefined framework describing how to analyse the domain and what design issues to take into account. This may facilitate a more comprehensive view on the domain.

\section{Slage based approaches}

Stage based or life-cycle approaches to KBS development divide the development process into separate phases. For each of these phases a set of activities is specified. Different life-cycle models have been presented such as the waterfall model (Royce, 1987), the Hayes-Roth feedback model (Hayes-Roth et al. 1983) and the spiral model (Boehm, 1988). The application of these stage based approaches can be difficult: what the intermediate results of each of the stages should include is only vaguely presented by the authors. Also techniques and tools for supporting the different phases are usually lacking (Fen and Veren, 1991).

A more detailed approach for a stage based development of KBSs is the Pragmatic Knowledge Acquisition Methodology (PKAM) (Grover, 1983). It consists of three phases in which a domain is defined, fundamental knowledge is acquired and the obtained knowledge is improved. This methodology mentions a set of issues to be addressed in each of the phases and contains a number of techniques for knowledge elicitation. Studies on the utility of this approach however are lacking.

\section{KADS}

A more comprehensive methodology is KADS (Knowledge Acquisition, Documentation and Structuring, Breuker and Wielinga 1984a). It describes procedures and documentation, as well as tools and methods for KBS development. KADS emphasises the pre-implementation phases. An extensive analysis of the knowledge is advocated before system design and implementation take place.

The analysis phase in the most recent version of KADS consists of two streams: the external and the internal stream (Breuker and Wielinga 1984ab, Breuker and Wielinga 1987, Breuker et al. 1987, Breuker and Winkels, 1989). In the external stream the analysis of requirements and constraints concerning the system takes place. The internal stream describes the analysis of expertise. The methodology of the external stream is hardly documented. The analysis of the expertise was considered most important (Hickman et al. 1989, Schreiber et al. 1993). Analysis of expertise on different levels of abstraction has to be performed:

- Linguistic analysis, the identification of domain concepts in verbal data

- Conceptual analysis; the integration of individual concepts through formalised description(s), e g. compound concepts, relations and models

- Epistemological analysis; the definition of structural properties of the conceptual knowledge 
- Logical analysis; the definition of the formalisms with which the knowledge and inference making is expressed

- Implementation analysis

The level of abstraction increases from the linguistic to the epistemological level and then decreases again. The most important description of expertise results from the epistemological analysis

In KADS the epistemological description of expertise consists of four layers each describing a different category of knowledge: domain, inference, task and strategic knowledge. The domain layer contains the concepts and rules of the domain without an indication of when and how they can be applied. The inference layer depicts the relation between groups of concepts and inference procedures without an indication of the sequence of their application. This sequence of invocation of the inference procedures is described at the task layer. As the problem solving process may consist of multiple tasks, each of these tasks and the procedure for their invocation is described at the strategic layer

KADS provides a set of tools for knowledge elicitation as well as two facilities that support the analysis of expertise at the epistemological level: a conceptual modelling language and interpretation models. The conceptual modelling language provides a set of primitives for bottom-up development and refinement of the inference layer. These primitives consist of so called metaclasses and knowledge sources. Metaclasses are descriptions of domain concepts in abstract terms, indicating their roles in the reasoning process. The knowledge sources contain the inference procedures. They can be viewed as abstract operations on the metaclasses (Breuker and Wielinga, 1987)

To support the description at the inference layer, interpretation models have been developed. An interpretation model is a domain independent description of a specific type of problem solving task (Wielinga and Breuker, 1987). It provides a structure for analysing the verbalised data. The use of interpretation models resembles the idea of generic tasks: a limited set of general problem solving approaches. These generic tasks describe structures for problem solving which can be instantiated for a specific domain. Brown and Chandrasekaran (1989) define generic tasks as an elementary combination of a problem, a representation and an inference strategy. In contrast, the interpretation models in KADS do not at all address issues related to implementation such as representation aspects. The descriptions of tasks and inference structures in KADS are comparable with data-flow diagrams in conventional software engineering (Schreiber and Wielinga, 1993)

Proponents of structured development advocate an extensive analysis of the domain before commencing the implementation of a DSS. Such an analysis clarifies the view on the domain for which a DSS is developed. Due to the separation of analysis of expertise and its implementation, design decisions can be postponed until a more complete view on the domain and tasks has been obtained. It is assumed that analysis of expertise before implementation assures a more complete description of the expertise and thus a more efficient implementation can be expected (Schreiber et al. 1993) 


\subsubsection{Selection of the development method}

The acceptance of a KBS will depend both on the quality of the advice provided and on the functionality of the system. Although both aspects are important, confidence in the advice is considered a primary issue of concern (O'Keefe et al. 1987, O'Keefe 1989, O'Moore et al. 1990, Wyatt 1991). In prototyping the implementation starts with only a part of the knowledge. This approach makes a less optimal implementation more likely. Incremental improvement of implemented knowledge can cause inconsistencies particularly in complex systems (Nguyen et al. 1985). Given these problems a structured approach for the analysis of expertise in the domain of anti-epileptic drug treatment was considered more appropriate. As KADS provides the most comprehensive approach for structured development of KBSs it was selected as the approach for analysis of expertise in our work.

Prototyping is useful within KADS when it is used as a technique to support analysis and design activities (Nobis and Siedka-Bauer 1988, Tansley and Hayball, 1993). It provides the expert with insight in the procedures which are followed by the system (Hickman et al. 1989). The modelling of expertise in our work was alternated with an implementation of the obtained knowledge to analyse and demonstrate the interaction of the different layers of the model of expertise.

\subsection{Knowledge elicitation}

The process of obtaining knowledge from a human expert is called knowledge elicitation. The extracted knowledge may be modelled, i.e interpreted using some conceptual framework. Although elicitation and modelling are different activities, they are closely related and may direct each other's focus to specific parts of the knowledge.

Indirect techniques such as card sorting, multi-dimensional scaling, cluster analysis, general weighted networks and repertory grids provide a low level approach to knowledge elicitation (Wellbank 1983, Cooke and McDonald 1986, 1987, Burton et al. 1987, Wright and Ayton 1987, Shaw 1988). They enable the exploration of the domain by creating maps displaying the structure of the concepts in the domain. This structure is often derived from a (complete) set of perceived distances between pairs of concepts. The usability of the result depends on the completeness of the set of concepts used for obtaining the distance measures. If the set is not complete bias may result. This will show up in an erroneous description of the structure of domain concepts. How the set of concepts shall (or can) be obtained is not defined. One could question whether the development of a KBS is appropriate when the knowledge of a domain is so little developed that the structure of the domain still has to be identified by these techniques.

Apart from the card sorting technique all of the indirect techniques require the use of a kind of distance matrix in which all possible pairs of concepts are presented. Filling in this matrix is a tedious procedure. The expert may not be convinced of the necessity of such an exercise. Furthermore, the use of indirect techniques will not reveal the actual usage of concepts in the problem solving process (Hoffman, 1987).

Focused interviews, structured interviews, introspection, dialogues, protocol analysis, interruption analysis and questionnaires are direct techniques for knowledge elicitation (Reitman Olsen and Rueter, 1987). With these techniques, the role of concepts and the 
structurs: of the problem solving is derived directly from the verbal descriptions (Breuker and Wielinga 1984b).

A critical issue of direct techniques is that a successful application depends on the observer's skills for interviewing the expert. These techniques are also time consuming. However, most of the burden is on the interviewer, not on the domain expert. The interviewer has to transcribe the recordings of the interviews and to analyse the information obtained. Direct techniques are applied most often (Cullen and Bryman, 1988).

There is no formal methodology for knowledge elicitation (Wellbank 1983, Shadbolt and Burton 1989). The applicability of a knowledge elicitation technique may differ across domains. The suitability of a technique may depend on the personality (introvert/extrovert) and cognitive style (field dependent/independent) of the expert (Burton et al. 1988).

We only used direct techniques for knowledge elicitation. They allow the expert to express his knowledge in a natural way. There was no need to establish a structure of concepts in the domain as this knowledge was available. Our main interest was to obtain insight in how the knowledge is applied when performing tasks in the management of epilepsy patients.

\subsection{Models of anti-epileptic drug treatment}

The interviews and discussions with the expert and the literature review demonstrated that the treatment of epilepsy has different phases

- During the first phase the objective is to obtain enough information to establish a diagnosis. More than one patient-physician contact may be needed.

- The second phase starts when the diagnosis is established. Here the treatment is discussed and selected.

- The third phase consists of the evaluation of the treatment. The drug treatment may be adjusted depending on the symptomatology.

The model of expertise is based on an analysis of this phased approach to the treatment of epilepsy. We focused on the knowledge concerning the initiation and modification of the treatment.

\subsection{Strategic layer}

The management of a chronic disease is composed of a set of procedures for the diagnosis, the initiation of a therapy, the monitoring of the effect of the therapy and if possible the withdrawal of the treatment (Figure 3.5-1). For epilepsy the strategy - followed after the establishment of the diagnosis - can be described as follows. A drug therapy will be initiated with the intention to relief the patient from the seizures. The effectiveness of the drug treatment is checked by monitoring the seizure frequency and the well-being of the patient. The effectiveness of a drug may vary among patients. If the drug treatment proves ineffective the treatment is adjusted (a change in the drug dose or the initiation of another anti-epileptic drug). The effect of the adjustment is monitored. The adjustment of the treatment will end when seizures are controlled i.e. they do not occur anymore. When control of seizures has been established the monitoring of the patient continues for at least two years. After this period tapering of the drug treatment may be started. The results of changes in therapy will again be monitored. If seizures remain controlled the reduction of drug treatment will continue. 


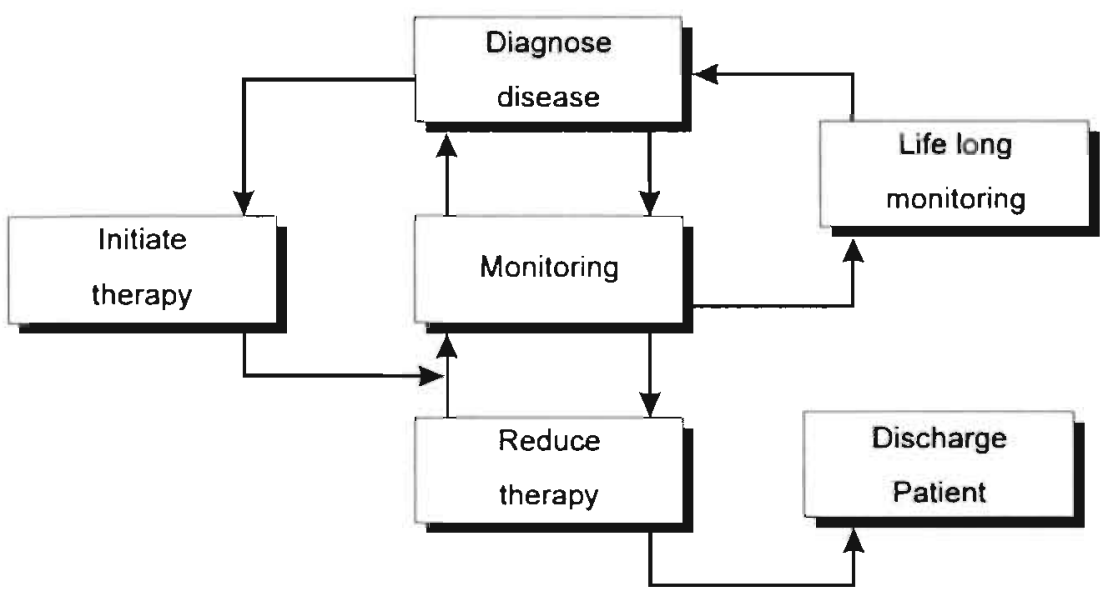

Figure 3.5-I: Strategy for management of epilepsy.

In case drug treatment is ineffective to control seizures completely or when tapering causes reoccurrence of seizures, life-long monitoring may be necessary: an approach in which adjustments of the treatment are made based on balancing the consequences of seizures and sideeffects.

\subsection{Inference structures}

The problem solving structures of both the initiation part and the monitoring part of the treatment were analysed. The analysis of the monitoring part of the treatment was guided by the KADS interpretation models for monitoring and assessment. Due to the lack of appropriate interpretation models for the process of initiating the treatment, we had to construct such a model bottom-up.

\subsubsection{Initiation of drug treatment}

The process for initiation of anti-epileptic drug treatment is decomposed into the subtasks of:

- Selecting the anti-epileptic drug

- Selecting the drug dose and

- Calculating the number of tablets and the intake scheme

For each of these subtasks the relevant domain concepts were extracted from the transcripts of the interviews and dialogues.

The epistemological analysis resulted in an identification of the various roles concepts have in the problem solving process (e.g. the group of main anti-epileptic drugs is used as a set of 
hypotheses from which the most appropriate can be selected; drug side-effects are used as constraints in this selection process). Concepts which have a similar role in the problem solving process are grouped in so-called metaclasses'.

Table 3.6-I: Description of the metaclasses used in the inference model for initiation of anti-epileptic drug treatment.

\begin{tabular}{|c|c|c|}
\hline METACLASS & CONCEPTS & ROL.E \\
\hline SKFLETAL MODEL & prescription data & template of a prescription \\
\hline COMPONENT & $\begin{array}{l}\text { generic drugname } \\
\text { brandname } \\
\text { dose of tablets } \\
\text { number of tablets } \\
\text { intake schemes }\end{array}$ & concepts to be assigned a value \\
\hline RESULT PART & $\begin{array}{l}\text { generic drugname } \\
\text { brundname } \\
\text { dose of tablets } \\
\text { number of tablets } \\
\text { intake schemes }\end{array}$ & instantiated components \\
\hline MODEL & prescription data & suggested drug trealment \\
\hline CASE DESCRIPTION & patient data & portrayal of diseased person \\
\hline HYPOTHESES & anti-epileptic drugs data & $\begin{array}{l}\text { providing options for selection of brand } \\
\text { providing options for selection of daily dose } \\
\text { providing options for selection of dose of tablets }\end{array}$ \\
\hline \multirow[t]{4}{*}{ CONSTRAINTS } & $\begin{array}{l}\text { type of epilepsy } \\
\text { type of seizures } \\
\text { co-medication } \\
\text { drug side-effects } \\
\text { ineflective drugs }\end{array}$ & restrictions for selection of drug \\
\hline & $\begin{array}{l}\text { weight } \\
\text { laboratory results }\end{array}$ & restrictious for selection of daily dose \\
\hline & $\begin{array}{l}\text { generic drugname } \\
\text { brandname } \\
\text { dose of tablets }\end{array}$ & $\begin{array}{l}\text { restrictions for selection of brand } \\
\text { restrictions for selection of dose of tablets } \\
\text { restrictions for assembling of intake scheme }\end{array}$ \\
\hline & $\begin{array}{l}\text { number of tablets } \\
\text { return date }\end{array}$ & $\begin{array}{l}\text { restriclions for assimbling of intake scheme/selection of } \\
\text { total number of tablets }\end{array}$ \\
\hline ALTERNATTVES & $\begin{array}{l}\text { optional generic drug } \\
\text { optional brandname } \\
\text { optional dose of tablets } \\
\text { optional number of lablets } \\
\text { optional intake scheme }\end{array}$ & choices for prescription \\
\hline
\end{tabular}

'The typographic conventions used in the description of inference structures are as follows:

- Tasks are printed in italicised capitals (OBTAIN_DRUGNAME)

- Metaclasses are printed in capitals (c.g. METACLASS)

- Knowledge sources are printed in sinall italicised letters (e.g. knowledge source)

- Individual concepts are printed in small bold capitals (e.g. CONCEPT)

- Values of concepts are printed in sinall capitals (c.g. Valuie)

We use the notation (CONCEPT VALUE) to indicate a concept and its value. 
The metaclasses used in the inference model for initiation of therapy are presented in Table 3.6-I. As concepts may have more than one role in the problem solving process, they can occur in different metaclasses. This also means that a concept (e.g. CARBAMAzEPINE) may occur as a value e.g. in (PRESCRIBED-DRUG CARBAMAZEPINE).

A second part of the elicited knowledge consists of rules, each describing a condition which leads to an assertion of a new fact or an action like the commencement of a task. In our case they are grouped by so called knowledge sources, a set of rules dealing with a specific inference. In an abstract sense a knowledge source is an inference process which instantiates concepts in the output metaclass given instantiated concepts in the input metaclass(es). The inference layer for the initiation of drug treatment is presented in Figure 3.6-I. Metaclasses are represented by rectangles and knowledge sources by ovals. An example of a knowledge source is assign-value which gives a concept in the metaclass RESULT PART a value (e.g. GENERIC DRUGNAME the value CARBAMAZEPINE) based on instantiated concepts in the metaclasses COMPONENT and ALTERNATIVES.

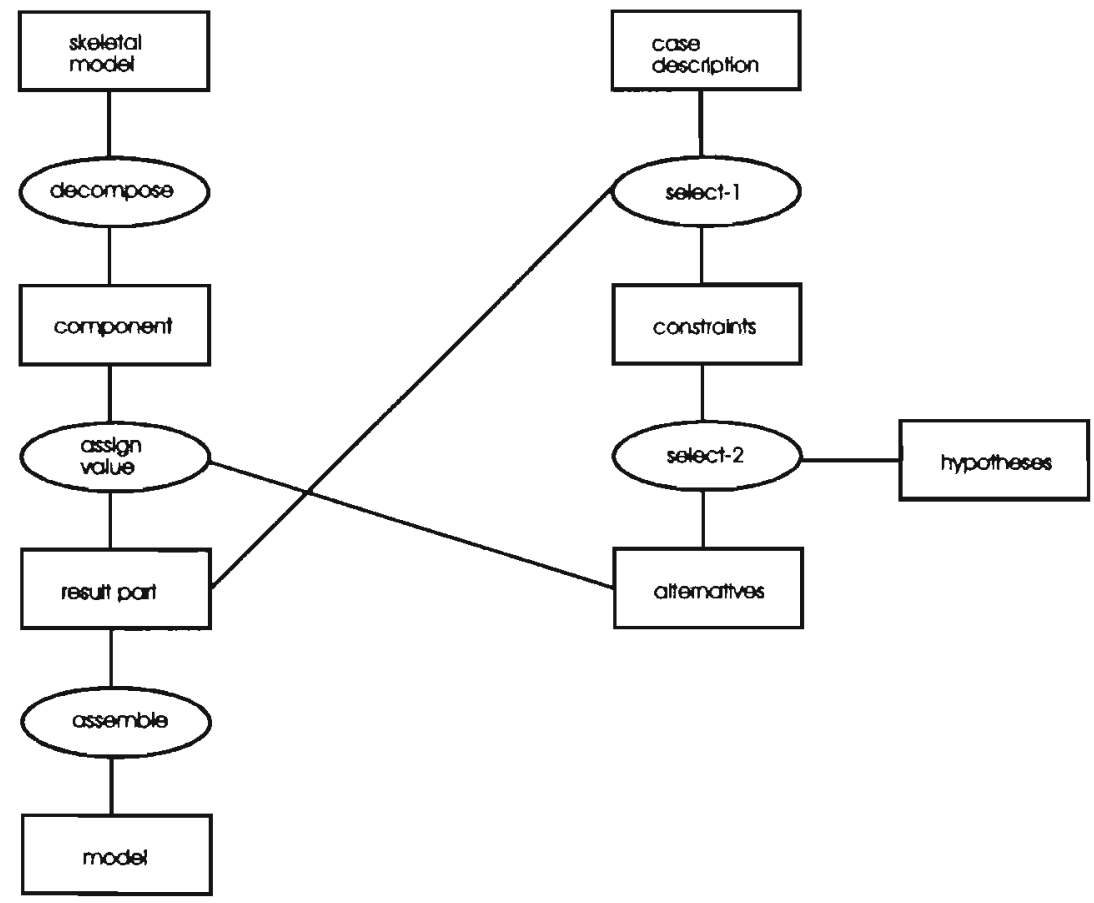

Figure 3.6-I: Inference layer for the initiation of anti-epileptic drug treatment, depicting the metaclasses (rectangles) and knowledge sources (ovals). 
The knowledge sources in Figure 3.6-I are composite ones. By decomposing these knowledge sources into primitive knowledge sources a more detailed view on the inference process can be obtained. Addressing the knowledge sources of the inference layer keeps the knowledge elicitation process focused.

The task structure for the initiation of anti-epileptic drug treatment is presented in Figure 3.6-II. In the task structure, the sequence of application of the knowledge sources is defined as well as the involved metaclasses. In the following the task structure for initiation of a therapy will be discussed and illustrated by the inference model of this task.

Figure 3.6-II: Task layer describing the control structure for the initiation of an anti-epileptic drug treatment.
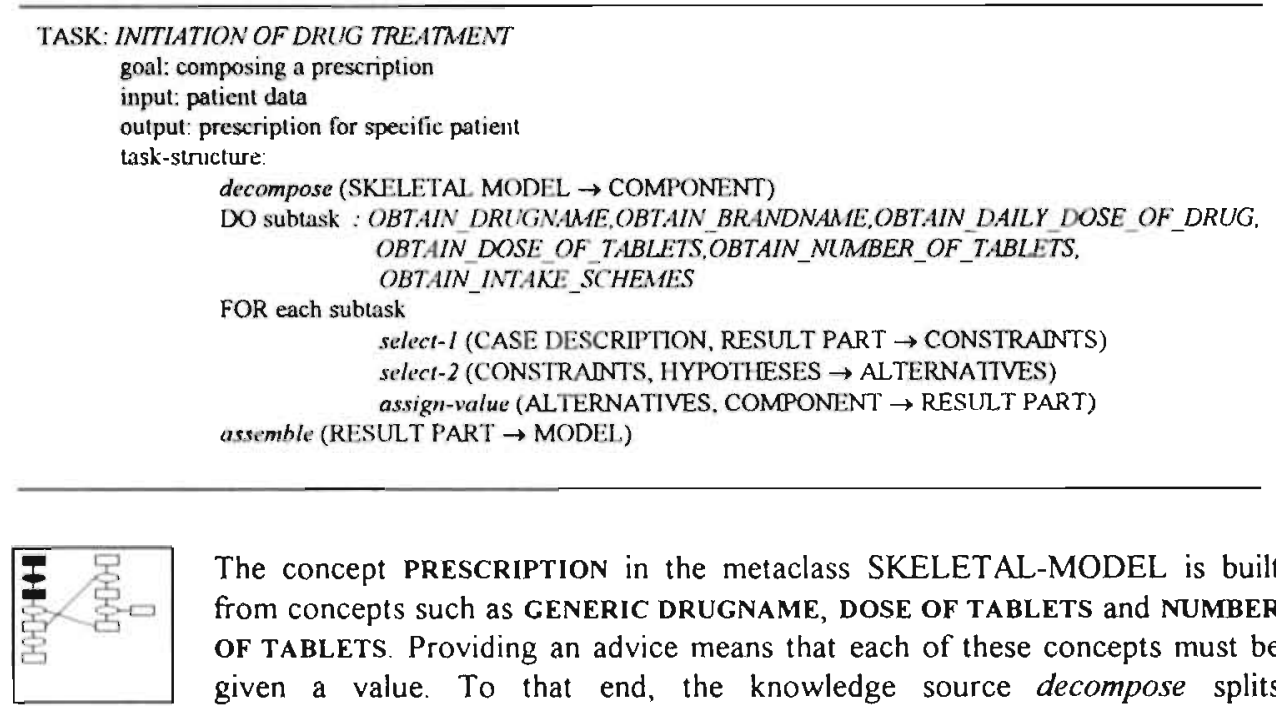

The concept PRESCRIPTION in the metaclass SKELETAL-MODEL is built from concepts such as GENERIC DRUGNAME, DOSE OF TABLETS and NUMBER OF TABLETS. Providing an advice means that each of these concepts must be given a value. To that end, the knowledge source decompose splits PRESCRIPTION into its constituent concepts. These concepts are all contained in the metaclass COMPONENT. ${ }^{2}$

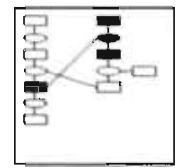

Next several subtasks are to be performed in sequence. As an example we describe the subtask OBTAIN DRUGNAME. This subtask assigns a specific drugname to the concept GENERIC DRUGNAME of the metaclass COMPONENT. To achieve this, information about the patient is extracted from the metaclass CASE DESCRIPTION. For example, the concept TYPE-OFEPILEPSY together with its value PARTIAL EPILEPSY is singled out by the knowledge source select-1. This concept is placed into the metaclass CONSTRAINTS, containing instantiated concepts which narrow down the choice of the drug. Note that the instantiated concepts in RESULT PART can influence the selection

${ }^{2}$ Black rectangles and ovals identify the metaclasses and knowledge sources discussed in the adjacent text. 


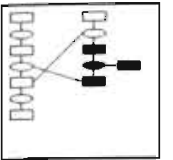

The metaclass HYPOTHESES contains data on the major anti-epileptic drugs. A set of first choice anti-epileptic drugs (CARBAMAZEPINE, PHENYTONN) is derived by select-2 using the information from CONSTRAINTS. This set of drugs becomes the value of the concept OPTIONAL GENERIC DRUGS in the metaclass ALTERNATIVES.

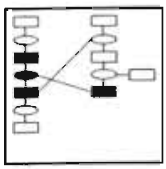

From this set of alternatives a drug (e.g. CARBAMAZEPINE) is selected. The knowledge source assign-value adds this piece of information to the GENERIC DRUGNAME in the metaclass RESULT PART.

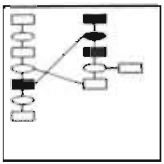

The process is repeated for the other subtasks which each add values to concepts in the metaclass COMPONENT, after obtaining information stored in CASE DESCRIPTION as well as from the intermediate conclusions from metaclass RESULT PART. For example, in the subtask OBTAIN DAILY DO$S E \_O F \_D R U G$, select-I takes the anti-epileptic drug from the GENERIC DRUGNAME in RESULT PART as well as additional restrictive information from the CASE DESCRIPTION such as the LABORATORY-RESULTS and WEIGHT. This information is stored in CONSTRANTS.

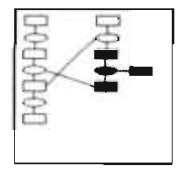

Next, information on DRUG DOSE IN MG/KG, MAXIMUM DOSE and AVAILABLE DOSES of TABLETS is obtained from the drug data in HYPOTHESES. This information is used by the composite knowledge source select-2 to obtain a drug dose for the patient. The dose is adjusted according to the MAXIMUM DOSE, the AVAILABLE DOSE OF TABLETS and the MINIMUM INTAKE FREQUENCY. The calculated dose serves as input for the calculation of values of the concepts OPTIONAL DOSE OF TABLETS and OPTIONAL NUMBER OF TABLETS in ALTERNATIVES.

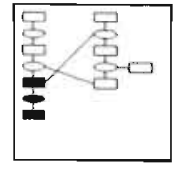

In this way each of the concepts in COMPONENT is assigned a value which is considered most appropriate given the status of the patient and the available medication. When the concepts belonging to the intake scheme are assigned a value, the individual concepts in RESULT PART are restructured by knowledge source assemble into the concept PRESCRIPTION in the metaclass MODEL.

\subsubsection{Monitoring drug treatment}

The KADS library has two interpretation models that are useful for modelling the monitoring of drug treatment: monitoring and assessment. In a monitoring task a comparison of individual data against a norm is made, followed by a classification of the difference, taking into account information from previous patient contacts In the assessment task changes in a group of concepts instead of in a single concept are classified. Table 3.6-III gives an example of the concepts contained by metaclasses in the monitoring process. 
Table 3.6-III: Examples of concepts contained by metaclasses defined in the monitoring process.

\begin{tabular}{|c|c|c|}
\hline METACLASS & CONCEPTS & ROLE \\
\hline CASE DESCRIPTION & patient data & description of cument state of the patient \\
\hline SELECTION CRITERLA & pathophysiological model & description of disease \\
\hline SYSTEM MODEL & physiological model & description of state of a healthy person \\
\hline VARLABLE SET & disease concepts & symptoms related to the patient \\
\hline PARAMETER SET & physiological data of healthy person & symptoms in case not diseased \\
\hline VARIABLE VALUE & $\begin{array}{l}\text { type of epilepsy } \\
\text { type of seizure } \\
\text { frequency of seizures etc. }\end{array}$ & values of symptoms when diseased \\
\hline PARAMETER VALUE & values of physiologic data & values of symptoms when not diseased \\
\hline DIFFERENCE & $\begin{array}{l}\text { presence of epilepsy } \\
\text { presence of seizure } \\
\text { presence of complaints etc. }\end{array}$ & $\begin{array}{l}\text { conclusions on symptoms relevant when } \\
\text { diseased }\end{array}$ \\
\hline HISTORICAL DATA & data from previous contacts & points of reference \\
\hline DISCREPANCY CLASSES & essential data on the patient & course of the disease \\
\hline MODEL SET & prototypical patient descriptions & profiles of standard patients \\
\hline NORM & $\begin{array}{l}\text { standard course of epilepsy } \\
\text { standard course of complaints } \\
\text { standard treaunent procedures etc. }\end{array}$ & $\begin{array}{l}\text { standard values of disease concepts and } \\
\text { treatment strategies }\end{array}$ \\
\hline DECISION CLASS & treatunent procedure & adjustment of treatment \\
\hline
\end{tabular}

Figure 3.6-111: Task structure of the anti-epileptic dng monitoring process.

TASK: MONITORING model-driven

goal: obtain conclusion on progress of disease

input: INITIATION OF DRLIG TREATMENT (MODEL)

output: DISCREPANCY CLASSES

task structure:

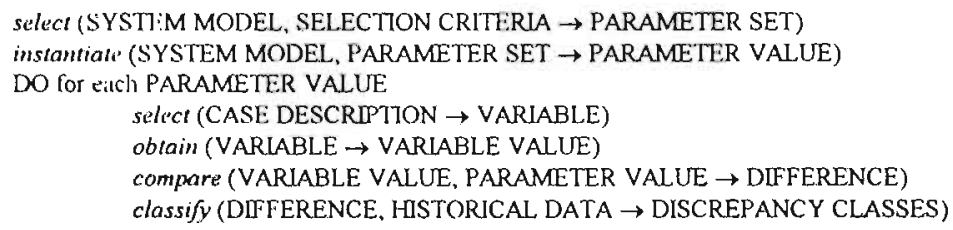

TASK : ASSESSMENT

goal: obtain a treatment procedure input MONITORING (DISCREPANCY CLASSES)

output: DECISION CLASS

task structure

specify (MODEL SET $\rightarrow$ NORMA)

match (DISCREPANCY CLASSES. NORM $\rightarrow$ DECISION CLASS) 


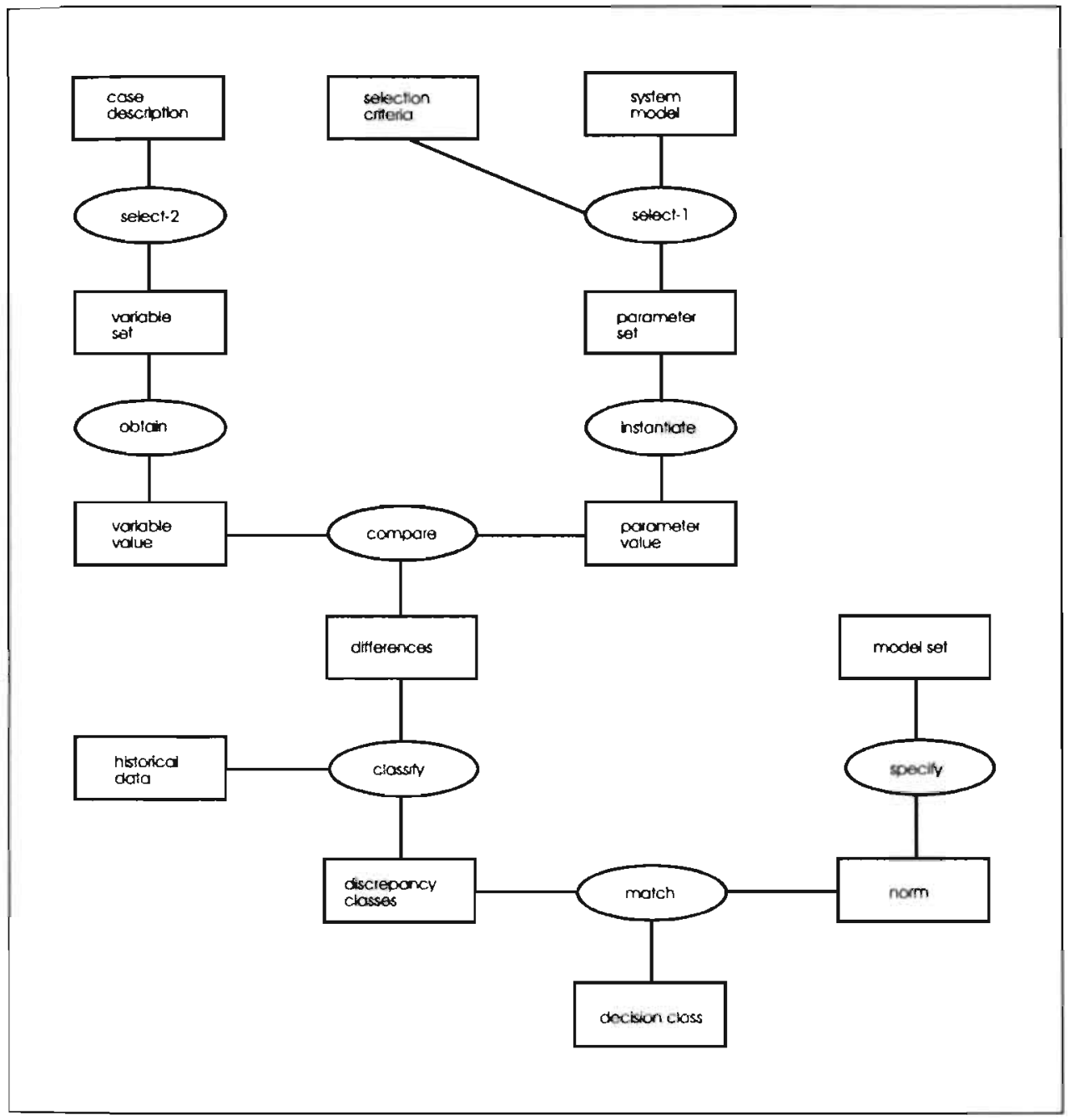

Figure 3.6-rV: Inference model for monitoring drug treatment of epilepsy.

From interviews and on-the-job-recordings it became clear that both a data-driven approach and a model-driven approach to monitoring may be applied. In a data-driven approach it is the patient who takes the initiative by starting to provide information on the effectiveness of the treatment. In the model-driven approach it is the physician who starts asking questions. In both approaches an identical set of issues - but not necessarily in the same sequence - is addressed for analysing the effectiveness of the treatment. In the following discussion the model-driven approach is used since this approach is more structured (see Figure 3.6-III for the task description). In Figure 3.6-IV the inference model of monitoring the drug treatment is presented. 
The interpretation models for the monitoring and assessment tasks are connected through metaclass DISCREPANCY CI.ASSES which contains the concepts that describe the course of the disease in the patient. The data from the concepts in this metaclass are matched with a set of profiles characterising possible courses of the disease. The prototypical profile matching the description of the disease course in the patient under consideration provides information for further treatment.

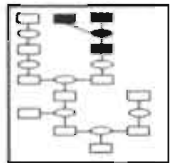

The concepts needed for monitoring the treatment process such as SEIZUREOCCURRENCE, TYPE-OF-SEIZURES, NUMBER-OF-SEIZURES, TYPE-OFEPILEPSY, COMPLAINTS, SIDE-EFFECTS, and COMPLIANCE are contained in the metaclass SELECTION CRITERIA. The SYSTEM MODEL represents the goal of the treatment e.g. the state of a healthy person. The knowledge source select $l$ singles out the relevant concepts from the metaclass SELECTION CRITERIA and obtains the values for these concepts from the SYSTEM MODEL. This piece of information is placed into the metaclass PARAMETER SET. For example, the metaclass PARAMETER SET would contain (SEIZURE-OCCURRENCE NO) for a healthy person.

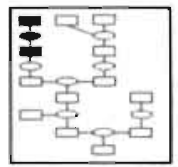

Next the corresponding concepts are singled out by select-2 from the metaclass CASE DESCRIPTION, which contains curTent information on the patient. As an example this could be (SEIZURE-OCCURRENCE YIS). This information is put into the VARIABLE SET

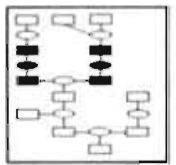

To compare these values, they are retrieved from the metaclasses VARIABLE SET and PARAMETER SET by respectively ubtuin and instantiate. The values are stored into the metaclass VARIABLE VALUE and PARAMETER VALUE respectively.

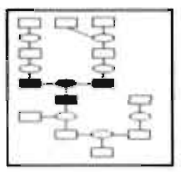

In our example the value of the concept SEIZURE-OCCURRENCE in the patient is different from the value in the physiological model. The conclusion of this comparison is denoted by the concept PRESENCE-OF-SEIZURES with the value PRESENT stored in the metaclass DIFFERENCES

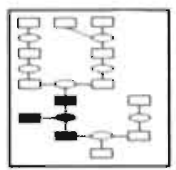

Information on the issue at stake may be compared with the values recorded during previous contacts listed in the metaclass HISTORICAL DATA. The result of the comparisons is contained by the metaclass DISCREPANCY CLASSES. In this case, if the value for PRESENCE-OF-SEIZURES in HISTORICAL DATA is ABSENT, the concept SEIZURE-COURSE in DISCREPANCY CLASSES receives the value INCREASED

By repeating this process for all concepts in metaclass SELECTION CRITERIA, all relevant aspects will be taken from the patient information and compared with historical data.

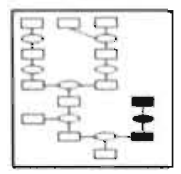

The 'status presence' of disease related items as stored in DISCREPANCY CLASSES is matched with similar items in a set of prototypical cases for which the treatment decision is known (Table 3.6-IV). The MODEL SET contains a description of these prototypical cases. From this metaclass concepts are derived and placed into the metaclass NORM by the knowledge source specify. 
Table 3.6-IV: An example of a description of a case as contained by the metaclass DISCREPANCY CLASSES and a prototypical case as contained by the metaclass NORM.

\begin{tabular}{ll}
\hline Abstract case description (DISCREPANCY CLASSES) & Prototypical case description (NORM) \\
\hline SEIZURE-COURSE INCREASED & SEIZURE-COURSE INCREASED \\
MINOR-SEIZURES ABSENT & MINOR-SEIZURES ABSENT \\
MAJOR-SEIZURES-FREQUENCY INCREASED & MAJOR-SEIZURES-FREQUENCY INCREASED \\
COMPLIANCE AS PRESCRIBED & COMPLIANCE AS PRESCRIBED \\
COMPLAINTS ABSENT & COMPLAINTS ABSENT \\
PLASMA-DRUG-LEVEL LOW THERAPEUTIC & PLASMA-DRUG-LEVEL LOW THERAPEUTIC \\
& TREATMENT-PROCEDURE INCREASEDOSE \\
\hline
\end{tabular}

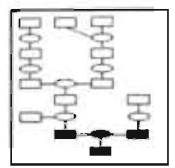

Each of the concepts in DISCREPANCY CLASSES is compared with the corresponding concept in NORM. A set of concepts in DISCREPANCY CLASSES equal to those of the prototypical patient leads to the storing of the concept TREATMENT-PROCEDURE together with its value from the prototypical case in DECISION CLASS. For example, the prototypical case description that matches the description in DISCREPANCY CLASSES contains among other concepts TREATMENT-PROCEDURE with the value INCREASE DOSE. This concept which indicates a further decision with respect to the treatment, is stored by the knowledge source match in the metaclass DECISION CLASS.

Two different types of decision classes were identified. While some decisions can be considered to be relevant only for the present contact, other treatment decisions could lead to treatment changes that have implications for future contacts. For example, switching the type of anti-epileptic drug or the decision to withdraw a drug may require a staged approach. In these procedures, the change in the treatment is stretched out over more than one contact thereby necessitating a monitoring of the consequences of the previous change.

\subsubsection{Additional treatment phases}

At the strategic level two additional phases were mentioned: the life-long monitoring phase and the phase of tapering drug treatment. Both phases are related to the monitoring and assessment tasks. The main difference between monitoring and life-long monitoring is a change in the system model. This is caused by a change in the goal of the drug treatment. Since in monitoring the goal is 'to cure' the patient by controlling the seizures, a physiological model is applied to compare disease concepts with. In life-long monitoring - with the exception of patients with well controlled seizures at a non-toxic level of medication who had recurrence of seizures and for whom therefore withdrawal of medication is no option (or who are known to get reoccurrence of seizures while tapering off the medication) - a balance between 'suppression of seizure and occurrence of side-effects' is required as curing the disease is less likely. Therefore the model is changed into a pathophysiological model of the patient. The withdrawal phase may also require a change in the system model. New seizures which may occur are due to withdrawing the drug instead of due to an ineffective treatment. 


\subsection{Implementation}

The two above described conceptual models were implemented in an experimental system using mul.ISPTM ${ }^{3}$. A layered structure separating the static parts of the knowledge from the procedural parts is used. The static knowledge of the domain layer is contained in frames. These are descriptions of concepts by means of their characteristics, so-called attributes and values. For example, the concept prescription has the attributes drugname, number of tablets and dose of tablets which may have the values carbamazepine, 2 and $200 \mathrm{mg}$, respectively. The domain concepts are arranged into three groups: information about medication, information about epilepsy and information about the patient.

Most of the heuristic knowledge expressed by the expert was captured in IF-THEN rules, which relate a condition to a specific action. Rules which are related to a specific section of the problem solving process are combined in rule sets. In this way the activation of only the relevant part of the knowledge base is possible. In the initiation part 14 of these rule sets with in total 66 rules were used for establishing a drug treatment. The monitoring part contains 965 rules which were combined into 59 rule sets. By using the frame and rule structures the composition of the knowledge as expressed by the expert was preserved in a familiar way. An assessment of the frames could be accomplished easily

Another part of the knowledge concerns the procedural aspects of the problem solving process. This is the task layer of the models described above. This knowledge is separated from the declarative part, but makes use of the information derived from the frames and rules.

A third part of the system is the user interface. The procedure driving the menus is described at the task layer. While in the initiation part we implemented some explanation on why a specific drug is used, in the monitoring part we can only provide a trace of the rules used for obtaining information about the reasoning process of the system.

In the initiation part as well as in the monitoring part a forward-chaining mechanism was used for reasoning. A simple conflict resolution scheme was implemented. The first rule of a set of rules of which the condition part matches the current information is identified. Its conclusion part is executed (an assertion added or a task executed). Next the rule is removed from the set and the process is repeated until the rule set is empty or none of the remaining rules fires.

\subsection{Discussion}

KADS has been presented by its developers as an approach for structured development of KBSs. In the original version of KADS the focus is on the analysis of cognitive processes. The main issue is the analysis of expertise which results in the definition of a model of expertise. This model of expertise is considered a high level specification of the expertise which is required for building the system (Breuker and Wielinga, 1987). This more cognitive oriented approach however widens the gap with implementing a working model. In a later version of KADS less emphasis is put on describing the expertise; a more pragmatic, implementation oriented view has been adopted. The model of expertise is seen as a model which is not a cognitive model of the expert but a specification of the system to be constructed by analysing the expert's problem solving behaviour (Schreiber et al. 1993).

\footnotetext{
3 muLISP is a trademark of Software House, Inc. 3660 Waialae Avenue. Honolulu, Hawaii 96816-3236.
} 
This change in view towards implementation can also be recognised in the changes in the contents of the conceptual model. In the early reports on KADS the conceptual model is a representation of the expertise, contained in a four layer model (Breuker et al. 1987, Schreiber et al. 1988). In the later version, a model of co-operation was added to the conceptual model. This model describes the system-user interaction; tasks to be accomplished by the user and by the system are indicated taking the competence of both agents into account (Breuker and de Greef, 1993). A model of co-operation and particularly a model of communication have been incorporated in the analysis phase (de Greef and Breuker 1992, Tansley and Hayball, 1993).

A third consequence resulting from this shift in attention towards implementation is a change in the project management approach. The early version of KADS is based on the waterfall model for conventional software design. In later reports on KADS the general structure as well as the life-cycle model were modified. A new life-cycle model $\mathrm{CONCH}$ (Client Oriented Normative Control Hierarchy) was introduced, based on the spiral life-cycle model (Boehm 1988, Taylor et al. 1989). A requirements analysis phase is now included. Guidelines and techniques for obtaining these requirements are absent, however (Hickman et al. 1989). More recently, a more detailed description of the processes in the analysis phase has been presented as well as a set of templates for modelling the organisational environment of the KBS (Tansley and Hayball 1993, Schreiber et al. 1994).

Although KADS advocates the use of models for the analysis of expertise, few guidelines with respect to the use and development of models are provided. The use of interpretation models is essential as they are a means for a top-down analysis of the elicited knowledge. Selection and application of these models, however, appears to be difficult. Some of the interpretation models have a very fine granularity. Other models, on the contrary, are less detailed and need extensive refinement. The interpretation models for assessment and monitoring could be used without significant refinement. This seems to be in agreement with the findings of others (Thiemann, 1989).

In case the model library has no interpretation model for the problem at hand, it may be constructed bottom-up. Development of an interpretation model for structuring the verbalised knowledge is supported by the conceptual modelling language. However, the set of primitives presented is only a tentative list of metaclasses and knowledge sources. An extension of the list is necessary, as has been recognised in the literature as well (Linster and Musen 1992, Woodward 1992).

Structured development improves the degree of completeness of the view on the domain but postpones the implementation process. In the knowledge acquisition phase, ideas verbalised by the expert have to be analysed, evaluated and refined. This process requires a spiral life-cycle approach, which enables an incremental evolution of a model of expertise. However, KADS does not offer tools to monitor this incremental process of modelling. Reviewing of intermediate results will require the interaction with a domain expert. In this process the model of expertise is of limited value as it is an abstract representation of his knowledge. Although the inference model will be important during the interaction with the expert, the terminology knowledge sources and metaclasses - as well as the names of the individual knowledge sources and metaclasses have a meaning of their own which is difficult to map on the concepts used by the expert to discuss the domain and his expertise. Also the interaction of the layers of a model will be difficult to comprehend. An extensive translation towards a detailed, less abstract model or an implementation of the model is required. 
As is the case with other approaches for software development KADS provides little support with respect to the evaluation of the quality of models. Work on metrication has taken place in KADS: process related and product related metrics, addressing issues such as duration and milestones, have been developed. A set of primitives which may be used for estimating costs has been defined (Readdie et al. 1989). Also in the new versions of KADS the evaluation of models of expertise needs further consideration. The iterative approach which enables the critiquing of evolving models - as in prototyping - should be included since this leads to a reduction of the risk of building an unsuitable system.

\subsection{References}

Bochm B.W., A spiral model of software development and enhancement, Computer, 3, 1988, pp. 6I72 .

Breuker J., de Greef P., Modelling system-user co-operation in KADS. In: KADS a principled approach to knowledge-based system development, (Schreiber, Wielinga, and Breuker, Eds.) Academic Press, London, 1993, pp. 47-70.

Breuker J., Wielinga B., van Someren M., de Hoog R., Schreiber G., de Greef P., Bredeweg B., Wielemaker J., Billeaut J.P., Davoodi M., Hayward S., Model driven knowledge acquisition, interpretation models, Deliverable task Al, Esprit Project 1098, Dept. Social Science Informatics, University of Amsterdam, 1987.

Breuker J., Wielinga B., Use of models in the interpretation of verbal data. In: Knowledge elicitation for experl systems, a handbook, (Kidd, Ed.), Plenum Press. New York, 1987.

Breuker J, Wiclinga B., Model-driven knowledge acquisition. interpretation models, Deliverable task Al Esprit Project 1098, Dept. Social Science Informatics, University of Amsterdam, 1984a.

Breuker J., Wielinga B., Techniques for knowledge elicitation and analysis, Report 1.5, Dept. Social Science Informatics, University of Amsterdam, 1984b.

Brcuker J., Winkels R., The use of the KADS methodology in designing an intelligent teaching system for diagnosis in physiotherapy. In: Lecture Noles in Medical Informatics 47, Knowledge based systems in medicine, (Talmon and Fox, Eds.), Springer-Verlag. Berlin, 1989, pp. 3-27.

Brown D.C., Chandrasekaran B, Design problem solving, knowledge structures and control strategies, Pitman Publishing, London, 1989.

Buchanan B.G., Shortliffe E.H., Rule-based expert systems, Addison-Wesley, Reading, Massachusetts, 1984

Burton A.M., Shadbolt N.R., Hedgecock A.P., Rugg G., A formal evaluation of knowledge elicitation techniques for expert systems: domain I. In: Research and development in expert systems $\mathrm{W}$, (Moralee, Ed), Cambridge University Press, 1987, pp. 136-145.

Burton A.M., Shadbolt N.R., Rugg G., Hedgecock A.P., Knowledge elicitation techniques in classification domains. In: Proceedings of ICAI-88, (Kodratoff, Ed), Pittman Publishing, London, 1988, pp. 85-90.

Cooke N.M., McDonald J.E., A formal methodology for acquiring and representing expert knowledge, Proceedings of the IEEE, 74, 1986, pp. 1422-1430. 
Cooke N.M., McDonald J.E., The application of psychological scaling techniques to knowledge based s elicitation for knowledge-based systems, International Journal of Man-Machine Studies, 26, 1987. pp.533-550

Cullen J., Bryman A., The knowledge acquisition bottleneck: time for reassessment?, Expert Systems, $5,1988, p p .216-225$

De Greef, P., Breuker J., Analysing system-user cooperation, Knowledge Acquisition, 4, 1992, pp. 89108 .

Diaper D., Designing expert systems, from Dan to Bursheba. In: Knowledge Elicitation, principles, techniques and applications, (Diaper, Ed), Ellis Horwood Limited, Chichester, 1989, pp. 17-46.

Dijk. J.E.M. van, Hilgevoord F.G., Jacques M.T., Otten G.A.M., Sittig A.C., Talmon J.L., Drie methoden voor kennis verwerving, Kennissystemen, 2, 1988, pp. 20-32.

Fen J.A., Veren L.C., Expert system development methodologics in theory and practice. In: Proceedings of the IEEE/ACM Conference on Developing and Managing Expert System Programs, Washington D.C., Sept. 1991, (Fcinstein, Ed), IEEE Computer Society Press, New York, 1991.

Grover M., A pragmatic knowledge acquisition methodology, TRW Defence Systems Group, Redondo Beach, Califomia, 1983.

Hayes-Roth F., Waterman D.A, Lenat D., Buiding expert systems, Addison-Wesley, Reading, Massachusetts. 1983.

Heng M.S.H., Why evolutionary development of expert systems appears to work, Future Generation Computer Systems, 3. 1987, pp. 103-109

Hickman F.R., Killin J.L., Land L., Mulhall T., Poner D. Taylor R.M., Analysis for knowledge-based systems, a practical guide to the KADS methodology, Ellis Horwood Limited, Chichester, 1989.

Hoffman R.R., The problem of extracting knowledge from experts from the perspective of experimental psychology, Artificial Intelligence Magazine. 2, 1987, pp. 53-67.

Jackson P., Introduction to expert systems, International Computer Science Series, Addison-Wesley Publishing Company, Reading, Massachusetts, 1986

Linster M., Musen M.A.. Use of KADS to create a conceptual model of the ONCOCIN task, Knowledge Acquisition, 4, 1992, pp. 55-87.

Mayhew P.J., Dearnley P.A., Organization and management of systems prototyping Information and Sofrware Technology, 32, 1990, pp. 245-252.

Nobis R., Siedka-Bauer H.G.. Prototyping and the LCM, Deliverable G13, NTE-GI3-PR-00I, ESPRIT P1098. NeuTech Entwicklungsgesellschaft mbH \& Co.KG, München, 1988.

Nguyen T., Perkins W.A., Laffey T.J., Checking an cxpert system's knowledge base for consistency and completeness. In: Proceedings of the I.JCAI. 1985, Los Angeles, pp. 375-378

O'Keefe R.M., Balci O., Smith E.P., Validating expert system performance, IEEE Expert, 4, 1987 , pp. $81-90$

O'Keefe R.M., The evaluation of decision-aiding systems: guidelines and methods, Informution and Management, 17, 1989, pp 217-226.

O’Moore R., Clarke K., Smeets R., Brender J., Nykänen P., McNair P., Grimson J., Barbeir B., Items of relevance for the evaluation of knowledge based expert systems and influence from domain characteristics, Deliverable EM-I.I, KAVAS (A I02I). Trinity College, Dublin, 1990.

Readdie M., Streng K.H., Wermser D., KADS metrication, Deliverable G10, SD-G10-R-001, ESPRIT PI098, SD Europe Ltd \& NeuTech Entwicklungsgesellschaft mbH \& Co.KG, München, 1989.

Reitman Olsen J., Rueter H.H., Extracting expertise from experts: methods for knowledge acquisition, Expert Systems, 4, 1987, pp. 152-168. 
Royce W.W., Managing the development of large software systems: concepts and techniques, Proceedings of the $9^{t h}$ International Conference on Sofiware Engineering, Computer Society Press, New York, 1987, pp. 121-125

Schreiber G., Wielinga B., KADS and conventional software engineering. In: KADS a principled approach to KBS development, (Scheiber, Wielinga and Breuker, Eds), Academic Press, London, 1993, pp. 151-165.

Schreiber G., Wielinga B., Breuker J., Introduction and overview on KADS, In: KADS a principled approach to KBS development, (Scheiber, Wielinga and Breuker, Eds), Academic Press, London, 1993, pp. 1-17.

Schreiber G., Wielinga B., Hoog R. de, Akkermans H., Velde W. van de, CommonKADS: a comprehensive methodology for KBS development, IEEE Expert, 12, 1994, pp.28-36.

Schreiber G., Breuker J., Bredeweg B., Wielinga B., Modelling in KBS development, paper presented at the Second Europcan Knowledge Acquisition Workshop '88, Bonn, June 1988.

Shadbolt N., Burton M., Knowledge elicitation. In: Evaluation of human work: practical ergonomics methodology, (Wilson and Corlett. Eds), Taylor and Francis, 1989.

Shaw M.L.G., Knowledge acquisition techniques for knowledge-based systems. In: Proceedings of the ( SC' 'Sl 88, Edmonton, 1988

Stephens M.A., Batıs P.E., Requirements engineering by prototyping: experiences in development of estimating system, Information and Software Technology, 32, 1990, pp. 253-257.

Tate G., Prototyping: helping to build the right software, Information and Software Technology, 32, 1990, pp. 237-244

Tanslcy D.S.W. Hayball C.C., Knowledge-based systems analysis and design: a KADS developer's handhook, Prentic: Hall, New York, 1993.

Taylor R.M., Porter D. Hickman F., Streng K.H., Tansley S., Dorbes G., System evolution, principles and methods (the life-cycle model). ESPRIT project 1098, deliverable Task G9, The KnowledgeBased Systems Centre of Touche Ross Management Consultants, London, 1989

Thiemann J., Wielinga over KADS: weglaten vierde niveau niet verwonderlijk, Kennissystemen, 3, 1989, pp. 3-5

Waterman D.A., A guide to expert systems, Addison-Wesley Publishing Company, Reading, Massachusetts, 1986.

Weitzel J.R., Kerschberg L., Developing knowledge-based systems: reorganizing the system development life cycle, Communications of the $A C M, 32,1989$. pp. 482-488.

Wellbank M., A review of knowledge acquisition techniques for expert systems, British Telecom Research, Martlesham Heath. 1983.

Wielinga B.J., Breuker J.A., Models of expertise. In: Advances in Artificial Intelligence 2, (du Boulay, Hogg, and Stecls. Eds.), Elsevier Science Publishers B.V., Amsterdam, 1987, pp. 497-509.

Woodward J.B., Developing K-ONCOCIN: a case study in the cognitive process of knowledge engineers, Knowledge Acquisition, 4, 1992, pp 237-258.

Wright G. Ayton P., Eliciting and modelling expert knowledge, Decision Support Systems, 3, 1987, pp. $13-26$ 


\section{Reference development}

\subsection{Method for system evaluation}

Evaluation $^{1}$ is generally considered as an essential part in the development of a DSS. Several methodologies have been proposed (Carlson 1974, Spiegelhalter 1983, Green and Keyes 1987, Lundsgaard 1987, Rushby 1988, O’Keefe et al. 1987, O'Keefe 1989, O'Leary and O'Keefe 1989, Rossi-Mori and Ricci 1988, Rossi-Mori et al. 1990, Hollnagel 1989, Preece 1990, Wyatt and Spiegelhalter 1990, O'Moore et al. 1990ab, Rector et al. 1990, Smeets and Talmon 1990, Engelbrecht et al. 1991, Sørgaard 1991, Bankowitz te al. 1992, Clarke et al. 1991, 1994) but none has been accepted as a standard. Usually validation ${ }^{2}$ of a DSS is considered the comerstone of the evaluation process as it addresses among other things the quality of the implemented knowledge. In our case correctness of the output of the system is the issue of interest.

The performance of the system and the neurologist(s) can be expressed by several quality concepts of which correctness and agreement are considered the most important. The correctness metric requires a reference with which the advice of the neurologists and the system can be compared. Correctness then is the percentage of cases which are assigned the reference value for an aspect of the treatment by either the system or a neurologist. Agreement is determined by comparing the treatment proposals of two or more observers without having recourse to a reference. The agreement beyond chance can be expressed by the kappa statistic.

The literature on anti-epileptic drug treatment can be used only as a guideline to assess the correctness of the advice of a system since the recommendations are too general. To assess the correctness of the prescription of an anti-epileptic drug treatment based on data of an individual patient, a reference for each case has to be available. The absence of detailed guidelines for the treatment of an individual patient necessitates consensus about what constitutes good treatment. Human expert(s) must be involved in the development of such a reference. To obtain a generally accepted opinion multiple experts have to be involved. To cope with multiple opinions a method for consensus formation is required.

\footnotetext{
'In general the definitions of ISO/IEC 9126: 1991 (E) are adhered to. The term evaluation incorporates the evaluation preparation - defined as metric selection, rating level definition and assessinent criteria definition - as well as the evaluation procedure i.e. the measurement, rating and assessment part of the evaluation procedure model.

2 Validation is a phase in the evaluation process which is focused on the correctness of the output of the system. It is concemed with the question whether the right system was built. It has to show that the program satisfies the user requirements and is considered to be a 'live' activity in which the software is tested.
} 
such records. In this process the case descriptions were evaluated on their clarity, fidelity and usability to assure that they contained sufficient information to enable diagnosis and treatment decision making. First we will describe the design of our 60 test cases in more detail. Secondly, it is described how from these 60 cases two reference sets for performance evaluation of the system were constructed.

\subsection{Test case design}

Written case descriptions were used in our studies. Although this approach does not mimic the daily patient-doctor contacts, physicians are used to paper cases when discussing and evaluating patients. Hence it is expected that an experimental set-up based on such paper cases will not introduce large biases. Also the fact that during a patient-doctor contact at the outpatient clinic, symptoms caused by an epileptic seizure are normally not observed but communicated verbally makes a written case description sufficiently similar to a patient-doctor contact. Therefore it is safe to assume that relevant information can be presented in writing (as if it were told by an eyewitness), without causing too much deformation of the problem solving process.

\subsubsection{Patient selection}

Patient records formed the basis for our paper cases. Epilepsy patients visiting the outpatient neurology clinic of the University Hospital of Maastricht who were 16 years or older were asked for consent to use anonymously their patient record. In total 70 patients were approached during a period of four months. Patients on anti-epileptic drug medication as well as patients off medication were included. Sixty-six patients gave their permission. Based on the availability of diagnostic information in the patient record 60 cases were finally used for our study.

Information normally gathered during the first few visits to a neurologist such as data concerning the admission of the patient, the patient history and hetero anamnestic information was extracted from the patient record. The family history, the tractus anamneses, psychosocial circumstances, the use of medication, intoxications and data from the physical examination, laboratory and other investigations such as EEG and CT-scans were included as well.

\subsubsection{Validity of paper cases}

The content of the paper cases was first assessed by two neurologists with more than 20 years of practical experience in epilepsy. One of them was professor and head of the Department of Neurology at the University Hospital Maastricht. The other a professor in epileptology and head of a special centre for epilepsy.

The paper cases were presented together with the original diagnosis. The assessors were asked to rate several aspects of the case on a five point scale.

\section{Clarity}

Clarity deals with how the case is presented: can the data be interpreted and is the information sufficient and not contradictory.

In 50 out of our 60 cases $(83.3 \%)$ both neurologists considered the case description to be clear. In five cases both stated that the case description was not clear. 
In the remaining five cases one of the participants did not provide a judgement, due to absence of relevant information or contradictions in the presented information. In three of these cases the other neurologist stated that the description was clear.

\section{Fidelity}

Fidelity deals with whether the case description could come from a real patient.

In fifty-three case descriptions both neurologists considered the case description realistic In four cases at least one neurologist disagreed with the statement that the case had a sufficient fidelity.

\section{Usability}

Usability deals with how well the case description is suited for our purpose. In 47 cases both experts agreed with the statement that the case was usable. In five cases at least one of the participants disagreed with the statement, while in eight cases both participants considered the case not usable.

On the basis of these results 13 case descriptions were refined taking the comments of the experts into account. The remarks mainly concerned unclear symptomatology of seizures and the lack of EEG-findings.

\section{Check on the usability}

After this refinement all 60 cases were assessed on their practical usability through the establishment of a diagnosis and prescription of a therapy by a second neurologist (Neurologist II) from the Neurology Department of the University Hospital in Maastricht.

Neurologist II diagnosed 51 cases as a specific type of epilepsy while in three cases the type of epilepsy was not certain. In six cases Neurologist II felt he needed additional information from a sleep-EEG and refrained from diagnosis.

In 50 of the 51 cases in which a type of epilepsy was diagnosed Neurologist II wrote a prescription. In one case he established a diagnosis but postponed drug treatment to enable registration of the EEG prior to medication.

For the three cases in which the type of epilepsy was uncertain no prescription was provided In the six cases in which Neurologist II requested a sleep-EEG, this information could not be provided and the cases were included unchanged in the set of test cases. The three cases in which the type of epilepsy was uncertain were not modified, as a treatment can be based on such a diagnosis.

\subsubsection{Description of the test cases}

The 60 cases concerned 34 male $(56.6 \%)$ and 26 female patients (43.3\%). The age of the patients ranged from 16 to 72 years. Forty-five percent of the patients was less than 30 years old, $36.6 \%$ was between 30 and 50 years of age while $18.4 \%$ was older than 50 years. Compared with Northern European (adult population) studies of active epilepsy our population is younger (Table 4.2-1). The age distribution resembles closer the adult population of an epilepsy centre (Meinardi 1990), which reflects the selection of problem cases from the Maastricht cohort. 
Table 4.2-I: Age distribution of the cases in our study compared with Northern European adult studies.

\begin{tabular}{llllllll}
\hline $\begin{array}{l}\text { Age } \\
\text { distribution }\end{array}$ & $\begin{array}{l}\text { Our study } \\
\mathrm{n}=60\end{array}$ & $\begin{array}{l}\text { Granf } \\
(1974) \\
\mathrm{n}=489\end{array}$ & $\begin{array}{l}\text { Wagner } \\
(1983) \\
\mathrm{n}=2572\end{array}$ & $\begin{array}{l}\text { Kerainen } \\
(198.9) \\
\mathrm{n}=1233\end{array}$ & $\begin{array}{l}\text { Sander } \\
(1990) \\
\mathrm{n}=425\end{array}$ & $\begin{array}{l}\text { Forsgren } \\
(1992) \\
\mathrm{n}=713\end{array}$ & $\begin{array}{l}\text { Meinardi* } \\
(1990) \\
\mathrm{n}=318\end{array}$ \\
\hline $15-19$ & 18 & 19 & 31.4 & 8.9 & 15.5 & 6.2 & 11.6 \\
$20-29$ & 22 & 30 & & 22.4 & 17.4 & 18.1 & 31.4 \\
$30-39$ & 20 & 13.7 & 34.0 & 18.0 & 14.6 & 21.3 & 17.6 \\
$40-49$ & 22 & 11.9 & & 16.6 & 8.2 & 15.4 & 15.1 \\
$50-59$ & 10 & 11.5 & 34.6 & 16.6 & 12.2 & 15.6 & 8.2 \\
$60-69$ & 8 & 8.2 & & 11.7 & 14.8 & 15.4 & 5.0 \\
$70-79$ & 2 & 5.7 & & 6.3 & 10.1 & 8.0 & \\
$>=80$ & & & & & 7.1 & & \\
\hline
\end{tabular}

Age distribution used: 16-20,21-30 etc

The following types of epilepsy (as validated by our two experts) were present:

- 28 cases $(47 \%)$ had partial epilepsy with secondary generalised partial seizures

- 16 cases (27\%) had primary (idiopathic) generalised epilepsy

- 9 cases $(15 \%)$ had partial epilepsy with complex partial seizures

- 2 cases $(3 \%)$ had partial epilepsy with simple partial seizures

- 5 cases $(8 \%)$ were diagnosed as partial epilepsy with complex partial seizures and partial epilepsy with secondary generalised partial seizures.

Using the classification of the International League Against Epilepsy 44 cases (73.3\%) had partial epilepsy while 16 cases (26.7\%) suffered from (idiopathic) generalised epilepsy.

Table 4.2-11: Distribution (in percentages) of epilepsies in diffenent populations as reported by Overweg (1985). For generalised epilepsy the percentage of cases with idiopathic generalised cpilepsy are presented as well.

\begin{tabular}{|c|c|c|c|c|}
\hline Type of epilepsy & study 1 & study 2 & study 3 & our study \\
\hline Generalised epilepsy & 45.8 & 23.0 & 18.2 & 26.7 \\
\hline Idiopathic & 4.3 .7 & 21.2 & 16.6 & 26.7 \\
\hline Other & 2.1 & 1.8 & 1.6 & 0.0 \\
\hline Partial epilepsy & 52.1 & 73.5 & 63.3 & 73.3 \\
\hline Unclassifiable & 1.7 & 3.4 & 18.4 & 0.0 \\
\hline
\end{tabular}

Overweg (1985) describes three studies which classify epilepsies according to the international classification of epilepsies of Gastaut (Table 4.2-I1). The first study describes the occurrence of epilepsies in a population referred to a single general hospital by primary care physicians. The two other studies describe patient populations in epilepsy centres.

Idiopathic generalised epilepsy occurred in respectively $16.6 \%$ and $21.2 \%$ of the patients of 15 years of age and older coming from epilepsy centres, while in the general hospital this occurred in $43.7 \%$ of the patients. The number of patients with partial epilepsies ranged from $63.3 \%$ up to 
$73.5 \%$. Also with respect to epilepsy syndromes our population resembles the more selected populations of epilepsy centres.

\subsection{Reference development}

Since no objective standard is available for assessing the system, the system was compared with the neurologists. Reference sets were created to obtain sets of cases with which the agreement between neurologists and the system could be assessed. In this section the procedure of constructing reference sets is presented. Two different reference sets were constructed: a diagnostic reference set containing cases for which at least two out of three neurologists agreed about the diagnosis and a treatment reference set for which both a majority agreement with respect to the diagnosis and treatment was obtained.

\subsubsection{Method for consensus formation}

In general two different approaches exist to reduce diversity in opinions among a group of individuals:

- Nominal techniques: a review of anonymous arguments/solutions

- Interactive techniques: a review of onymous arguments/solutions

Social and emotional factors which occur during an interactive group process may influence the way in which an agreement is reached. For example, dominance of some partners in the discussion, the tendency to embark quickly on a solution and the tendency to avoid conflicts, may induce nonoptimal solutions (van der Ven and Delbecq 1971, Delbecq et al. 1975). To avoid these problems, nominal techniques - the Nominal Group Technique (NGT) and the Delphi technique - were developed. In these approaches it remains unknown for the other participants who provided an opinion or solution. This anonymity of views makes it easier to discuss the various options without any prejudice. Nominal techniques have been applied successfully to establish objectives and standards (Loughlin and Moore 1979, Romm and Hulka 1979, Farrel and Scherer 1983, Quaglini et al. 1988, Kors et al. 1989, Kors and van Bemmel 1990), to explore risks and costs and to forecast events (Goodale and Gander 1976, Hill and Goodale 1981, Barret-Connor 1984, Arevalo and Washington 1988). The use of a modified Delphi approach has been shown to be valuable for developing references (Willems et al. 1985).

The NGT and the Delphi technique both consist of three steps: generating and pooling of ideas, feedback and evaluation. In the feedback round of the NGT, participants are expected to explain and discuss different views. In the Delphi procedure statistical information on the generated ideas is provided as feedback to the participants. In the third round participants still present their possibly revised opinions in an anonymous way (Delbecq and van der Ven 1975 , Linstone and Turoff 1975)

In our study we used a Delphi procedure in combination with a consensus meeting to obtain a reference for each case. The Delphi round was used to reduce the variation in prescribing. After the Delphi round the anonymous prescriptions were assessed by a peer review. The annotated prescriptions were discussed in the consensus meeting to reach a reference treatment. 


\subsubsection{Reference development/Acceptance testing}

Complete agreement among neurologists on diagnosis and treatment is expected difficult to achieve given the various opinions in the literature. A majority agreement among the participants was therefore considered to be sufficient for establishing a reference.

Although a neurologist may provide a prescription that is not equal to the reference it may still be considered an acceptable treatment. To assess the acceptability of the (possibly non-reference) prescriptions independent experts are needed to judge the quality of the prescriptions. To avoid to have to rely on the opinion of one single neurologist, each prescription was assessed by two experts

Table 4.3-I: Prescribing (P) and acceptability testing (A) scheme.

\begin{tabular}{cccccc}
\hline Data & Participant & & & & \\
set & A & B & C & D & E \\
\hline I & P & P & P & A & A \\
II & P & P & A & P & A \\
II & P & P & A & A & P \\
IV & P & A & P & P & A \\
V & P & A & P & A & P \\
VI & P & A & A & P & P \\
VII & A & P & P & P & A \\
VIII & A & P & P & A & P \\
IX & A & P & A & P & P \\
X & A & A & P & P & P \\
\hline
\end{tabular}

We designed a scheme for the reference development and acceptability testing procedure with the following properties (Table 4.3-1):

- each participant assessed the acceptability of prescriptions of cases for which he did not prescribe,

- each participant provided prescriptions for the same number of cases,

- each participant assessed the acceptability of the same number of prescriptions,

- each pair of participants was given the same number of cases to provide prescriptions,

- each pair of participants assessed the acceptability of the same number of prescriptions.

The 60 cases were divided into ten sets of six cases in such a way that each neurologist encountered approximately the same case mix (Table 4.3-11).

\subsubsection{Participating neurologists}

Five neurologists were asked to participate in this part of the study. The neurologists were working in neurology departments in five different general hospitals. Their clinical experience in neurology ranged from two to more than 20 years. Two of the participants received their training in neurology from the same supervisor. None of the participants was trained by the 
expert involved in the development of the system. All five neurologists were practising physicians, four as general neurologists, one with more emphasis on epilepsy care. Three participants saw an epilepsy patient every 1-3 days, 2 participants saw at least one epilepsy patient every day. Before the consensus meeting part of our study, one of the neurologists dropped out. In the follow-up part (see chapter 5) he was replaced by a colleague from the same hospital, having an equivalent number of years of clinical experience.

Table 4.3-II: Distribution of diagnoses presented to each participant for prescribing drug treatment.

\begin{tabular}{lrrrrr}
\hline Diagnosis* & N II & N IV & N V & N VI & N VII \\
\hline PEPCS & 6 & 6 & 5 & 5 & 5 \\
PESGPS & 17 & 17 & 17 & 16 & 17 \\
PEPCS+PESGPS & 3 & 4 & 3 & 2 & 3 \\
PESPS & 1 & 1 & 1 & 2 & 1 \\
IGE & 9 & 8 & 10 & 11 & 10 \\
Total & 36 & 36 & 36 & 36 & 36 \\
\hline
\end{tabular}

* PEPCS: partial epilepsy with complex parial seizures. PESGPS: partial epilcpsy with secondary generalised partial seizures, PESPS: partial epilepsy with simple partial seizurcs, IGE: primary (idiopathic) generalised epilepsy.

\subsubsection{Reference development process}

Since the diagnosis may determine the use of specific anti-epileptic drugs a majority agreement on the diagnosis has to be obtained before prescriptions can be compared. The cases for which no majority agreement (two out of three neurologists) on the general diagnosis (partial or generalised epilepsy) was reached were sent back to the neurologists. The various diagnoses established on such a case were given as feedback.

Cases for which the majority of the neurologists agreed on the diagnosis $(3 / 3$ or $2 / 3)$ but for which no majority agreement on the generic drug was obtained were retumed for review as well. Feedback was provided about the different drugs prescribed for the case. Furthermore, information on the frequency of suggested treatments in cases with the same general diagnosis, was given. Because the number of cases for which no agreement on the general diagnosis could be established was rather small, the reviewing of the diagnoses and the review of the generic drug were done simultaneously

After agreement was obtained on the generic drug, the prescribed daily doses were reviewed. For each drug the 5 and 95 percentiles of the daily dose in $\mathrm{mg} / \mathrm{kg}$ body weight were used to keep the number of cases to be reviewed within acceptable limits.

Prescriptions with a drug dose outside this range not caused by rounding off dosages towards an integer number of tablets were classified as outliers and returned for review

\subsection{Statistical analysis}

The $\chi^{2}$ test is used for analysing frequencies of cases in specific categories. It tests whether there is a significant difference between an observed number of cases in the categories and an expected number of cases based on the null hypothesis. The $\chi^{2}$ will be small if the observed and expected frequencies of cases are close to each other (Siegel and Castellan, 1988). 
The McNemar $\chi^{2}$ test is used for the analysis of 'before and after' designs in which the subjects are used as their own controls. The measurements should be made on an nominal or ordinal scale. The number of changes which occur are of importance. The test indicates whether the observed changes are significant (Siegel and Castellan, 1988).

For interpreting the significance of the results from the paired experiments on the acceptabilty scores of the prescriptions for a case the paired Student T-test is used. The differences were expected to be normally distributed. The degrees of freedom is $\mathrm{N}-1$, in which $\mathrm{N}$ is the number of pairs (Hayes, 1980).

The kappa statistic was used for obtaining information on the agreement beyond chance. This statistic was calculated for agreement among pairs of observers as well as for the agreement between one observer and a group (Schouten, 1985).

\subsection{Results}

In this paragraph the results are described of the agreement on the diagnosis and treatment before and after feedback. The agreement of the neurologists with the diagnostic and treatment reference is presented. Furthermore, the agreement between the diagnostic and consensus reference is described.

\subsubsection{Agreement on the diagnosis, generic drug and daily dose}

The degree of agreement among the neurologists on the diagnosis - both at a detailed and a general level - was established. In Table 4.5-I the different diagnostic categories at the general and detailed level are presented. This categorisation is based on the ILAE diagnostic classification (Commission on Classification and Terminology of the ILAE, 1989).

Table 4.5-I: General and detailed diagnoses

\begin{tabular}{ll}
\hline General type of epilepsy & Detailed type of epilepsy \\
\hline Generalised epilepsy & $\begin{array}{l}\text { Primary (idiopathic) generalised epilepsy } \\
\text { Myoclonic epilepsy } \\
\text { Lennox-Gastaut syndrome } \\
\text { West syndrome } \\
\text { Combinations }\end{array}$ \\
Partial epilepsy & $\begin{array}{l}\text { Partial epilepsy with complex partial seizures } \\
\text { Partial epilepsy with simple partial seizures } \\
\text { Partial epilepsy with secondary generalised seizures } \\
\text { Combinations }\end{array}$ \\
Combination & Combination of generalised and partial epilepsy \\
Other & Other suggestions \\
& $\begin{array}{l}\text { Withdrawal seizures } \\
\text { Multifocal epilepsy }\end{array}$ \\
Unknown & Diagnosis unknown or uncertain \\
& Diagnosis unknown or uncertain in combination with any of the above
\end{tabular}


Table 4.5-II presents the degree of agreement on the diagnosis and the prescribed generic drug before feedback. The table is divided into two sections. In the upper part of the table a breakdown of the cases is given according to the agreement with respect to the general and the detailed diagnosis.

Since for each case three neurologists provided a diagnosis and a prescription three $(3 / 3)$ or two neurologists $(2 / 3)$ can give the same diagnosis, or each neurologist may provide a different diagnosis (no). The third row of the upper part of the table presents the number of cases for each level of agreement.

The level of agreement between the three neurologists concerning the medication (stratified with respect to the degree of agreement on the diagnosis) is given in the lower part of the table (Therapy).

Total agreement with respect to the diagnosis (3/3) does not always lead to a total agreement with respect to the medication. Partial agreement with respect to the diagnosis $(2 / 3)$ can still result in total agreement with respect to the treatment, as the third neurologist who disagreed on the diagnosis may still prescribe the same generic drug. Of the 20 cases in which two out of three neurologists agreed on the general diagnosis, all three neurologists agreed about the generic drug in four cases. In 12 of these cases two out of three agreed on the treatment. In ten of these latter cases the pair of neurologists who agreed on the diagnosis also agreed on the generic drug.

Table 4.5-II: Agrecment on the general and detailed diagnosis and the type of generic drug before feedback.

\begin{tabular}{|c|c|c|c|c|c|c|c|c|}
\hline Diagnosis & \multicolumn{4}{|c|}{ general } & \multicolumn{4}{|c|}{ detailed } \\
\hline Agreement on type of epilepsy & $3 / 3$ & \multicolumn{2}{|c|}{$2 / 3$} & no & $3 / 3$ & \multicolumn{2}{|c|}{$2 / 3$} & no \\
\hline Number of cases & 36 & \multicolumn{2}{|c|}{20} & 4 & 20 & \multicolumn{2}{|c|}{26} & 14 \\
\hline Participants & All & Al! & $\begin{array}{l}\text { Diagn } \\
\text { agree }\end{array}$ & All & All & All & $\begin{array}{c}\text { Diagn } \\
\text { agree }\end{array}$ & All \\
\hline Therapy & & & & & & & & \\
\hline Agreement $3 / 3$ & 12 & 4 & 11.a. & 0 & 7 & 6 & ก.a. & 3 \\
\hline Agreement $2 / 3$ & 19 & 12 & 14 & 3 & 10 & 14 & 15 & 10 \\
\hline No ägreement & 5 & 4 & 6 & 1 & 3 & 6 & I! & l \\
\hline
\end{tabular}

Agreement on the diagnosis and the generic drug after feedhack

After the second round the number of cases with a majority agreement on the general diagnosis slightly increased (Table 4.5-III). In 58 out of 60 cases at least two out of three participants agreed on the general diagnosis. Also the agreement with respect to the therapy increased. A majority agreement on both the general diagnosis and the generic drug was reached in 55 out of 60 cases. 
Table 4.5-III: Agreement after foedluack.

\begin{tabular}{|c|c|c|c|c|c|c|c|c|}
\hline Diagnosis & \multicolumn{4}{|c|}{ general } & \multicolumn{4}{|c|}{ detailed } \\
\hline Agreement on type of epilepsy & $3 / 3$ & & & no & $3 / 3$ & & & no \\
\hline Number of cases & 36 & & & 2 & 20 & & & 13 \\
\hline Participants & All & All & $\begin{array}{l}\text { Dingn } \\
\text { agree }\end{array}$ & All & All & All & $\begin{array}{l}\text { Diagn } \\
\text { agree }\end{array}$ & All \\
\hline $\begin{array}{l}\text { Therapy } \\
\text { Agreement } 3 / 3\end{array}$ & 13 & 6 & n.a. & 0 & 8 & 8 & п.а. & 3 \\
\hline Agreement $2 / 3$ & 23 & 14 & 19 & I & 12 & 17 & 19 & 9 \\
\hline No agreement & 0 & 2 & 3 & 1 & 0 & 2 & 8 & 1 \\
\hline
\end{tabular}

For the detailed diagnosis level a majority agreement on both the diagnosis and treatment was reached in $65 \%$ of the cases as compared to $53 \%$ in the first round.

For the general diagnosis level after the first round there were $15(25.0 \%)$ cases with no agreement on either the general diagnosis or generic drug or both. This number decreased to $5(8.3 \%)$ cases after feedback (McNemar $\chi^{2}=8.1 \mathrm{p}<0.01$ ). In three cases disagreement remained with respect to the prescribed generic drug despite the fact that two neurologists agreed on the general type of epilepsy. In two other cases the participants disagreed on the general diagnosis. For the detailed diagnosis in 28 cases $(47 \%$ ) a majority agreement on either the general diagnosis, the generic drug or both was not reached initially. After feedback for 21 cases $(35 \%)$ a majority agreement was not reached.

Agreement on the drug dosage

The upper 95 percentile and the lower 5 percentile $\mathrm{mg} / \mathrm{kg}$ dosage of the drug were calculated for CBZ, VPA and PHT from the cases in which those drugs were prescribed in the second round. These dosages were then used to calculate the maximally and minimally acceptable dose for each case. In 10 cases the prescribed dose was outside these margins. In 7 of these cases the higher or lower dose could be attributed to rounding towards an integer number of tablets. In 3 cases a dose of CBZ was prescribed which was outside the obtained ranges. Review of these cases did not lead to a change in the prescribed dose.

\subsubsection{Description of the reference sets}

Diagnostic reference set

Whether an anti-epileptic drug has to be selected on the basis of a detailed diagnosis or whether a global description of the type of epilepsy is sufficient is not clear from the literature (chapter 2). Therefore, it was decided to use the set of 47 cases where the majority of the neurologists agreed about the detailed diagnosis for the assessment of the system.

The 47 cases in which the majority of the neurologists agreed about the detailed diagnosis is called the diagnosic reference sel. Although each neurologist saw 36 cases the reference cases are not equally divided over the individual neurologists. Table 4.5-IV gives the number of cases which each individual neurologist agreed with the detailed diagnosis for. 
Table 4.5-IV: Number of cases from the diagnostic reference set seen by each neurologist.

\begin{tabular}{|c|c|c|c|c|c|}
\hline Neurologist & III $(\mathrm{n}=36)$ & IV $(n=.36)$ & $V(n=36)$ & $V I(n=36)$ & VU $(n=36)$ \\
\hline Agreement with ref. & 23 & 25 & 21 & 22 & 23 \\
\hline detailed diagnosis & $64 \%$ & $69 \%$ & $58 \%$ & $61 \%$ & $64 \%$ \\
\hline
\end{tabular}

The reference cases were mainly diagnosed as partial epilepsies $(64 \%)$, while $30 \%$ of the cases were diagnosed as primary (idiopathic) generalised epilepsy. Furthermore, twice the neurologists agreed that the type of epilepsy was uncertain or unknown. Once a combination of partial and generalised epilepsy was diagnosed.

One hundred and fourteen prescriptions were generated for the 47 cases by the neurologists that agreed on the detailed diagnosis. In $51.8 \%, 24.6 \%$ and $10.5 \%$ of these prescriptions the selected drug was CBZ, VPA and PHT respectively. Eleven times a neurologist decided not to initiate anti-epileptic drug treatment. For the 47 cases a majority treatment was defined (if it existed) consisting of the opinions of those neurologists which agreed about the detailed diagnosis (Table 4.5-V)

Of the cases diagnosed as a kind of partial epilepsy $63 \%$ received a prescription of $\mathrm{CBZ}$, while in 10\% PHT was the drug prescribed by the majority. In seven cases (23\%) a majority agreement about the treatment was not obtained (No ref) despite the fact that the neurologists agreed about the diagnosis. In one case the reference was not to start drug treatment No drug)

The drug for cases diagnosed as a primary (idiopathic) generalised epilepsy was mainly VPA $(71 \%)$. CBZ was the treatment of the majority in $10 \%$ of these cases. In one case a majority treatment was not obtained.

For the combination of partial and generalised epilepsy $\mathrm{CBZ}$ was used by the majority. For the two cases in which the diagnosis was not certain anti-epileptic drug treatment was not initiated.

Table 4.5-V: Detailed diagnoses and treatment in the diagnostic reference set.

\begin{tabular}{|c|c|c|c|c|c|c|c|c|}
\hline Diagnosis & & Cuses & CBZ & VPA & PHT & No ref & No drug & $\%$ \\
\hline \multirow{4}{*}{$\begin{array}{l}\text { Partial } \\
\text { epilepsy }\end{array}$} & PECPS & 6 & 4 & & & 2 & & 13 \\
\hline & PESGS & 10 & 6 & & 2 & 2 & & 21 \\
\hline & PECPS+PI:SGS & 11 & 8 & & & 2 & 1 & 23 \\
\hline & PESIS & 3 & 1 & & 1 & I & & 6 \\
\hline $\begin{array}{l}\text { Gencralised } \\
\text { epilepsy }\end{array}$ & IGE & 14 & 3 & 10 & & 1 & & 30 \\
\hline Combi & IGIT+PECPS & 1 & 1 & & & & & 2 \\
\hline Unknown & & 2 & & & & & 2 & 4 \\
\hline Total & & 47 & 23 & 10 & 3 & 8 & 3 & 100 \\
\hline
\end{tabular}




\section{Treatment reference set}

In 39 out of the 47 cases a majority agreement on the prescribed treatment was reached. This subset of cases is called the trealmenl reference set.

Cases in which two neurologists agreed about the detailed diagnosis but disagreed about the prescribed drug were excluded, even if the third neurologist who disagreed about the diagnosis agreed on the prescribed drug.

In Table 4.5-VI the number of cases for which the individual neurologists agreed with the reference are presented. Because the neurologists may disagree with the diagnosis or with the prescribed drug the number of cases per neurologist differs.

Table 4.5-VI: Number of cases in which an individual ncurologist agrees with the treatment reference. The number of cases seen by each neurologist and belonging to the diagnostic reference set is indicated by $n$.

\begin{tabular}{llllll}
\hline Neurologist & III $(n=23)$ & IV $(n=25)$ & V $(n=21)$ & V $(n=22)$ & VI $(n=23)$ \\
\hline Agreement with reference drug & 18 & 20 & 14 & 16 & 18 \\
& $78.3 \%$ & $80.0 \%$ & $66.6 \%$ & $72.7 \%$ & $78.3 \%$ \\
\hline
\end{tabular}

Overall, in 18 of the 47 cases (38\%) the majority of the neurologists agreed on the detailed diagnosis as well as on the generic drug and daily dose. In three of these cases no drug treatment was started. In the remaining 15 cases a majority agreement on the generic drug as well as on the daily dose was observed

In 13 of the 14 cases diagnosed as primary (idiopathic) generalised epilepsy, the majority agreed about the drug. In eight of these 13 cases (57\%) at least two neurologists prescribed the same daily dose.

In 22 of the 30 cases diagnosed as a type of partial epilepsy the neurologists agreed about the prescribed drug. In six of these 22 cases $(27 \%)$ the majority agreed about the daily dose. This concerned five cases for which $\mathrm{CBZ}$ and one case for which PHT was prescribed. The neurologists also agreed on the prescribed drug and daily dose for the one case diagnosed as a combination of partial and generalised epilepsy.

\section{Consensus meeting reference}

After the three Delphi rounds the neurologists still varied with respect to the prescribed drug and the daily dose for a specific case. Therefore a consensus meeting was organised. The cases of the diagnostic reference set were discussed. Instead of a reference for an individual case in this consensus meeting guidelines were developed on how to approach a patient with epilepsy in general. A detailed outline of these guidelines is presented in chapter 5 . 
Table 4.5-VII: Comparison of the diagnostic reference set and the consensus.

\begin{tabular}{|c|c|c|c|c|c|c|}
\hline \multirow{8}{*}{$\begin{array}{l}\text { Diagnostic } \\
\text { reference set } \\
(n=47)\end{array}$} & \multirow[b]{2}{*}{ Drug } & \multicolumn{5}{|c|}{ Consensus drug } \\
\hline & & $\mathrm{CBZ}$ & VPA & PHT & No drug & Total \\
\hline & $\mathrm{CBZ}$ & 18 & 2 & 3 & & 23 \\
\hline & VPA & & 10 & & & 10 \\
\hline & $\mathrm{PHT}$ & 3 & & & & 3 \\
\hline & No drug & 1 & & & 2 & 3 \\
\hline & No ret & 4 & 2 & 2 & & 8 \\
\hline & Tota! & 26 & 14 & 5 & 2 & 47 \\
\hline
\end{tabular}

The guidelines defined in the consensus meeting were applied to the diagnostic reference set. Of the 34 cases with partial epilepsy, $26(84 \%)$ should receive CBZ while five cases ( $16 \%$ ) needed PHT according to the consensus (Table 4.5-VII). All 14 cases diagnosed as primary (idiopathic) generalised epilepsy received VPA. For cases in which the type of epilepsy was unknown or uncertain drug treatment was not initiated

In 30 cases (64\%) the majority drug was in accordance with the consensus. Cases in which the reference drug was PHT should have received $\mathrm{CBZ}$ according to the consensus. The case for which treatment was not started although the diagnosis was established should have been treated with CBZ. Using the guidelines a treatment could be defined for those cases for which no reference was obtained by means of the Delphi procedure.

\subsection{References}

Arevalo J.A., Washington E., Cost-effectiveness of the prenatal screening and immunization for hepatitis B virus, Journal of the American Medical Association, 259, 1988, pp 365-369.

Bankowitz R.A., Lave J R., McNcil M.A.. A method for assessing the impact of a computer based decision support system on health care outcomes, Methods of Information in Medicine, 31, 1992, pp.3-11.

Barret-Connor E., Natural history of intestinal parasites in asymptomatic adults, The Journal of Family Practitioners, 19, 1984, pp.635-639.

Carlson E.D., Evaluating the impact of information systems, Management Informatics, 3, 1974, pp.5767.

Clarke K., O'Moore R., Smects R., Talmon J., Brender J., McNair P., Nykänen P., Grimson J., Barber B., A methodology for evaluation of knowledge-based systems. In: Lecrure notes in Medical Informatics 45, Proceedings MIE 91, (Adlassnig, Graber, Bengtsson and Hansen, Eds.), SpringerVerlag, Berlin, 1991, pp. 361-365.

Clarke K., O`Moore R., Smects R., Talmon J., Brender J., McNair P., Nykänen P., Grimson J., Barber B., Methodology for evaluation of knowledge-bascd systems in medicine, Artificial Intelligence in Medicine, 6, 1994, pp.107-121.

Commission on Classification and Terminology of the Intemational League Against Epilepsy, Proposal for revised classification of epilepsies and epileptic syndromes, Epilepsia, 30, 1989, pp. 389-399.

Delbecq A.L., Ven A.H. van der, Gustafson D.H., Group lechniques for program planning: a guide 10 nominal group and Delphi processes, Scott Foresman, Glenvicw IL, 1975. 
Engelbrecht R., Fitter M., Rector A., Requirements for a medical workstation using user-centered design. In: Lecture notes in Medical Informatics 45. Proceedings MIE 91 . (Adlassnig, Graber, Bengtsson and Hansen. Eds.), Springer-Verlag, Berlin, 1991, pp. 140-144.

Farrel P., Scherer K., The Delphi technique as a method for selecting criteria to evaluate nursing care, Nursing Papers, 15, 1983, pp.51-60.

Forsgren L., Prevalence of epilepsy in adults in northem Sweden, Epilepsia, 33, 1992, pp. 450-458

Goodale F., Gander G.W., The future of pathology: a Delphi study by pathology dcpartment chairmen, Journal of Medical Education, 51, 1976, pp.897-903

Graaf A.S de, Epidemiological aspects of epilepsy in northem Norway, Epilepsia, 15, 1974, pp. 291 299.

Green C.J., Keyes M.M., Verification and validation of expert systems. In: Proceedings of the Western Conference on Expert Systems, IEEE Computer Society Press, New York, 1987, pp.38-43.

Hayes W.L., Statistics for the social sciences, $2^{\text {nd }}$ edition, Holt Rineman and Winston, London, 1980.

Hill R.B., Goodale F. The Delphi predictions of pathology chaimen; a six-year retrospective view, Journal of Medical Education, 56, 1981, pp.537-546.

Hollnagel E., Evaluation of expert systems. In: Topics in expert system design, eds. Guida G., Tasso C., Elsevier Science Publishers B.V. (North-Holland), Amsterdam, 1989, pp.377-416

Kors J., Bemmel J.H. van, The Delphi method: a review of its applications in medicine. In: Proceedings MEDINFO'89, (Eds.)Beijing Singapore, 1989, pp. 156-160.

Kors J., Sittig A.C.. Bemmel J.H. van, The Delphi method to validate diagnostic knowledge in computerized HCG interpretation. Methods of Informalion in Medicine. 29. 1990, pp.44-50.

Keränen T., Riekkinen P.J., Sillanpää M., Incidence and prevalence of epilepsy in adults in eastem Finland, Epilepsia, 30, 1989, pp.413-421

Linstone H.A., Turoff M., The Delphi method: techniques and applications, Reading, Mass. AddisonWesley, 1975.

Loughlin K.G., Moore L.F., Using Delphi to achieve congruent objectives and activities in a pediatrics department, Journal of Medical Education, 54, 1979, pp.101-106.

Lundsgaard H.P., Evaluating medical expert systems, Social Science in Medicine. 24, 1987, pp. 805819

Meinardi H., Pachlatko C.. Special centers for epilepsy. In: Comprehensive lipileptology, (Dam and Gram, Eds.), Raven Press, New York, 1990, pp. 769-779

O'Keefe R.M., Balci O., Smith E.P., Validating expert systems performance, IEEE Expert, 4, 1987, pp. 81-89.

O'Keefe R.M., The evaluation of decision-aiding systems: guidelines and methods, Information and Management, 17, 1989, pp.217-226.

O'Leary D., O'Keefe R.M. Verifying and validating expert systems. Tulorial at the IJAI-89, Detroit, 1989.

O'Moore R., Clarke K., Smeets R., Brender J., Nykänen P., McNair P., Grimson J., Barber B., ltems of relevance for the evaluation of knowledge basedlexpert systems and the influence of domain characteristics, KAVAS (A 1021), Report EM-1.1, Trinity College, Dublin, 1990

O'Moore R., Clarke K., Brender J., McNair P., Nykänen P., Smeets R., Talmon J., Grimson J., Barber B., Methodology for evaluation of knowledge based systems, KAVAS (AI021), Report EM-1.2, Trinity College, Dublin, 1990.

Ovenweg J., Withdrawal of anti-epileptic drigs in seizure-free adult patients. prediction of outcome, thesis, Amsterdam, 1985 
Preece A.D., Towards a methodology for evaluating expert systems, Expert Systems, 7, 1990, pp.215223

Quaglini S., Stefanelli M., Barosi G., Benzuini A., A performance evaluation of the expert system ANEMIA, Computers and Biomedical Research, 21, 1988, pp.307-323.

Rector A., Engelbrecht R., Diedrich T., Fieschi M., Fitter M., Moser W., Schneider J., Report on validation and verification requirements and operational criteria, Eurodiabeta (AIM 1019), Deliverable I2, DG-XIII of the Commission of the EC, Brussels, 1990.

Romm F.J., Hulka B.S., Developing criteria for quality of care assessment: effect of the Delphi technique, Health Service Research, 14, 1979, pp.309-312.

Rossi-Mori A., Ricci F.L., On the assessment of medical expert systems. In: Expert systems and decision support in medicine. (Rienhoff, Piccollo and Schneider, Eds.), Lecture notes in medical Informatics 36, Springer Verlag, Berlin, 1988, pp. 292-297.

Russi-Mori A., Pisanelli D.M., Ricci F.L., Evaluation stages and design stups for knowledge-base systems in medicine, Medical Informatics. 15, 1990, pp.191-204.

Rushby J., Quality measures and assurance for AI sofiware, NASA contractor report 4187, SRI Intemational, Menlo Park, 1988.

Sander J.W.A.S., Hart Y.M., Johnson A.L., Shorvon S.D., National General Practice Study of Epilepsy: newly diagnosed epileptic seizures in a general population, The Lancet, 336, 1990, pp. 1267-1271.

Siegel S., Castellan N.J., Nonparametric statistics for the behavioral sciences, $2^{\text {nd }}$ edition, McGrawHill Book Company, New York, 1988.

Schouten H.J.A., Statistical Measurement of interobserver agreement, analysis of agreements and disagreements between observers, thesis, Rotterdam, 1985.

Smeets R., Talmon J., General methodology for the assessment of decision support systems. In: A critique of the evaluation methodology, (Nolan, Ed.), KAVAS (AIM 102.1), Report EM-2.I, Hvidovre Hospital, Hvidovre, 1990.

Sorgaard P.. Evaluating expert system prototypes. Artificial Intelligence and Socicty, 5, 1991, pp.317.

Spiegelhalter D., Evaluation of clinical decision aids. with an application to a system for dyspepsia, Statistics in Medicine, 2, 1983, pp 207-216.

Ven A. van der., Delbecq A.L. Nominal versus group processes for committee decision-making effectiveness, Academy of Management Journal, 6, 1971, pp. 203-212.

Wagner A.L., A clinical and epidemiological study of adult patients with epilepsy, Acta Neurologica Scandinavia, 94 (Suppl.). 1983, pp. 63-72

Willems J.L., Amaud P., Bemmel J.H. van, Bourdillon P.J., Degani R, Dennis B. Harms F.M.A., Macfarlane P.W., Mazzocca G., Meyer J., Ritsema van Eck H.J., Robles de Medina E.O., Zywietz C.. Establishment of a reference library for evaluating computer ECG measurement programs, Computers and Biomedical Research, 18, 1985, pp.439-457.

Wyatt J., Spicgellialter D., Evaluating medical expert systems: what to test and how?. Medical Iniormatics, 15, 1990, pp. 205-217. 



\section{Results on initiation of treatment}

\subsection{Introduction}

In this chapter the results of the analysis of the performance of the system in prescribing antiepileptic drugs in the initiation phase are reported. The advice of the system is compared both with the advice of two neurologists from the same department, one of which provided the knowledge implemented in the system and with that of five neurologists from different neurology departments. In the latter case the final prescriptions of the neurologists as obtained via the Delphi procedure are compared with those of the system.

To assess the acceptability of the advice - independent of whether the advice was according to the majority agreement - the prescriptions were reviewed by two neurologists.

A consensus meeting was held to discuss and possibly reduce the variability among the neurologists that remained after the Delphi procedure. While in the Delphi procedure the objective was to obtain a majority decision for each individual case, a more global approach for treatment was defined in the consensus meeting. These guidelines are used as a reference to compare the system and the neurologists with.

\subsection{Study design}

The goal of the study is to establish the adequacy of the advice of the system by comparing it with the advice of neurologists. A staged approach was adopted for the evaluation.

Five different assessments of the advice of the system were made. For each of the 60 cases of our test set, the expert neurologist (Neurologist I) whose knowledge was implemented in the system, provided a diagnosis and a prescription. The advice of the system, based on the detailed diagnosis of the neurologist and the other relevant patient data, was compared with the advice of this neurologist. This assessment provides some insight in the quality of the knowledge acquisition process.

To assess the impact of local differences a second neurologist (Neurologist II) from the same department as Neurologist $\mathrm{l}$ also provided a detailed diagnosis and a prescription for the 60 cases. A comparison was made with the advice of the system using Neurologist II's diagnosis.

In a third experiment the drug and the daily dose proposed by the system are compared with the treatments suggested by the neurologists after the Delphi method. The diagnostic reference set was used for assessing the system's proposed generic drug, the treatment reference set for assessing the suggested daily dose. This comparison provides a more objective measure of the 
performance of the system as these neurologists did not belong to the department of the expert neurologist.

A prescription different from the majority decision may still be considered a good option for treatment. Therefore, the acceptability of the prescriptions for each case in the diagnostic reference set was assessed by two neurologists who did not provide prescriptions for such a case.

To reduce the interobserver variability to determine the system's correctness, discrepancies in prescriptions were discussed during a consensus meeting. In this meeting general guidelines were proposed by the neurologists. From these guidelines a reference prescription was derived for each case in our diagnostic reference set. The advice of the system and the prescriptions of the individual neurologists were compared with this reference.

In the above design the focus of the assessment gradually shifts from an assessment of the coverage of the implemented knowledge and the correctness of the implementation to an assessment and comparison of the system's and neurologists' performance using a set of cases with reference diagnoses and prescriptions

When discussing the results a difference is made between cases and prescriptions. One has to remember that for each case up to three neurologists prescribe. The sets of cases for which individual neurologists provide a prescription vary. Therefore in most analyses the number of prescriptions is used.

\subsection{Comparing the system with Neurologist I and Neurologist II}

In this paragraph the advice of the system is compared with that of Neurologists I and II, using the 60 test cases. The diagnosis established by the neurologist is entered into the system. Since both neurologists independently diagnose the same case, it is possible that a case receives two different diagnoses. These cases are then entered twice into the system and the treatment advice of the neurologist is compared with that of the system using the diagnosis of that neurologist. In addition to the diagnosis, information about seizures and previous successful treatments, side-effects, use of co-medication, liver and kidney function and weight is entered into the system and used in the reasoning process

\subsubsection{Agreement on the generic drug}

Neurologist 1 provided 59 prescriptions. Although he was able to diagnose the type of epilepsy by using the available information, in one case he did not prescribe a drug because he wanted an additional EEG registration to be made before commencing drug treatment. Monotherapy was used in all cases.

Table 5.3-I: Agreement between Neurologist I, Neurologist II and the system.

\begin{tabular}{|c|c|c|c|c|c|c|}
\hline \multirow{2}{*}{$\begin{array}{l}\text { Gencric drug } \\
\text { System }\end{array}$} & \multicolumn{3}{|c|}{ Neurologist I } & \multicolumn{3}{|c|}{ Neurologist II } \\
\hline & No drug & CBZ. & $\mathrm{Na}-\mathrm{VPA}$ & No drug & $\mathrm{CBZ}$ & Na-VPA \\
\hline No drug & 0 & 0 & 0 & 9 & 0 & 0 \\
\hline CBZ & l & 44 & 0 & 0 & 40 & 0 \\
\hline Na-VPA & 0 & 0 & 15 & 1 & 0 & 10 \\
\hline
\end{tabular}

$\mathrm{CBZ}=$ carbanazepine, $\mathrm{Na}-\mathrm{VPA}=$ sodium valproate 
In 59 out of the 60 cases the system prescribed the same generic drug as Neurologist I (Table 5.3-1). Although the detailed diagnosis is entered, the system reasons with the global diagnosis. Also Neurologist I assigns a particular drug on the basis of the global diagnosis. If the diagnosis was partial epilepsy CBZ was proposed. For the cases diagnosed as primary (idiopathic) generalised epilepsy Na-VPA was selected. In the one case in which the neurologist established a diagnosis but did not provide a prescription the system suggested CBZ since the diagnosis was partial epilepsy.

Neurologist II established a diagnosis in 51 cases while a prescription was provided in 50 cases. Monotherapy was used for all of these cases. In six cases Neurologist Il could not establish the diagnosis and in three cases he was not certain about the diagnosis. In these nine cases Neurologist II decided not to prescribe a drug. In one additional case the neurologist decided not to prescribe a drug although he reached the diagnosis primary (idiopathic) generalised epilepsy. The reason for this was that he wanted to have additional EEG data about the patient. Because anti-epileptic drugs can influence the recordings he decided not to prescribe any drugs until the EEG findings were available

In 50 out of 51 cases (Table 5.3-1) the system and Neurologist II agreed on the drug treatment. Also Neurologist II selects a specific drug on the basis of the global diagnosis. The 39 cases diagnosed by Neurologist II as partial epilepsy received CBZ. In the one case diagnosed as primary (idiopathic) generalised epilepsy and partial epilepsy with secondary generalised partial seizures CBZ was also prescribed. In all ten cases diagnosed as primary (idiopathic) generalised epilepsy Na-VPA was prescribed. The system prescribed the same drugs. For the nine cases in which the neurologist did not provide a diagnosis the system did not suggest a treatment. In the one case in which the neurologist established the diagnosis primary (idiopathic) generalised epilepsy but did not prescribe a drug, the system suggested Na-VPA.

\subsubsection{Agreement on tradename}

When prescribing a drug one can use a generic name, leaving the choice of the brand to the pharmacist, or a tradename. Both neurologists used only tradenames. The system was programmed to do likewise.

The neurologists and the system used only one brand for each of the generic drugs. In case of Na-VPA all three selected Depakine* EC. For CBZ all three used the same brand. Neurologist I used Tegretol ${ }^{\text {w }}$, while Neurologist Il used only the controlled release variant, Tegretol ${ }^{\text {in: }} \mathrm{CR}$. The system uses the same formulation as Neurologist $\mathrm{I}$, hence there is a consistent difference with Neurologist II on the formulation of CBZ.

\subsubsection{Agreement on daily dose}

Both formulations for $\mathrm{CBZ}$ contain the same biologically active substance. The drug is available in the same doses of tablets for both formulations. Therefore, one can compare the prescribed daily doses. In 48 of the 59 cases the system prescribed the same daily dose as Neurologist I. Although the system and the Neurologist II completely agreed in 50 cases about the drug, in only 3 cases the system proposed the same daily dose. Table 5.3-II and Table 5.3III present the results comparing the daily doses of CBZ and Na-VPA prescribed by Neurologist I, Neurologist II and the system. 
Table 5.3-II: Daily dose for CBZ suggested by the system and by Neurologists I and IL.

\begin{tabular}{|c|c|c|c|c|c|c|c|c|}
\hline \multirow[t]{2}{*}{ CBZ } & & \multicolumn{7}{|c|}{ Prescribed daily dose (mg) } \\
\hline & & Neuro & 300 & 400 & 500 & 600 & 700 & 800 \\
\hline \multirow{8}{*}{$\begin{array}{l}\text { System's } \\
\text { daily dose }\end{array}$} & 200 & I & 1 & & & & & \\
\hline & & II & & 2 & & & & \\
\hline & 300 & I & 24 & 3 & & & & \\
\hline & & $\amalg$ & & 1 & 15 & 7 & & \\
\hline & 400 & 1 & 3 & 11 & & 1 & & \\
\hline & & Ш & & & & 6 & 6 & 3 \\
\hline & 500 & I & & 1 & & & & \\
\hline & & II & & & & & & \\
\hline
\end{tabular}

Forty-four times Neurologist I provided a prescription for CBZ. The daily dose of CBZ prescribed by Neurologist 1 ranged from 300 to $600 \mathrm{mg}$ per day. The dosages recommended by the system for $\mathrm{CBZ}$ ranged from 200 to $500 \mathrm{mg}$ per day. In 35 of the 44 prescriptions the daily dose suggested by the system was identical. Five times the neurologist prescribed a higher dose than that proposed by the system. Twice a daily dose two thirds of that prescribed by the neurologist was suggested. Four times the system proposed a higher dose than the neurologist

Table 5.3-III: Daily dose for VPA suggested by the system and by Neurologists I and II.

\begin{tabular}{|c|c|c|c|c|c|c|}
\hline \multirow[t]{2}{*}{ VPA } & & \multicolumn{5}{|c|}{ Prescribed daily dose (mg) } \\
\hline & & Neuro & 900 & 1000 & 1200 & 1500 \\
\hline \multirow{4}{*}{$\begin{array}{l}\text { System's } \\
\text { daily dose } \\
\text { (mg) }\end{array}$} & 900 & 1 & 13 & & 1 & \\
\hline & & [1] & 3 & 4 & 2 & \\
\hline & 1050 & 1 & 1 & & & \\
\hline & & Il & & & & 1 \\
\hline
\end{tabular}

Neurologist II approaches the treatment of partial epilepsy differently. The daily dose for CBZ ranges from 400 to $800 \mathrm{mg}$. In all except one case the system suggested only $50-66 \%$ of the daily dose prescribed by the neurologist.

For Na-VPA Neurologist $\mathrm{l}$ used a daily dose between 900 and $1200 \mathrm{mg}$. The system recommended Na-VPA in a daily dose of 900 or $1050 \mathrm{mg}$ per day. Twice the system suggested a daily dose different from that of the neurologist.

Neurologist II again prescribed higher daily doses for the cases diagnosed as primary (idiopathic) generalised epilepsy. The daily doses ranged from 900 to $1500 \mathrm{mg}$ per day. Also the prescription of Na-VPA differed from that of the system although the differences are smaller as compared to the differences for CBZ. In three of the 10 cases a dose less than $90 \%$ of the neurologist's dose was suggested.

\subsection{Comparing the system with an external reference}

In this paragraph the advice of the system is assessed using the cases from the diagnostic and treatment reference sets (see chapter 4 par. 4.2). 


\subsubsection{Agreement about the drug using the diagnostic reference set}

The agreement of the system with the neurologists on the cases of the diagnostic reference set is presented in Table 5.4-]. The system is compared with only those neurologists who agreed on the detailed diagnosis.

Table 5.4-I: Agreement between the system and the neurologists on the prescribed generic drug using the diagnostic reference set.

\begin{tabular}{|c|c|c|c|c|c|c|c|}
\hline \multirow{3}{*}{\multicolumn{2}{|c|}{$\begin{array}{l}\text { Agreement on: } \\
\text { delailed diagnosis } \\
\text { prescribed drug }\end{array}$}} & \multicolumn{4}{|c|}{ Neurologists } & & \\
\hline & & \multicolumn{2}{|c|}{$3 / 3$} & \multicolumn{2}{|c|}{$2 / 3$} & \multirow{2}{*}{\multicolumn{2}{|c|}{ Total }} \\
\hline & & $3 / 3$ & $2 / 3$ & $2 / 2$ & no & & \\
\hline \multirow[b]{2}{*}{ Agreement } & 3 neuso's & 7 & & & & 7 & \multirow[b]{2}{*}{ Agroement } \\
\hline & 2 neuro's & & 8 & 17 & & 25 & \\
\hline system & I neuro & & 3 & & 8 & 11 & \\
\hline \multirow[t]{2}{*}{ with } & 0 neuro's & I & 1 & 2 & 0 & 4 & Disagreement \\
\hline & Total & 8 & 12 & 19 & 8 & 47 & \\
\hline
\end{tabular}

Full agreement on diagnosis

In 8 cases all three neurologists agreed on the detailed diagnosis and the generic drug (Table 5.4-1). The system agreed in 7 of these cases with the generic drug. The diagnosis of the remaining case was partial epilepsy with secondary generalised seizures. While all three neurologists prescribed PHT the system prescribed CBZ.

There were 12 cases in which two out of three neurologists agreed about the generic drug although all agreed about the detailed diagnosis. The system agreed with the majority in 8 of these cases. In three cases the system recommended the drug prescribed by the third neurologist. In one case the system prescribed a drug which was not prescribed by any of the experts. In this case, diagnosed as partial epilepsy with complex partial seizures and partial epilepsy with secondary generalised seizures, two of the neurologists recommended no drug treatment while the third neurologist decided to prescribe Na-VPA. The system prescribed CBZ.

\section{2/3 agreement on diagnosis}

In 27 cases two out of three neurologists agreed on the detailed diagnosis. In 19 cases these two neurologists also agreed on the generic drug. The system prescribed the same drug in 17 cases (Table 5.4-I). The system prescribed Na-VPA for a case diagnosed as primary (idiopathic) generalised epilepsy while both neurologists prescribed CBZ. In another case, diagnosed as partial epilepsy with simple partial seizures the system suggested CBZ while the neurologists both prescribed PHT for this case.

In 32 of the 47 cases, the generic drug suggested by the system was in agreement with the reference drug. In eleven cases the system proposed a generic drug which was prescribed by only one neurologist. In all eleven cases this neurologist prescribed a drug different from that prescribed by his colleague(s) despite agreement on the detailed diagnosis. In four cases the system suggested a drug not prescribed by any of the neurologists. 


\subsubsection{Variability in neurologist's prescriptions}

In 20 cases of the diagnostic reference set all three neurologists established the same detailed diagnosis and reached a majority agreement on the prescribed drug in all of these cases. In the subset of 27 cases in which 2/3 neurologists agreed on the detailed diagnosis $63 \%$ of the cases had a majority agreement on the prescribed drug. As the selection of the drug is mainly based on the diagnosis, we required total agreement about this issue when comparing the proposed drugs of the system with those of the individual neurologists. Therefore the earlier mentioned subset of 20 cases is used for assessment of the generic drug suggested by the system.

Table 5.4-II presents how often each of the individual neurologists prescribed a generic drug agreed upon by the majority of the neurologists in cases of this subset of 20 cases. All except one of the neurologists prescribed the majority drug in more than $80 \%$ of his cases. One neurologist agreed in only $46 \%$ of the cases.

Table 5.4-1I: Generic drug prescribed in accordance with the majority.

\begin{tabular}{|c|c|c|c|c|c|c|c|c|c|c|c|}
\hline \multicolumn{12}{|c|}{ Agreement of participants and system with drug in $N=20$} \\
\hline \multirow[t]{2}{*}{ Participant } & III & \$ & IV & $S$ & $\mathrm{~V}$ & $\$$ & $\mathrm{~V}$ & $\mathrm{~S}$ & V & $\mathrm{S}$ & system \\
\hline & \multicolumn{2}{|c|}{$n=13$} & \multicolumn{2}{|c|}{$\mathrm{n}=16$} & \multicolumn{2}{|c|}{$n=11$} & \multicolumn{2}{|c|}{$\mathrm{n}=\mathrm{ll}$} & \multicolumn{2}{|c|}{$n=9$} & $n=20$ \\
\hline Drug agreed & 11 & 9 & 15 & 10 & 5 & 9 & 9 & 10 & 8 & 6 & 15 \\
\hline$\%$ & 85 & 75 & 24 & 63 & 46 & 82 & 82 & 91 & 89 & 67 & 75 \\
\hline
\end{tabular}

The system agreed on the prescribed drug with at least two neurologists in 15 of these 20 cases. As a majority was established if a neurologist agreed with at least one of his colleagues it was also examined in what percentage the system agreed with at least one neurologist. In 18 cases $(90 \%)$ the system prescribed the same drug as at least one of the prescribing neurologists

The system's advice was compared with that of an individual neurologist on the basis of the cases seen by both. Three neurologists prescribed more in accordance with the majority than the system. This analysis is in favour of the neurologists as they defined the drug used by the majority, while the system did not

\subsubsection{Agreement about the daily dose}

To obtain insight in the adequacy of the suggested dosage the treatment reference set was used. In 32 out of the 39 cases the system prescribed the same drug. These 32 cases were used to evaluate the dosages prescribed. In Table 5.4-111 a breakdown is presented of how many of these cases are seen by each of the neurologists and by the system.

Table 5.4-III: Agreement with the reference daily Jose.

\begin{tabular}{lcccccc}
\hline Neurologist & $3(\mathrm{n}=14)$ & $4(\mathrm{n}=16)$ & $5(\mathrm{n}=13)$ & $6(\mathrm{n}=14)$ & $7(\mathrm{n}=14)$ & System $(\mathrm{n}=32)$ \\
\hline $\begin{array}{l}\text { Cases for daily dose } \\
\text { comparison }\end{array}$ & 6 & 5 & 6 & 5 & 4 & 11 \\
Agreed daily dose & $43 \%$ & $31 \%$ & $46 \%$ & $36 \%$ & $29 \%$ & 32 \\
\hline
\end{tabular}


A majority dosage (at least two neurologists prescribe the same daily dose) could be obtained in 11 cases (34\%). Within this set of 11 cases the system agreed with the majority of the neurologists in 5 cases (46\%). In $20(63 \%)$ out of the 32 cases the system agreed about the daily dose with at least one neurologist.

Table 5.4-IV:Differences in the daily dose of CBZ (in parentheses the dose prescribed by the system is given as a percentage of the dose prescribed by the neurologists). The number of $100 \mathrm{mg}$ tablets presented in the first row indicates the number of tablets the system preseribed too few or too many.

\begin{tabular}{|c|c|c|c|c|c|c|c|}
\hline $\begin{array}{l}\text { Daily dose } \\
\text { neurologist (mg) }\end{array}$ & -5 tabl. & -4 tabl. & -3 tabl. & -2 lathl & -1 tabl. & 0 tabl. & {$[t .(b)$.} \\
\hline 200 & & & & & & & $2(150 \%)$ \\
\hline 300 & & & & & & $2(100 \%)$ & \\
\hline 400 & & & & & $12(75 \%)$ & $6(100 \%)$ & \\
\hline 600 & & & $9(50 \%)$ & $9(66,7 \%)$ & & & \\
\hline 800 & $1(37.5 \%)$ & $3(50 \%)$ & & & & & \\
\hline 900 & $\mathrm{I}(44 \%)$ & & & & & & \\
\hline
\end{tabular}

In Table 5.4-IV and Table 5.4-V the differences in daily dose between the system and the neurologists are presented for the drugs CBZ and Na-VPA using the 32 cases from the treatment reference set. In this analysis we used the doses of the individual prescriptions of the neurologists.

In 20 of the 32 cases in which the system prescribed the same dug as the majority of the neurologists CBZ was prescribed. In total 45 prescriptions were provided for this drug by the neurologists. The daily dose ranged from 200 to $900 \mathrm{mg}$. A daily dose of more than $600 \mathrm{mg}$ is prescribed 5 times $(12 \%)$. One neurologist prescribed more than $800 \mathrm{mg} \mathrm{CBZ}$.

Table 5.4-V: Differences in prescribed daily dose of CBZ between system and 5 neurologists.

\begin{tabular}{lccccc}
\hline $\begin{array}{l}\text { Difference in CBZ } \\
\text { daily dose (mg) }\end{array}$ & N III & N IV & N V & N VI & N VI \\
\hline-500 & 2 & & & 2 & \\
-400 & 5 & 1 & 3 & 1 & \\
-300 & 1 & & 5 & 3 & \\
-200 & & 6 & & 3 & 3 \\
-100 & & 4 & 1 & 1 & 2 \\
0 & & & & & 2 \\
\hline
\end{tabular}

Table 5.4-IV presents the differences in daily dose for CBZ between the neurologists and the system. In $8(18 \%)$ of these 45 prescriptions the system and a neurologist agreed on the daily dose of CBZ. If a difference of 1 tablet of $100 \mathrm{mg}$ is still acceptable the number of acceptable doses of the system increases to $22(49 \%)$. The system agrees with the neurologists in the lower dosage range. The higher the dose prescribed by the neurologists the larger the 
difference becomes. In 14 prescriptions a neurologist recommended twice or more the daily dose prescribed by the system. Table $5.4-\mathrm{V}$ shows the difference in agreement between the individual neurologists and the system. The system tends to prescribe approximately the same daily doses for CBZ as Neurologist IV and VII.

In 10 cases the system prescribed Na-VPA according to the reference (Table 5.4-VI). Agreement between the neurologists and the system on the prescribed dose of (Na-)VPA occurs in 14 out of 22 prescriptions $(64 \%)$. There are 6 prescriptions where the dose suggested by the system is $90 \%$ of the prescribed dose of the neurologist. This difference in doses is caused by a difference in intake scheme: $3 \times 300 \mathrm{mg}$ versus $2 \times 500 \mathrm{mg}$. Including these cases leads to an agreement in 20 out of 22 prescriptions. In 2 prescriptions ( $9 \%$ ) the system recommended a daily dose which was only $60 \%$ the dose prescribed by one of the neurologists.

Table 5.4-VI: Differences in the daily dose of ( $\mathrm{Na}$-)VPA. The percentages present the dose of the system relative to that of the neurologist. The number of tablets presented in the first row indicates the number of $300 \mathrm{mg}$ tablets the system prescribed too few.

\begin{tabular}{llll}
\hline $\begin{array}{l}\text { Daily dose of } \\
\text { neurologist (mg) }\end{array}$ & -2 tabl. & $-1 / 3$ labl. & 0 tabl. \\
\hline 900 & & & $14(100 \%)$ \\
1000 & & $6(90 \%)$ & \\
1500 & $2(60 \%)$ & & \\
\hline
\end{tabular}

\subsection{Review of the prescriptions}

All prescriptions for each of the 47 cases in the diagnostic reference set were assessed by two neurologists who did not prescribe for such a case. The reviewers judged the overall acceptability of each of the prescriptions on a five point scale (excellent, sufficient, neutral, insufficient, poor). For those prescriptions judged as of possibly poor quality - scored as neutral, insufficient or poor acceptability - the various components of a prescription e.g. the generic drug and the dose of tablets were assessed separately.

\subsubsection{Overview of the results for system and individual neurologists}

In total 114 prescriptions from the neurologists and 47 from the system were reviewed. For all prescriptions of the system both reviewers assessed the overall quality, resulting in 94 reviews. For seven prescriptions of the neurologists a reviewer did not provide an overall judgement. So only 221 rather than 228 review reports for the neurologists' prescriptions are available

In Table 5.5-I more than half of the prescriptions of all but one of the neurologists were considered to be of good quality by the reviewers (range 51 to $63 \%$ ). In less than $20 \%$ (range 13 to $19 \%$ ) their prescriptions were considered inadequate. Compared with these four neurologists a lower percentage of prescriptions of the system was considered to be of good quality but also a smaller percentage of prescriptions were considered inadequate. 
Table 5.5-I: Overall judgements by each of the two reviewers on the prescriptions provided by an individual neurologist. At most two judgements per prescription are available.

\begin{tabular}{|c|c|c|c|c|c|c|c|c|c|c|c|c|}
\hline \multirow{2}{*}{$\frac{\text { Participant }}{\text { Judgement }}$} & \multicolumn{2}{|c|}{ m } & \multicolumn{2}{|c|}{ IV } & \multicolumn{2}{|c|}{$\mathrm{V}$} & \multicolumn{2}{|c|}{$\mathrm{Vl}$} & \multicolumn{2}{|c|}{ VII } & \multicolumn{2}{|c|}{ System } \\
\hline & $\mathrm{n}$ & $\%$ & $n$ & $\%$ & $n$ & $\%$ & $\mathrm{n}$ & $\%$ & $\mathrm{n}$ & $\%$ & $\mathbf{n}$ & $\%$ \\
\hline poor' & 6 & 13 & 8 & 16 & 12 & 29 & 8 & 20 & 8 & 18 & 10 & 11 \\
\hline neutral & 14 & 30 & 15 & $3 !$ & 16 & 39 & 7 & 17 & 14 & 31 & 39 & 42 \\
\hline $\operatorname{good}^{2}$ & 26 & 57 & 26 & 53 & 13 & 32 & 26 & 63 & 22 & 51 & 45 & 48 \\
\hline Total judgements & 46 & 100 & 49 & 100 & 41 & 100 & 41 & 100 & 44 & 100 & 94 & 100 \\
\hline Cases diagn.ref.set & 23 & & 25 & & 21 & & 22 & & 23 & & 47 & \\
\hline Missing judgem. & 0 & & I & & I & & 3 & & 2 & & 0 & \\
\hline
\end{tabular}

(1) poor consists of insufficient and poor, (2) good contains the classes: good and excellent of the five point scale.

In 147 of the 157 review reports indicating a poor or neutral overall acceptability assessments of the prescribed drug were presented. For three of the neurologists more than $50 \%$ of these judgements was positive (range $54 \%$ to $70 \%$ ). Also for the system most (67\%) of the assessments indicated that a proper drug was prescribed (Table 5.5-II). For four of the neurologists $17 \%$ to $36 \%$ of the prescribed drugs were considered not appropriate. The system and one of the neurologists suggested an inappropriate drug in less than $5 \%$ of the prescriptions.

Table 5.5-II: Judgements on the generic drug for prescriptions with an overall poor or neutral quality. For each prescriber the obtained number of judgements indicating that the prescribed drug is appropriate, neutral or inappropriate is presented.

\begin{tabular}{|c|c|c|c|c|c|c|c|c|c|c|c|c|}
\hline \multirow{2}{*}{$\begin{array}{l}\text { Participant } \\
\text { Judgement drug }\end{array}$} & \multicolumn{2}{|c|}{ III } & \multicolumn{2}{|c|}{ IV } & \multicolumn{2}{|c|}{ v } & \multicolumn{2}{|c|}{$\mathrm{VI}$} & \multicolumn{2}{|c|}{ VII } & \multicolumn{2}{|c|}{ System } \\
\hline & $\mathrm{n}$ & $\%$ & $n$ & $\%$ & $n$ & $\%$ & 川 & $\%$ & n & $\%$ & $\mathrm{n}$ & $\%$ \\
\hline inappropriate & 3 & 17 & I & 4 & 5 & 21 & 5 & 36 & 4 & 20 & 1 & 2 \\
\hline neutral & 8 & 44 & 6 & 26 & 6 & 25 & 1 & 7 & 7 & 35 & 15 & 31 \\
\hline appropriate & 7 & 39 & 16 & 70 & 13 & 54 & 8 & 57 & 9 & 45 & 32 & 67 \\
\hline Total judgements & 18 & 100 & 23 & 100 & 24 & 100 & 14 & 100 & 20 & 100 & 48 & 100 \\
\hline Poor/neutral judg. & 20 & & 23 & & 28 & & 15 & & 22 & & 49 & \\
\hline Missing & 2 & & 0 & & 4 & & 1 & & 2 & & 1 & \\
\hline
\end{tabular}

For the prescriptions with a neutral or poor overall quality and an appropriate drug, the quality of the prescribed dose was analysed (Table 5.5-III). For one of these prescriptions a judgement was not provided on this issue. The percentage of judgements suggesting an inappropriate dose ranged from $53 \%$ to $60 \%$ for three of the neurologists, while for two neurologists more than $85 \%$ the daily dose was inappropriate. The system suggested an inappropriate dose in $91 \%$ of these prescriptions. Three neurologists tend to prescribe a (too) high rather than a (too) low dose. Most of the dosages prescribed by the system were judged as being too low.

In 21 of the 157 prescriptions which were considered to be of a (possibly) poor quality the judgement could not be attributed to an inadequate generic drug or daily dose but was caused by an inadequate brand, dose of tablets and/or intake scheme. 
Table 5.5-1II: Judgements provided on the daily dose for prescriptions in which the prescribed drug was considered to be appropriate but the overall judgement indicated (possibly) poor quality. For each neurologist the number of obtained judgements indicating a (too) low, neutral and (too) high daily dose is provided.

\begin{tabular}{|c|c|c|c|c|c|c|c|c|c|c|c|c|}
\hline \multirow{2}{*}{$\frac{\text { Participant }}{\text { Judgm. daily dose }}$} & \multicolumn{2}{|c|}{ III } & \multicolumn{2}{|c|}{ IV } & \multicolumn{2}{|c|}{ V } & \multicolumn{2}{|c|}{$\mathrm{VI}$} & \multicolumn{2}{|c|}{ VII } & \multicolumn{2}{|c|}{ System } \\
\hline & $\mathrm{n}$ & $\%$ & $\mathrm{n}$ & $\%$ & $\mathrm{n}$ & $\%$ & $n$ & $\%$ & $\mathrm{n}$ & $\%$ & $\pi$ & $\%$ \\
\hline$(1 \infty)$ low & 0 & 0 & 7 & 47 & 3 & 23 & 2 & 25 & 6 & 67 & 28 & 88 \\
\hline neutral & 1 & 14 & 6 & 40 & 6 & 46 & 3 & 38 & 1 & 11 & 3 & 9 \\
\hline (too) high & 6 & 86 & 2 & 1.3 & 4 & $3 !$ & 3 & 38 & 2 & 22 & 1 & 3 \\
\hline Tolal judgements & 7 & 100 & 15 & 100 & 13 & 100 & 8 & 100 & 9 & 100 & 32 & 100 \\
\hline Judgen.drug appr. & 7 & & 16 & & 13 & & 8 & & 9 & & 32 & \\
\hline Missing & 0 & & I & & 0 & & 0 & & 0 & & 0 & \\
\hline
\end{tabular}

\subsubsection{Comparing overall scores of system and individual neurologists}

For each case two or three neurologists and the system provided a prescription. The score for a prescription of a neurologist can be compared with the score for the prescription of the system on the same case. The difference between the overall acceptability of the prescription of a neurologist and the system gives an indication which of the two prescriptions is favoured. A positive difference indicat "; that the reviewer favours the prescription of the neurologist, a negative difference is in favour of the system. In Table 5.5-IV the difference scores are presented as a function of

Table 5.5-IV: Difference in overall acceptability scores of the reviewer when comparing the scores of the neurologist with the score of the system.

\begin{tabular}{|c|c|c|c|c|c|c|c|c|c|c|c|c|c|c|c|}
\hline \multirow[t]{2}{*}{ Overall score } & \multicolumn{3}{|c|}{ Reviewer 3} & \multicolumn{3}{|c|}{ Reviewer 4} & \multicolumn{3}{|c|}{ Reviewer 5} & \multicolumn{3}{|c|}{ Reviewer 6} & \multicolumn{3}{|c|}{ Reviewer 7} \\
\hline & - & 0 & + & $=$ & 0 & + & . & 0 & + & - & 0 & + & - & 0 & + \\
\hline n3-syst & & & & 2 & 7 & 2 & l & 4 & 5 & 2 & 8 & 3 & 4 & 6 & 2 \\
\hline n4-syst & 3 & 8 & 0 & & & & 1 & 3 & 9 & 2 & $\bar{y}$ & 0 & 2 & 10 & 2 \\
\hline n5-syst & 4 & 4 & 3 & 4 & 4 & 1 & & & & 4 & 4 & 2 & 4 & 5 & 2 \\
\hline n6-syst & 1 & 9 & । & 3 & 3 & 1 & 3 & 1 & 5 & & & & 4 & 3 & 7 \\
\hline n7-syst & 3 & 6 & 4 & 3 & 4 & 2 & 4 & 3 & 3 & 1 & 7 & 4 & & & \\
\hline Total & 11 & 27 & 8 & 12 & 18 & 6 & 9 & 11 & 22 & 9 & 28 & 9 & 14 & 24 & 13 \\
\hline$\%$ & 24 & 59 & 17 & 33 & 50 & 17 & 21 & 26 & 52 & 20 & 60 & 20 & 28 & 47 & 26 \\
\hline
\end{tabular}

\begin{tabular}{lccccccc}
\hline Overall score & \multicolumn{3}{c}{ Total } & \multicolumn{4}{c}{ Percentage } \\
& \multicolumn{1}{c}{0} & \multicolumn{1}{c}{+} & \multicolumn{1}{c}{0} & + \\
\hline nj-syst & 9 & 25 & 12 & 20 & 54 & 26 \\
n4-syst & 8 & 30 & 11 & 16 & 61 & 23 \\
n3-syst & 16 & 17 & 8 & 39 & 42 & 20 \\
n6-syst & 11 & 16 & 14 & 27 & 39 & 34 \\
n7-syst & 11 & 20 & 13 & 24 & 44 & 29 \\
\hline Total & 55 & 108 & 58 & & & \\
$\%$ & & & & 25 & 49 & 26 \\
\hline
\end{tabular}


the reviewing neurologist. Since in total 221 prescriptions of the neurologists were reviewed, 221 differences could be computed

Twenty prescriber-reviewer combinations exist. In 10 of these combinations the system's prescription was favoured above the neurologists' prescription more often than the reverse. In 7 the neurologists prescription was favoured.

It appears that Neurologist $V$ judges the performance of the system much lower than the other neurologists do. In turn Neurologist $V$ is judged less positively by his colleagues than the system. Overall the system's advice is judged as being of comparable quality as the neurologists.

Exclusion of Neurologist $\mathrm{V}$ as reviewer and as prescriber does not change the conclusion that the system's prescriptions are just as acceptable as the neurologists' prescriptions.

\subsubsection{Variability of the reviewers}

The prescriptions for a case have been independently assessed by two reviewers. This allows the study of the variability in the assessments of the reviewers.

Again we determined for each case the difference between the overall scoring of the recipe of the neurologist and the overall scoring of the corresponding recipe of the system: + indicating that the system's recipe had a better score, 0 indicating equal scores and - indicating that the recipe of the system obtained a lower score than the neurologist's recipe. This comparative score is determined for the assessments of both reviewers

Table 5.5-V gives an overview of how often in the 109 prescriptions of the neurologists for which two assessments were available, two judges give a certain comparative score.

In $14(13 \%)$ prescriptions both reviewers disagreed completely on which prescription is of better quality: one reviewer considered the neurologist's recipe better than the system's, the other reviewer did the reverse. For 42 prescriptions (38\%) both reviewers provided the same assessment for the two recipes.

Table 5.5-V: Agreement between reviewers compa-

ring the overall score for the system

with that for the prescription of a

neu rologist.

\begin{tabular}{crrr}
\hline Judge 1 & Judge 2 & No. cases & $\%$ \\
\hline+ & + & 6 & 5.5 \\
+ & 0 & 32 & 29.4 \\
+ & - & 14 & 12.8 \\
0 & 0 & 2 & 24.8 \\
0 & - & 21 & 19.3 \\
- & - & 9 & 8.3 \\
\hline \multicolumn{2}{l}{ Tolal no or cases } & 109 & 100 \\
\hline
\end{tabular}

The prescription of the system can be considered to be of lower quality when one of the reviewers considered a recipe of the neurologist of better quality and the other reviewer says that the neurologist's recipe is equal or better than the system's prescription. This occurs for 
30 prescriptions of the neurologists (28\%). For 38 pairs of prescriptions $(35 \%)$, the system's prescription is considered better by one reviewer and of equal or better quality than the neurologist's recipe by the other reviewer

\subsection{Results of the consensus meeting}

In this section the results of the consensus meeting are presented. The meeting dealt with general principles and not with individual cases although the diagnostic reference set served as background material for the discussion. Only the issues that are related to the diagnosis, selection of drugs and the initial dose are presented. The discussion resulted in a reference against which the prescriptions of the neurologists and of the system can be compared.

\subsubsection{The use of diagnostic classification schemes}

The diagnostic classification schemes as presented in the literature are considered to be more detailed than necessary for daily medical practice. The reliability of a detailed classification of the epilepsy of a patient may be doubtful. Cases admitted for neurosurgery may require a more detailed classification. Also for research purposes a detailed classification is valuable.

\subsubsection{Initiation of drug treatment}

In the literature a diagnosis ex juvantibus approach as well as initiation of anticonvulsive treatment if the diagnosis is not certain are both considered to be inappropriate (Chadwick and Reynolds 1985, Porter 1984, 1989, Shorvon 1987). In our study drug treatment was initiated twice in the 11 instances that a neurologist could not establish a diagnosis. In the consensus meeting and also in the review process drug treatment was considered unacceptable when no diagnosis is established. It was agreed that one should wait and let time decide what the diagnosis is before drug treatment is started.

Although the neurologists are willing to make a trade-off between the burden of antiepileptic drug treatment and the conseguences and the severeness of the seizures, all agreed that drug treatment should be started as soon as the diagnosis is established and two or more seizures have occurred. Also in the review process refraining from drug treatment was considered unacceptable when the diagnosis was established. However, in daily practice the opinion of the patient should be taken into account. If a patient refuses anticonvulsive treatment, despite knowing the consequences and risks, close monitoring of the patient is required to start the treatment immediately in case he changes his opinion.

\subsubsection{Selection of generic drug}

Based on the literature we concluded that at present there exists no consensus on whether the selection of the drug should be based on the type of epilepsy or on the type of seizures. On the basis of the meeting the neurologists agreed that the type of epilepsy should be the basis for the selection of the anticonvulsive treatment. A differentiation between generalised and partial epilepsy was considered to be sufficient for initial drug selection.

The neurologists agreed that if treatment is started in a newly diagnosed patient monotherapy should be used. The advantages of monotherapy are: a clear relation between drug and 
side-effects, a narrow spectrum of interactions and a better compliance. Polytherapy may be used in those patients where monotherapy proved to be ineffective.

Cases where complete seizure control was achieved in the past with polytherapy while monotherapy was not tried or tried inappropriately, may pose a problem in case of a relapse. Also in these patients the use of monotherapy is preferred even when a relapse occurs

For treatment of primary (idiopathic) generalised epilepsy Na-VPA is preferred. The argument that VPA (Convulex ${ }^{(i)}$ ) does not increase the sodium intake did not outweigh the advantages of Depakine ${ }^{\text {in }}$ EC. Even in case of hypertension the use of VPA is not advised nor has hypertension been mentioned as a contraindication for Na-VPA (Meinardi 1993, Shorvon 1995, Brodie and Dichter 1996).

The neurologists preferred the use of CBZ for treatment of partial epilepsy. Little agreement was observed when to apply PHT. The consensus meeting led to the conclusion that PHT should be the drug of first choice only for patients older than sixty years mainly because of side-effects such as gingival hyperplasia and coarsing of features in younger patients. PHT is a time honoured drug. The therapeutic range can be reached quickly and its use is easy due to the long half-life. Furthermore, the use of PHT for older patients has the advantage that a change in therapy is not necessary in case of surgery as a formulation for parenteral administration is available

\subsubsection{Generic drug or brand, formulation}

All neurologists preferred the use of the brand when prescribing a drug. The advantages mentioned were:

- The quality of a brand is fairly constant. The components as well as the production process have met criteria of quality control

- Variability in drug effectiveness is reduced. The occurrence of seizures can be attributed to a number of known factors.

A special formulation of the drug may prevent the occurrence of side-effects. Fluctuation in the plasma drug level and the occurrence of seizures at a particular time during the day, for example only in the early morning, may be important factors determining the selection of a special formulation of the drug. Furthermore, the compliance of the patient influences the selection of the formulation.

The neurologists agreed that Na-VPA may be prescribed as an uncoated formulation as far as the effectiveness of the drug is concerned. However, they prefer the enteric coated (EC) formulation because gastric distress occurs less often. Besides the $\mathrm{EC}$ formulation the Chrono formulation may be prescribed. This formulation has the advantage of less fluctuation in the plasma drug level while the peak level is reached faster. Nevertheless the EC formulation was preferred, using $300 \mathrm{mg}$ tablets in case the drug is taken three times a day or $500 \mathrm{mg}$ tablets if the drug is taken twice a day

The short half-life of CBZ together with the fluctuations in the plasma drug level require either a frequent drug intake or a controlled release (CR) formulation for this drug. All participants agreed on the use of the CR formulation when $C B Z$ is prescribed. The reference dose of the tablets is considered to be $200 \mathrm{mg}$. Tablets of $400 \mathrm{mg}$ should only be used in case a daily dose of $800 \mathrm{mg}$ or more is prescribed. The use of tablets of $400 \mathrm{mg}$ when a daily dose of less than $800 \mathrm{mg}$ is prescribed is considered to be inappropriate. 


\subsubsection{Daily dose of the drug}

In chapter 2 it has been shown that different opinions exist in the literature about the appropriate daily dose for Na-VPA, CBZ and PHT. Apart from the weight of the patient, which is according to our neurologists the basis for the determination of the daily dose, other factors such as stability of the plasma drug level, the risk of adverse drug reactions, the wish of the patient or the temperament of the neurologist, may influence the prescribed dose.

Although a 'middle of the target range' approach may be applied in selecting the daily dose, all participants agreed that in newly diagnosed patients one should start with a low daily dose and gradually increase the dose based on clinical findings. This is in contrast with the prescriptions they provided in which also a high initial dose occurred.

The ranges of the daily doses for the different drugs used by the neurologists were 900 to $1500 \mathrm{mg}$ for Na-VPA, 200 to $900 \mathrm{mg}$ for CBZ and 150 to $300 \mathrm{mg}$ for PHT. During the discussion the neurologists reached the conclusion that the prescribed daily doses were rather high. They agreed that if:

- Na-VPA or VPA was prescribed one should start with a dose of 900 to $1000 \mathrm{mg}$ per day, depending on the intake frequency

- $\mathrm{CBZ}$ is prescribed and the weight of the patient was less than $80 \mathrm{~kg} 400 \mathrm{mg}$ per day is sufficient. If the weight of the patient is higher than $80 \mathrm{~kg}$ the initial daily dose of the patient should be $600 \mathrm{mg}$

- PHT is selected as the drug for treatment and the weight is less than $80 \mathrm{~kg}$ a daily dose of $200 \mathrm{mg}$ PHT should be prescribed. In case the weight of the patient is higher than $80 \mathrm{~kg}$ they preferred a daily dose of $300 \mathrm{mg}$

One of the reasons for the relatively low daily dose of $\mathrm{CBZ}$ was the risk of adverse drug reactions. Side-effects may have consequences for the compliance. $\mathrm{CBZ}$ strongly induces its own catabolism. Therefore the initial daily dose has to be lower than the daily dose required eventually. The risk of having another seizure was not sufficient for the neurologists to initiate treatment with a higher daily dose. The kindling effect of additional seizures was considered not proven. Nevertheless, they agreed that sufficient prevention against seizures should be guaranteed. In addition to this, the wish of the patient should be taken into account when determining the daily dose. Some of the patients oppose drug treatment and do not like to be reminded several times a day that they are suffering from epilepsy. Others are afraid of having additional seizures and prefer a high dose to suppress further seizures.

\subsubsection{Intake frequency}

To be able to comply. patients need an intake scheme that is easy to handle. Therefore, the neurologists should try to keep the number of intakes per day as low as possible. The drug half-life as well as the formulation are important determinants for establishing the intake frequency. Practical consequences of drug intake have to be considered. While some neurologists combine the intake of the drug with meals, others stated that most patients tend to forget the intake during lunch because of the social consequences. The neurologists agreed that a drug intake of twice a day is most optimal as far as patient compliance is concerned 


\subsubsection{Gradual increase of the dose}

Dose related side-effects can be prevented through a gradual increase of the daily dose. The use of a gradual increase procedure is considered relevant for $\mathrm{CBZ}$. The half-life of $\mathrm{CBZ}$ when starting the intake is about 16 hours while long-term use of the drug decreases the half-life to 8 hours. Side-effects of each dosage can be assessed in a steady state which is reached after five times the half-life after the increase. Theoretically one can increase the dose on the fifth day. However, the neurologists prefer to increase the dose once a week. A more complicated intake scheme may hamper patient compliance. In case of $400 \mathrm{mg}$ the final dose will be reached in the second week, while with $600 \mathrm{mg}$ the final dose will be reached in the third week. The neurologists agreed that reaching an intake of 400 or $600 \mathrm{mg}$ within one week is not appropriate.

\subsection{Comparing the system and the neurologists with the consensus meeting reference}

Based on the guidelines obtained in the consensus meeting, a consensus treatment was established for cases from the diagnostic reference set. The prescriptions of the neurologists as well as the prescriptions of the system were compared with this consensus reference treatment. For the neurologists the number of prescriptions in accordance with the reference was established. The system was compared with the individual neurologists using the same set of cases.

\subsubsection{Agreement on the generic drug}

The group of neurologists provided 114 treatments. Five different drugs were used by the neurologists, whereas in the consensus reference only three drugs were used. The system used only two drugs for treatment (Table 5.7-1).

Anti-epileptic drug therapy was initiated 103 times by the neurologists. Eleven times drug treatment was not started. Seven times this was not according to the consensus meeting reference as this reference stated that drug treatment should be started when the diagnosis is established

Table 5.7-I:Agreement between the group of neurologists and the system with the reference developed during the consensus mecting.

\begin{tabular}{l|rrrrrrr|rrrrr}
\hline $\begin{array}{l}\text { Reference } \\
\text { Treatunent }\end{array}$ & \multicolumn{7}{|c|}{ Neurologists } & \multicolumn{5}{c}{ System } \\
\hline & No & CBZ & PB & PHIT & VF'A & OCI3 & Tolal & No & CBZ & PHT & VPA & Total \\
No & 4 & 0 & 0 & 0 & 0 & 0 & 4 & 2 & 0 & 0 & 0 & 2 \\
CBZ & 6 & 42 & 0 & 9 & 2 & 3 & 62 & 0 & 26 & 0 & 0 & 26 \\
PHT & 0 & 9 & 0 & 2 & 1 & 0 & 12 & 0 & 5 & 0 & 0 & 5 \\
VPA & 1 & 8 & 1 & 1 & 25 & 0 & 36 & 0 & 0 & 0 & 14 & 14 \\
\hline & 11 & 59 & 1 & 12 & 28 & 3 & 114 & 2 & 31 & 0 & 14 & 47 \\
\hline
\end{tabular}

In 73 of the 114 prescriptions (64\%) the selected drug of a neurologist was in accordance with the consensus reference. In two cases two neurologists decided that the type of epilepsy was unknown. In these cases both neurologists did not start drug treatment. 
The system suggested in 42 of the 47 cases (89\%) a drug according to the reference. In 2 cases drug treatment was not started because the type of epilepsy was not certain.

All neurologists prescribed a drug in accordance with the reference in more than half of the cases (range $52 \%$ to $72 \%$ ). The system compared with the individual neurologists suggested a drug according to the consensus consistently more often (range $88 \%$ to $91 \%$ )(Table $5.7-\Pi$ ).

The system suggested CBZ according to the reference consistently more often than the individual neurologist, while the proposal to use VPA was consistently at least as good as that of a neurologist. From the consensus meeting it was concluded that PHT should be used as well. This drug was not recommended by the system. Before the consensus meeting the neurologists disagreed more about the prescription of PHT than about the use of CBZ and VPA. Only twice (17\%) a neurologist used the drug in accordance with the reference, compared to $68 \%$ for $\mathrm{CBZ}$ and $69 \%$ for VPA.

Table 5.7-II: Number of cases for which the system and the individual neurologist recommended the same prescription as the reference.

\begin{tabular}{lllllllllll}
\hline Participant & III & Syst & IV & Syst & $V$ & Syst & V & Syst & VI & Syst \\
\hline Cases & $\mathrm{n}=23$ & $\mathrm{n}=23$ & $\mathrm{n}=25$ & $\mathrm{n}=25$ & $\mathrm{n}=21$ & $\mathrm{n}=21$ & $\mathrm{n}=22$ & $\mathrm{n}=22$ & $\mathrm{n}=23$ & $\mathrm{n}=23$ \\
Correct & 15 & 21 & 18 & 22 & 11 & 19 & 13 & 20 & 16 & 21 \\
Total \% & 65 & 91 & 72 & 88 & 52 & 91 & 59 & 91 & 70 & 91 \\
\hline
\end{tabular}

\subsubsection{Agreement on the daily dose}

For those cases in which the detailed diagnosis established by the neurologist was in agreement with the reference diagnosis and the prescribed drug and the reference drug were the same, the daily doses were compared. In Table 5.7-III prescribed daily doses are expressed as percentages of the reference dose. Since in the case of VPA a range was considered appropriate, doses between $100 \%$ (equivalent to $900 \mathrm{mg}$ ) and $111 \%$ (equivalent to $1000 \mathrm{mg}$ ) are appropriate. For $\mathrm{CBZ}$ the daily dose depends on the weight of the patient.

\section{Total set of prescriptions}

If the neurologists prescribe a drug according to the reference they achieve a better agreement with the reference daily dose than the system (57\% vs $37 \%$ of the prescriptions had the same dosage). When VPA is prescribed little difference between the neurologists and the system was observed $(88 \%$ and $94 \%$ of the prescriptions had the same daily dose as the reference, respectively). The prescribed daily dose of the neurologists ranged from $100 \%$ to $167 \%$ of the reference dose. The system suggested a daily dose ranging from $100 \%$ to $117 \%$ of the reference (Table 5.7-III).

When $\mathrm{CBZ}$ is prescribed - 42 and 62 times by the neurologists and the system respectively the system suggested only twice (3\%) a daily dose equal to that of the consensus, while in $36 \%$ of the prescriptions of the neurologists the dose was according to the reference. In $21 \%$ of the prescriptions the neurologists prescribed a lower daily dose (ranging from $50 \%$ to $75 \%$ of the reference dose), in $43 \%$ the neurologists prescribed a higher daily dose than the reference (ranging from $133 \%$ to $225 \%$ of the reference daily dose). The dosages suggested by the system were lower than the reference in $97 \%$ of the prescriptions (the daily dose ranged from $67 \%$ to $75 \%$ of the reference dose) 
Table 5.7-III: Variations in daily dose for those cases for which the consensus treatment and the detailed diagnosis are agreed upon by the participant.

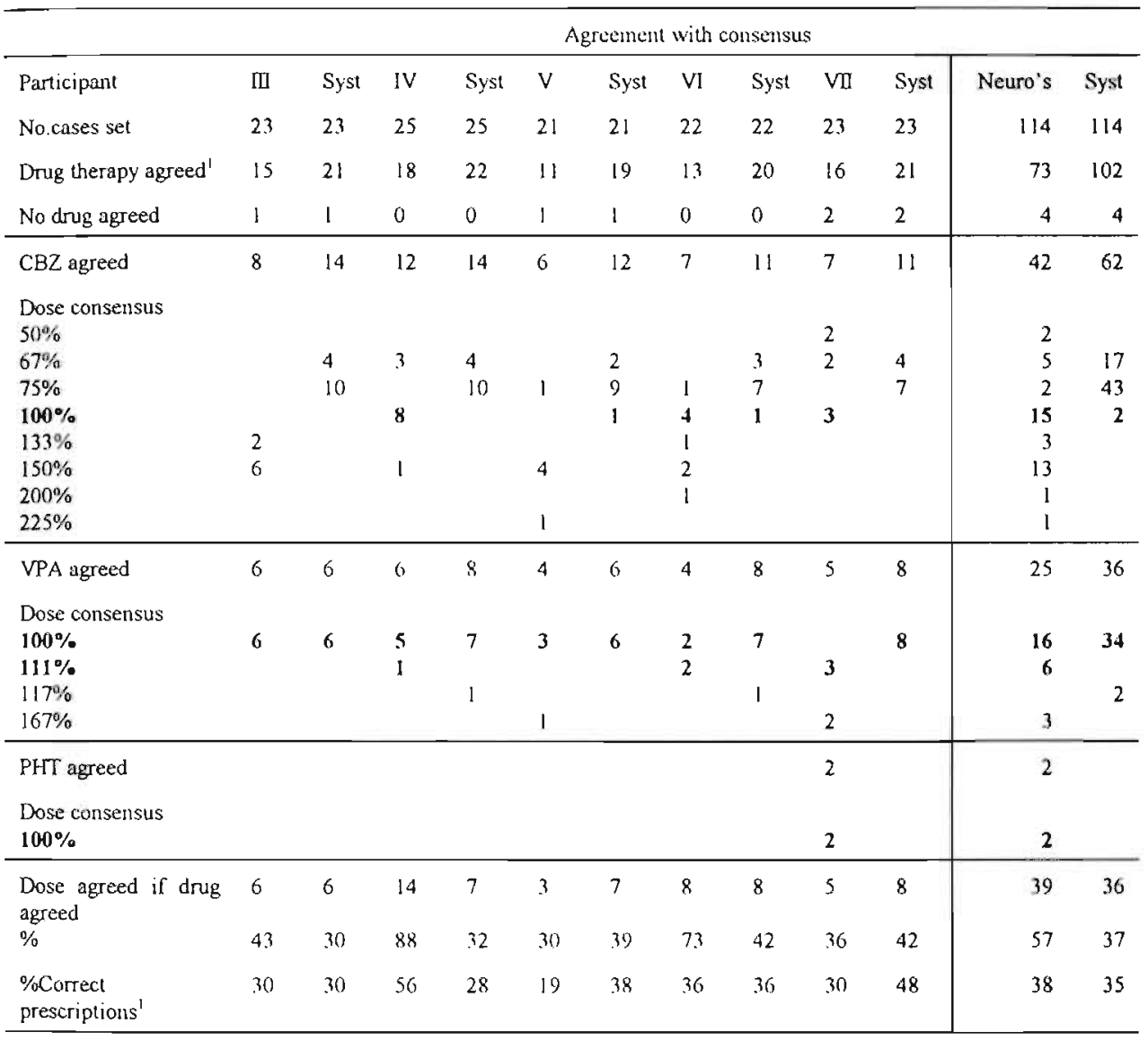

${ }^{1}$ This includes also the cuses for which the reference treatment was to provide no drug treatunent

Agreement in the sets of the individual nenrologist

In those cases in which the individual neurologist prescribes $\mathrm{CBZ}$ in agreement with the reference a daily dose according to the reference was suggested in 0 to $75 \%$ of the prescriptions. The system agreed in $10 \%$ or less for each of the sets seen by an individual neurologist. The system agreed with the reference in less cases than four of the neurologists.

Three neurologists prescribed VPA in a daily dose equal to the reference. Also the system prescribed VPA equal to the reference daily dose in three sets of the individual neurologist.

Agreement on drug and daily dose

All but one of the neurologists provided a prescription (drug and daily dose) according to the reference in less than $40 \%(19 \%$ to $36 \%)$. The other neurologist agreed with the reference in $56 \%$. The system provided a prescription according to the reference in $28 \%$ to $48 \%$ of the cases (for the various sets of cases common with the individual neurologists). Except for one 
of the neurologists the system consistently suggested an equal or higher number of prescriptions in accordance with the reference mainly because of a better agreement on VPA.

\subsection{References}

Chadwick D., Reynolds E.H., When do epileptic putients need treatment? Starting and stopping medication. British Medical Journal, 290. 1985, pp. 1885-1888.

Meinardi H., Epilepsie compendium, Katholicke Universiteit Nijmegen, 1993.

Porter R.J., Epilepsy, 100 elemenlary principles, W.B. Saunders Company Ltd., London, 1984.

Porter R.J. General principles how to use anti-epileptic drugs. In: Anti-epileptic drugs, $3^{\text {rd }}$ edition, (Levy, Mattson, Meldrum, Penry and F.E.Dreifuss, Eds.), Raven Press Ltd., New York, 1989, pp. 117-131.

Shorvon S.D., The trcatment of cpilepsy by drugs. In: Epilepsy. (Hopkins, Ed.), Chapman and Hall, London, 1987, pp. 229-282. 


\section{Results on follow-up of treatment}

\subsection{Introduction}

After a treatment is initiated its effectiveness is assessed during the next patient-doctor contacts; the follow-up. Based on this assessment a new therapy can be prescribed that may be different from the initial treatment. This chapter describes the results of our study concerning this second phase of anti-epileptic drug treatment.

First the treatment proposals provided by the neurologists based on 'paper cases' are compared with the advice provided by our system. The question to be answered is whether the treatments provided by the system are similar to the treatments provided by the neurologists. Several aspects of the treatment suggestions can be studied. We restrict ourselves to the global decisions to adjust the treatment: a continuation of the prescribed treatment ('Equal'), an adjustment of the daily dose of the drug ('Decrease' or 'Increase'), a switch to another antiepileptic drug ('Change') or adding an additional drug to the prescribed treatment ('Extra').

Since no objective standard exists for treatment of epilepsy we use the majority decision of a group of neurologists as the reference. To obtain insight in the variability of treatment decisions and to obtain information about possible alternatives without being dependent on the opinion of a single participant five neurologists were involved in defining a reference treatment for each case. Although consensus development by using a Delphi procedure has been considered, the time investment was deemed to be considerable. Therefore the majority decision among five neurologists was used for obtaining the reference treatment for each case. Comparing the correctness of the neurologists' treatment proposals with the system's output using this reference is disadvantageous for the system. Each of the neurologists has contributed to the reference and needs only to be in agreement with two other neurologists to have a correct proposal. The system needs to be in agreement with three neurologists to be correct. Therefore a second reference is defined by taking the majority of the treatment proposals of the system and the neurologists.

The adequateness of the system is evaluated in the following ways

- Comparison of the agreement between the system and neurologist( $s$ ). This analysis consists of two parts. Firstly the agreement between the system and the individual neurologists as well as among the individual neurologists was determined to establish the position of the system among the neurologists. In this study 265 cases (set 0) are used.

In the second analysis the prescriptions of each neurologist and the system are assessed by comparing them with prescriptions of the other four neurologists. Set 0 is used for this analysis. 
- Evaluation of the system and individual neurologists using the majority decision of the neurologists as the reference. This assessment provides information on how the neurologists and the system agree with a clinical reference. A reference was obtained in 219 of the 265 cases (set I).

- Evaluating the system and the neurologists using the majority decision of the neurologists and the system as the reference. In this study the set of 189 out of 265 cases for which such a reference was established was used (set II)

It is reasonable to assume that case characteristics determine which decision has to be taken. The fact that a majority decision is found in only 219 cases is, however, an indication that individual variation exists. In the second part of this chapter another type of analysis of the follow-ups is presented. Using a non parametric partitioning algorithm, a decision tree has been created based upon the case characteristics. In the ideal situation each terminal or endnode identifies cases of a certain (therapeutic) category. There may be several end nodes which point to the same category. The path from the root to the end-node contains the criteria to reach such an end-node. Four aspects of the derived tree are discussed

- The actual pureness of end-nodes in the decision tree

- The existence of cases with the same combination of patient characteristics that lead to an end-node, for which no reference treatment could be established

- The decision profiles within a impure end-node

- The decisions taken by the system for the cases of an end-node

Based upon this analysis we deduced a set of guidelines to be applied in the assessment of the initiated treatment that lead to specific treatment decisions.

\subsection{Design of test cases}

Like in our study on initiation of the drug treatment, paper cases were used. Information about the first follow-up after treatment initiation was added to the information in the paper cases of our previous study.

From patient records and interviews with neurologists it was determined which information is relevant for evaluating a patient who returns for an assessment of the prescribed treatment. Five characteristics were used to describe the course of the disease: the frequency of the seizures of various types, the compliance, the (kind of) complaints and the plasma drug level of the anti-epileptic drug. As in seizure severity scales the terminology (major and minor seizures) used for the seizure types is related to the severity of the seizures (Duncan and Sander 1991, Cramer 1993).

The following descriptors were used for these characteristics:

- The seizures (both major and minor) may be controlled (absent), decreased (the number of seizures decreased $\geq 50 \%$ ), equal (the number of seizures is unchanged, increased or decreased $<50 \%$ ) or not applicable (for a seizure type which was not present)

- There may be either a good compliance or non-compliance

- There can either be no complaints, dose related or idiosyncratic complaints

- The range of the plasma drug level can be either sub therapeutic, low therapeutic, therapeutic, high therapeutic or toxic 
In total 480 different combinations of these characteristics are possible.

We included - where relevant - other information related to the awareness of the patient, the occurrence of warnings, falling, incontinence, automatisms, convulsion, duration of the seizures and the duration before the patient is able to resume the activity he was pursuing before the seizure. The cause for non-compliance is presented where relevant. Furthermore, in case of complaints a description of the complaint together with information about the start and circumstances was presented. The prescription for the initiation of treatment was presented as well, including the brand of the medication, formulation, dose, number of tablets and number of intakes.

Apart from the detailed descriptors as discussed above a more global classification of the change in seizure frequency is used also (Table 6.2-I). In cases with several seizure types for which only one type of seizures was controlled or decreased in frequency, the reduction was still considered to be significant. Both case types were grouped into the class 'seizures decreased'.

Table 6.2-I: Overview of the seizure frequencies and the used terminology.

\begin{tabular}{|l|l|l|l|}
\hline Major seizures & Minor seizures & Litennediate & Global \\
\hline $\begin{array}{l}\text { equal } \\
\text { equal }\end{array}$ & $\begin{array}{l}\text { n.a. } \\
\text { equal } \\
\text { equal }\end{array}$ & uncontrolled & equal \\
\hline $\begin{array}{l}\text { decreased } \\
\text { n.a. } \\
\text { decreased } \\
\text { decreased } \\
\text { equal } \\
\text { equal } \\
\text { controlled }\end{array}$ & $\begin{array}{l}\text { n.a. } \\
\text { decreased } \\
\text { decreased } \\
\text { equal } \\
\text { decreased } \\
\text { controlled } \\
\text { equal }\end{array}$ & hurdly controlled & decreased \\
\cline { 1 - 2 } $\begin{array}{l}\text { controlled } \\
\text { decreased }\end{array}$ & $\begin{array}{l}\text { decreased } \\
\text { controlled }\end{array}$ & alinost controlled & \\
\hline $\begin{array}{l}\text { controlled } \\
\text { n.a. } \\
\text { controlled }\end{array}$ & $\begin{array}{l}\text { n.a. } \\
\text { controlled } \\
\text { controlled }\end{array}$ & controlled & absent \\
\hline
\end{tabular}

Of the 480 different case descriptions that can be constructed from the case characteristics, 270 follow-up contacts were actually used by:

- Reducing the number of cases with complete seizure control. Only few cases with complete seizure control were included as these cases usually do not entail difficult decisions

- Reducing the number of cases with only one type of seizures. Cases with two different types of seizures were considered of greater interest with regard to careful decision making

- Eliminating less realistic combinations of characteristics

\subsection{Description of the cases}

The 270 cases used in this study were derived from a subset of 43 case descriptions from the diagnostic test set of our study on the initiation of therapy. Each of these cases was the basis 
for one or more cases in the follow-up study. From 22 cases one to five different follow-up contacts were derived; 16 cases were the basis for six to ten different follow-ups. From five cases more than 10 follow-ups were created. The majority of the 43 cases had partial epilepsy. This selection was deliberate as this type of epilepsy is the most difficult to treat and has a larger variety of expressions of seizures (Elwes and Reynolds, 1990).

In 267 of the 270 cases all five neurologists provided a prescription. In the remaining three cases only four neurologists gave a prescription. In two of the 267 cases the system did not present a prescription. So 265 cases remained for comparison.

In this set of 265 cases 238 cases $(89.8 \%$ ) had a diagnosis of partial epilepsy, 12 cases (4.5\%) had idiopathic generalised epilepsy and 15 cases $(5.7 \%)$ had a combination of generalised and partial epilepsy. Three different drugs were prescribed in the 265 cases. CBZ was used in 219 cases $(82.6 \%)$, PHT was used in 36 cases (13.6\%) and Na-VPA was used in 10 cases $(3.8 \%)$.

Table 6.3-I: Distribution of the main characteristics of the paper cases used.

\begin{tabular}{|c|c|c|c|c|c|c|c|c|c|c|}
\hline \multirow{4}{*}{$\begin{array}{l}\text { Chancteristics } \\
\text { Compliance } \\
\text { Complaints } \\
\text { Seizures }\end{array}$} & \multicolumn{10}{|c|}{ Values and frequencies } \\
\hline & \multicolumn{4}{|c|}{ Compliant } & \multicolumn{4}{|c|}{ Non-compliance } & \multirow{2}{*}{\multicolumn{2}{|c|}{ Total }} \\
\hline & \multicolumn{2}{|c|}{ Absent } & \multicolumn{2}{|c|}{ Presient } & \multicolumn{2}{|c|}{ Absent } & \multicolumn{2}{|c|}{ Present } & & \\
\hline & $n$ & $\%$ & n & $\%$ & 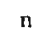 & $\%$ & $n$ & $\%$ & $\mathbf{n}$ & $\%$ \\
\hline controlled & 7 & 2.6 & 17 & 6.4 & 5 & 1.9 & II & 4.2 & 40 & 15.1 \\
\hline almost controlled & 8 & 3.0 & 15 & 5.7 & 6 & 2.3 & 13 & 4.9 & 42 & 15.8 \\
\hline hardly controlled & 20 & 7.6 & 39 & 14.7 & 22 & 8.3 & 34 & 12.8 & 115 & 43.4 \\
\hline uncontrolled & 11 & 4.2 & 18 & 6.8 & 22 & 8.3 & 17 & 6.4 & 68 & 25.7 \\
\hline Total & 46 & 17.4 & 89 & 33.6 & 55 & 20.8 & 75 & 28.3 & 265 & 100 \\
\hline
\end{tabular}

The set of cases is an enriched sample of what one would expect in daily clinical practice. Relatively more cases with (multiple) problems were included (e.g. cases in which seizures were not controlled by the initial treatment and/or cases with side-effects). In our set $95 \%$ of the cases had seizures which were not controlled (Table 6.3-I). This should be contrasted with a clinical setting where about one third of the patients may be controlled by the initial drug treatment (Elwes et al 1984, Beghi and Tognoni 1988,van Donselaar 1990, Collaborative Group for the Study of Epilepsy 1992). Hence it is to be expected that in our study the disagreement between neurologists concerning a specific treatment is larger than that found in daily practice.

\subsubsection{Description of reference set I}

In 219 of the 265 cases the majority of the five neurologists agreed about a specific global treatment decision. In Table 6.3-11 the distribution of the 219 cases with respect to the main characteristics is presented. Seizures were not completely controlled in the majority of these cases $(83.1 \%$ ). In more than half of the cases $(59.8 \%$ ) complaints were present. A combination of incompletely controlled seizures, presence of complaints and non-compliance is observed in $23.3 \%$ of the cases. 
Table 6.3-II: Overview of reference set I.

\begin{tabular}{|c|c|c|c|c|c|c|c|c|c|c|}
\hline \multirow{3}{*}{$\begin{array}{l}\text { Characteristics } \\
\text { Compliance } \\
\text { Complaints }\end{array}$} & \multicolumn{10}{|c|}{ Values and frequencies } \\
\hline & \multicolumn{4}{|c|}{ Compliant } & \multicolumn{4}{|c|}{ Non-compliant } & & \\
\hline & \multicolumn{2}{|c|}{ Absent } & \multicolumn{2}{|c|}{ Present } & \multicolumn{2}{|c|}{ Absent } & \multicolumn{2}{|c|}{ Present } & \multicolumn{2}{|c|}{ Total } \\
\hline Seizures & $\mathrm{n}$ & $\%$ & $\mathrm{n}$ & $\%$ & $\mathrm{n}$ & $\%$ & $\mathrm{n}$ & $\%$ & $\mathrm{n}$ & $\%$ \\
\hline controlled & 7 & 3.2 & 14 & 6.4 & 5 & 2.3 & 11 & 5.0 & 37 & 16.9 \\
\hline a most controlled & 8 & 3.7 & 11 & 5.0 & 5 & 2.3 & 7 & 3.2 & 31 & 14.2 \\
\hline hardly controlled & 16 & 7.3 & 28 & 12.8 & 19 & 8.7 & 27 & 12.3 & 90 & 41,1 \\
\hline uncontrolled & 10 & 4.6 & 16 & 7.3 & 18 & 8.2 & 17 & 7.8 & 61 & 27.9 \\
\hline Total & 41 & 18.7 & 69 & 31.5 & 47 & 21.5 & 62 & 28.3 & 219 & 100 \\
\hline
\end{tabular}

\section{Agreement profiles}

The degree of agreement among the neurologists may vary from case to case. The degree of agreement is indicated by means of agreement profiles. Each entry in the profile indicates the number of neurologists that agree about a treatment decision. For example a 3-2 profile indicates a majority of 3 neurologists while the other two neurologists disagreed with the majority but agreed among themselves.

Table 6.3-MI: Agreement profiles in the group of five neurologists.

\begin{tabular}{lrrrr|rrr}
\hline & \multicolumn{3}{c|}{ Reference set } & \multicolumn{3}{c}{ No reference } \\
\hline Profile & 5 & \multicolumn{1}{c}{$4-1$} & $3-2$ & $3-1-1$ & $2-2-1$ & $2-1-1-1$ & $1-1-1-1-1$ \\
Cases & 58 & 75 & 52 & 34 & 33 & 13 & 0 \\
$\%$ & 21.9 & 28.3 & 19.6 & 12.8 & 12.5 & 4.9 & 0 \\
\hline \multicolumn{3}{c}{ Total } & \multicolumn{3}{c}{$\mathrm{n}=219,82.6 \%$} & & \multicolumn{3}{c}{$\mathrm{n}=46.17 .4 \%$} \\
\hline
\end{tabular}

Table 6.3-IIl shows the distribution of the agreement profiles in the 265 cases. In 219 cases $(82.6 \%)$ a majority decision of the neurologists could be established. In 58 out of 265 cases all five neurologists completely agree on how to treat the patient.

\subsubsection{Description of reference set II}

In 189 of the 265 cases (71.3\%) a majority of at least four out of the six participants - the five neurologists and the system - agreed on the treatment decision. Table 6.3-IV presents the main characteristics of these 189 cases. When this set is compared with the set of 219 cases, minor changes are observed in the frequency of cases with seizures controlled and seizures uncontrolled. The system proposes an approach different from the majority of neurologists mainly in cases with.

- seizures almost controlled

- seizures hardly controlled and complaints

- seizures uncontrolled, complaints absent and non-compliance

- seizures controlled, complaints present and good compliance 
Table 6.3-IV: Overview of reference set II.

\begin{tabular}{|c|c|c|c|c|c|c|c|c|c|c|}
\hline \multirow{4}{*}{$\begin{array}{l}\text { Characteristics } \\
\text { Compliant } \\
\text { Complaints } \\
\text { Seizures }\end{array}$} & \multicolumn{10}{|c|}{ Values and frequencies } \\
\hline & \multicolumn{4}{|c|}{ Compliaut } & \multicolumn{6}{|c|}{ Non-compliant } \\
\hline & \multicolumn{2}{|c|}{ Absent } & \multicolumn{2}{|c|}{ Present } & \multicolumn{2}{|c|}{ Absent } & \multicolumn{2}{|c|}{ Present } & \multicolumn{2}{|c|}{ Total } \\
\hline & $\mathrm{n}$ & $\%$ & $\mathrm{n}$ & $\%$ & n & $\%$ & $\mathrm{n}$ & $\%$ & $\mathrm{n}$ & $\%$ \\
\hline controlled & 7 & 3.8 & 11 & 5.8 & 5 & 2.7 & 11 & 5.8 & 34 & 18.0 \\
\hline almost controlled & 5 & 2.7 & 10 & 5.3 & 5 & 2.7 & 5 & 2.7 & 25 & 13,2 \\
\hline hardly controlled & 15 & 7.9 & 22 & 11.6 & 17 & 9.0 & 21 & 11.1 & 75 & 39.7 \\
\hline uncontrolled & 9 & 4.8 & 16 & 8.5 & 13 & 6.9 & 17 & 9.0 & 55 & 29.1 \\
\hline Total & 36 & 19.1 & 59 & 31.2 & 40 & 21.2 & 54 & 28.6 & 189 & 100 \\
\hline
\end{tabular}

\section{Agreement profiles in reference cases}

The agreement profiles based on all six participants are shown in Table 6.3-V. Inclusion of the system in the judgement procedure yields reference cases in which either at least four neurologists or three neurologists and the system agree about the treatment approach.

Table 6.3-V: Comparison of the agreement in the group of five neurologists with that of the system and the group consisting only of five neurologists.

\begin{tabular}{|c|c|c|c|c|c|c|c|c|c|c|c|c|}
\hline \multirow{2}{*}{\multicolumn{2}{|c|}{$\begin{array}{l}\text { Agrecment among } \\
\text { five neurologists }\end{array}$}} & \multicolumn{11}{|c|}{ five neurologists and the system } \\
\hline & & \multicolumn{4}{|c|}{ Reference $\mathrm{N}=189.713 \%$} & \multicolumn{7}{|c|}{ No reference $\mathrm{N}=76,28.8 \%$} \\
\hline & Profile & 6 & $5-1$ & $4-2$ & $4-1-1$ & $3-3$ & $3-2-1$ & $3-1-1-1$ & $2-2-2$ & $2-2-1-1$ & $2-1-1-1-1$ & To \\
\hline Ref & 5 & 54 & 4 & & & & & & & & & 58 \\
\hline $\mathrm{N}=219$ & 4.1 & & 61 & 9 & 5 & & & & & & & 75 \\
\hline \multirow[t]{2}{*}{$82.6 \%$} & $3-2$ & & & 34 & & 16 & 2 & & & & & 52 \\
\hline & $3-1-1$ & & & & 22 & & 12 & & & & & 34 \\
\hline No ref & $2-2-1$ & & & & & & 26 & & 5 & 2 & & 37 \\
\hline $\begin{array}{l}N=46 \\
17.4 \%\end{array}$ & $2-1-1-1$ & & & & & & & 5 & & 7 & 1 & 13 \\
\hline \multicolumn{2}{|l|}{ Total } & 54 & 65 & 43 & 27 & 16 & 40 & 5 & 5 & 9 & 1 & 268 \\
\hline
\end{tabular}

In 54 of the 265 cases $(20.4 \%)$ the system completely agreed with the five neurologists (profile 6) In $\mathbf{3} 33$ cases the five neurologists had a 5 or 4 -1 agreement profile. When only the neurologists were used as a reference in 86 cases three out of five neurologists agreed about the treatment decision (profiles 3-2 and 3-1-1) Including the system resulted in the definition of a reference in 56 of these cases (profiles 4-2 and 4-1-1). In $16(13.5 \%)$ of the remaining 30 cases the system agreed with the minority of two neurologists. In 12 cases the system proposed a treatment decision equal to that of one of the neurologists.

In 14 of the 265 cases the system suggested a treatment different from any of the treatments suggested by the group of neurologists In nine of these cases there was a reference treatment (5-! and 4-I-! agreement profile), As a 3-3i agreement profile was not sufficient for a case to 
be incorporated as reference case, including the system could not cause cases not in set I to become part of set II. Where a reference had been based on a majority decision of three neurologists the system could either strengthen the agreement or refute it.

\subsubsection{Cases not included in the reference set II}

Reference set II contains 189 cases in which a majority decision was reached. In 76 cases no majority decision could be obtained. Table 6.3-VI gives an overview of the main characteristics of the cases for which a reference treatment could not be obtained. The results can be summarised as:

- In 51 of the 76 cases with no reference a complaint was present, which means that in $31.1 \%$ of the 164 cases in which a complaint was present a reference was not obtained In contrast, in 25 of the 101 cases $(24.8 \%$ ) without a complaint no reference was reached

- In 57 of the cases with no reference, seizures were hardly or almost completely controlled This means that in $36.3 \%$ of the 157 cases in which the anti-epileptic drug treatment seemed to have some but insufficient effect no majority decision could be achieved

- In 17 of the 42 cases $(40.5 \%)$ with seizures which were almost controlled a reference was not obtained.

- In 40 of 115 cases $(34.8 \%$ ) with seizures hardly controlled a reference could not be obtained.

- In 6 of the 40 cases $(15 \%)$ in which seizures were controlled a reference could not be defined. Also for less than one fitth of the cases $(19 \%)$ in which the seizure frequency remained equal to the previous visit a majority agreement could not be obtained

Table 6.3-VI: Description of the cases not included in the reference. In the column ' $\%$ ' the percentage of the total of 76 cases is presented. In the column $\%$ tor the percentage is related to the number of cases in the total test set with the same characteristics.

\begin{tabular}{|c|c|c|c|c|c|c|c|c|c|c|c|c|c|c|c|}
\hline \multirow{3}{*}{$\begin{array}{l}\text { Characteristic } \\
\text { Compliance } \\
\text { Complaints }\end{array}$} & \multicolumn{15}{|c|}{ Values and freyuencies } \\
\hline & \multicolumn{6}{|c|}{ Compliant } & \multicolumn{6}{|c|}{ Non-comp̣tiant } & \multirow{2}{*}{\multicolumn{3}{|c|}{ Total }} \\
\hline & & Ahsent & & & Frese: & & & Abser & & & Presen & & & & \\
\hline Seizures & n & $\%$ & $\% 10 t$ & $n$ & $\%$ & $\because$ tot & n & $\because$ & $\sim \operatorname{tot}$ & n & $\because$ & $*+104$ & $n$ & $\because$ & "stust \\
\hline centrolled & 0 & 0 & a & 6 & 7.9 & 35.1 & 0 & 0 & 0 & 4 & 0 & 0 & 6 & $7 \%$ & 150 \\
\hline almost cont. & 3 & 39 & 37.5 & 5 & 66 & 33.1 & 1 & 1.3 & 167 & $\mathrm{x}$ & 105 & 6.15 & 17 & $2: 4$ & 405 \\
\hline hardly con! & 5 & 6.6 & 25.0 & 17 & 22.4 & 436 & 9 & 66 & 227 & 13 & 171 & $1 \times 2$ & 40 & 826 & $34 x$ \\
\hline uncontr. & 2 & 2.6 & 18.2 & 2 & 2.6 & 111 & 91 & 118 & 409 & 0 & 4 & $\theta$ & 17 & 171 & 191 \\
\hline Totat & 10 & 132 & 217 & 30 & 395 & 337 & 15 & 197 & 273 & $2 !$ & 276 & $2 \times 0$ & 76 & 100 & 100 \\
\hline
\end{tabular}

Agreement profiles in non-reference cases

in $61(80.3 \%)$ of the 76 cases with no reference treatment three out of six participants agree about a specific treatment (profiles $3-3,3-2-1$ and $3-1-1-1$ ) (Table $63-\mathrm{V}$ )

The agreement of each participant with the other participants in these 76 cases is presented in Table 6.3-VII. 'Score' indicates the number of other participants which agree with the participant. 
Table 6.3-VII: Variability in agreement between participants in the 76 cases. The item 'Score' indicates the number of participants which agree with the decision taken by the neurologist or the system.

\begin{tabular}{lrrrrrrrrrrrrr}
\hline & \multicolumn{1}{c}{ NUI } & \multicolumn{3}{c}{ NIV } & \multicolumn{2}{c}{ NV } & \multicolumn{2}{c}{ NVI } & \multicolumn{2}{c}{ NVI } & \multicolumn{2}{c}{ System } \\
\hline Score & n & $\%$ & $\mathrm{n}$ & $\%$ & $\mathrm{n}$ & $\%$ & $\mathrm{n}$ & $\%$ & $\mathrm{n}$ & $\%$ & $\mathrm{n}$ & $\%$ \\
0 & 17 & 22.4 & 12 & 15.8 & 18 & 23.7 & 14 & 18.4 & 11 & 14.5 & 5 & 6.6 \\
1 & 27 & 35.5 & $3 !$ & 40.8 & 15 & 19.7 & 27 & 35.5 & 24 & 31.6 & 24 & 31.6 \\
2 & 32 & 42.1 & 33 & 43.4 & 43 & 56.6 & 35 & 46.1 & 41 & 54.0 & 47 & 61.8 \\
\hline
\end{tabular}

The percentage of cases for which a neurologist prescribed a treatment different from those prescribed by any of the other participants ranges from $14.5 \%$ to $23.7 \%$. The system provided an advice different from those of all neurologist's in only $6.6 \%$ of the cases.

Each of the neurologists agreed with two other participants in $42.1 \%$ to $56.6 \%$ of the cases. The system agreed with two neurologists in $61.8 \%$ of the cases.

\subsubsection{Relevance of characteristics for agreement among neurologists}

Various levels of agreement among the neurologists occur in the cases with a reference treatment (set l). The degree of agreement probably depends on the characteristics of the case. For each of the main features in the case descriptions we determined the level of agreement. The five out of five and four out of five agreement levels are considered to represent high degrees of agreement as changing the opinion of one of the neurologists will not affect the reference treatment.

\section{Seizure froyuency}

In $78 \%$ of the 37 cases in which seizures were completely controlled and in $65.6 \%$ of the 61 cases in which seizures remained equal to the previous visit there is a high degree of agreement on the reference treatment (Table 6.3-VIII). For the cases in which seizures were almost completely controlled a lower $(58.1 \%)$ percentage of cases with a high degree of agreement is observed.

Table 6.3-VII: Distribution of the agreement profiles for the frequencies of the type of seizures.

\begin{tabular}{|c|c|c|c|c|c|c|c|c|c|c|c|c|c|c|}
\hline Characteristic & \multicolumn{9}{|c|}{ Reference treatinent } & \multicolumn{5}{|c|}{ No reference treatmint } \\
\hline Profile & \multicolumn{2}{|c|}{5} & \multicolumn{2}{|c|}{$4-1$} & \multicolumn{2}{|c|}{$3-2$} & \multicolumn{2}{|c|}{$3-1-1$} & Tolal & \multicolumn{2}{|c|}{$2-2-1$} & \multirow{2}{*}{$\begin{array}{l}2-1-1-1 \\
N \%\end{array}$} & \multicolumn{2}{|c|}{ Total } \\
\hline Seizures: & $\mathrm{N}$ & $\%$ & $N$ & $\%$ & $N$ & $\%$ & $N$ & $\%$ & $N \%$ & $\mathrm{~N}$ & $\%$ & & $\mathrm{~N}$ & $\%$ \\
\hline controlled & 17 & 45.6 & 12 & 32.4 & 5 & 13.5 & 3 & 8.1 & 37100 & 3 & 100 & 00 & 3 & 100 \\
\hline almost contr & 8 & 25.8 & 10 & 32.3 & 4 & 12.9 & 9 & 29.0 & 31100 & 5 & 46 & 655 & 11 & 100 \\
\hline hardly contr & 19 & 21.1 & 27 & 30.0 & 32 & 35.6 & 12 & 13.3 & $90 \quad 100$ & 18 & 72 & 728 & 25 & 100 \\
\hline uncontr & 14 & 23.0 & 26 & 42.6 & 11 & 180 & 10 & 16.4 & 61100 & 7 & 100 & 0 & 7 & 100 \\
\hline Total & 58 & 26.5 & 75 & 34.3 & 52 & 23.7 & 34 & 15.5 & 219100 & 33 & 72 & 1328 & 46 & 100 \\
\hline
\end{tabular}


For cases that were hardly controlled the high degree of agreement decreases to $51.1 \%$. This drop in strong agreement can partially be explained by the fact that there seem to be two alternative treatments for certain cases. For example in $35.6 \%$ of the cases in which seizures are hardly controlled the two neurologists who propose a treatment different from the reference agree among themselves (the 3-2 agreement profile). This in contrast with a 3-2 agreement profile in at most $18 \%$ of the other types of seizure frequency.

\section{Compliance}

Compliance appears to have no effect on the degree of agreement among the neurologists (Table 6.3-IX). A high degree of agreement is found in $63.6 \%$ and $57.8 \%$ of the cases with a good compliance and non-compliance, respectively.

Table 6.3-IX: Dist ribution of the agreement profiles for the characteristic 'Compliance'.

\begin{tabular}{|c|c|c|c|c|c|c|c|c|}
\hline \multirow{3}{*}{$\begin{array}{l}\text { Characteristic } \\
\text { Profile } \\
\text { Compliance }\end{array}$} & \multicolumn{5}{|c|}{ Reference trealtnent } & \multicolumn{3}{|c|}{ No reference } \\
\hline & 5 & 4-1 & $3-2$ & $3-1-1$ & Total & $2-2-1$ & $2-1-1-1$ & Total \\
\hline & $N \%$ & $\mathrm{~N} \%$ & $\mathrm{~N} \%$ & $\mathrm{~N} \%$ & $N \quad \%$ & $\mathrm{~N} \%$ & $\mathrm{~N} \%$ & $\mathrm{~N} \%$ \\
\hline $\operatorname{good}$ & $35 \quad 31.8$ & 3531.8 & 2220.0 & $18 \quad 16.4$ & $110 \quad 100$ & 1872 & 728 & 25100 \\
\hline non-compliance & 2321.1 & $40 \quad 36.7$ & 3027.5 & $16 \quad 14.7$ & $109 \quad 100$ & 1571 & 29 & 21100 \\
\hline Total & $58 \quad 26.5$ & $\begin{array}{ll}75 & 34.3\end{array}$ & $52 \quad 23.7$ & $34 \quad 15.5$ & $219 \quad 100$ & 3372 & 1328 & 46100 \\
\hline
\end{tabular}

\section{Complaints}

In $63.7 \%$ of the cases where complaints are absent more than three neurologists agree on the treatment (Table 6.3-X). For cases in which idiosyncratic complaints are present strong agreement occurs in $83.1 \%$ of the cases.

Contrary to this, strong agreement is present in only $38.9 \%$ for cases with dosc related complaints. In $44.4 \%$ of the cases with dose related complaints, there is a $3-2$ agreement pattern, indicating that in these circumstances two alternative treatments may exist.

Table 6.3-X: Distribution of the agreement profiles for the characteristic 'Complaints'.

\begin{tabular}{|c|c|c|c|c|c|c|c|c|c|c|c|c|}
\hline \multirow{3}{*}{$\begin{array}{l}\text { Characteristic } \\
\text { Profile } \\
\text { Complaints }\end{array}$} & \multicolumn{9}{|c|}{ Reference treatınent } & \multicolumn{3}{|c|}{ No reference } \\
\hline & \multicolumn{2}{|r|}{5} & \multicolumn{2}{|c|}{$4-1$} & \multicolumn{2}{|c|}{$3-2$} & \multicolumn{2}{|c|}{$3-1-1$} & Total & $2-2-1$ & $2-1-1-1$ & \multirow{2}{*}{$\begin{array}{l}\text { Total } \\
\text { N } \%\end{array}$} \\
\hline & $N$ & $\%$ & $N$ & $\%$ & $\mathrm{~N}$ & $\%$ & $N$ & $\%$ & $N \%$ & $\mathrm{~N} \%$ & $\mathrm{~N} \%$ & \\
\hline ahsent & 24 & 27.3 & 32 & 36.4 & 17 & 19.3 & 15 & 17.1 & $88 \mathrm{l}(n)$ & 1077 & 323 & 13100 \\
\hline dose related & 6 & 8.3 & 22 & 30.6 & 32 & 44.4 & 12 & 16.6 & 72100 & 2067 & 1033 & 30100 \\
\hline idiosyncratic & 28 & 47.5 & 21 & 35.6 & 3 & 5.1 & 7 & 11.9 & $59 \quad 100$ & 3100 & 00 & 3100 \\
\hline Total & 58 & 26.5 & 75 & 34.3 & 52 & 23.7 & 34 & 15.5 & 219100 & 3372 & 1328 & 46. 100 \\
\hline
\end{tabular}

\section{Plasma drug level}

'Plasma drug level' hardly influences the degree of agreement. Strong agreement is found in more than $55 \%$ of the cases for all plasma drug levels except for levels in the toxic range. Even in the latter strong agreement was found in $50 \%$ of the cases (Table 6.3-XI). 
Table 6.3-XI: Distribution of the agreement profiles for the characteristic 'Plasma drug level'.

\begin{tabular}{|c|c|c|c|c|c|c|c|c|c|c|c|c|}
\hline \multirow{3}{*}{$\begin{array}{l}\text { Characteristic } \\
\text { Profile } \\
\text { Plasma drug level }\end{array}$} & \multicolumn{9}{|c|}{ Reference treatment } & \multicolumn{3}{|c|}{ No reference } \\
\hline & \multicolumn{2}{|c|}{$s$} & \multicolumn{2}{|c|}{$4-1$} & \multicolumn{2}{|c|}{$3-2$} & \multicolumn{2}{|c|}{$3-1-1$} & Total & $2-2-1$ & $2-1-1-1$ & Tola] \\
\hline & $\mathrm{N}$ & $\%$ & $N$ & $\%$ & $\mathrm{~N}$ & $\%$ & $\mathrm{~N}$ & $\%$ & $\mathrm{~N} \%$ & $\mathrm{~N} \%$ & $\mathrm{~N} \%$ & N \% \\
\hline sub ther & 15 & 33.3 & 14 & 31.1 & 12 & 26.7 & 4 & 8.9 & $45 \quad 100$ & 571 & 229 & $7 \quad 100$ \\
\hline low ther & 16 & 36.4 & 14 & 31.8 & 9 & 20.5 & 5 & 11.4 & $44 \quad 100$ & 6100 & 00 & 6100 \\
\hline ther & 11 & 20.8 & 21 & 39.6 & 12 & 22.6 & 9 & 17.0 & $53 \quad 100$ & 770 & 330 & $10 \quad 100$ \\
\hline high ther & 9 & 20.0 & 17 & 37.8 & 9 & 20.0 & 10 & 22.2 & $45 \quad 100$ & 975 & 325 & 12100 \\
\hline toxic & 7 & 21.9 & 9 & 28.1 & 10 & 31.3 & 6 & 18.8 & $32 \quad 100$ & 655 & 546 & 11100 \\
\hline Total & 58 & 26.5 & 75 & 34.3 & 52 & 23.7 & 34 & 15.5 & 219100 & 3372 & 1328 & $46 \quad 100$ \\
\hline
\end{tabular}

\subsubsection{Alternative treatments}

The previous section showed that in more than one third of the cases with hardly controlled seizures as well as in cases with dose related complaints a 3-2 agreement profile was frequently observed. For these two characteristics the alternative treatment is discussed.

\section{Seizure frequency}

In the 32 cases with hardly controlled seizures two neurologists propose an alternative treatment (see Table 6.3-XII). Change of drug is the most common alternative proposal for the reference treatments

Table 6.3-XII: Distribution of the reference treatments and the alternatives for the 32 cases in which a 3-2 agreement pattern was observed.

\begin{tabular}{|c|c|c|c|c|c|c|}
\hline \multirow[t]{2}{*}{ Treatment } & & \multicolumn{5}{|c|}{ Reference treatment } \\
\hline & Decision & Decrease & Equal & Increase & Change & Extra \\
\hline \multirow{4}{*}{$\begin{array}{l}\text { Altenative } \\
\text { tratunent }\end{array}$} & Decrease & & & & d & \\
\hline & Equal & 2 & & & 4 & \\
\hline & Increase & & & & 1 & \\
\hline & Change & 12 & 6 & 1 & & 2 \\
\hline
\end{tabular}

\section{Dose related complaints}

In 32 cases with dose related complaints (Table 6.3-XIII) in most cases (75\%) a change of drug was either the reference treatment or the alternative treatment. With dose related complaints in the history in all cases (9/9) the alternative treatment suggested was a change of drug when the reference decision was to decrease the dose, and also a change of drug in $7 / 12$ cases where the reference was to keep the medication constant. In five cases with dose related complaints where the reference was to continue the treatment, presumably because of seizure related reasons, the alternative was an increase of the dose.

A decision by either the reference or the alternative treatment to increase the dose was rarely observed in combination with an advice to change the drug (3/10) 
Table 6.3-XII: Distribution of the reference treatments and the altematives for the 32 cases with dose related complaints in which a 3-2 agreement pattern was observed.

\begin{tabular}{|c|c|c|c|c|c|c|}
\hline Treatment & & \multicolumn{5}{|c|}{ Reference treatment } \\
\hline \multirow{5}{*}{$\begin{array}{l}\text { Altemative } \\
\text { treatment }\end{array}$} & Decision & Decrease & Equal & Increase & Change & Extra \\
\hline & Decrease & & & & 1 & \\
\hline & Equal & & & 2 & 4 & \\
\hline & Increase & & 5 & & 2 & \\
\hline & Change & 9 & 7 & 1 & & 1 \\
\hline
\end{tabular}

\subsection{Agreement and correctness}

Two of the metrics mainly used when evaluating a DSS are agreement and correctness. The term agreement is used when comparing the advice of the system with that of a neurologist or a group of neurologists. Correctness is used when assessing the advice of the system using a previously defined reference.

\subsubsection{Overview of the decisions in the total set}

Eight different decisions were taken by the neurologists. The decisions to continue the prescribed treatment are grouped into the class 'Equal'. If the daily dose was decreased or increased the decisions are labelled as 'Decrease' and 'Increase'. The class 'Change' contains decisions in which a different anti-epileptic drug was selected for further treatment. This class does not include the addition of a second anti-epileptic drug to the prescribed treatment. The class 'Stop' contains the decision to discontinue the treatment immediately. The class 'Other' contains decisions which differed from adjustment of the treatment, e.g. a referral. Finally, two

Table 6.4-I: Overview of the frequencies of decisions for further treatment of the patient.

\begin{tabular}{|c|c|c|c|c|c|c|c|c|c|c|c|c|c|c|}
\hline \multirow[t]{2}{*}{ Decision } & \multicolumn{2}{|c|}{ Decruase } & \multicolumn{2}{|c|}{ Equal } & \multicolumn{2}{|c|}{ Increase } & \multicolumn{2}{|c|}{ Change } & \multicolumn{2}{|c|}{ Extra } & \multicolumn{2}{|c|}{ Stop } & \multicolumn{2}{|c|}{ Other } \\
\hline & $\mathrm{n}$ & $\%$ & $n$ & $\%$ & $n$ & $\%$ & $\mathrm{n}$ & $\%$ & $n$ & $\%$ & $\mathrm{n}$ & $\%$ & $\mathrm{n}$ & $\%$ \\
\hline III & 50 & 18.9 & 67 & 25.3 & 39 & 14.7 & 96 & 36.2 & 13 & 4.9 & 0 & 0 & 0 & 0 \\
\hline IV & 37 & 14.0 & 113 & 42.6 & 56 & 21.1 & 47 & 17.7 & 9 & 3.4 & 2 & 0.8 & I & 0.4 \\
\hline V & 13 & 4.9 & 30 & 11.3 & 52 & 19.6 & 154 & 58.1 & 14 & 5.3 & 2 & 0.8 & 0 & 0 \\
\hline $\mathrm{VI}$ & 31 & 11.7 & 62 & 23.4 & 65 & 24.5 & 107 & 40.4 & 0 & 0 & 0 & 0 & 0 & 0 \\
\hline VII & 2 & 0.8 & 91 & 34.3 & 34 & 12.8 & 1.35 & 50.9 & 2 & 0.8 & 1 & 0.4 & 0 & 0 \\
\hline System & 11 & 4.2 & 54 & 20.4 & 62 & 2.3 .4 & 136 & 51.3 & 0 & 0 & 2 & 0.8 & 0 & 0 \\
\hline
\end{tabular}

decisions were observed in combination with adding an additional drug to the existing treatment ('Extra'); a continuation of the existing treatment or an alteration of the existing daily dose. Table 6.4-I presents the distribution of the decisions of each of the five neurologists and the system on the 265 cases (in the table the eight types of decisions have been reduced to seven by combining 21 decisions in which an anti-epileptic drug was added to 
the existing treatment and 11 decisions in which both an anti-epileptic drug was added and the original dose was altered into the category 'Extra')

\subsubsection{Differences in treatment approaches}

There are clear differences in the approaches taken by the neurologists. Neurologist IV is the one that most often sticks to the initiated treatment. In $42.6 \%$ of the cases he does not make any adjustment in the dose or the drug. In only $17.7 \%$ he decides to change the treatment. This is in contrast with Neurologist $V$ who predominantly changes the therapy $(58.1 \%$ of the cases) and keeps the drug dose as it was in only a small fraction of the cases.

Also the fraction of cases in which the decision was taken to adjust the dose of the drug shows large variations. While Neurologist III decides to decrease the drug dose in almost one fifth of the cases, Neurologist VII decides to decrease the drug dose in less than $1 \%$.

An increase of the drug dose was decided upon by Neurologist VI in almost a quarter of the cases which is almost twice as often as Neurologist VII.

Adding a second anti-epileptic drug to the existing drug treatment or the immediate stopping of the prescribed drug are not very common decisions. All neurologists decide to switch to polytherapy in less than $6 \%$ of the cases, while the decision to stop the drug is taken by all neurologists in less than $1 \%$ of the cases.

On average, in $25 \%$ of the cases the same treatment was suggested, while in approximately $40 \%$ of the cases the drug was changed. These numbers clearly show that the cases are heavily biased towards cases that are more difficult to treat.

The system proposed to change the drug in more than $50 \%$ of the cases. In less than $5 \%$ of the cases a decrease of the dose of the drug was suggested, while an increase was suggested in almost one fourth of the cases. In $20 \%$ of the cases a continuation of the prescribed treatment was suggested.

Table 6.4-II: $k$-values for the individual neurologists and the system on the total set of 265 cases.

\begin{tabular}{lccccc}
\hline & Neuro II & Neuro IV & Neuro V & Neuro VI & Neuro VI \\
\hline Neuro IV & 0.42 & & & & \\
Neuro V & 0.40 & 0.28 & & & \\
Neuro VI & 0.40 & 0.41 & 0.44 & & \\
Neuro VII & 0.35 & 0.26 & 0.35 & 0.29 & \\
System & 0.42 & 0.35 & 0.56 & 0.46 & 0.40 \\
\hline
\end{tabular}

The agreement beyond what is to be expected by chance alone between two observers can be expressed with the kappa coefficient. $\kappa$-values between 0.21 and 0.40 are considered to indicate fair agreement, those ranging from 0.41 up to 0.60 are considered to denote moderate agreement, while the values between 0.61 and 0.80 and those larger than 0.81 indicate substantial and almost perfect agreement, respectively (Landis and Koch, 1977). 
The $\kappa$-values for the pair wise comparisons of the decisions of neurologists range from 0.26 to 0.44 , while the systen when compared with the individual neurologists has $\mathrm{K}$-values between 0.35 and 0.56 (Table 6.4-II). The agreement of the system with a neurologist is at least as good as the agreement of that neurologist with any of the other neurologists, except for Neurologist IV.

\subsubsection{Agreement between an individual and a group}

The agreement of each individual neurologist with the four remaining neurologists is compared with the agreement of the system with the same group of neurologists. The number of neurologists that took the same decision as the individual neurologist or the system is counted for each case. In Table 6.4-III the distribution of the degree of agreement for each of the neurologists and the system with the other four neurologists is shown for the 265 cases.

The system achieved a significantly higher mean score than all the individual neurologists (paired student t-test, $\mathrm{p}<0.05$ )

Table 6.4-1U: Overview of the scores comparing the decision concerning 265 cases of an individual neurologist with the remaining neurologists. The number of neurologists which agree with the decision for treatment of the individual neurologist is represented by the score.

\begin{tabular}{|c|c|c|c|c|c|c|c|c|c|c|c|c|}
\hline \multirow[b]{2}{*}{ Score } & \multicolumn{2}{|c|}{ N III } & \multicolumn{2}{|c|}{ System } & \multicolumn{2}{|c|}{ NIV } & \multicolumn{2}{|c|}{ System } & \multicolumn{2}{|c|}{$\mathrm{NV}$} & \multicolumn{2}{|c|}{ System } \\
\hline & n & $\%$ & $\mathrm{n}$ & $\%$ & 11 & $\%$ & $\mathrm{n}$ & $\%$ & $n$ & $\%$ & $\mathrm{n}$ & $\%$ \\
\hline 0 & 38 & 14.3 & 18 & 6.8 & 52 & 19.6 & 18 & 6.8 & 43 & 16.2 & 23 & 8.7 \\
\hline 1 & 53 & 20.0 & 42 & 15.8 & 66 & 24.9 & 43 & 16.2 & 42 & 15.8 & 53 & 20.0 \\
\hline 2 & 47 & 17.7 & 62 & 23.4 & 41 & 15.5 & 55 & 20.8 & 60 & 22.6 & 64 & 24.2 \\
\hline 3 & 69 & 26.0 & 84 & 31.7 & 48 & 18.1 & 72 & 27.2 & 62 & 23.4 & 63 & 23.8 \\
\hline 4 & 58 & 21.9 & 59 & 22.3 & 58 & 21.9 & 77 & 29.1 & 58 & 21.9 & 62 & 23.4 \\
\hline \multicolumn{5}{|c|}{$t=3.22 p<0.0005$} & \multicolumn{4}{|c|}{$t=5.96 p<0.0005$} & \multicolumn{4}{|c|}{$t=1.94 p<0.05$} \\
\hline
\end{tabular}

\begin{tabular}{lcccccccc}
\hline & \multicolumn{2}{c}{ Neuro VI } & \multicolumn{2}{c}{ System } & \multicolumn{2}{c}{ Ncuro VII } & \multicolumn{2}{c}{ Systen } \\
\hline Score & $\mathrm{n}$ & $\%$ & $\mathrm{n}$ & $\%$ & $\mathrm{n}$ & $\%$ & $\mathrm{n}$ & $\%$ \\
0 & 37 & 14.0 & 22 & 8.3 & 45 & 17.0 & 22 & 8.3 \\
$\mathrm{I}$ & 47 & 17.7 & 40 & 15.1 & 54 & 20.4 & 48 & 18.1 \\
2 & 55 & 20.8 & 67 & 25.3 & 55 & 20.8 & 61 & 23.0 \\
3 & 68 & 25.7 & 76 & 28.7 & 53 & 20.0 & 61 & 23.0 \\
4 & 58 & 21.9 & 60 & 22.6 & 58 & 21.9 & 73 & 27.5 \\
& & $\mathrm{t}=2.37 \mathrm{p}<0.025$ & & & $1=3.85$ & $0<0.0005$ \\
\hline
\end{tabular}

\section{Complete agreement}

Total agreement among the five neurologists occurs in $21.9 \%$ of the cases. The system takes more decisions which agree with any group of 4 neurologists. In $22.3 \%$ to $29.1 \%$ of the cases, groups of four neurologists agree with the system. The system agrees significantly more with the remaining neurologists than Neurologists IV and VII (McNemar $\chi^{2}=12 p<0.05$, McNemar $\chi^{2}=8.52 \mathrm{p}<0.05$, respectively) 


\section{Majority}

A majority decision was reached by the neurologists in 219 of the 265 cases $(82.6 \%)$. For each neurologist the number of cases in which he agrees with two or more of his colleagues is counted. Also for the system the number of cases in which the system reaches a majority decision with each group of the four remaining neurologists is counted. Each individual neurologist participated in majority agreements in $55.5 \%$ to $68.3 \%$ of the cases, as compared to $71.3 \%$ to $77.4 \%$ when the system replaces the neurologist.

The system achieved a majority agreement (system + two neurologists) significantly more often than four of the individual neurologists (McNemar $\chi^{2}=10.84 p<0.05 ; \chi^{2}=30.45 p<0.05$; $\chi^{2=1.26} \mathrm{NS} ; \chi^{2}=5.96 \mathrm{p}<0.05 ; \chi^{2}=10.74 \mathrm{p}<0.05 ; \mathrm{df}=1$, for Neurologists III to VII, respectively).

\section{Complete disagreement}

A neurologist may take a decision which is not made by any of his colleagues. This occurs in $14.0 \%$ to $19.6 \%$ of the cases for the individual neurologists, whereas for the system this percentage varies between $6.8 \%$ to $8.7 \%$ of the cases. The system had significantly fewer cases with which all neurologists disagreed than each of the neurologists (McNemar $\chi^{2}=8.21$ $p<0.05, \chi^{2}=18.78 p<0.05, \chi^{2}=8.21 p<0.05, \chi^{2}=4.78 p<0.05, \chi^{2}=10.76 p<0.05$ for neurologists III to VII, respectively).

Table 6.4-IV: Agreement of a particular ncurologist and the system with the remaining neurologists, expressed as a kappa value.

\begin{tabular}{lllllllllllll}
\hline \multicolumn{10}{c}{ Assessment of individual neurologist and system by } \\
\hline Group & $4-5-6-7$ & \multicolumn{3}{c}{$3-5-6-7$} & \multicolumn{3}{c}{$3-4-6-7$} & \multicolumn{3}{c}{$3-4-5-7$} & \multicolumn{3}{c}{$3-4-5-6$} \\
Prescriber & N III & Syst & N IV & Syst & N V & Syst & N VI & Syst & N VII & Syst \\
Kappa & 0.39 & 0.44 & 0.34 & 0.46 & 0.36 & 0.43 & 0.39 & 0.46 & 0.35 & 0.47 \\
\hline
\end{tabular}

Agreement beyond chance

The agreement between a particular neurologist and the remaining group of four colleagues can also be expressed by a $\kappa$-value (Schouten, 1985). The $\kappa$-values range from 0.34 to 0.39 (Table 6.3-IV). The agreement of the system with the groups of neurologists ranges from 0.43 to 0.47 and is in all cases higher than the kappa of each individual neurologist.

\subsubsection{Correctness}

In the previous paragraphs the advice of the system was compared with the decisions of individual neurologists. This gave an indication about the capability of the system to make decisions similar to those made by individual neurologists. The correctmess of the decisions of the neurologist and of the system has been assessed using both reference sets.

For set I - the reference based on the neurologists alone - the correctness of the system is somewhat higher than the average correctness of the neurologists $(77.4 \%)$. However, three neurologists score better than the system (Table 6.4-V)

Including the system when establishing the reference increased the correctness for all participants. The system provided a correct advice more often than each individual neurolo- 
gist. This was significantly better for three of the five neurologists $\left(\mathrm{McNemar} \chi^{2}=4.5 \mathrm{p}<0.025\right.$; $\chi^{2}=24.85 \mathrm{p}<0.0005 ; \chi^{2}=0.52 \mathrm{NS} ; \chi^{2}=1.84 \mathrm{NS} ; \chi^{2}=9.13 \mathrm{p}<0.005 ; \mathrm{df}=1$, for neurologists III to VII respectively).

Table 6.4-V: Correctness with the reference of set I (i.e. majority of five neurologists) and set II (i.e. majority of five neurologists and the system).

\begin{tabular}{l|lcccccc}
\hline Set & Participants & N III & N IV & N V & N VI & N VI & System \\
\hline Set I, 3/5 neur. & Cases & 174 & 147 & 180 & 181 & 166 & 171 \\
N=219 & Correciness & 79.5 & 67.1 & 82.2 & 82.6 & 75.8 & 78.1 \\
\hline Set II, 4/6 neur.-syst. & Cases & 155 & 128 & 166 & 161 & 148 & 171 \\
N=189 & Correctness & 82.0 & 67.7 & 87.8 & 85.2 & 78.3 & 90.5 \\
\hline
\end{tabular}

\subsubsection{Variability in agreement in set II}

Since the reference treatment procedure is based on a majority, individual participants may disagree with the reference. In this paragraph the degree of agreement of the individual participants with his colleagues is discussed by.

- Comparing the mean of the scores obtained by each individual participant

- Addressing for each participant the disagreement with the reference treatment

- Studying the complete disagreement of each participant with the rest of the participants Furthermore, for each reference treatment the decisions taken by the group of neurologists and the system are presented.

\section{Overall score}

For the reference cases Table 6.4-VI provides a break-down of the number of participants that agree with a specific neurologist or the system. 'Score' indicates the number of participants a neurologist or the system agrees with. A score of 2 cannot be obtained because the reference contains only the agreement profiles $6,5-1,4-2$ and $4-1-1$. The system achieved a significantly higher score than each individual neurologist does (paired T-test $t=10.68 p<0.05, t=19.62$ $\mathrm{p}<0.05, \mathrm{t}=9.79 \mathrm{p}<0.05, \mathrm{t}=10.11 \mathrm{p}<0.05, \mathrm{t}=15.18 \mathrm{p}<0.05)$.

\section{Disagreement with the reference}

As the reference is defined by at least four participants a score less than 3 indicates disagreement of the participant with the reference. This is observed in 18 cases $(9.5 \%)$ for the system up to 61 cases $(32.3 \%)$ for neurologist IV. The agreement of the system with more than two other participants is better than that of three of the individual neurologists. 
Table 6.4-VI: Variability in the degree of agreement between participants for set II $(n=189)$. The item 'Score' indicates the number of participants which agree with the decision taken by the neurologist or the system.

\begin{tabular}{lrrrrrrrrr}
\hline & \multicolumn{3}{c}{ Neurologist III } & \multicolumn{3}{c}{ Neurologist IV } & \multicolumn{3}{c}{ Neurologist V } \\
\hline Score & $\mathrm{n}$ & $\%$ & cum & $\mathrm{n}$ & $\%$ & cum & $\mathrm{n}$ & $\%$ & cum \\
0 & 17 & 9.0 & 9.0 & 36 & 19.1 & 19.1 & 16 & 8.5 & 8.5 \\
1 & 17 & 9.0 & 18.0 & 25 & 13.2 & 32.3 & 7 & 3.7 & 12.2 \\
3 & 41 & 21.7 & 39.7 & 32 & 16.9 & 49.2 & 55 & 29.1 & 41.3 \\
4 & 60 & 31.7 & 71.4 & 42 & 22.2 & 71.4 & 57 & 30.2 & 71.4 \\
5 & 54 & 28.6 & 100 & 54 & 28.6 & 100 & 54 & 28.6 & 100 \\
Total & 189 & 100 & & 189 & 100 & & 189 & 100 & \\
mean & 3.44 & & & $2.9 \%$ & & & 3.55 & & \\
\hline
\end{tabular}

\begin{tabular}{lrrrrrrrrr}
\hline & \multicolumn{3}{c}{ Neurologist VI } & \multicolumn{4}{c}{ Neurologist VII } & \multicolumn{3}{c}{ System } \\
\hline Score & $\mathrm{n}$ & $\%$ & cum & $\mathrm{n}$ & $\%$ & cum & $\mathrm{n}$ & $\%$ & cum \\
0 & 15 & 7.9 & 7.9 & 26 & 13.8 & 13.8 & 9 & 4.8 & 4.8 \\
$\mathrm{l}$ & 13 & 6.9 & 14.8 & 15 & 7.9 & 21.7 & 9 & 4.8 & 9.5 \\
3 & 48 & 25.4 & 40.2 & 48 & 25.4 & 47.1 & 56 & 29.6 & 39.2 \\
4 & 59 & 31.2 & 71.4 & 46 & 24.3 & 71.4 & 61 & 32.3 & 71.4 \\
5 & 54 & 28.6 & 100 & 54 & 28.6 & 100 & 54 & 28.6 & 100 \\
Total & 189 & 100 & & 189 & 100 & & 189 & 100 & \\
mean & 3.51 & & & 3.24 & & & 3.65 & & \\
\hline
\end{tabular}

\section{('omplete disagreement with the other participants}

The number of cases for which a neurologist disagreed with all other participants (Score 0) ranges from $15(7.9 \%)$ to 36 cases $(19.1 \%)$. In nine cases $(4.8 \%)$ the system proposes a treatment different from any proposal of the neurologists

Table 6.4-VII also gives an overview of the agreement profiles for those cases where the individual participant disagreed completely with the rest of the group. The percentage of cases in which a participant disagreed while all other participants agreed about the treatment decision ranged from $2.7 \%$ to $12.2 \%$ of the cases (three neurologists caused a $5-1$ agreement profile in less than $5 \%$, two neurologists in more than $10 \%$ of the cases). In four cases (2.1\%) the system did not agree with the treatment procedure although all five neurologists did agree completely. Each neurologist as well as the system disagreed completely with the five other participants of which a group of four agreed among themselves (agreement profile 4-1-1) in less than $10 \%$ of the cases 
Table 6.4-VII: Distribution of cases for which the individual participant disagreed completely with the other five participants in cases from set II.

\begin{tabular}{lrrrrrrrrrrrr}
\hline Participant & \multicolumn{2}{c}{$\mathrm{N} \mathrm{WI}$} & \multicolumn{2}{c}{$\mathrm{N} \mathrm{IV}$} & \multicolumn{2}{c}{$\mathrm{N} \mathrm{V}$} & \multicolumn{2}{c}{$\mathrm{N} \mathrm{VI}$} & \multicolumn{2}{c}{$\mathrm{N} \mathrm{VM}$} & \multicolumn{2}{c}{ System } \\
\hline Agreement profile & $\mathrm{n}$ & $\%$ & $\mathrm{n}$ & $\%$ & $\mathrm{n}$ & $\%$ & $\mathrm{n}$ & $\%$ & $\mathrm{n}$ & $\%$ & $\mathrm{n}$ & $\%$ \\
$5-1$ & 5 & 2.7 & 23 & 12.2 & 8 & 4.2 & 6 & 3.2 & 19 & 10.1 & 4 & 2.1 \\
$4-1-1$ & 12 & 6.4 & 13 & 6.9 & 8 & 4.2 & 9 & 4.8 & 7 & 3.7 & 5 & 2.7 \\
Total & 17 & 9.0 & 36 & 19.0 & 16 & 8.5 & 15 & 7.9 & 26 & 13.8 & 9 & 4.8 \\
\hline
\end{tabular}

\section{Difference in treatment decisions}

Four different reference treatments were suggested in the 189 cases. In 10 cases (5.3\%) the dose is decreased, in 38 cases $(20.1 \%)$ the prescribed treatment is continued without any changes, in 41 cases $(21.7 \%)$ the dose is increased and in 100 cases (52.9\%) another drug was selected. The decisions of all neurologists and the system were compared with the reference decision (Table 6.4-VIII). The numbers in the cells on the diagonal (shaded grey) show the agreement of the neurologists and the system with the reference decision. In each cell the following information is provided: the number of decisions, the percentage of these decisions related to the total number of decisions taken ( 945 for the neurologists, 189 for the system) and the percentage of the decisions related to the number of cases in the reference treatment.

Table 6.4-VIn: Overview of all 945 ( $5 \times 189$ ) decisions of the neurologists and the system on the 189 reference cases. Each cell contains information on the number of times a decision is made, as well as the percentage of the total number of decisions and the percentage of the decisions taken (in)correctly. For each cell in the column 'Total' the first row presents the number of times a specific reference decision is made. The second row presents for a specific reference decision the percentage of the total number of decisions (945). The third row presents for the neurologists and the system the percentage of decisions according to the reference.

\begin{tabular}{|c|c|c|c|c|c|c|c|c|c|c|c|c|}
\hline \multirow{3}{*}{$\begin{array}{l}\mathrm{N}=945 \\
\text { Decision } \\
\text { Reference }\end{array}$} & \multicolumn{12}{|c|}{ Participants } \\
\hline & \multicolumn{2}{|c|}{ decrease } & \multicolumn{2}{|c|}{ equal } & \multicolumn{2}{|c|}{ increase } & \multicolumn{2}{|c|}{ change } & \multicolumn{2}{|c|}{ rest } & \multicolumn{2}{|c|}{ Total } \\
\hline & N3-7 & $\mathrm{s}$ & N3-7 & $s$ & N3-7 & $\mathrm{s}$ & N3-7 & $\mathrm{s}$ & N3-7 & $\mathrm{s}$ & N3.7 & $\mathrm{S}$ \\
\hline decrease & 40 & 9 & 5 & 1 & 0 & 0 & 5 & 0 & 0 & 0 & 50 & 10 \\
\hline$\%$ total & 4.2 & 4.8 & 0.5 & 0.5 & 0 & 0 & 0.5 & 0 & 0 & 0 & $s$ & \\
\hline$\%$ (dis)agree & 80.0 & 90.0 & 10.0 & 10.0 & 0 & 0 & 10.0 & 0 & 0 & 0 & 80.0 & 90.0 \\
\hline equal & 4 & 0 & 161 & 31 & 12 & 6 & 11 & 1 & 2 & 0 & 190 & 38 \\
\hline$\%$ total & 0.4 & 0 & 17.0 & 16.4 & 1.3 & 3.2 & 12 & 0.5 & 0.2 & 6 & 20 & \\
\hline$\%$ (dis)agree & 2.1 & 0 & 84.7 & 81.6 & 6.3 & 15.8 & 58 & 2.6 & 1.1 & 0 & 84.7 & 81.6 \\
\hline increase & 0 & 0 & 31 & 0 & 162 & 37 & 8 & 4 & 4 & 0 & 205 & 41 \\
\hline$\%$ total & 0 & 0 & 3.3 & 0 & 17.1 & 19.6 & 0.9 & 2.1 & 0.4 & 0 & 21 & \\
\hline$\%$ (dis)agree & 0 & 0 & 15.1 & 0 & 79.0 & 90.2 & 3.9 & 9.8 & 2.0 & 0 & 79.0 & 90.2 \\
\hline change & 33 & 0 & 44 & 2 & 18 & 2 & 395 & 94 & 10 & 2 & 500 & 100 \\
\hline$\%$ total & 3.5 & 0 & 4.7 & 1.1 & 1.9 & 1.1 & 41.8 & 49.7 & 1.1 & 1.1 & 52 & \\
\hline$\%$ (dis)agree & 6.6 & 0 & 8.8 & 2 & 3.6 & 2.0 & 79.0 & 94.11 & 2.0 & 20 & 79.0 & 94.0 \\
\hline
\end{tabular}


In cases in which the reference treatment was to decrease the drug dose the system made more correct decisions than the neurologists. However, the number of cases in this group is rather small.

The neurologists and the system do not differ much with respect to their correctness for cases in which the reference treatment decision is to continue the existing treatment (equal). For cases with this reference decision almost half $(48.3 \%)$ of the decisions different from the reference were taken by one particular neurologist. In $6(15.8 \%)$ of the 38 cases in which the reference decision was to continue the treatment the system suggested to increase the dose.

The system also made more correct decisions than the neurologists for cases where the reference indicated to increase the drug dose. Of the incorrect decisions proposed by the neurologists $20(46.5 \%)$ were taken by one neurologist. The system decided to change the prescribed drug in $4(9.8 \%)$ of the 41 cases.

The largest difference $(15 \%)$ in correct decisions between the system and the neurologists is found for the reference 'Change'. Again almost half (47.6\%) of the incorrect decisions could be attributed to one particular neurologist.

\subsection{Decision criteria}

It may be expected that characteristics of a case are important for reaching a decision. For each of the main characteristics of a case it was determined whether it could predict the reference treatment. Set I was used for this purpose.

\subsubsection{Treatments indicated by a single attribute}

Only in cases in which idiosyncratic complaints occur there is an almost unanimous decision to change the drug treatment. In only one of the 59 cases the reference treatment was to keep the treatment unchanged. In this case seizures were completely controlled, there existed a good compliance and a high therapeutic plasma drug level was observed (Table 6.5-I)

Table 6.5-I: Breakdown of the reference treatment decisions as a function of the presence of complaints.

\begin{tabular}{lcccccc}
\hline Complaints & \multicolumn{2}{l}{ Absent } & \multicolumn{2}{c}{ Dose relaled } & \multicolumn{2}{l}{ Idiosyncratic } \\
\hline Reftreatment & $\mathrm{N}$ & $\%$ & $\mathrm{~N}$ & $\%$ & $\mathrm{~N}$ & $\%$ \\
decrease & & & 14 & 19.4 & & \\
equal & 34 & 38.6 & 16 & 22.2 & 1 & 1.7 \\
increase & 43 & 48.9 & 1 & 1.4 & & \\
chunge & 10 & 11.4 & 41 & 56.9 & 58 & 98.3 \\
extra & 1 & 1.1 & & & & \\
\hline
\end{tabular}

The system's advice was to change to another drug in 57 of the 59 cases. In the other two cases the system suggested to stop the treatment immediately. In both cases all five neurologists agreed that the drug should be changed and immediate discontinuation of the drug was not deemed necessary. 
As the decision to change the drug when idiosyncratic complaints are present may obscure the possible relation between other case characteristics and treatment decisions, we further analysed the 160 cases without idiosyncratic complaints. In these cases, however, no single characteristic could be identified that leads to a specific treatment decision.

\subsubsection{Treatments ruled out by a single attribute}

Although an attribute may not indicate a specific treatment decision, it may rule out a particular treatment decision. For the 160 reference cases without idiosyncratic complaints, the absence of treatment decisions in the reference is described.

\section{Seizure frequency}

Even when seizures are controlled, one could consider to increase the dose when the plasma drug level is below the therapeutic range. However, when considering cases with completely controlled seizures this decision is not made. Also the decision to change the drug due to e.g. dose related complaints is not made in these cases (Table 6.5-II).

In those cases in which seizures were either almost completely or hardly controlled the dose hardly ever was decreased despite the fact that in more than two thirds of these cases a complaint was present.

The decision to decrease the drug dose is never made when the seizure frequency remains uncontrolled. The presence of complaints in these cases does not lead to a reduction of the dose but mainly to a change of the drug.

The system did not suggest to change or increase the dose in cases in which seizures were completely controlled. For cases in which seizures were still present the system did never decide to decrease the dose of the drug. In the three cases in which the reference treatment was to decrease the drug dose, the system proposed to change to another drug.

Table 6.5-II: Distribution of reference treatments when related to seizure frequency in 160 cases without idiosyncratic complaints.

\begin{tabular}{lcccccccc}
\hline Seizures & \multicolumn{2}{c}{ controlled } & \multicolumn{2}{c}{ almost contr } & \multicolumn{2}{c}{ hardly contr } & \multicolumn{2}{c}{ uncontrolled } \\
\hline Ref treatment & $\mathrm{N}$ & $\%$ & $\mathrm{~N}$ & $\%$ & $\mathrm{~N}$ & $\%$ & $\mathrm{~N}$ & $\%$ \\
decrease & 11 & 36.7 & 2 & 9.5 & 1 & 1.5 & & \\
equal & 19 & 63.3 & 12 & 57.1 & 15 & 22.4 & 4 & 9.5 \\
increase & & 4 & 19.0 & 26 & 38.8 & 14 & 33.3 \\
change & & 3 & 14.3 & 25 & 37.3 & 23 & 54.8 \\
extra & & & & & & 1 & 2.4 \\
\hline
\end{tabular}

\section{Complaints}

When complaints are absent, the decision to decrease the drug dose is never taken by the neurologists or the system.

In only one case with a dose related complaint the neurologists decided to increase the drug dose. The decision profile for this exceptional case was 3-2; two of the neurologists as well as the system decide to change the drug. In none of the cases with a dose related complaint the system proposed to increase the dose. 


\section{Plasma drug level}

For each plasma drug level the four major reference treatments were present. The system applied all but one of the decisions for each of the plasma drug levels. For cases in which the plasma drug level was in the sub therapeutic range the system never decreased the drug dose.

Table 6.5-III: Distribution of the reference treatment decisions when related to the range of the plasma drug level.

\begin{tabular}{lrrrrrrrrrr}
\hline Plasma drug level & \multicolumn{1}{c}{ sub ther. } & \multicolumn{2}{c}{ low ther. } & \multicolumn{2}{c}{ therapeutic } & \multicolumn{2}{c}{ high ther. } & \multicolumn{2}{c}{ toxic } \\
\hline Ref.treatment & $\mathrm{N}$ & $\%$. & $\mathrm{N}$ & $\%$. & $\mathrm{N}$ & $\%$ & $\mathrm{~N}$ & $\%$. & $\mathrm{N}$ & $\%$. \\
decrease & 2 & 5.9 & 1 & 3.2 & 1 & 2.6 & 5 & 16.7 & 5 & 18.5 \\
equal & 10 & 29.4 & 9 & 29.0 & 17 & 44.7 & 8 & 26.7 & 6 & 22.2 \\
increase & 14 & 41.1 & 13 & 41.9 & 13 & 34.2 & 3 & 10.0 & 1 & 3.7 \\
change & 8 & 23.5 & 8 & 25.8 & 7 & 18.4 & 13 & 43.3 & 15 & 55.6 \\
extra & & & & & & & 1 & 3.3 & & \\
\hline
\end{tabular}

\subsection{Treatments indicated by combinations of attributes}

To identify combinations of characteristics which predict the reference treatment an induction algorithm was used to analyse the 219 cases in set 1 . The algorithm iteratively partitions the high-dimensional feature space such that in principle each end-node of the resulting classification tree groups together (almost) only cases with one type of reference treatment. The algorithm has as additional stop criterion that a split has to provide sufficient information. If that is not the case, an impure node is not further splitted (Talmon, 1986).

When we assign to each end-node the treatment decision of the majority of cases in that node the tree has a correctness of $85.4 \%$ on set 1 . In total 14 different end-nodes were created (see figure on next page). In this paragraph first the 'pure' end-nodes, containing cases with the same reference treatment, are discussed. Next the mixed end-nodes, with two different treatments are reviewed.

For the cases in each end-node the treatments suggested by the neurologists and by the system are presented. To assure that there is sufficient support to decide that a combination of case characteristics leads to a specific treatment decision the set of cases for which no majority treatment decision exists was classified according to the criteria described in the decision tree. The assigned treatments were compared with the suggested treatments for these cases. Support for a single treatment decision was considered strong for nodes in which:

- The number of cases from a reference group was relatively large $(\mathrm{N} \geq 5)$

- The number of cases with a treatment other than the main treatment among the cases at an end node is relatively small $(<20 \%)$ and

- The non-reference group that fulfilled the criteria was relatively small when compared with the number of cases from the reference group ( $<20 \%$ of the cases)

Finally the nodes with the largest variation in decisions are discussed briefly. For these nodes little can be concluded about the use of a single treatment given the occurrence of three decisions. 


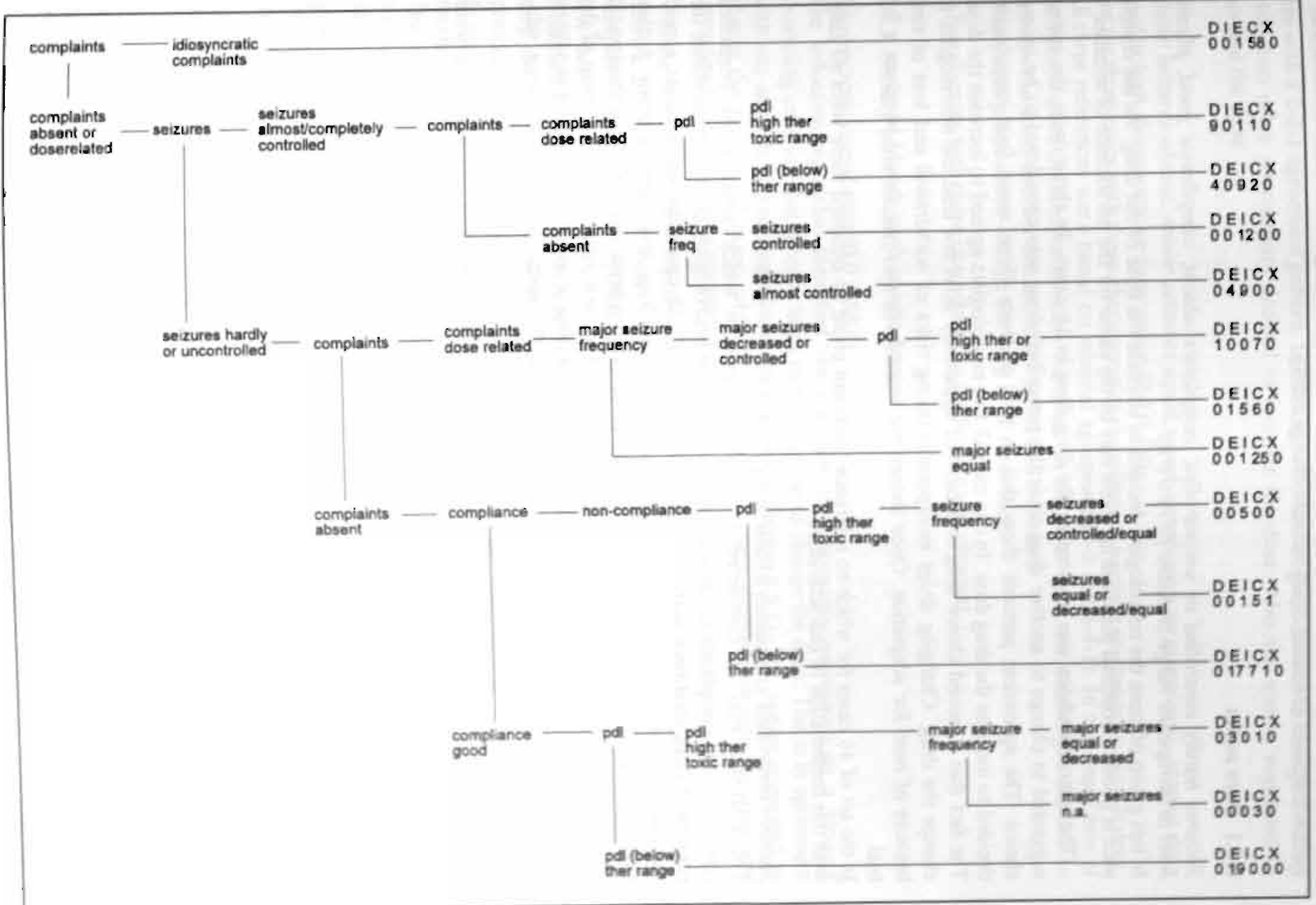


In the following each node is represented by a vector indicating the number of cases with a certain treatment (decrease equal increase change extra).

\subsubsection{Pure nodes}

Seizures hardly controlled or uncontrolled, complaints absent, compliance good, plasma level in therapeutic range or below $(019000)^{1}$

In this group of cases the neurologists decided to increase the dose of the drug. As the compliance is good, suboptimal plasma drug levels may be the cause for the persistence of seizures.

The system's advice was to increase the drug dose in 16 cases. In three cases the system suggested to change to another drug since the prescribed drug was considered to be second choice. The agreement patterns show that in two of these three cases four neurologists decided to increase the drug dose. In one case all five neurologists agreed to increase the dose. The fact that a second choice drug is prescribed apparently does not lead the neurologists to change the drug. Changing drugs incorporates taking risks of withdrawal and loss of time because of need for adaptation. Once chosen the second choice drug should be given a full trial.

In the set of 46 cases for which no reference treatment could be obtained there were no cases with this combination of characteristics.

\section{Seizures controlled, complaints absemt $\left(\begin{array}{lllll}0 & 0 & 12 & 0 & 0\end{array}\right)$}

The treatment may be considered successful in cases in which seizures are controlled completely and complaints are absent. In these cases the neurologists decided to continue the prescribed drug treatment without further changes. In all 12 cases the neurologists agreed completely. The system also decided to continue the drug treatment without further adjustments in all cases. In five of these cases the plasma drug level was below the therapeutic range, while in two cases the plasma drug level was in the toxic range. An adjustment of the drug dose was not suggested. This indicates that plasma drug levels are not applied rigidly to modify the treatment when it is effective in controlling the seizures without inducing sideeffects.

Among the 46 cases without a reference no cases with the same characteristics were observed.

\section{Seizures hardly controlled, non-compliance, plasma drug level high therapeutic or toxic

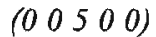

In cases in which seizures were hardly controlled, with non-compliance and a plasma drug level in the high therapeutic or toxic range, the neurologists decided to continue the prescribed treatment. In these five cases the system also decided to continue the prescribed drug treatment.

Non-compliance may explain the persistence of seizures. In these cases it is preferred to improve the compliance as this may lead to improved seizure control.

\footnotetext{
'Decisions taken by the neurologists (Decrease Equal increase Change Extra) are presented in parentheses (D E I C X). In

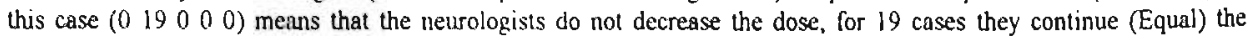
treatment without change, they do not increase, change or add an additional drug.
} 
In the set of cases without a reference treatment the same characteristics were found in one case with a 2-2-1 agreement pattern. In this case two neurologists decided to change the drug while two other neurologists decided to increase the dose. Also the system suggested to increase the dose.

Major seizures absent, minor seizures equal, goad compliance, no complaints, plasma drug level in high therapeutic or toxic range $\left(\begin{array}{lllll}0 & 0 & 0 & 3 & 0\end{array}\right)$

For the three cases with these characteristics the reference suggests to change to another drug. All three references were based on minimal agreement (3-2 and 3-1-1). In two cases two of the neurologists decided to increase the dose. In the other case one neurologist decided to increase the dose, another continued the prescribed treatment.

The system proposed to change the drug in all three cases.

For all cases with these characteristics a reference was obtained

\subsubsection{Nearly pure nodes; two decisions}

\section{Idiosyncratic complaints (0 0 I 580$)$}

The induction algorithm identified the presence of idiosyncratic complaints as a kind of overruling criterion that directly leads to the decision to change the drug. This is in agreement with our analysis of the single case characteristics (section 6.5.1). The system suggested to change the drug in 57 cases, while in two cases the system proposed to stop the treatment immediately as the complaints were very severe.

In the 46 cases of the non reference set, three cases had idiosyncratic complaints. In all three cases a 2-2-1 agreement pattern was present. In two of these cases in which seizures were completely controlled, compliance was good and the plasma drug level was in the sub- and low-therapeutic range two of the neurologists decided to change the drug. An alternative treatment for these cases was proposed by two other neurologists. In one case they decided to stop the prescribed drug without replacement, and in the other case they continued the prescribed drug without adjustments.

In the third case seizures decreased, compliance was good and the plasma drug level was low therapeutic. For this case only one of the neurologists decided to change the prescribed drug. One pair of neurologists decided to increase the drug dose. The other pair continued the treatment without adjustments.

In all three cases the system suggested to change to another drug.

\section{Major seizures equal or minor seizures equal or decreased, and dose related complaints $(001250)$}

In 25 of 26 cases a change of the drug was decided upon. In one case a continuation of the prescribed treatment was the reference. For this case there was a 3-2 agreement profile, in which the two remaining neurologists decided to change the drug

The system used two different approaches for these cases. In 21 cases the system also decided to change the drug, while in 5 cases a continuation of the prescribed treatment was suggested. In all five cases dose related complaints occurred with a plasma drug level below or in the therapeutic range. In two of these cases in which the seizure frequency did not change four of the neurologists decided to change the drug, while the fith chose to continue the 
treatment. In the three remaining cases in which the frequency of minor seizures decreased, a 3-2 decision profile was found, in which continuation of the prescribed treatment was the alternative used by two of the neurologists.

In the set of 46 cases without a reference five cases were found with the same characteristics all of which had a plasma drug level in or above the therapeutic range. In four of the cases (three times 2-2-1 and once a 2-1-1-1 agreement profile) two neurologists agreed to change the drug. In two of these cases two neurologists decided to continue the treatment, while in one case two neurologists decided to decrease the dose. In the remaining case (2-1-1-1) two neurologists decided to continue the prescription.

The system proposed to change the drug in four cases while in one case with a low plasma drug level a continuation of the treatment was suggested, which was also decided upon by two of the neurologists.

While the presence of incompletely or uncontrolled seizures and dose related complaints seems to lead the neurologists towards a change of the drug, the presence of low therapeutic plasma drug levels modifies the decision of the system towards continuation of the treatment.

Major seizures decreased and minor seizures decreased, equal, or absent, or major seizures controlled and minor seizures equal, dose related complaints and plasma level in the high therapeutic or toxic range (1 0070$)$

This node contains eight cases, in seven of which the reference was to change the drug.

A 3-2 decision profile was found in 6 of the cases for which change was the reference. In four of these cases a decrease of the dose was proposed, in one case the dose was decreased and an additional drug was added, in one case a continuation was proposed by the two other neurologists. In one case with seizures hardly controlled, good compliance, dose related complaints and a plasma drug level in the toxic range, a 4-1 agreement profile was observed. In one case with both seizure types decreased, a good compliance and a plasma drug level in the high therapeutic range, three neurologists decided to decrease the dose, while two changed to another drug.

In all 8 cases the system decided to change the drug.

In the set of cases without a reference two cases were found with the same characteristics In both cases two neurologists decided to change the drug while two other neurologists decided to decrease the dose and one neurologist decided to continue the treatment.

In both cases the system decided to change the drug.

Despite some success in controlling the seizures, the presence of dose related complaints and high or toxic range plasma drug levels mainly causes the neurologists to adjust the treatment by changing the drug. As a decrease could lead to further continuation of seizures, the system also suggests to change the drug.

Major seizures equal or major and minor seizures decreased, good compliance, no complaints and plasma drug level in the high therapeutic or toxic range $\left(\begin{array}{lllll}0 & 3 & 0 & 1 & 0\end{array}\right)$

Four cases were found with these characteristics in the set of 219 cases with a reference. In three of these cases the reference was to increase the drug dose (agreement profile 3-1-1 or 41) In one case, with major and minor seizures which remained equal a change of the drug was decided upon by three neurologists, the other two neurologists increased the dose 
In two cases the system decided to increase the dose, while in two cases a change was proposed.

In the 46 cases without a reference, four cases were observed with the same characteristics. In all four cases a 2-2-1 agreement profile was present. In total three different treatment decisions were taken by a pair of neurologists i.e. increase, change or equal.

The system decided to increase the dose in three cases in which seizures were hardly controlled. A change was proposed in a case in which the seizures did not change and the plasma drug level was in the toxic range.

The number of cases in this node is small and an equal number of cases was found in the non reference set. Little more can be concluded than that for these characteristics the neurologists mainly try to achieve complete seizure control by either increasing the dose or changing to another drug.

Seizures almost controlled, complaints absent $(04900)$

A group of 13 cases was identified in which either an increase in the dose or a continuation of the treatment was agreed upon. In 6 cases major seizures were controlled. In five of these the neurologists decided to continue the treatment, although the minor seizures were still incompletely controlled. In one case with a sub therapeutic plasma drug level, the dose was increased. While for cases in which major seizures are controlled the neurologists tend to continue the treatment, for minor seizures the treatment decision is less clear.

The system proposed in all 8 cases with a good compliance to increase the dose. In cases with non-compliance either an increase of the dose -three cases with a (below) therapeutic plasma drug level- or a continuation of the prescribed treatment -two cases with a high therapeutic plasma drug level- was suggested.

In the set of cases without a reference treatment one case was found with the same characteristics. For this case two neurologists agreed about the treatment to continue the treatment. The system also proposed to continue the treatment without adjustments as noncompliance and a toxic range plasma drug level were present.

The system increases the dose if seizures are not completely controlled unless noncompliance and high therapeutic or toxic plasma drug levels are present. The neurologists mainly tend to continue the prescription although seizures are not completely controlled and compliance is good.

\subsubsection{Mixed nodes; three decisions}

In five nodes covering 70 cases three different reference treatment decisions were observed. The system suggested the same treatment as the reference in 45 cases (64\%). In nine cases the system agreed with two neurologists who did not agree with the reference but agreed among themselves on a different treatment (Table 6.6-I). 
Table 6.6-I: Overview of the reference decisions and the advice of the system concerning the 70 cases in the 5 nodes in which three different decisions were observed.

\begin{tabular}{l|lccccc}
\hline Treatment & \multicolumn{6}{|c}{ Reference } \\
\hline \multirow{3}{*}{ System } & Decisions & Decreasc & Equal & Increase & Change & eXtra \\
& Decrease & 9 & 2 & & & \\
& Equal & 2 & 12 & 1 & 4 & \\
& Increase & & 6 & 16 & 3 & 1 \\
& Change & 2 & 3 & 1 & 8 & \\
\hline
\end{tabular}

In two of the five nodes the correctness of the system is more than $70 \%$, in one node more than $60 \%$ was reached, while twice the system achieved a correctness of less than $60 \%$. In all nodes except node $\left(\begin{array}{lllll}9 & 0 & l & 1 & 0\end{array}\right)$ more than $50 \%$ of the reference treatments is based on a minimal agreement pattern (3-2 or 3-1-1). In (901llo) this was $27 \%$.

Only for node $\left(\begin{array}{lllll}9 & 0 & 1 & 1 & 0\end{array}\right)$ one can speak of an indication for a reference treatment (Table 6.6-II). In this node with seizures almost or completely controlled, dose related complaints and a plasma drug level in the high therapeutic or toxic range, 3 cases have a minimal agreement pattern (3-1-1 or 3-2). For 7 of the 9 cases in which both seizure types are completely controlled and compliance is good the system as well as the neurologists decided to decrease the drug dose. No cases with these characteristics were observed in the non-reference group. In the case in which both the neurologists and the system decided to change to another drug, the seizures were almost controlled and a high therapeutic plasma drug level was observed. Four neurologists agreed on this treatment.

Table 6.6-II: Distribution of the reference decision and the treatment decision suggested by the system for node $\left(\begin{array}{lllll}9 & 0 & 1 & 1 & 0\end{array}\right)$

\begin{tabular}{l|lccc}
\hline Treatment & \multicolumn{4}{|c}{ Reference } \\
\hline \multirow{3}{*}{ System } & Decisions & Decrease & Equal & Change \\
& Decrease & 7 & 1 & \\
& Equal & 1 & & \\
& Change & 1 & & 1 \\
\hline
\end{tabular}

The non-reference group of this node contained five cases ( $31 \%$ of the total set) with the same characteristics. All of these cases had one type of seizures controlled while the other decreased

In node ( $\left.\begin{array}{lllll}4 & 0 & 9 & 2 & 0\end{array}\right)$ with cases with seizures almost or completely controlled, dose related complaints and a plasma drug level in or below the therapeutic range, the neurologists as well as the system mainly decide to continue the treatment. The dose was decreased in cases in which compliance was good and complaints were severe. In case of severe complaints and non-compliance a change of the drug was suggested. One single approach for treatment for 
these cases is not indicated. The system and the neurologists agree in $73 \%$ of the cases covering three different decisions.

Table 6.6-III: Decisions of the neurologists and the system for node $(40920)$.

\begin{tabular}{l|lccc}
\hline Treatment & \multicolumn{4}{|c}{ Reference } \\
\hline \multirow{3}{*}{ System } & Decisions & Decrease & Equal & Change \\
& Decrease & 2 & 1 & \\
& Equal & 1 & 8 & 1 \\
& Change & 1 & & 1 \\
\hline
\end{tabular}

Differences in treatment between the system and the neurologists were observed for three nodes $\left(\begin{array}{llll}0 & 17 & 7 & 1\end{array}\right),\left(\begin{array}{lllll}0 & 0 & 5 & 1\end{array}\right)$ and $(01560)$

In node $\left(\begin{array}{llll}0 & 17 & 7 & 1\end{array}\right)$, the neurologists used one treatment decision in $68 \%$ of the cases in which seizures are hardly or uncontrolled, complaints are absent, non-compliance and a plasma drug level in or below the therapeutic range (Table 6.6-IV). The neurologists decided mainly to increase the dose, while in 7 cases the treatment is continued without further adjustments. In $68 \%$ of the cases agreement between the system and the reference was observed. The system adheres to a larger extent to the decision to increase the dose for these cases ( $92 \%$ ).

Table 6.6-IV: Decisions of the neurologists and the system for node $\left(\begin{array}{llllll}0 & 17 & 7 & 1 & 0\end{array}\right)$.

\begin{tabular}{l|lccc}
\hline Treatment & \multicolumn{4}{|c}{ Reference } \\
& Decisions & Equal & Increase & Change \\
System & Equal & 1 & 1 & \\
& Increase & 6 & 16 & 1 \\
\hline
\end{tabular}

In node $\left(\begin{array}{lllll}0 & 0 & 1 & 5 & 1\end{array}\right)$ in which seizures are hardly controlled, non-compliance is present and a plasma drug level in the high therapeutic or toxic range is observed the main reference treatment is a change of the drug. In one case the dose was kept equal, while in another case a second anti-epileptic drug was added. The system did not suggest a specific treatment for the cases at this node. In three cases the system decided to continue the prescription, in three other cases an increase of the dose was proposed. In one case a change of the drug was suggested. Two of these decisions coincide with the reference. 
Table 6.6-V: Overview of the decisions taken by the neurologists and the system on node ( 01560 )

\begin{tabular}{l|lccc}
\hline \multirow{2}{*}{ Treatment } & \multicolumn{4}{c}{ Reference } \\
\hline \multirow{3}{*}{ System } & Decisions & Equal & Increase & Change \\
& Equal & 2 & 1 & 1 \\
& Change & 3 & 5 \\
\hline
\end{tabular}

In node ( $\left.\begin{array}{lllll}0 & 1 & 5 & 6 & 0\end{array}\right)$ in which seizures are hardly controlled, dose related complaints are present and the plasma drug level was in or below the therapeutic range all reference treatments were based on minimal agreement (3-2 and 3-1-1).

The single decision to increase the dose was taken by three of the neurologists, while the other two proposed to change the drug. The system suggests only two different treatments of which nine $(75 \%)$ are a change of the drug. The neurologists however use a continuation of the treatment almost as often as a change of the treatment (Table 6.6-V).

\subsection{References}

Beghi E., Tognoni G., Prognosis of epilepsy in newly referred patients: A multicentre prospective study, Epilepsia, 29, 1988, pp. 236-243.

Collaborative Group for Study of Epilepsy, Prognosis of epilepsy in newly referred patients: a multicintre prospective study of the effects of monotherapy on the long-term course of epilepsy, Epilepvia, 33, 1992, pp. 45-51.

Cramer J.A., A clinimetric approach to assessing quality of life in epilepsy, Epilepsia, 34, 1993, (Suppl.), 4, s8-s13.

Donselaar C. van, Idioparhic first seizure in adult life, thesis, Rotterdam, 1990.

Duncan J.S., Sander J.W.A.S., The Chalfont seizure severity scale. Journal of Neurology, Neurosurgery and Psychiatry, 54, 1991, pp. 873-876.

Elwes R.D., Johnson C.B., Shorvon S.D., Reynolds E.H., The prognosis for seizure control in newly diagnosed epilepsy, The New England Journal of Medicine, 40, 1984, pp. 944-947.

Elwes R.D.C., Reynolds E.H., The early prognosis of epilepsy. In: Comprehensive Epileptology, (Dam and Gram, Eds.), Ravens Press, New York, 1990, pp. 715-727.

Landis J.R., Koch G.G., The measurement of ubserver agreement for categorical data, Biometrics, 1977,33 , pp. 159-174

Schouten H.J.A., Statistical measurement of inlerobserver agreement, analysis of agreements and disagreements between observers, thesis, Rotterdam, 1985.

Talmon J.L., A multiclass nonparametric partitioning algorithm, Pattern Recognition Letters, 1986, 4, pp. 31-38. 


\section{Conclusions and discussion}

\subsection{Introduction}

In this thesis the development and assessment of a computerised model of expert knowledge for prescribing anti-epileptic drugs was described.

The research question investigated was:

- Is it possible to develop a decision support system that provides adequate advice concerning anti-epileptic drug therapy?

Both the initiation of anti-epileptic drug treatment and the adjustment of the treatment during a first follow-up contact were studied.

\subsection{System development}

A structured approach for system development, the KADS methodology, was used At the time we used KADS, its orientation was mainly towards the analysis of the cognitive process. In the more recent literature on KADS, this orientation shifted towards a more pragmatic approach regarding the implementation of models (Schreiber et al. 1993, Tansley and Hayball 1993).

In the early versions of KADS a waterfall-based model for system development was used. Limited attention was paid to project management in these versions. Later, a spiral life-cycle model was introduced for project management (Hickman et al. 1989)

KADS provides little support with respect to the evaluation of the quality of the developed models. The little work on metrication that has taken place was mainly directed towards project management (Readdie et al. 1989). It is the philosophy of KADS that because of the extensive analysis of knowledge there is a better chance that the right implementation is made. However, we recognised that also implementation of the (partial) model of expertise could be of value to support elicitation and analysis. In this context it would be appropriate if KADS would include a reference to a recommended evaluation methodology.

Several problems were encountered with the application of KADS. The use of interpretation models appeared to be difficult. This could party be attributed to differences in granularity 
between models. Furthermore, the model library was incomplete, requiring the design of additional interpretation models. Although development of interpretation models is supported by a conceptual modelling language, this language only provides a tentative list of metaclasses and knowledge sources. Moreover, due to the high level of abstraction of the models they need to be translated before they can be presented to a domain expert.

The goal of our study was the development of a computerised model for prescribing antiepileptic drug treatment. The expertise for such a knowledge-based system may be obtained from a single expert or multiple experts. In our case we decided to use only one expert because of the availability of the extensive literature. It was expected that one neurologist was enough to disambiguate any problems found in the literature and to offer additional information. The plan was to assess and - if necessary to adapt - the developed models using experts that are successively less involved in the development of the system and less aware of the working procedures of the expert who provided the knowledge. In retrospect, we expect that involvement of multiple experts during the development could have reduced some of the evaluation efforts and would have made us aware of some of the observed problems earlier in the development process

The developed models for the initiation and monitoring of anti-epileptic drug treatment were implemented in an experimental system using muLISP ${ }^{\mathrm{rm}}$. The system has a layered structure separating the static parts of the knowledge from the procedural parts. A frame and rule based structure was used. Rules related to a specific section of the problem solving process were combined in rule sets which can be used when a knowledge source is activated. In the initiation part as well as in the monitoring part a forward-chaining mechanism was used for reasoning. Most of the rule sets belong to the assessment task of the monitoring part of drug treatment. The use of the frame and rule structures preserved the structure of the knowledge in a way recognisable to the expert. Therefore, a static assessment was possible.

\subsection{Reference development procedure}

Detailed guidelines for anti-epileptic drug treatment of individual patients are not available. Therefore a reference had to be developed to assess the system's advice. This reference was also used to obtain insight in the variability of the treatment decisions of neurologists. Paper cases based on real patient data were designed and used for the determination of the reference. The ultimate goal was to have a set of cases with for each case an agreed upon diagnosis and an agreed upon prescription. The majority decision among three neurologists - if it existed was taken as reference.

A Delphi-like approach was used to reduce the variability and remove erroneous treatment suggestions of the neurologists. The use of statistical feedback significantly increased the number of cases in which a majority decision on the drug was reached.

Due to the variation in the prescribed daily dose among the neurologists we applied a range of acceptable daily doses to reduce the outliers. Feedback was less successful in this situation because part of the outliers could be attributed to rounding off dosages toward an integer number of tablets and part of the outliers concerned mainly one participant who did not change his prescriptions 
In our initial set of 60 cases the neurologists achieved a majority on the detailed diagnosis in 47 cases (the diagnostic reference set). In 39 of these 47 cases they achieved also a majority decision on the selected drug (the treatment reference set)

A prescription not equal to that of the majority may still be considered an acceptable treatment. Therefore, after the Delphi round the prescriptions were assessed on their acceptability by two independent neurologists.

A consensus meeting was organised to establish objective decision criteria. The annotated prescriptions containing information on the acceptability were discussed in a consensus meeting. For each of the 47 cases in the diagnostic reference set a reference treatment could be obtained on the basis of guidelines. The discussion led to a better agreement among the neurologists with respect to the use of phenytoin.

The Delphi-approach could also have been used to establish a reference for the assessment of the treatment decisions. However, the number of cases needed to investigate the decision process would require too much time from the neurologists to achieve a reference. Instead of this, the decisions of five neurologists on 265 cases are used to obtain two reference subsets: a clinical reference consisting of the majority decision of the five neurologists and a reference consisting of the majority decision of both the neurologists and the system. Whereas the clinical reference indicates the minimal level of agreement of the system with the neurologists as the system was not involved in defining the reference, the second reference enables a more balanced assessment of the neurologists' and the system's performance.

\subsection{Initiating anti-epileptic drug treatment}

For the evaluation of the initiation of drug treatment a staged approach was used. The focus gradually shifts from an assessment of the coverage and correctness of the implemented knowledge to an evaluation of the system's and neurologists' performance using reference diagnoses and prescriptions.

\section{Quality of the knowledge acquisition}

The prescriptions of the neurologist who provided the implemented knowledge and the advice of the system were compared. The 60 paper cases were used. In all but one case the neurologist and the system agreed on the generic drug as well as on the selected brand and formulation.

In $80 \%$ of the prescriptions for carbamazepine the daily dose was identical to that of the neurologist. The system suggested a lower dose for carbamazepine $(66 \%-75 \%$ of the daily dose) in $11 \%$ of the cases and a higher dose (125-133\% of the daily dose) in $9 \%$ of the cases.

When prescribing sodium-valproate the neurologist and the system differed in two of the fifteen cases (13\%). Once a higher dose (116\% of the daily dose) and once a lower dose (75\% of the daily dose) was prescribed.

In one case the neurologist did not provide a prescription (because although a diagnosis was established he wanted additional information from the EEG). This showed that despite the fact that a diagnosis is established, drug treatment need not be started. The system was programmed to initiate a drug treatment when a diagnosis is established.

From this experiment it was concluded that the knowledge acquisition was appropriate. 


\section{Local differences}

The same 60 cases were presented to another neurologist (Neurologist II) from the same department as the expert neurologist.

Neurologist II and the system use the same drugs for treatment of epilepsy. The neurologist did not initiate drug treatment when the diagnosis was unknown. Again in one case the system suggested to start anti-convulsive treatment as a diagnosis was established. The neurologist, however, refrained from drug treatment as he also felt he needed additional information about the EEG

A difference in the formulation as well as in the daily dose was observed for carbamazepine. Neurologist II and the system do not seem to agree as far as the daily dose is concerned. The system suggested $50 \%$ to $75 \%$ of the daily doses for carbamazepine prescribed by the neurologist. With respect to the formulation the neurologist preferred the controlled release variant

The daily dose for sodium-valproate differed less. In three out of 10 cases an identical dose was prescribed, while in 4 cases $90 \%$ of the daily dose of the neurologist was prescribed. This difference in the daily dose of sodium-valproate could be attributed to the use of a different intake scheme. The neurologist preferred a tablet intake twice a day, thereby being able to prescribe tablets of $500 \mathrm{mg}$ instead of $300 \mathrm{mg}$. Still there were three cases in which Neurologist II preferred to start with a higher dose. In these cases, 70-75\% of the dose of the neurologist was suggested by the system.

The experiment showed that even within the same department different approaches exist. Neurologist 1 starts with a relatively low dose which may be increased when treatment proves not to be effective. Neurologist II on the other hand seems to adhere more to a 'middle of the target range' approach, reducing the dose when side-effects occur.

Some investigators suggest that anti-epileptic drugs have a certain specificity for certain types of epilepsy (Porter 1984, Mattson et al. 1988, 1992). However, conclusions about differences in efficacy of anti-epileptic drugs in controlling particular seizure types or epilepsies are difficult to draw, due to differences in patient populations and study designs (Gram et al. 1982, Chadwick and Tumbull 1985, Chadwick 1994ab, Brodie and Dichter, 1996). Both our neurologists consistently used different drugs for the different types of epilepsy: carbamazepine for treatment of partial epilepsy and sodium-valproate for treatment of idiopathic generalised epilepsy

Little difference was expected with respect to the daily dose; both neurologists worked in the same department and agreed when selecting the anti-epileptic drug for treatment. However, the different approaches - start with a low dose and increase when necessary versus start with a high dose and decrease in case of side-effects - suggests differences in the assessment of the risk of occurrence and consequences of seizures and complaints. Differences in treatment policy were also observed in recent literature (Mattson et al. 1992, Reynolds et al. 1993, Heller et al. 1995).

To be able to cope with such differences - given that consensus on this issue not yet exists systems that provide therapeutic advice should be tuneable to different treatment styles. Whether the dosage schemes for a given treatment style are 'hand-coded' or user-adjustable remains an issue for discussion 


\section{Impact of local preferences}

The cases in the diagnostic reference set were used to assess the prescribed drug.

The system prescribed a drug in accordance to this reference in $75 \%$ of the cases (range neurologists $46 \%$ to $94 \%$ ). Since the system was not involved in the establishment of the reference a bias in favour of the neurologists is present as each neurologist

- was allowed to change his prescription in the Delphi procedure

- only had to agree with one of his two colleagues to define a reference.

In $90 \%$ of the cases there was at least one neurologist that prescribed the same generic drug as the system did.

In 32 of the 39 cases of the treatment reference set the system prescribed the same generic drug as the treatment reference. A reference for the daily dose (defined as a dose prescribed by two neurologists who agreed on the prescribed drug) could be established in a subset of 11 cases. The system agreed in 5 of these cases (46\%). In $62.5 \%$ of these cases the system suggested a daily dose identical to that of at least one of the neurologists. The individual neurologists agreed with the reference daily dose in $75 \%$ to $100 \%$ of the cases.

Differences in doses mainly occurred in cases in which carbamazepine was the reference drug. The neurologists seem to adhere more to a 'middle of the target range' approach, whereas the system prescribed according to a 'minimal therapy' principle. The fact that carbamazepine induces its own catabolism, which may necessitate a dose higher than the initial dose for controlling the seizures, may have been a reason for the higher daily dose prescribed by the neurologists. Another reason for the difference may be due to the risks of dose related complaints after an initial dose of carbamazepine. The balancing of the risks of having another seizure and the occurrence of side-effects may cause variations in the prescribed daily dose. The system does not balance these risks explicitly

\section{Judgemen of quality of prescriptions}

The overall acceptability (as judged by two independent reviewers) of the system's advice was compared with the acceptability of the advice of each of the neurologists. Little difference was observed between the overall acceptability of the prescriptions of the system and those of the prescribing neurologists. The system obtained a lower number of judgements indicating a poor acceptability $(11 \%)$ than the neurologists (range 13\% to $29 \%$ ). On the other hand also the number of prescriptions with a good acceptability (48\%) were fewer than those of most of the neurologists (range $32 \%$ to $63 \%$ ).

\section{Judgement on selected drug and daily dose}

It is apparent from the literature that there exists disagreement about the choice of antiepileptic drugs. Also our study showed disagreement about the appropriateness of the prescribed drug. A 'neutral' or 'poor' overall acceptability judgement was given 157 (49.8\%) times. For these prescriptions judgements were also given with respect to the prescribed drug and the daily dose. In 62 (39.5\%) of these reviews the drug selection was judged 'neutral' or 'inappropriate'. For three of the neurologists more than $50 \%$ of the judgements indicated that an appropriate drug was prescribed (range $54 \%$ to $70 \%$ ). Also the system mainly $(67 \%)$ obtained a positive judgement for the suggested drug. Except for one of the neurologists the drug was considered inappropriate in $17 \%$ to $36 \%$. For the remaining neurologist as well as for the system less than $5 \%$ of the judgements indicated that an inappropriate drug was prescribed. 
The system's choice of a specific drug is therefore less often the cause for a low acceptability of the prescriptions than for four of the neurologists.

The doses to control seizures recommended in the literature vary. The initial doses for carbamazepine ranges from 200 to $800 \mathrm{mg}$ whereas the dose for sodium-valproate ranged from $600 \mathrm{mg}$ to $3000 \mathrm{mg}$ (Sonnen 1979, Shorvon et al. 1980, Aird et al. 1984, Lechtenberg 1985, Shorvon 1987, Rimmer and Richens 1988, Brodie and Dichter 1996). Also in our study differences in the daily doses of the prescriptions of the neurologists were observed. For the individual neurologists the percentage of judgements indicating an inappropriate dose ranged from $53 \%$ to $60 \%$ for three of the neurologists, while for two neurologists more than $85 \%$ of the judgements indicated that the daily dose was inappropriate. For the system in $91 \%$ of these judgements the dose was considered inappropriate. All but one of the judgements indicated that the daily dose was (too) low. Overall there is little difference between the acceptability of the advice of the system and that of the neurologists.

It has been recently suggested that the main anti-epileptic drugs differ little with respect to their effectiveness of controlling seizures (Richens et al. 1994, Heller et al. 1995). Nevertheless, the approach in which carbamazepine and phenytoin are the first choice drugs for partial epilepsy and valproate for generalised epilepsy is favoured in daily practice (Chadwick 1994ab, Shorvon 1995, Brodie and Dichter 1996). This was also observed in our study. As far as the proposed drug is concerned the advice of the system appears to be sound

Although doses of anti-epileptic drugs are not predictive for efficacy (Shorvon 1987, Commission on Anti-epileptic Drugs 1993, Lammers 1994) disagreement about the prescribed daily dose in our study turned out to be the main reason for a low acceptance of prescriptions of the system.

\section{Consensus meeting}

To establish an objective reference, differences among the neurologists were discussed during a consensus meeting. It was concluded that the initial daily dose for carbamazepine and sodiumvalproate as prescribed by the neurologists was rather high. Consensus was reached about the fact that the initial daily dose of carbamazepine for patients with a body weight up to $80 \mathrm{~kg}$ should be $400 \mathrm{mg}$, while patients with a higher body weight should receive $600 \mathrm{mg}$ per day. An appropriate initial dose of sodium-valproate was considered to be 900 to $1000 \mathrm{mg}$ depending on whether two or three intakes per day are used. For the system this means an adjustment of its 'minimal effective dose' with respect to carbamazepine, while the system's sodiumvalproate minimal effective dose agreed with the one proposed by the neurologists.

A relation between the drug dose and the efficacy of the drug has not been found so far (I.ammers, 1994). It has been suggested that the policy to achieve therapeutic plasma levels soon after the onset of treatment may induce side-effects (Reynolds et al. 1993, Heller et al. 1995). The adverse drug effects may contribute significantly to decreased patient compliance (Allen 1995). Increasing attention is paid to quality-of-life issues such as behavioural and cognitive effects and the ability to sustain as normal a life as possible while receiving medication (Pellock 1994). These arguments together with reports from the literature which show that the view with respect to the prognosis of epilepsy is changing from a chronic outcome towards a more benign course with a good chance of achieving a remission, lead to the conclusion that there is little room left for a 'middle of the target' policy in drug treatment of epilepsy. The consensus on the daily dose as achieved in our study is near the lower range of 
the daily doses found in the literature, which suggests that also the neurologists in our study agree most with the treatment policy of 'minimal therapy'.

\section{Comparing the proposed drug with the consensus}

The guidelines from the consensus meeting were used for establishing a consensus meeting reference prescription for each case. Comparing prescriptions of the neurologists and the advice of the system with this consensus meeting reference showed that all neurologists prescribed a drug in accordance with the reference in more than half of the cases (range $52 \%$ to $72 \%$ ). The system reached a consistently better agreement (range $88 \%$ to $91 \%$ ) with the reference drug on the subsets of cases seen by the individual neurologists.

The consensus meeting advised that phenytoin should be used as well. This drug was not recommended by the system as a first choice. The neurologists disagreed more with the consensus meeting reference about the prescription of phenytoin than about the use of carbamazepine and valproate. Only twice (17\%) a neurologist used the drug in accordance with the consensus meeting reference, compared to $68 \%$ for carbamazepine and $69 \%$ for valproate.

\section{Comparing the proposed daily dose with the consensus}

If the group of neurologists prescribed a drug according to the consensus meeting reference, they achieved a better agreement with the reference daily dose than when the system agreed with the drug reference. When valproate is prescribed according to the consensus, little difference between the neurologists and the system was observed ( $88 \%$ vs. $94 \%$ ). For carbamazepine the system suggested only twice (3\%) a daily dose equal to that of the reference, while in $36 \%$ of the prescriptions of the neurologists the dose was in accordance with the consensus meeting reference.

\section{Correctness of the prescribed drug and the daily dose}

All but one of the neurologists provided a prescription - taking also the decision "no drug treatment' into account - according to the consensus (drug and daily dose) in less than $40 \%$ (19\% to $36 \%$ ). The other neurologist agreed with the reference in $56 \%$. The system prescribed according to the consensus reference in $28 \%$ to $48 \%$ of the cases (for the various sets of cases common with the individual neurologists), which was equal to or better than most of the neurologists did

Since the dosage prescribed by the system can be easily corrected to agree with the consensus, it can be concluded that the system is suitable for providing advice regarding the initiation of anti-epileptic drug treatment.

\subsection{Follow-up of treatment}

The second evaluation of the system concerned the adjustment of the treatment based on information obtained in the first follow-up contact. The global decisions of the system (continuation, increase or decrease of the daily dose or change of the prescribed drug) were compared with the decisions taken by five neurologists. Three different analyses were performed. 
First, the agreement between the system and the neurologists was studied on a set of 265 cases. The system had a moderate agreement (as measured with the kappa-statistic) with three individual neurologists on the treatment decision. Only two pairs of neurologists showed such a moderate agreement, all other combinations of neurologists had a lower level of agreement.

The system reached a fair agreement beyond chance with each of the subgroups of four neurologists, whereas the individual neurologists only reach a moderate agreement with their group of peers. Also the system takes less decisions with which the neurologists completely disagree.

In a second analysis a clinical reference was developed, being the majority decision of the five neurologists. A reference treatment decision was obtained in 219 cases (set I). Although this reference was positively biased towards the neurologists as they contributed to the reference, the system reached a correctness of $78.1 \%$ for these cases which was comparable with the average correctness of the neurologists $77.4 \%$ (range $67.1 \%$ to $82.6 \%$ )

To correct for the bias a second reference of 189 cases was developed based on the treatment decisions of the neurologists as well as of the system (set II). In this set the system reached a correctness of $90.5 \%$, whereas the average correctness within the group of neurologists was $81.9 \%$ (range $67.7 \%$ to $87.8 \%$ ). The system achieved a significantly higher correctness than three of the neurologists.

Also for the set of 76 cases for which no reference treatment decision could be obtained, the system proposed fewer treatment decisions (5\%) which were different from the decisions taken by any of the neurologists than any of the individual neurologists did (range $8 \%$ to 19\%).

\section{Criteria for adjustment of the therapy}

Set I was used to investigate whether criteria could be derived that are used by the neurologists for certain treatment decisions. With the help of machine learning techniques a decision tree was constructed. The algorithm tries to build a tree such that the end nodes contain sets of cases with a single therapy. Stop criteria may prevent splits that have insufficient predictive value. Hence, impure end nodes may occur. The internal nodes contain criteria indicating which way to proceed in the tree. The tree had 14 end nodes. Support for a treatment decision was considered to be strong for six nodes. From the criteria in the tree that lead to these nodes the following guidelines for adjustment of the treatment were derived:

- In cases in which seizures are completely controlled and complaints are absent the prescribed treatment is continued. In the literature it is mentioned that an increase of the drug dose was a common decision if drug plasma levels were low (Meijer, 1991). In our study, however, as also has been recommended in the literature (Shorvon, 1995), neurologists did not apply serum levels as rigidly in these cases. Also the system decided to continue the treatment without adjustments for these cases.

- In cases with idiosyncratic complaints a change of the drug is appropriate. For the neurologists as well as for the system the presence of idiosyncratic complaints leads to the use of another drug.

- The drug dose is increased when seizures are hardly controlled (the seizure frequency decreased, or one type of seizures remained the same, while the other type decreased or was controlled) or uncontrolled (the frequency of the seizure type(s) did not change), in combination with absence of complaints, a good compliance and a plasma drug level below or in the therapeutic range. It is stated in the literature that often the dose is 
increased without checking the plasma drug level (Meijer 1991). In our study however, neurologists appear to take the plasma drug level as well as information about complaints and compliance into account to decide whether or not to increase the dose. The system reached the same treatment decision in all except three cases in which the prescribed drug was considered to be second choice. Receni studies indicate that as far as controlling the seizures is concerned little difference is observed in effectiveness of the main anti-epileptic drugs (Richens et al 1994, Heller et al. 1995). Given the risks of drug withdrawal and time loss, once a second choice drug has been initiated it should be given a full trial.

- In cases with uncontrolled major seizures and dose related complaints the prescribed drug is changed. In 21 of the 26 cases the system also decides to change the drug. In all five cases dose related complaints occurred at a (below) therapeutic plasma drug level immediately after initiation of the treatment. These complaints may be transient in contrast with those occurring in combination with a high or toxic range plasma dnug level. A continuation of the treatment was proposed by the system and agreed at by at least one of the neurologists.

- The prescribed drug is changed in cases in which some success is achieved in controlling the seizures (either major seizures decreased while minor seizures were not controlled or major seizures controlled and minor seizures remained equal), dose related complaints are present and the plasma drug level is in the high therapeutic or toxic range. These findings are in accordance with the findings of others who considered an increase of the dose in case of a (near) toxic serum concentration to be a dubious decision (Meijer, 1991). The plasma drug level is used as indication whether toxicity develops and additional anticonvulsive action is small (Shorvon, 1995). The system decided to change the drug in all eight cases.

- The drug treatment is continued in cases in which the seizure type(s) decreased or in which one type of seizures was controlled while the other remained equal, with noncompliance and a plasma drug level in the high therapeutic or toxic range

For $39.3 \%$ of the cases in set I criteria leading to a single therapy could not be obtained. The reason could be the way in which the cases were described and analysed, or the existence of alternative treatments. The first reason can largely be rejected as a detailed analysis did not show any discriminative factors not taken into account in the analysis. There is some evidenci that alternative treatments may exist for some subgroups. For some groups of cases a single reference treatment does not seem to exist, although in individual cases a reference treatment was defined by the neurologists

\subsection{General conclusion and future prospects}

The question whether it is possible to provide adequate advice for guiding anti-epileptic drug treatment by means of a computerised model can be answered in the affirmative

1. For the initiation of the therapy and the monitoring of the treatment, the agreement of the system with individual neurologists was in general at least as good as the agreement among the neurologists

2. For the monitoring of the treatment the correctness of the system on a clinical reference was as good as the correctness of the neurologists even though the system was not involved in establishing the reference while the neurologists were.

It was possible to elicit the necessary knowledge from an expert neurologist. 
With respect to the question whether the system suggested an adequate anti-epileptic drug it was concluded that the system proposed an adequate anti-epileptic drug when initiating drug treatment. When comparing the overall acceptability (as judged by two independent reviewers) of the prescriptions there was little difference between the neurologists and the system. The neutral or poor quality overall judgement was in the case of the system due to a (too) low prescribed dose.

It was apparent that even after a two-staged Delphi procedure there existed a relatively large inter-individual variability between the neurologists. A similar variability was observed among the reviewers. Because of this variability in opinions with respect to the way how to initiate the therapy a consensus meeting was organised. When assessing the correctness of the prescriptions (in relation to the reference obtained in the consensus meeting) the system's proposals for initiating anti-epileptic drug treatment were better than those of most of the individual neurologists.

With respect to the follow-up the system appears to be as good as the neurologists in taking treatment decisions. There is a moderate agreement between the treatment proposals of individual neurologists and the system. The system had a consistently better agreement with a group of four neurologists than each other neurologist individually. Comparison of the system's prescriptions with a reference defined by the group of neurologists (clinical reference) showed that the system reached a correctness comparable to that of the individual neurologists Compared with the reference based on the decisions of the neurologist as well as the system, the system reached a better correctness than three of the neurologists.

As far as the criteria for adjustment of the treatment are concerned, six guidelines for adjustment were identified. For each of these guidelines the system seems to apply the same treatment decisions.

The described system is an implementation of the elicited knowledge on anti-epileptic drug treatment rather than a prototype of a system that can be used in clinical practice. It lacks the interface with a clinical information system as well as a user intertace that will meet the requirements of the clinician. Also relevant functionalities for clinical use are lacking. One of the aspects which will require further development is the explanation facility. Although a trace of the rules used for obtaining an advice may be provided, they have to be reworded to enable interpretation of the reasoning process of the system by a neurologist.

Despite these shortcomings our studies indicate that the developed models are fit for integration with other software such as electronic medical records or clinical information systems. The model also would be eminently suited for incorporation into an interactive teaching system for neurological residents. The cases used in the consensus meeting could also be used for this purpose.

Because of the observed inter-individual variability it is advisable to further develop the system such that it can be installed in a neurologist's office initially for testing purposes. In these circumstances the reasons for deviating from the system's advice should be analysed. In this way information can be obtained that will assist in the further development of guidelines for treatment of epilepsy. At the same time such guidelines become available in computerised form to ease their application for individual patients. Affer the system is released for use and knowledge with respect to the treatment is maintained the system can be used by the neurologists for a second opinion. 
Also the system could be extended to incorporate the diagnostic process. It can be expected that future changes in the diagnostic criteria will be less than in the past. The current criteria have been reviewed and established over the years and are generally accepted.

An organisation should volunteer to organise regular consensus meetings in order to keep diagnosis and treatment protocols up to date. This knowledge could be stored in a knowledge base and be distributed among the neurologists.

\subsection{References}

Aird R.B., Masland R.L., Woodbury D.M., The epilepsies a critical review, Raven Press, New York, 1984.

Allen F.H., Entering a new drug era: practical issues in the treatment of epilepsy, Neurology, 45, 1995, (Suppl 2), s5-s6.

Brodie M.J., Dichter M.A., Antiepileptic drugs. The New England Journal of Medicine, 334, 1996, pp.168-175.

Chadwick D., Epilepsy, Journal of Neurology, Neurosurgery and Psychiatry, 57, 1994a, pp.264-277.

Chadwick D., Standard approach to anticpileptic drug treatment in the United Kingdom, Epilepsia, 35 (Suppl.4), 1994b, s3-s 10.

Chadwick D., Tumbull D.M., The comparative efficacy of antiepileptic drugs for partial and tonicclonic seizures, Journal of Neurology, Neurosurgery and Psychiairy, 48, 1985, pp. 1073-1077.

Commission on Antiepileptic Drugs of the International League Against Epilepsy, Guidelines for therapeutic monitoring on antiepileptic drugs, Epilepsia, 34, 4, 1993, pp 585-587.

Dreifuss F.E., Risk benefit analysis in the management of the child with cpilepsy. In: Modern approach 10 antiepileptic drug treatment, improving quality of life, (Dam, Gram, Pcdersen and orum, Eds.), The Danish Epilepsy Society, Kobenhavn, 1985.

Dreifuss F.E, The epilepsies: clinical implications of the international classification, Epilepsia, 31 , (Suppl. 3), 1990, s3-s10.

Gram L., Drachmann Bentsen K., Parnas J., Flachs H., Controlled trials in epilepsy: a review, Epilepsia, 23. 1982, pp. 49I-519.

Heller A.J., Chesteman P., Elwes R.D., Crawford P., Chadwick D., Johnson A.L., Reynolds E.H., Phenobarbitone, phenytoin, carbamazepine, or sodium valproatc for newly diagnosed adult epilcpsy: a randomised comparative monotherapy trial, Journal of Neurology. Neurosurgery and Psychiary, 38,1995 , pp. 44-50.

Hicknan F.R., Killin J.L, Land L., Mulhall T., Porter D., Taylor R.M., Analysis for knowledge-based systems, a practical guide to the KADS methodology, Ellis Horwood Limited, Chichester, 1989.

Lammers M.W., Clinimetrics in epileprology, thesis, Nijmegen, 1994.

Lechtenberg R., The diagnosis and ireatment of epilepsy, Macmillan Publishing Company, New York, 1985.

Mattson R.H., Cramer J.A., Collins J.F., VA Epilepsy Cooperative Study Group, Aspects of compliance; taking drugs and keeping clinic appointments. In: Compliance in Epilepsy (Schmidt and Leppik, Eds.), Elsevier Science Publishers B.V., Amsterdam, 1988, pp. III-117.

Mattson R.H., Cramer J A. Collins J.F., and the Dept. of Veterans Affairs Epilepsy Cooperative Study No 264 Group, A comparison of valproate with carbamazepine for the treatment of complex partial seizures and secondarily generalised tonic-clonic seizures in adults, The New England Journal of Medicine, 327, 1992, pp. 765-771 
Meijer J.W.A., Knowledge, attitude and practice in antiepileptic drug monitoring, thesis, Nijmegen, 1991

Pellock J.M., Standard approach to antiepileptic drug treatment in the United States, Epilepsia, 35(Suppl.4), 1994, s11-s 18.

Porter R.J., Epilepsy, 100 elementary principles, W.B. Saunders Company Ltd., London, 1984.

Principe J.C., Smith J.R., Microcomputer-based system for the detection and quantification of petit mal epilepsy, Computers in Biology and Medicine, 1982, 12, pp. 87-95

Readdie M., Streng K.H., Wermser D, KADS metrication, Deliverable G10. SD-G10-R-001, ESPRT P1098, SD Europe Ltd \& NeuTech Entwicklungsgesellschaft mbH \& Co.KG, 1989.

Reynolds E.H., Heller A.J., Chadwick D., Valproate versus carbamazepine for seizures, The New England Journal of Medicine, 328, 1993, pp. 207-208.

Richens A., Davidson D.L.W., Cartlidge N.E.F., Easter D.J., A multicentre comparitive trial of sodium valproate and carbamazepine in adult onset epilepsy. Journal of Neurology. Neurosurgery and Psychiatry, 1994, 57, pp. 682-687.

Rimmer E.M., Richens A., Clinical phamacology and medical treatment. In: A textbook of epilepsy, (Laidlaw, Richens and Oxley, Eds.), $3^{\text {rd }}$ edition, Churchill Livingstone, Edinburgh, 1988, pp. 421483.

Scheiber G., Wielinga B., Breuker J., KADS a principled approach to KBSS development, Academic Press, London, 1993.

Shorvon S.D., Galbraith A.W.. Laundy M., Vydelingum L., Reynolds E.H., Monotherapy for epilepsy. In: Antiepileptic therapy: advances in drug monitoring. (Johannessen, Pippenger, Schmidt, Morselli, Richens and Meinardi, Eds.). Raven Press, New York, 1980, pp. 213-220.

Shorvon S.D., The treatment of epilepsy by drugs. In: Epilepsy (Hopkins, Ed.), Chapman and Hall, London, 1987, pp. 229-282.

Shorvon S., The drug treatment of epilepsy. In: Epilepsy, (Hopkins, Shorvon and Cascino, Eds.), $2^{\text {nd }}$ edition, Chapman and Hall Medical, London, 1995.

Sonnen A.E.H., De medicamenteuze behandeling van epilepsie, Nedcrlands Tijdschrift voor de Geneeskunde, 123, 1979, pp. 485-490.

Tansley D.S.W., Hayball C.C., Knowledge-based systems analysis and design, a KADS developer's handbook, Prentice Hall, New York, 1993.

Ward C.D., Decision making in refractory epilepsy: an analysis based on computer simulation, Journal of Neurology: Neurosurgery and Psychiatry, 1989, 49, pp. 781-784. 


\section{Summary}

Given the ageing of the population and the expected growth in the number of patients with multiple problems, an increase can be expected in the amount of information that has to be processed when dealing with an individual patient. Simultaneously there is a pressure to practice medicine in a cost-effective way. The clinician who wants to do the best for his patient must try to maximise the expected utility of his actions. The expectations of patients with respect to the capabilities of medicine complicate this process as conflicts may arise between the requests of individual patients and societal needs. Physicians need tools to assist them in mastering the growing amount of increasingly more complex medical knowledge to assure optimal care. Information technology may assist the physician with clinical decision making in daily practice to achieve best medical practice. Also in the domain of epilepsy - a disorder of the brain which manifests itself through brief usually unprovoked stereotypical disturbances of behaviour, emotion, motor function or sensation - supporting the doctor by a 'computer fed by a team of specialists' was considered as an option to improve patient management.

In this thesis the development of a computerised model of the expert knowledge used for prescribing anti-epileptic drugs is described. It was studied whether such a model can provide advice of a good quality. The advice concerns:

1. the initiation of anti-epileptic drug treatment after the diagnosis has been established and

2. the adjustment of the drug treatment after assessment of its effectiveness.

\subsection{The domain of epilepsy}

In chapter 2 a review of the literature with respect to diagnosis and treatment of epilepsy is presented. Problems discussed are related to

- the definition of epilepsy which can be considered as a framework rather than a fixed set of criteria for diagnostic interpretation of symptoms.

- the presence of different approaches for deciding on drug treatment.

- the disagreement on therapeutic ranges of drugs and the usage of plasma drug levels

- the psychological and social consequences of the long-term character of the treatment procedures. 
Although guidelines with respect to diagnosis and treatment of epilepsy are available the actual context for their application is usually missing. Guidelines to enhance the decision making process of the physician in managing a specific patient could therefore be of value.

\subsection{Building a model of expertise}

The KADS structured approach for knowledge based systems development was used. Two inference models describing the initiation and the monitoring of the treatment in patients with epilepsy were developed (Chapter 3 )

At the time we used KADS two main problems were encountered:

- KADS orientation towards the analysis of the cognitive process instead of the implementation of a model of expertise.

- the lack of support for the evaluation of the quality of the models.

Over the years the orientation of KADS has shifted from the analysis of cognitive processes towards a more pragmatic approach for implementation of models. Some work on metrication has also taken place. This work, however, is mainly directed towards project management. Little has been done with respect to the evaluation of models. Integrating KADS with an evaluation methodology could enhance user acceptance of a decision support system.

\subsection{Reference development}

The evaluation of computerised models is a difficult but essential part of the development of a decision support system. Several methodologies have been proposed in the literature. Most methodologies consider validation of a decision support system as the most important part of the evaluation process. The assessment of the correctness of the output of the system is the main issue in this phase. An important problem in the evaluation of decision support systems is the development of a reference for assessment of the system's advice. As described in chapter 2 an objectively correct treatment for an individual patient is not available. This means that a reference for evaluating the advice of the system had to be developed by a group of neurologists.

\section{Reference for the initiation of treatment}

For the initiation of the treatment sixty paper cases were constructed describing the clinical presentation of a patient's first two or three visits (Chapter 4). For each case three neurologists were asked to provide a prescription. By means of a two step Delphi-like approach addressing the diagnosis and the prescribed drug, the variability in prescribing could be reduced as far as the drug was concerned. The feedback round on the daily dose was less successful in reducing the variance in dosage.

Two reference sets were developed, a diagnostic reference set for the assessment of the prescribed drug and a treatment reference set for the assessment of the daily dose.

Because a treatment not equal to that provided by the majority may still be considered an acceptable treatment, the acceptability of the prescriptions was assessed by two independent neurologists after the Delphi round.

The variability among the neurologists made it difficult to obtain a set of cases for which the neurologists agreed about the diagnosis as well as about the treatment. Therefore a consensus 
meeting was organised to obtain more objective decision criteria. For the cases of the diagnostic reference set a consensus reference treatment was obtained on the basis of the guidelines defined in the meeting. The consensus meeting led to a better agreement among the neurologists with respect to the use of phenytoin.

\section{Reference for the follow-up of treatment}

The assessment of the advice in a first follow-up contact also required the use of a majority decision of a group of neurologists as no objective standard existed. Two reference sets were obtained (Chapter 6). A clinical reference consisted of cases about which the majority of the neurologists agreed. A second reference consisted of cases about which a majority decision of both the neurologists and the system could be obtained.

In addition to this more global approach we identified case characteristics, using machine learning techniques, which the neurologists may have used when deciding about the adjustment of the treatment.

\subsection{Evaluation of the initiation of the treatment}

Comparing the system's advice for cases in the diagnostic reference set with the prescriptions of the neurologist showed that the system prescribed an anti-epileptic drug in accordance with the reference as least as often as an individual neurologist (Chapter 5).

The system prescribed a daily dose in accordance to the reference for sodium-valproate. For carbamazepine the system predominantly prescribed a dose lower than the neurologists did. A difference in treatment approach apparently existed. While the neurologists adhered more to a 'middle of the target' approach the system prescribed according to the 'minimal therapy' principle which means that initially a low dose is used which is increased if seizures do not remit.

The acceptability analysis showed little difference in overall acceptability between the prescriptions of the system and the neurologists. The system received a lower number of judgements indicating a poor acceptability than the individual neurologists. On the other hand the number of prescriptions for which a good acceptability was observed was lower than those of most of the neurologists. The system suggested more often an acceptable drug than most of the neurologists. If the acceptance of the suggested prescription of the system was judged low this was mainly due to a too low daily dose.

This evaluation of the system's advice for the reference cases and the assessment of the acceptability provided an indication of the performance of the system but the variability in prescribing among the neurologists did not enable us to make a firm statement about the system's correctness. To reduce the variability a consensus meeting was organised. It appeared that the daily dose initially prescribed by the neurologists was rather high

The system as well as the group of neurologists for sodium-valproate mainly prescribed a daily dose in accordance with the consensus guidelines. For carbamazepine the system prescribed less often a daily dose in accordance with the consensus guidelines than the group of neurologists.

Except for one of the neurologists who prescribed according to the consensus in more than half of the cases, the system suggested an equal or higher number of prescriptions (drug and daily dose) according to the consensus than the other neurologists did. 


\subsection{Evaluation of the follow-up of the treatment}

To assess whether the system takes adequate decisions during monitoring the drug treatment, the global treatment decisions of the system were compared with the decisions of the five neurologists.

The system reached a moderate agreement on the treatment decision - as expressed with the $\kappa$-statistic - with three of the individual neurologists on the treatment decision. Only twice a pair of neurologists showed a moderate agreement, while the other combinations of two neurologists had a lower level of agreement. This shows that the system agreed better with each individual neurologist than any of the neurologist with their colleagues.

Comparing the agreement between the system and a group of four neurologists with that of an individual neurologist with the same four neurologists showed that the system reached a fair agreement, while each of the individual neurologists moderately agreed with their four colleagues.

A clinical reference was developed, based on the treatment decisions of the majority of the five neurologists. For 219 out of 265 cases such a majority decision could be obtained. The system reached a correctness comparable to that of the individual neurologist on this set of cases. This reference was positively biased towards the neurologists, as they contributed to the definition of the reference treatment decision while the system did not. Including the system's treatment decision in the reference creation process led to a subset of 189 cases in which a majority decision could be obtained. Within this subset the system reached a higher correctness than the neurologists. The analysis of the set of 76 cases for which no reference treatment could be obtained showed that the system suggested fewer treatment decisions different from the decisions taken by any of the neurologists, than each of the individual neurologists did.

Case characteristics may be expected to be important for reaching specific treatment decisions. To obtain insight in the decision making process of the group of neurologists a decision tree was constructed with the help of machine learning techniques. The tree consists of branches which describe a path in theory leading to one specific treatment. Each path may use different case characteristics. An end-node presents the number of cases for each different treatment that fulfil the criteria belonging to the specific branch.

An assessment of the criteria for adjustment of the therapy showed that for six branches the end-nodes contained cases of predominantly one treatment. These criteria were taken as guidelines for follow-up of the treatment. The system uses the same treatment decisions for cases described by each of these guidelines

\subsection{General conclusion}

The question whether a computerised model based on expert knowledge can provide quality advice on anti-epileptic drug treatment can be answered in the affirmative. Comparing the system with the diagnostic reference and the consensus reference showed that it provides a correct advice for initiating anti-epileptic drug treatment. Poor acceptability of an advice could be attributed to a low dose. This part of the knowledge base can easily be corrected.

Also for the adjustment of the initiated treatment the system proposed treatment decisions which were in good agreement with those of the individual neurologists as well as with those 
of a group of neurologists. Because of the detected inter-individual differences it is advisable to further develop the system (including a user-friendly user interface) and install it for testing in a neurologist's office to analyse reasons for deviations from the system's advice. When the knowledge base is maintained over time the system's advice can be used as a second opinion or for educational purposes 


\section{Samenvatting}

Door de stijgende leeftijd van de bevolking en de verwachte groei van het aantal patienten met meer dan één gezondheidsprobleem, kan men een toename verwachten van de hoeveelheid informatie die verwerkt moet worden bij het behandelen van een individuele patient. Gelijktijdig wordt er druk uitgeoefend om zo kosten-effectief mogelijk te werken in de gezondheidszorg. De clinicus die het beste voor zijn patient wil bereiken moet trachten de verwachte opbrengst van zijn activiteiten te maximaliseren. De verwachtingen van patienten ten aanzien van de mogelijkheden van de geneeskunde bemoeilijken dit proces doordat er conflicten kunnen optreden tussen wensen van de individuele patient en het maatschappelijke belang. Artsen hebben behoefte aan methoden die helpen bij het verwerken van de groeiende hoeveelheid van steeds complexere medische kennis om optimale zorg te waarborgen. Informatietechnologie kan de arts bij zijn dagelijkse klinische besluitvorming helpen optimale zorg te verlenen. Ook op het gebied van epilepsie - een aandoening van de hersenen welke zich manifesteert door korte, meestal niet geprovoceerde stereotypische verstoringen van gedrag, emotie, motoriek of gevoel - werd ondersteuning van de arts door 'een computer gevoed door een team van specialisten' gezien als mogelijkheid om het beleid met betrekking tot de patient te verbeteren.

In dit proefschrift wordt de ontwikkeling van een geautomatiseerd model van de kennis van een expert met betrekking tot het voorschrijven van anti-epileptische medicatie beschreven. $\mathrm{Er}$ werd onderzocht of een dergelijk model een kwalitatief goed advies kan geven. Dit advies betreft:

1. het starten van de behandeling met anti-epileptica nadat de diagnose gesteld is en

2. de aanpassing van de medicamenteuze behandeling na beoordeling van de effectiviteit.

\section{Epilepsie}

In hoofdstuk 2 wordt een overzicht van de literatuur met betrekking tot diagnostick en behandeling van epilepsie gepresenteerd. Verschillende problemen worden besproken:

- de definitie van epilepsie, die eerder beschouwd kan worden als een raamwerk in plaats van een vastgestelde set van criteria voor diagnostische interpretatie van symptomen

- de verschillende benaderingen met betrekking tot beslissingen over de medicamenteuze behandeling

- het verschil in mening betreffende de therapeutische range van de medicamenten en het gebruik van plasmaspiegels bij de instelling en bewaking van de therapie

- de psychische en sociale consequenties van het lange termijn karakter van de behandelingsprocedure

Ofschoon richtlijnen voor diagnostiek en behandeling van epilepsie beschikbaar zijn, ontbreekt hierin meestal de context voor de toepassing ervan. Ondersteuning van het besluitvormingsproces van de arts middels uitgebreidere richtlijnen betreffende de behandeling van de specifieke patient kunnen van waarde zijn. 


\section{Het bouwen van een kennismodel}

KADS, een gestructureerde benadering voor de ontwikkeling van kennissystemen, werd gebruikt om de kennis over de behandeling van epilepsie te modelleren. Twee inferentie modellen - een voor de initiatie en een voor het bewaken van de medicamenteuze behandeling van patienten met epilepsie - werden ontwikkeld.

Bij het gebruik van KADS werden twee belangrijke problemen geconstateerd:

- de gerichtheid van KADS op het analyseren van het cognitieve proces in plaats van op de implementatie van een kennismodel.

- het ontbreken van ondersteuning voor het evalueren van de kwaliteit van de modellen.

Door de jaren heen is de nadruk van KADS op de analyse van cognitieve processen steeds meer verschoven in de richting van een meer pragmatische benadering voor implementatie van modellen. Verschillende metrieken zijn ontwikkeld. Dit werk was voornamelijk gericht op projectmanagement. Met betrekking tot de evaluatie van modellen heeft weinig onderzoek plaatsgevonden. Integratie van KADS met een evaluatiemethode kan de acceptatie van een besluitvormingondersteunend systeem door de gebruiker verbeteren

\section{Ontwikkeling van referentie sets}

De evaluatie van een implementatie van een kennismodel is een moeilijk maar essentieel onderdeel van de ontwikkeling van een besluitvormingondersteunend systeem. Verschillende methoden zijn beschreven in de literatuur. De meeste methoden beschouwen validatie van het besluitvormingondersteunend systeem als het belangrijkste onderdeel van het evaluatieproces. De beoordeling van de correctheid van de output van het systeem is hierbij het belangrijkste onderwerp. Voor de evaluatie van een besluitvormingondersteunend systeem is de ontwikkeling van een referentie, waarmee het advies van het systeem kan worden beoordeeld, belangrijk. Zoals beschreven in hoofdstuk 2 zijn er geen objectieve criteria voor correcte behandeling van een individuele patient beschikbaar. Dit betekent dat een referentie voor de evaluatie van het systeem ontwikkeld moet worden door een groep van op dit gebied deskundige neurologen

\section{Referentie voor het initieren van de behandeling}

Voor het initierren van de behandeling werden zestig papieren casus ontwikkeld, die elk een klinische presentatie van de eerste twee of drie poliklinische bezoeken van de patient beschrijven (hoofdstuk 4). Voor elk van deze casus werden drie neurologen verzocht een recept te schrijven. Met behulp van een twee stappen Delphi-achtige benadering, die zowel de diagnose als het voorgeschreven medicament betrof, kon de variabiliteit in voorschrijven van het soort medicament worden beperkt. De feedbackronde voor de dagelijkse dosering was minder succesvol in het reduceren van de variabiliteit

Twee referentie sets werden ontwikkeld, een diagnostiek referentie set voor de beoordeling van het voorgeschreven medicament en een therapeutische referentie set die gebruikt werd voor de beoordeling van de dagelijkse dosering.

Omdat een behandeling die niet in overeenstemming is met die voorgeschreven door de meerderheid nog steeds acceptabel kan zijn, werd na de Delphi ronde de aanvaardbaarheid van de recepten beoordeeld door twee onafhankelijke neurologen 
De variabiliteit onder de neurologen maakte het moeilijk een set van casus te verkrijgen met volledige overeenstemming op zowel de diagnose als de behandeling. Daarom werd een consensus bijeenkomst georganiseerd om een objectief criterium te verkrijgen. Voor de casus uit de diagnostiek referentie set werd een consensus referentie behandeling opgesteld op basis van de richtlijnen van de consensus bijeenkomst. De consensus bijeenkomst leidde tot een betere overeenstemming onder de neurologen met betrekking tot het gebruik van met name phenytoine.

\section{Referentie voor de follow-up van de behandeling}

Voor de beoordeling van het advies in een eerste follow-up contact moest een meerderheidsbeslissing van een groep van neurologen gebruikt worden aangezien hiervoor geen objectieve standaard bestaat. Twee referentie sets werden verkregen (hoofdstuk 6). Een klinische referentie set bestaande uit casus waarbij de meerderheid van de neurologen het eens was en een tweede referentie set bestaand uit casus waarbij een meerderheidsbeslissing verkregen werd op basis van zowel de uitspraken van de neurologen en de adviezen van het systeem Verder identificeerden we karakteristieken van de casus, middels machinaal leren, die de neurologen gebruikt kunnen hebben bij het beslissen over het aanpassen van de behandeling.

\section{Evaluatie van de initiatie van de behandeling}

Een vergelijking van de adviezen van het systeem, voor de casus uit de diagnostiek referentie set, met de recepten van de neurologen toonde aan dat het systeem ten minste net zo vaak als een individuele neuroloog anti-epileptica voorschrijft in overeenstemming met de referentie (hoofdstuk 5).

Het systeem adviseert voor natrium-valproaat een dagelijkse dosering in overeenstemming met de referentie. Voor carbamazepine suggereert het systeem vournamelijk een lagere dosering dan de neurologen. Klaarblijkelijk bestond er een verschil in benadering bij de behandeling. Terwijl de neurologen meer aansluiten bij een 'middle of the target' aanpak, adviseerde het systeem meer volgens het 'minimale therapie' principe, hetgeen betekent dat bij aanvang een lage dosering van het medicament wordt geadviseerd die verhoogd wordt indien de toevallen niet afnemen.

De analyse van de aanvaardbaarheid van de medicamenteuze adviezen toonde op globaal niveau weinig verschil in acceptatie tussen de recepten van het systeem en die van de neurologen. Het systeem had een kleiner aantal oordelen met een lage graad van acceptatie dan de individuele neurologen. Echter het aantal recepten met een hoge acceptatie was lager dan dat van de meeste neurologen. Het systeem adviseerde vaker een acceptabel medicament dan de meeste neurologen. Indien de acceptatiegraad van het door het systeem geadviseerde recept laag was werd dit meestal veroorzaakt door een te lage dagelijkse dosering

De evaluatie van de adviezen van het systeem voor de referentie casus en de beoordeling van de aanvaardbaarheid gaven een indruk van de prestatie van het systeem. De variabiliteit onder de neurologen maakte het echter moeilijk een harde uitspraak te doen over de correctheid van het systeem. Om de variabiliteit te verminderen werd een consensus bijeenkomst gehouden. Hierbij kwam naar voren dat de dagelijkse dosering die initieel door de neurologen werd voorgeschreven, vrij hoog was. 
Zowel het systeem als de groep van neurologen schreven voor natrium-valproaat meestal een dagelijkse dosering voor in overeenstemming met de consensus richtlijn. Voor carbamazepine adviseerde het systeem minder vaak een dagelijkse dosering in overeenstemming met de consensus richtlijnen dan de groep neurologen

Het systeem suggereerde een gelijk of hoger aantal recepten (medicament en dagelijkse dosering) in overeenstemming met de consensus dan vrijwel alle neurologen. Slechts een neuroloog schreef in meer dan de helft van de casus een recept in overeenstemming met de consensus voor en was hierbij beter dan het systeem.

\section{Evaluatie van de follow-up van de behandeling}

Om te beoordelen of het systeem adequate beslissingen neemt tijdens het bewaken van de medicamenteuze behandeling, werden de globale beslissingen betreffende de behandeling zoals voorgeschreven door het systeem vergeleken met de beslissingen van de vijf neurologen.

Het systeem bereikte een matige overeenstemming met betrekking tot het behandelingsbesluit - uitgedrukt in de $\mathrm{k}$-waarde - met drie van de individuele neurologen. Slechts twee paren van neurologen hadden een matige overeenstemming; de overige combinaties van twee neurologen hadden een lagere graad van overeenstemming. Het systeem stemde dus betere overeen met elk van de individuele neurologen dan elk van de neurologen met zijn collega's

De vergelijking van de overeenstemming tussen het systeem en een groep van vier neurologen met de overeenstemming tussen een individuele neuroloog en dezelfde groep van vier neurologen, wees uit dat het systeem een vrij goede overeenstemming bereikte, terwijl elk van de individuele neurologen matig overeenstemde met zijn vier collega's

Een klinische referentie werd ontwikkeld, gebaseerd op de behandelingsbesluiten van de meerderheid van de vijf neurologen. Voor 219 van de 265 casus kon een dergelijke meerderheidsbeslissing verkregen worden. Op deze set van casus bereikte het systeem een correctheid vergelijkbaar met die van de individuele neuroloog. Deze referentie heeft echter een bias ten gunste van de neurologen doordat deze laatsten bijdragen aan de definitie van de referentie voor de behandeling, terwijl het systeem dit niet deed. Door het advies van het systeem te betrekken bij de definitie van de referentie behandeling werd voor een subset van 189 casus een meerderheidsoordeel verkregen. Binnen deze subset van casus bereikte het systeem een betere correctheid dan de neurologen. De analyse van de 76 casus waarbij geen referentie verkregen kon worden, toonde aan dat het systeem minder vaak dan elk van de individuele neurologen een behandelingsbesluit adviseerde dat door geen van de neurologen was voorgesteld.

Karakteristieken van een casus kunnen belangrijk zijn voor het bereiken van specifieke behandelingsbesluiten. Om meer inzicht te krijgen in het besluitvormingsproces van de groep van neurologen werd een beslisboom geconstrueerd met behulp van machinaal leren. De boom bestaat uit paden die elk leiden tot een eindknoop waaraan in principe één specifieke behandeling toegewezen kan worden. Elk pad gebruikt verschillende karakteristieken van een casus. Een eindknoop geeft het aantal casus weer voor elk van de verschillende behandelingsbesluiten, dat aan de criteria voldoet behorende bij het specifieke pad, dat naar deze eindknoop leidt. 
Een analyse van de criteria voor aanpassing van de therapie toonde aan dat bij zes paden de eindknopen casus bevatten met voornamelijk één behandelingsbesluit. Deze criteria werden gebruikt als richtlijnen voor de follow-up van de behandeling. Voor elk van deze richtlijnen adviseer het systeem dezelfde behandelingsbesluiten als de groep neurologen

\section{Algemene conclusies}

De vraag of een geautomatiseerd model gebaseerd op kennis van een expert een kwalitatief goed advies voor de medicamenteuze behandeling van epilepsie kan geven kan bevestigend beantwoord worden. Een vergelijking van de adviezen met de diagnostiek referentie en de consensus referentie toonde aan dat het systeem een correct advies geeft voor het initiëren van de behandeling met anti-epileptica. Een lage graad van aanvaardbaarheid van een advies kon worden toegeschreven aan een (te) lage dagelijkse dosering. Dit deel van het kennisbestand kan gemakkelijk aangepast worden.

Ook voor de aanpassing van de geïnitieerde behandeling stelde het systeem behandelingsbesluiten voor die een goede overeenstemming hadden met zowel de individuele neurologen als groepen van neurologen.

Vanwege de waargenomen inter-individuele verschillen is het raadzaam het systeem verder te ontwikkelen (inclusief een gebruikersvriendelijke interface) en het voor verdere tests te installeren in een neurologische polikliniek, om zo de redenen voor het niet volgen van het advies van het systeem te kunnen analyseren. Als het kennisbestand van het systeem onderhouden wordt, kan het gebruikt worden voor een second opinion, of voor onderwijsdoeleinden. 


\section{Tot slot}

Het schrijven van een proefschrift is niet mogelijk zonder de hulp en de steun van anderen. Dit is reeds vele malen geschreven in het dankwoord van een proefschrift. Ook bij het schrijven van dit proefschrift werd het wederom proefondervindelijk vastgesteld. Daarom wil ik hier enkele mensen speciaal bedanken die voor mij van groot belang zijn geweest in de afgelopen jaren.

Wijlen Prof.dr.van der Lugt voor zijn bereidwilligheid tot een reflecterende kijk op het medisch denken en handelen en het stimuleren van een idee van een tweede jaars student geneeskunde.

Prof.dr.ir.Hasman: Beste Arie, het idee medische kennis te standaardiseren vond bij jou gehoor. Bedankt voor je interesse en de deskundige begeleiding met name in de laatste fase van het proefschrift.

Prof.dr.Meinardi: Beste Harry, je interesse, je praktische kijk, je gevoel voor humor en je inzet hebben me vaak verwonderd. Bedankt voor je stimulerende invloed.

Prof dr.Troost: Beste Jaap, bedankt voor je positieve commentaar en de duwtjes in de goede richting

Dr.ir. Talmon: Beste Jan, een speciaal woord van dank geldt jou. Je enorme energie en je flexibiliteit zijn essentieel voor een promovendus.

Drs. Groothuis en drs. Tange: Beste Siebren en Huibert, gelukkig was er ook nog iets anders dan promoveren. Ik wens jullie alle twee veel succes met jullie promotie.

Drs.Kubben, internist. Beste Frank, je belangstelling en vertrouwen is voor menig mens een verademing. Ik wens je veel succes met je promotie.

Ir.Stallenberg: Beste Gerard, je nuchtere kijk op zaken en je gevoel voor humor worden zeer op prijs gesteld. Onze wandelingen waren een welkome afwisseling. Arigato.

Verder wil ik de neurologen die aan het onderzoek hebben meegewerkt bedanken voor het beschikbaar stellen van hun tijd en hun bereidwilligheid om op een andere manier naar medisch handelen te kijken. Dr.H.Schouten en dr. A.van Kesteren wil ik bedanken voor hun statistische adviezen

Dat de laatste loodjes het zwaarst wegen heefl naast enkele goede vrienden met name de fam. moeten ervaren. Mijn dank gaat dan ook uit naar hen wier steun ik heb ontvangen, in het bijzonder naar mijn moeder, voor al haar goede zorgen, mijn vader, voor al zijn inspanningen, en Marjo, Desirée en Vincent voor hun warme belangstelling.

Tot slot wil ik Eline, mijn vrouw, bedanken voor haar steun en bereidheid mijn werk te laten prevaleren boven andere belangrijke zaken. Dankzij jouw laatste zetjes is het gelukt. Het is af. 
